\title{
Optimization principles and constraints shaping visual cortical architecture
}

\author{
Dissertation \\ (Cumulative Dissertation) \\ for the award of the degree \\ "Doctor rerum naturalium" \\ Division of Mathematics and Natural Sciences \\ of the Georg-August-Universität Göttingen
}

submitted by

Wolfgang Keil

from Karl-Marx-Stadt (Chemnitz)

Göttingen 2012 


\section{Examination committee}

Prof. Dr. Fred Wolf

(Thesis supervisor and 1st reviewer)

Department of Nonlinear Dynamics, Max Planck Institute (MPI) for Dynamics and Self-Organization, Göttingen

Bernstein Center for Computational Neuroscience, Göttingen

Prof. Dr. Theo Geisel

(2nd reviewer)

Department of Nonlinear Dynamics, MPI for Dynamics and Self-Organization, Göttingen

Institute for Nonlinear Dynamics, Georg August University, Göttingen

Bernstein Center for Computational Neuroscience, Göttingen

Prof. Dr. Eberhard Bodenschatz

Department of Fluid Dynamics, Pattern Formation and Nanobiocomplexity,

MPI for Dynamics and Self-Organization, Göttingen

Dr. Alexander Gail

German Primate Center, Göttingen

Bernstein Center for Computational Neuroscience, Göttingen

Prof. Dr. Matthias Kaschube

Frankfurt Institute for Advanced Studies, Frankfurt

Johann Wolfgang Goethe University, Frankfurt

Prof. Dr. Ulrich Parlitz

Department of Fluid Dynamics, Pattern Formation and Nanobiocomplexity, MPI for Dynamics and Self-Organization, Göttingen

Date of the oral examination: 24th April 2012 


\section{Statement of originality}

I hereby ensure that this dissertation entitled "Optimization principles and constraints shaping visual cortical architecture" has been written independently and with no other sources and aids than quoted.

Göttingen, 8th March 2012

Wolfgang Keil 


\begin{abstract}
Neurons in the mammalian primary visual cortex (V1) are often preferentially driven by only one of the two eyes and respond best to oriented bar stimuli at a particular position in the visual field. Most carnivorans and primates exhibit ordered columns of eye preference referred to as ocular dominance maps (ODMs). Similarly, orientation preferences in the V1 of these species are arranged into smooth and roughly periodic orientation preference maps (OPMs). Glires (rodents and lagomorphs), although in part clearly visual animals, apparently lack any orderly arrangement of preferred orientations. It is still controversial whether and how these dichotomic V1 functional architectures can be explained by the self-organized activity-dependent development of neuronal circuits and to what degree their development is influenced or dominated by biological constraints. In this dissertation, I investigate how simple optimization dynamics and biological constraints can be used to understand quantitative features of the V1 circuits in carnivorans and primates on the one side and Glires on the other. First, I focus on the interaction between postnatal brain growth and the development of ODMs in cat V1. I find that cat ODMs reorganize during a period of considerable postnatal growth. Characteristic features of this reorganization as well as the time scale on which it evolves are in good agreement with the predicted brain-growth-induced reorganizations in an optimization model for the activity-dependent formation of ODMs. Second, I develop a general mathematical formalism to analytically calculate OPMs predicted by optimization models for OPM development. I apply this formalism to one of the most prominent optimization models for OPMs, the Elastic Network model. It is shown that in all previously studied regimes the predicted OPM layouts are perfectly periodic and do not reproduce the experimentally observed common OPM layout. In an extreme and biologically unrealistic limit, aperiodic OPMs quantitatively resembling experimental observations emerge. Stabilization of these layouts results from strong non-local interactions. Third, I reconcile two contradictory recent findings on OPM layouts: On the one hand, they have been shown to exhibit aperiodic statistics, apparently invariant among species widely separated in eutherian evolution and in good agreement with prediction from self-organization models with long-range suppressive interactions; on the other hand a recent study identifies a hexagonal arrangement underlying OPM layouts which is explained by constraints arising from subcortical inputs. By analyzing a large data set of experimentally measured OPMs, I find that the degree of hexagonal order in real maps is statistically indistinguishable from the one in aperiodic isotropic control maps. Hence, there is currently no empirical evidence for subcortical constraints on the development of OPM layouts in primates and carnivorans. Fourth, I consider optimization models for the coordinated development of OPMs and the system of long-range tangential connections in V1. I show that, independent of the precise structure of the long-range coupling, the spatial organization of predicted OPMs is in good agreement with experiments, provided that the coupling exceeds a critical strength and range. Consequently, according to these theories, only experimental or genetic perturbations that substantially weaken tangential interactions or restrict their range may alter the spatially complex layout of OPMs. Finally, I for the first time show that very similar optimization approaches can be invoked to explain both the emergence of disordered selectivity organizations in Glires and of the ordered ones in carnivorans and primates. In the models studied, dominant short-range, effectively inhibitory interactions are crucial for the transition from ordered to disordered arrangements.
\end{abstract}




\section{Zusammenfassung}

Die meisten Neuronen im primären visuellen Kortex (V1) der Großhirnrinde von Säugetieren reagieren bevorzugt auf orientierte visuelle Stimuli, die sich an einer bestimmen Position im Gesichtsfeld befinden und in einem bestimmten Auge registriert werden. Bei Primaten und Raubtieren variieren sowohl Augen- als auch Orientierungspräferenzen systematisch über der Sehrinde und sind in sogenannten Augendominanz- bzw. Orientierungskarten angeordnet. Bei Nagern jedoch zeigt die Anordnung von Orientierungspräferenzen keinerlei offensichtliche räumliche Struktur. Es ist immer noch umstritten, ob und wie diese verschiedenen funktionalen V1 Architekturen durch Optimierungsprinzipien für die selbstorganisierte aktivitätsabhängige Entwicklung neuronaler Schaltkreise erklärt werden können und inwieweit biologische Zwangsbedingungen ihre Entwicklung beeinflussen oder sogar dominieren. Im ersten Teil dieser Arbeit analysiere ich die Wechselwirkung zwischen der Herausbildung von Augendominanzkarten (ODMs) und postnatalem Hirnwachstum am Beispiel des V1 von Katzen. Ich zeige, dass sich ODMs zeitgleich mit der stärksten Wachstumsphase umordnen. Charakteristische Eigenschaften dieser Reorganisation sowie deren zeitlicher Verlauf sind in guter Übereinstimmung mit wachstumsinduzierten Reorganisationsprozessen in Optimierungsmodellen für die aktivitätsabhängige Herausbildung von ODMs. Zweitens entwickle ich einen allgemeinen mathematischen Formalismus zur Untersuchung von Optimierungsmodellen für die Entwicklung von OPMs. Mittels dieses Formalismus untersuche ich eines der meist verwendeten Optimierungsmodelle, das sogenannte Elastische Netzwerk. Dabei stellt sich heraus, dass in allen bisher untersuchten Parameterbereichen lediglich räumlich periodische Muster zu erwarten sind, die die experimentell bestimmte universelle Statistik nicht reproduzieren können. Lediglich in einem extremen und biologisch nicht realistischen Bereich werden aperiodische Muster vorhergesagt. Im dritten Teil löse ich den Widerspruch zwischen zwei jüngst erschienen Forschungsergebnissen zur Struktur von OPMs auf: Eine Studie zeigt, dass OPMs eine universelle räumlich aperiodische Statistik aufweisen, die gut mit den Vorhersagen von Selbstorganisationsmodellen mit langreichweitigen Kopplungen übereinstimmt. Eine andere Studie kommt zum Ergebnis, dass OPMs hexagonal geordnet sind. Diese Struktur lieBe sich durch die räumliche Anordnung afferenter Verbindungen in V1 erklären. Anhand eines großen Satzes funktionaler bildgebender Daten zeige ich, dass der Grad an hexagonaler Ordnung in OPMs statistisch ununterscheidbar von isotropen räumlich aperiodischen Kontrollkarten ist. Das Muster von Orientierungspräferenzen in Primaten und Raubtieren ist demnach nicht durch die räumliche Struktur subkortikaler Verbindungen bestimmt. Ausgehend von obigen Ergebnissen untersuche ich viertens, wie räumlich aperiodische Muster in Optimierungsmodellen für eine gekoppelte Entwicklung von langreichweitigen Tangentialverbindungen in V1 und OPMs stabilisiert werden können. Es zeigt sich, dass die Statistik optimaler OPMs gut mit den experimentellen Daten übereinstimmt, solange lediglich Reichweite und Stärke der langreichweitigen Kopplung kritische Werte überschreiten. Diesen Theorien zufolge kann also die Struktur von OPMs nur dann wesentlich durch experimentelle Manipulation beeinflusst werden, wenn entweder Reichweite oder Stärke der lateralen Wechselwirkung substanziell reduziert werden können. Schließlich zeige ich, dass sich sowohl die Herausbildung räumlich ungeordneter Selektivitäten in Nagern als auch die Entwicklung systematisch variierender Präferenzen in Primaten und Raubtieren durch sehr ähnliche Optimierungsprinzipien beschreiben lassen. Entscheidend für einen Übergang von geordneten zu ungeordneten räumlichen Anordnungen in den untersuchten Modellen ist eine dominante kurzreichweitige effektiv inhibitorische Wechselwirkung. 


\section{Papers submitted for this thesis}

Keil W, Schmidt K-F, Löwel S, and Kaschube M Reorganization of columnar architecture in the growing visual cortex. Proc Nat Acad Sci USA 2010, 107(27):12293-12298.

(C2010 Keil, Schmidt, Löwel, and Kaschube

Keil W and Wolf F Coverage, continuity, and visual cortical architecture. Neural Syst E Circ 2011 1:17 doi:10.1186/2042-1001-1-17.

(C)2011 Keil and Wolf

Keil W, Schnabel M, Coppola D M, Löwel S, White L E, Kaschube M, and Wolf F Orientation maps lack hexagonal organization. Under review.

(C)2012 Keil, Schnabel, Coppola, Löwel, White, Kaschube, and Wolf 


\section{Contents}

$\begin{array}{ll}\text { Introduction } & 1\end{array}$

1 Fundamentals 3

1.1 The early visual pathway $\ldots \ldots \ldots \ldots \ldots \ldots \ldots \ldots \ldots \ldots \ldots$

1.2 Columnar organizations of visual response properties in primates and carnivorans . 5

1.3 Columnar versus salt-and-pepper arrangements . . . . . . . . . . . 8

1.4 Development and plasticity of visual cortical representations . . . . . . . . . . . . . 9

1.5 Modeling the development of visual cortical architecture . . . . . . . . . . . . . 11

1.5.1 The Wolf model . . . . . . . . . . . . . . . . . . . . 13

1.5.2 The Elastic Network model . . . . . . . . . . . . . . . . . . . . . 18

1.6 Retinal constraints on the spatial structure of orientation preference maps . . . . . 20

2 Reorganization of columnar architecture in the growing visual cortex 23

Manuscript . . . . . . . . . . . . . . . . . . 25

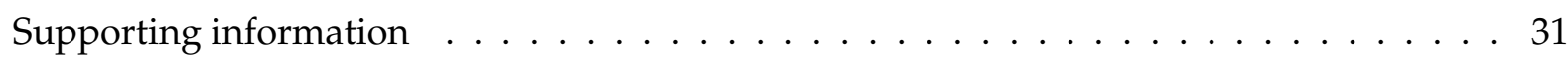

3 Coverage, continuity, and visual cortical architecture 63

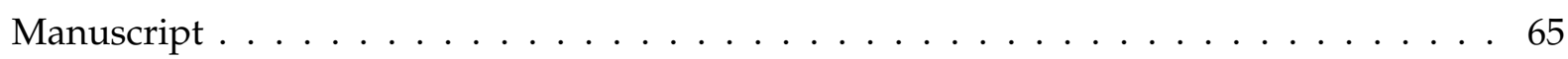

4 Orientation maps lack hexagonal organization $\quad 121$

Manuscript . . . . . . . . . . . . . . . . . . . . . . . . 123

Supporting information . . . . . . . . . . . . . . . . . . . . . . . 129

5 Robustness of pinwheel stabilization by long-range interactions 137

5.1 Motivation . . . . . . . . . . . . . . . . . . . . . . 137

5.2 The development of orientation preference maps and long-range connections as dynamical optimization process . . . . . . . . . . . . . . . . . 138

5.2.1 Weakly nonlinear analysis of the long-range interaction model . . . . . . 141 
5.3 Numerical procedures . . . . . . . . . . . . . . . . . . . . . . . . . . . . 144

5.4 Specific models and their phase diagrams . . . . . . . . . . . . . . . . 145

5.4 .1 Gaussian interactions . . . . . . . . . . . . . . . . . . 145

5.4.2 Interactions derived from the statistics of long-range horizontal connections . 147

5.4 .3 Non-orientation-selective local connectivity . . . . . . . . . . . . . . 150

5.4 .4 Power-law interactions mediated by visual input? . . . . . . . . . . . . . 153

5.5 Simultaneous development of horizontal connections and OPMs . . . . . . . . 157

5.6 General stability ranges for the one-parametric long-range interaction models . . . . 160

5.6 .1 Intrinsic and extrinsic stability boundaries . . . . . . . . . . . . 160

5.6 .2 Asymptotic stability boundaries . . . . . . . . . . . . . . . . . 162

5.7 Summary . . . . . . . . . . . . . . . . . . . . 166

6 Stimulus-driven optimization models for rodent visual cortical development 167

6.1 Motivation . . . . . . . . . . . . . . . . . . . . . . . . . 167

6.2 Model outline . . . . . . . . . . . . . . . . . . . . . 168

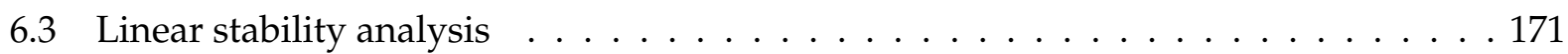

6.4 The dynamics of the model . . . . . . . . . . . . . . . . . 172

6.4.1 Methods . . . . . . . . . . . . . . . . . . . . 172

6.4.2 The spontaneous emergence of disordered selectivity organizations . . . . . . 173

6.4 .3 Starting from ordered initial conditions . . . . . . . . . . . . . 174

6.4.4 Quantifying the spatial structure of orientation preference patterns in the

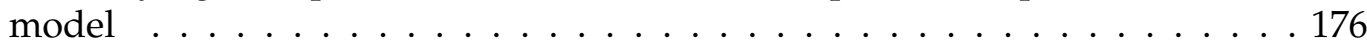

6.4.5 Modeling an initial bias in preferred orientations . . . . . . . . . . 180

6.5 Adding lateral excitation to the model . . . . . . . . . . . . . . . . . . . 182

6.5.1 Linear stability analysis and numerical simulations . . . . . . . . . . . . . 184

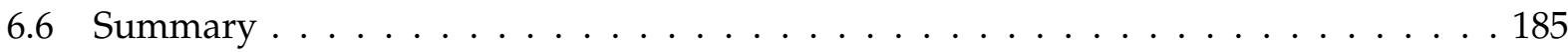

$\begin{array}{llr}7 & \text { Summary and Outlook } & 189\end{array}$

$\begin{array}{ll}\text { Bibliography } & 201\end{array}$

$\begin{array}{ll}\text { Acknowledgements } & 219\end{array}$ 


\section{Introduction}

Are biological structures such as cells, organs such as the lung and the brain, or even entire organisms designed to optimally serve a specific functional demand? Wooden structures, for example, seem to be laid out to obtain high twist-to-bend ratios [1]. The progression of branch diameters in the vascular tree appears to minimize both the mass of blood needed to fill the vessel and the energy required to transport blood to the targets against flow resistance [2-4]. Similarly, the average diameter of the airway elements in the human lung follows a "best dimension scaling law" [5]. Arbor sizes and branching of neurons in mammalian brains fit a global volume-minimization model $[6,7]$. The spatial positions of neuronal elements in biological circuits such as the nervous system of Caenorhabditis elegans and Drosophila melanogaster can be explained by wiring optimization principles $[8,9]$. Similar rules have even been invoked to explain the arrangement of functional areas of the mammalian cerebral cortex [10]. Whenever one refers to optimization principles in biology, the hope is to find potentially simple rules which underlie the design of the overwhelmingly rich variety and complexity of shapes found in nature, ranging from unicellular algae to probably the most complex structure on earth - the primate brain. The above examples suggest that complex biological structures may indeed, at least to some extent, be optimally adjusted to simple functional needs.

However, optimization approaches to biology have raised intense debates [4] and certainly have to be viewed with skepticism. First, there are numerous reasons why biological systems may not be optimal. Organisms are neither "designed" nor "engineered". Constraints and trade-offs may be pervasive, thus completely preventing any kind of global or general optimality. Biological materials have limitations and natural selection is constrained to pre-existing structures, which might not be perfectly suited for a particular novel functional requirement. Available resources have to be parsimoniously used. Adequacy or sufficiency of biologically designed structures might hence completely dominate optimality. Second, biological structures in most cases are multifunctional. Observing that a structure or a system found in nature appears to violate seemingly obvious optimality requirements might merely reflect the fact that it predominantly serves a purpose yet undiscovered or that it represents a complex compromise among a diverse set of requirements. Third, theoretical predictions of optimality principles are often not unique, i.e. different optimality criteria may yield very similar outcomes, in which case they are of limited predictive power.

Despite all these caveats, optimization principles can be very useful to understand certain aspects of the function of a biological system or its emergence in the course of evolution and development in idealized terms. With life sciences becoming increasingly quantitative, optimization principles are particularly informative if their predictions, i.e. their respective optima, can be quantitatively established. This, however, poses serious and often intractable mathematical problems. 
This thesis is devoted to a quantitative understanding of aspects of postnatal development, structure, and function of the primary visual cortex (V1), the largest and best-studied cerebral cortical area involved in early visual processing, in terms of simple optimization principles and constraints. For this particular brain region, recent comparative data on its functional architecture $[11,12]$ have raised the urgency of investigating whether there are general layout principles that govern the design of its neural circuits. A quantitative comparison of functional V1 circuit design in a large data set from three species widely separated in the evolution of eutherian mammals revealed that its aperiodic spatial statistics are quantitatively invariant [11]. This quantitative match is particularly puzzling in view of the fact, that an entire mammalian taxon branch, namely that of rodents and lagomorphs, has been recently discovered to obey apparently opposing rules [13-15]. Have different general requirements for a versatile and powerful cortical circuit architecture resulted in this dichotomy of layout designs during evolution? How are these designs influenced by developmental constraints? Can similar types of self-organization mechanisms for constructing visual cortical circuitry explain the emergence of both functional architectures during postnatal development? What is constitutive of their layout principles? Do the different types of circuit architecture in some way optimally process visual information? These are the core questions underlying the present thesis. Some optimization approaches when considered with suitable biological constraints will turn out to describe postnatal development of the primary visual cortex surprisingly well. Other widely used principles will turn out to be too simple to adequately model biological reality. And some seemingly obvious constraints on functional circuit design do not appear to play any role at all in shaping V1 functional architecture.

Chapter 1 briefly outlines the neuroanatomical properties of the early visual system as well as previous mathematical modeling approaches relevant to this work. In Chapter 2, I focus on the interaction between the biological constraint of postnatal brain growth and the self-organized development of functional architecture in cat V1. In Chapter 3, I develop a general mathematical formalism to analytically calculate the optima of models for visual cortical development and apply this formalism to one of the most prominent optimization models, the Elastic Network model. In Chapter 4, I reconcile two contradictory recent findings on the spatial structure of V1 functional architecture in primates and carnivorans: aperiodic statistics from self-organized development on the one hand, and hexagonal order arising from subcortical constraints on the other. Chapter 5 is devoted to an analysis of models for the coordinated development of functional architecture and the system of long-range tangential connections in V1. Finally, in Chapter 6, I show that very similar optimization approaches can be invoked to explain both the development of disordered selectivity organization in rodent and lagomorphs and of ordered representations in carnivorans and primates. 


\section{Chapter 1}

\section{Fundamentals}

In this chapter, I will first briefly sketch the neuroanatomical and physiological properties of the early visual system relevant to this work. Particular emphasis will be placed on aspects of its postnatal development and differences between carnivorans and primates on the one hand and rodents and lagomorphs on the other. For a more comprehensive overview, I refer to [16]. Second, I will outline two mathematical modeling approaches to the development of visual cortical circuit architecture, which form the basis of the mathematical analyses in the present thesis.

\subsection{The early visual pathway}

Visual information processing in the mammalian nervous system begins in the retinas of the two eyes. Light enters through the lenses and falls onto a layer of photo-receptors which convert the light signals into electrical signals. These electrical signals are then transformed into a series of stereotyped electrical pulses, the action potentials, emitted by the retinal ganglion cells (RGCs). The axons of these cells form the optic nerve and conduct the RGC signals to the brain. In the transfer of visual signals to the brain, the visual pathway (Fig. 1.1.1), the retina is vertically divided in two parts, a temporal half (nearer to the temple) and a nasal half (nearer to the nose). The axons from the nasal half cross the brain at the optic chiasma to join with axons from the temporal half of the other eye before passing into the lateral geniculate nuclei (LGN). The signals from RGCs are relayed through the LGN without substantial modification. Therefore receptive field properties of LGN neurons are qualitatively similar to the receptive fields of retinal ganglion cells $([18,19]$, but see also [20]). The visual input into the two LGN is organized in such a way that information from the right parts of the two retinas is relayed by the left LGN and vice versa. However, no binocular responses are observed in LGN neurons. Instead, the LGN consist of several layers of neurons, each containing cells that are driven by either the right or the left eye exclusively.

From the LGN, visual information is projected to the left and right primary visual cortex (V1) via the optic radiation (Fig. 1.1.1). V1 is a part of the neocortex of about $2 \mathrm{~mm}$ thickness and several $\mathrm{cm}^{2}$ area in humans. It is the largest of all visual areas and often considered to be the first stage of true visual information processing. Whereas the number of RGC cells and the number of LGN neurons is of roughly the same order, the number of neurons in V1 is about a thousandfold higher than the number of projection neurons in the LGN. Due to the organization of the LGN, 


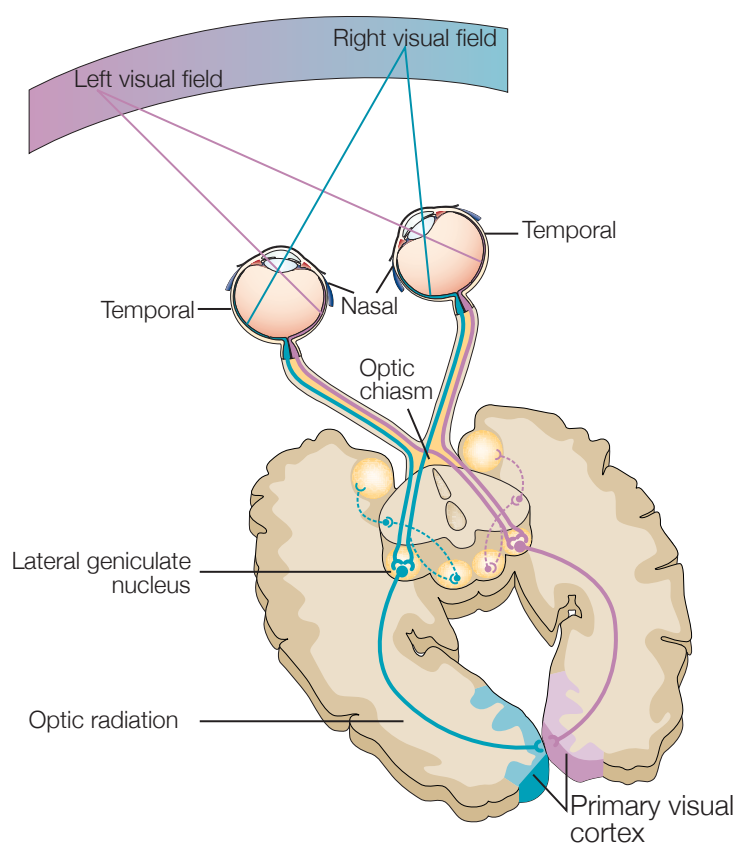

Figure 1.1.1: Schematic illustration of the early visual pathway in carnivorans and primates. The visual pathway begins with the eyes and extends through several interior brain structures before ascending to the primary visual cortex (V1). At the optic chiasm, the optic nerves cross over partially so that each hemisphere of the brain receives input from the temporal and nasal visual field of both eyes. The lateral geniculate nucleus (LGN), consists of layers of nerve cells that each respond to stimuli from one eye only (mod. from [17]).

both hemispheres receive input from both eyes. In fact, neurons in V1 are the first along the visual pathway to respond to stimuli from both eyes. However, while being responsive to both eyes, many neurons are preferentially driven by the left or the right eye, a property called ocular dominance (OD).

The sheet of neural tissue that is formed by the primary visual cortex contains a topographic representation of visual space. This means that neighboring neurons in V1 respond to stimuli coming from neighboring spots in the visual field. Along the vertical axis through the cortical layers, spatial receptive field properties of neurons are very similar. Hence, there is a more or less ordered mapping, called retinotopic map $\mathbf{R}(\mathbf{x})$ which maps the 2-dimensional cortical surface $\mathbf{x}$ onto visual field coordinates $\mathbf{R}$. While in some animals, e.g. tree shrews and mice, this mapping is rather uniform [21-24], it has been found to be rather non-uniform in cats [25] and macaque monkeys [26].

In contrast to neurons in the retina and LGN which are well-characterized by their spatial receptive field, neurons in V1 exhibit much more complex response properties. One such property, namely ocular dominance, has already been mentioned above. A second important property is the response to visual contours or bar stimuli of a particular orientation within the spatial receptive field $[27,28]$. This property is called orientation preference. The present thesis is exclusively concerned with the spatial organization of the three response properties - spatial position, ocularity, and orientation - of neurons in the primary visual cortex. 


\subsection{Columnar organizations of visual response properties in primates and carnivorans}

In the primary visual cortex of carnivorans and primates, the response to orientations is spatially organized into a 2-dimensional array of modules, called orientation columns ${ }^{1}$. An orientation column is a local population of neurons extending through the entire cortical thickness that respond to the same or similar orientation. Parallel to the cortical surface, orientation preference varies smoothly from column to column [27, 28] apart from so-called pinwheel centers, around which columns activated by different stimulus orientations are radially arranged like the spokes of a wheel [40,45-47]. The spatial pattern with which the preferred orientation changes parallel to the cortical surface in carnivorans and primates is called orientation preference map (OPM). Figure 1.2.1 shows a typical example of such an orientation map as revealed by optical imaging of intrinsic signals $[21,46,48]$ in tree shrew primary visual cortex. Figure 1.2.1a depicts the responses $E(\mathbf{x} \mid \phi)$ to gratings recorded from V1 with orientations $\phi=\left\{0^{\circ}, 45^{\circ}, 90^{\circ}, 135^{\circ}\right\}$. One can summarize these responses by constructing a complex-valued field

$$
z(\mathbf{x})=\sum_{j} e^{2 i \phi_{j}} E\left(\mathbf{x} \mid \phi_{j}\right)=e^{2 i \theta(x)}|z(\mathbf{x})| .
$$

The phase $\theta(\mathbf{x})=\frac{1}{2} \arg z(\mathbf{x})$ then represents the preferred orientation at cortical position $\mathbf{x}$, i.e. the OPM. The magnitude $|z(\mathbf{x})|$ is a measure for the selectivity of the responses at position $\mathbf{x}$. The OPM is visualized by plotting $\theta(\mathbf{x})$ and colorcoding the preferred orientation (Fig. 1.2.1b). The OPM exhibits a roughly repetitive arrangement of preferred orientations, in which adjacent columns preferring the same orientation are separated by a typical distance in the millimeter range $[21,40,46,47,49]$. Within these repetitive arrangements, so-called linear zones (Fig. 1.2.1c, left) with smooth progression of the preferred orientations as well as numerous pinwheel centers (Fig. 1.2.1c, right) are present. Mathematically, pinwheels are the zeros of the field $z(\mathbf{x})$ and pointsingularities of the field $\theta(\mathbf{x})$ [50]. They come in two chiralities, depending on whether the preferred orientation rotates clockwise or counterclockwise around the pinwheel (Fig. 1.2.1c, right, [50]).

Similar to orientation preference, ocular dominance (OD) in primates and carnivorans is organized into columnar patterns. Figure 1.2.1d depicts the overall pattern of OD columns visualized by transneural labeling of the afferents in layer IV from the ipsilateral eye with ${ }^{3} \mathrm{H}$-proline $[29,51]$. Mathematically, the pattern of OD can be described by a real-valued field $o(\mathbf{x})$ which is positive at positions dominated by the contralateral eye, negative at positions dominated by the ipsilateral eye, and close to zero for regions that are binocularly driven.

The spatial layout of OD columns is qualitatively different in cats compared to macaque monkeys and humans (Fig. 1.2.1e,f). While in monkeys and humans, OD columns form parallel bands with stripe-width in the millimeter range in large parts of the V1 surface area, they are more patchy and spatially irregular in cat throughout the entire V1 [29, 51-53]. However, in both cats and monkeys,

\footnotetext{
${ }^{1}$ More precisely, orientation columns have been found in several species of the primate order such as the squirrel monkey [32], the macaque [33-35] and owl monkey [36, 37], the galago [38] and humans [39] as well as of the orders Scandentia (tree shrews) [21] and Carnivora (carnivorans) such as the cat [28, 40] and the ferret [41-44]. Throughout this thesis, primates and carnivorans is written instead of primates, carnivorans and tree shrews, for reasons of readability.
} 


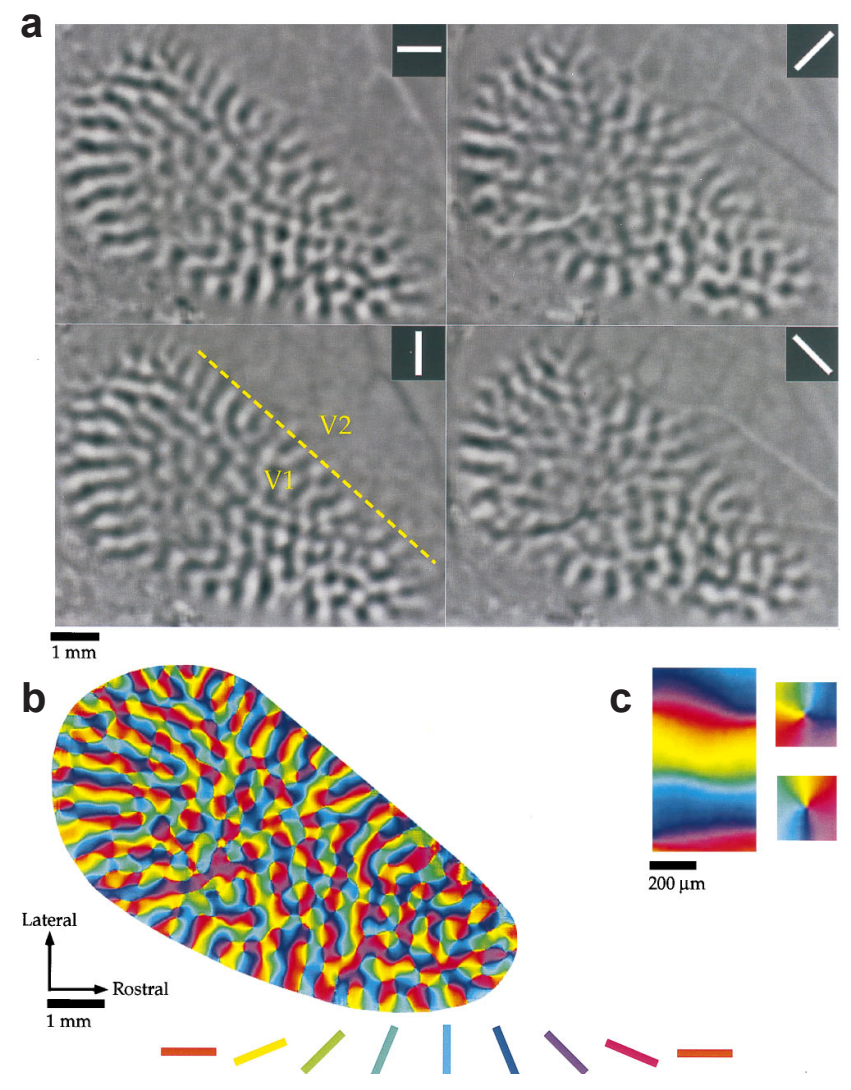

d

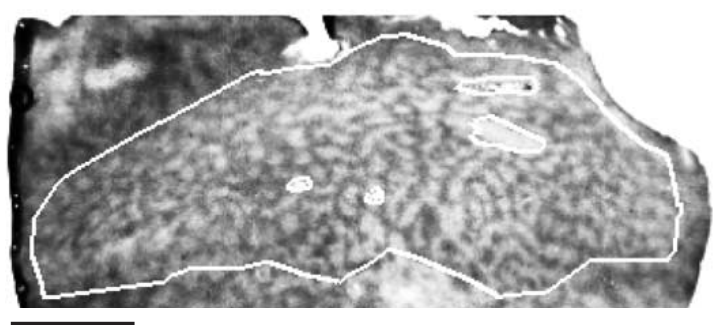

e
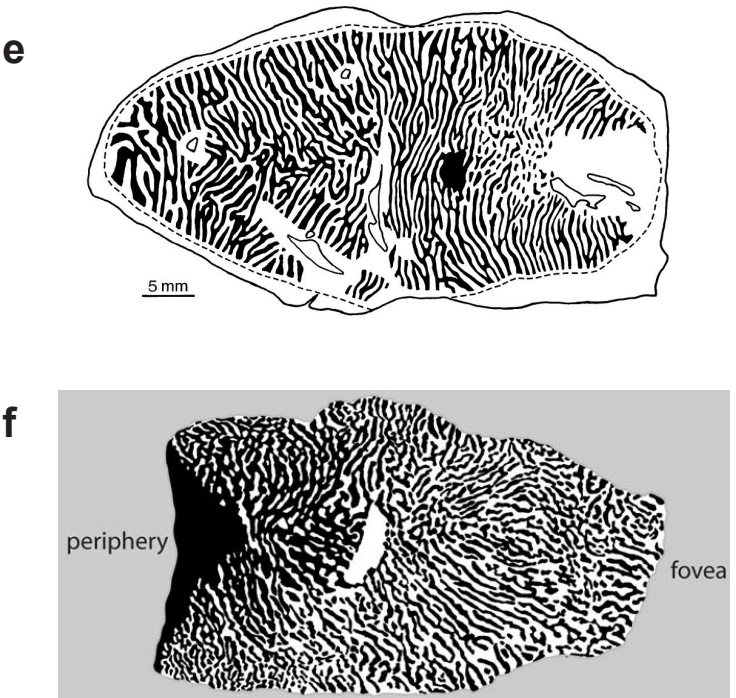

Figure 1.2.1: Orientation preference maps (OPMs) and ocular dominance (OD) maps in carnivoran and primate V1. (a) Difference images obtained for the four angles $0^{\circ}, 45^{\circ}, 90^{\circ}, 135^{\circ}$ of drifting gratings as revealed by optical imaging of intrinsic signals in tree shrew V1 (mod. from [21]). Dark areas of each panel indicate areas that were preferentially activated by a given stimulus. Light gray areas indicate areas that were active during presentation of the orthogonal angle. (b) OPM obtained by vector summation of data in a. Orientation preference of each location is color-coded according to the key below. (c) linear zones (left) and pinwheel arrangements (right) as common features in OPMs. (d) Overall pattern of OD columns in cat visual cortex, visualized by transneural labeling of the afferents in layer IV from the ipsilateral eye with ${ }^{3} \mathrm{H}$-proline. Scale bar equals $10 \mathrm{~mm}$. (mod. from[29]). (e) Overall pattern of OD columns in macaque monkey, visualized with ${ }^{3} \mathrm{H}$-proline labeling (mod. from [30]). (f) Overall pattern of OD columns in human V1, visualized by cytochrome oxidase histochemistry in subjects with monocular vision loss (mod. from [31]). 
a

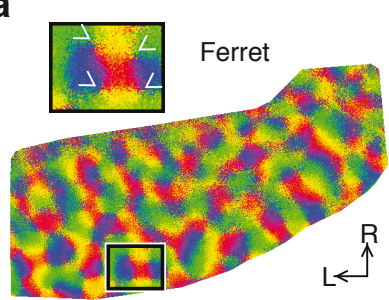

d

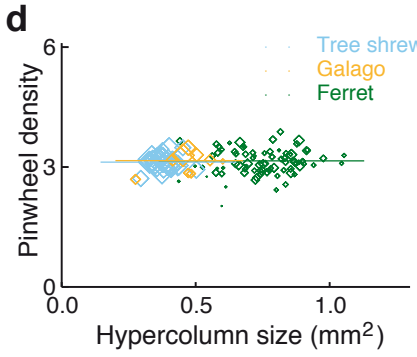

b

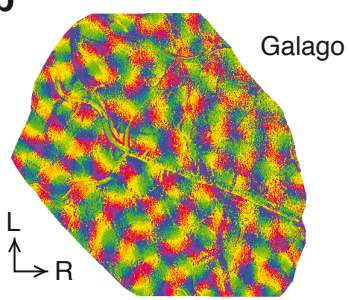

e

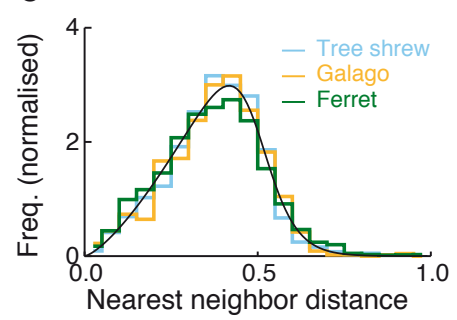

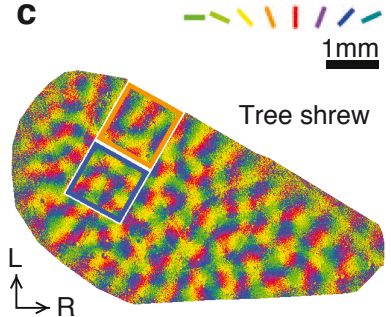

f

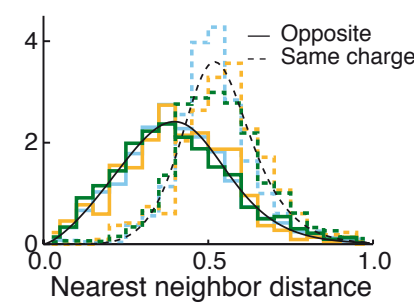

Figure 1.2.2: Common design of orientation maps in ferret, tree shrew and galago. (a-c) Orientation maps recorded with optical imaging of intrinsic signals in (a) ferret, (b) galago, and (c) tree shrew primary visual cortex. (d) Pinwheel densities versus hypercolumn size in the three species. Solid lines indicate average pinwheel densities of the three species. (e, f) Nearest neighbor distances for pinwheels of (e) arbitrary and (f) opposite and equal topological charge. Black curves indicate fits to the data (mod. from [11]).

the pattern of ocular dominance columns exhibits a pronounced inter-individual variability [29] and may even be qualitatively different in individuals of the same species [54, 55].

In contrast to the extreme inter-species variability in the spatial layout of ocular dominance patterns, the pattern of orientation columns is very similar in the different species that have been investigated so far $[11,21,44,46,47,49,56]$. Orientation columns always appear as organized by a spatially complex aperiodic array of pinwheel centers. The arrangement of these pinwheel centers, although spatially irregular, has been shown to be statistically distinct from a pattern of randomly positioned points [11] as well as from patterns of phase singularities in a random pattern of preferred orientations $[11,57,58]$ with spatial correlations identical to experimental observations $[11,59]$. This suggests that the layout of orientation columns and pinwheels, although spatially aperiodic, follows a definite system of layout rules. In fact, recent comparative data strongly indicates such a system of layout rules. Kaschube et al. [11] quantitatively compared pinwheel arrangements in a large data set from ferret, galago, and tree shrew - three species widely separated in the evolution of eutherian mammals (Fig. 1.2.2a-c). These authors found that the spatial statistics of pinwheels are surprisingly invariant. In particular, the overall pinwheel density (Fig. 1.2.2d) was found to be virtually identical. Characterizing pinwheel layout on the scale of individual hypercolumns, they found the distributions of nearest-neighbor pinwheel distances to be almost indistinguishable in the three species (Fig. 1.2.2e,f). Similarly, the spatial configuration of the superficial patch system [60] and the responses to drifting grating stimuli were recently found to be very similar in cat and macaque monkey primary visual cortex [12], which further supports common layout rules for orientation columns in carnivorans and primates.

Cortical columns can, in principle, exhibit almost perfectly repetitive order as exemplified by oc- 
a

Figure 1.3.1: Columnar arrangements vs. salt-andpepper layouts. (a) Cell-based orientation map from $230 \mu \mathrm{m}$ depth in cat V1 obtained with twophoton calcium imaging in vivo (from [61]). Selective cells are colored according to their preferred orientation. Orientation preferences are highly ordered around a pinwheel center. (b) Orientation preferences as revealed by two-photon imaging in rat visual cortex (from [13]) with no discernible local spatial structure.

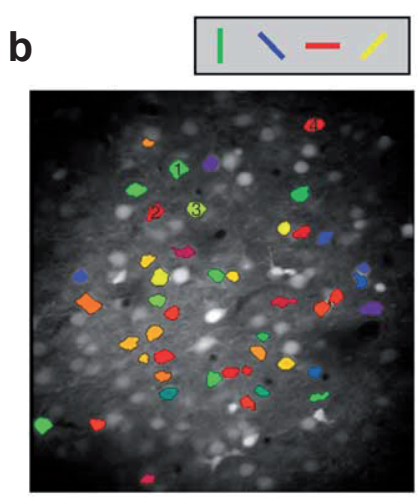

ular dominance (OD) bands in the primate primary visual cortex (Fig. 1.2.1e,f). Therefore it is a fundamental question for understanding visual cortical architecture, whether there are functional requirements or constraints which lead to layout principles that prohibit a spatially exactly periodic organization of orientation columns in primates and carnivorans and, instead, enforce complex arrangements of these columns. This question is at the core of several chapters of the present thesis.

\subsection{Columnar versus salt-and-pepper arrangements}

Recent progress in imaging techniques has revealed a fascinating dichotomy between carnivorans and primates on the one hand and Glires on the other. Through two-photon calcium imaging $[62,63]$, Ohki et al. were able to show [13] that while carnivorans and primates exhibit beautifully ordered orientation preference maps up to single-cell scale (Fig. 1.3.1a), Glires apparently lack any orderly arrangement of preferred orientations ${ }^{2}$ (Fig. 1.3.1b). The receptive fields of V1 cells in rodents and lagomorphs, however, are very similar to those found in carnivorans and primates $[14,66]$. In particular the primary visual cortex of mice has recently attracted a lot of attention, not least because of the promising genetic tools and methods available in mice to dissect cortical circuits. The circuits underlying orientation selectivity in mice have been found to be intriguingly unspecific. Orientation-selective neurons receive inputs with very different tunings even on the same neuronal arbor (dendrite) so that adjacent dendritic segments are tuned to distinct orientations [68]. Inputs that share the same orientation preference are widely distributed throughout the dendritic tree [68]. Somewhat contrary, connection probability between neurons seems related to the similarity of visually driven neuronal activity. In mouse V1, neurons with the same preference for oriented stimuli connect at higher rates, yet there is still a substantial fraction of strongly connected neurons with orthogonal preferred orientation [15].

The apparent randomness of visual cortical networks in rodents and lagomorphs compared to the highly ordered arrangements observed in carnivorans and primates together with their similarities regarding response features is puzzling in many ways. How do the different types of

\footnotetext{
2 The lack of orderly arrangement of orientation preferences has been demonstrated since the 1970s in several species of both Glires orders: (i) Rodentia (rodents), such as the rat [13, 64], the grey squirrel [14], and the mouse [65, 66] and (ii) Lagomorpha (lagomorphs) such as the rabbit [67].
} 
a

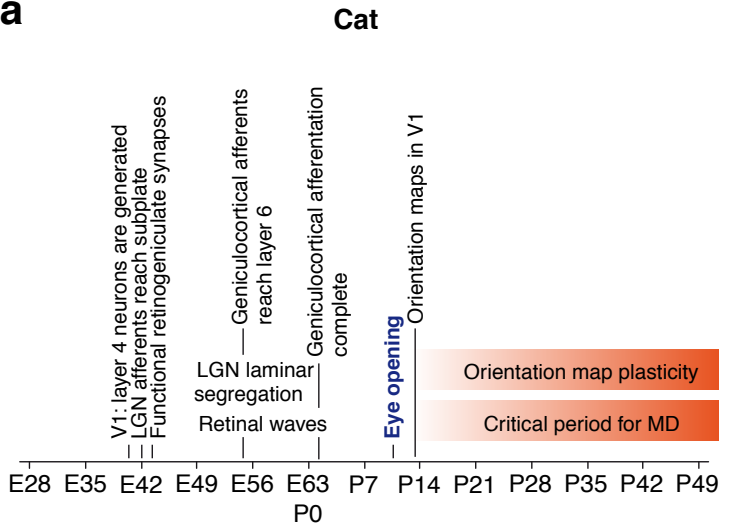

b

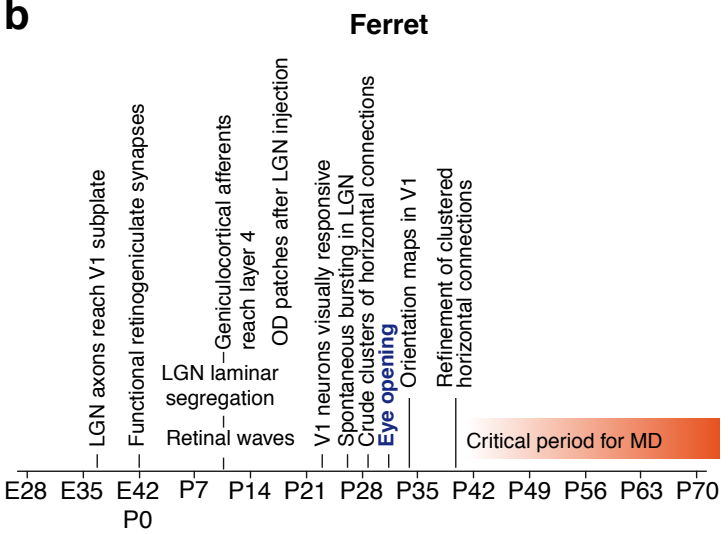

Figure 1.4.1: Timeline of development of the visual system in (a) cats and (b) ferrets. Major events are plotted against embryonic (E) and postnatal (P) age. At birth, the development of the ferret lags that of the cat by about 3 weeks. In both species, plasticity dependent on visually driven activity sets on around the time of eye-opening, i.e. shortly after maturation of the major response properties is completed (mod. from [69]).

circuit architecture process visual information? Can similar and well-established neural learning mechanisms be taken as a basis to explain the two different designs? Have different general requirements for a versatile and powerful cortical circuit architecture resulted in two fundamentally different orientation preference layout designs during evolution? While numerous experimental studies have quantitatively characterized the functional architecture of V1 in rodents and lagomorphs, modelers so far still owe adequate explanations for their apparently random arrangement of selectivities as well as their functional role. The results presented in Chapter 6 aim at closing this gap, by constructing and analyzing models for the activity-dependent development of orientation-selective cell arrangements that seemingly lack any spatial order.

\subsection{Development and plasticity of visual cortical representations}

How do the different types of functional organization emerge during the development of the animal? In fact, many aspects of the thalamo-cortical pathway in general and primary visual cortical architecture in particular only emerge around the time of birth or thereafter. It is one of the oldest and most controversial questions in neurobiology of vision to what extent the structure and function of visual cortical circuits are determined by intrinsic factors such as genetic disposition, and to what extent they can be influenced and shaped by the environment. Figure 1.4.1 illustrates some milestones of visual cortical development in the two most comprehensively studied carnivoran species: cats (Fig. 1.4.1a) and ferrets (Fig. 1.4.1b). In both cats and ferrets, during normal postnatal development, column representations emerge around the time of eye-opening [42, 43, 70-72]. In the cat, ocular dominance can already be visualized at postnatal week 2 [71] and a mature pattern of OD columns can be labeled over the full extent of V1 as early as week 3 [73, 74]. Orientation maps arise over roughly the same period [75]. In the ferret, Crowley and Katz demonstrated segregated ocular dominance columns only few days after the innervation of cortical layer 4 . The emergence of cortical maps is accompanied by the formation and refinement of a clustered network of long-range horizontal connections [21, 44, 76-78]. These patchy con- 
nections originate from an initially diffuse pattern of unbranched neuronal arbors and emerge gradually through a process of selective axon elaboration and addition of new collaterals $[79,80]$. Activity-dependent mechanisms guide this refinement $[79,81]$, yet the precise interplay between the development of clustered horizontal projections and the emergence of orientation selectivity is not understood. In Chapter 5, I study models in which the development of horizontal connections and orientation preference maps is viewed as a joint optimization (minimization) process of an abstract energy functional. The model can also be used to examine what types of biologically plausible long-range interactions are able to mediate the stabilization of realistic orientation preference maps during development.

Both, orientation preference maps and ocular dominance maps have been shown to remain plastic over a phase of at least several weeks after their emergence [70,75]. During this so-called critical period, primary visual cortical circuits are particularly susceptible to artificial manipulations of visual inputs $[69,71,82,83]$. For instance, stripe-rearing of kittens during this period can cause up to twice as much cortical surface area to be devoted to the experienced orientations $[84,85]$. Similarly, if vision is occluded in one of the two eyes, geniculocortical afferents from the deprived eye shrink and afferent arbors from the non-deprived eye show significant expansion [86, 87]. In the same line, in macaque monkeys, periods of monocular lid-suture can result in dramatic changes in the distribution of input from the two eyes into cortical layer 4 in favor of the open eye if applied during the critical period [88, 89].

It is highly remarkable that the onset of the critical period is delayed relatively to the onset of visual experience $[69,90]$ (cf. Fig. 1.4.1a,b), and key features of neuronal response properties are already present in almost adult form well before the onset of the critical period [44, 70, 71, 78, 91]. However, this poses a long-standing enigma: What is the functional role of relatively strong plasticity at such a late stage of development and how is this plasticity during normal postnatal development involved in shaping the adult cortical circuitry [92, 93]? A recent study has offered an interesting possible answer to this question, suggesting that one function of critical-period plasticity is to progressively coordinate the functional architectures of different cortical areas even across hemispheres [94]. In Chapter 2, I explore an alternative or complementary hypothesis based on the observation that the primary visual cortex after establishing mature cortical feature representations is still growing in size.

Growth represents a natural constraint arising during development, yet it has received relatively little attention when considering visual cortical development of mammals. Cortical area increase can be considerable during postnatal development. The human brain, for instance, weighs on average $350 \mathrm{~g}$ in newborns and 1,400 $\mathrm{g}$ in adult males [95]. The neocortical volume of the cat increases postnatally from $\approx 1,000 \mathrm{~mm}^{3}$ to $\approx 4,500 \mathrm{~mm}^{3}$ in adulthood [96]. Consistently, the surface area of cat V1 increases postnatally by a factor of 2.5 between week 1 and week 12 [97]. Importantly, this growth takes place without a significant change in the number of neurons but is mainly due to the generation of glial cells, the addition of more vasculature and connective tissue, and the myelinization of axons. To a lesser extent it also reflects the outgrowth and elaboration of axonal and dendritic processes [98]. Therefore, the distance between any two neuronal cell bodies in cat V1, for instance, grows on average by a factor of 1.6 during postnatal development. In Chapter 2, I study a simple yet fundamental question: What happens to cortical columns when the cortex is growing in size? Do neuronal processes such as axons and dendrites simply elongate and columns merely expand or are larger changes needed to accommodate this growth? To answer 
these questions, I combine the analysis of imaging data from acute and chronic imaging experiments in cats, obtained during a period of substantial postnatal growth with the investigation of a classical optimization model for the activity-dependent formation of OD columns considered under the constraint of a growing domain [99].

Is the development of salt-and-pepper arrangements in rodent and lagomorph V1 distinct from the development of columnar representations in carnivorans and primates? Several early studies have shown that essential features of postnatal cortical maturation are very similar to carnivorans and primates. Response properties develop gradually during the first weeks after birth and dramatic shifts of the ocular dominance distributions obtained by monocular deprivations as well as shifts in orientation preference obtained by dark rearing have been observed during a critical period delayed relatively to the emergence of feature responsiveness [100, 101]. Similarly, a critical period for monocular deprivation has been identified [102, 103]. In the mouse, critical periods might even extend over weeks and month after mature selectivities have been established $[104,105]$. There seem to be, however, some interesting differences between the postnatal development of rodents and carnivorans or primates. In mouse, for example, activity-dependent changes induced by normal visual experience during the critical period, have been shown to gradually match eye-specific inputs in the cortex [106]. In this way, neurons can rotate their orientation preferences up to at least $45^{\circ}$ during postnatal development. Another such difference concerns direction selectivity, i.e. the selective response of neurons not just to gratings with a particular orientation but drifting into a particular direction. In contrast to observations in ferret V1 [91], in mouse just after eye-opening nearly all orientation-selective neurons appear to be also direction selective and direction selectivity develops normally in dark-reared animals [107]. In addition, at eye-opening, most selective cells respond to gratings of drifting in anterodorsal directions. The fraction of neurons responding to drifting gratings, however, increases during the following weeks of normal development and an equal representation of all orientations is observed in mature animals. As shown in Chapter 6, these findings can easily be included into a mathematical model for the self-organization of orientation-selective responses in rodent and lagomorph V1 by choosing appropriate initial conditions for the developmental dynamics.

\subsection{Modeling the development of visual cortical architecture}

Modeling the emergence of columnar patterns in the primary visual cortex (V1) of carnivorans, primates, and their close relatives has been a challenging task since their discovery. Hubel and Wiesel shortly after their discovery of orientation selectivity [27] proposed genetical predetermination of the required circuitry [108]. This view, however, was hard to reconcile with the high degree of plasticity of the functional cortical architecture demonstrated later in numerous types of deprivation experiments (see above). Additionally, as already recognized by von der Malsburg in 1973 [109], the amount of genetic information necessary to specify all the synaptic contacts between the different neuronal layers involved very likely exceeds the capacity of the DNA. Therefore, starting with the seminal paper by von der Malsburg [109], the study of mechanisms of self-organization has been a promising route to understand cortical development. Three main self-organization modeling approaches have been pursued to understand the development of visual cortical representation, which mainly differ in their degree of realism. 
In the first approach, networks of a large number of individual neuronal elements are modeled by specifying initial feed-forward and lateral excitatory and inhibitory synaptic weights as well as activity-dependent learning rules for the updates of these [109-118]. As the high degree of realism in these models comes hand in hand with a high degree of complexity, it is then mostly assessed by numerical simulations whether the specified mechanisms of self-organization are capable of generating the experimentally observed functional architecture in the cortical layer. Furthermore, the often large number of parameters in these models makes it hard to reveal simple and general principles which might suffice to explain the emergence of functional V1 circuits. In the second approach, one takes the opposite view and starts with general principles of neural learning and observations about the emergence of visual cortical representations to postulate simple and highly abstract mathematical models for the development of feature maps in V1 (e.g. [50,119-130]). Very often, only the development of a single feature representation, e.g. the orientation preference map, is considered $[11,50,122,123,126]$ and plausible symmetry assumptions, such as translation symmetry, rotation symmetry or eye inversion symmetry are used to restrict the functional form of possible dynamical equations and to enable the analytical calculation of stationary solutions. It is argued that essential features of the dynamics of pattern forming systems are robust with respect to the precise form of the equations. The power of such order parameter approaches lies in their simplicity and analytical tractability. Their disadvantage, however, is that the mechanisms postulated are often general to such degree that they constrain properties of possible mechanisms of cortical development without specifying whether these mechanism are activity-dependent or genetically based or both [131]. Additionally, despite some of the models being variational, i.e. the dynamics minimizes (optimizes) a certain cost function, only little can be learned about the functional role of feature maps in V1 because cost functions are too abstract to relate them to cortical function.

The third type of approach aims at striking a balance between the complexity of detailed network models and the lack of specificity of abstract order parameter models. Here, one proposes simple optimization principles, such as the minimization of the total length of neuronal arbors $[132,133]$, and compares (mostly numerically) obtained optimal solutions to experimentally observed cortical maps. A paradigmatic example of such an approach that is studied in great detail in this dissertation is called dimension reduction. In dimension reduction approaches, the receptive fields of individual units are also described by only a few variables, called features. The columnar cortical organization is then assumed to emerge as an optimal trade-off between the coverage of the space of visual stimulus features and the continuity of their cortical representation [134-142]. On the one hand, each combination of stimulus features should be well represented in a cortical map to avoid 'blindness' to stimuli with particular feature combinations. On the other hand, the wiring cost to establish connections within the feature map should be kept low. This can be achieved if neurons that are physically close in the cortex tend to have similar stimulus preferences. These two design goals generally compete with each other. The better a cortical representation covers the stimulus space, the more discontinuous it has to be. Some of the dimension reduction models, such as the Elastic Network model [134, 142, 143] or the model by Scherf et al. [144] possess an explicit energy functional to minimize. Others, like Kohonen's self-organizing feature map (SOFM) [145, 146] have been proven to lack such a functional [147, 148], yet are still conceived as some kind of optimization model. An explicit variational structure of models for the development of cortical representations allows for the formulation of a gradient descent dynamics. The emergence of cortical selectivity patterns and their convergence toward a minimal 
energy state in this dynamics often serves as a model for an optimization process taking place in postnatal development. Interestingly, the developmental dynamics of dimension reduction models, although biologically not very detailed, constitute a straightforward idealization of the two most important aspects of activity-dependent modifications: (i) afferent activity patterns induce activity patterns in the visual cortex and (ii) the selectivities of activated neurons are modified as a function of presynaptic and postsynaptic activity according to Hebbian learning rules [149]. Despite the great amount of idealization in dimension reduction models, a rigorous mathematical analysis of optimal solutions of these models and their dynamics has often been impeded by the mathematical difficulties of these problems.

This thesis is exclusively concerned with models of the second and third type. In Chapter 3, I develop a mathematical formalism to analytically calculate the optimal solutions predicted by one of the most prominent dimension reduction model, the Elastic Network model. In Chapter 5, I study generalizations of an abstract order parameter model, introduced by Wolf [123], to investigate how different types of biologically plausible long-range interactions might influence the formation of OPMs and shape their layout during development. Both models are shortly outlined below.

Finally, it needs to be pointed out that there are currently no simple optimization principles available which could explain the emergence and stabilization of salt-and-pepper layouts in rodents and lagomorphs. The only two modeling studies on rodent and lagomorph V1 architecture have addressed this issue using detailed neural networks with balanced excitatory and inhibitory activity [150-152].

\subsubsection{The Wolf model}

\section{Model definition}

Wolf approached the problem of modeling orientation map formation and development from a rather abstract dynamical systems perspective. An initially non-selective 2-dimensional sheet of neurons acquires a roughly periodic pattern of selectivities that stabilizes after its emergence. This process is reminiscent of many systems exhibiting a pattern forming instability, such as in Rayleigh-Benard convection, where a homogeneous state loses stability with respect to a finite band of Fourier modes. Wolf employed the fact that essential properties of pattern forming systems are often independent of model-specific details but rather depend on the symmetries (or equivariances) of the system $[153,154]$. Starting with an equation of the type

$$
\partial_{t} z(\mathbf{x})=F[z(\cdot)]
$$

equivariances restrict possible choices of $F[z(\cdot)]$. Wolf assumed equivariance with respect to translations

$$
\hat{T}_{\mathbf{y}} z(\mathbf{x})=z(\mathbf{x}+\mathbf{y})
$$

rotations

$$
\hat{R}_{\beta} z(\mathbf{x})=e^{2 i \beta} z\left(\Omega_{-\beta} \mathbf{x}\right)
$$


with $2 \times 2$ rotation matrix

$$
\Omega_{\beta}=\left(\begin{array}{cc}
\cos \beta & -\sin \beta \\
\sin \beta & \cos \beta
\end{array}\right)
$$

and reflections

$$
\hat{P} z(\mathbf{x})=\bar{z}(\Psi \mathbf{x}),
$$

where $\Psi=\operatorname{diag}(-1,1)$ is the $2 \times 2$ reflection matrix. In terms of the right hand side of the dynamics (Eq. (1.5.1)), equivariance means that

$$
\begin{aligned}
\hat{T}_{\mathbf{y}} F[z] & =F\left[\hat{T}_{\mathbf{y}} z\right] \\
\hat{R}_{\beta} F[z] & =F\left[\hat{R}_{\beta} z\right] \\
\hat{P} F[z] & =F[\hat{P} z] .
\end{aligned}
$$

It immediately follows that solutions of the model which can be converted into one another by translation, rotation, or reflection of the cortical layers represent equivalent model solutions, by construction. Wolf furthermore assumed the dynamics to be equivariant with respect to shifts in orientation $S_{\phi} z(\mathbf{x})=e^{i \phi} z(\mathbf{x})$, i.e.,

$$
e^{i \phi} F^{z}[z, \mathbf{r}]=F^{z}\left[e^{i \phi} z, \mathbf{r}\right] .
$$

Thus, two patterns are also equivalent solutions of the model if their layout of orientation domains is identical, but the preferred orientations differ everywhere by the same constant angle. To specify an analytically tractable instance of $F[z(\cdot)]$, Wolf incorporated basic features of intracortical connectivity into the model and restricted himself to a linear and a cubic term:

$$
\partial_{t} z(\mathbf{x})=L_{S H}[z]+N_{3}[z, z, \bar{z}] .
$$

The linear term $L_{S H}$ was chosen as the Swift-Hohenberg operator $L_{S H}[z]=r-\left(k_{c}+\Delta\right)^{2}$, wellknown to phenomenologically describe a system exhibiting a supercritical bifurcation where the homogeneous state loses stability with respect to a finite wavenumber [153-155]. As cubic nonlinearities, Wolf included both local and long-local interaction terms, inspired by the long-range orientation-selective horizontal connectivity found in V1 (e.g. [21, 44, 156-158]):

$$
N_{3}[z, z, \bar{z}]=(1-g)|z(\mathbf{x})|^{2} z(\mathbf{x})-\frac{(2-g)}{2 \pi \sigma^{2}} \int d^{2} y e^{-(\mathbf{x}-\mathbf{y})^{2} / 2 \sigma^{2}}\left(|z(\mathbf{y})|^{2} z(\mathbf{x})+\frac{1}{2} \bar{z}(\mathbf{x}) z^{2}(\mathbf{y})\right) .
$$

The Wolf model is a variational model, i.e. $\partial_{t} z(\mathbf{x})=-\frac{\delta}{\delta \bar{z}(\mathbf{x})} \mathcal{F}[z]$, with energy functional

$$
\begin{aligned}
\mathcal{F}[z]= & -\int d^{2} \mathbf{x} r|z(\mathbf{x})|^{2}+\int d^{2} \mathbf{x}\left(\left|\left(\Delta+k_{c}^{2}\right) z(\mathbf{x})\right|^{2}-\frac{(1-g)}{2}|z(\mathbf{x})|^{4}\right) \\
& +\frac{(2-g)}{4 \pi \sigma^{2}} \iint d^{2} y d^{2} x\left(|z(\mathbf{x})|^{2} e^{-(\mathbf{x}-\mathbf{y})^{2} / 2 \sigma^{2}}|z(\mathbf{y})|^{2}+\frac{1}{2} \bar{z}^{2}(\mathbf{x}) \int d^{2} y e^{-(\mathbf{x}-\mathbf{y})^{2} / 2 \sigma^{2}} z^{2}(\mathbf{y})\right) .
\end{aligned}
$$

The Wolf model is the first model for OPM development which was rigorously shown to exhibit quasiperiodic optima that quantitatively resemble experimentally observed OPMs [11, 123, 159]. 

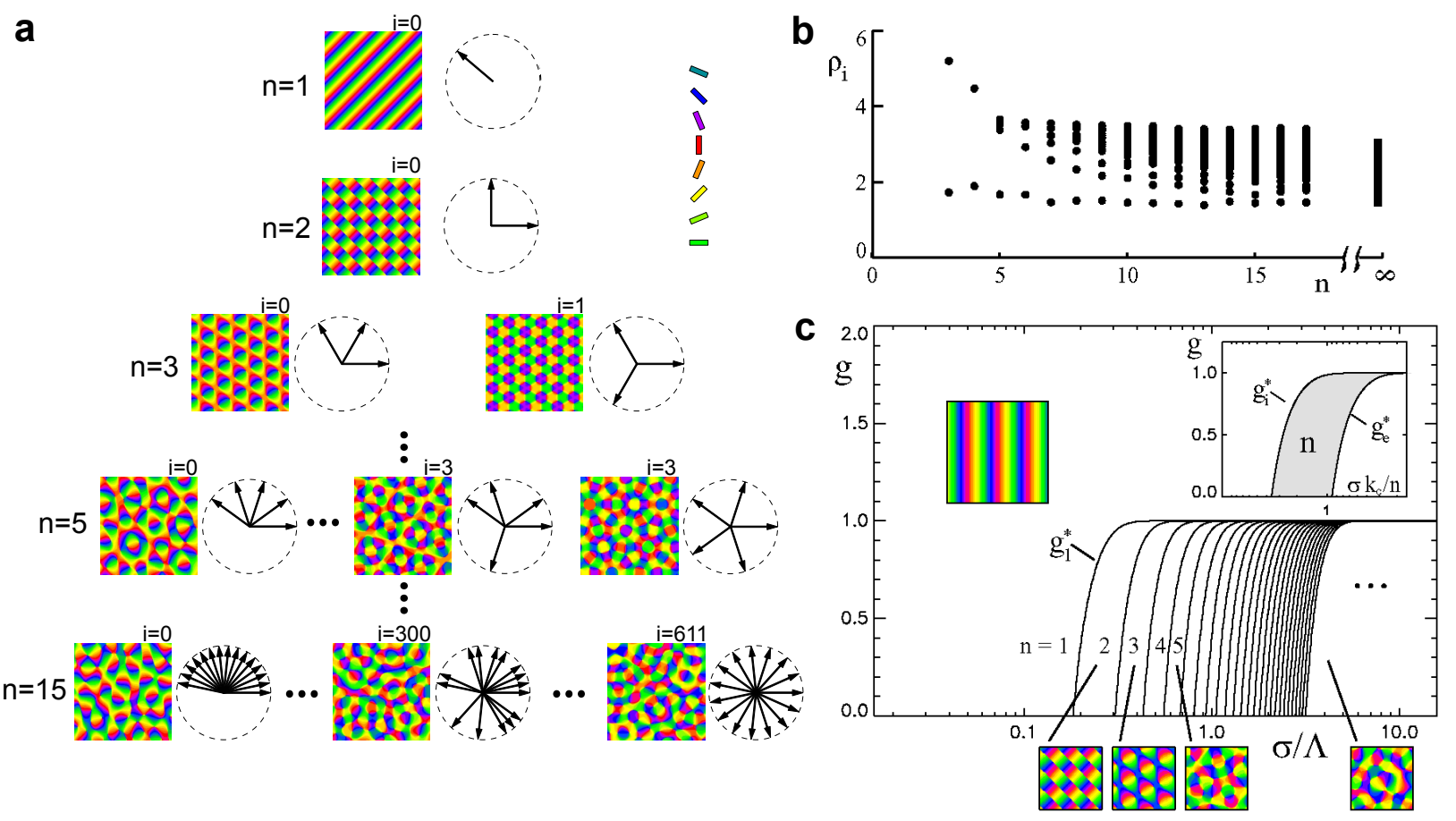

Figure 1.5.1: Essentially complex planforms (ECPs) and their stabilization by long-range interactions in the Wolf-model. (a) The family of ECPs (redrawn from [123]). The number of nonzero wave vectors is indicated by $n$. The index $i$ enumerates non-equivalent configurations of wave vectors with the same $n$, starting with $i=0$ for the most anisotropic planform. For $n=3,5$, and 15, there are 2, 4, and 612 different ECPs, respectively. OPM layouts become more irregular with increasing $n$. (b) Pinwheel densities of $n$-ECPs (from [123]). (c) Phase diagram of the Wolf-model. The graph shows regions in the $g-\sigma / \Lambda$-plane, where $n$-ECPs have minimal energy.

Because of the simplicity of the model, its approximate stationary solutions close to pattern formation threshold as well as their stability properties can be characterized completely by a mathematical formalism called weakly nonlinear analysis, which is outlined in the following.

\section{Weakly nonlinear analysis}

The order parameter model proposed by Wolf represents a partial integro-differential equation that is in general impossible to solve analytically. However, the symmetries (Eqs. (1.5.2)-(1.5.5)) can be employed to analytically determine two simple fixed-points of both dynamics: (i) the homogeneous non-selective state $z(\mathbf{x})=0$ and (ii) the single Fourier mode pinwheel free orientation stripe pattern $z(\mathbf{x})=A_{0} e^{i \mathbf{k x}}$ (Fig. 1.5.1a). Other solutions may only be determined in approximate form. To compute additional fixed points of (1.5.6), Wolf examined the stability of so-called planforms $[153,154,160]$, i.e. patterns that are composed of a finite number of Fourier components:

$$
z(\mathbf{x})=\sum_{j} A_{j}(t) e^{i \mathbf{k x}} .
$$

When the dynamics is close to a finite wavelength instability, the essential Fourier components of the emerging pattern are located on the critical circle $\left|\mathbf{k}_{j}\right|=k_{c}$. For a discrete number of $N$ 
Fourier components of $z(\mathbf{x})$ whose wave vectors lie equally spaced on the critical circle, the most general system of amplitude equations compatible with the symmetry assumptions has the form $[123,161]$

$$
\dot{A}_{i}=r A_{i}-A_{i} \sum_{j=1}^{N} g_{i j}\left|A_{j}\right|^{2}-\bar{A}_{i^{-}} \sum_{j=1}^{N} f_{i j} A_{j} A_{j^{-}},
$$

where $j^{-}$denotes the index of the mode antiparallel to the mode $j, \mathbf{k}_{j}=-\mathbf{k}_{j^{-}}$, and the coefficients $(\hat{g})_{i j}=g_{i j}=\left(1-\frac{1}{2} \delta_{i j}\right) g\left(\left|\alpha_{i}-\alpha_{j}\right|\right)$ and $(\hat{f})_{i j}=f_{i j}=\left(1-\delta_{i j}-\delta_{i^{-} j}\right) f\left(\left|\alpha_{i}-\alpha_{j}\right|\right)$ only depend on the angle $\left|\alpha_{i}-\alpha_{j}\right|$ between mode $i$ and $j$. The angle-dependent interaction functions $g(\alpha)$ and $f(\alpha)$ are obtained from the cubic nonlinearity $N_{3}^{z}[z, z, \bar{z}]$ by a multi-scale expansion $[153,160]$ as

$$
\begin{aligned}
g(\alpha)= & -e^{-i \mathbf{k}_{0} \mathbf{x}}\left[N_{3}^{z}\left(e^{i \mathbf{k}_{0} \mathbf{x}}, e^{i \mathbf{h}(\alpha) \mathbf{x}}, e^{-i \mathbf{h}(\alpha) \mathbf{x}}\right)\right. \\
& \left.+e^{-i \mathbf{k}_{0} \mathbf{x}} N_{3}^{z}\left(e^{i \mathbf{h}(\alpha) \mathbf{x}}, e^{-i \mathbf{h}(\alpha) \mathbf{x}}, e^{i \mathbf{k}_{0} \mathbf{x}}\right)\right] \\
f(\alpha)= & -\frac{1}{2} e^{-i \mathbf{k}_{0} \mathbf{x}}\left[N_{3}^{z}\left(e^{i \mathbf{h}(\alpha) \mathbf{x}}, e^{-i \mathbf{h}(\alpha) \mathbf{x}}, e^{i \mathbf{k}_{0} \mathbf{x}}\right)\right. \\
& \left.+e^{-i \mathbf{k}_{0} \mathbf{x}} N_{3}^{z}\left(, e^{-i \mathbf{h}(\alpha) \mathbf{x}}, e^{i \mathbf{h}(\alpha) \mathbf{x}}, e^{i \mathbf{k}_{0} \mathbf{x}}\right)\right],
\end{aligned}
$$

where $\mathbf{k}_{0}=k_{c}(1,0)$ and $\mathbf{h}(\alpha)=k_{c}(\cos \alpha, \sin \alpha)$. By definition, both angle-dependent interaction functions are $2 \pi$-periodic. The function $f(\alpha)$ is also periodic with a period of $\pi$ since the right hand side of Equation (1.5.9) is invariant with respect to the transformation $\mathbf{h}(\alpha) \rightarrow \mathbf{h}(\alpha+\pi)=$ $-\mathbf{h}(\alpha)$. If the cubic nonlinearity $g_{s r} N_{l o c a l}[z]+g_{l r} N_{3}[z, z, \bar{z}]$ obeys the so-called permutation symmetry

$$
N_{3}^{z}[u, v, w]=N_{3}^{z}[w, u, v],
$$

the function $g(\alpha)$ is $\pi$-periodic as well (cf. also [162]). In this case, it can be shown that all realvalued solutions of the amplitude equations are intrinsically unstable [123, 161]. The dynamics of the amplitude as given by Equation (1.5.7) is potential if $g_{i j}$ and $f_{i j}$ are real-valued. The corresponding energy is

$$
U_{A}=-r \sum_{i=1}^{N}\left|A_{i}\right|^{2}+\frac{1}{2} \sum_{i, j=1}^{N} g_{i j}\left|A_{i}\right|^{2}\left|A_{j}\right|^{2}+\frac{1}{2} \sum_{i, j=1}^{N} f_{i j} \bar{A}_{i} \bar{A}_{i^{-}} A_{j} A_{j^{-}} .
$$

What are nontrivial stationary solutions of these dynamic equations? The above orientation stripe solution with one single nonzero wave vector $z(\mathbf{x})=A_{0} e^{i \mathbf{k x}}$ and amplitude

$$
\left|A_{0}\right|^{2}=\frac{r}{g_{i i}}
$$

is the simplest such solution. In [123], the family of so-called essentially complex planforms (ECPs) has been introduced as solutions of Eq. (1.5.7). This solution class encompasses a large variety of quasiperiodic OPM layouts and is therefore an suitable candidate solution class for models of 
OPM development. An $n$-ECP solution is given by

$$
z(\mathbf{x})=\sum_{j=1}^{n} A_{j} e^{i l_{j} \mathbf{k}_{j} \mathbf{x}},
$$

with $n$ wave vectors $\mathbf{k}_{j}=k_{c}(\cos (\pi j / n), \sin (\pi j / n))$ distributed equidistantly on the upper half of the critical circle, complex amplitudes $A_{j}$ and $l_{j}= \pm 1$ determining whether the mode with wave vector $\mathbf{k}_{j}$ or $-\mathbf{k}_{j}$ is active (nonzero). As these planforms cannot realize a real-valued function they are called essentially complex [123]. For an $n$-ECP, the third term on the right hand side of Equation (1.5.7) vanishes and the amplitude equations for the active modes $A_{i}$ reduce to a system of Landau equations

$$
\dot{A}_{i}=r A_{i}-A_{i} \sum_{j=1}^{n} g_{i j}\left|A_{j}\right|^{2},
$$

where $g_{i j}$ is the $n \times n$-coupling matrix for the active modes. Consequently, the stationary amplitudes obey

$$
\left|A_{i}\right|^{2}=r \sum_{j=1}^{n}\left(\mathbf{g}^{-1}\right) i j
$$

The energy of an $n$-ECP is given by

$$
U_{\mathrm{ECP}}=-\frac{1}{2} \sum_{i, j}\left(\mathbf{g}^{-1}\right)_{i j}
$$

The family of $n$-ECP solutions is depicted in Figure 1.5.1a. The 1-ECP corresponds to the pinwheelfree stripe pattern. For fixed $n \geq 3$, there are multiple planforms not related by symmetry operations, which considerably differ in their spatial layouts. For $n \geq 4$, the patterns are spatially quasi-periodic. Figure 1.5.1b depicts the pinwheel densities of planform solutions as a function of the number of active modes. For $n \rightarrow \infty$, the average pinwheel density of the set of $n$-ECPs approaches $\pi[11,94,163]$. For $n \geq 8$, the family of $n$-ECPs has been shown to accurately reproduce the pinwheel statistics found in experimentally measured OPMs (see Fig. 1.2.2 and [11, 159]). Fig. 1.5.1c shows the phase diagram of the Wolf model as obtained with the above introduced weakly nonlinear analysis. Shown are the regions in parameter space in which $n$-ECPs have minimal energy. ECP solutions with the same number of active modes are energetically degenerate due to the permutation symmetry (Eq. (1.5.10)) exhibited by the model [123]. The main observation is that realistic aperiodic OPM layouts are stable solutions of the dynamics (Eq. (1.5.6)) if non-local interactions are sufficiently strong and long-ranged. In Chapter 5, I generalize and extend these results by considering optimization models for the joint development of the system of long-range tangential connections and OPMs which include a variety of long-range interaction types.

I conclude this section by noting that the Shift symmetry assumption (Eq. (1.5.5)) has been challenged by recent investigations $[58,122,124,126,164,165]$ and dropping this assumption appears to have interesting effects on pattern formation dynamics and the spatial layout of stationary solutions [58, 126]. However, for reasons of simplicity, throughout this thesis, Shift-symmetric model instances are considered. 
Figure 1.5.2: Retinotopic distortions and OPMs together form a dimension reducing mapping in the classical Elastic Network (EN) model (mod. from [134]). Left: Map in visual space, simultaneously showing the retinotopic distortions and the rate of change of preferred orientation (filled squares). The distorted grid represents the cortical array of cells. Each grid intersection is at the receptive field center of the corresponding cell. The size of the squares is proportional to the rate of change of the preferred orientations. Right: OPM obtained in a the same numerical simulation of the EN model.
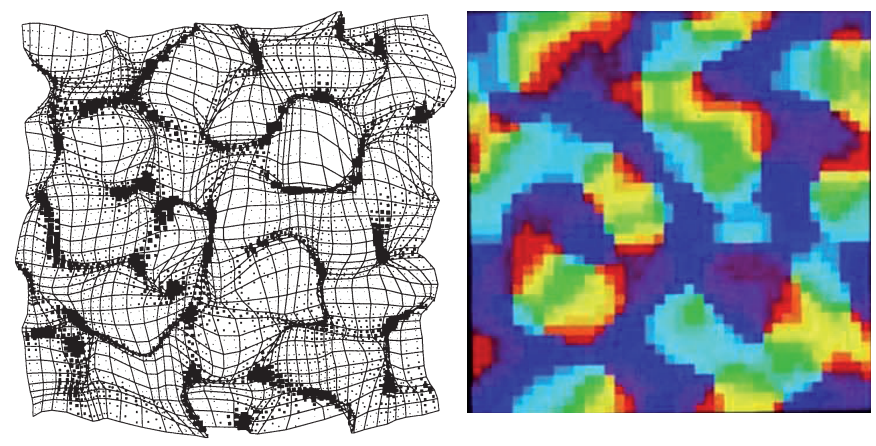

\subsubsection{The Elastic Network model}

The Wolf model establishes that the refinement of the visual cortical architecture and the postnatal emergence of orientation preference maps can be modeled as a dynamical optimization process. Yet, given its high level of abstraction, many questions remain unanswered: How does the typical scale arise during postnatal development? What functional significance does it have? Does the observed aperiodic layout of OPMs serve a specific purpose in visual information processing? These questions can only be answered by more detailed optimization models which postulate explicit functional requirements for primary visual cortical architecture and whose analysis turns out to be more difficult. One of the most promising optimization models of such type is the so-called Elastic Network (EN) model first introduced by Durbin and Mitchison [134]. In this dimension reduction model, the columnar cortical organization is hypothesized to represent an optimal trade-off between the coverage of the space of visual stimulus features and the continuity of their cortical representation [134-142] (cf. Sec. 1.5). The EN model has been used in numerous numerical studies, and good qualitative agreement between the layout of numerically obtained dimension reducing maps and experimental observations have been reported (e.g. [134, 140, 141, 143], cf. Fig. 1.5.2). Yet comprehensive analytical investigations of this model and, in particular, the determination of its optimal solutions are still missing.

In Chapter 3, I give a mathematical analysis of the EN model for the joint optimization of position and orientation selectivity. In this classical version of the EN model, the retinotopic map $(\mathrm{RM})$ is represented by a mapping $\mathbf{R}(\mathbf{x})=\left(R_{1}(\mathbf{x}), R_{2}(\mathbf{x})\right)$ which describes the receptive field center position of a neuron at cortical position $\mathbf{x}$. Decomposing the retinotopic map into an affine transformation $\mathbf{x} \mapsto \mathbf{X}$ from cortical to visual field coordinates and a superimposed vector-field of retinotopic distortions $\mathbf{r}(\mathbf{x})$ yields

$$
\mathbf{R}(\mathbf{x})=\mathbf{X}+\mathbf{r}(\mathbf{x})
$$

with appropriately chosen units for $\mathbf{x}$ and $\mathbf{R}$. The OPM is represented by a second complexvalued scalar field $z(\mathbf{x})$ as introduced above. Solving the EN model requires to find pairs of maps $\{\mathbf{r}(\mathbf{x}), z(\mathbf{x})\}$ that represent an optimal compromise between stimulus coverage and map continuity (Fig. 1.5.2). This is achieved by minimizing a free energy functional

$$
\mathcal{F}[z, \mathbf{r}]=\sigma^{2} \mathcal{C}[z, \mathbf{r}]+\mathcal{R}[z, \mathbf{r}]
$$

in which the functional $\mathcal{C}$ measures the coverage of a stimulus space and the functional $\mathcal{R}$ the continuity of its cortical representation. The stimulus space is defined by an ensemble $\{\mathbf{S}\}$ of 
idealized point-like stimuli, each described by two features: $s_{z}=\left|s_{z}\right| e^{2 i \theta}$ and $\mathbf{s}_{r}=\left(s_{x,}, s_{y}\right)$ which specify the orientation $\theta$ of the stimulus and its position in visual space $\mathbf{s}_{r} . \mathcal{C}$ and $\mathcal{R}$ are given by

$$
\begin{aligned}
\mathcal{C}[z, \mathbf{r}] & =-\left\langle\ln \int d^{2} y e^{-\left(\left|s_{z}-z(\mathbf{y})\right|^{2}+\left|\mathbf{s}_{r}-\mathbf{X}-\mathbf{r}(\mathbf{y})\right|^{2}\right) / 2 \sigma^{2}}\right\rangle_{\mathbf{s}} \\
\mathcal{R}[z, \mathbf{r}] & =\frac{1}{2} \int d^{2} y \eta\|\nabla z(\mathbf{y})\|^{2}+\eta_{r} \sum_{j=1}^{2}\left\|\nabla r_{j}(\mathbf{y})\right\|^{2},
\end{aligned}
$$

with $\nabla=\left(\partial_{x}, \partial_{y}\right)^{T}$, and $\eta \in[0,1]$. The ratios $\sigma^{2} / \eta$ and $\sigma^{2} / \eta_{r}$ control the relative strength of the coverage term versus the continuity term for OPM and RM, respectively. $\langle\cdots\rangle_{\text {S }}$ denotes the average over the ensemble of stimuli. Minima of the energy functional $\mathcal{F}$ are stable fixed points of the gradient descent dynamics

$$
\begin{aligned}
& \partial_{t} z(\mathbf{x})=-2 \frac{\delta \mathcal{F}[z, \mathbf{r}]}{\delta \bar{z}(\mathbf{x})}=\left\langle\left[s_{z}-z(\mathbf{x})\right] e(\mathbf{x}, \mathbf{S}, z, \mathbf{r})\right\rangle_{\mathbf{S}}+\eta \triangle z(\mathbf{x}) \\
& \partial_{t} \mathbf{r}(\mathbf{x})=-\frac{\delta \mathcal{F}[z, \mathbf{r}]}{\delta \mathbf{r}(\mathbf{x})}=\left\langle\left[\mathbf{s}_{r}-\mathbf{X}-\mathbf{r}(\mathbf{x})\right] e(\mathbf{x}, \mathbf{S}, z, \mathbf{r})\right\rangle_{\mathbf{S}}+\eta_{r} \triangle \mathbf{r}(\mathbf{x}),
\end{aligned}
$$

where $e(\mathbf{x}, \mathbf{S}, z, \mathbf{r})$ is given by

$$
e(\mathbf{x}, \mathbf{S}, z, \mathbf{r})=\frac{e^{-\left(\left|\mathbf{s}_{r}-\mathbf{X}-\mathbf{r}(\mathbf{x})\right|^{2}\right) / 2 \sigma^{2}} e^{-\left(\left|s_{z}-z(\mathbf{x})\right|^{2}\right) / 2 \sigma^{2}}}{\int d^{2} y e^{-\left(\left|\mathbf{s}_{r}-\mathbf{X}-\mathbf{r}(\mathbf{y})\right|^{2}\right) / 2 \sigma^{2}} e^{-\left(\left|s_{z}-z(\mathbf{y})\right|^{2}\right) / 2 \sigma^{2}}} .
$$

and $\Delta$ is the Laplacian operator. This dynamics will be referred to as EN dynamics throughout this thesis. When Equations (1.5.16,1.5.17) are viewed as a learning dynamics, $e(\mathbf{x}, \mathbf{S}, z, \mathbf{r})$ represents the activity pattern, evoked by a stimulus $\mathbf{S}=\left(\mathbf{s}_{r}, s_{z}\right)$ in a model cortex with retinotopic distortions $\mathbf{r}(\mathbf{x})$ and OPM $z(\mathbf{x})$. The development of the two cortical maps is then driven by an ensemble of stimuli. Each stimulus $\mathbf{S}$ is assumed to cause a small plastic change in the receptive field parameters at activated locations, which is proportional to their response $e(\mathbf{x}, \mathbf{S}, z, \mathbf{r})$. The change is such that the matching between receptive fields and afferent activity patterns is enhanced. This represents a classical Hebbian learning scheme $[142,149,166]$. The Laplacian term can be derived from interactions mediated by lateral spread of activity through excitatory cortical connections [144].

In Chapter 3, I develop a mathematical formalism to compute approximate fixed-points of the above coupled dynamics of retinotopic distortions and orientation preference maps via weakly nonlinear analysis (cf. Sec. 1.5.1). This enables a precise characterization of optimal dimension reducing mappings predicted by the EN model and a quantitative comparison to experimentally measured OPMs. 


\subsection{Retinal constraints on the spatial structure of orientation prefer- ence maps}

In an approach somehow opposing the various self-organization models for cortical development, orientation-selective responses in V1 are viewed as a constraint imposed by the nature of retinal inputs (relayed by the LGN) to the primary visual cortex. Recently, it has been shown that this modeling approach makes distinct predictions as to the spatial arrangement of V1 orientation columns [168]. To understand these implications, it is necessary to review some basic facts about the response properties and spatial arrangements of RGCs in the mammalian retina.

There are two principle types of RGCs: those with ON-center receptive field that respond at the onset of a small bright light centered in the right position (Fig. 1.6.1a, upper panels) and those with OFF-center receptive field that respond at the offset of a small bright light (Fig. 1.6.1a, lower panels). ON-center cells also respond at the offset of an annulus of light and OFF-center cells respond at the onset of the annulus of light. Neither of the two cells responds well to an overall change in brightness covering a large area. As Figure 1.6.1a illustrates, ON- and OFF-center ganglion cells with superimposed receptive fields give complementary responses. When the ON-center cell responds strongly, the OFF-center cell is quiet and vice versa. ON- and OFF-receptive fields of RGCs have been shown to be well-described by a difference of Gaussians [169]. Regarding their spatial arrangement, Wässle and his collaborators have shown that RGCs of the cat are arranged in a lattice-like fashion (also called RGC mosaics) with regular cell-to-cell spacings [167, 170] (see Fig. 1.6.1b). ON-center and OFF-center RGCs form their own lattices, and the ON- and OFF-lattices are superimposed independently of each other [171]. Assuming that the signals from RGCs are relayed through the LGN without substantial modification (but see [20]), the ON/OFF-RGC mosaic represents the pattern of visual input to the cortex. Thus, as already noticed by Wässle et al. [167], the RGC mosaic may play an important role in constraining the construction of receptive fields in visual cortical areas such as V1 [167]. Based on the results by Wässle and co-workers, Soodak modeled receptive fields at visual pathway stages beyond the retina as a convergence of Gaussian subunits [172]. He showed that a great variety of realistic receptive fields including orientationselective ones can be obtained by just a linear summation of Gaussian subunit responses. Using the lattice structure of the ON-cell and OFF-cell mosaics, Soodak furthermore demonstrated that such a purely feedforward theory of orientation preference also predicts its continuous spatial arrangement as observed in V1 of carnivorans and primates [173]. Experimental support for OPM models based on the receptive field arrangement of ON- and OFF-LGN inputs was provided by a study in which the preferred orientation of a V1 column was shown to be rather well-predicted by calculating the population receptive field of its thalamic inputs [174] (see also [175]). In a series of papers, Ringach and co-workers have further elaborated Soodak's hypothesis to account for the precise functional organization of iso-orientation domains in V1 into OPMs [168, 176, 177]. Finally, a very recent study by Paik and Ringach advances the above theoretical results by proposing how maps with a typical column spacing can arise in feedforward models for orientation preference via a Moiré interference mechanism between the mosaic of ON-cells and the mosaic of OFF-cells [168, 178] (cf. Fig. 1.6.1c). Assuming that both of these mosaics have hexagonal statistics, the resulting Moiré interference pattern exhibits a hexagonal arrangement of orientation columns [179] with a typical column spacing, an example of which is depicted in Figure 1.6.1d. In support of their model, Paik and Ringach presented evidence for hexagonal order in cortical OPMs of tree 
a
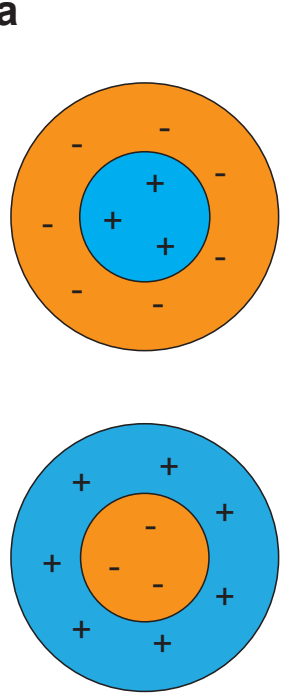

center

surround

center \& surround

\section{stimulus \\ center \\ surround \\ center \& center \&
surround}
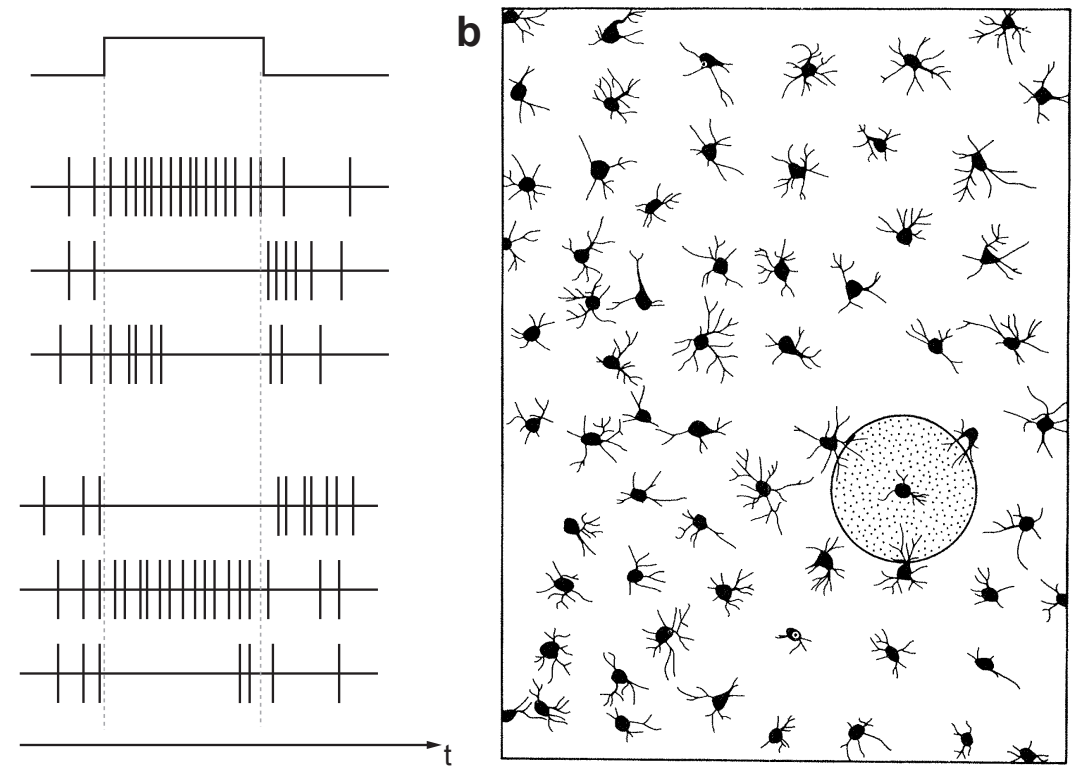

C

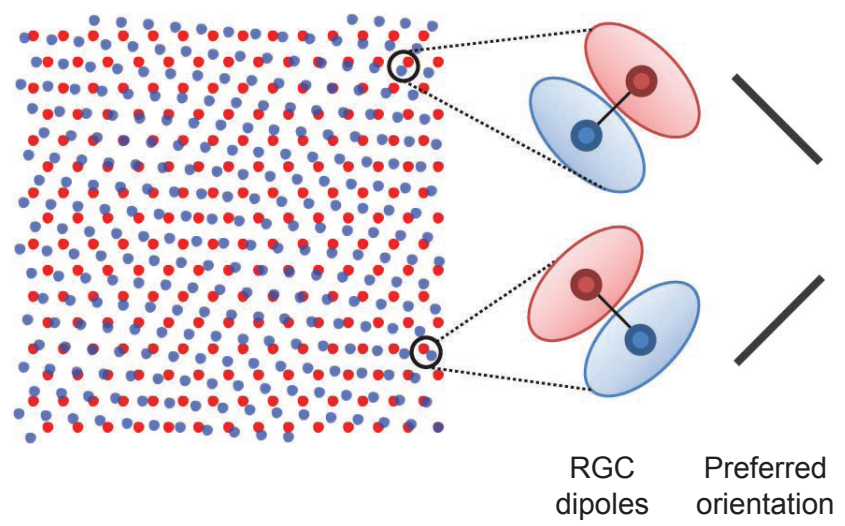

d

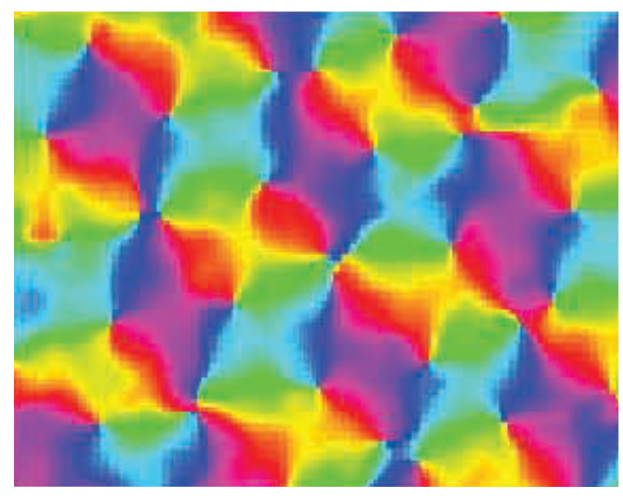

Figure 1.6.1: Hexagonal orientation maps by Moiré interference between ON- and OFF-RGC mosaics. (a) Schematic illustration of the two principle response types of ON-center (upper) and OFF-center RGCs. The upper curve on the right shows a time course of a light stimulus with light either in the center, the surround, or both. Spike trains below indicate typical responses of ON- and OFF-cells. (b) Mosaic formed by the ON-cells in the cat retina (from [167]). Cell positions are arranged in a lattice-like mosaic with regular cell-to-cell spacings. (c) (Left) The superposition of two hexagonal lattices results in a periodic, hexagonal interference pattern. (Right) Pooling of inputs from an RGC dipole results in oriented simple-cell receptive fields with side-by-side ON- and OFF-subregions (mod. from [168]). (d) Hexagonal orientation map, predicted by the Moiré interference patterns between ON- and OFF-RGC mosaics (mod. from [168]). 
shrews, macaque monkeys, ferrets, and cats [168].

It is noteworthy that both theories, the self-organization model with long-range interactions by Wolf [123] and the theory of Moiré interference patterns by Ringach and co-workers [168] make very different predictions about the spatial arrangement of orientation columns. In the Wolf model, aperiodic pinwheel-rich states that lack any hexagonal symmetry by definition are predicted. The average pinwheel density for these states approaches $\pi$ and distinct nearest neighbor pinwheel statistics are predicted. These predictions were quantitatively confirmed in a large data set of orientation maps of tree shrews, galagos, and ferrets [11, 159]. In contrast, Paik \& Ringach observed a predominantly hexagonal spatial arrangement of orientation maps in a small set of experimental maps. Reconciling the above contradictory findings represents an obvious essential step for understanding the general principles that shape the layout of functional visual cortical organization. This step is carried out in Chapter 4 through the analysis of a large data set of orientation maps ( $>90$ maps) from tree shrews, galagos, and ferrets in the search for signatures of hexagonal organization. It should be emphasized at this point that about half of the theoretical studies on the self-organization of OPMs in the past have predicted different types of hexagonal organizations [109, 118, 125, 129], including the earliest one almost 40 years ago [109]. Hence, hexagonal structure in OPMs, if present, does not distinguish the feedforward theory of OPMs as Moiré interference patterns from many other models for self-organized orientation map development. In view of this, the results of Chapter 4 are not only of importance for resolving the puzzling contradictions between the findings of Paik \& Ringach and Kaschube et al.. They also check the predictions of a large number of past theoretical studies on the development of OPMs. 


\section{Chapter 2}

\section{Reorganization of columnar architecture in the growing visual cortex}

\section{Citation}

Keil W, Schmidt K-F, Löwel S, and Kaschube M Reorganization of columnar architecture in the growing visual cortex. Proc Nat Acad Sci USA 2010 107(27):12293-12298.

(C2010 Keil, Schmidt, Löwel, and Kaschube

\section{Original contribution}

I conceived and designed the study together with M. Kaschube and S. Löwel. I contributed the analysis of the experimental data and performed the theoretical analyses and numerical simulations. I wrote the manuscript as well as the Supplementary Information together with M. Kaschube and Siegrid Löwel. 



\title{
Reorganization of columnar architecture in the growing visual cortex
}

\author{
Wolfgang Keila,b,c, Karl-Friedrich Schmidtt ${ }^{\mathrm{d}}$, Siegrid Löwel ${ }^{\mathrm{d}}$, and Matthias Kaschube ${ }^{\mathrm{b}, \mathrm{c}, 1}$ \\ ${ }^{a}$ Max Planck Institute for Dynamics and Self-Organization, Göttingen 37073, Germany; 'bewis-Sigler Institute for Integrative Genomics, Princeton \\ University, Princeton, NJ 08544; 'Physics Department, Princeton University, Princeton, NJ 08544; and Institute of General Zoology and Animal Physiology, \\ Friedrich Schiller University, Jena 07743, Germany
}

Edited* by Charles F. Stevens, The Salk Institute for Biological Studies, La Jolla, CA, and approved May 25, 2010 (received for review November 17, 2009)

Many cortical areas increase in size considerably during postnatal development, progressively displacing neuronal cell bodies from each other. At present, little is known about how cortical growth affects the development of neuronal circuits. Here, in acute and chronic experiments, we study the layout of ocular dominance (OD) columns in cat primary visual cortex during a period of substantial postnatal growth. We find that despite a considerable size increase of primary visual cortext, the spacing between columns is largely preserved. In contrast, their spatial arrangement changes systematically over this period. Whereas in young animals columns are more band-like, layouts become more isotropic in mature animals. We propose a novel mechanism of growth-induced reorganization that is based on the "zigzag instability," a dynamical instability observed in several inanimate pattern forming systems. We argue that this mechanism is inherent to a wide class of models for the activity-dependent formation of OD columns. Analyzing one representative of this class, the Elastic Network model, we show that this mechanism can account for the preservation of column spacing and the specific mode of reorganization of OD columns that we observe. We conclude that column width is preserved by systematic reorganization of neuronal selectivities during cortical expansion and that this reorganization is well described by the zigzag instability. Our work suggests that cortical circuits may remain plastic for an extended period in development to facilitate the modification of neuronal circuits to adjust for cortical growth

cortical growth | ocular dominance columns | postnatal development | critical period | zigzag instability

$T^{\prime}$

he brain of most mammalian species grows substantially during postnatal development without a significant change in the number of neurons. The human brain, for instance, weighs on average $350 \mathrm{~g}$ in newborns and $1,400 \mathrm{~g}$ in adult males (1). In cat, the neocortical volume increases from $\approx 1,000 \mathrm{~mm}^{3}$ at birth to $\approx 4,500 \mathrm{~mm}^{3}$ in adulthood (2). Consistently, the surface area of cat primary visual cortex (area $17=\mathrm{V} 1$ ) increases postnatally by a factor of 2.5 between week 1 and week 12 (3). This size increase implies that the distance between any two neuronal cell bodies grows on average by a factor of 1.6 during postnatal development. Do neuronal processes such as axons and dendrites simply elongate or are larger changes needed to accommodate this growth? It is not known at present how the brain achieves permanen adjustment of its functional wiring to the changing physical proportions while at the same time being fully functional at every moment. Whereas the importance of mechanical factors is appreciated in a number of growth-related phenomena in biology such as morphogenesis (4), heart development (5), and tumor growth (6), their possible impact on functional aspects of neural circuits has received relatively little attention.

In cat $\mathrm{V} 1$, much of the growth takes place during a period in which most parts of the visual field are already represented (7) and many neurons have already reached fairly mature levels of selectivity. For instance, the selective response of visual cortical neurons to inputs from one eye or the other, called ocular dominance (OD), can already be visualized at postnatal week
2 in cat V1 (8) (Fig. 1A). OD is organized into columns that can be labeled over the full extent of V1 as early as week 3 (9) (Fig. $1 B$ ). For these properties, this system is well suited for studying the impact of cortical growth on neural circuitry.

What happens to cortical columns when the cortex is growing in size? The seemingly simplest scenario, in which new columns are inserted into the cortex, appears rather implausible because the number of neurons remains largely constant during this period (10). In fact, most of the area increase is due to the generation of glial cells, the addition of more vasculature and connective tissue, and the myelinization of axons. To a lesser extent it also reflects the outgrowth and elaboration of axonal and dendritic processes (11). Therefore, a different scenario has been suggested, sometimes referred to as the "balloon effect," in which columns expand by a similar factor as the surrounding cortical tissue [see, e.g., (3)]. In this study, we start out by testing the balloon hypothesis for the case of OD columns in cat visual cortex. We show that the expected expansion of columns during cortical growth does not take place. Instead, columnar layouts reorganize over the considered period and become more isotropic in older animals. These observations strongly argue against a simple balloon-like expansion and imply that cortical circuits can respond to the constraints arising during growth by a different as yet unknown mechanism.

In order to account for our empirical observations, a fraction of neurons must either shift their relative spatial location or, alternatively, alter their functional response properties. Although appealing, the former possibility is difficult to address at present, because little is known about coherent motion of groups of neurons in response to mechanical tension (12-14). In contrast, a large body of experimental and theoretical work exists addressing phenomena related to cortical plasticity and demonstrating the impressive susceptibility of neural circuits to changes in activity patterns, frequently in the context of $\operatorname{OD}(15,16)$. Furthermore, it is noteworthy that in the two most intensely studied animal models for cortical plasticity, namely the cat and the mouse, the period of brain and body growth coincide with and end at about the same time as the period that allows for intense restructuring of neuronal connections (17-19). In this study, we therefore explore the latter possibility and analyze the predicted reorganization in models for the activity-dependent formation of OD columns. Based on general properties of these models, we develop a scenario of growth-induced cortical reorganization. Characteristic features of this reorganization as well as the time scale on which it evolves are in good agreement with the changes in columnar layout we observe during postnatal growth in cat V1.

Author contributions: W.K., S.L., and M.K. designed research; W.K., K.-F.S., S.L., and M.K. performed research; W.K. and M.K. analyzed data; and W.K., S.L., and M.K. wrote the paper.

The authors declare no conflict of interest.

This Direct Submission article had a prearranged editor.

'To whom correspondence should be addressed. E-mail: kaschube@princeton.edu. This article contains supporting information online at www.pnas.org/lookup/supp// doi:10.1073/pnas.0913020107/-IDCSupplemental. 


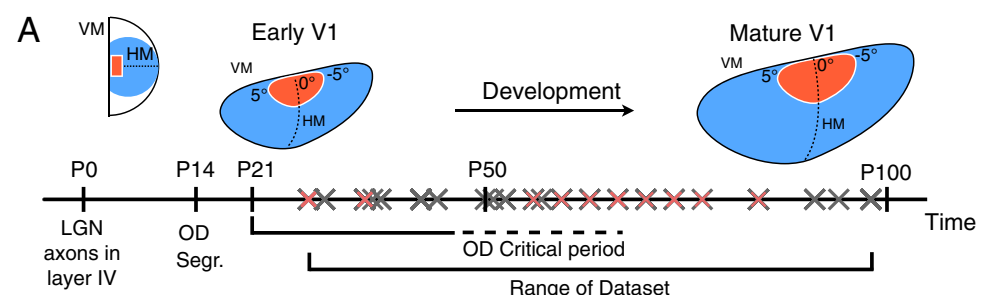

B

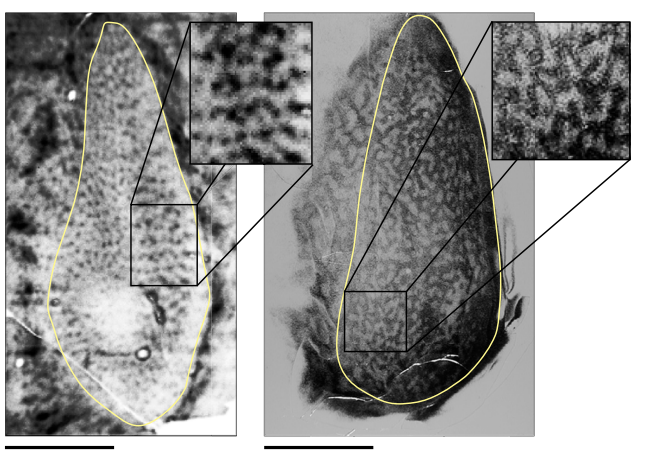

E
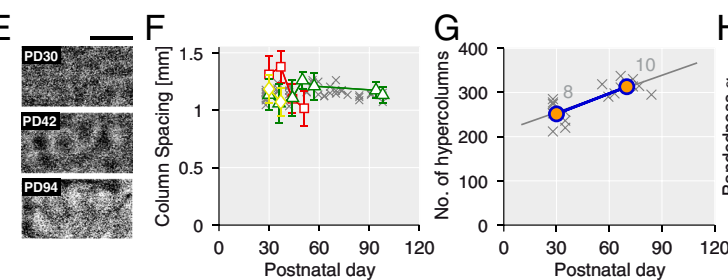

Results

The Spacing of OD Columns Is Preserved Over a Period of Cortical

Growth. We first measured the size increase of cat V1 during early postnatal development (Fig. 1A). We labeled complete layouts of OD columns in V1 visualized by either $2-\left[{ }^{14} \mathrm{C}\right]$-deoxyglucose (2-DG) or $\left.{ }^{3} \mathrm{H}\right]$-proline in kittens at different ages between postnatal day (PD) 28 and PD98 $(N=18$ hemispheres, Fig. $1 B)$. V1 is readily discernible by its distinctive columnar activation pattern in comparison to the labeling in surrounding cortical areas (20). In particular, V1 is distinguished from the secondary visual cortex (area $18=\mathrm{V} 2$ ) based on its considerably smaller column spacing. We observed a size increase of about a factor of 1.3 between two groups centered at PD30 and PD70 $(r=0.62, p<0.006)$ (Fig. 1C). To reduce possible influences of genetic variability (21), we analyzed a littermate couple on PD30 and PD72. Consistent with our previous results, V1 area is a factor of 1.46 larger in the older kitten. Thus, our analyses confirm previous studies $(3,9)$ by observing a considerable size increase of V1 during cat postnatal development.

We next asked whether the spacing of OD columns increases by a corresponding factor over this period. First, we measured the column spacing $\Lambda$ of 2-DG/proline-labeled OD patterns in $N=$ 41 hemispheres between PD28 and PD98 (data includes the $N=$ 18 hemispheres used for the analyses of V1 sizes). To obtain accurate estimations of column spacings, we used the wavelet method introduced in $(21,20)$ (SI Appendix). As shown in Fig. $1 D$, column spacings vary between $1.05 \mathrm{~mm}$ and $1.28 \mathrm{~mm}$, but do not show a significant increase over this period $(r=-0.034, p<$ 0.83 ). Consistent with this observation, the column spacings of size of $46 \%$.
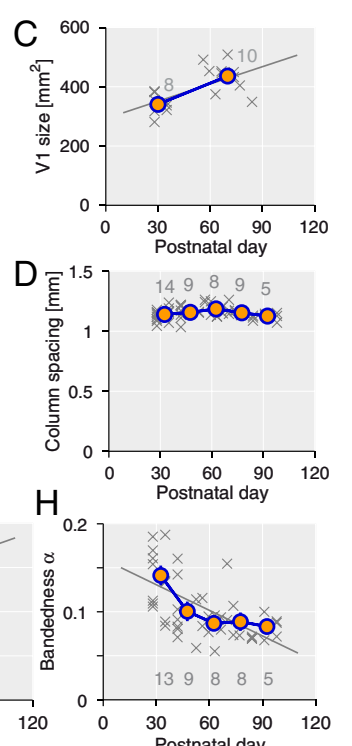

Fig. 1. Reorganization of $O D$ columns in cat V1 over a period of cortical growth. (A) Growth of cat V1, the representation of the visual hemifield (blue area, V1; red area, central visual field representation; $\mathrm{HM}$, horizontal meridian; VM, vertical meridian), and the time line of cat $O D$ development. Our dataset includes 2-DG/prolinelabeled hemispheres from kittens between PD28 and PD98 (2-DG, $N=37$; proline, $N=4)$ and chronic optical recordings between PD30 and PD98 (crosses mark indivi-
dual data points). (B) Two representative examples of OD layouts. Left, at PD30 (2$\mathrm{DG}) ;$ right, at PD60 (proline). Yellow lines mark $\mathrm{V} 1$ borders (scale bar, $10 \mathrm{~mm}$ ). (C) Area sizes of $\mathrm{V} 1$ for the $N=18$ kittens with complete reconstructions of $\mathrm{V} 1$ (red-marked crosses in A). Blue-orange dots represent averages over pools of sizes denoted by the gray numbers. Gray crosses mark values for individual animals. Gray line shows linear regression $(r=0.62, p<0.006)$. ( $D)$ OD column spacings $A$ do not increase over this ceriod ( $r=-0.034, p<0.83$ ). (E) OD columns in cat V1 by intrinsic signal optical imaging [same animal; high-pass filtered (SI Appendix); scale bar, $1.5 \mathrm{~mm}$ ]. (F) Column spacings $\Lambda$ for the case in (E) (green triangles; error bars by bootstrapping) and for two other cases (red boxes, yellow diamonds) corroborating thes, yellow dia(shown in light gray for comparison). (G and $H$ ) Whereas the number of hypercolumns $N_{\mathrm{HC}}$ increases over this period $(G)(r=$ $0.77, p<0.0002)$, the bandedness $\alpha$ decreases considerably $(H) \quad(r=-0.58, p<$ $6 \cdot 10^{-5}$ ). Note that error bars for pool averages averages in $C, D, G$, and $H$ are smaller than
the symbol size.

the two littermates differ by $<10 \%$, despite their difference in V1

To follow the development of column spacings in individual hemispheres, we visualized OD columns by chronic optical imaging $(N=3$ hemispheres; total age range PD30-PD98) (Fig. $1 E)$. We quantified their spacings by the above wavelet method (Fig. $1 F$ ). Whereas column spacings based on optical recordings exhibit larger variability compared to the $2 \mathrm{DG} /$ proline data (Fig. $1 F$ ), we found no systematic increase of column spacings in individual animals, thus confirming the conclusions drawn from the $2 \mathrm{DG} /$ proline data. Increased variability might be explained by the substantial intraareal variability of OD column spacings (20) together with the fact that the imaged regions were much smaller than V1 and may have shifted with age.

Taken together, both the 2-DG/proline data and the chronic optical recordings demonstrate that the postnatal growth of cat V1 is not accompanied by a corresponding increase in the spacing of OD columns, strongly arguing against the balloon scenario.

OD Columns Reorganize During Cortical Growth. An increase of area without a change in column spacing indicates an increase in the number of hypercolumns. The concept of a hypercolumn is related to that of a functional module and denotes a cortical unit containing a full set of values for any given set of receptive field parameters (22). We roughly estimated the typical size of a hypercolumn by $\Lambda^{2}$ (SI Appendix) and defined the number of hypercolumns in a map by $N_{\mathrm{HC}}=A / \Lambda^{2}(20)$, where $A$ is its total area. Fig. $1 G$ shows that for the $N=18$ completely reconstructed hemispheres from Fig. $1 C$ the number of hypercolumns $N_{\mathrm{HC}}$ increases significantly $(r=0.77, p<0.0002)$. At PD28, V1 contains 


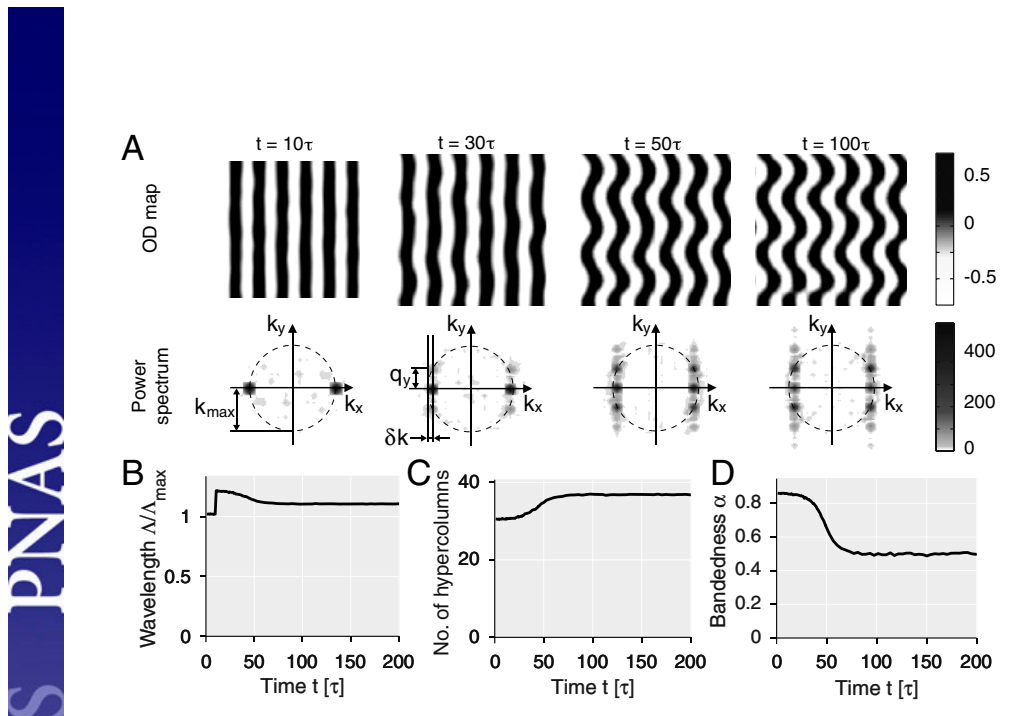

Fig. 2. Expansion-induced reorganization in models for $O D$ formation. (A) Snapshots of a simulation of the EN model (23) starting from a near steady state solution; i.e., a stripe-like OD pattern (upper row) corresponding to a single Fourier mode in the power spectrum (lower row) $(\eta=0.025, r=0.15)$. After instantaneous area increase (linear extent by a factor of 1.18 ; i.e., $\delta k / k_{\max }=-0.15$; at $10 \tau)$, OD domains bend sinusoidally and additional Fourier modes appear at $\approx\left(k_{\max }+\delta k\right) \overrightarrow{\mathbf{x}} \pm q_{y} \overrightarrow{\mathbf{y}}$. $(B-D)$ This reorganization is captured by the column spacing $\Lambda(B)$, the number zation is captured by the column spacing $\Lambda(B)$, the number
of hypercolumns $N_{\mathrm{HC}}(C)$, and the bandedness $\alpha(D)$ (time in units of the time scale $\tau$ of OD segregation). on average $260 \pm 40$ hypercolumns $(N=5)$, increasing to $319 \pm$ $17(N=6)$ at PD72 (increase of $23 \%$ ).

To reveal more directly the reorganization of OD columns, we analyzed a third parameter called bandedness $\alpha$ that characterizes the structural properties of local pattern elements $(21,20)$ (Fig. S1). Large values of $\alpha$ indicate layouts composed of regular stripe-like parallel domains, whereas small values indicate more isotropic layouts such as bended stripes or patches. Such quantitative evaluation of the spatial organization of columns was possible in $N=39$ hemispheres. We found that the bandedness $\alpha$ decreases by almost a factor of 2 from an average of $0.14 \pm$ $0.02(N=13)$ at PD35 to an average of $0.083 \pm 0.006$ at PD95 $(N=5)\left(r=-0.58, p<6 \cdot 10^{-5}\right)($ Fig. $1 H)$. This systematic decrease in bandedness indicates that OD columns, while largely preserving their initial spacing, reorganize and develop more isotropic layouts over time.

Modeling OD Column Formation with Growth. To understand these experimental observations, we studied cortical growth in model for the activity-dependent self-organization of OD columns. For specificity, we focussed on the well-studied Elastic Network (EN) model (23-25) (Materials and Methods and SI Appendix). Solutions in the absence of growth are shown in Fig. S2 and Movie S1. Linear stability analysis around the initially nonselective cortex (ref. 24 and SI Appendix) identifies a control parameter $r$ describing the distance from the pattern formation instability threshold. A pattern of OD columns forms for $r>0$. The analysis also defines an intrinsic timescale $\tau=1 / r$, on which the segregation of columns takes place, and a spatial scale $\Lambda_{\max }$ that is roughly equal to the column spacing of the developing OD pattern. As in other models for the self-organization of OD columns (25-28), this spatial scale arises from the effective recurrent interactions that have a "Mexican-hat" structure (local facilitation, nonlocal suppression). In agreement with previous work $(24,25)$, we find in simulations that an OD pattern emerges after a few $\tau$ (Fig. S2 and Movie S1). The only steady state solutions we observe are parallel OD stripes.

As a simple way to mimic cortical growth, we started from steady state solutions and abruptly increased isotropically the size of the simulated system without changing the other model parameters (i.e., without increasing the width of the Mexican-hat) (Materials and Methods and SI Appendix). Fig. 2 displays snapshots of a typical example of such a simulation (see also Movie S2). Upon size increase at $t=10 \tau$, stripes start to bend sinusoidally (Fig. $2 A$, upper row). In the power spectrum, this corresponds to the growth of new Fourier modes on both sides of the original mode of the stripe pattern (Fig. $2 A$, lower row).
We quantitatively analyzed this reorganization by the wavelet method used above. The column spacing $\Lambda$ increases abruptly at $10 \tau$, but subsequently decreases to close its initial value (Fig. $2 B$ ). The number of hypercolumns $N_{\mathrm{HC}}$ increases persistently (Fig. 2C), whereas the bandedness $\alpha$ decreases significantly over this period (Fig. 2D). Thus, the growth-induced bending of OD columns largely restores the initial spacing and results in a bandedness drop similar to what we observe in experiment (Fig. 1).

A General Mechanism of Growth-Induced Reorganization. We argue that this type of expansion-induced reorganization of OD columns in the EN model is caused by a zigzag (ZZ) instability (29), a type of dynamical instability that has been widely studied in the theory of pattern formation $(30,31)$. Fig. $3 A$ and $B$. This instability is typical for the wide class of relaxational, rotationally symmetric models in which a two-dimensional pattern forms by a finite wavelength instability (30). This class includes the EN model, as we outline in the SI Appendix, and many other OD models (e.g., refs. 25-28).

A theory $(31,32)$ for this model class exists predicting the regime of the $\mathrm{ZZ}$ instability (Fig. 3C, Inset). However, strictly speaking, this theory is valid only in a narrow parameter region close to the point of instability threshold at $r=0$. We therefore analyzed numerically the behavior of the EN model further away from threshold by probing systematically a large set of instantaneous size increases and testing for growing $\mathrm{ZZ}$ modes (Fig. S3 and SI Appendix). We observed that the regime of $\mathrm{ZZ}$ instability is very large (Fig. $3 C$ ). Similar to the theoretical predictions $(31,32)$, even a slight expansion results in a ZZ instability and its regime increases parabolically with the control parameter $r$. Moreover, the induced reorganization evolves on a time scale $\tau_{\mathrm{ZZ}}$ that for small expansions exceeds the time scale $\tau$ of OD column segregation by more than an order of magnitude.

Realistic Growth Scenarios. Finally, we show that growth-induced reorganization shows signatures of the $\mathrm{ZZ}$ instability even if the initial OD layout is not a simple stripe pattern and the increase in system size follows a continuous growth scenario. To approximate realistic conditions, we initialized our simulations with the nonselective state and linearly increased the linear extent of the simulated regions by a factor of 1.6 (factor of 2.56 in area increase) between $t=0 \tau$ and $t=100 \tau$. Fig. $4 A$ shows that layouts appear to be more bended in a ZZ-fashion when compared to simulations for which we stopped growth after $t=10 \tau$. Typically, the column spacing $\Lambda$ in growing systems increases only transiently (Fig. $4 B$ ) implying that the hypercolumn number $N_{\mathrm{HC}}$ increases persistently (Fig. 4C). The bandedness $\alpha$ is relatively 

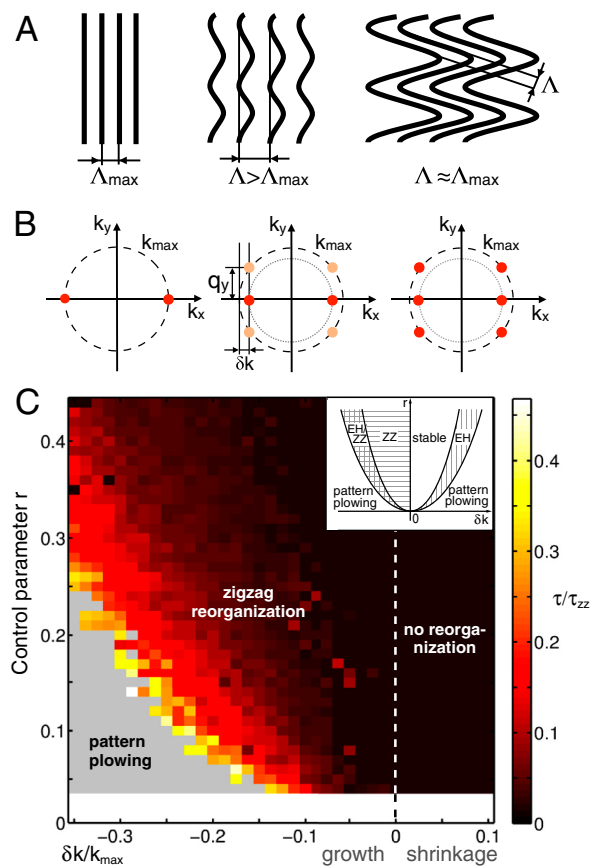

Fig. 3. The $Z Z$ instability provides a general framework for understanding growth-induced cortical reorganization. $(A)$ The $Z Z$ mechanism: Initially, OD stripes exhibit the spacing $\Lambda_{\max }$ (Left). Upon expansion (Center), OD stripes recover their initial spacing locally through bending of OD stripes (Right). (B) In Fourier space: Two new Fourier modes grow at $\left(k_{\max }+\delta k\right) \overrightarrow{\mathbf{x}} \pm q_{y} \overrightarrow{\mathbf{y}}$ restoring the initial wave number $k_{\max }$ of the pattern. (C) Regime and time scale of ZZ instability in the EN model. Instantaneous isotropic expansion (regime left of white dashed line) induces a ZZ reorganization that evolves on a timescale $\tau_{\mathrm{ZZ}} \gg \tau$ (SI Appendix). For very large expansion (gray region), a complete new pattern forms (pattern plowing). For area decrease (right of white dashed line) no reorganization was observed. No simulations were carried out in the white region, because the simulation time diverges for $r \rightarrow 0$ (SI Appendix). (Inset) Predicted regime of ZZ instability for two-dimensional, relaxational, isotropic dynamics close to instability threshold $(r \ll 1)$ [redrawn from (31): horizontally striped region, ZZ instability; vertically striped region, Eckhaus (EH), instability; i.e., insertion/elimination of a stripe (SI Appendix)].

variable across solutions reflecting the large diversity of the evolving OD layouts (Fig. $4 D$ and Fig. S2). However, whereas in virtually all simulations without growth $\alpha$ increases nearly monotonically (Fig. S2 and Movie S1), in growing systems $\alpha$ typically drops considerably reflecting the ZZ-type reorganization of OD columns (Fig. $4 A$ and Movie S3).

We systematically studied the growth-induced reorganization by varying the control parameter $r$ and testing different area increases (factor of 1, 1.44, 1.96, 2.56, and 3.24, Fig. $4 \mathrm{~F}-\mathrm{H}$ and SI Appendix). We measured the difference $\Delta \alpha$ between the first maximum in bandedness and the subsequent minimum and the time interval $\Delta t$ between these two bandedness extrema [Fig. $4 E$, based on eighth-order polynomial least square fit (SI Appendix)]. Whereas drops with $\Delta \alpha>0.05$ and $\Delta t>15 \tau$ occurred in only $<6 \%$ of the nongrowing systems, already with moderate growth, they are present in a large fraction of systems (Fig. $4 F$ ). For smaller values of $r$ the drop is generally more pronounced. Both the average size $\Delta \alpha$ and the duration $\Delta t$ depend only weakly on the total area increase and drops last on average $>40 \tau$ (Fig. $4 G$ and $H$ ).

Thus, also for more realistic growth scenarios, the induced reorganization exhibits key features of a ZZ instability, in particular the only mild and transient increase in column spacing and the prominent and long lasting drop in bandedness. Intriguingly, these features also describe the mode of reorganization we observe in experiment (Fig. 1). Moreover, if we identify the model time unit $\tau$ with roughly $1 \mathrm{~d}$ in cat postnatal development-an assumption that may be justified by experiments showing that OD columns segregate within a few days (e.g., ref. 8)-we observe that even the time scales on which these changes evolve, agree fairly well between model and experiment. This suggests that the reorganization we observe in experiments is caused by cortical expansion through a mechanism that is based on the ZZ instability.

\section{Discussion}

Cortical Expansion, the Absence of the Balloon Effect and the Range of the Mexican Hat. Our empirical data provides evidence against the so-called balloon effect (3) by showing that OD columns do not simply expand during cortical growth, but largely maintain their spacing. At first sight, a balloon-like expansion may seem plausible. For instance, in vitro connected neurons, when moderately pulled apart, readily extend their axonal arbors to prevent disruption, thereby achieving neurite growth rates of up to $1 \mathrm{~cm} /$ day (13). However, mechanical tension on one axonal branch can strongly influence the arborization of other branches of the same neuron (14), indicating that expansion-induced responses can be rich and may lead to nontrivial collective behavior in expanding networks of interconnected neurons.

Models for the activity-dependent formation of OD columns can reproduce the absence of the balloon effect if the width of the lateral interactions is kept fixed during growth as we assume in this study. In the EN model, an effective intracortical interaction of Mexican-hat type (Fig. S2A) arises from the interplay between the coactivation of cortical regions and a tendency for neighboring neurons to acquire similar response properties (23). Even if the interaction range increases by only half the rate of the cortex, we observe a ZZ-type reorganization accompanied by a bandedness drop (SI Appendix and Fig. S8). However, in this case also the column spacing increases systematically. Thus, these models can be reconciled with our data only if the interaction range does increase only little during growth.

There are several possibilities of why the range of effective lateral interactions might not increase during growth. It is conceivable that the width of interaction could depend on the lateral spread of dendritic arbors. Limits on an increase of the arbor size during growth might be imposed by a tendency of neuronal circuits to minimize the total length of wiring (e.g., ref. 32) and could be achieved by synaptic pruning (10). Alternatively, interactions of Mexican-hat type could arise if the time scales of the dominant inhibitory synapses are small compared to excitatory synapses (33). Thus, a possible shift from a dominance of smaller toward larger synaptic timescales during the period of growth could partly compensate for the increase of the distance between neurons. Finally, Mexican-hat type interactions could arise by excitatory connections that at larger distances preferentially target inhibitory interneurons. In this case, the width of interactions may depend on the strength of inhibition (16), which, appropriately adjusted, could keep the range of the Mexican-hat constant during growth.

Reorganization vs. Displacement. In the scenario of growth-induced reorganization proposed in this paper, increasing distances between cell bodies alter the effective lateral interactions between neurons, thereby inducing shifts in the response properties in a fraction of them. Alternatively, one may explore a scenario in which neuronal response properties are preserved, and the mature columnar layout is obtained by an inhomogeneous displacement of cells. Strong intracolumnar connections may provide the necessary mechanical stability for keeping cells within columns closer to one another. However, the ability of neurons to rapidly 

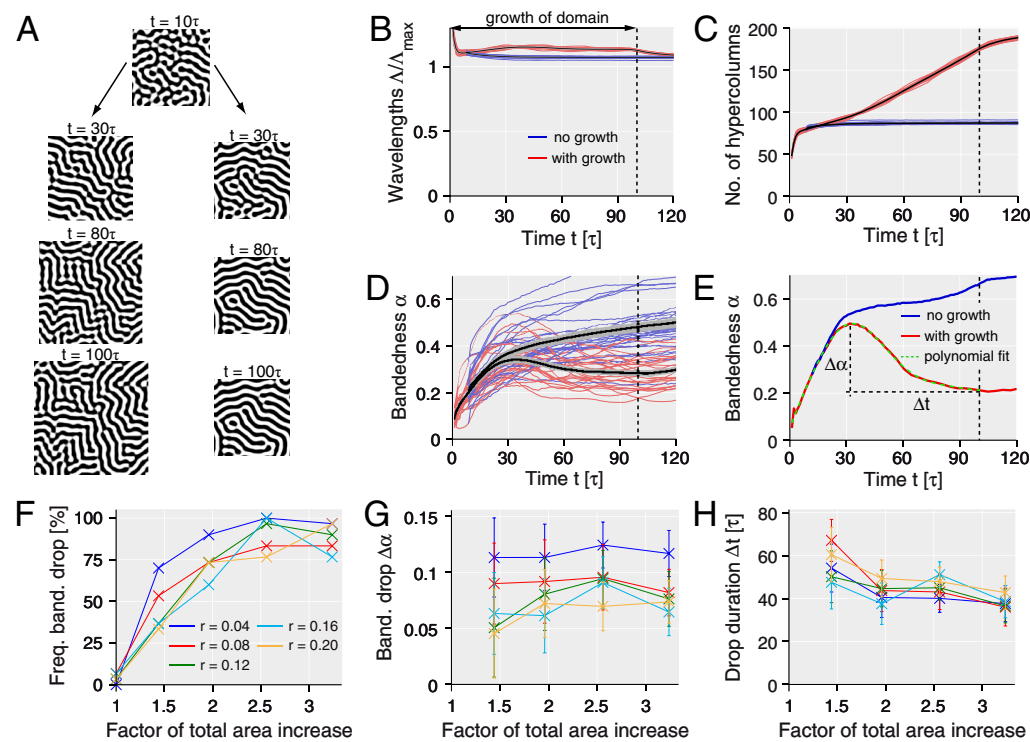

Fig. 4. ZZ-type reorganization of $O D$ columns in a realistic growth scenario. $(A)$ Snapshots of EN model simulations with isotropic linear area increase by a factor of 2.56 between $t=0$ and $t=100 \tau$ (left column) and without size increase after $t=10 \tau$ (right column) $(r=0.16, \eta=0.025)$. (B-D) Time courses of column spacing $\Lambda(B)$, number of hypercolumns $N_{\mathrm{HC}}(C)$, and bandedness $\alpha(D)$ for 30 pairs of simulations as in $A$. Black curves are averages, gray regions SEM. Despite the large area increase, growing systems display an only mild and transient increase in $\Lambda$ implying a strong increase in $N_{\text {HC }}$, while $\alpha$ typically drops considerably. $(E)$ Quantification of bandedness drop by its strength $\Delta \alpha$ and duration $\Delta t$. $(F-H)$ The percentage of simulations showing a substantial bandedness drop (defined by $\Delta \alpha>0.05, \Delta t>15 \tau)(F)$, the average size $\Delta \alpha$ of such drops $(G)$ and their average duration $\Delta t(H)$ evaluated for various total area increases and control parameters $r(N=50$ simulations per data point, error bars indicate SEM).

extend their axonal arbors in response to mechanical tension (13) raises doubts about expansion-induced forces being strong enough to promote inhomogeneous displacement of cells. Following the spatial positions as well as functional response properties of many cells experimentally (e.g., by chronic 2-photon microscopy) could help to disentangle these two hypotheses. Moreover, experiments that, instead of altering the patterns of neural activity, measure and/or apply mechanical stress to cortical tissue or individual neuronal processes in vivo may reveal further insights into the role of mechanical tension in cortical development. A better understanding of the interplay between tensionmediated and activity-driven mechanisms in shaping neural circuits in vivo could shed new light on normal developmen and cortical growth, but potentially also on the response of neura network function to cortical lesions and brain tumors.

The two scenarios have different implications for the hypercolumn as a functional cortical unit. A shift in OD in individual neurons would alter the set of stimulus representations in a hypercolumn. It would be interesting to monitor simultaneously other neuronal selectivities and test whether they codevelop in a systematic fashion; e.g., by improving coverage uniformity (34) over time. On the other hand, a pure displacement of groups of neurons would distort the original hypercolumn and result in systematic inhomogeneities in the cortical representation of the visual field position. Such inhomogeneities may be detectable in the mature cortex even without the necessity of technically very challenging chronic experiments.

Relation to Previous Work. A longitudinal optical imaging study (35) of OD columns in a single strabismic cat reported an increase of OD column spacing between PD27 and PD61 consistent with the slight but not significant increase we observe over this period (Fig. 1D). A chronic imaging study in ferret reported a fairly stable spatial organization of orientation columns between PD30 and PD55 (36). However, a more recent study (37) analyzing orientation columns in the cat between PD35 and PD105 ob- served changes in local column spacing that were coordinated between V1 and V2. Consistent with the present study, the average spacings in V1 and V2 remained largely constant over this period. A theoretical study (38) of a one-dimensional model of OD development during cortical growth predicts a splitting of OD stripes analogous to the Eckhaus instability (SI Appendix). As we show here, two-dimensional models exhibit a much richer dynamics and behave qualitatively differently.

A Novel Function of Plasticity in Normal Development. The impressive ability of cortical circuits to reorganize during and after the critical period has been demonstrated in numerous studies by artificially manipulating cortical activity; e.g., by monocular deprivation (see ref. 16 for a review). However, relatively little is known at present about the role of cortical plasticity for normal cortical development $(8,15)$, but see refs. 37,39 . As we point out in this study, the period of cortical plasticity in cat visual cortex overlaps with the period of postnatal cortical growth (17). Whereas the peak of the classical critical period is around PD30 (40), cortical plasticity does not cease after the critical period, but rather declines gradually (16). It is readily conceivable that this plasticity may be exploited by the cortex to accommodate for growth-induced changes. Interestingly, the reorganization we report here is largest close to the peak of the critical period. (Fig. 1H). Furthermore, key features of this reorganization are reproduced by modeling OD formation as self-organization based on cortical plasticity. Thus, we conclude that cortical plasticity may play an important role in normal development through facilitating growth-related modifications of neuronal circuits.

\section{Materials and Methods}

Experiment. OD patterns were labeled with $2-\left[{ }^{14} \mathrm{C}\right]$-deoxyglucose (2-DG) autoradiography after monocular stimulation of the animals or by $\left[{ }^{3} \mathrm{H}\right]$-proline autoradiography after injection of the labeled proline into one eye that labels the thalamocortical afferents of that eye in cortical layer IV 
(see ref. 20 and therein). OD columns were recorded by intrinsic signal optical imaging following ref. 41

Model. OD is described by a real valued field $o(\mathbf{x}, t)$, where $\mathbf{x}$ represents the position on the cortical surface and $t$ time. Negative/positive values of $o(x, t)$ indicate a preference for inputs from the ipsilateral/contralateral eye. The dynamics of this field is given by

$$
\partial_{t} o(\mathbf{x}, t)=\left\langle\left[s_{o}-o(\mathbf{x}, t)\right] A_{\sigma}(\mathbf{x}, \mathbf{S}, o(\cdot, t))\right\rangle_{\mathbf{S}}+\eta \Delta o(\mathbf{x}, t),
$$

where

$$
A_{\sigma}(\mathbf{x}, \mathbf{S}, o(\cdot, t))=\frac{e^{-\left(\left|\mathbf{s}_{r}-\mathbf{x}\right|^{2}+\left|s_{o}-o(\mathbf{x}, t)\right|^{2}\right) / 2 \sigma^{2}}}{\int d^{2} y e^{-\left(\left|\mathbf{s}_{r}-\mathbf{y}\right|^{2}+\left|s_{o}-o(\mathbf{y}, t)\right|^{2}\right) / 2 \sigma^{2}}}
$$

is the cortical activity pattern, $\sigma$ controls the receptive field size in the stimulus parameter space, $\langle\cdot\rangle$ denotes the average of the ensemble of visual stimuli $\{\boldsymbol{S}\}, \eta$ measures the strength of lateral interactions and $\Delta$ is the two-dimensional Laplacian. Visual stimuli $\mathbf{S}=\left(\mathbf{s}_{r}, s_{0}\right)$ are point-like and characterized by a location $s_{r}$ and an OD value $s_{0}$, which describes whether the activated units are forced to prefer the ipsilateral $\left(s_{0}<0\right)$ or the contralateral $\left(s_{0}>0\right)$ eye.

Numerical Integration. Simulations were performed on a $64 \times 64$ grid with periodic boundary conditions. We used at least 4 grid points per $\Lambda_{\max }$ and an integration time step $\delta t=\min \left\{1 /\left(20 \eta k_{\max }^{2}\right), \tau / 10\right\}$. The first term on the right hand side of Eq. $\mathbf{1}$ was treated by an Adams-Bashforth scheme, the second term by spectral integration. $\mathbf{s}_{r}$ and $s_{0}$ were uniformly distributed with $\left\langle s_{o}^{2}\right\rangle=1$. Typically, between $4 \times 10^{4}$ and $2 \times 10^{5}$ stimuli were used per integration step.

1. Dekaban AS, Sadowksy D (1978) Changes in brain weight during the span of a human life: Relation of brain weight to body heights and body weights. Ann Neurol 4:345-356.

2. Villabanca JR, Schmanke TD, Crutcher HA, Sung AC, Tavabi K (2000) The growth of the feline brain from fetal into adult life I. A morphometric study of neocortex and white matter. Dev Brain Res 122:11-20.

3. Duffy KR, Murphy KM, Jones DG (1998) Analysis of the postnatal growth of visual cortex. Vis Neurosci 15:831-839.

4artin AC, Gelbart M, Fernandez-Gonzalez R, Kaschube M, Wieschaus EF (2010) Integration of contractile forces during tissue invagination. $J$ Cell Biol 188:735-749. 5. Vermot J, et al. (2009) Reversing blood flows act through klf2a to ensure normal valvulogenesis in the developing heart. PLOS Biol 7:e1000246.

6. Kumar S, Weaver VM (2009) Mechanics, malignancy, and metastasis: The force journey of a tumor cell. Cancer Metastasis Rev 28:113-127.

7. Sireteanu R, Maurer D (1982) The development of the kitten's visual field. Vision Res 22:1105-1111.

8. Crair MC, Horton JC, Antonini A, Stryker MP (2001) Emergence of ocular domi nance columns in cat visual cortex by 2 weeks of age. J Comp Neurol 430:235-249.

9. Rathjen S, Schmidt KE, Löwel S (2003) Postnatal growth and column spacing in cat primary visual cortex. Exp Brain Res 149:151-158.

10. Cragg BG (1975) The development of synapses in the visual system of the cat. J Comp Neurol 160:147-166.

11. Purves D (1994) Neural activity and the growth of the brain (Cambridge University Press, Cambridge, UK).

12. van Essen DC (1997) A tension based theory of morphogenesis and compact wiring in the central nervous system. Nature 385:313-318.

13. Smith DH (2009) Stretch growth of integrated axons tracts: Extremes and exploitations. Prog Neurobiol 89:231-39.

14. Anava S, Greenbaum A, Jacob EB, Hanein $Y$, Ayali A (2009) The regulative role of neurite mechanical tension in network development. Biophys $J$ 96:1661-1670.

15. Katz L, Crowley J (2002) Development of cortical circuits: Lessons from ocular dominance columns. Nat Rev Neurosci 3:34-42.

16. Hensch TK (2005) Critical period plasticity in local cortical circuits. Nat Rev Neurosci 6:877-888.

17. Daw NW, Fox K, Sato H, Czepita D (1992) Critical period for monocular deprivation in the cat visual cortex. I Neurophysiol 67:197-202.

18. Lehmann $\mathrm{K}$, Löwel $\mathrm{S}$ (2008) Age dependent ocular dominance plasticity in adult mice. PLoS One 3:e3120.

19. Gall GAE, Kyle WH (1968) Growth of the Laboratory mouse. Theor Appl Genet 38:304-308.

20. Kaschube $M$, et al. (2003) The pattern of ocular dominance columns in cat primary visual cortex: Intra and interindividual variability of column spacing and its visual cortex: Intra and interindividual variability of column

21. Kaschube M, Wolf $F$, Geisel T, Löwel $S$ (2002) Genetic influence on quantitative features of neocortical architecture. $J$ Neurosci 22:7206-17.
Instantaneous Area Increases. We rescaled the system length $L$ as determined from the desired value of $\delta k / k_{\max }$ (no change in number of grid points: see $S /$ Appendix). We adjusted the number of stimuli, $N_{s}$, and, because $\Delta \sim 1 / L^{2}$, the matrix for the spectral integration step. The numerical value of $\sigma$ remained constant.

Continuous Area Increases. We linearly increased the linear extent $L$ of the simulated regions between $t=0 \tau$ and $t=100 \tau$ and updated the Laplacian $\Delta$ and the number of stimuli $N_{s}$ at every integration step.

Data Analysis Method. Column spacing $\Lambda$ and bandedness $\alpha$ of both data and simulations, were analyzed using the wavelet method introduced in ref. 21. An overcomplete basis of complex Morlet wavelets at various scales and orientations was compared to the OD pattern at each spatial location. $\Lambda$ was estimated by the scale of the best matching wavelet, $\alpha$ by the angular variance of matching at that scale (SI Appendix).

Statistics. $r$-values denote Pearson's linear correlation coefficient; $p$-values were obtained with Student's $t$ tests.

All methods are described in detail in the SI Appendix.

ACKNOWLEDGMENTS. We thank F. Wolf for many inspiring discussions, and M. Huang and M. Schnabel for help with numerical procedures. We thank S. Palmer and P. Mehta for helpful comments on the manuscript. W. K. and M. K. acknowledge financial support from Grant 01GQ0430 from the German Ministry for Education and Science (BMBF) via the Bernstein Center for Computational Neuroscience (BCCN), Göttingen. M.K. was supported by Grant P50 GM071508 from the National Institute of Health and the National Institute of General Medical Sciences. K.-F. S. and S. L. acknowledge financial support from BMBF Grants 01GQ07111 and 01GQ0921.

22. Horton JC, Adams DL The cortical column: A structure without a function. Philos $T R$ Soc $B$ 360:837-862.

23. Durbin R, Mitchison $G$ (1990) A dimension reduction framework for understanding cortical maps. Nature 343:644-647.

24. Wolf F, Geisel T (1998) Spontaneous pinwheel annihilation during visual develop ment. Nature 395:73-78.

25. Goodhill GJ, Cimponeriu A (2000) Analysis of the elastic net model applied to the formation of ocular dominance and orientation columns. Network-Comp Neural 11:153-168.

26. Swindale NV (1980) A model for the formation of ocular dominance stripes. $P$ Roy Soc Lond B Bio 208:243-264.

27. Miller KD, Keller JB, Stryker MP (1989) Ocular dominance column development: Analysis and simulation. Science 245:605-615.

28. Obermayer K, Blasdel G, Schulten K (1992) Statistical mechanical analysis of self organization and pattern formation during the development of visual maps. Phys $\operatorname{Rev} A$ 45:7568-7589.

29. Newell AC, Whitehead JA (1969) Finite bandwidth, finite amplitude convection. J Fluid Mech 38:279-303.

30. Cross MC, Hohenberg PC (1993) Pattern formation outside of equilibrium. Rev Mod Phys 65:851-1112.

31. Cross MC, Greenside H (2009) Pattern Formation and Dynamics in Nonequilibrium Systems (Cambridge University Press, Cambridge, UK).

32. Chklovskii DB, Schikorski T, Stevens CF (2002) Wiring optimization in cortical circuits. Neuron 34(3):341-347.

33. Kang K, Shelley M, Sompolinsky H (2003) Mexican hats and pinwheels in visual cortex. Proc Natl Acad Sci USA 100:2848-2853.

34. Swindale NV, Shoham D, Grinvald A, Bonhoeffer T, Hubener M (2000) Visual cortex maps are optimized for uniform coverage. Nat Neurosci 3:822-826.

35. Müller T, et al. (2000) An analysis of orientation and ocular dominance patterns in the visual cortex of cats and ferrets. Neural Comput 12:2573-2595.

36. Chapman B, Stryker MP, Bonhoeffer T (1996) Development of orientation preference maps in ferret primary visual cortex. J Neurosci 16(20):6443-6453.

37. Kaschube $M$, Schnabel $M$, Wolf $F$, Löwel $S$ (2009) Interareal coordination of columnar architectures during visual cortical development. Proc Natl Acad Sci USA 106:17205-17210.

38. Oster AM, Bressloff PC (2006) A developmental model of ocular dominance column formation on a growing cortex. B Math Biol 68:73-98.

39. Wang BS, Sarnaik R, Cang J (2010) Critical period plasticity matches binocular orientation preference in the visual cortex. Neuron 65:246-256.

40. Olson CR, Freeman RD (1980) Profile of the sensitive period for monocular deprivation in kittens. Exp Brain Res 39:17-21.

41. Schmidt KF, Löwel S (2006) Optical imaging in cat area 18: Strabismus does not enhance the segregation of ocular dominance domains. Neuroimage 29(2):439-445. 


\title{
Supporting Information - Reorganization of columnar architecture in the growing visual cortex
}

\author{
Wolfgang Keil ${ }^{1,3}$, Karl-Friedrich Schmidt ${ }^{2}$, \\ Siegrid Löwel ${ }^{2}$, Matthias Kaschube ${ }^{3 *}$ \\ ${ }^{1}$ Max-Planck-Institute for Dynamics and Self-Organization \\ Bunsenstrasse 10, 37073, Göttingen, Germany. \\ ${ }^{2}$ Institute of General Zoology and Animal Physiology, Friedrich-Schiller-University \\ Erbertstrasse. 1, 07743 Jena, Germany. \\ ${ }^{3}$ Lewis-Sigler Institute for Integrative Genomics, and Physics Department, \\ Princeton University, 261 Carl-Icahn Laboratory, Princeton NJ, 08544, USA.
}

*To whom correspondence should be addressed:

Matthias Kaschube

Princeton University

261 Carl-Icahn Laboratory

Princeton NJ, 08544, USA

Phone: +1 (609) 258-1937

Fax: $+1(609)$ 258-7070

E-mail: kaschube@princeton.edu

Information on the Supporting Material:

30 pages, 8 figures, 3 movies 


\section{Optical imaging data}

Surgery. Anaesthesia was induced with an intramuscular injection of ketamine $(10 \mathrm{mg} / \mathrm{kg} \mathrm{Ke}-$ tanest $\AA$, Parke-Davis, Berlin, Germany) and xylazine hydrochloride (Rompun $\AA$, Bayer AG, Leverkusen, Germany) and maintained throughout the experiment using nitrous oxide/oxygen anaesthesia $\left(50 \% \mathrm{~N}_{2} \mathrm{O} / 50 \% \mathrm{O}_{2}\right)$, supplemented with halothane (0.8-1.2\%, Eurim Pharma, Germany). The ECG, pulmonary pressure, end tidal $\mathrm{CO}_{2}(3-4 \%)$, and rectal temperature $\left(37-38^{\circ}\right)$ were continuously monitored. The animal's head was fixed in a stereotactic frame by means of a metal nut cemented to the skull. For optical imaging of $\mathrm{V} 1$ a craniotomy was performed centered at Horsley-Clarke coordinate P4. All experiments were performed when the animals were between 28 and 94 days old. Successive experiments in the same animal were performed with intervals of 7 days and in one case 42 days. All animal experiments have been performed according to the German Law on the Protection of Animals and the corresponding European Communities Council Directive of November 24, 1986 (86/609/EEC).

Visual Stimulation. Animals were stimulated monocularly with high-contrast square-wave gratings (subtending $90^{\circ} \times 60^{\circ}$ visual field) of four orientations $\left(0^{\circ}, 45^{\circ}, 90^{\circ}\right.$, and $\left.135^{\circ}\right)$ moving at a speed of $2 c y c / s$ with a spatial frequency of $0.5 c y c / d e g$. Stimuli were generated by EZV-Stim software (Optical Imaging Inc., Rehovot, Israel) and presented on a LG Electronics Flatron 295 LCD-monitor (luminosity $180 \mathrm{~cd} / \mathrm{m}^{2}$; contrast $300: 1$; refresh rate $85 \mathrm{~Hz}$; resolution $1600 \times 1200$ pixel) at a distance of $25 \mathrm{~cm}$. The eyes were treated with atropine and Neosynephrine ${ }^{\circledR}$ and refracted appropriately using corrective corneal contact lenses with artificial pupils with a diameter of $3 \mathrm{~mm}$.

Data Acquisition. The cortical surface was illuminated by means of two adjustable light guides attached to a tungsten-halogen lamp (Spindler \& Hoyer, Göttingen, Germany) equipped with interference filters for different wavelengths. The vascular pattern of the cortex was visualized at $546 \mathrm{~nm} \pm 10 \mathrm{~nm}$ (green), cortical activity maps at $707 \mathrm{~nm} \pm 1 \mathrm{~nm}$ (red). During data acquisition of intrinsic signals, the camera was focused $650-750 \mu \mathrm{m}$ below the cortical surface. A tandem-lense was used for imaging [1]. The ORA 2001 system (Optical Imaging Inc.), equipped with a cooled Theta CCD system ( $384 \times 288$ pixel chip from Thomson-CSF) was used for collecting the intrinsic 
signals. We acquired a series of frames every $12 \mathrm{~s}$, whereby a grating of a given orientation was presented for $2 \mathrm{~s}$ in a static mode, followed by $4.2 \mathrm{~s}$ of data acquisition during which the grating was moved in both directions along the axis orthogonal to its orientation. We used episodic stimulation during data acquisition (7 frames of $600 \mathrm{~ms}$ duration). The first frames were excluded from further analysis. The stimulus presentation was monocular, an eye shutter was used to conceal the eyes. A single stimulus trial consisted of $2 \times 8$ stimulus conditions ( 4 grating orientations for the left and the right eye) and 8 isoluminant blanks presented in a random sequence. Twelve trials were usually presented to obtain a map, so that every stimulus was shown 24 times. We first calculated 'single condition maps' in which the images acquired during presentation of a particular stimulus were divided by the sum of all different stimulus conditions ('cocktail blank procedure') $[2,3]$. Differential maps for ocular dominance (OD) were calculated by summing all left eye activity maps and subtracting all right eye activity maps.

Data Preprocessing. All computed differential maps $I^{\prime}(\mathbf{x})$ were preprocessed in order to remove overall variations in signal strength and measurement noise. We calculated a high-pass filtered map $I(\mathbf{x})=I^{\prime}(\mathbf{x})-J(\mathbf{x})$ by subtracting the regional mean

$$
J(\mathbf{x})=\frac{1}{W(\mathbf{x})} \mathcal{F}^{-1}\left\{\tilde{K}_{h p}(\mathbf{k}) \tilde{I}^{\prime}(\mathbf{k})\right\},
$$

where $\mathcal{F}$ denotes the Fourier transform and the Fermi-function

$$
\tilde{K}_{h p}(\mathbf{k})=\frac{1}{1+e^{-\left(k_{h p}-|\mathbf{k}|\right) / \beta_{h p}}}
$$

is parametrized by the high-pass cutoff frequency $k_{h p}$ and the steepness $\beta_{h p}$. After transforming back to real space, the signal outside the region of interest (ROI) was discarded. Normalizing by $W(\mathbf{x})=\int_{R O I} d^{2} x^{\prime} K_{h p}\left(\mathbf{x}-\mathbf{x}^{\prime}\right)$ accounted for the boundary of the ROI. We used a Fermi filter with $\beta=0.2 k_{h p}$ and $k_{h p}=2 \pi / \lambda_{h p}$ with $\lambda_{h p}=1.8 \mathrm{~mm}$. Lowpass filtering was done with a second Fermi filter $\tilde{K}_{l p}(\mathbf{k})$ with parameters $\beta=0.2 k_{l p}$ and $k_{l p}=2 \pi / \lambda_{l p}$ with $\lambda_{l p}=0.7 \mathrm{~mm}$.

The resulting pattern $I(\mathbf{x})$ was then centered to yield $\int_{R O I} d^{2} x I(\mathbf{x})=0$ and its variance was normalized to one. This overall bandpass filtering ensured that structures on a scale between 0.7 $\mathrm{mm}$ and $1.8 \mathrm{~mm}$ were only weakly attenuated by the preprocessing and enabled us to do further quantitative analysis. 


\section{Data analysis methods}

Column spacing. For each preprocessed OD pattern $I(\mathbf{x})$, we calculated a wavelet representation, using wavelets which covered only a few hypercolumns but exhibited a strong periodicity. These representations were obtained from

$$
\hat{I}(\mathbf{x}, \theta, l)=\int_{R O I} d^{2} y I(\mathbf{y}) \psi_{\mathbf{x}, \theta, l}(\mathbf{y})
$$

where $\mathbf{x}, \theta, l$ are the position, orientation and scale of the wavelet $\psi_{\mathbf{x}, \theta, l}$ and $\hat{I}(\mathbf{x}, \theta, l)$ denotes the array of wavelet coefficients. We used complex Morlet wavelets defined by

$$
\psi(\mathbf{x})=\exp \left(-\frac{\left(x_{1}^{2}+\sigma_{2}^{-2} x_{2}^{2}\right)}{2}\right) e^{i \mathbf{k}_{\psi} \mathbf{x}}
$$

and

$$
\psi_{\mathbf{x}, \theta, l}(\mathbf{y})=l^{-1} \psi\left(\Omega^{-1}(\theta) \frac{\mathbf{y}-\mathbf{x}}{l}\right)
$$

where

$$
\Omega(\theta)=\left(\begin{array}{cc}
\cos \theta & -\sin \theta \\
\sin \theta & \cos \theta
\end{array}\right)
$$

is the two-dimensional rotation matrix. The characteristic wavelength of a wavelet with scale $l$ is $\Lambda_{\psi} l$ with $\Lambda_{\psi}=2 \pi /\left|\mathbf{k}_{\psi}\right| . \sigma_{2}$ denotes the anisotropy of the wavelet. We used relatively large isotropic wavelets $\left(\mathbf{k}_{\psi}=(7,0), \sigma_{2}=1\right)$ to estimate local column spacing $\Lambda(\mathbf{x})$. We used 12 equally spaced orientations and 16 scales $l_{j}$ between $0.5 \mathrm{~mm}$ and $2.0 \mathrm{~mm}$. For computational efficiency, 9 equally spaced orientation were used to analyze model cortices. Test analyses with 12 orientation led to almost indistinguishable results. We first calculated

$$
\bar{I}(\mathbf{x}, l)=\int_{0}^{\pi} \frac{d \theta}{\pi}|\hat{I}(\mathbf{x}, \theta, l)|
$$

of the wavelet coefficients for every position $\mathbf{x}$ and then determined the scale by computing $\bar{l}(\mathbf{x})=$ $\arg \max _{l}(\bar{I}(\mathbf{x}, l))$. The corresponding characteristic local wavelength was obtained by $\Lambda(\mathbf{x})=$ $l(\mathbf{x}) \Lambda_{\psi} \cdot \bar{l}(\mathbf{x})$ was estimated as the maximum of a polynomial of 6 th degree in $l$, fitting the $\bar{I}\left(\mathbf{x}, l_{j}\right)$ (least square fit) for a given position $\mathbf{x}$. Based on the local column spacing $\Lambda(\mathbf{x})$, we calculated the mean column spacing $\Lambda=\langle\Lambda(\mathbf{x})\rangle_{x}$, where here and in the following, $\langle\cdot\rangle_{x}$ denotes the average over the ROI (e.g. V1 or simulated domain). In the case of 2-DG maps, we analyzed column 
spacings in $N=4$ different brain slices for each hemisphere and averaged over slices to obtain more accurate estimations of the mean column spacing $[5,4]$. This procedure also provides an estimation of the measurement error. On average errors were below $0.02 \mathrm{~mm}$ implying a relative error (error/column spacing) of less than $2 \%$. For the optical imaging data, errors were estimated by bootstrap resampling. For each map, a bootstrap sample of $\mathrm{N}=100$ maps was generated from the individual trials. The column spacing was calculated for each map in the sample. The error was estimated by the standard deviation of the bootstrap distribution of column spacings.

Number of hypercolumns. We defined the number of hypercolumns $N_{H C}$ in an area $A$ by $N_{H C}=$ $A / \Lambda^{2}$, where $\Lambda=\langle\Lambda(\mathbf{x})\rangle_{x}$ is the mean column spacing in $\mathrm{A}$. Note that we do not assume a specific shape of the hypercolumn, but solely that its size is equal to $c \Lambda^{2}$ where $c$ is a constant close to 1. For simplicity, and because we are primarily interested in relative changes in $N_{H C}$, we set $c=1$. Note further that for simplicity we are assuming $\langle\Lambda(\mathbf{x})\rangle_{x}^{-2} \approx\left\langle\Lambda(\mathbf{x})^{-2}\right\rangle_{x}$. Typical standard deviations $\sigma_{\Lambda}=\sqrt{\left\langle\Lambda(\mathbf{x})^{2}\right\rangle_{x}}$ of OD column spacings in the cat are $\approx 0.1 \Lambda$ [6] and in the model $<0.03 \Lambda$. Based on these values, this approximation is accurate up to $\approx 3 \%$.

Bandedness. The orientation dependence of the wavelet coefficients was used to calculate a parameter measuring the anisotropy of local pattern elements as shown in Fig. S1. For a pattern consisting of parallel stripes the magnitude of the wavelet coefficients depends strongly on the wavelet orientation and is largest if the orientation of the wavelet matches the orientation of the bands (Fig. S1, A and C). For a pattern consisting of more bended stripes or isotropic patches, the wavelet coefficients depend only weakly on the orientation of the wavelet (Fig. S1, B and D). Therefore, using only wavelets of wavelength $\Lambda(\mathbf{x})$, we calculated

$$
s^{\prime}(\mathbf{x})=\int_{0}^{\pi} d \theta|\hat{I}(\mathbf{x}, \theta)|^{2} e^{i 2 \theta} / \int_{0}^{\pi} d \theta|\hat{I}(\mathbf{x}, \theta)|^{2},
$$

where we used relatively small and anisotropic wavelets $\left(\mathbf{k}_{\psi}=(2,0), \sigma_{2}=1.5\right)$ in order to resolve the layout locally. We defined the local bandedness as

$$
s(\mathbf{x})=\int_{R O I} d^{2} y K(\mathbf{x}-\mathbf{y}) s^{\prime}(\mathbf{y}) / \int_{R O I} d^{2} y K(\mathbf{x}-\mathbf{y}),
$$

where $K(\mathbf{x})=\frac{1}{2 \pi \sigma^{2}} \exp \left(-\mathbf{x}^{2} / 2 \sigma^{2}\right)$ and $\sigma=1.3 \Lambda$. To estimate (1) and (2), wavelet coefficients were computed for 9 equally spaced orientations for both model cortices and data. Test analyses 

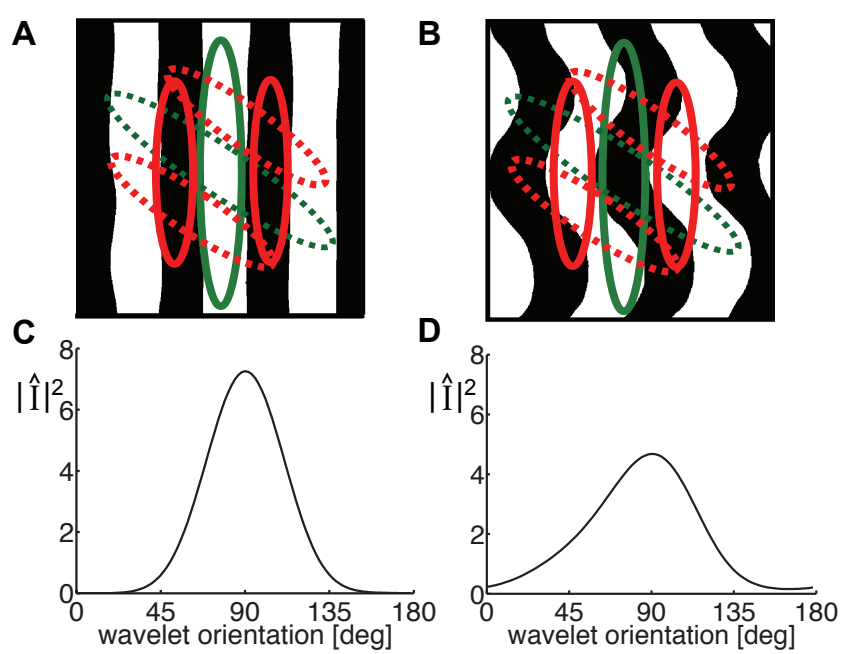

Figure S1. Wavelet analysis of columnar layouts. (A) Examples of two wavelets superimposed on a stripe-like region of a simulated OD pattern. The real parts of the complex-valued wavelet are depicted. Positive regions are delineated by red lines, negative regions are delineated by green lines. The two wavelets exhibit the best matching spatial frequency. One wavelet (solid line) also exhibits an optimal orientation. (B) Wavelets superimposed on sinusoidally bended stripe patterns, characteristic for the $\mathrm{ZZ}$ reorganization. (C-D) The normalized squared modulus of the wavelet coefficients as a function of orientation $\theta$ for the pattern in $A(C)$ and for the pattern in $B(D)$. Note that for the stripe-like pattern $|\hat{I}(\mathbf{x}, \theta)|^{2}$ is strongly modulated and exhibits a pronounced peak, whereas the sinusoidally bended stripes lead to a broader $\theta$-dependence and a less pronounced peak. This difference is captured by the bandedness parameter $\alpha$. 
with 12 equally spaced orientations led to almost indistinguishable results. With the above choice of $\sigma$, the function $s(\mathbf{x})$ is sensitive to the occurrence of band-like regions that extend over the size of more than a hypercolumn. Based on the local bandedness $s(\mathbf{x})$, we computed the bandedness $\alpha=\langle s(\mathbf{x})\rangle_{x}$ to characterize the overall layout of simulated and measured OD patterns. To estimate measurement errors for 2-DG maps, the bandedness was calculated for $N=4$ brain slices and then averaged $[5,4]$. Relative errors for bandedness estimation were below $\approx 5 \%$.

\section{The Elastic Network model - linear stability analysis and numerical procedures}

\subsection{Model definition and linear stability analysis}

In the continuous version [7] of the Elastic Network (EN) model [8, 9], the OD map in V1 at a given time $t$ is described by a real valued field $o(\mathbf{x}, t)$, where $\mathbf{x}$ represents the position on the cortical surface. Negative values of $o(\mathbf{x}, t)$ indicate a preference for input from the ipsilateral eye, positive values a preference for the contralateral eye. This field follows a dynamics

$$
\partial_{t} o(\mathbf{x}, t)=\left\langle\left[s_{o}-o(\mathbf{x}, t)\right] A_{\sigma}(\mathbf{x}, \mathbf{S}, o(\cdot, t))\right\rangle_{\mathbf{S}}+\eta \triangle o(\mathbf{x}, t),
$$

where

$$
A_{\sigma}(\mathbf{x}, \mathbf{S}, o(\cdot, t))=\frac{e^{-\left(\left|\mathbf{s}_{r}-\mathbf{x}\right|^{2}+\left|s_{o}-o(\mathbf{x}, t)\right|^{2}\right) / 2 \sigma^{2}}}{\int d^{2} y e^{-\left(\left|\mathbf{s}_{r}-\mathbf{y}\right|^{2}+\left|s_{o}-o(\mathbf{y}, t)\right|^{2}\right) / 2 \sigma^{2}}}
$$

is the cortical activity pattern, $\sigma$ controls the receptive field size in the stimulus parameter space, $\langle\cdot\rangle_{\mathbf{S}}$ denotes the average of the ensemble of visual stimuli $\{\mathbf{S}\}, \eta$ measures the strength of lateral interactions and $\Delta$ is the two-dimensional Laplacian. Selectivities $o(\mathbf{x})$ are modified through the cumulative effect of a large number of activity events, evoked by the complete stimulus ensemble. Visual stimuli $\mathbf{S}=\left(\mathbf{s}_{r}, s_{o}\right)$ are point-like and characterized by a location $\mathbf{s}_{r}$ and an OD value $s_{0}$ which describes whether the activated units are forced to prefer the ipsilateral $\left(s_{o}<0\right)$ or the contralateral $\left(s_{o}>0\right)$ eye. The stimulus parameters $\mathbf{s}_{r}$ and $s_{o}$ are uniformly distributed with densities $\rho_{\mathbf{s}_{r}}$ and $\rho_{s_{o}}$ such that $\left\langle s_{o}^{2}\right\rangle=1$.

Linear stability analysis around nonselective fixed point. By linear stability analysis, we show in the following that $\mathrm{OD}$ columns segregate by a finite wavelength instability. We linearize eq. (3) 
around the homogeneous nonselective state $o(\mathbf{x})=0$ [7]. Inserting $o_{\text {hom }}(\mathbf{x}) \equiv 0$ into eq. (4) yields

$$
A_{\sigma}\left(\mathbf{x}, \mathbf{S}, o_{\mathrm{hom}}\right)=\frac{e^{-\frac{\left|\mathbf{s}_{r}-\mathbf{x}\right|^{2}}{2 \sigma^{2}}}}{2 \pi \sigma^{2}} .
$$

After averaging over the ensemble of stimuli with normalized uniform densities $\rho_{\mathbf{s}_{r}}$ and $\rho_{s_{o}}$, we obtain

$$
\left.\partial_{t} o(\mathbf{x})\right|_{o_{\mathrm{hom}}}=\int d^{2} s_{r} \rho_{\mathbf{s}_{r}} \frac{e^{-\frac{\left|\mathbf{s}_{r}-\mathbf{x}\right|^{2}}{2 \sigma^{2}}}}{2 \pi \sigma^{2}} \int d s_{o} \rho_{s_{o}} s_{o}=0 .
$$

This shows that $o_{\text {hom }}(\mathbf{x}) \equiv 0$ is a fixed point of the dynamics in eq. (3). The stability of this fixed point can be studied by linearizing the r.h.s of eq. (3) [7, 10],

$$
\mathcal{F}[o, \mathbf{x}, \sigma, \mathbf{S}] \approx \mathcal{F}\left[o_{\mathrm{hom}}, \mathbf{x}, \sigma, \mathbf{S}\right]+\left.\int d^{2} y \frac{\delta \mathcal{F}}{\delta o(\mathbf{y})}\right|_{o_{\mathrm{hom}}} o(\mathbf{y})
$$

This yields

$$
\begin{aligned}
\partial_{t} o(\mathbf{x}) \approx & \eta \Delta o(\mathbf{x})-o(\mathbf{x}) \iint d^{2} s_{r} d s_{o} d^{2} y \rho_{s_{o}} \rho_{\mathbf{s}_{r}} A_{\sigma}\left(\mathbf{y}, \mathbf{S}, o_{\mathrm{hom}}\right) \\
& +o(\mathbf{x}) \iint d^{2} s_{r} d s_{o} d^{2} y \rho_{s_{o}} \rho_{\mathbf{s}_{r}} A_{\sigma}\left(\mathbf{y}, \mathbf{S}, o_{\mathrm{hom}}\right) \frac{s_{o}^{2}}{\sigma^{2}} \\
& -\iint d^{2} s_{r} d s_{o} d^{2} y \rho_{s_{o}} \rho_{\mathbf{s}_{r}} \frac{s_{o}^{2}}{4 \pi^{2} \sigma^{6}} e^{-\left(\left|\mathbf{s}_{r}-\mathbf{x}\right|^{2}+\left|\mathbf{s}_{r}-\mathbf{y}\right|^{2}\right) / 2 \sigma^{2}} o(\mathbf{y}) .
\end{aligned}
$$

Assuming a uniform stimulus density $\rho_{\mathbf{s}_{r}}$ across the cortical surface, this results in

$$
\partial_{t} o(\mathbf{x})=\left(\eta \triangle+\frac{\left\langle s_{o}^{2}\right\rangle}{\sigma^{2}}-1\right) o(\mathbf{x})-\frac{\left\langle s_{o}^{2}\right\rangle}{4 \pi \sigma^{4}} \int d^{2} y e^{-\frac{(\mathbf{x}-\mathbf{y})^{2}}{4 \sigma^{2}}} o(\mathbf{y}) .
$$

Note that the linearization is only governed by the variance of the stimulus ensemble, and not by higher order statistical moments.

Due to translation and rotation invariance of the model, the eigenfunctions of its linearized dynamics are Fourier modes $\sim e^{i \mathbf{k x}}$ with eigenvalues $[7,10]$

$$
\lambda(k)=-1+\frac{\left\langle s_{o}^{2}\right\rangle}{\sigma^{2}}\left(1-e^{-k^{2} \sigma^{2}}\right)-\eta k^{2}
$$

only depending on the absolute value $|\mathbf{k}|=k$ (see Fig. S2A for an illustration). The homogeneous nonselective state $o_{\text {hom }}(\mathbf{x}) \equiv 0$ is unstable, if some eigenvalue with $k>0$ is larger than zero. The amplitude of any small perturbation containing a Fourier mode with wave vector $\mathrm{k}$ will evolve $\sim \exp (\lambda(k) t)$, and therefore spatial frequencies with $\lambda(k)<0$ are exponentially damped, whereas 
those with $\lambda(k)>0$ grow exponentially (shaded region in Fig. S2A). For $\eta>0$ and $\sigma>0, \lambda(k)$ has a single maximum at $k_{\max }=\frac{1}{\sigma} \sqrt{\ln (1 / \eta)}$. The maximum $\lambda\left(k_{\max }\right)$ is positive if the width $\sigma$ of the activation function is smaller than $\sigma^{*}=\sqrt{1-\eta+\eta \ln \eta}$. Importantly, the maximum positive eigenvalue $r=\lambda\left(k_{\max }\right)$ defines a time scale $\tau=1 / r$ on which OD columns segregate. We refer to $r$ as the control parameter. For $r>0$, the homogeneous nonselective state is unstable and the maximum is at some finite wavelength $\Lambda_{\max }=2 \pi / k_{\max }$. This spatial scale is roughly the spacing of columns in the developing OD map. Confirming this linear stability analysis, we find numerically that the early pattern consists of Fourier modes with wavelength $\approx \Lambda_{\max }$ (see below). Thus, in the EN model, OD columns segregate because the nonselective state $o(\mathbf{x})=0$ becomes unstable and Fourier modes with period $\sim \Lambda_{\max }$ grow exponentially if $\sigma$ is below a critical value.

Generality of model definition. In the above definition of the Elastic Network model, the widths of the activation function $A_{\sigma}(\mathbf{x}, \mathbf{S}, o(\cdot, t))$ in $\mathrm{OD}$ space and retinotopic space are identified and set to $\sigma$. In the following, we show that this can always be achieved by a proper rescaling of cortical space, and hence does not imply a loss of generality.

With two different widths, $\sigma_{r}$ for retinotopic space and $\sigma_{o}$ for OD, the EN model becomes

$$
\partial_{t} o(\mathbf{x}, t)=\left\langle\left[s_{o}-o(\mathbf{x}, t)\right] A_{\sigma_{o}, \sigma_{r}}(\mathbf{x}, \mathbf{S}, o(\cdot, t))\right\rangle_{\mathbf{S}}+\eta \triangle o(\mathbf{x}, t)
$$

where

$$
A_{\sigma_{o}, \sigma_{r}}(\mathbf{x}, \mathbf{S}, o(\cdot, t))=\frac{e^{-\left(\left(\left|\mathbf{s}_{r}-\mathbf{x}\right|^{2}\right) / 2 \sigma_{r}^{2}+\left(\left|s_{o}-o(\mathbf{x}, t)\right|^{2}\right) / 2 \sigma_{o}^{2}\right)}}{\int d^{2} y e^{-\left(\left(\left|\mathbf{s}_{r}-\mathbf{x}\right|^{2}\right) / 2 \sigma_{r}^{2}+\left(\left|s_{o}-o(\mathbf{x}, t)\right|^{2}\right) / 2 \sigma_{o}^{2}\right)}} .
$$

For the spectrum of eigenvalues of the linearized dynamics we obtain

$$
\lambda(k)=-1+\frac{\left\langle s_{o}^{2}\right\rangle}{\sigma_{o}^{2}}\left(1-e^{-k^{2} \sigma_{r}^{2}}\right)-\eta k^{2} .
$$

and hence the critical wavenumber (i.e. the expected wavenumber of the emerging OD pattern) is given by

$$
k_{c}=\frac{1}{\sigma_{r}} \sqrt{\ln \left(\frac{\left\langle s_{o}^{2}\right\rangle}{\sigma_{o}^{2}} \frac{\sigma_{r}^{2}}{\eta}\right)} .
$$


By a rescaling of cortical space according to

$$
\begin{aligned}
\mathbf{x} & \rightarrow \mathbf{x}^{\prime}=\alpha \mathbf{x} \\
\mathbf{k} & \rightarrow \mathbf{k}^{\prime}=\frac{1}{\alpha} \mathbf{k} \\
\sigma_{r} & \rightarrow \sigma_{r}^{\prime}=\alpha \sigma_{r} \\
\eta & \rightarrow \eta^{\prime}=\alpha^{2} \eta,
\end{aligned}
$$

where the last transformation rescales the laplacian term in the r.h.s of eq. (6), we obtain

$$
k_{c}^{\prime}=\frac{1}{\alpha} k_{c}
$$

and hence $\Lambda_{\max }^{\prime}=\alpha \Lambda_{\max }$. The typical column spacing of the emerging OD pattern is properly transformed under the above rescaling. Importantly, the maximal growth rate

$$
\begin{aligned}
r^{\prime}=\frac{1}{\tau^{\prime}}=\lambda^{\prime}\left(k_{c}^{\prime}\right) & =-1+\frac{\left\langle s_{o}^{2}\right\rangle}{\sigma_{o}^{2}}\left(1-e^{-k_{c}^{\prime 2} \sigma_{r}^{\prime 2}}\right)-\eta^{\prime} k_{c}^{\prime 2} \\
& =\lambda\left(k_{c}\right)=\frac{1}{\tau}=r
\end{aligned}
$$

is unaffected by the scaling transformation. In fact, one can show that not only the linearized dynamics, but also all higher order terms determining pattern selection, are unchanged by this rescaling.

These considerations imply that the numerical value of $\sigma_{r}$ can always be identified with the value of $\sigma_{o}$ without loss of generality. In a final step, we choose to measure space in dimensionless units which allows for setting $\sigma_{o}=\sigma_{r}=\sigma$ and we arrive at the model definition as given in eq. (1) of our manuscript.

The linear as well as higher order terms exclusively depend on the ratio between $\left\langle s_{o}^{2}\right\rangle$ and $\sigma_{o}^{2}$, meaning that $\left\langle s_{o}^{2}\right\rangle$ just sets an arbitrary scale in OD space. For convenience, we chose $\left\langle s_{o}^{2}\right\rangle=1$ in our analytics as well as in our numerics.

In our simulations, we have applied the opposite direction of the above argumentation: fixing $r$ and $\eta$ (e.g., when computing the diagram in Fig. 3C), implied a certain ratio between $\left\langle s_{o}^{2}\right\rangle / \sigma^{2}$ as well as a numerical value of $\Lambda_{\max }$. The numerical value, $L$, of the system size was then set to a multiple of $\Lambda_{\max }, L=\gamma \Lambda_{\max }$, with $\gamma \in \mathbb{R}$ chosen according to the size of the cortical subregion (in terms of hypercolumns) we wanted to simulate (see below). 

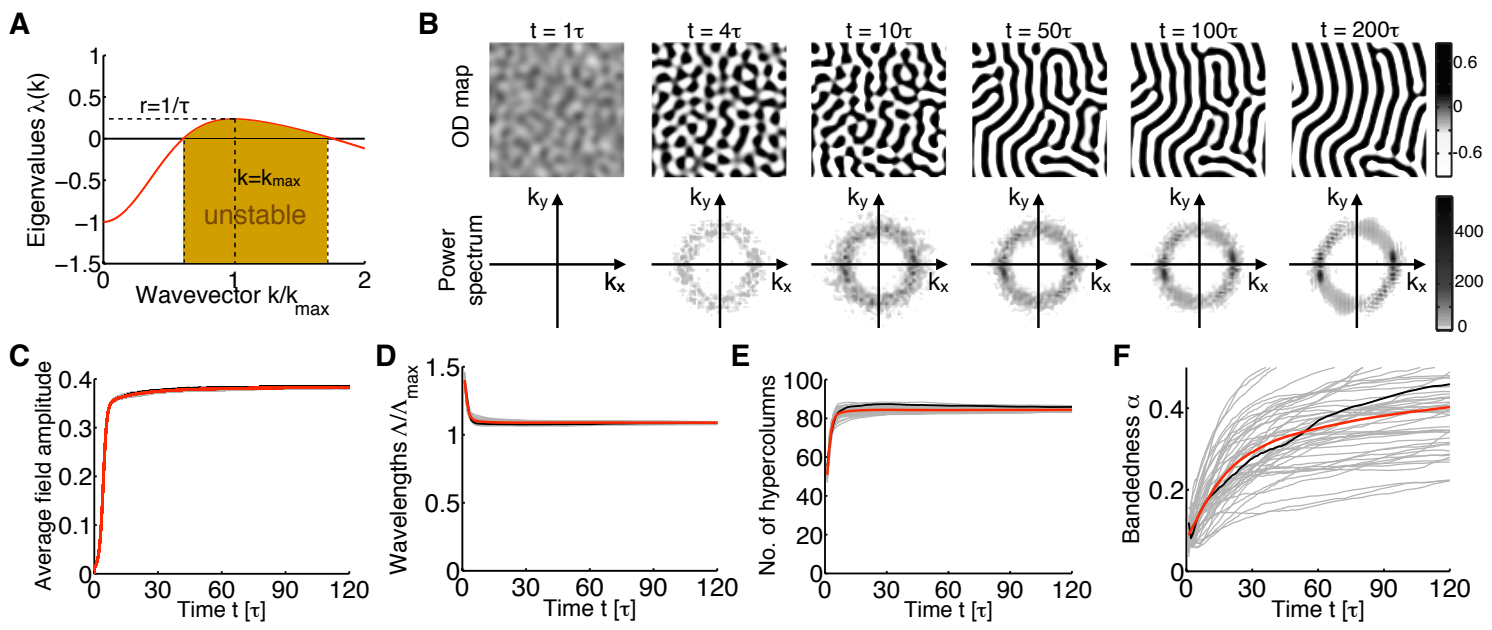

Figure S2. Development of OD column layout in the Elastic Network model without growth. (A) Eigenvalues of the linearized dynamics around the homogeneous nonselective state, $o(\mathbf{x})=0$. For maximal eigenvalue $r(\sigma, \eta)>0$, Fourier modes with positive growth rate form a band around $k_{\max }$, corresponding to an annulus in 2D. OD columns with spatial scale $\Lambda_{\max }=2 \pi / k_{\max }$ segregate on a time scale $\tau=1 / r$. (B) Development of OD columns starting from initial condition $o(\mathbf{x}, t=0)=0(\eta=0.025, r=0.2)$. At $t=4 \tau$, the emerging pattern already exhibits characteristics of OD columns. After $10 \tau$, columns start to merge and progressively reorganize towards a stripe-like layout. (C-F) Time courses of average OD segregation $\mathcal{A}(C)$ and of three parameters, characterizing OD layouts during development: (D) mean column spacing $\Lambda$ (E) number of hypercolumns $N_{H C}(\mathrm{~F})$ mean bandedness $\alpha(\mathrm{N}=50$ realizations, gray: individual traces, black: example from B, red: mean value). Whereas $\mathcal{A}, \Lambda$, and $N_{H C}$ reach mature levels around $10 \tau$ and exhibit only little variability, the bandedness $\alpha$ increases during the entire time course, expressing the fact that solutions slowly converge towards ideal OD stripes. Furthermore, $\alpha$-time courses display a large variability, capturing differences between individual realizations of OD map development. 


\subsection{Numerical procedures}

Simulations were performed on a $64 \times 64$ grid with periodic boundary conditions. Simulated systems were spatially discretized with at least 4 grid points per $\Lambda_{\max }$ to achieve sufficient resolution in space. Test simulations with larger grid sizes $(128 \times 128,256 \times 256)$ did not lead to significantly different results. Progression of time was measured in units of the intrinsic time scale $\tau$. Here, the integration time step $\delta t$ is bounded by the relevant decay time constant of the Laplacian in eq. (3) around $k_{\max }$ and by the intrinsic time scale $\tau$ of the system. We used $\delta t=\min \left\{1 /\left(20 \eta k_{\max }^{2}\right), \tau / 10\right\}$ to ensure good approximation to the temporally continuous changes of the OD patterns. Note that, since $\tau=1 / r$, simulation time diverges for $r \rightarrow 0$. The EN model dynamics was simulated using an Adams-Bashforth scheme for the first term on the r.h.s. of eq. (3). The second term was treated by spectral integration, exhibiting unconditional numerical stability. To approximate the stimulus ensemble, a large random sample of pointlike stimuli was drawn at each time step. Different realizations of $O D$ development were obtained by presenting different stimulus samples. The stimulus parameters $\mathbf{s}_{r}$ and $s_{o}$ were chosen to be uniformly distributed with densities $\rho_{\mathbf{s}_{r}}$ and $\rho_{s_{o}}$ such that $\left\langle s_{o}^{2}\right\rangle=1$ (see model definition above). The stimulus average in eq. (3) was approximated by choosing a random representative sample of $N_{s}$ stimuli at each integration time step, with

$$
N_{s}=\frac{N_{0} \Gamma^{2}}{\delta t} \varepsilon_{s}^{-n} \sqrt{\tau g_{00}}
$$

where $n$ is the number of dimensions of the feature space (in our case, $n=3), \Gamma^{2}=\left(L / \Lambda_{\max }\right)^{2}$ the size of the simulated system in units of $\Lambda_{\max }^{2}, \varepsilon_{s}$ the resolution in feature space, $N_{0}$ the number of stimuli we required to sufficiently approximate the cumulative effect of the ensemble of stimuli within each feature space voxel, and $g_{00}=\frac{9(\eta-1)^{4}}{40 \sigma^{6}}$ a factor that depends on the specific form of nonlinear competition between Fourier modes in the EN model. $\sqrt{\tau g_{00}}$ is proportional to the inverse of the expected mean amplitude of the OD pattern. With $N_{0}=50$ and $\varepsilon_{s}=0.15$, we ensured a low amplitude to noise ratio for all the simulations. Typical values for $N_{s}$ were between 40000 and 200000 . To model development prior to OD segregation, we initialized simulations with $o(\mathbf{x}, t=0)=0$. 


\section{Formation of ocular dominance in the Elastic Network model with- out growth.}

In this section, we briefly summarize the behavior of the Elastic Network model without cortical expansion. Fig. 2B shows a typical simulation of OD development $(\eta=0.025, r=0.2$, no increase in area). A structure strongly resembling the OD pattern observed in cat $\mathrm{V} 1$ emerges after a few time steps. Already at $t=4 \tau$ the OD pattern exhibits a visible characteristic spacing which becomes more dominant in the later time course. As a measure of the average cortical selectivity, we monitored the mean field amplitude $\mathcal{A}(t)=\int d^{2} x|o(\mathbf{x}, t)|$. It shows a rapid increase during the first $10 \tau$ and saturates thereafter (Fig. S2C).

We quantified each simulated OD development by the wavelet-method applied to our experimental data and compute three parameters characterizing the map layouts. First, we estimated the mean column spacing $\Lambda$. We find, that it reaches a constant value after approximately $10 \tau$ close to the predicted value $\Lambda_{\max }$ (Fig. S2D). Next, we computed the number of hypercolumns $N_{H C}$ in a simulated area. This number changes only very little after OD columns have segregated (Fig. S2E). Finally, we calculated the bandedness, $\alpha$, which quantifies the tendency of OD domains to form elongated parallel stripes. In all our simulations, the initial phase of OD segregation is followed by a long stage of slow rearrangements leading to progressively more stripe-like OD layouts. This behavior is captured by a monotonically increasing bandedness $\alpha$ (Figure S2F). In fact, we numerically find that OD stripes, for which the bandedness is maximal, are stable solutions of the EN model. We do not find any other stable solution.

\section{Simulating growth}

\subsection{Instantaneous area increases}

We started simulations with a predefined system length of $L_{0}$ and used a parameter-dependent sine-wave initial condition

$$
o(\mathbf{x})=1 / \sqrt{\tau g_{00}} \sin \left(k_{\max } x\right)
$$

with periodicity $\Lambda_{\max }=2 \pi / k_{\max }$ and amplitude close to the amplitude of the expected stationary state. The system was integrated keeping the length of the system fixed for the first $10 \tau$. Within this 
period the amplitude $\mathcal{A}(t)=\int d^{2} x|o(\mathbf{x}, t)|$ of the OD stripe patterns relaxed towards a stationary value while OD layout underwent only minor random fluctuations. Next, we rescaled the system length to $L_{0}+\delta L$ (no change in number of grid points), where

$$
\delta L=\frac{\left(\delta k / k_{\max }\right)}{\left(\delta k / k_{\max }\right)-1} L_{0}
$$

is determined from the desired value of $\delta k / k_{\max }$, thereby increasing $(\delta L>0)$ or decreasing $(\delta L<0)$ the total area size covered by the OD pattern. Accordingly, we adjusted the number of stimuli, $N_{s}$, and, since $\Delta \sim 1 / L^{2}$, the matrix for the spectral integration step. Note that the numerical value of $\sigma$, i.e. the width of the activation function $A_{\sigma}(\mathbf{x}, \mathbf{S}, o(\cdot, t))$, remains unchanged by this rescaling, but its extension relative to $L$ decreases (and the number of grid points per activation blob decreases as well). After rescaling, we continued simulations for another $190 \tau$. Upon instantaneous isotropic area increase, OD stripes typically display a zigzag-like bending of domains (Fig. S3A). As outlined below (sec. 6.3), a ZZ instability is characterized by the growth of two Fourier modes (representing the zig and the zag respectively) with wave vectors $\tilde{\mathbf{k}}_{Z Z}=\left(k_{\max }+\delta k\right) \tilde{\mathbf{x}} \pm q_{y} \tilde{\mathbf{y}}$, where $k_{\max } \tilde{\mathbf{x}}$ is the wave vector of the original stripe pattern. A ZZ instability can therefore be reliably identified by monitoring the power spectrum along the axis $k_{x}=k_{\max }+\delta k$ and searching for two characteristic peaks corresponding to the $Z Z$ modes.

To estimate the time scales $\tau_{Z Z}$ of this reorganization, we followed the growth of the two $Z Z$ modes over time (see Fig. S3B and C). At each time point, we fitted Gaussians (least square fit) of variable size, width, and position to the original mode and the two growing $Z Z$ modes along the expected axis of the ZZ modes in Fourier space (Fig. S3B). The time scale of ZZ reorganization was extracted by linear fitting (least square fit) the logarithm of the ratio between the peak height of the $\mathrm{ZZ}$ modes, $p_{Z Z}$, and the central peak height stemming from the original pattern, $p_{c}$ (Fig. $\mathrm{S} 3 \mathrm{C})$. We constrained the fit to values between $15 \%$ and $60 \%$ of the maximal peak ratio to capture best the regime of exponential growth of the $Z Z$ modes. The fitting procedure was only applied to simulations with maximal peak ratio of at least 0.05 . In Figure $3 \mathrm{C}$ of the manuscript, the inverse of the estimated growth rate, i.e. the time scale of the $Z Z$ instability, $\tau_{Z Z}$, is compared to the intrinsic time scale $\tau$ of OD segregation for various changes of area $(\delta k)$ and different values of the control parameter $r$. 
A $\quad t=10 \tau$
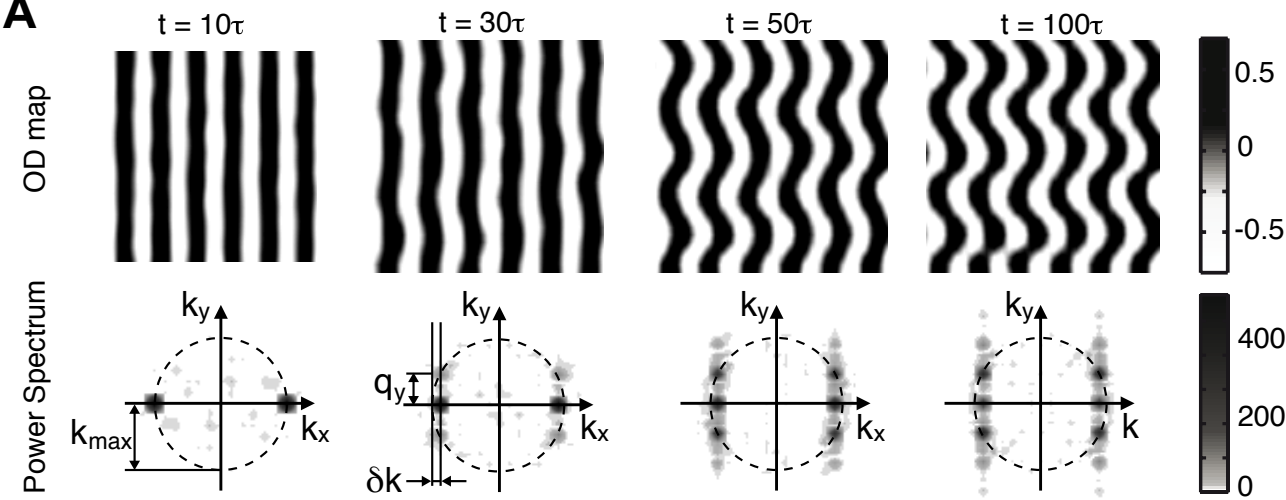

B
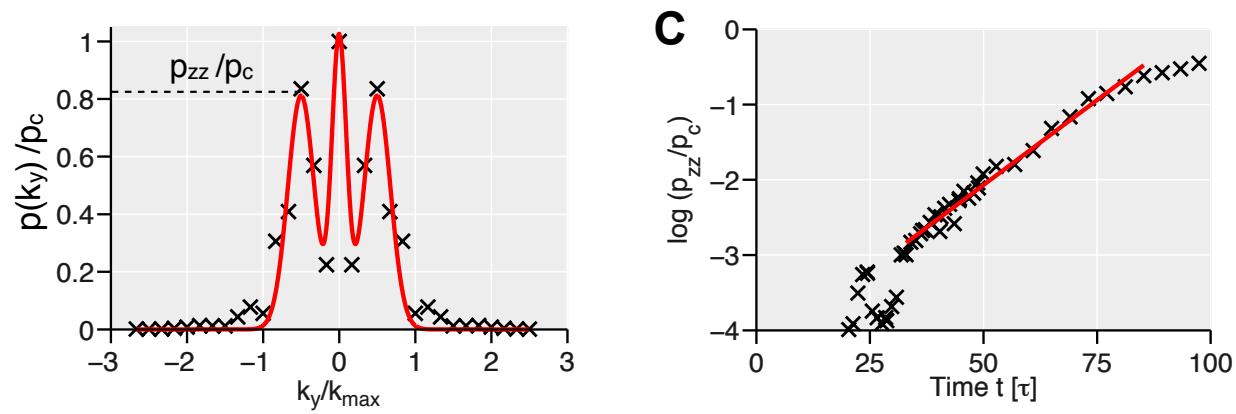

Figure S3. Measuring the time scale of ZZ reorganization in the EN model. (A) Snapshots of a simulation starting from a near steady state solution of the Elastic Network (EN) model [8], a stripelike OD pattern (upper row) corresponding to a single mode in the power spectrum (lower row) $(\eta=0.025, r=0.15)$ (redrawn from Fig. $2 \mathrm{~A}$ in the manuscript). After instantaneous area increase (by a factor of 1.18 (linear extent), i.e. $\delta k / k_{\max }=-0.15$, at $10 \tau$ ), OD domains bend sinusoidally and additional modes appear at $\left(k_{\max }+\delta k\right) \tilde{\mathbf{x}} \pm q_{y} \tilde{\mathbf{y}}$. (B) Measuring the growth rate of zigzag modes for the simulation in A. Black crosses mark the power spectrum $p\left(k_{x}=k_{\max }+\delta k, k_{y}\right)$. Most power is concentrated around the origin $k_{y}=0$, stemming from the initial stripe-like OD pattern, and around two secondary peaks corresponding to the emerging ZZ modes (compare to A, lower row). Red curve is a fit of three Gaussians (least square fit). (C) Ratios between the height $p_{c}$ of the central peak and height $p_{Z Z}$ of the side peaks at different time points (black crosses). The growth rate of $Z Z$ modes is estimated by fitting a linear slope to the logarithm of $p_{Z Z} / p_{c}$ in the region of exponential growth (least square fit). 


\subsection{Continuous area increases}

Different simulation protocols for realistic cortical growth. We tested three different simulation protocols to approximate the postnatal growth of cat V1 (see Fig. S4A, B):

(i) linear increase of the linear extent with slope $\alpha$, starting from $L_{0}$,

$$
L(t)=L_{0}+\alpha t
$$

(ii) sublinear increase of the linear extent $L$, such that the simulated area $A$ increases linearly with slope $\beta$,

$$
L(t)=\sqrt{L_{0}^{2}+\beta t}
$$

(iii) and logistic increase [19] of the linear extent $L$

$$
L(t)=L_{0} \frac{e^{\varepsilon t}}{1+\xi^{-1}\left(e^{\varepsilon t}-1\right)} \quad \lim _{t \rightarrow \infty} L(t)=\xi L_{0} .
$$

In each protocol, we tested total increases between $t=0 \tau$ and $t=100 \tau$ by factors of $1.2,1.4,1.6$ and 1.8 in linear extent of the simulated region (corresponding to isotropic total area increases by a factor of 1.44, 1.96, 2.56 and 3.24). Note that according to [20], the area of cat V1 increases between week 1 and week 12 by approximately a factor of 2.5 and thus our range of parameters includes realistic growth conditions. In our simulations of OD development, we find that different growth protocols yield similar results. In fact, the linear and sublinear increases describe virtually identical growth scenarios (Fig. S4, A and B) and provide a close match to the data on V1 growth shown in [20]. Our systematic quantitative characterization of OD development in the EN model with growth (Fig. 4 in the manuscript) was based on the scenario of linear increase of the linear extent (red traces in Fig. S4, A and B).

To model realistic growth conditions in our simulations, we linearly increased the linear extent $L$ of the simulated regions between $t=0 \tau$ and $t=100 \tau$. System length $L$, the Laplacian $\Delta$, and the number of stimuli $N_{s}$ were updated at each integration step. After $100 \tau$, integration was continued for another $100 \tau$ with fixed linear extent.

To capture and quantify the size of bandedness drops, we calculated their strength $\Delta \alpha$ and their duration $\Delta t$, i.e. the time over which $\alpha$ persistently decreased (Fig. 4E). We fitted an 8th- 

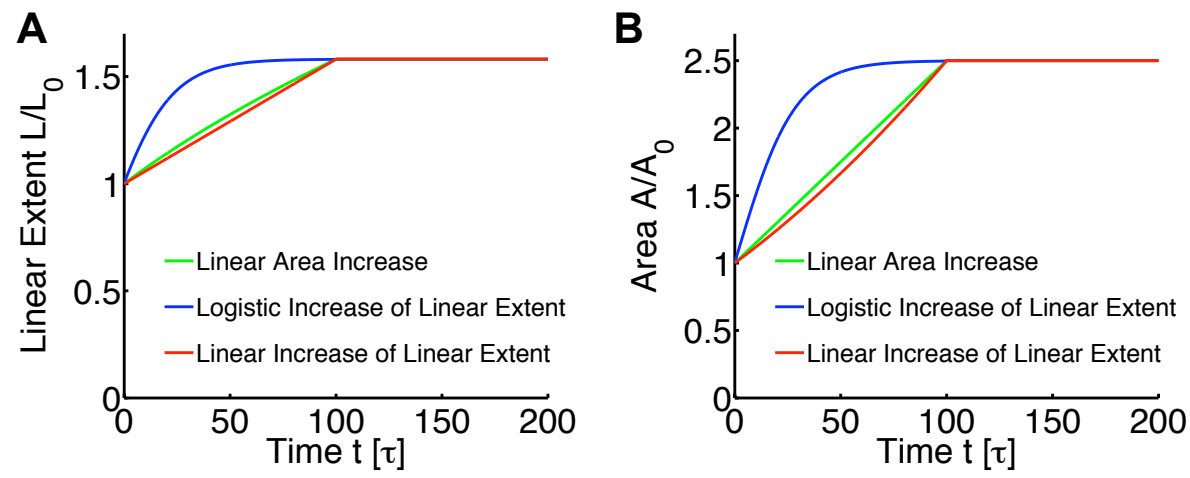

Figure S4. Three different simulation protocols of realistic cortical growth. (A and $B$ ) Time courses of the linear extent $L / L_{0}(\mathrm{~A})$ and the area size $A / A_{0}(\mathrm{~B})$ for linear increase of area (green traces), linear increase of the linear extent (red traces) and logistic growth (blue traces). Simulations of OD development for all three growth protocols yield qualitatively similar time courses of column spacing $\Lambda$, number of hypercolumns $N_{H C}$ and bandedness $\alpha$. Linear area increase and linear increase of the linear extent are virtually identical and both provide a reasonable fit to the data on cat $\mathrm{V} 1$ growth in [20] when setting 1 day $\approx 1 \tau$. Our quantitative characterization of OD development in the EN model with growth (Fig. 4 in the manuscript, Fig. S7) was carried out using linear increase of the linear extent (red traces).

order polynomial to each $\alpha$-time series (least square fit). Using an 8th-order polynomial, we ensured that the fit closely followed the coarse-grained bandedness time course for all simulations. In our simulations, we approximated the stimulus average in EN model equation (eq. (3)) by a representative sample of stimuli (typically between $4 \times 10^{4}$ and $2 \times 10^{5}$ ) drawn at each integration step. Thus, our simulations provided a stochastic approximation to the deterministic dynamics of the EN model as analyzed here. The time courses of wavelengths, bandedness and number of hypercolumns were fluctuating around the "real" time course. Specifically, for simulations carried out for small $r$ ), bandedness fluctuations were around 0.01 . To reliably detect growth-induced reorganizations, we only considered bandedness drops of minimum size $\Delta \alpha>0.05$. Furthermore, as growth-induced reorganization is expected to evolve on timescales several fold larger than the intrinsic time scale $\tau$ of OD segregation (see Fig. 2 and 3 in the manuscript), we only included drops with $\Delta t>15 \tau$ in our analysis. The results presented in Fig. $4 \mathrm{~F}-\mathrm{H}$ of the manuscript were not sensitive to this particular choice of parameters. 


\section{Zigzag and Eckhaus instability - parameter regimes}

In this section, we discuss two fundamental instabilities of stripe patterns subject to size increase or decrease, namely the zigzag (ZZ) instability and the Eckhaus instability. We describe their basic mechanisms and their expected parameter regime of occurrence. As it turns out, the ZZ instability constitutes the generic behavior upon isotropic continuous area increase.

\subsection{Zigzag instability}

The $\mathrm{ZZ}$ instability has been observed in many inanimate dynamical pattern forming systems as, for instance, Rayleigh-Benard convection, when these systems experience an instantaneous isotropic size increase $[13,14,15]$. As pointed out in our manuscript, the result of a $Z Z$ instability can be understood best by considering a simple pattern of OD columns consisting of alternating stripes (Fig. 3A in the manuscript). After an isotropic area increase, the spacing between the OD stripes is larger than the spacing set by the Mexican-Hat. Subsequently, the structure rearranges in a sinusoidal fashion: Through a zigzag-like bending of stripes the original spacing is largely recovered (see manuscript for details).

\subsection{Eckhaus instability}

The Eckhaus instability is a dynamical instability observed in systems that undergo drastic changes in system size $[13,14,15]$. Rearrangements of domains consist either of inserting new domains (area increase) or removing existing ones (area decrease). Even in two-dimensional systems, this instability gives rise to an effectively one-dimensional reorganization. It does not require rotation symmetry of the instability mechanism. A recent one-dimensional cortical growth study focused on the Eckhaus instability [18]. However, in our simulations, we did not observe an Eckhaus instability, even in the case of large instantaneous increase or decrease of area size.

The result of an Eckhaus instability can be understood best by considering a simple pattern of OD columns consisting of alternating stripes (Fig. S5). After an isotropic area increase, the spacing between the OD stripes is larger than the spacing set by the Mexican-Hat. Subsequently, new stripes of $\mathrm{OD}$ domains emerge between existing ones by which the original spac- 


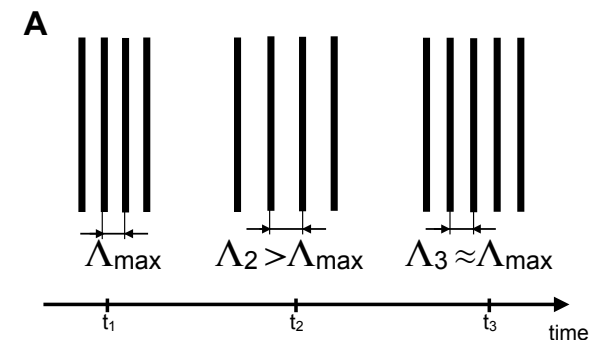

B

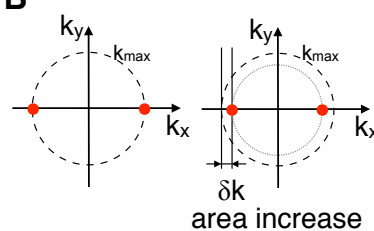

C

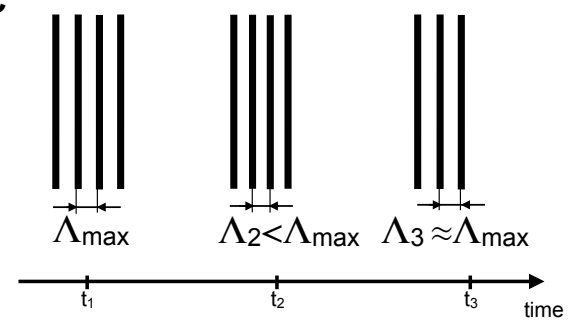

D

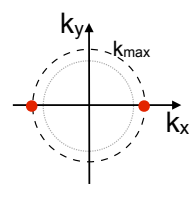

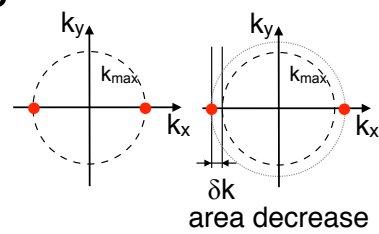

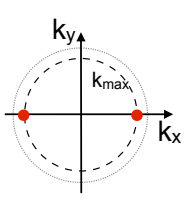

Figure S5. Eckhaus reorganization upon large isotropic area increase (A, B) or area decrease (C, D). Upon area increase/decrease, OD stripes recover their initial spacing $\Lambda_{\max }$ by insertion/removal of OD stripes. (A) During isotropic area increase, the OD pattern transiently acquires a spacing $\Lambda_{2}=\Lambda_{\max }+\delta \Lambda$, with $\delta \Lambda>0$. By insertion of an new stripe the system recovers a spacing $\Lambda_{3}$, roughly equal to $\Lambda_{\max }$. (B) In Fourier-space, the initial pattern is centered at $\pm k_{\max } \tilde{\mathbf{x}}$. $\delta \Lambda>0$ corresponds to a shift of $\delta k<0$. The initial spacing is recovered by the growth of new Fourier modes close to modes of the original pattern $\pm k_{\max } \tilde{\mathbf{x}}$. (C) During isotropic area decrease, the OD pattern transiently acquires a spacing $\Lambda_{2}=\Lambda_{\max }+\delta \Lambda$, with $\delta \Lambda<0$. By removal of a stripe the system recovers a spacing $\Lambda_{3}$, roughly equal to $\Lambda_{\max }$. (D) In Fourier-space, $\delta \Lambda<0$ corresponds to a shift of $\delta k>0$. The initial spacing is recovered by the growth of new Fourier modes at the center of the original pattern, i.e. $\pm k_{\max } \tilde{\mathbf{x}}$. Note that these modes of reorganization require strong and sudden changes in area. 
ing is eventually recovered (Fig. S5A). Fig. S5B illustrates this in Fourier space. The initial stripe pattern is described by a wave $\sim e^{i \tilde{\mathbf{k}}_{\max } \hat{\mathbf{x}}}$ with spatial wave vector $\tilde{\mathbf{k}}_{\max }$ and wave num$\operatorname{ber}\left|\tilde{\mathbf{k}}_{\max }\right|=k_{\max }=2 \pi / \Lambda_{\max }$. Upon area increase, its wavelength increases, and therefore its wave number differs by an amount $\delta k<0$ from $k_{\max }$. During an Eckhaus instability, two Fourier modes with wave vector $\pm k_{\max } \tilde{\mathbf{x}}$ grow, representing the new OD pattern with similar spacing as the original pattern.

Similarly, after an isotropic area decrease, the spacing between the OD stripes is smaller than the spacing set by the Mexican-Hat. Subsequently, a fraction of the OD stripes is removed and the original spacing is eventually recovered (Fig. S5C). Fig. S5D illustrates this in Fourier space. Upon area decrease, the OD pattern's wave number differs by an amount $\delta k>0$ from $k_{\max }$. Two Fourier modes grow with wave vector $\pm k_{\max } \tilde{\mathbf{x}}$, representing the new OD pattern in Fig. S5C with similar spacing as the original pattern.

\subsection{Parameter regimes}

In this section, we discuss the generic regions of occurrence of zigzag and Eckhaus instability in the $(\delta k, r)$-parameter space, where $r$ is the control parameter (or bifurcation parameter) of the system expressing the distance from the instability threshold of pattern formation, i.e. the distance from the primary finite wavelength instability, and $\delta k$ controls the size of area increase/decrease. Assuming a plane wave solution $A(x, y, t) e^{i k_{\max } x}$, close to the finite wavelength instability its behavior is governed by the Newell-Whitehead equation [16]

$$
\partial_{t} A=r A+\left(\partial_{x}-i \partial_{y^{2}}\right)^{2} A-|A|^{2} A .
$$

The form of Eq. (7) is independent of the microscopic details of the instability mechanism [14, 15]. Inserting the ansatz $A=|A| e^{i\left(\delta k x+\phi_{0}\right)}$ yields a uniform solution $|A|=\sqrt{r-\delta k^{2}}$ as long as $|\delta k|<\sqrt{r}$. The wave number of this solution differs by an amount $\delta k$ from $k_{\max }$, i.e. the characteristic spacing of the solution is either smaller or larger than $\Lambda$. Linear stability analysis yields the behavior of this solution depending on the control parameter $r$ and the difference in wave vector $\delta k$ [17]. Considering general perturbations of the form $\delta A \sim e^{i q_{y} y} e^{i q_{x} x}$, the uniform 


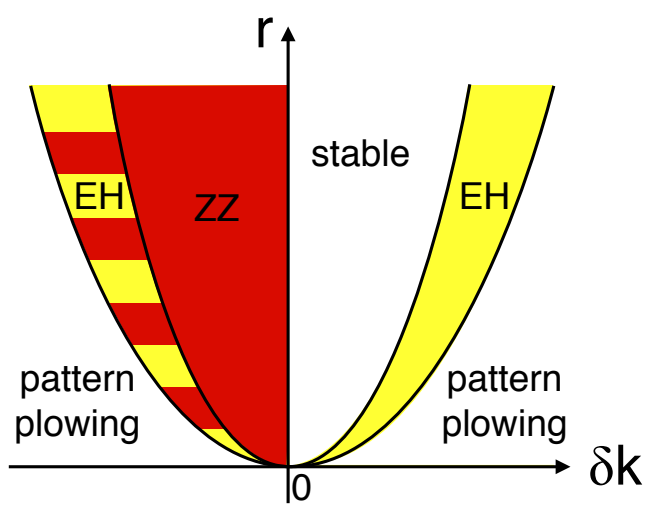

Figure S6. Generic regions of linear instabilities (Busse Balloon) of a stripe pattern in the $(\delta k, r)$-plane (redrawn from [13, 15]). The diagram applies to the general model class of 2dimensional relaxational, isotropic dynamics with linear Mexican-Hat type interactions. The region of the zigzag instability (ZZ, red) is to the left of the $\delta k=0$-axis, and is bounded by a parabola. $\mathrm{EH}$ (yellow) denotes the regions of the Eckhaus instability (see text) where a new OD stripe is inserted $(\delta k<-\sqrt{r / 3})$ or withdrawn $(\delta k>\sqrt{r / 3})$. For $-\sqrt{r}<\delta k<-\sqrt{r / 3}$ these two regions overlap (striped region). Note that the $\mathrm{ZZ}$ instability occurs for an arbitrarily small area increase. Therefore, it is the generic behavior of models for OD column formation upon continuous isotropic area increase.

solution is found to be unstable against perturbations with $q_{x}=0$ as soon as

$$
\delta k<0 .
$$

This defines the domain of the ZZ instability (red regions in Figure S6). In contrast, for $q_{y}=0$ the uniform solution is only unstable if $|\delta k|>\sqrt{r / 3}$. This is the domain of the so-called Eckhaus instability (EH; yellow regions in Figure S6). For $-\sqrt{r}<\delta k<-\sqrt{r / 3} \mathrm{EH}$ and ZZ overlap (striped region in Fig. S6) and model specific properties determine which of the two behaviors is observed in this regime. For $|\delta k|>\sqrt{r}$, Eq. (7) has no uniform stationary solution. In this regime, the initial stripe pattern decays exponentially and a new OD pattern emerges (pattern plowing, see Fig. S6). In summary, the $\mathrm{ZZ}$ instability is expected to dominate the response of OD patterns if the area increase is moderate. The Eckhaus instability is expected to occur only in systems with abrupt and dramatic size changes. We note that the form of the diagram in Figure 6 is very general, applying to the class of two dimensional translation and rotation symmetric relaxational dynamics in which the pattern forming process is based on a finite wavelength instability $[14,15]$. 


\section{$7 \quad \mathrm{ZZ}$ reorganization during continuous isotropic area increase start- ing from OD stripes}

In our manuscript, we analyzed OD stripes subject to instantaneous size increase and continuous expansion starting from the nonselective cortex in the EN model. In this section, we probe the reorganization of $\mathrm{OD}$ stripes under continuous increase of the system size for two different values of the control parameter $r$ (Fig. S7). We initialized our simulations with a sinusoidal pattern $o(\mathbf{x}) \sim \sin \left(k_{\max } x\right)$. After a brief relaxation period of $20 \tau$, we linearly increased the linear extent of the simulated regions by a factor of 1.2, 1.4, 1.6 and 1.8 between $t=20 \tau$ and $t=120 \tau$ (corresponding to isotropic total area increases by a factor of $1.44,1.96,2.56$ and 3.24 ). In these simulations, columnar layouts generally show reorganization on similar time scales but more irregular reorganization when compared to the scenario of instantaneous area increases (Fig. 2, 3 in the manuscript). As for the case of instantaneous size increase, the column spacing $\Lambda$ increases

only transiently (Fig. S7, C and $\mathrm{H}$ ). Thus, the number of hypercolumns $N_{H C}$ strongly increases over the simulated time period (Fig. S7, D and I). The bandedness $\alpha$ decreases after onset of reorganization as expected from the sinusoidal bending of OD domains (Fig. S7, E and J). Thus, a sinusoidal bending of OD stripes which is characteristic for a $Z Z$ instability also occurs upon continuous increase of the system size.

\section{Increasing the effective intracortical interaction width during sim- ulations of realistic growth scenarios}

To investigate growth-induced reorganization in our manuscript, we assumed constant EN model parameters in simulations of cortical growth. This implies that the range of lateral interaction does not change in size during cortical expansion. These interactions are of Mexican-Hat (MH) type and arise from the interplay between co-activation of cortical regions of roughly columnar size and a tendency of neighboring neurons to acquire similar response properties.

In this section, we examine the behavior of the EN model if the interaction width, i.e. the width of the $\mathrm{MH}$, is also expanding during cortical growth. We start by considering the extreme case, where both, cortical size and interaction width increase at the same rate. Fig. S8A shows the 

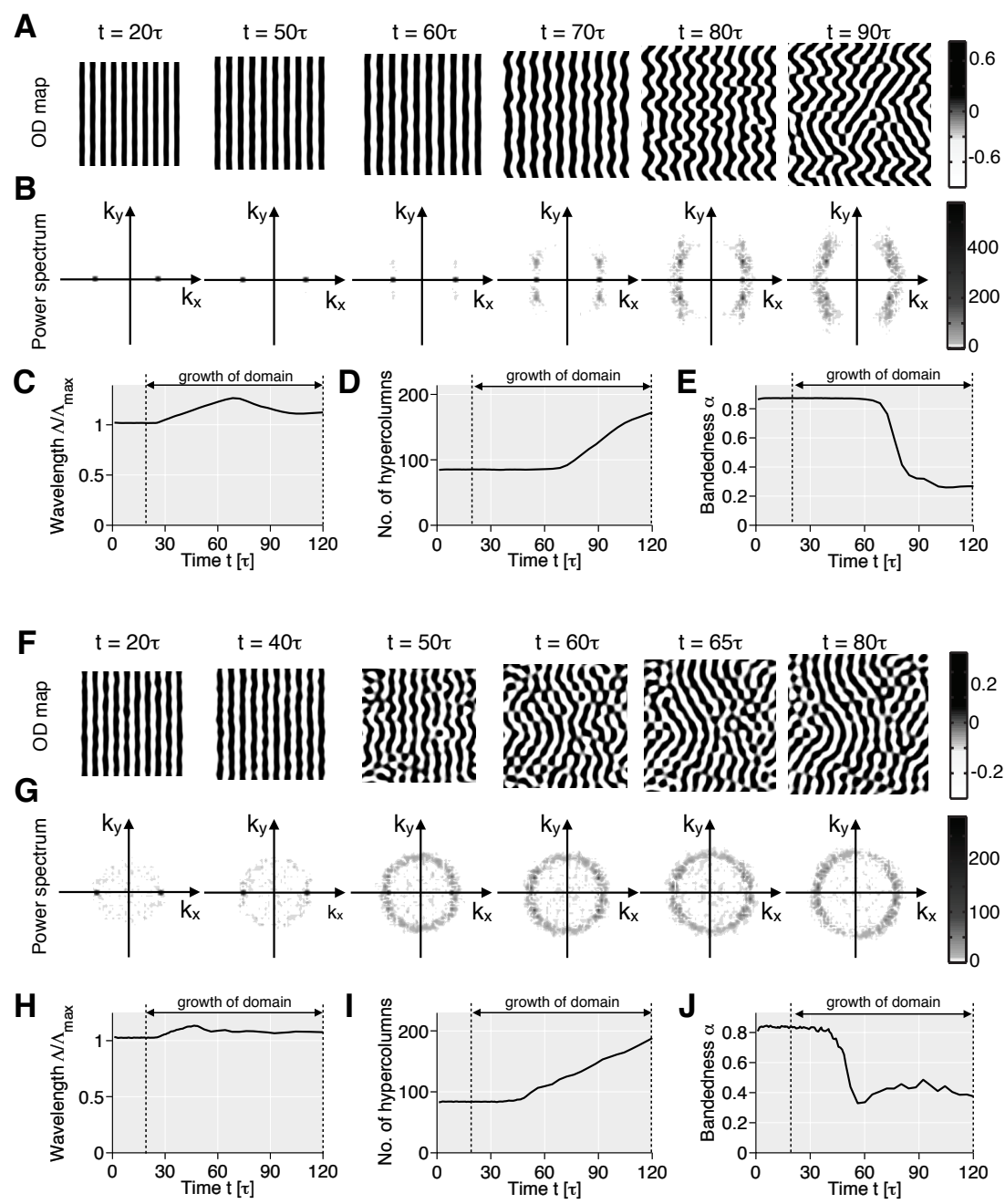

Figure S7. ZZ reorganization during continuous isotropic area increase starting from OD stripes. (A and B) Snapshots of OD layouts (A) and power spectra (B) of EN model simulations with continuous expansion starting from OD stripes in a parameter regime relatively far away from the pattern formation instability threshold $(r=0.16, \eta=0.025$, total area increase by factor of 2.56 between $t=20 \tau$ and $t=120 \tau)$. Until $t=50 \tau$ the OD stripes remain almost unchanged. Subsequently, a ZZ-type reorganization is apparent from the sinusoidal bending of domains $(A)$ and the growth of Fourier Modes at $k_{\max } \tilde{\mathbf{x}} \pm q \tilde{\mathbf{y}}(\mathrm{B})$. ( $\mathrm{C}$ to $\left.\mathrm{E}\right)$ Time courses of column spacing $\Lambda$ (C, normalized), number of hypercolumns $N_{H C}(\mathrm{D})$, and bandedness $\alpha(\mathrm{E})$. (F) A simulation in a regime closer to the pattern formation threshold $(r=0.04)$. Compared to $\mathrm{A}$, the pattern acquires a more isotropic layout over time. (G) Power spectra for (F). (H to J) Column spacing (H), hypercolumn number $N_{H C}(\mathrm{I})$, and bandedness $\alpha(\mathrm{J})$. Note, that despite a rather complex reorganization, key signatures of a ZZ instability are visible, such as a sinusoidal bending of domains. 
development of OD layouts for one such simulation $(r=0.16, \eta=0.025$, total area increase by a factor of 2.56 between $t=0 \tau$ and $t=100 \tau)$. By mere visual inspection, an increase in column spacing can be observed. Furthermore, the OD layout rapidly becomes stripe-like during the time course. Quantifying these observations, the mean column spacing $\Lambda$ increases considerably (Fig. $\mathrm{S} 8 \mathrm{~B}$ ), the number of hypercolumns $N_{H C}$ is roughly constant (Fig. S8C) and the bandedness $\alpha$ increases persistently (Fig. S8D) as in the simulations without growth (Fig. S2F).

What happens if the interaction width increases at lower rate than the cortex? Fig. S8E shows the typical development of OD layouts in a simulation for which $\mathrm{MH}$ growth rate was set to half of the cortical area growth rate $(r=0.16, \eta=0.025$, total area increase by a factor of 2.56 between $t=0 \tau$ and $t=100 \tau)$. In contrast to Fig. S8A, the OD layout acquires a more bended and ZZ-type shape during the time course, strikingly similar to Fig. 4A in the manuscript (see red frames in Fig. 8E). The mean column spacing $\Lambda$ increases considerably (Fig. S8F), though less than in Fig. S8B. The number of hypercolumns $N_{H C}$ increases (Fig. S8G), however less than in Fig. $4 \mathrm{C}$ of the manuscript. The bandednesses $\alpha$ exhibits a pronounced decrease, similar to the growing systems simulated in Fig. 4 - a characteristic feature of the ZZ instability.

The above observations are consistent with our results on simulations with fixed interaction width (see manuscript). There we have shown that even moderate cortical expansion rates can induce substantial cortical reorganization, both in simulations with abrupt size increases as well as in realistic growth scenarios. However, in the case at hand, this is accompanied by an increase in mean column spacing, and a relatively small increase in the number of hypercolumns. As we did not observe any increase in column spacing in cat $\mathrm{V} 1$ between week 4 and 14, this suggests that the effective intracortical interaction width stays approximately constant (see discussion in the manuscript). 

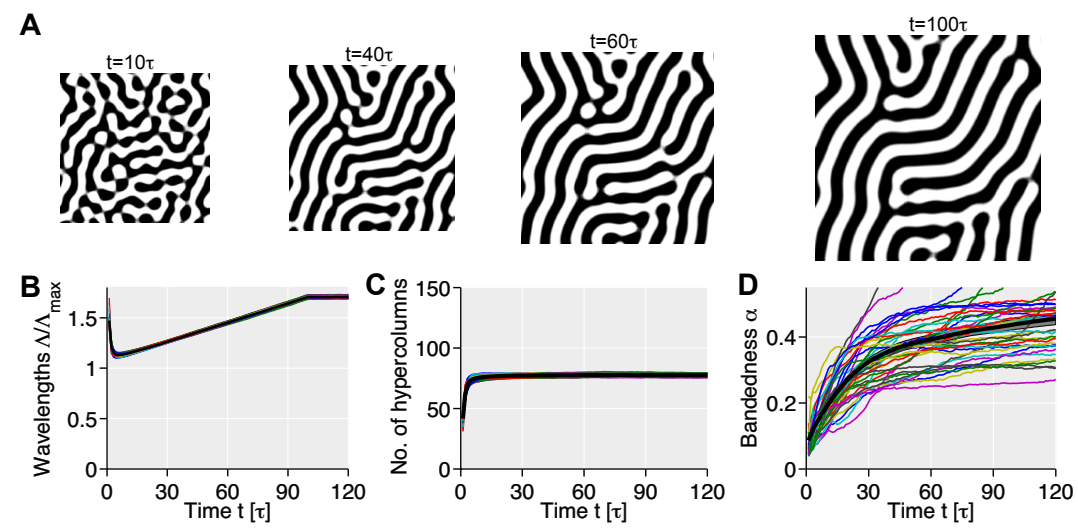

E
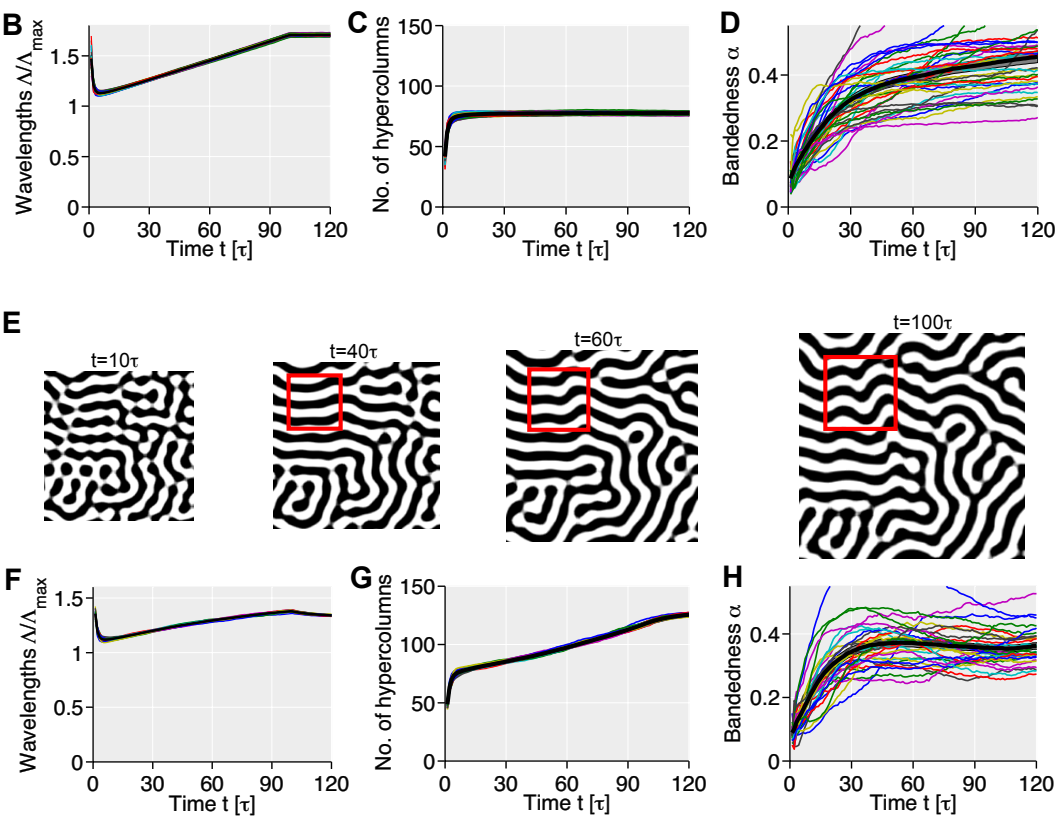

Figure S8. Increasing the width of interactions in the EN model during simulations of realistic growth scenarios. (A) Snapshots of OD layouts of EN model simulations with continuous cortical expansion and continuously increasing width of the effective intracortical interactions (Mexican-hat) with the same rate $(r=0.16, \eta=0.025$, total area increase by a factor of 2.56 between $t=0 \tau$ and $t=100 \tau)$. (B to $\mathrm{D})$ Time courses of column spacing $\Lambda(\mathrm{B})$, number of hypercolumns $N_{H C}(\mathrm{C})$, and bandedness $\alpha(\mathrm{D})$ for $\mathrm{N}=30$ simulations (parameters as in $\mathrm{A}$ ). The mean column spacing $\Lambda$ increases strongly between $t=10 \tau$ and $t=100 \tau$. Consequently, the number of hypercolumns $N_{H C}$ remains roughly constant. Cortical expansion does not induce a drop in bandedness. $\alpha$-time-courses show a monotonous increase as in simulations with non-growing cortical size (Fig. S2). (E) Snapshots of OD layouts of EN model simulations with continuous expansion and continuously increasing width of the effective intracortical interactions with half the cortical growth rate $(r=0.16, \eta=0.025$, total area increase by a factor of 2.56 between $t=0 \tau$ and $t=100 \tau)$. ( $\mathrm{F}$ to $\mathrm{H}$ ) Time courses of column spacing $\Lambda(\mathrm{F})$, number of hypercolumns $N_{H C}$ $(\mathrm{G})$, and bandedness $\alpha(\mathrm{H})$ for $\mathrm{N}=30$ simulations $(r=0.16, \eta=0.025$, total area increase by a factor of 2.56 between $t=0 \tau$ and $t=100 \tau)$. Growth typically induces a considerable drop in $\alpha$. However, in contrast to a fixed Mexican-Hat size (Fig. 4B in the manuscript), the mean column spacing $\Lambda$ increases considerably between $t=10 \tau$ and $t=100 \tau$. Consequently the number of hypercolumns $N_{H C}$ only increases moderately. 


\section{Movies}

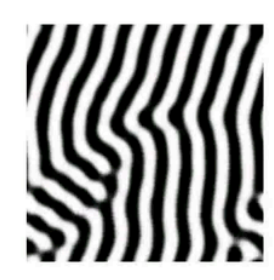

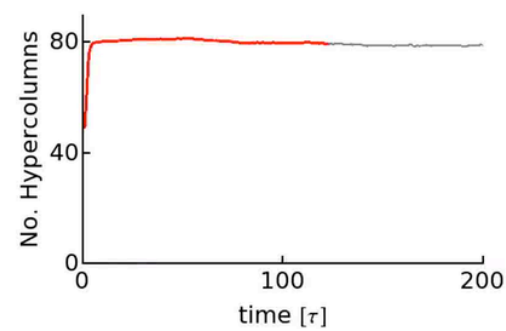

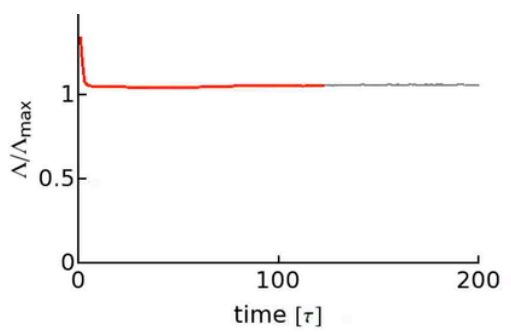

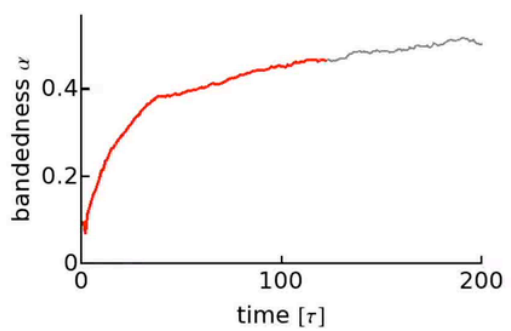

Movie S1. Development of OD column layout in the EN model without growth, starting from nonselective initial condition $o(\mathbf{x}, t=0)=0(\eta=0.025, r=0.16)$. The video displays OD maps (upper left) as well as the time courses of the parameters column spacing $\Lambda$ (upper right), number of hypercolumns $N_{H C}$ (lower left), and bandedness $\alpha$ (lower right), characterizing OD layouts during development. At $t=4 \tau$ OD columns have segregated. After $10 \tau$, columns start reorganizing and merge progressively towards a stationary stripe-like layout. Whereas $\Lambda$ and $N_{H C}$ reach near mature levels around $10 \tau$, the bandedness $\alpha$ increases during the entire time course capturing the slow reorganization of OD columns towards stripes. 


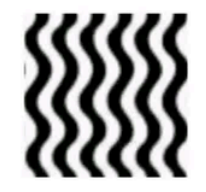
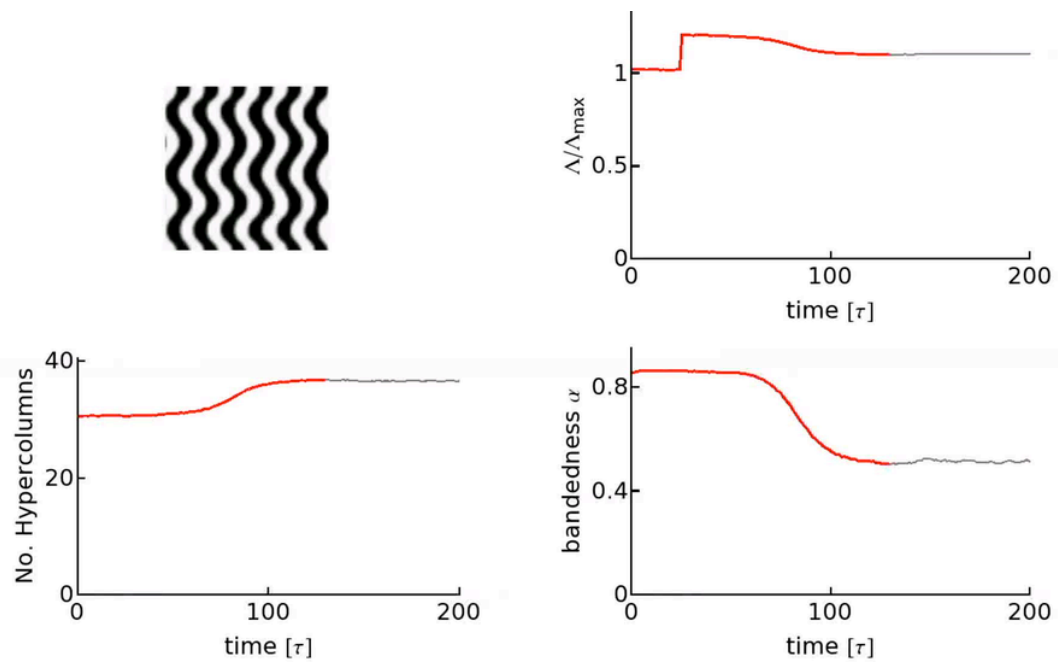

Movie S2. Zigzag reorganization in the EN model after instantaneous isotropic area increase $(\eta=0.025, r=0.16$ ). The video displays OD maps (upper left) as well as the time courses of the column spacing $\Lambda$ (upper right), the number of hypercolumns $N_{H C}$ (lower left), and the bandedness $\alpha$ (lower right). After instantaneous area increase at $t=10 \tau$, OD stripes bend sinusoidally, a behavior characteristic for ZZ instability. A jump in $\Lambda$ is observed shortly after $t=10 \tau$. Subsequently, $\Lambda$ decreases to almost the initial value and, consequently, $N_{H C}$ increases. The bandedness $\alpha$ decreases reflecting the bending of OD domains.
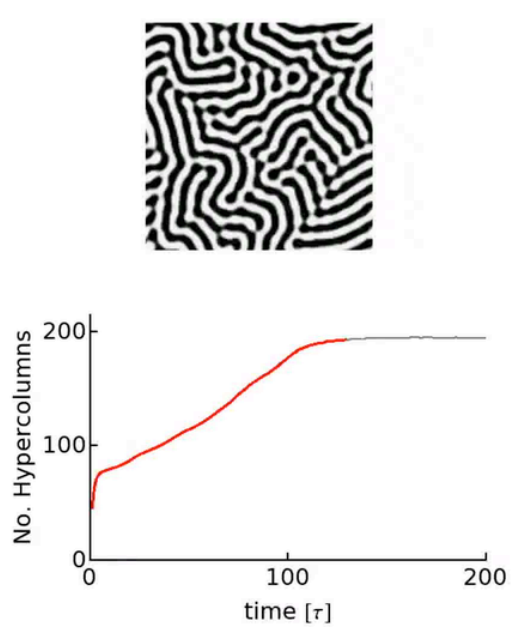
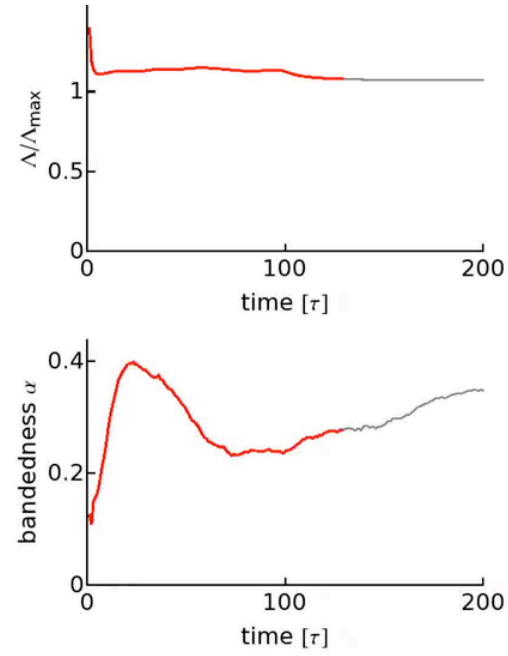

Movie S3. OD development in the EN model with growth at near realistic growth rate. (total area increase by a factor of 2.56 between $t=0$ and $t=100 \tau, \eta=0.025, r=0.16$ ). The video displays OD maps (upper left) as well as the time courses of the column spacing $\Lambda$ (upper right), the number of hypercolumns $N_{H C}$ (lower left), and the bandedness $\alpha$ (lower right). Despite the large area increase, the column spacing $\Lambda$ increases only slightly and transiently, implying a strong increase in $N_{H C}$. Note that growth-induced reorganization of OD columns shows signatures of the ZZ instability as captured by a drop in $\alpha$. 


\section{References}

[1] Ratzlaff E H, Grinvald A (1991) A tandem-lens epifluorescence macroscope: Hundred-fold brightness advantage for wide field imaging. J Neurosci 36:127-37.

[2] Bonhoeffer T, Grinvald A (1996) Optical imaging based on intrinsic signals: The methodology. In: Brain Mapping. The Methods. Toga A, Mazziotta J (editors) (New York Academic Press).

[3] Engelmann R, Crook J M, Löwel S (2002) Optical imaging of orientation and ocular dominance maps in area 17 of cats with convergent strabismus. Vis Neurosci 19:39-49.

[4] Kaschube $\mathrm{M}$ et al. (2003) The pattern of ocular dominance columns in cat primary visual cortex: Intra- and interindividual variability of column spacing and its dependence on genetic background. Eur J Neurosci 18:3251-66.

[5] Kaschube M, Wolf F, Geisel T, Löwel S (2002) Genetic influence on quantitative features of neocortical architecture. J Neurosci 22:7206-17.

[6] Kaschube M, Schnabel M, Wolf F, Löwel S (2009) Interareal coordination of columnar architectures during visual cortical development. PNAS 106:17205-10.

[7] Wolf F, Geisel T (1998) Spontaneous pinwheel annihilation during visual development. Nature 395:73-78.

[8] Durbin R, Mitchison G (1990) A dimension reduction framework for understanding cortical maps. Nature 343:644-47.

[9] Goodhill G J, Cimponeriu A (2000) Analysis of the elastic net model applied to the formation of ocular dominance and orientation columns. Network 11:153-68.

[10] Scherf O, Pawelzik K, Wolf F, Geisel T (1999) Theory of ocular dominance pattern formation. Phys Rev E 59:6977-93.

[11] Crair M C, Horton J C, Antonini A, Stryker M P (2001) Emergence of ocular dominance columns in cat visual cortex by 2 weeks of age. J Comp Neurol 430(2):235-49. 
[12] Rathjen S, Löwel S (2000) Early postnatal development of functional ocular dominance columns in cat primary visual cortex. Neuroreport 11:2363-67.

[13] Manneville P (1990) Dissipative Structures And Weak Turbulence. (Academic Press).

[14] Cross M C, Hohenberg P C (1993) Pattern formation outside of equilibrium. Rev Mod Phys 65:854-1086.

[15] Cross M C, Greenside H (2009) Pattern Formation and Dynamics in Nonequilibrium Systems. (Cambridge University Press).

[16] Newell A C, Whitehead J A (1969) Finite bandwidth, finite amplitude convection. J Fluid Mech 38:279-303.

[17] Busse F, Whitehead J A (1971) Instabilities of convection rolls in a high prandtl number fluid. J Fluid Mech 47:305-20.

[18] Oster A M, Bressloff P C (2006) A developmental model of ocular dominance column formation on a growing cortex. Bull Math Biol 68:73-98.

[19] Crampin E J, Gaffney E A, Maini P K (1999) Reaction and diffusion on growing domains: Scenarios for robust pattern formation. Bull Math Biol 61(6):1093-120.

[20] Duffy K R, Murphy K M, Jones D G (1998) Analysis of the postnatal growth of visual cortex. Vis Neurosci 15(5):831-39.

[21] Cragg B G (1975) The development of synapses in the visual system of the cat. J Comp Neurol 160(2), 147-66.

[22] LeVay S, Stryker M P, Shatz C J (1978) Ocular dominance columns and their development in layer IV of the cat's visual cortex: A quantitative study. J Comp Neurol 179(1):223-44.

[23] Luskin M B, Shatz C J (1985) Studies of the earliest generated cells of the cat's visual cortex: Cogeneration of subplate and marginal zones. J Neurosci 5(4):1062-107. 
[24] Luskin M B, Shatz C J (1985) Neurogenesis of the cat's primary visual cortex. J Comp Neurol 242(4):611-31.

[25] Purves D (1994) Neural activity and the growth of the brain. (Cambridge University Press). 


\section{Supporting Information}

\section{Keil et al. 10.1073/pnas.0913020107}

SI Movies
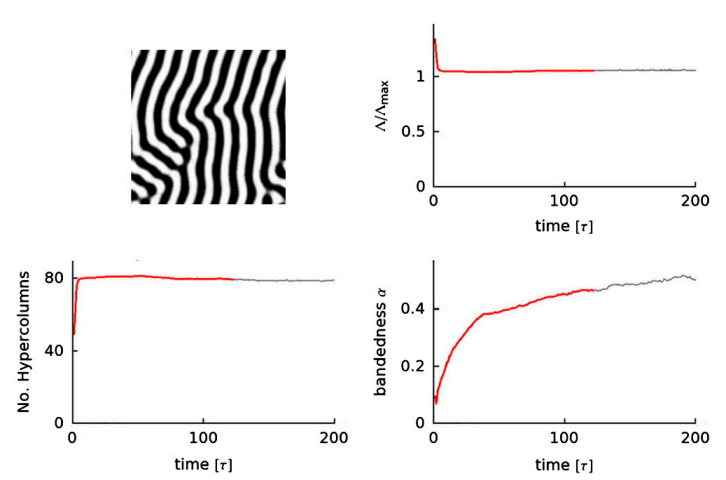

Movie S1 Development of ocular dominance (OD) column layout in the Elastic Network (EN) model without growth, starting from nonselective initial condition $\mathrm{o}(\mathrm{x}, t=0)=0(\eta=0.025, r=0.16)$. The video displays OD maps (Upper Left) as well as the time courses of the parameters column spacing $\Lambda$ (Upper Right), number of hypercolumns $N_{\mathrm{HC}}$ (Lower Left), and bandedness $\alpha$ (Lower Right), characterizing OD layouts during development. At $t=4 \tau$ OD columns have segregated. After $10 \tau$, columns start reorganizing and merge progressively toward a stationary stripe-like layout. Whereas $\Lambda$ and $N_{\mathrm{HC}}$ reach near mature levels around $10 \tau$, the bandedness $\alpha$ increases during the entire time course capturing the slow reorganization of OD columns toward stripes.

Movie S1 (MP4)
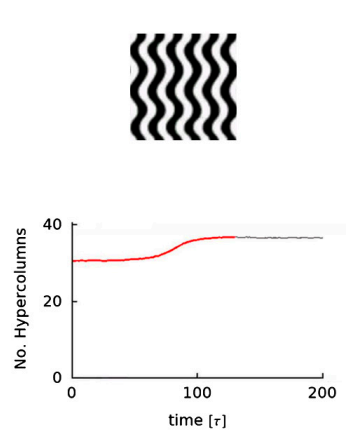
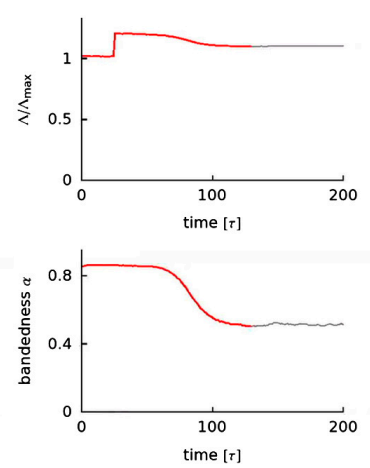

Movie S2 Zigzag (ZZ) reorganization in the EN model after instantaneous isotropic area increase $\eta=0.025, r=0.16$ ). The video displays OD maps (Upper Left) as well as the time courses of the column spacing $\Lambda$ (Upper Right), the number of hypercolumns $N_{\mathrm{HC}}$ (Lower Left), and the bandedness $\alpha$ (Lower Right). After instantaneous area increase at $t=10 \tau, \mathrm{OD}$ stripes bend sinusoidally, a behavior characteristic for ZZ instability. A jump in $\Lambda$ is observed shortly after $t=10 \tau$. Subsequently, $\Lambda$ decreases to almost the initial value and, consequently, $N_{\mathrm{HC}}$ increases. The bandedness $\alpha$ decreases reflecting the bending of OD domains. Movie S2 (MP4) 



\section{Chapter 3}

\section{Coverage, continuity, and visual cortical architecture}

\section{Citation}

Keil W and Wolf F Coverage, continuity, and visual cortical architecture. Neural Syst E Circ 2011, 1:17 doi:10.1186/2042-1001-1-1.

C2011 Keil and Wolf

\section{Original contribution}

I conceived and designed the study together with F. Wolf. I performed the theoretical analyses and numerical simulations. I wrote the manuscript together with F. Wolf. 



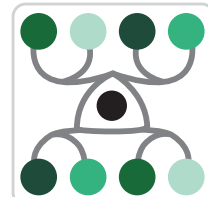

\section{Neural Systems \& Circuits}

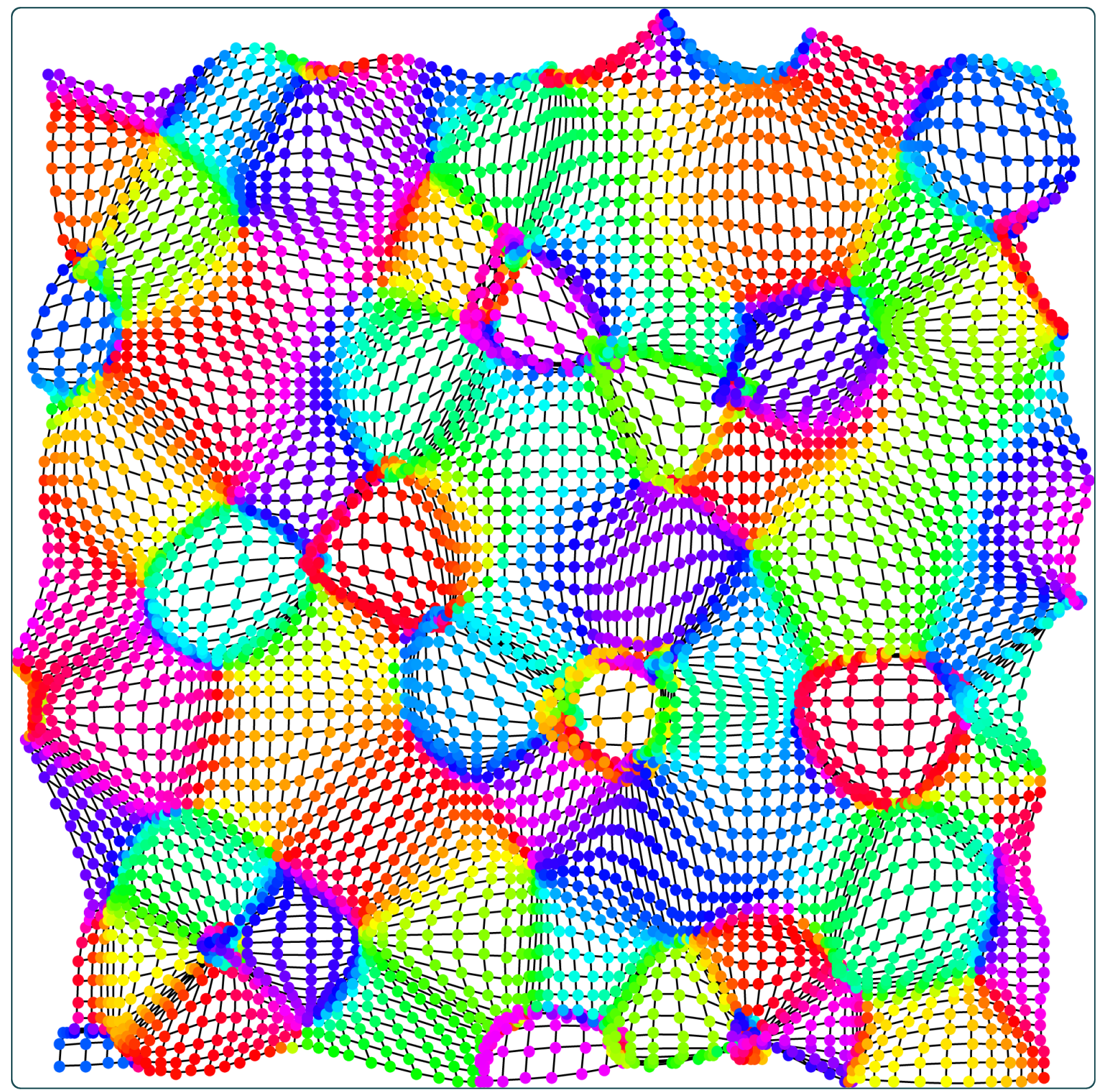

\section{Coverage, continuity, and visual cortical architecture}

Keil and Wolf

C Biomed Central 


\title{
Coverage, continuity, and visual cortical architecture
}

\author{
Wolfgang Keil ${ }^{1,2,3,4^{*}}$ and Fred Wolf $1,2,3,4$
}

\begin{abstract}
Background: The primary visual cortex of many mammals contains a continuous representation of visual space, with a roughly repetitive aperiodic map of orientation preferences superimposed. It was recently found that orientation preference maps (OPMs) obey statistical laws which are apparently invariant among species widely separated in eutherian evolution. Here, we examine whether one of the most prominent models for the optimization of cortical maps, the elastic net (EN) model, can reproduce this common design. The EN model generates representations which optimally trade of stimulus space coverage and map continuity. While this model has been used in numerous studies, no analytical results about the precise layout of the predicted OPMs have been obtained so far.

Results: We present a mathematical approach to analytically calculate the cortical representations predicted by the EN model for the joint mapping of stimulus position and orientation. We find that in all the previously studied regimes, predicted OPM layouts are perfectly periodic. An unbiased search through the EN parameter space identifies a novel regime of aperiodic OPMs with pinwheel densities lower than found in experiments. In an extreme limit, aperiodic OPMs quantitatively resembling experimental observations emerge. Stabilization of these layouts results from strong nonlocal interactions rather than from a coverage-continuity-compromise.

Conclusions: Our results demonstrate that optimization models for stimulus representations dominated by nonlocal suppressive interactions are in principle capable of correctly predicting the common OPM design. They question that visual cortical feature representations can be explained by a coverage-continuity-compromise.
\end{abstract}

\section{Introduction}

The pattern of orientation columns in the primary visual cortex (V1) of carnivores, primates, and their close relatives are among the most intensely studied structures in the cerebral cortex and a large body of experimental (e. g., [1-13]) and theoretical work (e.g., [14-39]) has been dedicated to uncovering its organization principles and the circuit level mechanisms that underlie its development and operation. Orientation preference maps (OPMs) exhibit a roughly repetitive arrangement of preferred orientations in which adjacent columns preferring the same orientation are separated by a typical distance in the millimeter range $[2-5,10]$. This range seems to be set by cortical mechanisms both intrinsic to a particular area [40] but potentially also involving interactions

\footnotetext{
* Correspondence: wolfgang@nld.ds.mpg.de

Max-Planck-Institute for Dynamics and Self-organization, Am Fassberg 17, D37077 Göttingen, Germany

Full list of author information is available at the end of the article
}

between different cortical regions [41]. The pattern of orientation columns is however not strictly periodic because the precise local arrangement of preferred orientation never exactly repeats. Instead, OPMs appear as organized by a spatially complex aperiodic array of pinwheel centers, around which columns activated by different stimulus orientations are radially arranged like the spokes of a wheel $[2-5,10]$. The arrangement of these pinwheel centers, although spatially irregular, is statistically distinct from a pattern of randomly positioned points [38] as well as from patterns of phase singularities in a random pattern of preferred orientations $[32,36,38,42]$ with spatial correlations identical to experimental observations $[38,42]$. This suggests that the layout of orientation columns and pinwheels although spatially aperiodic follows a definite system of layout rules. Cortical columns can in principle exhibit almost perfectly repetitive order as exemplified by ocular dominance (OD) bands in the macaque monkey primary 
visual cortex $[43,44]$. It is thus a fundamental question for understanding visual cortical architecture, whether there are layout principles that prohibit a spatially exactly periodic organization of orientation columns and instead enforce complex arrangements of these columns.

Recent comparative data have raised the urgency of answering this question and of dissecting what is constitutive of such complex layout principles. Kaschube et al. [38] quantitatively compared pinwheel arrangements in a large dataset from three species widely separated in the evolution of eutherian mammals. These authors found that the spatial statistics of pinwheels are surprisingly invariant. In particular, the overall pinwheel density and the variability of pinwheel densities in regions from the scale of a single hypercolumn to substantial fractions of the entire primary visual cortex were found to be virtually identical. Characterizing pinwheel layout on the scale of individual hypercolumns, they found the distributions of nearest-neighbor pinwheel distances to be almost indistinguishable. Further supporting common layout rules for orientation columns in carnivores and primates, the spatial configuration of the superficial patch system [45] and the responses to drifting grating stimuli were recently found to be very similar in cat and macaque monkey primary visual cortex [46].

From an evolutionary perspective, the occurrence of quantitatively similar layouts for OPMs in primate tree shrews and carnivorous species appears highly informative. The evolutionary lineages of these taxa diverged more than 65 million years ago during the basal radiation of eutherian mammals [47-49]. According to the fossil record and cladistic reconstructions, their last common ancestors (called the boreo-eutherial ancestors) were small-brained, nocturnal, squirrel-like animals of reduced visual abilities with a telencephalon containing only a minor neocortical fraction $[47,50]$. For instance, endocast analysis of a representative stem eutherian from the late cretaceous indicates a total anterior-posterior extent of $4 \mathrm{~mm}$ for its entire neocortex $[47,50]$. Similarly, the tenrec (Echinops telfari), one of the closest living relatives of the boreoeutherian ancestor [51,52], has a neocortex of essentially the same size and a visual cortex that totals only $2 \mathrm{~mm}^{2}$ [47]. Since the neocortex of early mammals was subdivided into several cortical areas [47] and orientation hypercolumns measure between 0.4 and $1.4 \mathrm{~mm}^{2}$ [38], it is difficult to envision ancestral eutherians with a system of orientation columns. In fact, no extant mammal with a visual cortex of such size is known to possess orientation columns [53]. It is therefore conceivable that systems of orientation columns independently evolved in laurasiatheria (such as carnivores) and in euarchonta (such as tree shrews and primates). Because galagos, tree-shrews, and ferrets strongly differ in habitat and ecologically relevant visual behaviors, it is not obvious that the quantitative similarity of pinwheel layout rules in their lineages evolved driven by specific functional selection pressures (see [54] for an extended discussion). Kaschube et al. instead demonstrated that an independent emergence of identical layout rules for pinwheels and orientation columns can be explained by mathematically universal properties of a wide class of models for neural circuit selforganization.

According to the self-organization scenario, the common design would result from developmental constraints robustly imposed by adopting a particular kind of self-organization mechanism for constructing visual cortical circuitry. Even if this scenario is correct, one question still remains: What drove the different lineages to adopt a similar self-organization mechanism? As pointed out above, it is not easy to conceive that this adoption was favored by the specific demands of their particular visual habitats. It is, however, conceivable that general requirements for a versatile and representationally powerful cortical circuit architecture are realized by the common design. If this was true, the evolutionary benefit of meeting these requirements might have driven the adoption of large-scale self-organization and the emergence of the common design over evolutionary times.

The most prominent candidate for such a general requirement is the hypothesis of a coverage-continuitycompromise (e.g., $[19,21,55,56])$. It states that the columnar organization is shaped to achieve an optimal tradeoff between the coverage of the space of visual stimulus features and the continuity of their cortical representation. On the one hand, each combination of stimulus features should be well represented in a cortical map to avoid 'blindness' to stimuli with particular feature combinations. On the other hand, the wiring cost to establish connections within the map of orientation preference should be kept low. This can be achieved if neurons that are physically close in the cortex tend to have similar stimulus preferences. These two design goals generally compete with each other. The better a cortical representation covers the stimulus space, the more discontinuous it has to be. The tradeoff between the two aspects can be modeled in what is called a dimension reduction framework in which cortical maps are viewed as two-dimensional sheets which fold and twist in a higher-dimensional stimulus space (see Figure 1) to cover it as uniformly as possible while minimizing some measure of continuity $[21,57,58]$.

From prior work, the coverage-continuity-compromise appears to be a promising candidate for a principle to explain visual cortical functional architecture. First, many studies have reported good qualitative agreement between the layout of numerically obtained dimension 


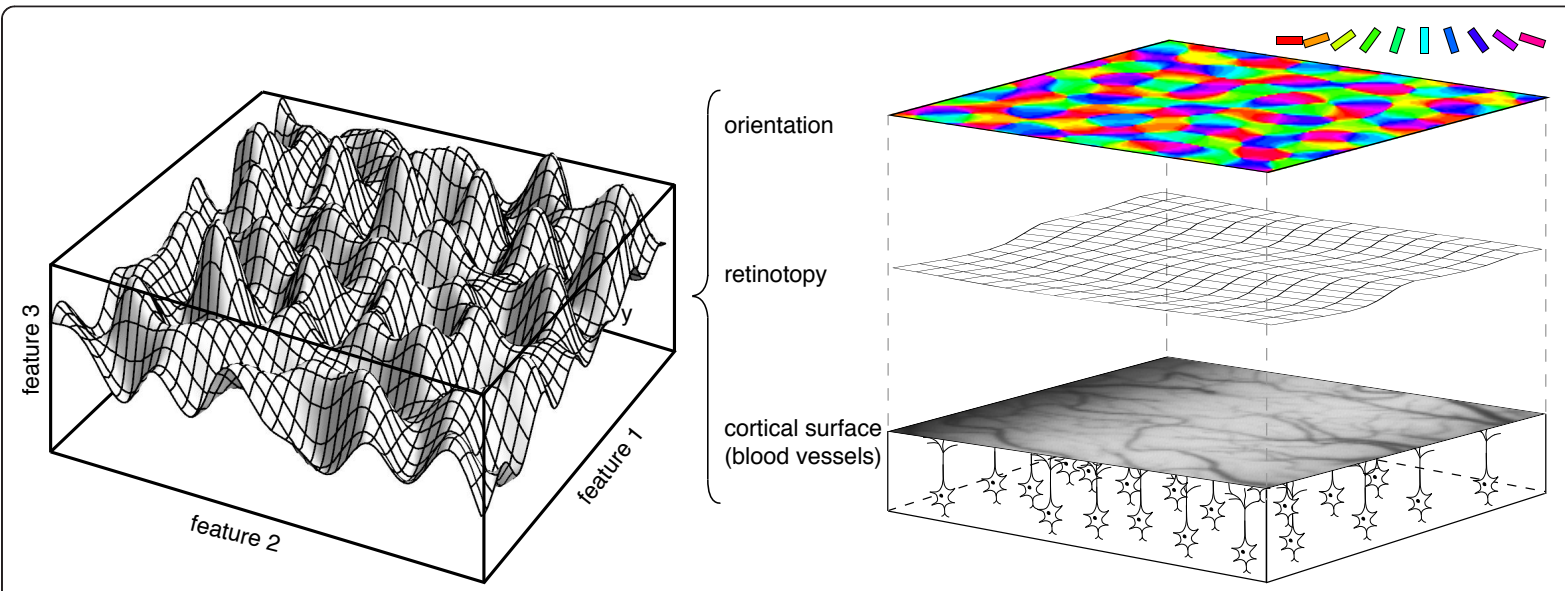

Figure 1 The dimension reduction framework. In the dimension reduction framework, the visual cortex is modeled as a two-dimensional sheet that twists in a higher-dimensional stimulus (or feature) space to cover it as uniformly as possible while minimizing some measure of continuity (left). In this way, it represents a mapping from the cortical surface to the manifold of visual stimulus features such as orientation and retinotopy (right).

reducing maps and experimental observations $[19,21,42,55-57,59-71]$. Second, geometric relationships between the representations of different visual features such as orientation, spatial frequency, and OD have been reproduced by dimension reduction models $[25,56,63-65,67,68,72]$.

Mathematically, the dimension reduction hypothesis implies that the layouts of cortical maps can be understood as optima or near optima (global or local minima) of a free energy functional which penalizes both 'stimulus scotomas' and map discontinuity. Unfortunately, there is currently no dimension reduction model for which the precise layouts of optimal or nearly optimal solutions have analytically been established. To justify the conclusion that the tradeoff between coverage and continuity favors the common rules of OPM design found in experiment, knowledge of optimal dimensionreducing mappings however appears essential.

Precise knowledge of the spatial organization of optimal and nearly optimal mappings is also critical for distinguishing between optimization theories and frozen noise scenarios of visual cortical development. In a frozen noise scenario, essentially random factors such as haphazard wiring [73], the impact of spontaneous activity patterns [74], or an idiosyncratic set of visual experiences [75] determine the emerging pattern of preferred orientations. This pattern is then assumed to be 'frozen' by an unknown mechanism, capable of preventing further modification of preferred orientations by ongoing synaptic turnover and activity-dependent plasticity. Conceptually, a frozen noise scenario is diametrically opposed to any kind of optimization theory. Even if the reorganization of the pattern prior to freezing was to follow a gradient descent with respect to some cost function, the early stopping implies that the layout is neither a local nor a global minimum of this functional. Importantly, the layout of transient states is known to exhibit universal properties that can be completely independent of model details [25,32]. As a consequence, an infinite set of distinct optimization principles will generate the same spatial structure of transient states. This implies in turn that the frozen transient layout is not specifically shaped by any particular optimization principle. Map layouts will thus in principle only be informative about design or optimization principles of cortical processing architectures if maps are not just frozen transients.

In practice, however, the predictions of frozen noise and optimization theories might be hard to distinguish. Ambiguity between these mutually exclusive theories would result in particular, if the energy landscape of the optimization principle is so 'rugged' that there is essentially a local energy minimum next to any relevant random arrangement. Dimension reduction models are conceptually related to combinatorial optimization problems like the traveling salesman problem (TSP) and many such problems are believed to exhibit rugged energy landscapes [76-78]. It is therefore essential to clarify whether paradigmatic dimension reduction models are characterized by a rugged or a smooth energy landscape. If their energy landscapes were smooth with a small number of well-separated local minima, their predictions would be easy to distinguish from those of a frozen noise scenario.

In this study, we examine the classical example of a dimension reduction model, the elastic network (EN) 
model. Since the seminal work of Durbin and Mitchison [21], the EN model has widely been used to study visual cortical representations [25,42,62-65,69-72,79]. The EN model possesses an explicit energy functional which trades off a matching constraint which matches cortical cells to particular stimulus features via Hebbian learning, with a continuity constraint that minimizes Euclidean differences in feature space between neighboring points in the cortex [63]. In two ways, the EN model's explicit variational structure is very appealing. First, coverage and continuity appear as separate terms in the free energy which facilitates the dissection of their relative influences. Second, the free energy allows for the formulation of a gradient descent dynamics. The emergence of cortical selectivity patterns and their convergence toward a minimal energy state in this dynamics might serve as a model for an optimization process taking place in postnatal development.

Following Durbin and Mitchison, we consider the EN model for the joint mapping of two visual features: (i) position in visual space, represented in a retinotopic map (RM) and (ii) line orientation, represented in an OPM. To compute optimal dimension-reducing mappings, we first develop an analytical framework for deriving closed-form expressions for fixed points, local minima, and optima of arbitrary optimization models for the spatial layout of OPMs and RMs in which predicted maps emerge by a supercritical bifurcation as well as for analyzing their stability properties. By applying this framework to different instantiations of the EN model, we systematically disentangle the effects of individual model features on the repertoire of optimal solutions. We start with the simplest possible model version, a fixed uniform retinotopy and an orientation stimulus ensemble with only a single orientation energy and then relax the uniform retinotopy assumption incorporating retinotopic distortions. An analysis for a second widely used orientation stimulus ensemble including also unoriented stimuli is given in Appendix 1. Surprisingly, in all cases, our analysis yields pinwheel-free orientation stripes (OSs) or stereotypical square arrays of pinwheels as local minima or optimal orientation maps of the EN model. Numerical simulations of the EN confirm these findings. They indicate that more complex spatially aperiodic solutions are not dominant and that the energy landscape of the EN model is rather smooth. Our results demonstrate that while aperiodic stationary states exist, they are generally unstable in the considered model versions.

To test whether the EN model is in principle capable of generating complex spatially aperiodic optimal orientation maps, we then perform a comprehensive unbiased search of the EN optima for arbitrary orientation stimulus distributions. We identify two key parameters determining pattern selection: (i) the intracortical interaction range and (ii) the fourth moment of the orientation stimulus distribution function. We derive complete phase diagrams summarizing pattern selection in the EN model for fixed as well as variable retinotopy. Small interaction ranges together with low to intermediate fourth moment values lead to pinwheel-free OSs, rhombic, or hexagonal crystalline orientation map layouts as optimal states. In the regime of large interaction ranges together with higher fourth moment, we find irregular aperiodic OPM layouts with low pinwheel densities as optima. Only in an extreme and previously unconsidered parameter regime of very large interaction ranges and stimulus ensemble distributions with an infinite fourth moment, optimal OPM layouts in the EN model resemble experimentally observed aperiodic pinwheelrich layouts and quantitatively reproduce the recently described species-invariant pinwheel statistics. Unexpectedly, we find that the stabilization of such layouts is not achieved by an optimal tradeoff between coverage and continuity of a localized population encoding by the maps but results from effectively suppressive long-range intracortical interactions in a spatially distributed representation of localized stimuli.

We conclude our reexamination of the EN model with a comparison between different numerical schemes for the determination of optimal or nearly optimal mappings. For large numbers of stimuli, numerically determined solutions match our analytical predictions, irrespectively of the computational method used.

\section{Results and discussion}

\section{Model definition and model symmetries}

We analyze the EN model for the joint optimization of position and orientation selectivity as originally introduced by Durbin and Mitchison [21]. In this model, the $\mathrm{RM}$ is represented by a mapping $\mathbf{R}(\mathbf{x})=\left(R_{1}(\mathbf{x}), R_{2}(\mathbf{x})\right)$ which describes the receptive field center position of a neuron at cortical position $\mathbf{x}$. Any RM can be decomposed into an affine transformation $\mathbf{x} \mapsto \mathbf{X}$ from cortical to visual field coordinates, on which a vector-field of retinotopic distortions $\mathbf{r}(\mathbf{x})$ is superimposed, i.e.:

$$
\mathbf{R}(\mathbf{x})=\mathbf{X}+\mathbf{r}(\mathbf{x})
$$

with appropriately chosen units for $\mathbf{x}$ and $\mathbf{R}$.

The OPM is represented by a second complex-valued scalar field $z(\mathbf{x})$. The pattern of orientation preferences $\vartheta$ $(\mathbf{x})$ is encoded by the phase of $z(\mathbf{x})$ via

$$
\vartheta(\mathbf{x})=\frac{1}{2} \arg (z(\mathbf{x})) \text {. }
$$

The absolute value $|z(\mathbf{x})|$ is a measure of the average cortical selectivity at position $\mathbf{x}$. Solving the EN model 
requires to find pairs of maps $\{\mathbf{r}(\mathbf{x}), z(\mathbf{x})\}$ that represent an optimal compromise between stimulus coverage and map continuity. This is achieved by minimizing a free energy functional

$$
\mathcal{F}=\sigma^{2} \mathcal{C}+\mathcal{R}
$$

in which the functional $\mathcal{C}$ measures the coverage of a stimulus space and the functional $\mathcal{R}$ the continuity of its cortical representation. The stimulus space is defined by an ensemble $\{\mathbf{S}\}$ of idealized point-like stimuli, each described by two features: $s_{z}=\left|s_{z}\right| e^{2 i \theta}$ and $\mathbf{s}_{r}=\left(s_{x}, s_{y}\right)$ which specify the orientation $\theta$ of the stimulus and its position in visual space $\mathbf{s}_{r}$ (Figure $2 \mathrm{~b}$ ). $\mathcal{C}$ and $\mathcal{R}$ are given by

$$
\begin{aligned}
\mathcal{C}[z, \mathbf{r}] & =-\left\langle\ln \int d^{2} y e^{-\left(\left|s_{z}-z(\mathbf{y})\right|^{2}+\left|\mathbf{s}_{r}-\mathbf{X}-\mathbf{r}(\mathbf{y})\right|^{2}\right) / 2 \sigma^{2}}\right\rangle_{\mathbf{S}} \\
\mathcal{R}[z, \mathbf{r}] & =\frac{1}{2} \int d^{2} y \eta\|\nabla z(\mathbf{y})\|^{2}+\eta_{r} \sum_{j=1}^{2}\left\|\nabla r_{j}(\mathbf{y})\right\|^{2},
\end{aligned}
$$

with $\nabla=\left(\partial_{x}, \partial_{y}\right)^{T}$, and $\eta \in[0,1]$. The ratios $\sigma^{2} / \eta$ and $\sigma^{2} / \eta_{r}$ control the relative strength of the coverage term versus the continuity term for OPM and RM, respectively. $\langle\ldots\rangle_{\mathbf{S}}$ denotes the average over the ensemble of stimuli.

Minima of the energy functional $\mathcal{F}$ are stable fixed points of the gradient descent dynamics

$$
\begin{aligned}
\partial_{t} z(\mathbf{x}) & =-2 \frac{\delta \mathcal{F}[z, \mathbf{r}]}{\delta \bar{z}(\mathbf{x})} \equiv F^{z}[z, \mathbf{r}](\mathbf{x}) \\
\partial_{t} \mathbf{r}(\mathbf{x}) & =-\frac{\delta \mathcal{F}[z, \mathbf{r}]}{\delta \mathbf{r}(\mathbf{x})} \equiv \mathbf{F}^{r}[z, \mathbf{r}](\mathbf{x})
\end{aligned}
$$

called the EN dynamics in the following. These dynamics read

$$
\begin{aligned}
& \partial_{t} z(\mathbf{x})=\left\langle\left[s_{z}-z(\mathbf{x})\right] e(\mathbf{x}, \mathbf{S}, z, \mathbf{r})\right\rangle_{\mathbf{S}}+\eta \Delta z(\mathbf{x}) \\
& \partial_{t} \mathbf{r}(\mathbf{x})=\left\langle\left[\mathbf{s}_{r}-\mathbf{X}-\mathbf{r}(\mathbf{x})\right] e(\mathbf{x}, \mathbf{S}, z, \mathbf{r})\right\rangle_{\mathbf{S}}+\eta_{r} \Delta \mathbf{r}(\mathbf{x}),(4)
\end{aligned}
$$

where $e(\mathbf{x}, \mathbf{S}, z, \mathbf{r})$ is the activity-pattern, evoked by a stimulus $\mathbf{S}=\left(\mathbf{s}_{r}, s_{z}\right)$ in a model cortex with retinotopic distortions $\mathbf{r}(\mathbf{x})$ and OPM $z(\mathbf{x})$. It is given by

$$
e(\mathbf{x}, \ldots)=\frac{e^{-\left(\left|\mathbf{s}_{r}-\mathbf{X}-\mathbf{r}(\mathbf{x})\right|^{2}\right) / 2 \sigma^{2}} e^{-\left(\left|s_{z}-z(\mathbf{x})\right|^{2}\right) / 2 \sigma^{2}}}{\int d^{2} y e^{-\left(\left|\mathbf{s}_{r}-\mathbf{X}-\mathbf{r}(\mathbf{y})\right|^{2}\right) / 2 \sigma^{2}} e^{-\left(\left|s_{z}-z(\mathbf{y})\right|^{2}\right) / 2 \sigma^{2}}} .
$$

Figure 2 illustrates the general features of the EN dynamics using the example of a single stimulus. Figure 2a shows a model orientation map with a superimposed uniform representation of visual space. A single pointlike, oriented stimulus $\mathbf{S}=\left(\mathbf{s}_{r}, s_{z}\right)$ with position $\mathbf{s}_{r}=\left(s_{x}\right.$, $s_{y}$ ) and orientation $\theta=1 / 2 \arg \left(s_{z}\right)$ (Figure $2 \mathrm{~b}$ ) evokes a cortical activity pattern $e(\mathbf{x}, \mathbf{S}, z, \mathbf{r}$ ) (Figure 2c). The activity-pattern in this example is of roughly Gaussian shape and is centered at the point, where the location $\mathbf{s}_{r}$ of the stimulus is represented in cortical space. However, depending on the model parameters and the stimulus, the cortical activity pattern may assume as well a more complex form (see also 'Discussion' section). According to Equations $(3,4)$, each stimulus and the evoked activity pattern induce a modification of the orientation map and RM, shown in Figure 2d. Orientation preference in the activated regions is shifted toward the orientation of the stimulus. The representation of visual space in the activated regions is locally contracted toward the position of the stimulus. Modifications of cortical selectivities occur due to randomly chosen stimuli and are set proportional to a very small learning rate. Substantial changes of cortical representations occur slowly through the cumulative effect of a large number of activity patterns and stimuli. These effective changes are described by the two deterministic equations for the rearrangement of cortical selectivities equations $(3,4)$ which are obtained by stimulus-averaging the modifications due to single activity patterns in the discrete stimulus model [25]. One thus expects that the optimal selectivity patterns and also the way in which cortical selectivities change over time are determined by the statistical properties of the stimulus ensemble. In the following, we assume that the stimulus ensemble satisfies three properties: (i) The stimulus locations $\mathbf{s}_{r}$ are uniformly distributed across visual space. (ii) For the distribution of stimulus orientations, $\left|s_{z}\right|$ and $\theta$ are independent. (iii) Orientations $\theta$ are distributed uniformly in $[0, \pi]$.

These conditions are fulfilled by stimulus ensembles used in virtually all prior studies of dimension reduction models for visual cortical architecture (e.g., $[19,21,25,64,65,71,72,80,81])$. They imply several symmetries of the model dynamics equations $(3,4)$. Due to the first property, the EN dynamics are equivariant under translations

$$
\begin{aligned}
& \hat{T}_{\mathbf{y}} z(\mathbf{x})=z(\mathbf{x}+\mathbf{y}) \\
& \hat{T}_{\mathbf{y}} \mathbf{r}(\mathbf{x})=\mathbf{r}(\mathbf{x}+\mathbf{y}),
\end{aligned}
$$

rotations

$$
\begin{aligned}
& \hat{R}_{\beta} z(\mathbf{x})=e^{2 i \beta} z\left(\Omega_{-\beta} \mathbf{x}\right) \\
& \hat{R}_{\beta} \mathbf{r}(\mathbf{x})=\Omega_{\beta} \mathbf{r}\left(\Omega_{-\beta} \mathbf{x}\right)
\end{aligned}
$$

with $2 \times 2$ rotation matrix

$$
\Omega_{\beta}=\left(\begin{array}{cc}
\cos \beta & -\sin \beta \\
\sin \beta & \cos \beta
\end{array}\right),
$$



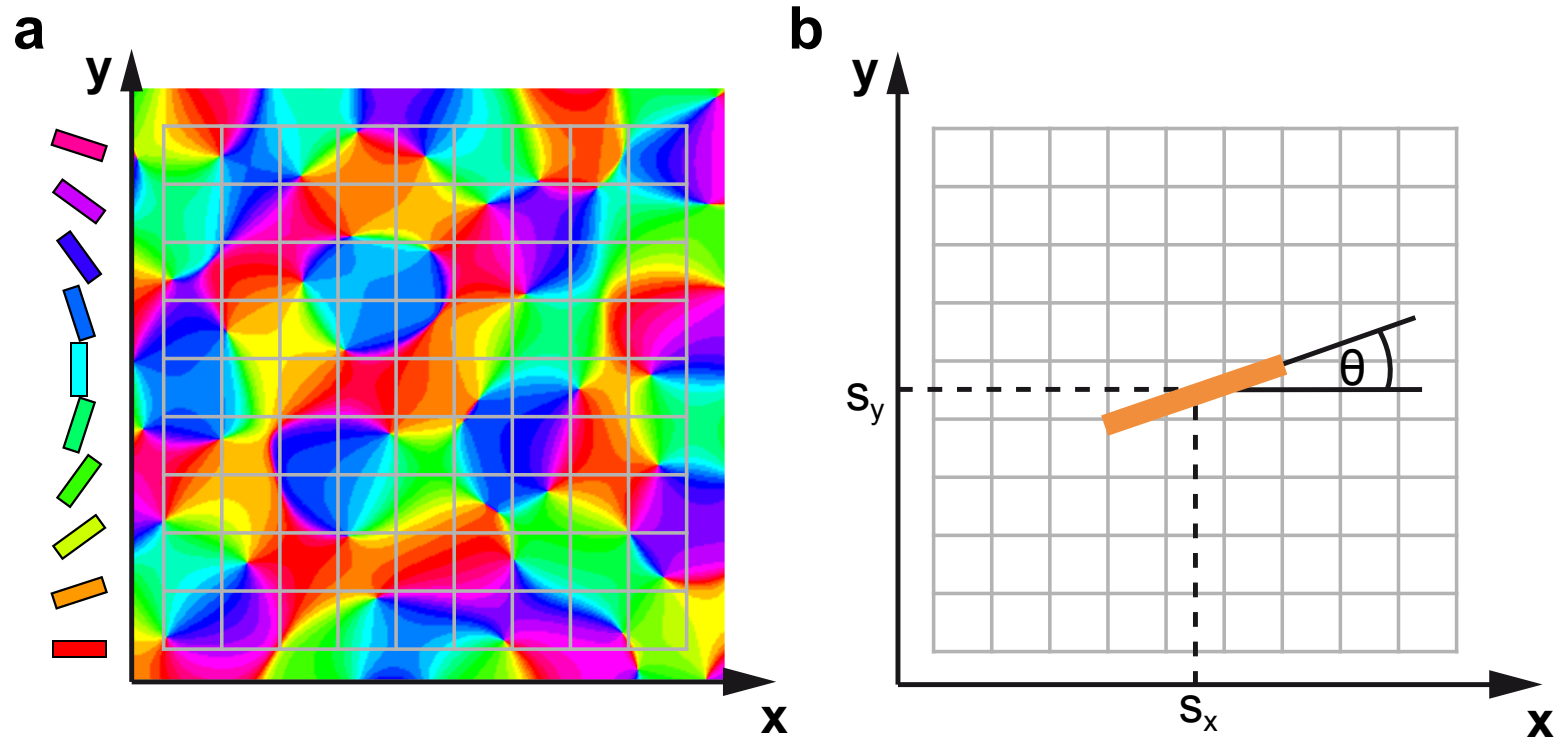

C
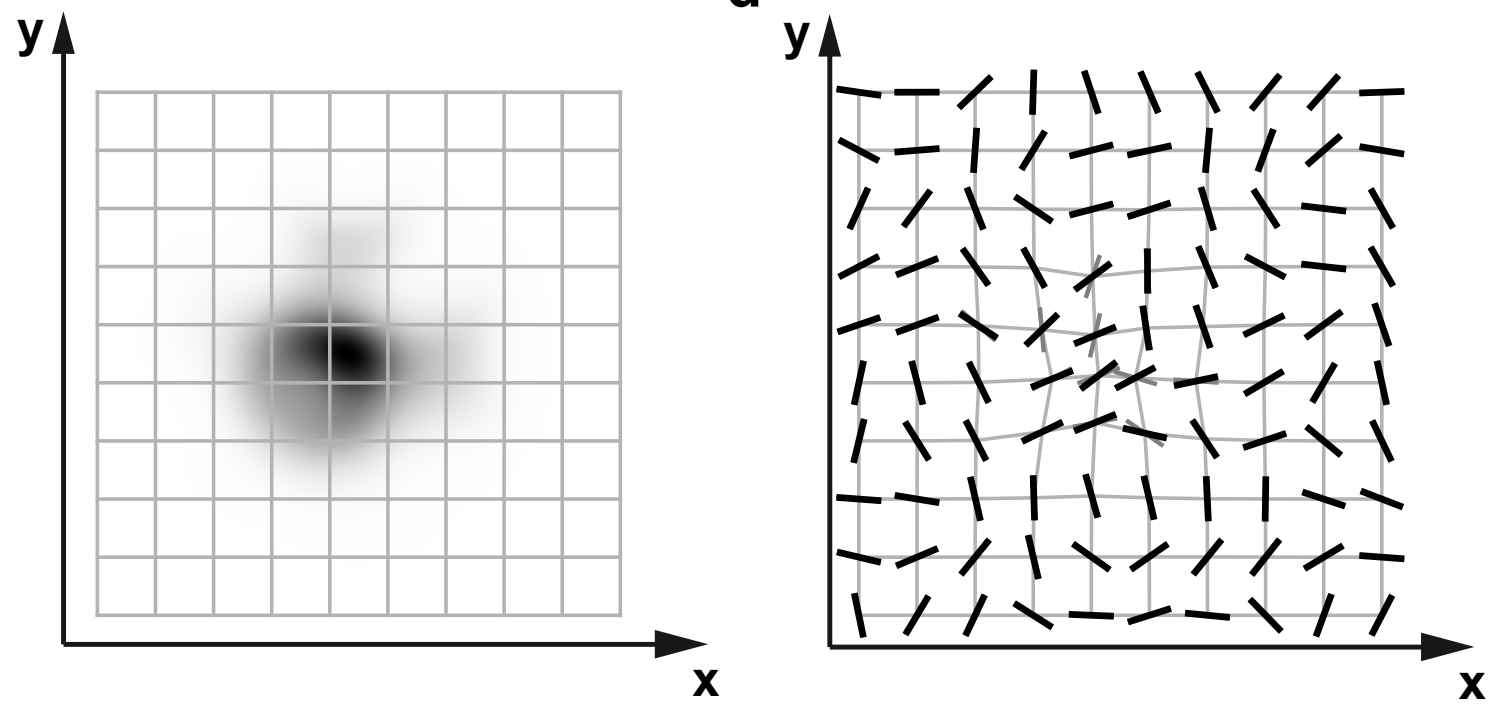

Figure 2 The EN model. (a) Example OPM (color code) together with a uniform map of visual space (RM) (grid lines). (b) Position $\mathbf{s}_{r}=\left(s_{x}, s_{y}\right)$ and orientation $\theta$ of a 'pointlike' stimulus. (c) Cortical activity, evoked by the stimulus in $\mathbf{b}$ for the model maps in $\mathbf{a}$. Dark regions are activated. Note, that in contrast to SOFM models, the activity pattern does not exhibit a stereotypical Gaussian shape. (d) Modification of orientation preference and retinotopy, caused by the stimulus in $\mathbf{b}$. Orientation preferences prior to stimulus presentation are indicated with grey bars, after stimulus presentation with black bars. Most strongly modified preferences correspond to thick black bars. Modifications of orientation preferences and retinotopy are displayed on an exaggerated scale for illustration purposes.

and reflections

$$
\begin{aligned}
& \hat{P} z(\mathbf{x})=\bar{z}(\Psi \mathbf{x}) \\
& \hat{P} \mathbf{r}(\mathbf{x})=\Psi \mathbf{r}(\Psi \mathbf{x}),
\end{aligned}
$$

where $\Psi=\operatorname{diag}(-1,1)$ is the $2 \times 2$ reflection matrix. Equivariance means that

$$
\hat{T}_{\mathrm{y}} \mathrm{F}^{z}[z, \mathbf{r}]=F^{z}\left[\hat{T}_{y} z, \hat{T}_{y} \mathbf{r}\right]
$$

$$
\hat{R}_{\beta} F^{z}[z, \mathbf{r}]=F^{z}\left[\hat{R}_{\beta} z, \hat{R}_{\beta} \mathrm{r}\right]
$$

$\hat{P} F^{z}[z, \mathbf{r}]=F^{z}[\hat{P} z, \hat{P} \mathbf{r}]$, 
with mutatis mutandis the same equations fulfilled by the vector-field $\mathbf{F}^{r}[z, \mathbf{r}]$.

As a consequence, patterns that can be converted into one another by translation, rotation, or reflection of the cortical layers represent equivalent solutions of the model equations $(3,4)$, by construction. Due to the second assumption, the dynamics is also equivariant with respect to shifts in orientation $S_{\varphi} z(\mathbf{x})=e^{i \varphi} z(\mathbf{x})$, i.e.,

$$
\begin{aligned}
& e^{i \phi} F^{z}[z, \mathbf{r}]=F^{z}\left[e^{i \phi} z, \mathbf{r}\right] \\
& \mathbf{F}^{r}[z, \mathbf{r}]=\mathbf{F}^{r}\left[e^{i \phi} z, \mathbf{r}\right] .
\end{aligned}
$$

Thus, two patterns are also equivalent solutions of the model, if their layout of orientation domains and retinotopic distortions is identical, but the preferred orientations differ everywhere by the same constant angle.

Without loss of generality, we normalize the ensembles of orientation stimuli such that $\left\langle\left|s_{z}\right|^{2}\right\rangle_{S}=\left\langle\left|s_{z}\right|^{2}\right\rangle=2$ throughout this article. This normalization can always be restored by a rescaling of $z(\mathbf{x})$ (see $[25,69]$ ).

Our formulation of the dimension-reduction problem in the EN model utilizes a continuum description, both for cortical space and the set of visual stimuli. This facilitates mathematical treatment and appears appropriate, given the high number of cortical neurons under one square millimeter of cortical surface (e.g., roughly 70000 in cat V1 [82]). Even an hypothesized neuronal monolayer would consist of more than $20 \times 20$ neurons per hypercolumn area $\Lambda^{2}$, constituting a quite dense sampling of the spatial periodicity. Treating the feature space as a continuum implements the concept that the cortical representation has to cover as good as possible the infinite multiplicity of conceivable stimulus feature combinations.

\section{The orientation unselective fixed point}

Two stationary solutions of the model can be established from symmetry. The simplest of these is the orientation unselective state with $z(\mathbf{x})=0$ and uniform mapping of visual space $\mathbf{r}(\mathbf{x})=\mathbf{0}$. First, by the shift symmetry (Equation (8)), we find that $z(\mathbf{x})=0$ is a fixed point of Equation (3). Second, by reflectional and rotational symmetry (Equations $(5,7)$ ), we see that the right-hand side of Equation (4) has to vanish and hence the orientation unselective state with uniform mapping of visual space is a fixed point of Equations $(3,4)$.

This homogeneous unselective state thus minimizes the EN energy functional if it is a stable solution of Equations $(3,4)$. The stability can be determined by considering the linearized dynamics of small deviations $\{\mathbf{r}(\mathbf{x}), z$ $(\mathbf{x})\}$ around this state. These linearized dynamics read

$$
\begin{aligned}
& \partial_{t} \mathbf{r}(\mathbf{x}) \simeq \mathbf{L}_{r}[\mathbf{r}]=\frac{1}{16 \pi \sigma^{4}} \int d^{2} y e^{-\frac{(\mathbf{x}-\mathbf{y})^{2}}{4 \sigma^{2}}} \hat{\mathbf{A}} \mathbf{r}(\mathbf{y})+\eta_{r} \Delta \mathbf{r}(\mathbf{x}) \\
& \partial_{z} z(\mathbf{x}) \simeq L_{z}[z]=\left(\frac{1}{\sigma^{2}}-1\right) z(\mathbf{x})+\eta \Delta z(\mathbf{x})-\frac{1}{4 \pi \sigma^{4}} \int d^{2} y e^{-\frac{(\mathbf{x}-\mathbf{y})^{2}}{4 \sigma^{2}}} z(\mathbf{y}),
\end{aligned}
$$

where $(\hat{\mathbf{A}})_{i j}=\left(x_{i}-y_{i}\right)\left(x_{j}-y_{j}\right)-2 \sigma^{2} \delta_{i j}$ with $\delta_{i j}$ being Kronecker's delta. We first note that the linearized dynamics of retinotopic distortions and orientation preference decouple. Thus, up to linear order and near the homogeneous fixed point, both cortical representation evolve independently and the stability properties of the unselective state can be obtained by a separate examination of the stability properties of both cortical representations.

The eigenfunctions of the linearized retinotopy dynamics $\mathbf{L}_{r}[\mathbf{r}]$ can be calculated by Fourier-transforming Equation (10):

$$
\partial_{t} \tilde{r}_{i}(\mathbf{k})=-\sum_{j=1}^{2}\left(\sigma^{2} e^{-k^{2} \sigma^{2}} k_{i} k_{j}+\eta_{r} k^{2} \delta_{i j}\right) \tilde{r}_{j}(\mathbf{k}),
$$

where $k=|\mathbf{k}|$ and $i=1,2$. A diagonalization of this matrix equation yields the eigenvalues

$$
\lambda_{L}^{r}=-k^{2}\left(\eta_{r}+e^{-\sigma^{2} k^{2}} \sigma^{2}\right), \lambda_{T}^{r}=-\eta_{r} k^{2}
$$

with corresponding eigenfunctions (in real space)

$$
\begin{aligned}
& \mathbf{r}_{L}(\mathbf{x})=\mathbf{k}_{\phi} e^{i \mathbf{k}_{\phi} \mathbf{x}}+\text { c.c. } \\
& \mathbf{r}_{T}(\mathbf{x})=\mathbf{k}_{\phi+\pi / 2} e^{i \mathbf{k}_{\phi} \mathbf{x}}+\text { c.c. },
\end{aligned}
$$

where $\mathbf{k}_{\varphi}=|\mathbf{k}|(\cos \varphi, \sin \varphi)^{T}$. These eigenfunctions are longitudinal $(\mathrm{L})$ or transversal $(\mathrm{T})$ wave patterns. In the longitudinal wave, the retinotopic distortion vector $\mathbf{r}$ (x) lies parallel to $\mathbf{k}$ which leads to a 'compression wave' (Figure $3 \mathrm{~b}$, left). In the transversal wave pattern (Figure $3 \mathrm{~b}$, right), the retinotopic distortion vector is orthogonal to $\mathbf{k}$. We note that the linearized Kohonen model [61] was previously found to exhibit the same set of eigenfunctions [80]. Because both spectra of eigenvalues $\lambda_{T}^{r}$, $\lambda_{L}^{r}$ are smaller than zero for every $\sigma>0, \eta_{r}>0$, and $k>0$ (Figure 3a), the uniform retinotopy $\mathbf{r}(\mathbf{x})=\mathbf{0}$ is a stable fixed point of Equation (4) irrespective of parameter choice.

The eigenfunctions of the linearized OPM dynamics $L_{z}[z]$ are Fourier modes $\sim e^{i \mathbf{k x}}$ by translational symmetry. By rotational symmetry, their eigenvalues only depend on the wave number $k$ and are given by

$$
\lambda^{z}(k)=-1+\frac{1}{\sigma^{2}}\left(1-e^{-k^{2} \sigma^{2}}\right)-\eta k^{2}
$$




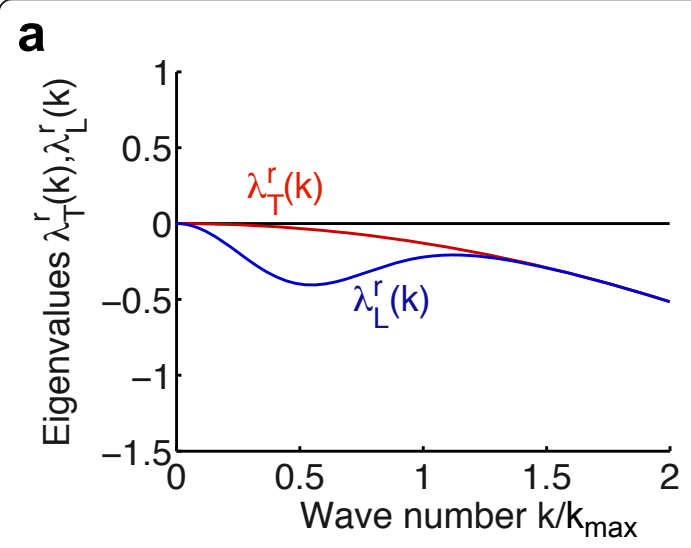

C

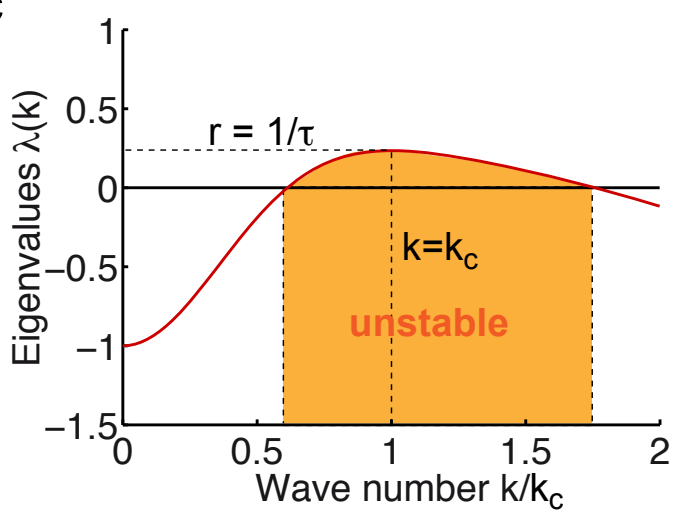

b

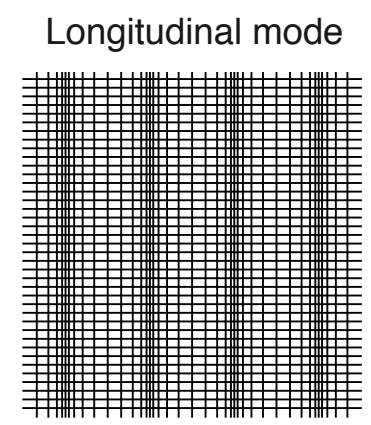

Transversal mode

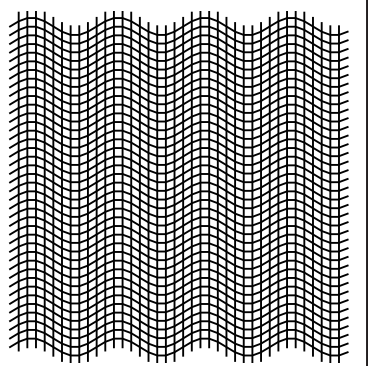

d

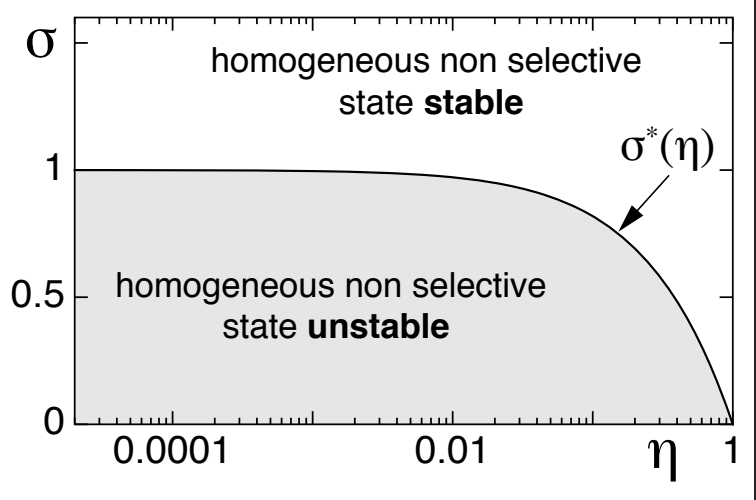

Figure 3 The linearization of the EN model dynamics around the unselective fixed point. (a) Eigenvalue spectra of the linearized retinotopy dynamics for longitudinal mode $\left(\lambda_{L}^{r}(k)\right.$, blue trace) and transversal mode $\left(\lambda_{T}^{r}(k)\right.$, red trace). (b) Longitudinal mode

$\sim \mathbf{k}_{\phi} e^{i \mathbf{k}_{\phi} \mathbf{x}}+$ c.c. (left) and transversal mode $\sim \mathbf{k}_{\phi+\pi / 2} e^{i \mathbf{k}_{\phi} \mathbf{x}}+$ c.c. (right). (c) Spectrum of eigenvalues of the linearized OPM dynamics (red trace) for $\sigma<\sigma^{*}(\eta)$. Orange region marks the unstable annulus of Fourier modes (critical circle). (d) Stability regions of the nonselective state in the EN model. The stability border is given by $\sigma^{*}(\eta)$ (Equation (12)).

(see [25]). This spectrum of eigenvalues is depicted in Figure 3c. For $\eta>0, \lambda^{z}(k)$ has a single maximum at $k_{c}=\frac{1}{\sigma} \sqrt{\ln (1 / \eta)}$. For

$$
\sigma>\sigma^{*}(\eta)=\sqrt{1+\eta \ln \eta-\eta},
$$

this maximal eigenvalue $r=\lambda^{z}\left(k_{c}\right)$ is negative. Hence, the unselective state with uniform retinotopy is a stable fixed point of Equations $(3,4)$ and the only known solution of the EN model in this parameter range. For $\sigma<$ $\sigma^{*}(\eta)$, the maximal eigenvalue $r$ is positive, and the nonselective state is unstable with respect to a band of Fourier modes $\sim e^{i \mathbf{k x}}$ with wave numbers around $|\mathbf{k}| \approx$ $k_{c}$ (see Figure 3c). This annulus of unstable Fourier modes is called the critical circle. The finite wavelength instability [83-85] (or Turing instability [86]) leads to the emergence of a pattern of orientation preference with characteristic spacing $\Lambda=2 \pi / k_{c}$ from the nonselective state on a characteristic timescale $\tau=1 / r$.
One should note that as in other models for the selforganization of orientation columns, e.g., [15,57], the characteristic spatial scale $\Lambda$ arises from effective intracortical interactions of 'Mexican-hat' structure (shortrange facilitation, longer-ranged suppression). The short-range facilitation in the linearized EN dynamics is represented by the first two terms on the right-hand side of Equation (11). Since $\sigma<1$ in the pattern forming regime, the prefactor in front of the first term is positive. Due to the second, Laplacian term, it is favored that neighboring units share selectivity properties, a process mediated by short-range facilitation. Longer-ranged suppression is represented by the convolution term in Equation (11).

Mathematically, this term directly results from the soft-competition in the 'activity-dependent' coverage term of Equation (1). The local facilitation is jointly mediated by coverage (first term) and continuity (second term) contributions. 
Figure $3 \mathrm{~d}$ summarizes the result of the linear stability analysis of the nonselective state. For $\sigma>\sigma^{*}(\eta)$, the orientation unselective state with uniform retinotopy is a minimum of the EN-free energy and also the global minimum. For $\sigma<\sigma^{*}(\eta)$, this state represents a maximum of the energy functional and the minima must thus exhibit a space-dependent pattern of orientation selectivities.

\section{Orientation stripes}

Within the potentially infinite set of orientation selective fixed points of the model, one class of solutions can be established from symmetry: $\left\{\mathbf{r}(\mathbf{x})=\mathbf{0}, z(\mathbf{x})=A_{0} e^{i \mathbf{k x}}\right\}$. In these pinwheel-free states, orientation preference is constant along one axis in cortex (perpendicular to the vector $\mathbf{k}$ ), and each orientation is represented in equal proportion (see Figure 4a). Retinotopy is perfectly uniform. Although this state may appear too simple to be biologically relevant, we will see that it plays a fundamental role in the state space of the EN model. It is therefore useful to establish its existence and basic characteristics. The existence of OS solutions follows directly from the model's symmetries (Equations (5) to (9)). Computing

$$
T_{\mathrm{y}}\left[F^{z}\left[e^{i \mathbf{k x}}, 0\right]\right]=F^{z}\left[T_{\mathbf{y}}\left[e^{i \mathbf{k x}}\right], T_{\mathbf{y}}[0]\right]=F^{z}\left[e^{i \mathbf{k y}} e^{i \mathbf{k x}}, 0\right]=e^{i \mathbf{k y}} F^{z}\left[e^{i \mathbf{k x}}, 0\right]
$$

demonstrates that $F^{z}\left[e^{i \mathbf{k x}}, \mathbf{0}\right]$ is proportional to $e^{i \mathbf{k x}}$. This establishes that the subspace of functions $\sim e^{i \mathbf{k x}}$ is invariant under the dynamics given by Equation (3). For $A_{0}=0$, we recover the trivial fixed point of the EN dynamics by construction, as shown above. This means that within this subspace $A_{0}=0$ is either a minimum or a maximum of the EN energy functional (Equation (1)). Furthermore, for $A_{0} \rightarrow \infty$ the EN energy tends to infinity. If the trivial fixed point is unstable, it corresponds to a maximum of the EN energy functional. Therefore, there must exist at least one minimum with $A_{0} \neq 0$ in the subspace of functions $\sim e^{i \mathbf{k x}}$ which then corresponds to a stationary state of the EN dynamics.

Regarding the dynamics of retinotopic deviations, the model's symmetries equations can be invoked to show that for the state $\left\{\mathbf{0}, A_{0} e^{i \mathbf{k x}}\right\}$, the right-hand side of Equation (4) has to be constant in space:

$$
T_{\mathbf{y}}\left[\mathbf{F}^{r}\left[e^{i \mathbf{k x}}, 0\right]\right]=\mathbf{F}^{r}\left[T_{\mathbf{y}}\left[e^{i \mathbf{k x}}\right], T_{\mathbf{y}}[0]\right]=\mathbf{F}^{r}\left[e^{i \mathbf{k y}} e^{i \mathbf{k x}}, 0\right]=\mathbf{F}^{r}\left[e^{i \mathbf{k x}}, 0\right]
$$

If this constant was nonzero the RM would drift with constant velocity. This, however, is impossible in a variational dynamics such that this constant must vanish. The OS solution (Figure 4a) is to the best of our knowledge the only exact nontrivial stationary solution of Equations $(3,4)$ that can be established without any approximations.

\section{Doubly periodic and quasi-periodic solutions}

In the EN model as considered in this study, the maps of visual space and orientation preference are jointly optimized to trade off coverage and continuity leading to mutual interactions between the two cortical representations. These mutual interactions vanish in the rigid retinotopy limit $\eta_{r} \rightarrow \infty$ and the perfectly uniform retinotopy becomes an optimal solution for arbitrary orientation column layout $z(\mathbf{x})$. As it is not clear how essential the mutual interactions with position specificity are in shaping the optimal orientation column layout, we continue our investigation of solution classes by considering global minima of optimization models with fixed uniform retinotopy. The mutual interactions will be taken into account in a subsequent step.

In the rigid retinotopy limit, minima of the energy functional are stable stationary states of the dynamics of the OPM (Equation (3)) with $\mathbf{r}(\mathbf{x})=\mathbf{0}$. To compute orientation selective stationary solutions of this OPM dynamics, we employ that in the vicinity of a supercritical bifurcation where the nonselective fixed point $z(\mathbf{x})=$ 0 becomes unstable, the entire set of nontrivial fixed points is determined by the third-order terms of the Volterra series representation of the operator $F^{z}[z, \mathbf{0}]$ $[35,84,85,87]$. The symmetries given by Equations (5) to (9) restrict the general form of such a third-order approximation for any model of OPM optimization to

$$
\partial_{t} z(\mathbf{x}) \approx L_{z}[z]+N_{3}^{z}[z, z, \bar{z}],
$$

where the cubic operator $N_{3}^{z}$ is written in trilinear form, i.e.,

$$
N_{3}^{z}\left[\sum_{j} \alpha_{j} z_{j}, \sum_{k} \beta_{k} z_{k}, \sum_{l} \gamma_{l} \bar{z}_{l}\right]=\sum_{j, k, l} \alpha_{j} \beta_{k} \gamma_{l} N_{3}^{z}\left[z_{j}, z_{k}, \bar{z}_{l}\right] .
$$

In particular, all even terms in the Volterra Series representation of $F^{z}[z, 0]$ vanish due to the Shift-Symmetry (Equations $(8,9)$ ). Explicit analytic computation of the cubic nonlinearities for the EN model is cumbersome but not difficult (see 'Methods' section) and yields a sum

$$
N_{3}^{z}[z, z, \bar{z}]=\sum_{j=1}^{11} a_{j} N_{3}^{j}[z, z, \bar{z}]
$$

The individual nonlinear operators $N_{3}^{j}$ are with one exception nonlocal convolution-type operators and are given in the 'Methods' section (Equation (38)), together with a detailed description of their derivation.

Only the coefficients $a_{j}$ depend on the properties of the ensemble of oriented stimuli. 
b

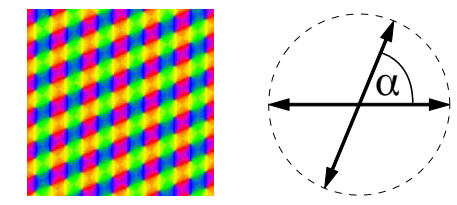

1

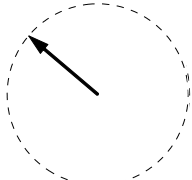

a
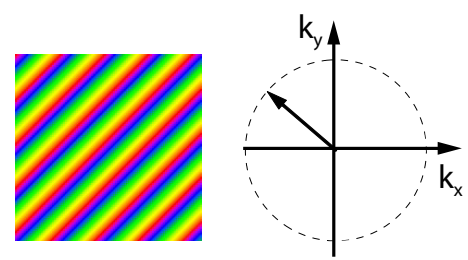

C

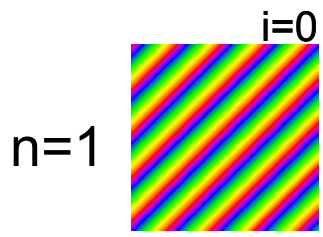

$i=0$
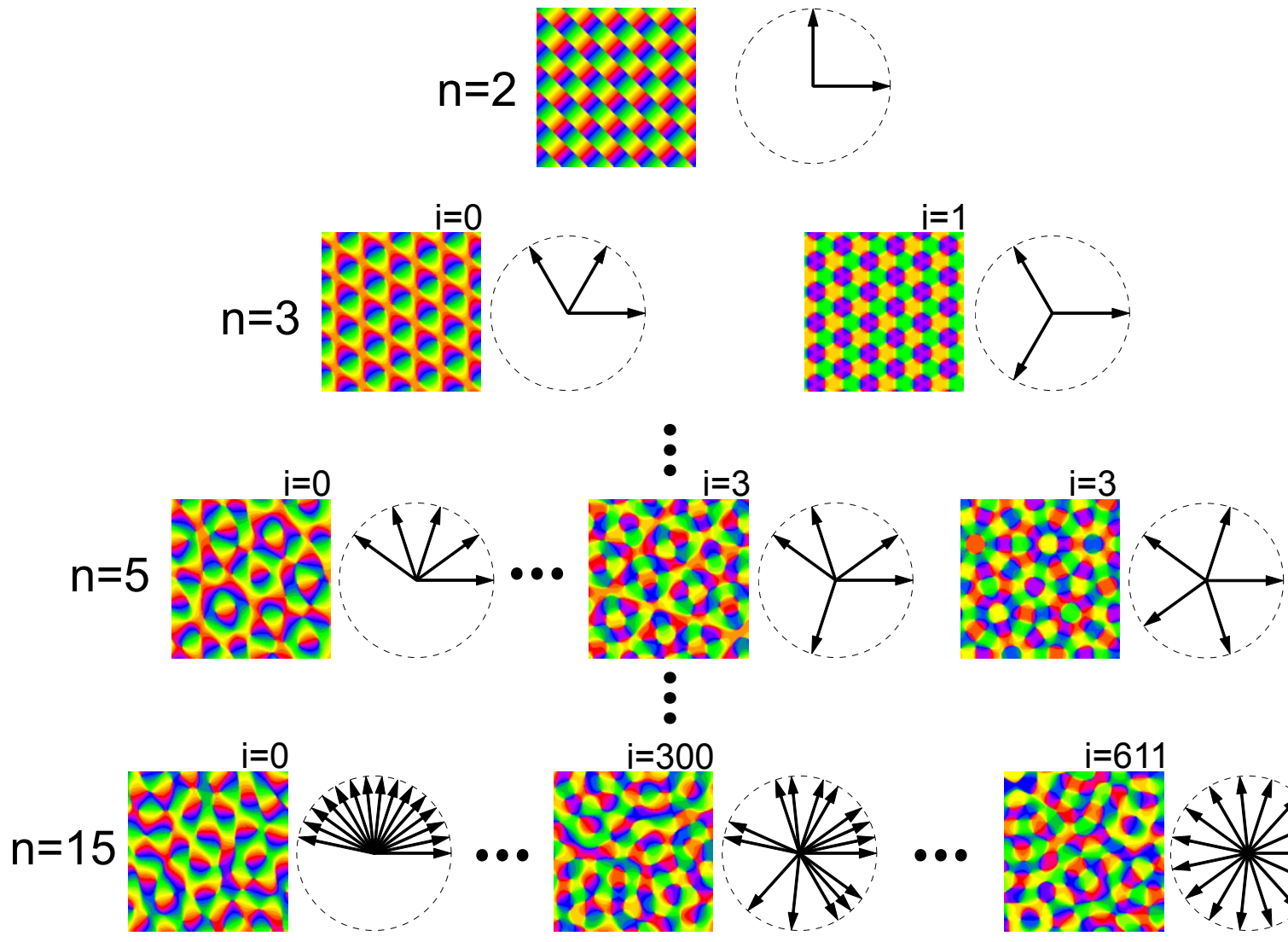

$i=300$
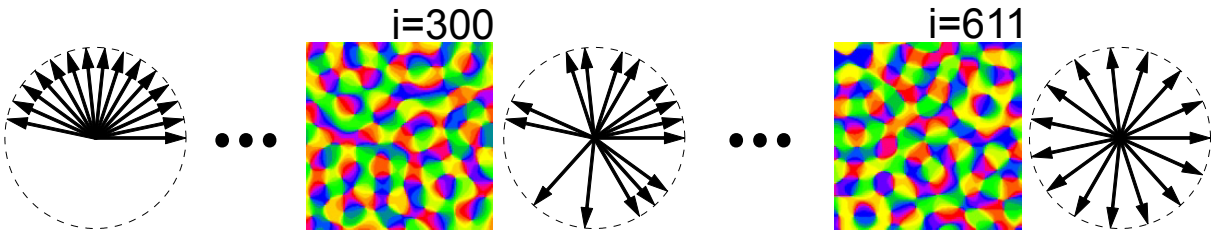

Figure 4 Exact and approximate orientation selective fixed points of OPM optimization models. (a) Pinwheel-free OS pattern. Diagram shows the position of the wave vector in Fourier space. (b) rPWC with four nonzero wave vectors. (c) Essentially complex planforms (ECPs). The index $n$ indicates the number of nonzero wave vectors. The index $i$ enumerates nonequivalent configurations of wave vectors with the same $n$, starting with $i=0$ for the most anisotropic planform. For $n=3,5$, and 15 , there are 2, 4, and 612 different ECPs, respectively. OPM layouts become more irregular with increasing $n$. 
To calculate the fixed points of Equation (13), we use a perturbative method called weakly nonlinear analysis that enables us to analytically examine the structure and stability of inhomogeneous stationary solutions in the vicinity of a finite-wavelength instability. Here, we examine the stability of so-called planforms [83-85]. Planforms are patterns that are composed of a finite number of Fourier components, such as

$$
z(\mathbf{x})=\sum_{j} A_{j}(t) e^{i \mathbf{k}_{\mathbf{j}} \mathbf{x}}
$$

for a pattern of orientation columns. With the above planform ansatz, we neglect any spatial dependency of the amplitudes $A_{j}(t)$ for example due to long-wave deformations for the sake of simplicity and analytical tractability. When the dynamics is close to a finiite wavelength instability, the essential Fourier components of the emerging pattern are located on the critical circle $\left|\mathbf{k}_{j}\right|=k_{c}$. The dynamic equations for the amplitudes of these Fourier components are called amplitude equations. For a discrete number of $N$ Fourier components of $z(\mathbf{x})$ whose wave vectors lie equally spaced on the critical circle, the most general system of amplitude equations compatible with the model's symmetries (Equations (5) to (9)) has the form [35,87]

$$
\dot{A}_{i}=r A_{i}-A_{i} \sum_{j=1}^{N} g_{i j}\left|A_{j}\right|^{2}-\bar{A}_{i^{-}} \sum_{j=1}^{N} f_{i j} A_{j} A_{j^{-}},
$$

with $r>0$. Here, $g_{i j}$ and $f_{i j}$ are the real-valued coupling coefficients between the amplitudes $A_{i}$ and $A_{j}$. They depend on the differences between indices $|i-j|$ and are entirely determined by the nonlinearity $N_{3}^{z}[z, z, \bar{z}]$ in Equation (13). If the wave vectors $\mathbf{k}_{i}=\left(\cos \alpha_{i}, \sin \alpha_{i}\right) k_{c}$ are parameterized by the angles $\alpha_{i}$, then the coefficients $g_{i j}$ and $f_{i j}$ are functions only of the angle $\alpha=\left|\alpha_{i}-\alpha_{j}\right|$ between the wave vectors $\mathbf{k}_{i}$ and $\mathbf{k}_{j}$. One can thus obtain the coupling coefficients from two continuous functions $g(\alpha)$ and $f(\alpha)$ that can be obtained from the nonlinearity $N_{3}^{z}[z, z, \bar{z}]$ (see 'Methods' section for details). In the following, these functions are called angle-dependent interaction functions. The amplitude equations are variational if and only if $g_{i j}$ and $f_{i j}$ are real-valued. In this case they can be derived through

$$
\dot{A}_{j}(t)=-\frac{\partial U_{A}}{\partial \bar{A}_{j}}
$$

from an energy

$$
U_{A}=-r \sum_{i=1}^{N}\left|A_{i}\right|^{2}+\frac{1}{2} \sum_{i, j=1}^{N} g_{i j}\left|A_{i}\right|^{2}\left|A_{j}\right|^{2}+\frac{1}{2} \sum_{i, j=1}^{N} f_{i j} \bar{A}_{i} \bar{A}_{i-} A_{j} A_{j-} .
$$

If the coefficients $g_{i j}$ and $f_{i j}$ are derived from Equation (1), the energy $U_{A}$ for a given planform solution corresponds to the energy density of the EN energy functional considering only terms up to fourth-order in $z(\mathbf{x})$.

The amplitude equations (15) enable to calculate an infinite set of orientation selective fixed points. For the above OS solution with one nonzero wave vector $z(\mathbf{x})=$ $A_{0} e^{i \mathbf{k} \mathbf{x}}$, the amplitude equations predict the so far undetermined amplitude

$$
\left|A_{0}\right|^{2}=\frac{r}{g_{i i}}
$$

and its energy

$$
U_{\mathrm{OS}}=-\frac{r}{2 g_{i i}} \text {. }
$$

Since $g_{i i}>0$, this shows that OS stationary solutions only exist for $r>0$, i.e., in the symmetry breaking regime. As for all following fixed-points, $U_{\mathrm{OS}}$ specifies the energy difference to the homogeneous unselective state $z(\mathbf{x})=0$.

A second class of stationary solutions can be found with the ansatz

$$
z(\mathbf{x})=A_{1} e^{i \mathbf{k}_{1} \mathbf{x}}+A_{2} e^{i \mathbf{k}_{2} \mathbf{x}}+A_{3} e^{-i \mathbf{k}_{1} \mathbf{x}}+A_{4} e^{-i \mathbf{k}_{2} \mathbf{x}}
$$

with amplitudes $A_{j}=\left|A_{j}\right| e^{i \varphi_{j}}$ and $\angle\left(\mathbf{k}_{1}, \mathbf{k}_{2}\right)=\alpha>0$. By inserting this ansatz into Equation (15) and assuming uniform amplitude $\left|A_{1}\right|=\left|A_{2}\right|=\left|A_{2}\right|=\left|A_{4}\right|=\mathcal{A}$, we obtain

$$
\mathcal{A}^{2}=\frac{r}{g_{00}+g_{0 \pi}+g_{0 \alpha}+g_{0 \pi-\alpha}-2 f_{0 \alpha}} .
$$
by

The phase relations of the four amplitudes are given

$$
\begin{aligned}
& \phi_{1}+\phi_{3}=\phi_{0} \\
& \phi_{2}+\phi_{4}=\phi_{0}+\pi .
\end{aligned}
$$

These solutions describe a regular rhombic lattice of pinwheels and are therefore called rhombic pinwheel crystals (rPWCs) in the following. Three phases can be chosen arbitrarily according to the two above conditions, e.g., $\varphi_{0}, \Delta_{0}=\varphi_{1}-\varphi_{3}$ and $\Delta_{1}=\varphi_{2}-\varphi_{4}$. For an rPWC parameterized by these phases, $\Delta_{0}$ shifts the absolute positions of the pinwheels in $x$-direction, $\Delta_{1}$ shifts the absolute positions of the pinwheels in $y$-direction, and $\varphi_{0}$ shifts all the preferred orientations by a constant angle. The energy of an rPWC solution is

$$
U_{\mathrm{rPWC}}=-\frac{2 r}{g_{00}+g_{0 \pi}+g_{0 \alpha}+g_{0 \pi-\alpha}-2 f_{0 \alpha}} .
$$


An example of such a solution is depicted in Figure $4 \mathrm{~b}$. We note that rPWCs have been previously found in several other models for OPM development $[27,31,37,39,88]$. The pinwheel density $\rho$ of an rPWC, i. e., the number of pinwheels in an area of size $\Lambda^{2}$, is equal to $\rho=4 \sin \alpha$ [54]. The angle $\alpha$ which minimizes the energy $U_{\mathrm{rPWC}}$ can be computed by maximizing the function

$$
s(\alpha)=g_{0 \alpha}+g_{0 \pi-\alpha}-2 f_{0 \alpha}
$$

in the denominator of Equation (20).

The two solution classes discussed so far, namely OS and rPWCs, exhibit one prominent feature, absent in experimentally observed cortical OPMs, namely perfect spatial periodicity. Many cortical maps including OPMs do not resemble a crystal-like grid of repeating units. Rather the maps are characterized by roughly repetitive but aperiodic spatial arrangement of feature preferences (e.g., $[5,10])$. This does not imply that the precise layout of columns is arbitrary. It rather means that the rules of column design cannot be exhaustively characterized by mapping a 'representative' hypercolumn.

Previous studies of abstract models of OPM development introduced the family of so-called essentially complex planforms (ECPs) as stationary solutions of Equation (15). This solution class encompasses a large variety of realistic quasi-periodic OPM layouts and is therefore a good candidate solution class for models of OPM layouts. In addition, Kaschube et al. [38] demonstrated that models in which these are optimal solutions can reproduce all essential features of the common OPM design in ferret, tree-shrew, and galago. An $n$-ECP solution can be written as

$$
z(\mathbf{x})=\sum_{j=1}^{n} A_{j} e^{i l_{j} \mathbf{k}_{j} \mathbf{x}},
$$

with $n=N / 2$ wave vectors $\mathbf{k}_{j}=k_{c}(\cos (\pi j / n), \sin (\pi j / n))$ distributed equidistantly on the upper half of the critical circle, complex amplitudes $A_{j}$ and binary variables $l_{j}=$ \pm 1 determining whether the mode with wave vector $\mathbf{k}_{j}$ or $-\mathbf{k}_{j}$ is active (nonzero). Because these planforms cannot realize a real-valued function they are called essentially complex [35]. For an $n$-ECP, the third term on the right-hand side of Equation (15) vanishes and the amplitude equations for the active modes $A_{i}$ reduce to a system of Landau equations

$$
\dot{A}_{i}=r A_{i}-A_{i} \sum_{j=1}^{n} g_{i j}\left|A_{j}\right|^{2},
$$

where $g_{i j}$ is the $n \times n$-coupling matrix for the active modes. Consequently, the stationary amplitudes obey

$$
\left|A_{i}\right|^{2}=r \sum_{j=1}^{n}\left(\mathbf{g}^{-1}\right)_{i j} .
$$

The energy of an $n$-ECP is given by

$$
U_{\mathrm{ECP}}=-\frac{r}{2} \sum_{i, j}\left(\mathrm{~g}^{-1}\right)_{i j} .
$$

We note that this energy in general depends on the configuration of active modes, given by the $l_{j}$ 's, and therefore planforms with the same number of active modes may not be energetically degenerate.

Families of $n$-ECP solutions are depicted in Figure 4c. The 1-ECP corresponds to the pinwheel-free OS pattern discussed above. For fixed $n \geq 3$, there are multiple planforms not related by symmetry operations which considerably differ in their spatial layouts. For $n \geq 4$, the patterns are spatially quasi-periodic, and are a generalization of the so-called Newell-Pomeau turbulent crystal $[89,90]$. For $n \geq 10$, their layouts resemble experimentally observed OPMs. Different $n$-ECPs however differ considerably in their pinwheel density. Planforms whose nonzero wave vectors are distributed isotropically on the critical circle typically have a high pinwheel density (see Figure 4c, $n=15$ lower right). Anisotropic planforms generally contain considerably fewer pinwheels (see Figure $4 \mathrm{c}, \mathrm{n}=15$ lower left). All large $n$-ECPs, however, exhibit a complex quasi-periodic spatial layout and a nonzero density of pinwheels.

In order to demonstrate that a certain planform is an optimal solution of an optimization model for OPM layouts in which patterns emerge via a supercritical bifurcation, we not only have to show that it is a stationary solution of the amplitude equations but have to analyze its stability properties with respect to the gradient descent dynamics as well as its energy compared to all other candidate solutions.

Many stability properties can be characterized by examining the amplitude equations (15). In principle, the stability range of an $n$-ECPs may be bounded by two different instability mechanisms: (i) an intrinsic instability by which stationary solutions with $n$ active modes decay into ones with lower $n$. (ii) an extrinsic instability by which stationary solutions with a 'too low' number of modes are unstable to the growth of additional active modes. These instabilities can constrain the range of stable $n$ to a small finite set around a typical $n[35,87]$. A mathematical evaluation of both criteria leads to precise conditions for extrinsic and intrinsic stability of a planform (see 'Methods' section). In the following, a planform is said to be stable, if it is both extrinsically and intrinsically stable. A planform is said to be an optimum (or optimal solution) if it is stable and possesses 
the minimal energy among all other stationary planform solutions.

Taken together, this amplitude equation approach enables to analytically compute the fixed points and optima of arbitrary optimization models for visual cortical map layout in which the functional architecture is completely specified by the pattern of orientation columns $z(\mathbf{x})$ and emerges via a supercritical bifurcation. Via a third-order expansion of the energy functional together with weakly nonlinear analysis, the otherwise analytically intractable partial integro-differential equation for OPM layouts reduces to a much simpler system of ordinary differential equations, the amplitude equations. Using these, several families of solutions, OSs, rPWCs, and essentially complex planforms, can be systematically evaluated and comprehensively compared to identify sets of unstable, stable and optimal, i.e., lowest energy fixed points. As already mentioned, the above approach is suitable for arbitrary optimization models for visual cortical map layout in which the functional architecture is completely specified by the pattern of orientation columns $z(\mathbf{x})$ which in the EN model is fulfilled in the rigid retinotopy limit. We now start by considering the EN optimal solutions in this limit and subsequently generalize this approach to models in which the visual cortical architecture is jointly specified by maps of orientation and position preference that are matched to one another.

\section{Representing an ensemble of 'bar'-stimuli}

We start our investigation of optimal dimension-reducing mappings in the EN model using the simplest and most frequently used orientation stimulus ensemble, the distribution with $s_{z}$-values uniformly arranged on a ring with radius $r_{s_{z}}=\sqrt{2}[57,64-66,91]$. We call this stimulus ensemble the circular stimulus ensemble in the following. According to the linear stability analysis of the nonselective fixed point, at the point of instability, we choose $\sigma=\sigma^{*}(\eta)$ such that the linearization given in Equation (11) is completely characterized by the continuity parameter $\eta$. Equivalent to specifying $\eta$ is to fix the ratio of activation range $\sigma$ and column spacing $\Lambda$

$$
\sigma / \Lambda=\frac{1}{2 \pi} \sqrt{\log (1 / \eta)}
$$

as a more intuitive parameter. This ratio measures the effective interaction-range relative to the expected spacing of the orientation preference pattern. In abstract optimization models for OPM development a similar quantity has been demonstrated to be a crucial determinant of pattern selection $[35,87]$. We note, however, that due to the logarithmic dependence of $\sigma / \Lambda$ on $\eta$, a slight variation of the effective interaction range may correspond to a variation of the continuity parameter $\eta$ over several orders of magnitude. In order to investigate the stability of stationary planform solutions in the EN model with a circular orientation stimulus ensemble, we have to determine the angle-dependent interaction functions $g(\alpha)$ and $f(\alpha)$. For the coefficients $a_{j}$ in Equation (14) we obtain

$$
\begin{array}{lll}
a_{1}=\frac{1}{4 \sigma^{6}}-\frac{1}{\sigma^{4}}+\frac{1}{2 \sigma^{2}} & a_{2}=\frac{1}{4 \pi \sigma^{6}}-\frac{1}{8 \pi \sigma^{8}} & a_{3}=-\frac{1}{16 \pi \sigma^{8}}+\frac{1}{8 \pi \sigma^{6}} \\
a_{4}=-\frac{1}{8 \pi \sigma^{8}}+\frac{1}{4 \pi \sigma^{6}}-\frac{1}{8 \pi \sigma^{4}} & a_{5}=-\frac{1}{16 \pi \sigma^{8}} & a_{6}=\frac{1}{8 \pi \sigma^{6}}-\frac{1}{16 \pi \sigma^{8}} \\
a_{7}=\frac{1}{12 \pi^{2} \sigma^{10}}-\frac{1}{12 \pi^{2} \sigma^{8}} & a_{8}=\frac{1}{24 \pi^{2} \sigma^{10}} & a_{9}=-\frac{3}{64 \pi^{3} \sigma^{12}} \\
a_{10}=\frac{1}{12 \pi^{2} \sigma^{10}}-\frac{1}{12 \pi^{2} \sigma^{8}} & a_{11}=\frac{1}{24 \pi^{2} \sigma^{10}} . &
\end{array}
$$

The angle-dependent interaction functions of the EN model with a circular orientation stimulus ensemble are then given by

$$
\begin{aligned}
& g(\alpha)=\frac{1}{\sigma^{4}}\left(1-2 e^{-k_{c}^{2} \sigma^{2}}-e^{2 k k_{\sigma}^{2} \sigma^{2}(\cos \alpha-1)}\left(1-2 e^{-k_{c}^{2} \sigma^{2} \cos \alpha}\right)\right) \\
& \quad+\frac{1}{2 \sigma^{2}}\left(e^{2 k_{c}^{2} \sigma^{2}(\cos \alpha-1)}-1\right)+\frac{8}{\sigma^{6}} e^{-2 k_{c}^{2} \sigma^{2}} \sinh ^{4}\left(1 / 2 k_{c}^{2} \sigma^{2} \cos \alpha\right) \\
& f(\alpha)=\frac{1}{\sigma^{4}}\left(1-e^{-2 k_{c}^{2} \sigma^{2}}\left(\cosh \left(2 k_{c}^{2} \sigma^{2} \cos \alpha\right)+2 \cosh \left(k_{c}^{2} \sigma^{2} \cos \alpha\right)\right)+2 e^{-k_{c}^{2} \sigma^{2}}\right) \\
& \quad+\frac{1}{2 \sigma^{2}}\left(e^{-2 k_{c}^{2} \sigma^{2}} \cosh \left(2 k_{c}^{2} \sigma^{2} \cos \alpha\right)-1\right)+\frac{4}{\sigma^{6}} e^{-2 k_{c}^{2} \sigma^{2}} \sinh ^{4}\left(1 / 2 k_{c}^{2} \sigma^{2} \cos \alpha\right) .
\end{aligned}
$$

These functions are depicted in Figure 5 for two different values of the interaction range $\sigma / \Lambda$. We note that both functions are positive for all $\sigma / \Lambda$ which is a sufficient condition for a supercritical bifurcation from the homogeneous nonselective state in the EN model.

Finally, by minimizing the function $s(\alpha)$ in Equation (21), we find that the angle $\alpha$ which minimizes the energy of the rPWC fixed-point is $\alpha=\pi / 2$. This corresponds to a square array of pinwheels (sPWC). Due to the orthogonal arrangement oblique and cardinal orientation columns and the maximized pinwheel density of $\rho=4$, the square array of pinwheels has the maximal coverage among all rPWC solutions.

\section{Optimal solutions close to the pattern formation threshold}

We first tested for the stability of pinwheel-free OS solutions and the sPWCs, by analytical evaluation of the criteria for intrinsic and extrinsic stability (see 'Methods' section). We found both, OSs and sPWCs, to be intrinsically and extrinsically stable for all $\sigma / \Lambda$. Next, we tested for the stability of $n$-ECP solutions with $2 \leq n \leq 20$. We found all $n$-ECP configurations with $2 \leq n \leq 20$ to be intrinsically unstable for all $\sigma / \Lambda$. Hence, none of these planforms represent optimal solutions of the EN model with a circular stimulus ensemble, while both OSs and sPWC are always local minima of the energy functional.

By evaluating the energy assigned to the sPWC (Equation 20) and the OS pattern (Equation 18), we next identified two different regimes: (i) For short interaction range $\sigma / \Lambda \leqslant 0.122$ the sPWC possesses minimal energy and is therefore the predicted global minimum. (ii) For $\sigma / \Lambda \gtrsim 0.122$ the OS pattern is optimal.

Figure 6a shows the resulting simple phase diagram. sPWCs and OSs are separated by a phase border at $\sigma / \Lambda$ 

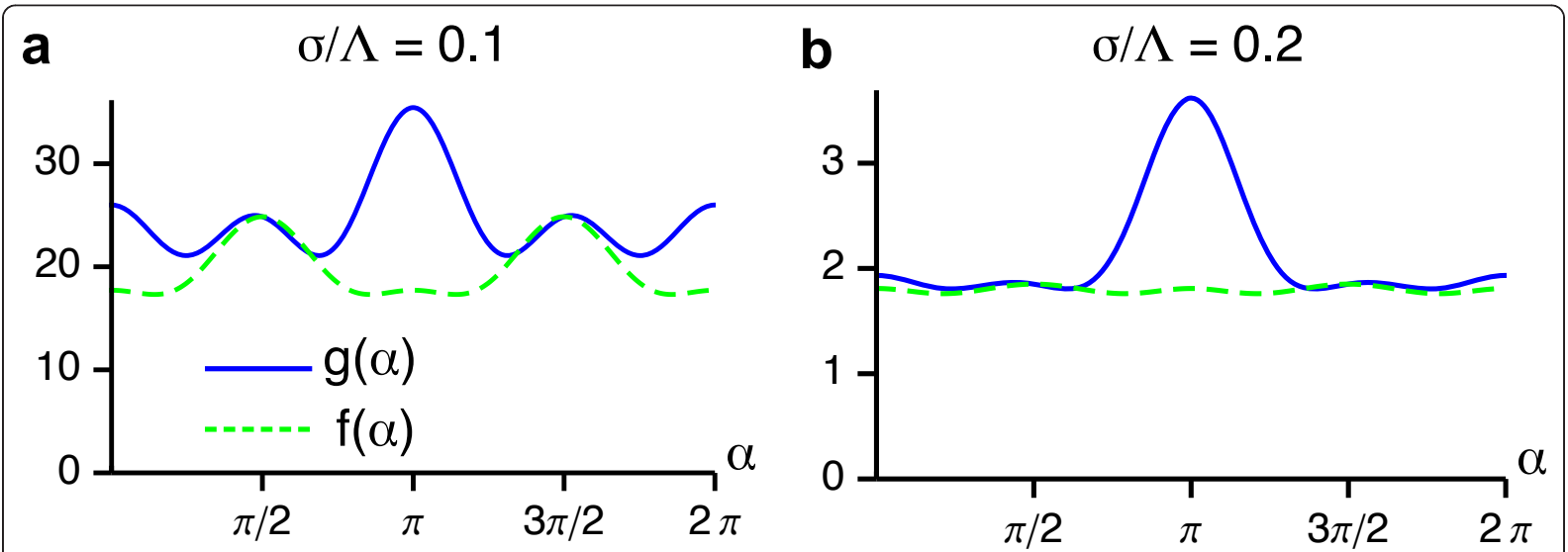

Figure 5 Angle-dependent interaction functions for the EN model with fixed retinotopy and circular orientation stimulus ensemble. (a, b) $g(\alpha)$ and $f(\alpha)$ for $\sigma / \Lambda=0.1(\mathbf{a})$ and $\sigma / \Lambda=0.2(\mathbf{b})$.

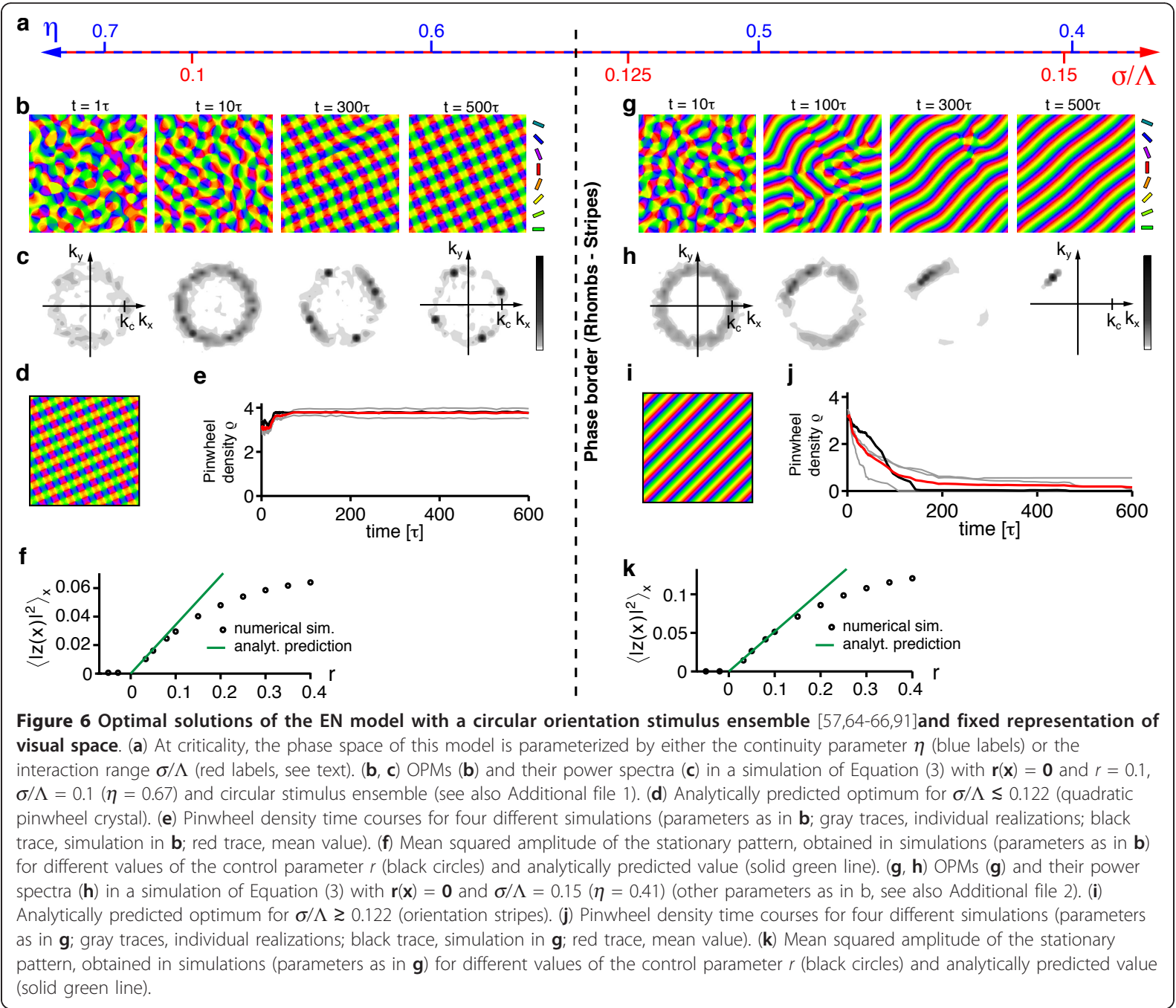


$\approx 0.122$. We numerically confirmed these analytical predictions by extensive simulations of Equation (3) with $\mathbf{r}$ $(\mathbf{x})=\mathbf{0}$ and the circular stimulus ensemble (see 'Methods' section for details). Figure $6 \mathrm{~b}, \mathrm{c}$ shows snapshots of a representative simulation with short interaction range $(r=0.1, \sigma / \Lambda=0.1(\eta=0.67))$ (see also Additional file $1)$. After the phase of initial pattern emergence (symmetry breaking), the OPM layout rapidly approaches a square array of pinwheels, the analytically predicted optimum (Figure 6d). Pinwheel density time courses (see 'Methods' section) display a rapid convergence to a value close to the predicted density of 4 (Figure 6e). Figure $6 f$ shows the stationary mean squared amplitudes of the pattern obtained for different values of the control parameter $r$ (black circles). For small control parameters, the pattern amplitude is perfectly predicted by Equation (19) (solid green line). Figure 6g,h shows snapshots of a typical simulation with longer interaction range $(r=0.1, \sigma / \Lambda=0.15(\eta=0.41))$ (see also Additional file 2). After the emergence of an OPM with numerous pinwheels, pinwheels undergo pairwise annihilation as previously described for various models of OPM development and optimization $[25,27,35]$. The OP pattern converges to a pinwheel-free stripe pattern, which is the analytically computed optimal solution in this parameter regime (Figure 6i). Pinwheel densities decay toward zero over the time course of the simulations (Figure 6j). Also in this parameter regime, the mean squared amplitude of the pattern is well-predicted by Equation (17) for small $r$ (Figure 6k).

In summary, the phase diagram of the EN model with a circular stimulus ensemble close to threshold is divided into two regions: (i) for a small interaction range (large continuity parameter) a square array of pinwheels is the optimal dimension-reducing mapping and (ii) for a larger interaction range (small continuity parameter) OSs are the optimal dimension-reducing mapping. Both states are stable throughout the entire parameter range. All other planforms, in particular quasi-periodic $n$-ECPs are unstable. At first sight, this structure of the EN phase diagram may appear rather counterintuitive. A solution with many pinwheel-defects is energetically favored over a solution with no defects in a regime with large continuity parameter where discontinuity should be strongly penalized in the EN energy term. However, a large continuity parameter at pattern formation threshold inevitably leads to a short interaction range $\sigma$ compared to the characteristic spacing $\Lambda$ (see Equation (24)). In such a regime, the gain in coverage by representing many orientation stimuli in a small area spanning the typical interaction range, e.g., with a pinwheel, is very high. Our results show that the gain in coverage by a spatially regular positioning of pinwheels outweighs the accompanied loss in continuity above a certain value of the continuity parameter.

\section{EN dynamics far from pattern formation threshold}

Close to pattern formation threshold, we found only two stable solutions, namely OSs and sPWCs. Neither of the two exhibits the characteristic aperiodic and pinwheelrich organization of experimentally observed OPMs. Furthermore, the pinwheel densities of both solutions ( $\rho$ $=0$ for OSs and $\rho=4$ for sPWCs) differ considerably from experimentally observed values [38] around 3.14. One way toward more realistic stable stationary states might be to increase the distance from pattern formation threshold. In fact, further away from threshold, our perturbative calculations may fail to correctly predict optimal solutions of the model due to the increasing influence of higher order terms in the Volterra series expansion of the right-hand side in Equation (3).

To asses this possibility, we simulated Equation (3) with $\mathbf{r}(\mathbf{x})=\mathbf{0}$ and a circular stimulus ensemble for very large values of the control parameter $r$. Figure 7 displays snapshots of such a simulation for $r=0.8$ as well as their pinwheel density time courses for two different values of $\sigma / \Lambda$. Pinwheel annihilation in the case of large $\sigma / \Lambda$ is less rapid than close to threshold (Figure $7 \mathrm{a}, \mathrm{b})$. The OPM nevertheless converges toward a layout with rather low pinwheel density with pinwheelfree stripe-like domains of different directions joined by domains with essentially rhombic crystalline pinwheel arrangement. The linear zones increase their size over the time course of the simulations, eventually leading to stripe-patterns for large simulation times. For smaller interaction ranges $\sigma / \Lambda$, the OPM layout rapidly converges toward a crystal-like rhombic arrangement of pinwheels, however containing several dislocations (Figure 24a in Appendix 1) [84]. Dislocations are defects of roll or square patterns, where two rolls or squares merge into one, thus increasing the local wavelength of the pattern $[83,85]$. Nevertheless, for all simulations, the pinwheel density rapidly reaches a value close to 4 (Figure 7c) and the square arrangement of pinwheels is readily recognizable. Both features, the dislocations in the rhombic patterns and domain walls in the stripe patterns, have been frequently observed in pattern-forming systems far from threshold $[84,85]$.

In summary, the behavior of the EN dynamics with circular stimulus ensemble far from pattern formation threshold agrees very well with our analytical predictions close to threshold. Again, orientation stripes and square pinwheel crystals are identified as the only stationary solutions. Aperiodic and pinwheel-rich patterns which resemble experimentally OPM layouts were not observed. 


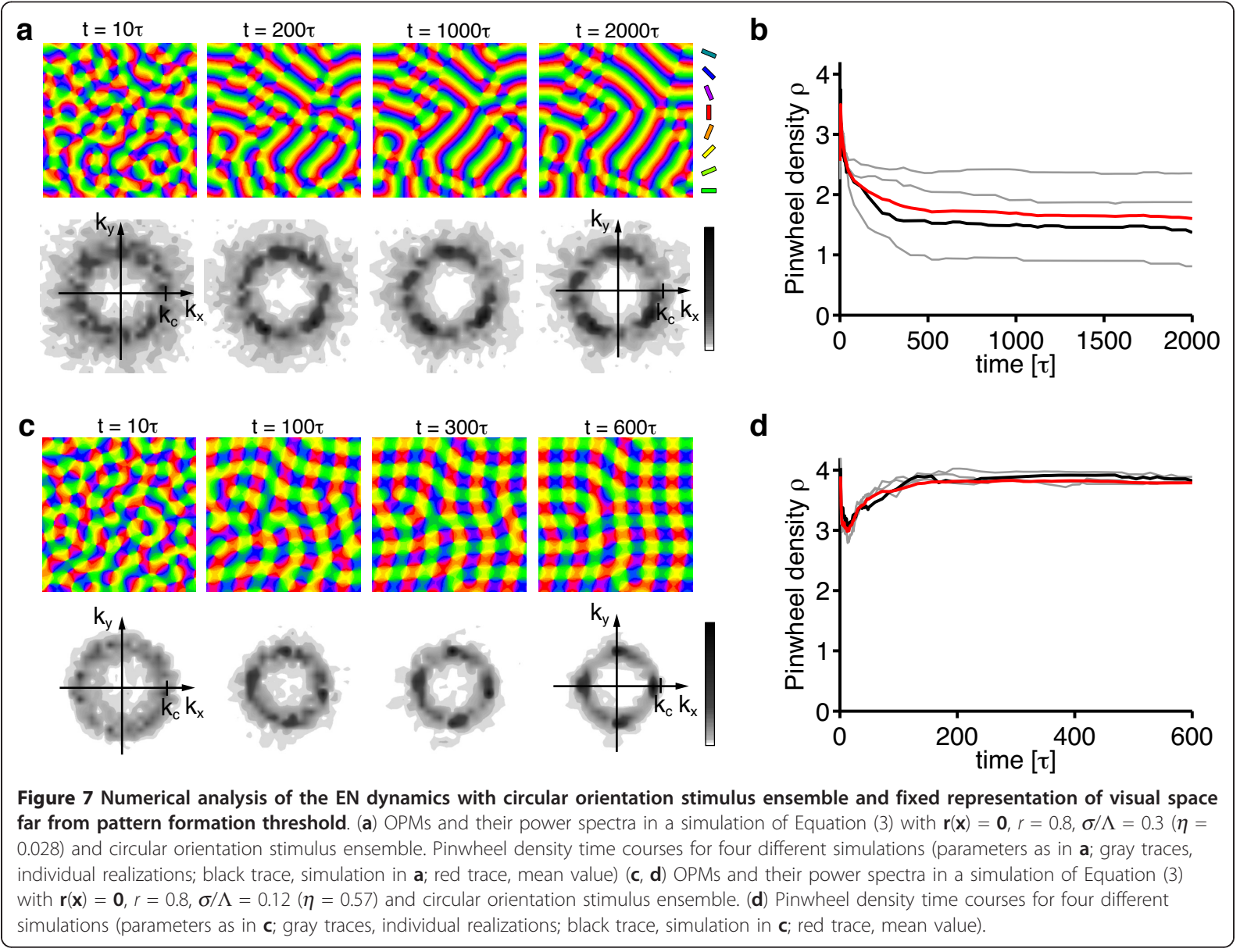

\section{Taking retinotopic distortions into account}

So far, we have examined the optimal solutions of the EN model for the simplest and most widely used orientation stimulus ensemble. Somewhat unexpected from previous reports, the optimal states in this case do not exhibit the irregular structure of experimentally observed orientation maps. Our treatment however differs from previous approaches in that the mapping of visual space so far was assumed to be undistorted and fixed, i.e., $\mathbf{r}(\mathbf{x})=\mathbf{0}$. We recall that in their seminal publication, Durbin and Mitchison [21] in particular demonstrated interesting correlations between the map of orientation preference and the map of visual space. These correlations suggest a strong coupling between the two that may completely alter the model's dynamics and optimal solutions.

It is thus essential to clarify whether the behavior of the EN model observed above changes or persists if we relax the simplifying assumption of undistorted retinotopy and allow for retinotopic distortions. By analyzing the complete EN model dynamics (Equations $(3,4)$ ), we study the EN model exactly as originally introduced by Durbin and Mitchison [21].

We again employ the fact that in the vicinity of a supercritical bifurcation where the nonorientation selective state becomes unstable, the entire set of nontrivial fixed points of Equations $(3,4)$ is determined by the third-order terms of the Volterra series representation of the nonlinear operators $F^{z}[z, \mathbf{r}]$ and $\mathbf{F}^{r}[z, \mathbf{r}]$. The model symmetries equations (5) to (9) restrict the general form of the leading order terms for any model for the joint optimization of OPM and RM to

$$
\begin{aligned}
& \partial_{t} z(\mathbf{x})=L_{z}[z]+Q^{z}[\mathbf{r}, z]+N_{3}^{z}[z, z, \bar{z}]+\cdots \\
& \partial_{t} \mathbf{r}(\mathbf{x})=\mathbf{L}_{r}[\mathbf{r}]+\mathbf{Q}^{r}[z, \bar{z}]+\cdots
\end{aligned}
$$

Because the uniform retinotopy is linearly stable, retinotopic distortions are exclusively induced by a coupling of the RM to the OPM via the quadratic vector-valued operator $\mathbf{Q}^{r}[z, \bar{z}]$. These retinotopic distortions will in turn alter the dynamics of the OPM via the quadratic 
complex-valued operator $Q^{z}[\mathbf{r}, z]$. Close to the point of pattern onset $(r \ll 1)$, the timescale of OPM development, $\tau=1 / r$, becomes arbitrarily large and retinotopic deviations evolve on a much shorter timescale. This separation of timescales allows for an adiabatic elimination of the variable $\mathbf{r}(\mathbf{x})$, assuming it to always be at the equilibrium point of Equation (27):

$$
\mathbf{r}(\mathbf{x})=-\mathbf{L}_{r}^{-1}\left[\mathbf{Q}^{r}[z, \bar{z}]\right] .
$$

We remark that as $\lambda_{T / L}^{r}(k)<0$ for all finite wave numbers $k>0$, the operator $\mathbf{L}_{r}[\mathbf{r}]$ is indeed invertible when excluding global translations in the set of possible perturbations of the trivial fixed point. From Equation (28), the coupled dynamics of OPM and RM is thus reduced to a third-order effective dynamics of the OPM:

$$
\begin{aligned}
\partial_{t} z(\mathbf{x}) & \approx L_{z}[z] \underbrace{-Q^{z}\left[\mathbf{L}_{r}^{-1}\left[\mathbf{Q}^{r}[z, \bar{z}]\right], z\right]}_{N_{3}^{r}[z, z, \bar{z}]}+N_{3}^{z}[z, z, \bar{z}] \\
& =L_{z}[z]+N_{3}^{r}[z, z, \bar{z}]+N_{3}^{z}[z, z, \bar{z}] .
\end{aligned}
$$

The nonlinearity $N_{3}^{r}[z, z, \bar{z}]$ accounts for the coupling between OPM and RM. Its explicit analytical calculation for the EN model is rather involved and yields a sum

$$
N_{3}^{r}[z, z, \bar{z}]=\sum_{j=1}^{12} a_{r}^{j} N_{r}^{j}[z, z, \bar{z}] .
$$

The individual nonlinear operators $N_{r}^{j}$ are nonlinear convolution-type operators and are presented in the 'Methods' section together with a detailed description of their derivation. Importantly, it turns out that the coefficients $a_{r}^{j}$ are completely independent of the orientation stimulus ensemble.

The adiabatic elimination of the retinotopic distortions results in an equation for the OPM (Equation (29)) which has the same structure as Equation (13), the only difference being an additional cubic nonlinearity. Due to this similarity, its stationary solutions can be determined by the same methods as presented for the case of a fixed retinotopy. Again, via weakly nonlinear analysis we obtain amplitude equations of the form Equation (15). The nonlinear coefficients $g_{i j}$ and $f_{i j}$ are determined from the angle-dependent interaction functions $g(\alpha)$ and $f(\alpha)$. For the operator $N_{3}^{r}[z, z, \bar{z}]$, these functions are given by

$$
\begin{aligned}
& g_{r}(\alpha)=\frac{\left(\left(1-\sigma^{2}-2 e^{-k_{c}^{2} \sigma^{2}}\right) e^{2 k_{c}^{2} \sigma^{2}(\cos \alpha-1)}+e^{-k_{c}^{2} \sigma^{2}}\right)^{2}}{2 \sigma^{4}\left(\eta_{r}+\sigma^{2} e^{-2 k_{c}^{2} \sigma^{2}(\cos \alpha-1)}\right)} \\
& f_{r}(\alpha)=\frac{1}{2}\left(g_{r}(\alpha)+g_{r}(\alpha+\pi)\right),
\end{aligned}
$$

verifying that, $N_{3}^{r}[z, z, \bar{z}]$ is independent of the orientation stimulus ensemble. Besides the interaction range $\sigma /$ $\Lambda$ the continuity parameter $\eta_{r} \in[0, \infty]$ for the $\mathrm{RM}$ appears as an additional parameter in the angle-dependent interaction function. Hence, the phase diagram of the EN model will acquire one additional dimension when retinotopic distortions are taken into account. We note, that in the limit $\eta_{r} \rightarrow \infty$, the functions $g_{r}(\alpha)$ and $f_{r}$ $(\alpha)$ tend to zero and as expected one recovers the results presented above for fixed uniform retinotopy. The functions $g_{r}(\alpha)$ and $f_{r}(\alpha)$ are depicted in Figure 8 for various interaction ranges $\sigma / \Lambda$ and retinotopic continuity parameters $\eta_{r}$.

\section{Coupled essentially complex n-planforms}

In the previous section, we found that by an adiabatic elimination of the retinotopic distortions in the dynamics equations $(26,27)$ the system of partial integro-differential equations can be reduced to a single equation for the OPM. In this case, the stationary solutions of the OPM dynamics are again planforms composed of a discrete set of Fourier modes

$$
z(\mathbf{x})=\sum_{j}^{N} A_{j} e^{i \mathbf{k}_{j} \mathbf{x}},
$$

with $|\mathbf{k}|=k_{c}$. However, each of these stationary planform OPM solutions induces a specific pattern of retinotopic distortions by Equation (28). The joint mapping $\{\mathbf{X}+\mathbf{r}(\mathbf{x}), z(\mathbf{x})\}$ is then an approximate stationary solution of Equations $(26,27)$ and will be termed coupled planform solution in the following. In contrast to other models for the joint mapping of orientation and visual space (e.g., $[31,33,92])$, the coupling between the representation of visual space and orientation in the EN model is not induced by model symmetries but a mere consequence of the joint optimization of OPM and RM that requires them to be matched to one another.

For planforms given by Equation (30), it is possible to analytically evaluate Equation (28) and compute the associated retinotopic distortions $\mathbf{r}(\mathbf{x})$. After a somewhat lengthy calculation (see 'Methods' section), one obtains

$$
\begin{aligned}
\mathbf{r}(\mathbf{x})=- & \sum_{k=1, j<k}^{n} \frac{\boldsymbol{\Delta}_{j k}}{\lambda_{L}^{r}\left(\left|\Delta_{j k}\right|\right)}\left(\frac{1}{\sigma^{2}}\left(e^{-k_{c}^{2} \sigma^{2} / 2}-e^{-\Delta_{j k}^{2} \sigma^{2} / 2}\right)^{2}-e^{-\Delta_{j k}^{2} \sigma^{2}}\right) \\
& *\left(\Im\left(A_{j} \bar{A}_{k}\right) \cos \left(\boldsymbol{\Delta}_{j k} \mathbf{x}\right)+\Re\left(A_{j} \bar{A}_{k}\right) \sin \left(\boldsymbol{\Delta}_{j k} \mathbf{x}\right)\right),
\end{aligned}
$$

with $\Delta_{j k}=\mathbf{k}_{j}-\mathbf{k}_{k}$ and $\lambda_{L}^{r}(k)=-k^{2}\left(\eta_{r}+e^{-\sigma^{2} k^{2}} \sigma^{2}\right)$. These retinotopic distortions represent superpositions of longitudinal modes (see Figure 3b). Hence, coupled planform stationary solutions of the EN dynamics do not contain any transversal mode components. According to Equation (31), the pinwheel-free coupled 1-ECP state has the functional form $\left\{\mathbf{r}(\mathbf{x})=\mathbf{0}, z(\mathbf{x})=A_{0} e^{i \mathbf{k x}}\right\}$. 

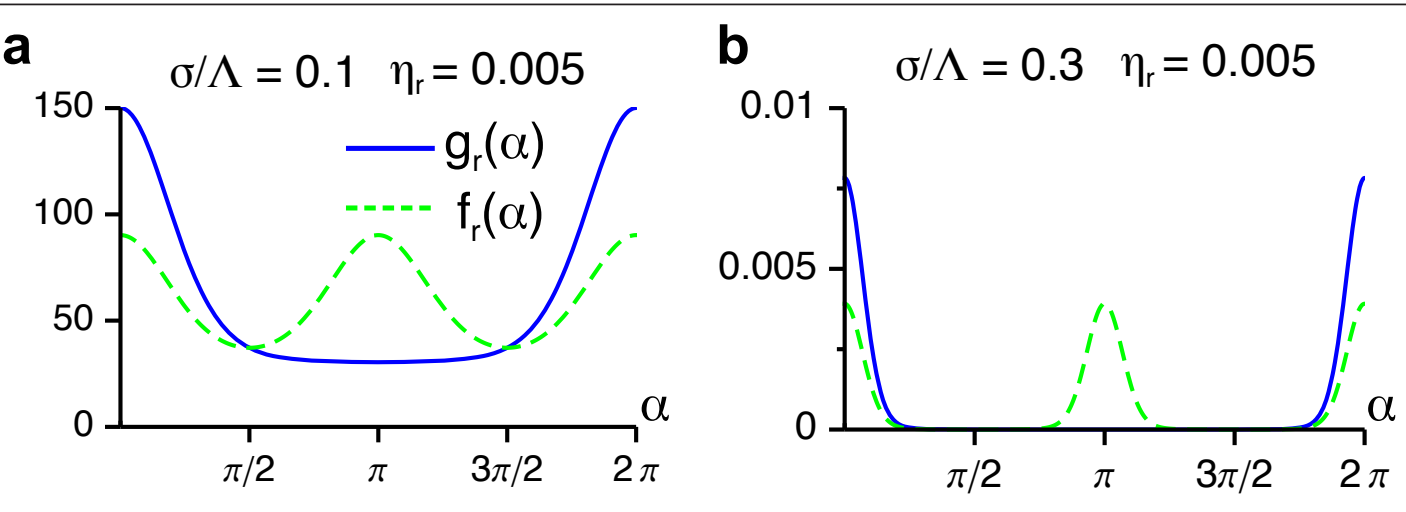

d
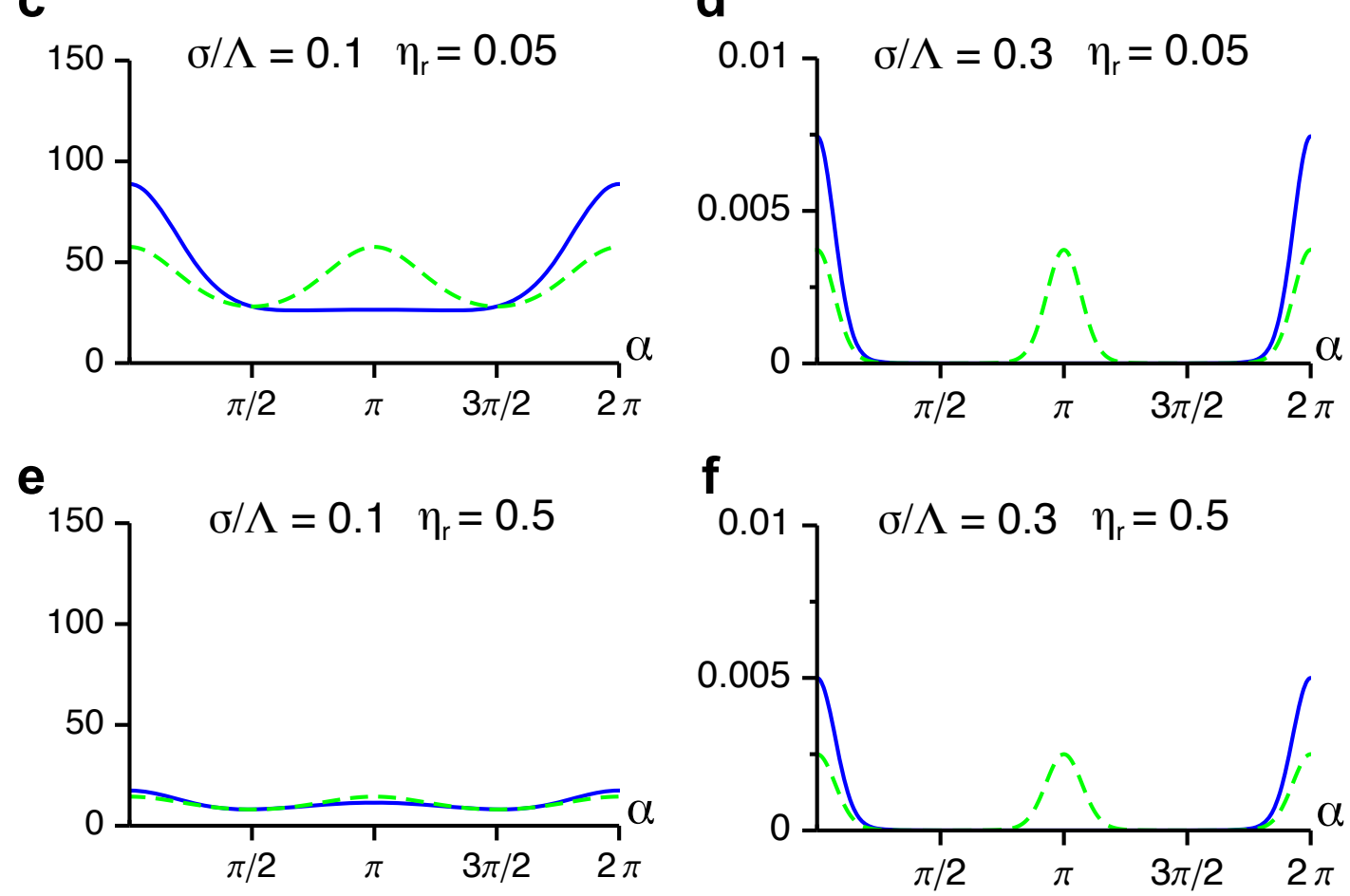

Figure 8 Angle-dependent interaction functions for the coupling between OPM and RM in the EN model. $(\mathbf{a}, \mathbf{b}) g_{r}(\alpha)$ and $f_{r}(\alpha)$ for $\eta_{r}=$ 0.005 and $\sigma / \Lambda=0.3(\mathbf{a})$ and $0.1(\mathbf{b})$. (c, d) $g_{r}(\alpha)$ and $f_{r}(\alpha)$ for $\eta_{r}=0.05$ and $\sigma / \Lambda=0.3$ (c) and 0.1 (d). (e, f) $g_{r}(\alpha)$ and $f_{r}(\alpha)$ for $\eta_{r}=0.5$ and $\sigma / \Lambda=$ $0.3(\mathbf{e})$ and 0.1 (f).

This means that the OS solution does not induce any deviations from the perfect retinotopy as shown previously from symmetry. This is not the case for the square pinwheel crystal (sPWC)

$$
z_{\mathrm{sPWC}}(\mathrm{x}) \propto \sin \left(k_{c} x_{1}\right)+i \sin \left(k_{c} x_{2}\right),
$$

the second important solution for undistorted retinotopy. Inserting this ansatz into Equation (31) and neglecting terms of order $\mathcal{O}\left(\left(e^{-k_{c}^{2} \sigma^{2}}\right)^{2}\right)$ or higher, we obtain

$$
\mathbf{r}_{s \mathrm{PWC}}(\mathbf{x}) \propto \frac{e^{-k_{c}^{2} \sigma^{2}}}{\sigma^{2} \lambda_{2}^{r}\left(2 k_{c}\right)}\left(\begin{array}{l}
k_{c} \sin \left(2 k_{c} x_{1}\right) \\
k_{c} \sin \left(2 k_{c} x_{2}\right)
\end{array}\right) .
$$

These retinotopic distortions are a superposition of one longitudinal mode in $x$-direction and one in $y$ direction, both with doubled wave number $\sim 2 k_{c}$. The doubled wave number implies that the form of retinotopic distortions is independent of the topological charge of the pinwheels. Importantly, the gradient of the retinotopic mapping $\mathbf{R}(\mathbf{x})=\mathbf{X}+\mathbf{r}_{\mathrm{sPWC}}(\mathbf{x})$ is reduced at all pinwheel locations. The coupled sPWC 
is therefore in two ways a high coverage mapping as expected. First, the representations of cardinal and oblique stimuli (real and imaginary part of $z(\mathbf{x})$ ) are orthogonal to each other. Second, the regions of highest gradient in the orientation map correspond to low gradient regions in the RM.

In Figure 9, the family of coupled $n$-ECPs is displayed, showing simultaneously the distortions of the RM and the OPM. Retinotopic distortions are generally weaker for anisotropic $n$-ECPs and stronger for isotropic $n$ ECPs. However, for all stationary solutions the regions of high gradient in the orientation map coincide with low gradient regions (the folds of the grid) in the RM. This is precisely what is generally expected from a dimension-reducing mapping $[21,62,63,91]$. In the following section, we will investigate which of these solutions become optimal depending on the two parameters $\sigma / \Lambda$ and $\eta_{r}$ that parameterize the model.

\section{The impact of retinotopic distortions}

According to our analysis, at criticality, the nontrivial stable fixed points of the EN dynamics are determined by the continuity parameter $\eta \in(0,1)$ for the OPM or, equivalently, the ratio $\sigma / \Lambda=\frac{1}{2 \pi} \sqrt{\log (1 / \eta)}$ and the continuity parameter $\eta_{r}$ for the mapping of visual space. We first tested for the stability of pinwheel-free orientation stripe (OS) solutions and rPWC solutions of Equation (15), with coupling matrices $g_{i j}$ and $f_{i j}$ as obtained from the nonlinearities in Equation (29). The angle which minimizes the energy $U_{\mathrm{rPWC}}$ (Equation (20)) is not affected by the coupling between retinotopic and OPM and is thus again $\alpha=\pi / 4$. By numerical evaluation of the criteria for intrinsic and extrinsic stability, we found both, OSs and sPWCs, to be intrinsically and extrinsically stable for all $\sigma / \Lambda$ and $\eta_{r}$.

Next, we tested for the stability of coupled $n$-ECP solutions for $2 \leq n \leq 20$. We found all coupled $n$-ECP configurations with $n \geq 2$ to be intrinsically unstable for all $\sigma / \Lambda$ and $\eta_{r}$. Evaluating the energy assigned to sPWCs and OSs, we identified two different regimes: (i) for shorter interaction range $\sigma / \Lambda$ the sPWC is the minimal energy state and (ii) for larger interaction range $\sigma /$ $\Lambda$ the optimum is an OS pattern as indicated by the phase diagram in Figure 10a. The retinotopic continuity parameter has little influence on the energy of the two fixed points. The phase border separating stripes from rhombs runs almost parallel to the $\eta_{r}$-axis. We numerically confirmed these analytical predictions by extensive simulations of Equation $(3,4)$ (see 'Methods' section for details). Figure 10c shows snapshots of a representative simulation with small interaction range $(r=0.1, \sigma / \Lambda=$ $\left.0.1(\eta=0.67), \eta_{r}=\eta\right)$. After the initial symmetry breaking phase, the OPM layout rapidly converges toward a crystalline array of pinwheels, the predicted optimum in this parameter regime (Figure 10c). Retinotopic deviations are barely visible. Figure 10b displays pinwheel density time courses for four such simulations. Note that in one simulation, the pinwheel density drops to almost zero. In this simulation, the OP pattern converges to a stripe-like layout. This is in line with the finding of bistability of rhombs and stripes in all parameter regimes. Although the SPWC represents the global minimum in the simulated parameter regime, OSs are also a stable fixed point and, depending on the initial conditions, may arise as the final state of a fraction of the simulations. In the two simulations with pinwheel densities around 3.4, patterns at later simulation stages consist of different domains of rhombic pinwheel lattices with $\alpha<\pi / 2$.

Figure 10d,e shows the corresponding analysis with parameters for larger interaction range $r=0.1, \sigma / \Lambda=$ $0.15(\eta=0.41), \eta_{r}=\eta$. Here after initial pinwheel creation, pinwheels typically annihilate pairwisely and the OPM converges to an essentially pinwheel-free stripe pattern, the predicted optimal solution in this parameter regime (Figure 10e). Retinotopic deviations are slightly larger. The behavior of the EN model for the joint optimization of RM and OPM thus appears very similar compared to the fixed retinotopy case. Perhaps surprisingly, the coupling of both feature maps has little effect on the stability properties of the fixed points and the resulting optimal solutions.

As in the previous case, the structure of the phase diagram in Figure 10a appears somewhat counterintuitive. A high coverage and pinwheel-rich solution is the optimum in a regime with large OPM continuity parameter where discontinuities in the OPM such as pinwheels should be strongly penalized. A pinwheel-free solution with low coverage and high continuity is the optimum in a regime with small continuity parameter. As explained above, a large OPM continuity parameter at pattern formation threshold implies a small interaction range $\sigma / \Lambda$ (see Equation (24)). In such a regime, the gain in coverage by representing many orientation stimuli in a small area spanning the typical interaction range, e.g., with a pinwheel, is very high. Apparently this gain in coverage by a regular positioning of pinwheels outweighs the accompanied loss in continuity for very large OPM continuity parameters. This counterintuitive interplay between coverage and continuity thus seems to be almost independent of the choice of retinotopic continuity parameters.

The circular orientation stimulus ensemble contains only stimuli with a fixed and finite 'orientation energy' or elongation $\left|s_{z}\right|$. This raises the question of whether the simple nature of the circular stimulus ensemble might restrain the dynamics of the EN model. The EN 


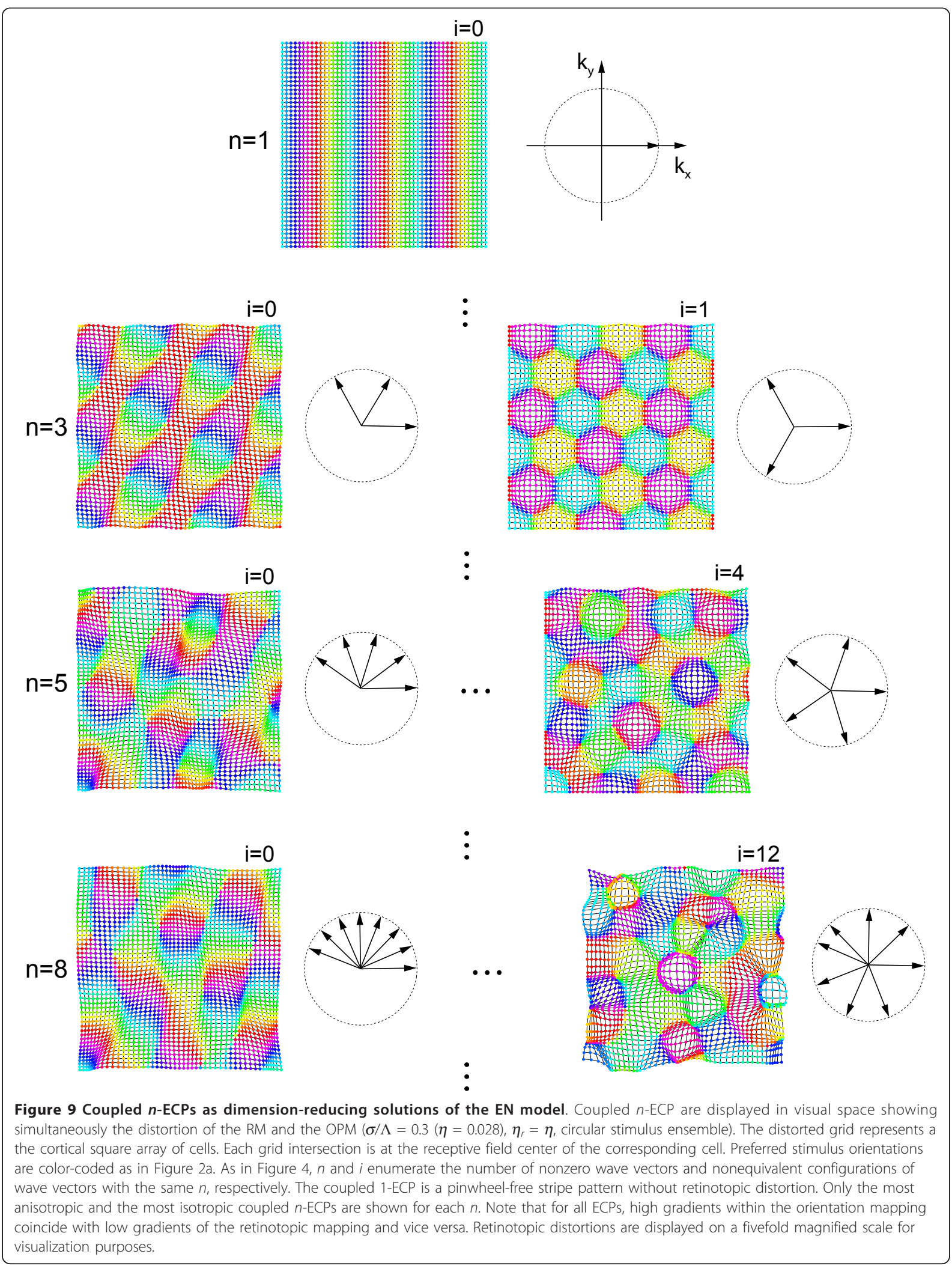




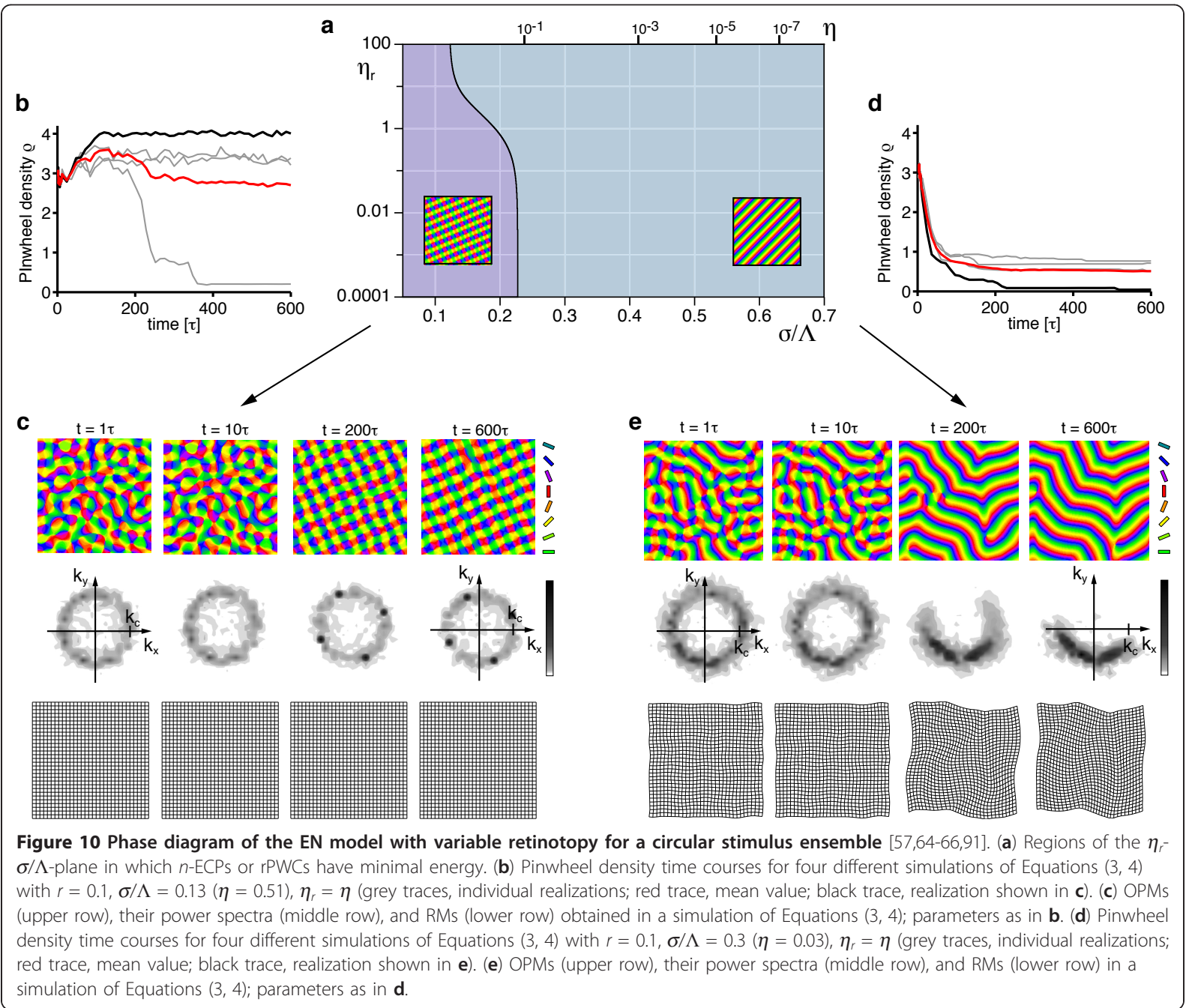

dynamics are expected to depend on the characteristics of the activity patterns evoked by the stimuli and these will be more diverse and complex with ensembles containing a greater diversity of stimuli. Therefore, we repeated the above analysis of the EN model for a richer stimulus ensemble where orientation stimuli are uniformly distributed on the disk $\left\{s_{z},\left|s_{z}\right| \leq 2\right\}$, a choice adopted by a subset of previous studies, e.g., [19,25,81]. In particular, this ensemble contains unoriented stimuli with $\left|s_{z}\right|=0$. Intuitively, the presence of these unoriented stimuli might be expected to change the role of pinwheels in the optimal OPM layout. Pinwheels' population activity is untuned for orientation. Pinwheel centers may therefore acquire a key role for the representation of unoriented stimuli. Nevertheless, we found the behavior of the EN model when considered with this richer stimulus ensemble to be virtually indistinguishable from the circular stimulus ensemble. Details of the derivations, phase diagrams and numerically obtained solutions are given in Appendix 1.

\section{Are there stimulus ensembles for which realistic,} aperiodic maps are optimal?

So far, we have presented a comprehensive analysis of optimal dimension-reducing mappings of the EN model for two widely used orientation stimulus distributions (previous sections and Appendix 1). In both cases, optima were either regular crystalline pinwheel lattices or pinwheel-free orientation stripes. These results might indicate that the EN model for the joint optimization of OPM and RM is per se incapable of reproducing the structure of OPMs as found in the visual cortex. Drawing such a conclusion is suggested in view of the apparent insensitivity of the model's optima to the choice of stimulus ensemble. The two stimulus ensembles considered so far however do not exhaust the infinite space of 
stimulus distributions that are admissible in principle. From the viewpoint of 'biological plausibility' it is certainly not obvious that one should strive to examine stimulus distributions very different from these, as long as the guiding hypothesis is that the functional architecture of the primary visual cortex optimizes the joint representation of the classical elementary stimulus features. If, however, stimulus ensembles were to exist, for which optimal EN mappings truly resemble the biological architecture, their characteristics may reveal essential ingredients of alternative optimization models for visual cortical architecture.

Adopting this perspective raises the technical question of whether an unbiased search of the infinite space of stimulus ensembles only constrained by the model's symmetries (Equations (5) to (9)) is possible. To answer this question, we examined whether the amplitude equations (15) can be obtained for an arbitrary orientation stimulus distribution. Fortunately, we found that the coefficients of the amplitude equations are completely determined by the finite set of moments of order less than 5 of the distributions. The approach developed so far can thus be used to comprehensively examine the nature of EN optima resulting for any stimulus distribution with finite fourth-order moment. While such a study does not completely exhaust the infinite space of all eligible distributions, it appears to only exclude ensembles with really exceptional properties. These are probability distributions with diverging fourth moment, i.e., ensembles that exhibit a heavy tail of essentially 'infinite' orientation energy stimuli.

Since the coupling between OPM and RM did not have a large impact in the case of the two classical stimulus ensembles, we start the search through the space of orientation stimulus ensembles by considering the EN model with fixed retinotopy $\mathbf{r}(\mathbf{x})=\mathbf{0}$. The coefficients $a_{i}$ for the nonlinear operators $N_{i}^{3}[z, z, \bar{z}]$ in Equation (14) for arbitrary stimulus ensembles are given by

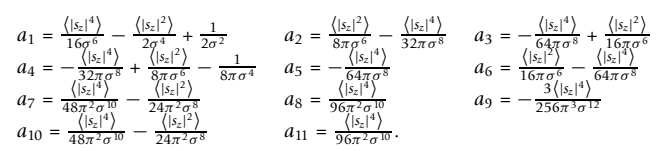

The corresponding angle-dependent interaction functions are given by (see 'Methods' section)

$$
\begin{aligned}
& g(\alpha)=\frac{\left\langle\left|z_{z}\right|^{2}\right\rangle}{2 \sigma^{4}}\left(1-2 e^{-k_{c}^{2} \sigma^{2}}-e^{2 k_{c}^{2} \sigma^{2}(\cos \alpha-1)}\left(1-2 e^{-k_{c}^{2} \sigma^{2} \cos \alpha}\right)\right) \\
& +\frac{1}{2 \sigma^{2}}\left(e^{2 k_{c}^{2} \sigma^{2}(\cos \alpha-1)}-1\right)+\frac{2\left\langle\left|s_{z}\right|^{4}\right\rangle}{\sigma^{6}} e^{-2 k_{c}^{2} \sigma^{2}} \sinh ^{4}\left(1 / 2 k_{c}^{2} \sigma^{2} \cos \alpha\right) \\
& f(\alpha)=\frac{\left.\left.|| s_{z}\right|^{2}\right\rangle}{2 \sigma^{4}}\left(1-e^{-2 k_{c}^{2} \sigma^{2}}\left(\cosh \left(2 k_{c}^{2} \sigma^{2} \cos \alpha\right)+2 \cosh \left(k_{c}^{2} \sigma^{2} \cos \alpha\right)\right)+2 e^{-k_{c}^{2} \sigma^{2}}\right) \\
& +\frac{1}{2 \sigma^{2}}\left(e^{-2 k_{c}^{2} \sigma^{2}} \cosh \left(2 k_{c}^{2} \sigma^{2} \cos \alpha\right)-1\right)+\frac{\left.\left.|| s_{z}\right|^{4}\right\rangle}{\sigma^{6}} e^{-2 k_{c}^{2} \sigma^{2}} \sinh ^{4}\left(1 / 2 k_{c}^{2} \sigma^{2} \cos \alpha\right)
\end{aligned}
$$

Again, without loss of generality, we set $\left\langle\left|s_{z}\right|^{2}\right\rangle=2$. At criticality, both functions are parameterized by the continuity parameter $\eta \in(0,1)$ for the OPM or, equivalently, the interaction range $\sigma / \Lambda=\frac{1}{2 \pi} \sqrt{\log (1 / \eta)}$ and the fourth moment $\left\langle\left|s_{z}\right|^{4}\right\rangle$ of the orientation stimulus ensemble. The fourth moment, is a measure of the peakedness of a stimulus distribution. High values generally indicate a strongly peaked distribution with a large fraction of nonoriented stimuli $\left(\left|s_{z}\right|^{4} \approx 0\right)$, together with a large fraction of high orientation energy stimuli $\left(\left|s_{z}\right|^{4}\right.$ large).

The dependence of $g(\alpha)$ on the fourth moment of the orientation stimulus distribution and $f(\alpha)$ suggests that different stimulus distributions may indeed lead to different optimal dimension-reducing mappings.

The circular stimulus ensemble possesses the minimal possible fourth moment, with $\left\langle\left|s_{\mathrm{z}}\right|^{4}\right\rangle=\left(\left\langle\left|s_{\mathrm{z}}\right|^{2}\right\rangle\right)^{2}=4$. The fourth moment of the uniform stimulus ensemble is \langle| $\left.\left.s_{\mathrm{z}}\right|^{4}\right\rangle=16 / 3$. The angle-dependent interaction functions for both ensembles (Equation (25), Figure 22 in Appendix 1) are recovered, when inserting these values into Equation (33).

To simplify notation in the following, we define

$$
s_{4}=\left\langle\left|s_{z}\right|^{4}\right\rangle-\left\langle\left|s_{z}\right|^{2}\right\rangle^{2}=\left\langle\left|s_{z}\right|^{4}\right\rangle-4
$$

as the parameter characterizing an orientation stimulus distribution. This parameter ranges from zero for the circular stimulus ensemble to infinity for ensembles with diverging fourth moments. Figure 11 displays the angle-dependent interaction functions for different values of $\sigma / \Lambda$ and $s_{4}$. In all parameter regimes, $g(\alpha)$ and $f(\alpha)$ are larger than zero. The amplitude dynamics are therefore guaranteed to converge to a stable stationary fixed point and the bifurcation from the nonselective fixed point in the EN model is predicted to be supercritical in general.

By evaluating the energy assigned to the rPWC and $n$ ECPs, we investigated the structure of the two-dimensional phase space of the EN model with an arbitrary orientation stimulus distribution. First, it is not difficult to show that the angle $\alpha$ which minimizes the energy $U_{\mathrm{rPWC}}$ (Equation (20)) of an rPWC is $\alpha=\pi / 4$ for all $\sigma /$ $\Lambda$ and $s_{4}$. Hence, a square lattice of pinwheels (sPWC) is in all parameter regimes energetically favored over any other rhombic lattice configuration of pinwheels. Figure 12 displays the phase diagram of the EN model with an arbitrary orientation stimulus distribution. For orientation stimulus distributions with small fourth moments, optimal mappings consist of either parallel pinwheel-free stripes or quadratic pinwheel crystals. These distributions include the circular and the uniform stimulus ensembles with $s_{4}=0$ and $s_{4}=4 / 3$. Above a certain value of the fourth moment around $s_{4}=6, n$ - 


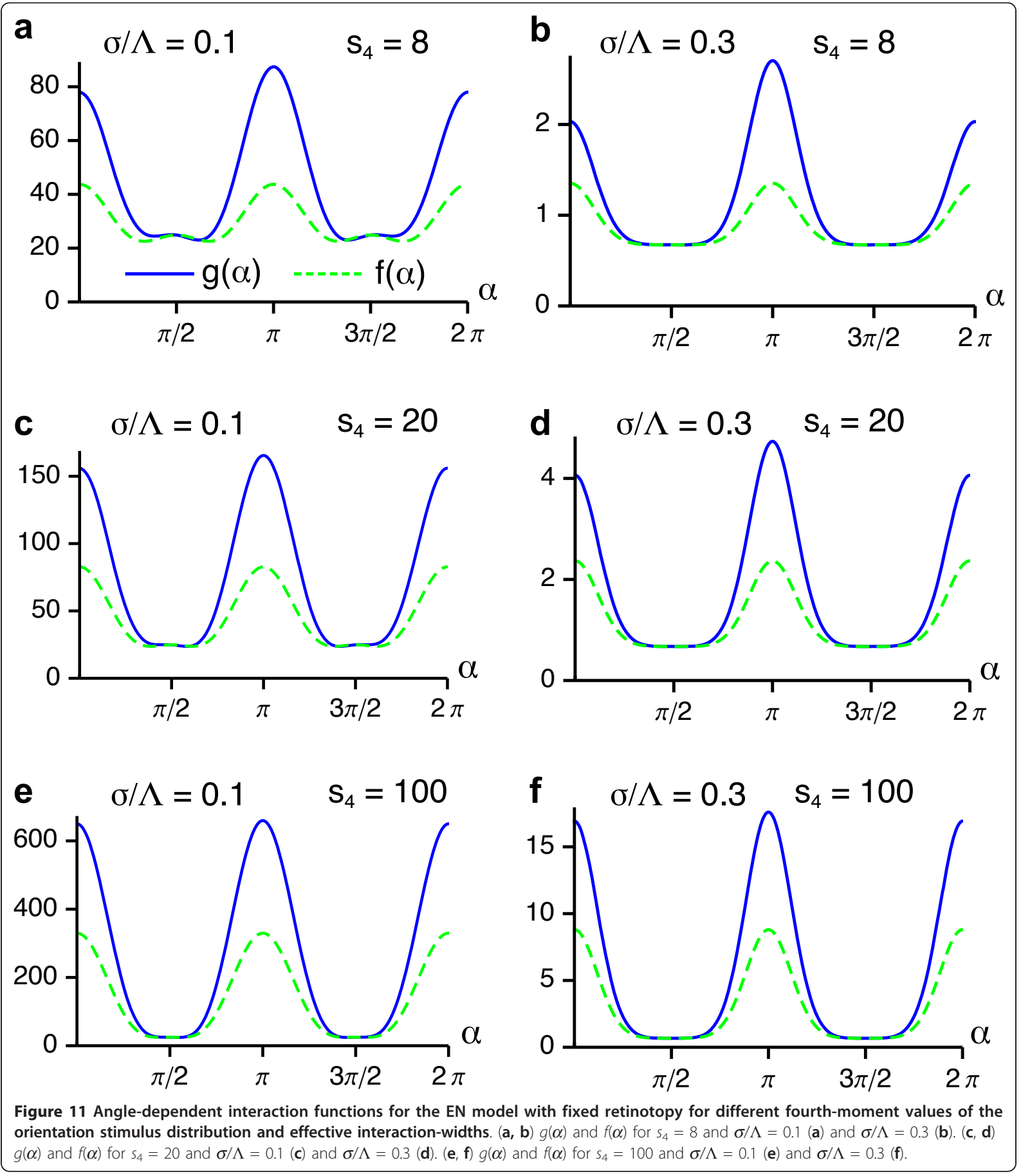

ECPs with $n>2$ become optimal mappings. For a short interaction range $\sigma / \Lambda$, hexagonal pinwheel crystals dominate the phase diagram in a large region of parameter space. With increasing interaction range, we observe a sequence of phase transitions by which higher $n$-ECPs become optimal. For $n>3$, these optima are spatially aperiodic. In all parameter regimes, we found that the $n$-ECP with the most anisotropic mode configuration (Figure 4c, left column) is the energetically favored state for $n>3$. Pinwheel densities of these planforms are indicated in Figure 12 and are typically smaller than 2.0. We note that this is well below experimentally observed 


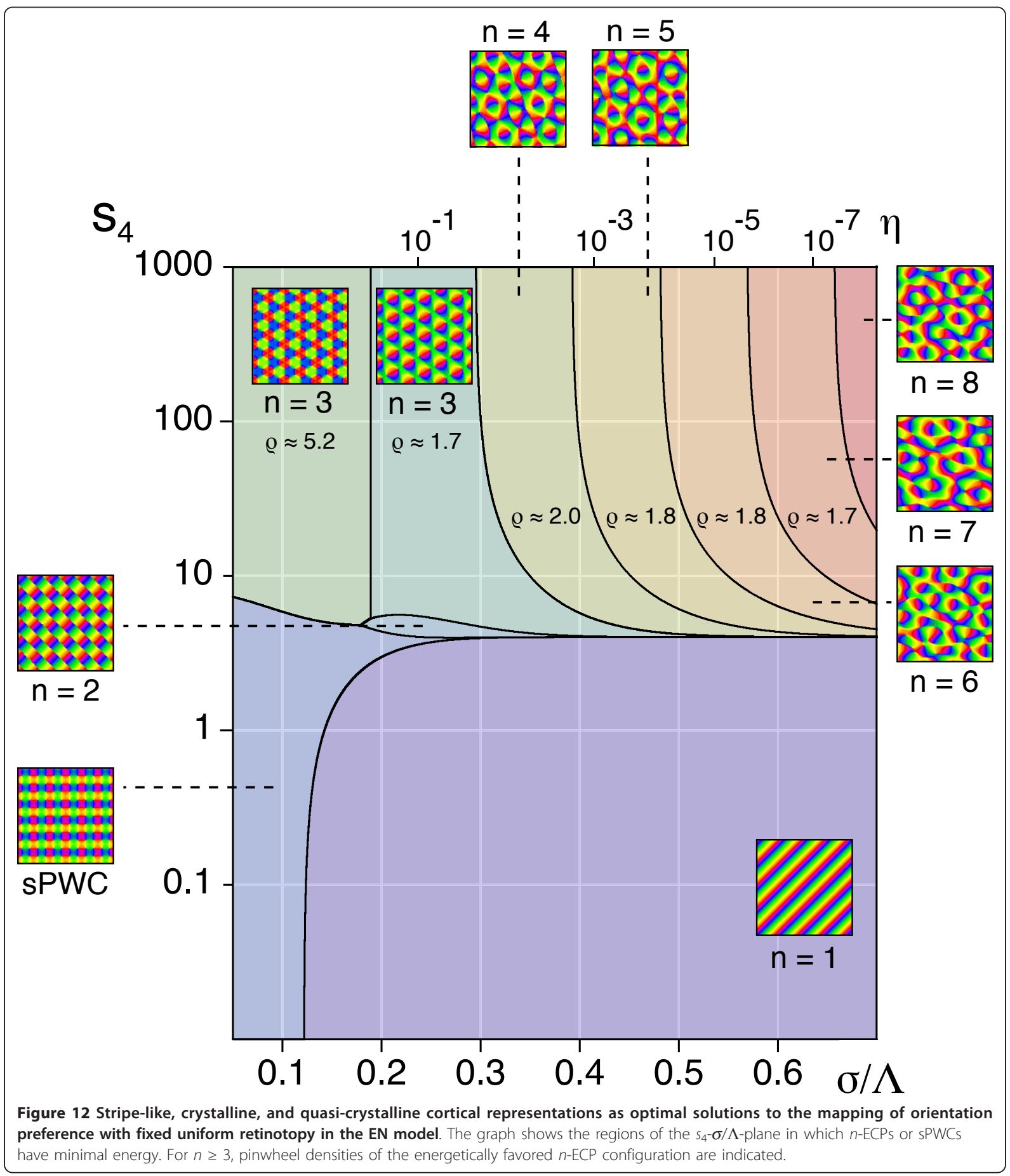

pinwheel density values [38]. Optimal mappings of orientation preference for finite fourth moment in the EN model are thus either orientation stripes, periodic arrays of pinwheels (hexagonal, square), or aperiodic pinwheel arrangements with low pinwheel density.
We numerically tested these analytical predictions by simulations of Equation (3) $(\mathbf{r}(\mathbf{x})=\mathbf{0})$ with two additional stimulus ensembles with $s_{4}=6$ and $s_{4}=8$ (see 'Methods' section). Figure 13a shows snapshots of a simulation with $(r=0.1, \sigma / \Lambda=0.2(\eta=0.2))$ and $s_{4}=6$ 


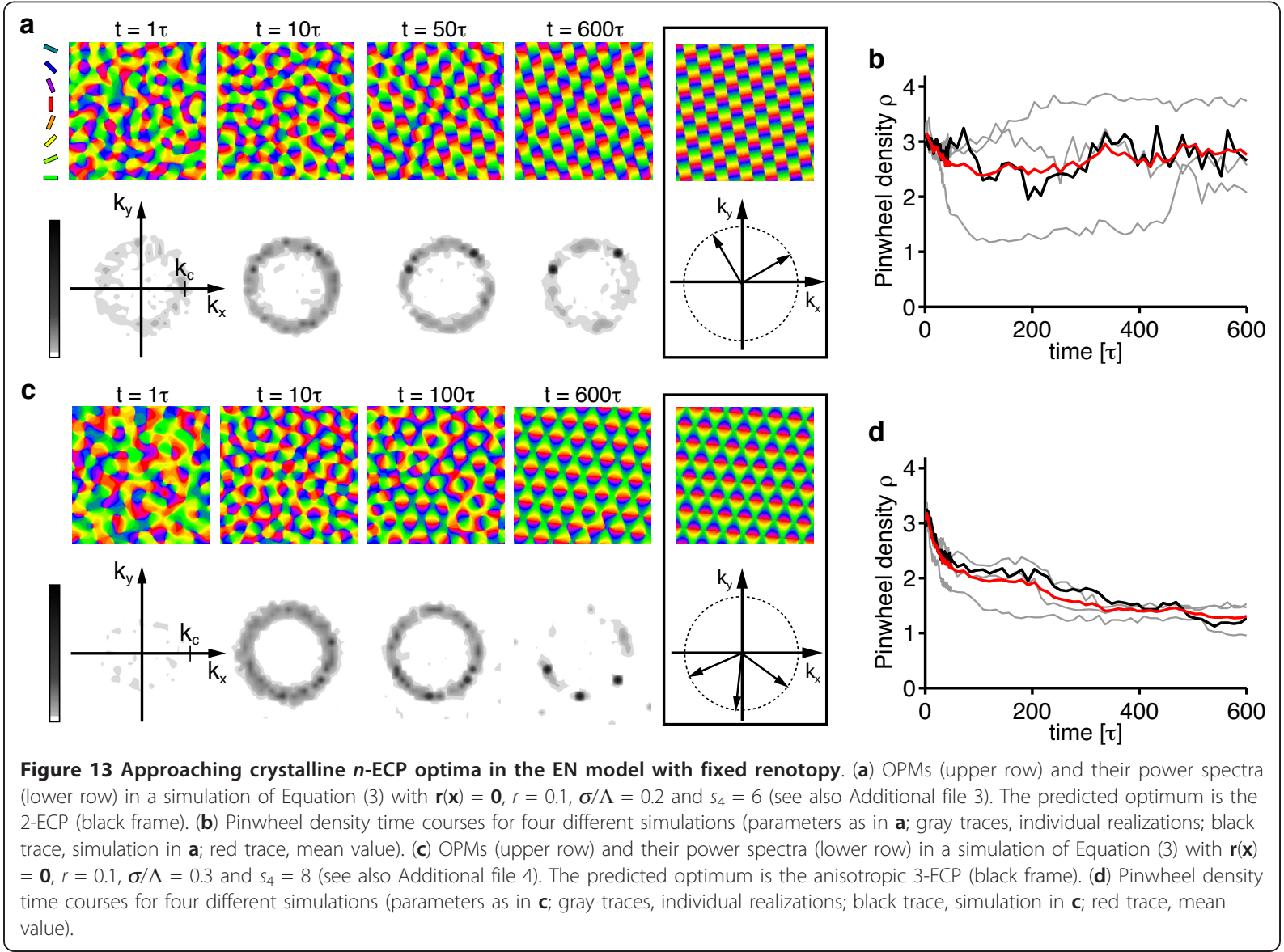

(see also Additional file 3). After the initial phase of pattern emergence, the OPM layout converges toward an arrangement of fractured stripes which resembles the 2ECP state (Figure 13a, most right), the optimum predicted in this regime. In the power spectra, two distinct peaks of the active modes are clearly visible in the final stages of the simulation (Figure 13a, lower row). The 2ECP state is exotic in the sense that it is the only $n$-ECP containing line defects and thus the pinwheel density is not a well-defined quantity. This explains the pronounced numerical variability in the measured pinwheel densities in simulations during the convergence toward a 2-ECP state (Figure 13b).

Figure 13c shows snapshots of a simulation with $(r=$ $0.1, \sigma / \Lambda=0.2(\eta=0.2))$ and $s_{4}=8$, Gaussian stimulus ensemble) (see also Additional file 4). After the initial phase of pattern emergence, the OPM layout converges toward a regular hexagonal arrangement of pinwheels which resembles the anisotropic 3-ECP (Figure 13c, far right), the optimum predicted in this regime. In the power spectra, three distinct peaks forming an angle of 60 degrees are clearly visible in the later stages of the simulation (Figure 13c, lower row). Pinwheel densities in the simulations consistently approach the theoretically predicted value of $2 \cos (\pi / 6) \simeq 1.73$ (Figure 13d).

\section{Permutation symmetric limit}

In the previous section, we uncovered a parameter regime for the EN model in which optimal solutions are spatially aperiodic. This can be viewed as a first step toward realistic optimal solutions. In the identified regime, however, among the family of $n$-ECPs only those with pinwheel densities well below experimentally observed values [38] are energetically favored (see Figure 12). In this respect, the repertoire of aperiodic optima of the EN model differs from previously considered abstract variational models for OPM development $[35,36,38,39]$. In these models, an energetic degeneracy of aperiodic states with low and high pinwheel densities has been found which leads to a pinwheel statistics of the repertoire of optimal solutions that quantitatively reproduces experimental observations $[38,93]$. What is the reason for this difference between the two models? In [35], the energetic degeneracy of aperiodic states with low and high pinwheel densities was derived from a so- 
called permutation symmetry

$$
N_{3}^{z}[u, v, w]=N_{3}^{z}[w, u, v],
$$

of the cubic nonlinearities of the model. It can be easily seen, that the cubic nonlinearities obtained in the third order expansion of the EN model do not exhibit this permutation symmetry (see 'Methods' section). As shown by Reichl [94], the absence of permutation symmetry can lead to a selection of a subrange of pinwheel densities in the repertoire of optima of OPM models. Depending on the degree of permutation symmetry breaking, the family of optima of such models, albeit encompassing aperiodic OPM layouts, may consist of layouts with either unrealistically low or high pinwheel densities. Furthermore, for very strong permutation symmetry breaking, stationary solutions from solution classes other than the $n$-ECPs and rPWCs with low or high pinwheel densities may become optima of models for OPM development. In order to determine a regime in which the EN model optima quantitatively resemble experimentally observed OPM layouts, it is therefore important to quantify the degree of permutation symmetry breaking in the EN model and to examine whether permutation symmetric limits exist. As shown in the 'Methods' section, any cubic nonlinearity $N_{3}^{z}[z, z, \bar{z}]$ that obeys Equation (34) has a corresponding angle-dependent interaction function $g(\alpha)$ which is $\pi$ periodic. Therefore, we examine the degree of permutation symmetry breaking in the EN model by comparing the angle-dependent interaction function $g(\alpha)$ of its third order expansion (see Equation 33 and Figure 11) to the $\pi$-periodic function $g_{p m}(\alpha)=1 / 2(g(\alpha)+g(\alpha+$ $\pi)$ ). This 'permutation-symmetrized' part of the angledependent interaction function of the EN model for general orientation stimulus ensembles reads

$$
\begin{aligned}
& g_{p m}(\alpha)=\frac{2\left\langle\left|s_{z}\right|^{4}\right\rangle}{\sigma^{6}} e^{-2 \mid k_{c}^{2} \sigma^{2}} \sinh ^{4}\left(1 / 2 k_{c}^{2} \sigma^{2} \cos \alpha\right)
\end{aligned}
$$

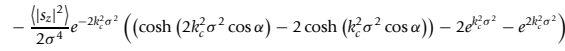

$$
\begin{aligned}
& +\frac{1}{2 \sigma^{2}}\left(1+e^{-2 k k_{c}^{2} \sigma^{2}} \cosh \left(2 k_{c}^{2} \sigma^{2} \cos \alpha\right)\right)
\end{aligned}
$$

A comparison between $g_{p m}(\alpha)$ and $g(\alpha)$ is depicted in Figure 14a-d. It shows that essentially insensitive to the interaction range $\sigma / \Lambda$, at large values of the fourth moment original and permutation symmetrized angledependent interaction functions converge. We quantified the degree of permutation symmetry breaking with the parameter

$$
d=\frac{\left\|g-g_{p m}\right\|_{2}}{\|g\|_{2}} \operatorname{sgn}(g(0)-g(\pi)) .
$$

This parameter is zero in the case of a permutation symmetric cubic nonlinearity. In the case of a g-function completely antisymmetric around $\alpha=\pi / 2$, the parameter is either plus or minus one, depending on whether the maximum of $g_{p m}$ is at zero or $\pi$. If $d$ is smaller than zero, low pinwheel densities are expected to be energetically favored and vice versa. The values of $d$ in parameter space is depicted in Figure 14e. It is smaller than zero in the entire phase space, implying a tendency for low pinwheel density optimal states, in agreement with the phase diagram in Figure 12. Permutation symmetry breaking is largest for $\sigma / \Lambda$ around 0.25 and small fourth moment values of the orientation stimulus distribution. It decays to zero for large fourth

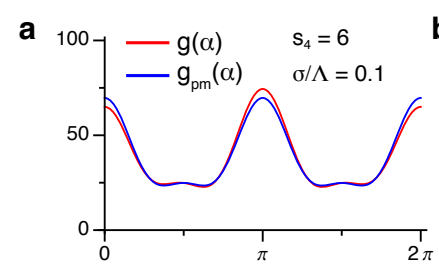

C
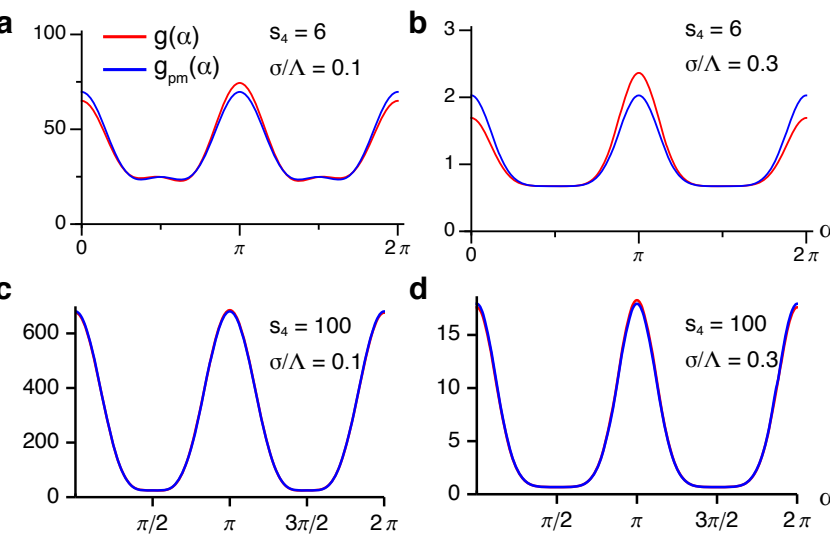

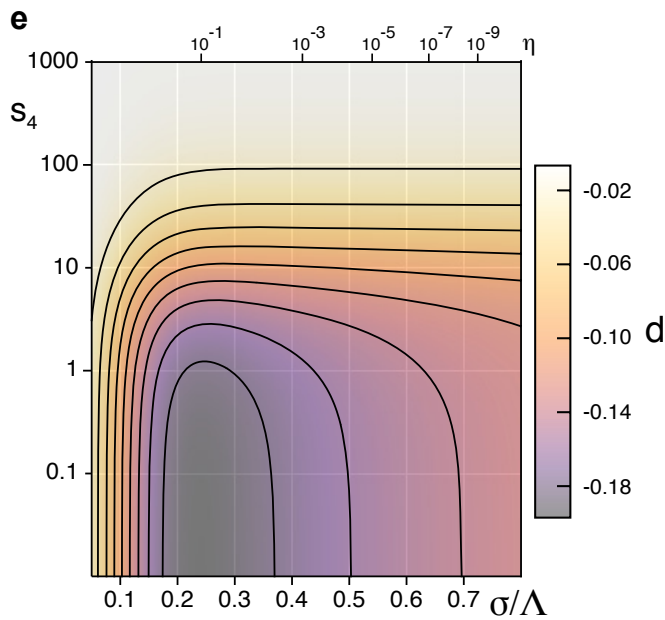

Figure 14 Quantifying permutation symmetry breaking in the EN model. (a-d) $g(\alpha)$ (red traces) and the 'permutation symmetrized' function $g_{p m}(\alpha)=1 / 2(g(\alpha)+g(\alpha+\pi))$ (blue traces, see Equation (35)) for $\sigma / \Lambda=0.1$ and 0.3 and $s_{4}=6$ and 100 . (e) Permutation symmetry parameter $\mathbf{d}$ (Equation 36 ) in the EN model with fixed retinotopy. Permutation symmetry breaking is largest for $\sigma / \Lambda \approx 0.25$ and small $s_{4}$. In the limit $s_{4} \rightarrow \infty$, permutation symmetry is restored. 
moments proportionally to $1 / s_{4}$ as can be seen by inserting Equations (33) and (35) into Equation (36). In the infinite fourth moment limit $s_{4} \rightarrow \infty$, the cubic nonlinearities of the third-order expansion of the EN model become permutation symmetric.

In this case, the EN model is parameterized by only one parameter, the effective intracortical interaction range $\sigma / \Lambda$ and we obtain a rather simple phase diagram (Figure 15). Optimal solutions are $n$-ECPs for increasing $\sigma / \Lambda$ and we observe a sequence of phase transitions toward a higher number of active modes and therefore more complex spatially aperiodic OPM layouts. Importantly, for a subregion in the phase diagram with given number of active modes, all possible $n$-ECP mode configurations are energetically degenerate. It is precisely this degeneracy that has been previously shown to result in a pinwheel statistics of the repertoire of aperiodic optima which quantitatively agrees with experimental observations [38]. Therefore, our unbiased search in fact identified a regime, namely a very large effective interaction range and infinite fourth moment of the orientation stimulus ensemble, in which the EN model formally predicts which quantitatively reproduce the experimentally observed V1 architecture.

Unexpectedly, however, this regime coincides with the limit of applicability of our approach. Permutation symmetry is exactly obtained by approaching stimulus distribution with diverging fourth moment for which the amplitude equations may become meaningless. We would generally expect that the EN for very large but finite fourth moment can closely resemble a permutation symmetric model. However, to consolidate the relevance of this regime, it appears crucial to establish the robustness of the limiting behavior to inclusion of retinotopic distortions.

\section{Optimal solutions of the EN model with variable retinotopy and arbitrary orientation stimulus ensembles} In the EN model for the joint mapping of visual space and orientation preferences, the angle-dependent interaction functions depend on four parameters: $\eta, \sigma$, the fourth moment $\left\langle\left|s_{z}\right|^{4}\right\rangle$ of the stimulus ensemble and $\eta_{r}$. By setting $\sigma=\sigma^{*}(\eta)$, we are left with three free parameters at criticality. Therefore, a three-dimensional phase diagram now completely describes pattern selection in the EN model. For better visualization, in Figure 16, we show representative cross sections through this three-dimensional parameter space for fixed $\eta_{r}$. First, we note the strong similarity between the phase diagram for fixed retinotopy (Figure 12) and the cross sections through the phase diagrams for the joint mappings shown in Figure 16. This expresses the fact that retinotopic mapping and OPM are only weakly coupled or mathematically, $g_{r}(\alpha) \ll g(\alpha)$ in all parameter regimes (see Appendix 2). Again, for distributions with small fourth moment, optimal mappings consist of either pinwheel-free orientation stripes or sPWCs. Above a certain fourth moment value around $s_{4}=6$, higher coupled $n$-ECPs are optimal. For small interaction range $\sigma / \Lambda$, hexagonal pinwheel crystals (coupled 3-ECPs) represent optimal mappings in a large fraction of parameter space. With increasing $\sigma / \Lambda$, we observe a sequence of phase transitions by which higher $n$-ECPs become optimal. Anisotropic planforms at the lower end of the spectrum

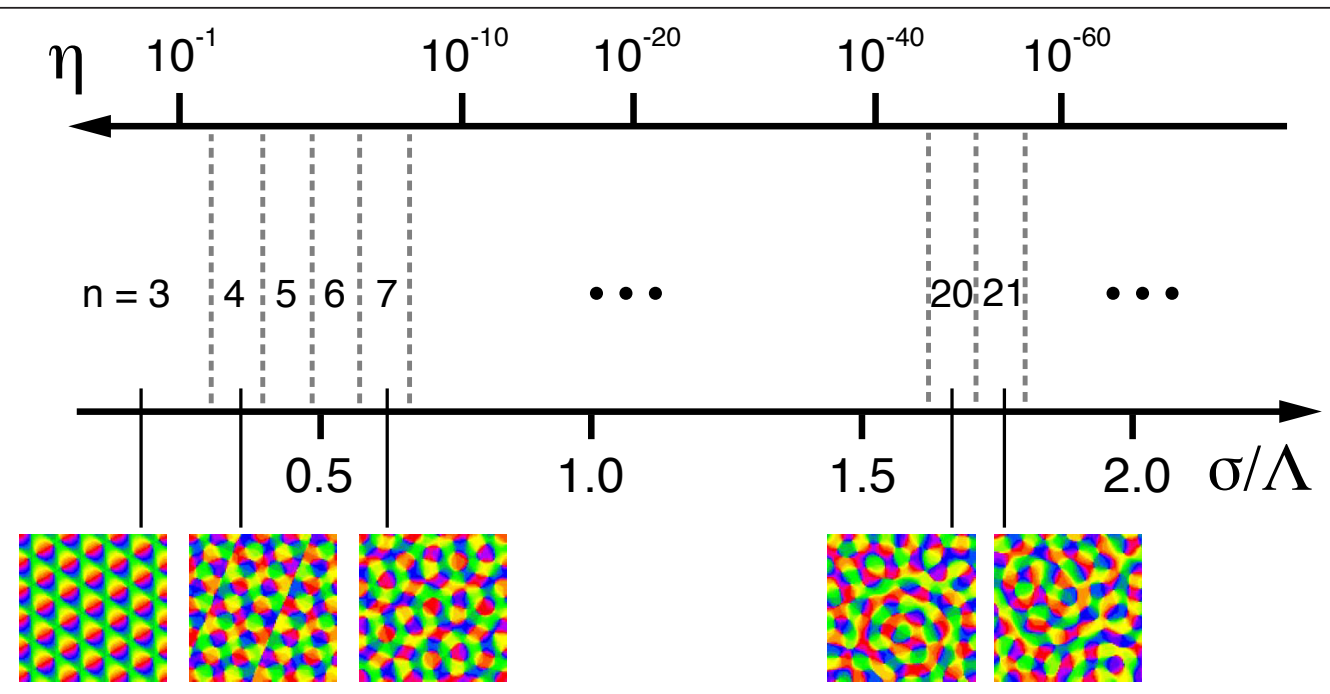

Figure 15 Phase diagram of the EN model with fixed retinotopy in the permutation symmetric limit $s_{4} \rightarrow \infty$. The graphs show the regions on the $\sigma / \Lambda$-axis (lower axis) and the corresponding $\eta$-axis (upper axis), where $n$-ECPs or sPWCs have minimal energy. High $n$-ECPs ( $n \geq$ 10) exhibit universal pinwheel statistics. Note however the extremely small $\eta$-values for large $\sigma / \Lambda$. 
of pinwheel densities are always energetically favored over high pinwheel density layouts. The only difference between the cross sections is that the region covered by sPWCs increases for decreasing $\eta_{r}$. The phase diagram for large $\eta_{r}=10$ is virtually indistinguishable from the phase diagram in Figure 16.

Optimal mappings of orientation preference are thus either orientation stripes, periodic arrays of pinwheels (hexagonal, quadratic) or quasi-periodic pinwheel arrays with low pinwheel density. Retinotopic distortions lead to lower gradients of the retinotopic mapping at high gradient regions of the OPM. This is in line with some of the experimental evidence $[55,95]$ but contradicts others [96].

Most importantly, we note that the results on permutation symmetry breaking in the fixed retinotopy case are not altered by allowing for retinotopic distortions. Since $g_{r}(\alpha)$ does not depend on the fourth moment of the orientation stimulus distribution, non-permutation symmetric terms decay as $1 / s_{4}$ for large $s_{4}$.

Hence, in the limit $s_{4} \rightarrow \infty$, permutation symmetry is restored and we recover the phase diagram in Figure 15 also for the EN model with variable retinotopy independent of $\eta_{r}$. As the energy contribution of retinotopic deviations $\mathbf{r}(\mathbf{x})$ becomes negligible in the infinite fourth moment limit, the optima are then simply the corresponding coupled $n$-ECPs and these states are energetically degenerate for fixed $n$. For very large effective interaction range and infinite fourth moment of the orientation stimulus ensemble, the EN model with variable retinotopy is able to quantitatively reproduce the experimentally observed pinwheel statistics in OPMs. It furthermore predicts reduced gradients of the visual space mapping at high gradient regions of the OPM.

Finite stimulus samples and discrete stimulus ensembles Our reexamination of the EN model for the joint optimization of position and orientation selectivity has been so far carried out without addressing the apparently fundamental discrepancy between our results and the large majority of previous reports. Since the seminal publication of Durbin and Mitchison [21], numerous studies have used the EN model to simulate the development of visual cortical maps or to examine the structure of optimal mappings by numerical simulation $[58,62-65,79,97,98]$. These studies have either used the circular or the uniform orientation stimulus ensemble for which, to the best of our knowledge, the only two nontrivial stationary solutions are square pinwheel crystals or orientation stripes. Furthermore, we found that the gradient descent dynamics seems to readily converge to the respective minima of the EN free energy. This indicates that other local minima and more complex intrinsically aperiodic states are not dominant in this model. In fact, we found that all aperiodic stationary solutions we could perturbative calculate analytically are unstable and thus represent hyperbolic saddle points and not local minima. As these stable solutions barely resemble experimentally observed OPMs, it is not obvious how the EN model in all of these studies could appear as a model well suited to describe the complex layout of real cortical orientation maps. Prior studies however often used computational methods different from our fixed parameter steepest descent simulations.

Two alternative approaches have been used predominantly to study dimension reducing mappings for cortical representations. These methods have been applied to both the EN model and the other widely used dimension reduction model, the self-organizing feature map (SOFM), originally introduced by Kohonen [59]. The simplest way to compute mappings from a high dimensional feature space onto the two-dimensional model cortex is by iterating the following procedure for a large number of randomly chosen stimuli (e.g., $[56,57,66-68,99,100])$ : (i) Stimuli are chosen one at a time randomly from the complete feature space. (ii) The activation function for a particular stimulus is computed. In the case of the EN model, this activation function can acquire a rather complex form with multiple peaks (see 'Discussion' section). In the case of an SOFM, this activation function is a $2 \mathrm{D}$-Gaussian. (iii) The preferred features of the cortical grid points are updated according to a discretized version of Equations $(3,4)$ or the corresponding equations for the SOFM model. Typically, this procedure is repeated on the order of $10^{6}$ times. The resulting layout is then assumed to at least approximately solve the dimension reduction problem. In many studies, small stimulus sets have been chosen presumably for computational efficiency and not assuming specifically that the cortex is optimized for a discrete finite set of stimuli. In [21] for instance, a set of 216 stimuli was used, that was likely already at the limit of computing power available at this time.

In a more refined approach, the EN model as well as Kohonen's SOFM model have been trained with a finite set of stimuli (typically with on the order of $10^{3}$ to $10^{4}$ ) and the final layout of the model map has been obtained by deterministic annealing [101], i.e., by gradually reducing the numerical value of $\sigma$ in a numerical minimization procedure for the energy functional $\mathcal{F}$ at each value of $\sigma$ (see e.g., $[21,64,65,79]$ and see 'Methods' section). In such simulations, often nonperiodic boundary conditions were used. One might suspect in particular the second approach to converge to OPM layouts deviating from our results. It is conceivable in principle, that deterministic annealing might track stationary solutions across parameter space that are systematically 


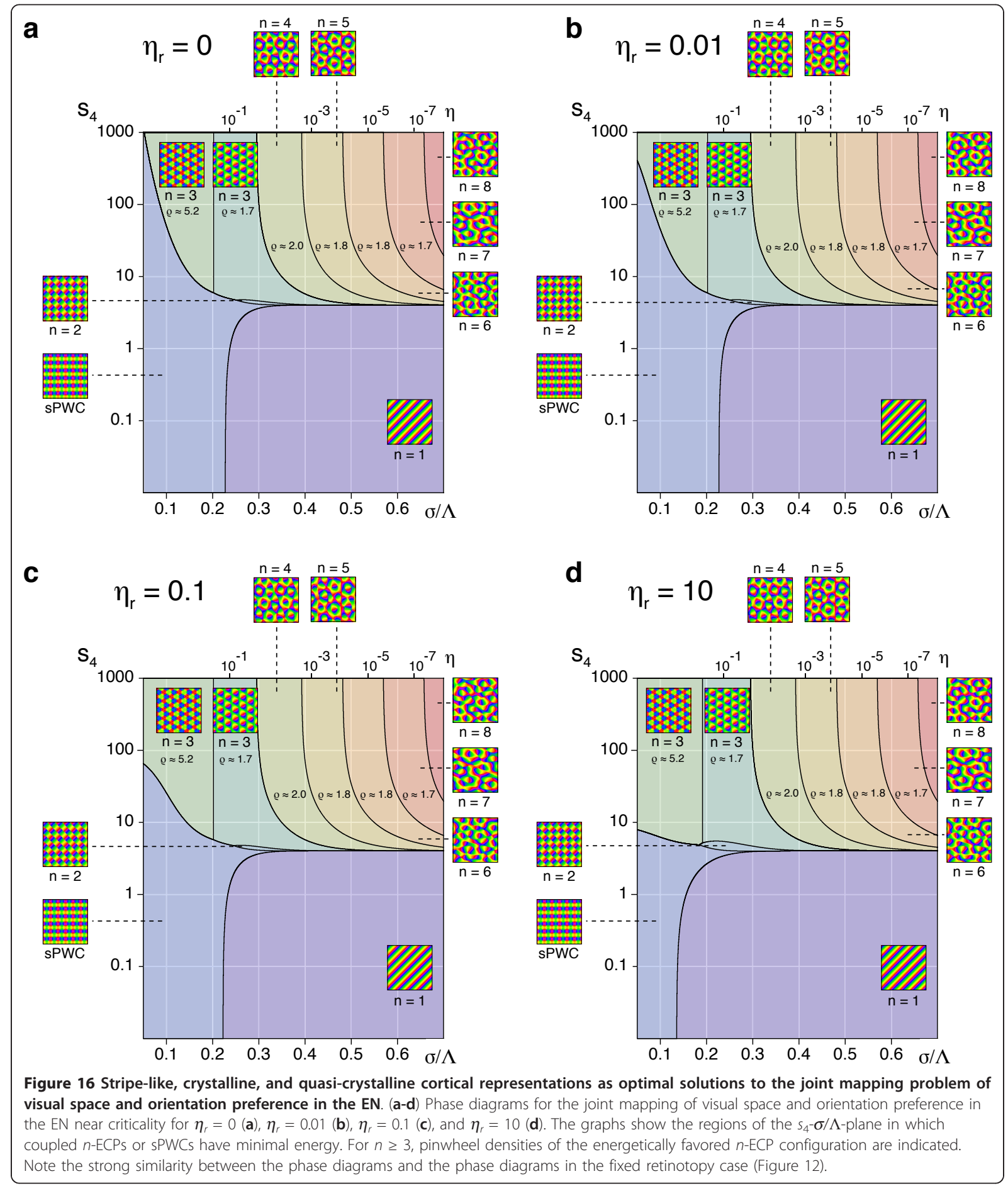

missed by both, our continuum limit analytical calculations as well as our descent numerical simulations.

To assess the potential biases of the different approaches, we implemented (i) finite stimulus sampling in our gradient descent simulations and (ii) studied the results of deterministic annealing simulations varying both the size of the stimulus set as well as the type of boundary conditions applied. 
We simulated Equations $(3,4)$ with finite sets of stimuli of different sizes (see 'Methods' section), drawn from the circular stimulus ensemble. Following [21,25], $\eta$ was set to a small value $(\eta=0.025)$ such that the optimal configuration for the joint mapping of visual space and orientation preference is the coupled 1-ECP (see Figure 10), i.e., a pattern of parallel orientation stripes without any retinotopic distortion (see Figure 9). Figure 17 displays representative simulations for stimulus sets of size $N=216$ (as used in [21]) (a), $N=10^{5}$ (b), $N=10^{6}$ (c) stimuli. Simulation time $t$ is measured in units of the intrinsic time scale $\tau$ (see 'Methods' section). For $N=216$ stimuli, RM and OPM quickly reach an apparently stationary configuration with a large number of pinwheels at around $t=20 \tau$. Power is distributed roughly isotropically around the origin of Fourier space $(\mathbf{k}=0)$. The stable OPM lacks a typical length scale and, expressing the same fact, the power spectrum lacks the characteristic ring of enhanced Fourier amplitude. Retinotopic distortions are fairly pronounced. Both obtained maps resemble the configurations reported in [21].

For $N=10^{5}$ stimuli, we find that OPMs exhibit a characteristic scale (see dark shaded ring in the power spectrum) and a dynamic rearrangement of the maps persists at least until $t=200 \tau$. Stripe-like OP domains are rapidly generated via pairwise pinwheel annihilation for $t>10 \tau$. Retinotopic distortions are fairly weak. For $N$ $=10^{6}$ stimuli, again OPMs exhibit a characteristic scale (see dark shaded ring in the power spectrum) and the map dynamics persists beyond $t=200 \tau$. A larger fraction of the pinwheels annihilate pairwisely compared to

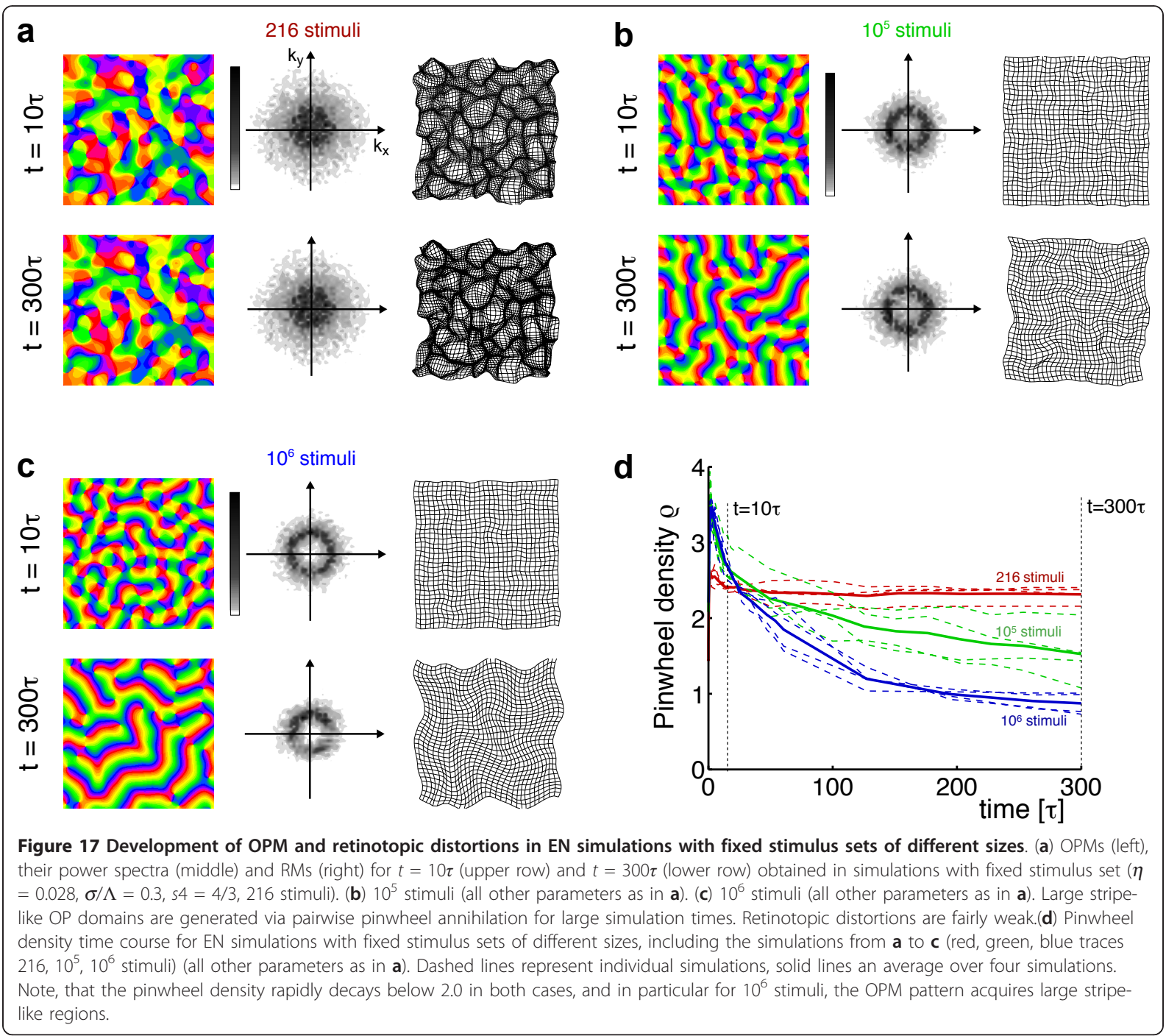


$N=10^{5}$ stimuli, leading progressively to a pattern with large stripe-like domains. Retinotopic distortions are fairly weak. For both cases with massive stimulus sampling $\left(N=10^{5}, N=10^{6}\right)$, the pinwheel density rapidly drops below the range observed in tree shrews, galagos and ferrets and than further decreases during subsequent map rearrangement. In summary, the more stimuli are chosen for the optimization procedure, the less pinwheels are preserved in the pattern of orientation preference and the more the resulting map resembles the analytically obtained optimal solution. Deterministic annealing approaches which change parameters of the energy functional during the computational minimization process differ more fundamentally from our gradient descent simulations than the iterative schemes used with fixed parameters. Studies using deterministic annealing in addition frequently used nonperiodic boundary conditions (e.g. [64,65,79]). To study all potential sources of deviating results, we implemented deterministic annealing for the EN energy function (see 'Methods' section, Equation (46)) for periodic boundaries, nonperiodic boundary conditions as well as random and grid-like finite stimulus ensembles (see 'Methods' section). We closely follow the refined methods used in $[64,65,79]$ and performed deterministic annealing simulations for the EN model with retinotopic distortions and stimuli drawn from the circular stimulus ensemble.

Figures 18a and 19a display representative simulations for random stimulus sets of size $N=10^{3}, N=10^{4}$ and $N=10^{5}$ for periodic boundary conditions (Figures 18a) and nonperiodic boundary conditions (Figures 19a). Furthermore depicted are the pinwheel densities of stationary solutions as well as their energies, relative to the energy of a pinwheel-free stripe solution (see 'Methods' section) for different annealing rates $\xi$ (Figures $18 \mathrm{~b}-\mathrm{d}$ and $19 \mathrm{~b}-\mathrm{d})$. Figures $18 \mathrm{e}-\mathrm{g}$ and $19 \mathrm{e}-\mathrm{g}$ additionally show the statistics of nearest neighbor (NN) pinwheel distances as well as the SD of the pinwheel densities for randomly selected subregions in the OPM as introduced in [38], averaged over four simulations with $N=10^{5}$. To facilitate comparison, we superimposed fits to the experimentally observed statistics [38] for orientation maps in tree shrews, ferrets and galagos.

When annealing with periodic boundary conditions, the maps found with deterministic annealing essentially resemble our gradient descent dynamics simulations. The larger the set of stimuli, the more stripe-like are the OPMs obtained (Figure 18a,b). Furthermore, the more carefully we annealed, the lower the pinwheel density of the obtained layouts (Figure 18c). For $N=$ $10^{5}$, the pinwheel density averaged over four simulations with annealing rate 0.999 was $\rho=2.04$ As expected, the energy of the final layouts decreased with slower annealing rates (Figure 18d). However, when starting from random initial conditions, the energy of the final layouts found was always higher compared to the energy of a pinwheel-free stripe solution (see 'Methods' section for details), which is the predicted optimum for the circular stimulus ensemble. $\mathrm{NN}$-pinwheel distance histograms are concentrated around half the typical column spacing and in particular pinwheel pairs with short distances are lacking completely (Figure 18e,f). For nonperiodic boundary conditions and random stimuli, we found that retinotopic distortions are more pronounced than for periodic boundary conditions. They however decreased with increasing number of stimuli. For large the stimulus numbers, we observed stripe-like orientation preference domains which are interspersed with latticelike pinwheel arrangements (see Figure 19c), lower row, upper left corner of the OPM). For $N=10^{5}$, the pinwheel density averaged over four simulations with annealing rate 0.999 was $\rho=2.71$.

Similarly to the results for periodic boundary conditions, short distance pinwheel pairs occur less frequently than in the experimentally observed maps, indicating an increased regularity in the pinwheel distances compared to real OPMs (Figure 19e,f). This regularity is further indicated by a smaller exponent of the SD compared to the Poisson process (Figure 19g). The perfect stripe-like solution is not the optimum for nonperiodic boundaries. The energy of the map layouts found with very slow annealing rates is slightly lower than the energy of the pinwheel-free OPM layout (Figure 19d). We note that the layout of the OPM at the boundaries does not differ substantially from the layout inside the simulated domain, suggesting that boundary effects affect the entire simulated domain for the relatively small region treated. Finally, we performed simulations with grid-like stimulus patterns as e.g., used in [64,65]. These simulations displayed a strong tendency toward rhombic pinwheel arrangements, i.e., the second stable stationary solution found for the circular stimulus ensemble. We refer to Appendix 3 for further details. In summary, our results for the discrete EN model with deterministic annealing largely agree with the analytical results. Irrespective of the numerical methodology, the emerging map structure for large numbers of stimuli is confined to the states predicted by our analytical treatment of the continuum formulation of the EN. This behavior is expected because the energies underlying the deterministic annealing and the steepest descent simulations are mathematically equivalent (see 'Methods' section). In any kind of deterministic annealing simulation we tested, resulting patterns were patchworks of the two fundamental stable solutions identified by the analytical treatment: pinwheel free stripes and square lattices of 


\section{Periodic boundary conditions}
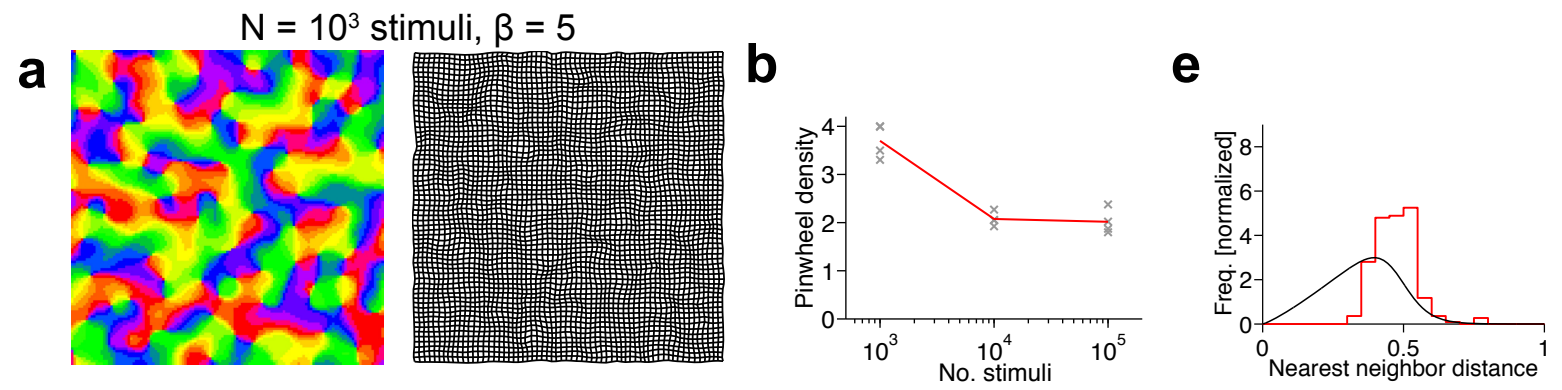

$N=10^{4}$ stimuli, $\beta=50$
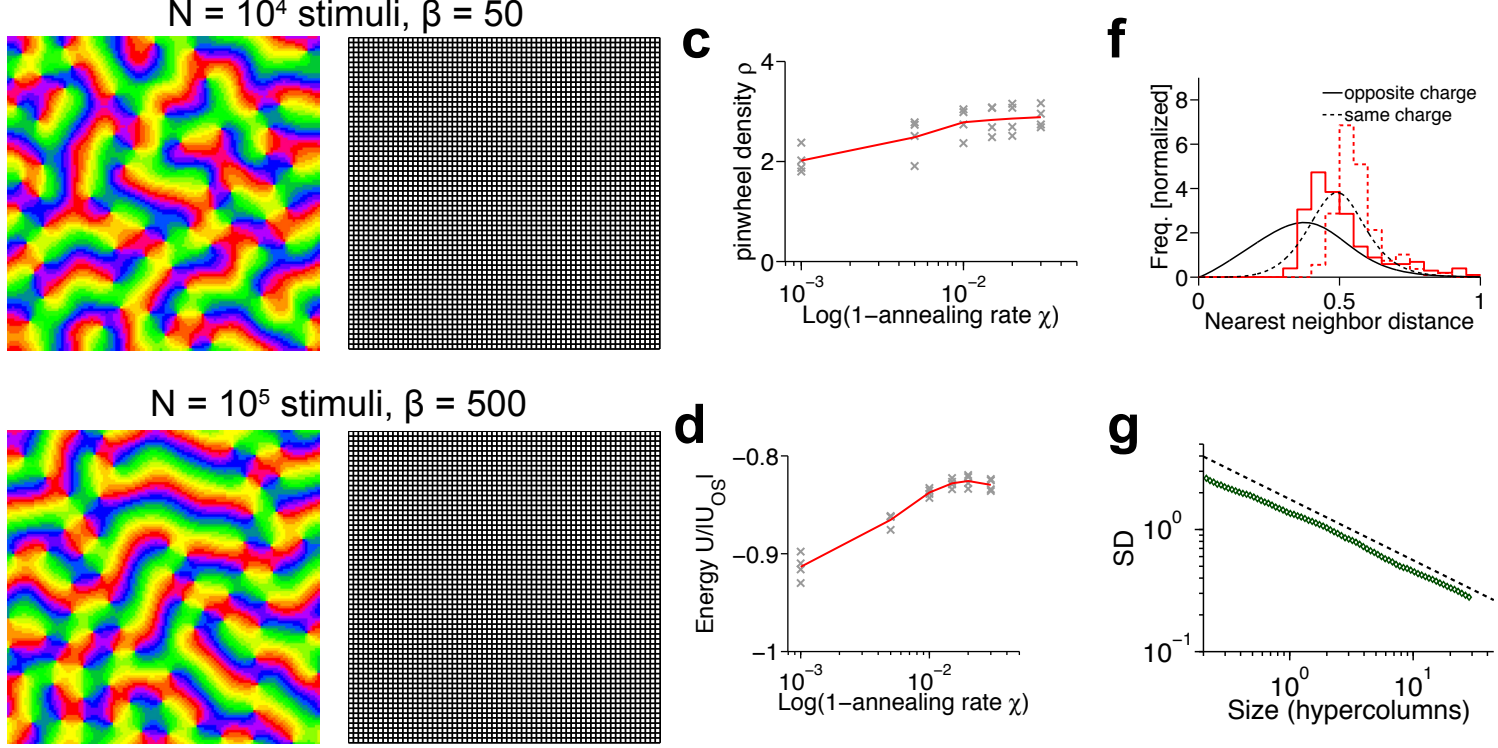

Figure 18 The EN model with periodic boundary conditions, solved with deterministic annealing. (a) OPMs (left) and RMs (right) for $N=$ $10^{3}$ (upper row), $N=10^{4}$ (middle row) and $N=10^{5}$ (lower row) random stimuli and periodic boundary conditions (annealing rate $\chi=0.999$, see 'Methods' section). $\beta$ is the continuity parameter in the conventional definition of the EN model (see 'Methods' section, Equation 46) and is scaled, such that a comparable number of columns is emerging in the simulations for each size of the stimulus set. (b) Pinwheel densities of EN solutions for different numbers of stimuli, $\chi=0.999$. (c) Pinwheel densities of EN solutions for $10^{5}$ stimuli and different annealing rates. (d) Energies of solutions for $10^{5}$ stimuli, relative to the energy of a pinwheel-free stripe solution (see 'Methods' section) for different annealing rates. (b-d) Crosses mark individual simulations, red line indicates average values. (e, f) Statistics of nearest neighbor pinwheel distances for pinwheels of (e) arbitrary and (f) opposite and equal charge for $10^{5}$ random stimuli and periodic boundary conditions, averaged over four simulations (red curves). Black curves represent fits to the experimental data from [38]. (g) Standard deviations (SD) of pinwheel densities estimated from randomly selected regions in the OPM. Black dashed curve indicates SD for a two-dimensional Poisson process of equal density.

pinwheels. Such patchworks are spatially more complicated than perfect stripes or crystals. Nevertheless, they qualitatively differ in numerous respects from the experimentally observed spatial arrangements (see Figures 18, 19, and 28 in Appendix 3). How the fundamental stable solutions are stitched together somewhat differs between the different kinds of simulations. For instance, using a grid-like stimulus ensemble with nonperiodic boundary conditions apparently energetically favors the rPWC compared to the pinwheel-free stripe regions (see Figure 27 in Appendix 3). In summary, while some of the patterns obtained by deterministic annealing might be called 'good-looking' maps, all of them substantially deviate from the characteristics of experimentally observed pinwheel arrangements.

We conclude that the differences between our results and those of previous studies are most likely due to the small finite stimulus samples used largely for reasons of computational tractability. Deterministic annealing using stimulus samples that fill the feature space converges to the same types of patterns found by perturbation theory. We further conclude that our methods do not systematically miss biologically relevant local minima of the classical EN energy function. 


\section{Non-periodic boundary conditions}
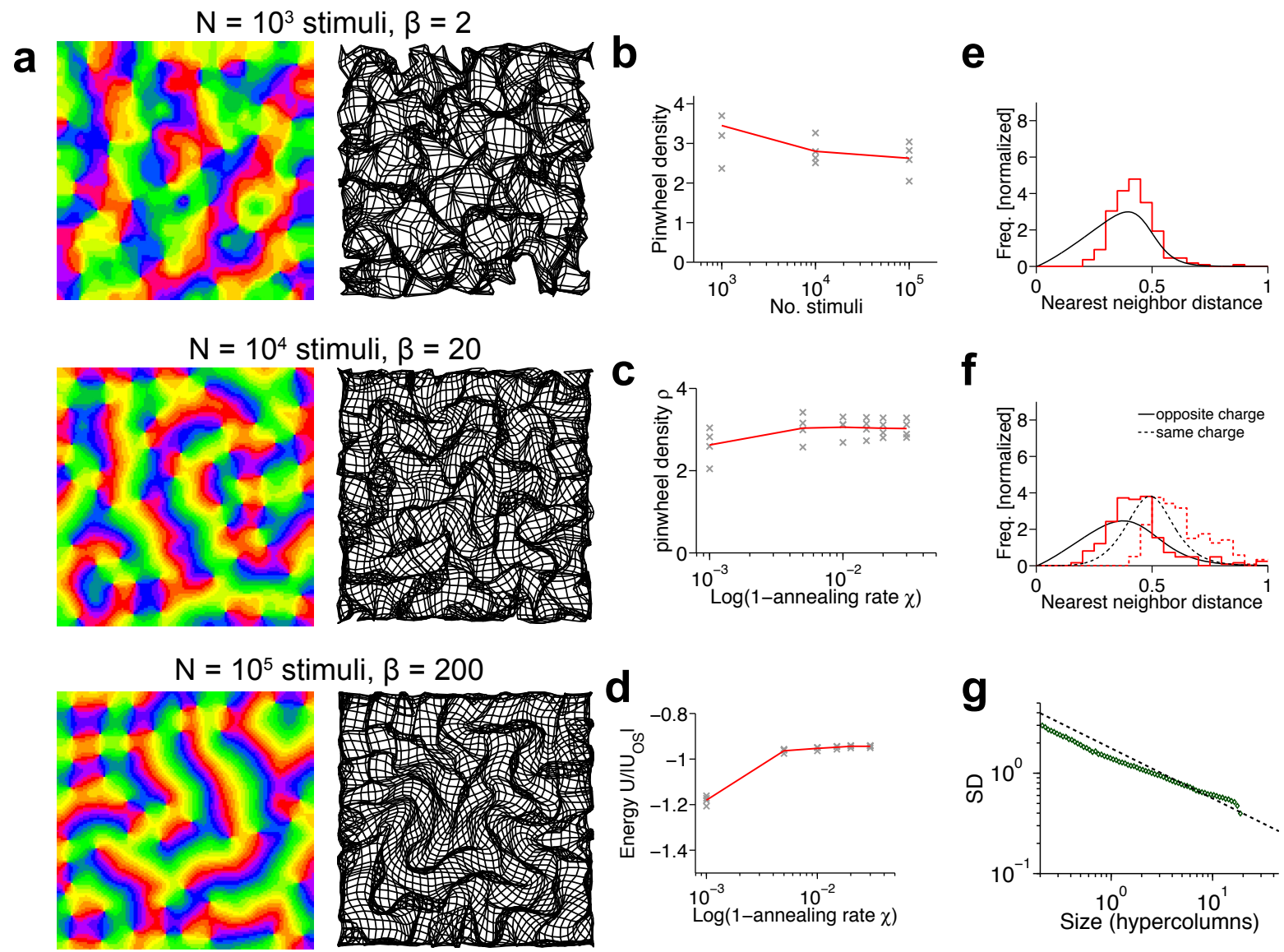

Figure 19 The EN model with nonperiodic boundary conditions, solved with deterministic annealing. (a-g) As Figure 18, but for nonperiodic boundary conditions.

\section{Discussion}

\section{Summary}

In this study, we examined the solutions of what is perhaps the most prominent optimization model for the spatial layout of orientation and RMs in the primary visual cortex, the EN model. We presented an analytical framework that enables us to derive closed-form expressions for hyperbolic fixed points, local and global minima, and to analyze their stability properties for arbitrary optimization models for the spatial layout of OPMs and RMs. Using this framework, we systematically reexamined previously used instantiations of the EN model, dissecting the impact of stimulus ensembles and of interactions between the two maps on optimal map layouts. To our surprise, the analysis yielded virtually identical results for all of these model instantiations that substantially deviate from previous numerical reports. Pinwheel-free orientation stripes and crystalline square lattices of pinwheels are the only optimal dimension-reducing OPM layouts of the EN model. Both states are generally stable but exchange their roles as optima and local minima at a phase border. Numerical simulations of the EN gradient descent dynamics as well as simulations utilizing deterministic annealing confirmed our analytical results. For both processes, the initially spatially irregular layouts rapidly decayed into a patchwork of stripe-like or crystal-like local regions that then became globally more coherent on longer timescales. Pinwheel-free solutions were approached after an initial phase of pattern emergence by pairwise pinwheel annihilation. Crystalline configurations were reached by the generation of additional pinwheels and pinwheel annihilation together with a coordinated rearrangement toward a square lattice. These results indicate that layouts which represent an optimal compromise of coverage and continuity for retinotopy and orientation do not 
per se reproduce the spatially aperiodic and complex structure of orientation maps in the visual cortex.

To clarify whether the EN model is in principle capable of reproducing the biological observations, we performed an unbiased comprehensive inspection of EN optima for arbitrary stimulus distributions possessing finite fourth moments. This analysis identified two key parameters determining pattern selection: (i) the effective intracortical interaction range and (ii) the fourth moment of the orientation stimulus distribution. We derived complete phase diagrams summarizing pattern selection in the EN model for fixed as well as variable retinotopy. Small interaction ranges together with low fourth moment values lead to either pinwheel-free orientation stripes, rhombic or hexagonal crystalline orientation map layouts as optimal states. Large interaction ranges together with orientation stimulus distributions with high fourth moment values lead to the stabilization of irregular aperiodic OPM layouts. These solutions belong to a class of solutions previously called $n$-ECPs. This solution class encompasses a large variety of OPM layouts and has been identified as optimal solutions of abstract variational models of OPM development [35]. We showed that in the EN model due to a lack of a so-called permutation symmetry, among this family of solutions, states with low pinwheel densities are selected as global minima. In the extreme and previously unexplored parameter regime of very large effective interaction ranges and stimulus ensemble distributions with infinite fourth moment, permutation symmetry is restored and spatially aperiodic OPM layouts with higher pinwheel density are included in the repertoire of optimal solutions. Only in this limit, the repertoire of optima reproduces the recently described species-insensitive OPM design [38] and quantitatively matches experimentally observed orientation map layouts. None of these findings depend on whether the EN model is considered with variable or fixed retinotopy.

\section{Comparison to previous studies}

It is an important and long-standing question, whether the structure of cortical maps of variables such as stimulus orientation or receptive field position can be explained by a simple general principle. The concept of dimension reduction is a prominent candidate for such a principle (see e.g., $[58,102]$ for reviews) and the qualitative agreement between experimental data and previous numerical results from dimension reduction models [21,42,60,62-66,68,98,102-104] can be viewed as evidence in favor of the dimension reduction hypothesis. Yet comprehensive analytical investigations of dimension reduction problems and in particular the determination of their optimal and nearly optimal solutions have been impeded by the mathematical complexity of these problems. For the EN algorithm applied to the TSP, previous analytical results established the unselective fixed point above the first bifurcation point as well as the parameters at which this solution becomes unstable [105]. Subsequent work extended these results to the EN model for cortical map formation. The periodicity of solutions depending on the model parameters has been obtained by computing the eigenvalues of the Hessian matrix of the energy function $[63,97,106]$. Hoffsümmer et al. [72] confirmed these results, and computed the periodicity of the emerging patterns in the continuous EN model formulation by linear stability analysis of the EN gradient descent dynamics as used in this study. Our results extend these findings and for the first time provide analytical expressions for the precise layout of optimal and nearly optimal dimension-reducing maps.

In the light of the qualitative agreement between experimental data and numerical solutions of the EN model previously described, it is perhaps our most surprising result that the model's optimal dimension-reducing maps are regular periodic crystalline structures or pinwheel-free stripe patterns in large regions of parameter space. In particular, the species-insensitive pinwheel statistics observed experimentally [38] are not exhibited by optimal solutions of the classical EN in any of the previously considered parameter regimes.

Our comparison of different numerical approaches indicates that the differences to previous studies are mainly attributable to differences in the sampling of the stimulus manifold in the numerical optimization procedures. In their seminal publication, Durbin and Mitchison used sets of 216 stimuli from the circular stimulus ensemble and applied a Gauss-Seidel procedure to obtain stationary configurations [21]. A similar procedure was used in [104]. Quite frequently, the number of stimuli used for optimization is of the same order of magnitude as the number of model neurons or centroids in feature space. This provides a relatively sparse sampling of the stimulus manifold [63-65]. Finite stimulus sampling effects are expected to worsen when feature spaces of higher dimension are considered.

The choice of small stimulus sets in previous dimension reduction studies was imposed mainly by the limitations of computing power. Using a parallelized implementation of the Cholesky method for deterministic annealing [62-65] on a multicore architecture with 2 TB working memory, we explored the dependence of the obtained near optimal solutions on the sampling of the feature space manifold over two orders of magnitude. We find that, the more stimuli are sampled, the closer the numerically obtained configurations resemble our analytical predictions. Our results on the classical EN model with deterministic annealing suggest that in 
the limit of large stimulus numbers, one would perfectly recover our analytical results both for periodic conditions or nonperiodic boundary conditions with realistic system sizes. This dense stimulus sampling limit is also readily visible in our reproduction of the original Durbin and Mitchison sampling and the modification of the predicted map structure with stimulus number (Figure 17). The finding that computational limitations prevented Durbin and Mitchison from obtaining the genuine predictions of their dimension reduction model should not be viewed as diminishing the importance of their contribution. The dimension reduction approach has played a unique and extremely productive role in guiding the conceptualization of cortical functional architecture. It has established an abstract view on cortical representations without which most of our current theoretical knowledge about candidate theories for cortical architectures could not have been obtained.

Our results about optimal states of the EN for the circular and uniform stimulus ensembles however agree with some prior work. In [25], the gradient descent dynamics of the EN model was used as a model for the emergence and refinement of cortical maps during development. Simulated visual stimulus features included retinotopy, orientation and eye dominance. The numerical procedures were similar to the one developed in this study. Parameters were chosen such that $s_{4}=4 / 3$ and $\sigma / \Lambda \approx 0.366$. This study found that an initially large number of pinwheels decayed via pairwise annihilation of pinwheels with opposite topological charge. Our analysis predicts a stripe-like OP pattern as optimal solution in this regime, both in the case of a fixed uniform retinotopy as well as with variable retinotopy. In our simulations, this state is reached after an initial phase of symmetry breaking with the generation of numerous pinwheels via pairwise pinwheel annihilation. Our analytical and numerical results thus confirm, explain, and generalize these previous findings.

The previous results also indicated that the inclusion of eye dominance in the EN model slightly slows down but does not stop the pinwheel annihilation process (see [25], Figure 3). This raises the possibility that the main features of our analysis of optimal solutions for the EN model may persist when additional feature dimensions are taken into account. Reichl et al. in fact observed that models with interacting OPM and OD maps (ODMs) exhibit a transition from pinwheel-free stripes to periodic pinwheel crystals similar to the transitions found in the EN [37] and demonstrated that this transition is a general feature of models with interacting OPM and ODMs [107]. A rigorous characterization of map structures predicted by the simultaneous optimization of multiple periodic feature representations such as orientation preference and OD constitutes an important goal for future studies. The recent study by Reichl et al. [37] suggests that this issue can successfully be approached using concepts from the nonlinear dynamics of pattern formation. Finally, one recent study used the continuous formulation of the EN model to investigate the impact of postnatal cortical growth on the formation of OD columns in cat visual cortex [69]. Consistent with our results, this study also observed perfectly regular stripelike patterns as stationary states in gradient descent simulations. The dynamics of the convergence of the ODMs toward the stripes was modified by including cortical growth into the model. However, as soon as growth terminated, simulated ODM layouts readily converged toward regular stripes. How cortical growth interacts with the formation of orientation columns is currently not understood and represents a further interesting topic for future studies.

\section{Geometric relationship between retinotopic distortions and OPMs}

Experimental results on the geometric relationships between the map of visual space and the map of orientation preference are ambiguous. Optical imaging experiments in cat V1 suggested a systematic covariation of inhomogeneities in the RM with singularities in the pattern of orientation columns in optical imaging experiments [96]. Regions of high gradient in the map of visual space preferentially appeared to overlap with regions of high gradient of the OPM. In ferret, however, it has been reported that high gradient regions of the map of visual space correspond to regions of low gradient in the OPM [67]. In tree shrew V1, no local relationships between the mapping of stimulus orientation and position seem to exist and the map of visual space appears to be ordered up to very fine scales [108]. In line with this, single unit recordings in cat area 17 revealed no correlation between receptive-field position scatter and orientation scatter across local cell ensembles $[109,110]$.

Our analysis of the EN model shows that its optimal states exhibit a negative correlation between the rates of change of orientation preference and retinotopic position, similar to what has been observed in the ferret [67]. This is expected from the principle of dimension reduction and in agreement with the original numerical results by Durbin and Mitchison [21]. However, both in simulations of the gradient descent dynamics and in deterministic annealing simulations with periodic boundary conditions as well as in analytically obtained optimal solutions, deviations from a perfectly uniform mapping of visual space are surprisingly weak (see Figures 9, 10, 18, and 25 in Appendix 1).

Deterministic annealing simulations with open nonperiodic boundary conditions showed a substantially 
increased magnitude of retinotopic distortions. This raises the possibility that different behaviors observed in different experiments might be at least partially related to the influence of boundary effects. The influence of boundary effects is expected to decline into the interior of an area, in particular for large areas as V1 (see [111]). In the bulk of V1, we thus expect only a weak coupling of orientation map and retinotopic distortions according to the EN model. In this regime, the predictions from models with reduced rotational symmetry (so-called Shift-Twist symmetry [112]) about the coupling between retinotopic distortions and OPMs [33] appear to be more promising than the weak effects resulting from the coverage-continuity-compromise. Consistent with the measurements of Das and Gilbert [96], such models predict small but significant positive correlations between the rates of change of orientation preference and retinotopic position [33]. Moreover, the form of the retinotopic distortions in such models is predicted to differ for pinwheels with positive and negative topological charge
[92]. This interesting prediction of OPM models with Shift-Twist symmetry deserves to be tested by measuring the receptive field center positions around the two types of pinwheels with single cell resolution [12].

\section{Aperiodic OPMs reflect long-range intracortical} suppression

Our unbiased search through the space of stimulus ensembles with finite fourth moment revealed the existence of spatially aperiodic optimal solutions in the EN. It is important to realize that the selection of these solutions is not easily viewed as resulting from an optimal compromise between coverage and continuity. In fact, the continuity parameter in the respective parameter regime is so small that solutions essentially maximize coverage (see Figures 12, 15, and 16). Instead, this phenomenon reflects a different key factor in the stabilization of pinwheel-rich aperiodic layouts, namely the dominance of long-ranged and effectively suppressive interactions. This is illustrated in Figure 20 which

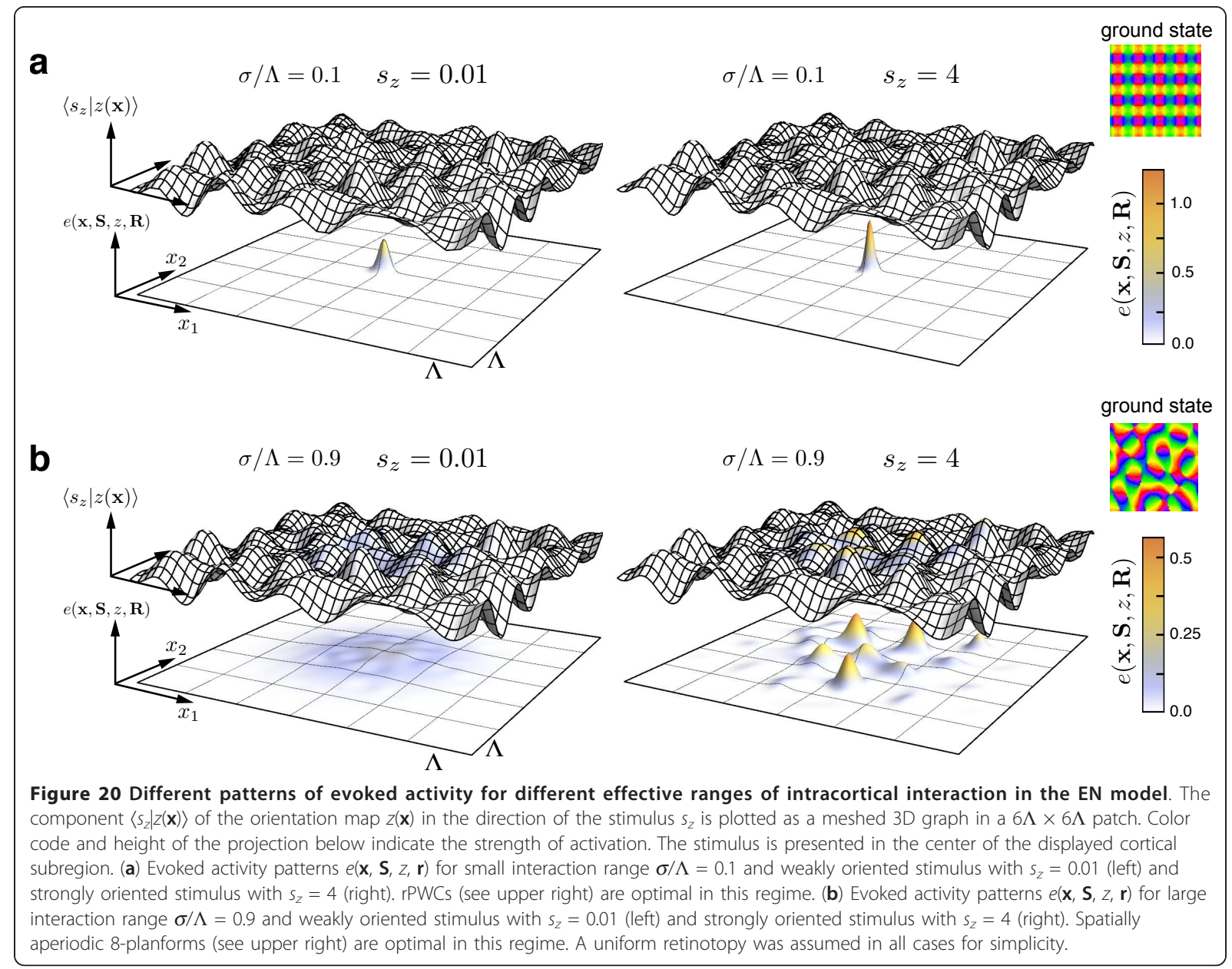


depicts different forms of stimulus-evoked activity patterns in the EN model. For a short-range interaction (Figure 20a), the activity evoked by low as well as high orientation energy stimuli is an almost Gaussian activity peak located near the stimulus position. The peak is shallow for low (left) and sharp for high 'orientation energy' (right). In the corresponding parameter regime, square pinwheel crystals are the optimal solution of the EN. For a longer range of interaction where aperiodic OPM layouts are the optimal states, the activity evoked by a single point-like stimulus is qualitatively different. Here, the activity pattern is extended and spans several hypercolumns (Figure 20b). It is weakly modulated for low orientation energy stimuli (left) and consists of several distinct peaks for high orientation energy stimuli (right). In this regime, neurons at a distance of several columns compete for activity through the normalization term in the EN which leads to a nonlocal and effectively suppressive intracortical interaction.

It is presumably not a mere coincidence that recent studies of abstract variational models of OPM development $[35,38,93]$ mathematically identified this type of interaction as a key mechanism for stabilizing realistic OPM layouts. It has been shown that all models for OPM development that share the basic symmetries (i) translational symmetry (ii) rotational symmetry (iii) shift symmetry and (iv) permutation symmetry and in addition are dominated by long-range suppressive interactions, form a universality class that generates maps exhibiting a universal and realistic pinwheel statistics. In such models, suppressive long-range interactions are key to stabilizing irregular arrangement of pinwheels, which otherwise largely disappear or crystalize during optimization. We have stressed that the EN model as considered here obeys the symmetries (i) to (iii). In the limit of infinite orientation stimulus ensemble fourth moment, permutation symmetry (iv) is restored. The EN can thus be tuned into the above universality class by sending the orientation stimulus distribution fourth moment to infinity and choosing an exponentially small continuity parameter to realize effective long-range coupling. Indeed, the phase diagrams for abstract variational models of OPM development [35] and those of the EN model found here are structurally very similar. In both cases, a rather large orientation stripe phase is complemented by a cascade of phase transitions toward more complex, aperiodic and pinwheel-rich OPM layouts induced by long-range suppressive interactions. Using abstract variational models, it has been shown recently that the stabilization of regular crystalline pinwheel layouts can alternatively be achieved by a strong coupling between the map of orientation and the map of eye dominance $[37,107]$. The structure of the phase diagrams of such models however appears fundamentally different from the structure of the EN phase diagrams.
The parameter regime in which the EN model's optimal solutions exhibit the experimentally observed pinwheel statistics is not at all intuitive and in our opinion questions the conventional interpretation of the EN model for the formation of cortical feature maps. Firstly, the extremely small continuity parameter questions the fundamental role of a tradeoff between coverage and continuity. We note that such a parameter regime is currently not accessible to numerical simulations. In addition, an apparently fundamental property for any adequate model for OPM optimization or development, namely a Turing-type finite wavelength instability of the unselective state [32], is lost in the limit $\eta \rightarrow 0$. At first sight the infinite fourth value required may appear reminiscent of the power-law distributions for orientation energy found in the statistics of natural images [113,114]. However, as visualized in Figure 20b, the essential property of the EN model in the infinite fourth moment regime is the occurrence of patterns of activity spatially extended beyond a single hypercolumn representing spatially localized point-like stimuli. These activity patterns mediate the long-range interactions between distant orientation columns which in turn cause the stability of realistic pinwheel-rich aperiodic OPM layouts. It is obvious that spatially extended stimuli provide a much more plausible and realistic source of extended activity patterns in models for visual cortical development (for an extended discussion see [54]). Optimization models for cortical maps based on the representation of more complex spatially extended visual stimuli, such as natural scenes, rather than a model based on point-like stimuli with extreme statistics would then be a more appropriate basis for understanding visual cortical functional architecture.

\section{Comparison to the SOFM model}

Several alternatives to the EN model have been proposed as optimization approaches that can account for the structure of visual cortical maps. One prominent alternative dimension reduction model is the so-called SOFM, originally introduced by Kohonen [59]. It is widely believed that this model, albeit lacking an exact energy functional [115], implements a competition between coverage and continuity very similar to the EN model $[56,57,66,115]$. The SOFM has been reported to reproduce many of the experimentally observed geometric properties of visual cortical feature maps (e.g., [56,57,61,66-68]). The numerical procedures used in all of these studies were either the deterministic annealing procedure or the nonrecurring application of a stimulus set without systematic assessment of pattern convergence. An analysis of the nontrivial stationary states of a dynamical systems formulation of the SOFM model is currently lacking. The main difference between the SOFM model and the EN model is that the activation function by definition has the form of a stereotypical Gaussian and competition is incorporated by a 
hard winner-takes-all mechanism. As a consequence, it is not obvious that a long-range suppressive interaction regime can be realized in this model. According to our analysis, one would thus expect orientation stripes and rPWCs as nontrivial stationary states of the SOFM model. In a very recent study of the SOFM algorithm that used a numerical procedure similar to the gradient descent simulations developed in this article, both pinwheel annihilation and rhombic pinwheel crystallization have been observed [116]. In addition, one study that examined the SOFM model for orientation and retinotopy found a fast convergence to pinwheel-free stripe-like solutions for a wide parameter range [25]. In view of these results, it seems worthwhile to also reexamine the SOFM model with respect to its stationary states.

\section{Rugged or smooth energy landscape}

As for many optimization problems in biology, the optimization of visual cortical functional architecture has been considered a problem characterized by a rugged energy landscape [76]. In case of the EN model the expectation of a rugged energy landscape at first sight seems quite plausible. Originally, the elastic network algorithm was invented as a fast analogue method to approximately solve NP-hard problems in combinatorial optimization such as the TSP $[77,78]$. In the TSP, the stimulus positions correspond to the locations of cities a salesman has to visit on the shortest possible tour. In problems such as the TSP, the energy functionals to be minimized are known to possess many local minima and the global minimization of these functionals generally represents an extremely difficult problem [78]. Our analysis reveals that the trade-off between coverage and continuity for the mapping of a continuous feature space manifold leads to a much simpler structure of the energy landscape. This is also indicated by the fact that almost all of our gradient descent dynamics simulations readily converged to the predicted global minimum of the energy functional. Figure 21 illustrates the smooth structure of the EN energy landscape close to pattern formation threshold for different model parameters for a one dimensional path through the state space. In this landscape, the small set of stable planforms correspond to local minima of the EN energy functional, and unstable planforms to saddle points in the energy landscape. The optimal states correspond to global minima. Note that along the depicted state space path, unstable stationary solutions may appear as local minima if the unstable directions along which the energy decreases are orthogonal to the path.

What is the origin of this qualitative difference in the shape of the energy landscapes? In the traveling salesman problem, the finite repertoire of possible tours consists of all permutations of the $N$ cities that the salesman has to visit. By self-organized competition between the aim to visit all cities and the aim to minimize the path length, the elastic network algorithm converges to a specific ordering of the cities that eventually yields a very short tour. Most likely, the qualitative difference to the EN model for visual cortical map architecture originates from the transition from a finite number of cities to a continuum. When the elastic network algorithm is considered with an ensemble of cities (or stimuli) distributed according to a continuous probability density function, there is no discrete repertoire of tours. Both, the repertoire of tours as well as the path through the landscape of cities or equivalently the space of visual stimulus features are determined by self-organization. The first is generated by the symmetry breaking mechanism that leads to the instability of the homogeneous state. The second corresponds to the selection of one of the many nontrivial stable steady states.

An interesting property of the EN model dynamics that can be inferred from the energy landscape depicted in Figure 21 is the type of competition between two stable stationary states, where both are present in the system with a wall or a domain boundary between them. The motion of the wall or domain boundary is predicted to proceed in the direction that increases the fraction of the pattern with lower energy. An example of such competition can be seen in Figure $23 \mathrm{~g}$ in Appendix. At $t=100 \tau$, a small domain with an sPWC state is present. The area of this region is gradually reduced over the time course of the simulation until the pinwheel-free optimal state is reached.

\section{Are simple OPM layouts an artifact of model simplicity?}

The perfectly periodic types of stationary solutions (stripes, crystals) that appear to dominate the classical EN model for retinotopy and orientation have been found in other models of visual cortical layouts that are relatively abstract. One might therefore suspect that they represent a mere artifact of model simplicity. One conceptually appealing approach where perfectly periodic layouts have been found is wiring-length minimization [27]. According to this hypothesis, the structure of an OPM can be understood by minimizing the total length of dendritic and axonal processes. Maps obtained by stopping minimization of wire length exhibited qualitatively realistic layouts (see Figure 6 in [27]). Complete optimization, however, leads to either stripe-like pinwheel-free patterns or rPWCs, identical to the ones obtained in our investigation of optimal solutions of the EN model [27]. Similarly, stripe-like and rhombic optima have been found in several abstract vector-field approaches for OPM development [31,33,117].

It is ruled out by two observations, that the crystalline and perfectly periodic optima observed in all four optimization models, the EN model, the SOFM, the wiringlength minimization model, and the vector-field models are a mere artifact of the abstract order parameter field 


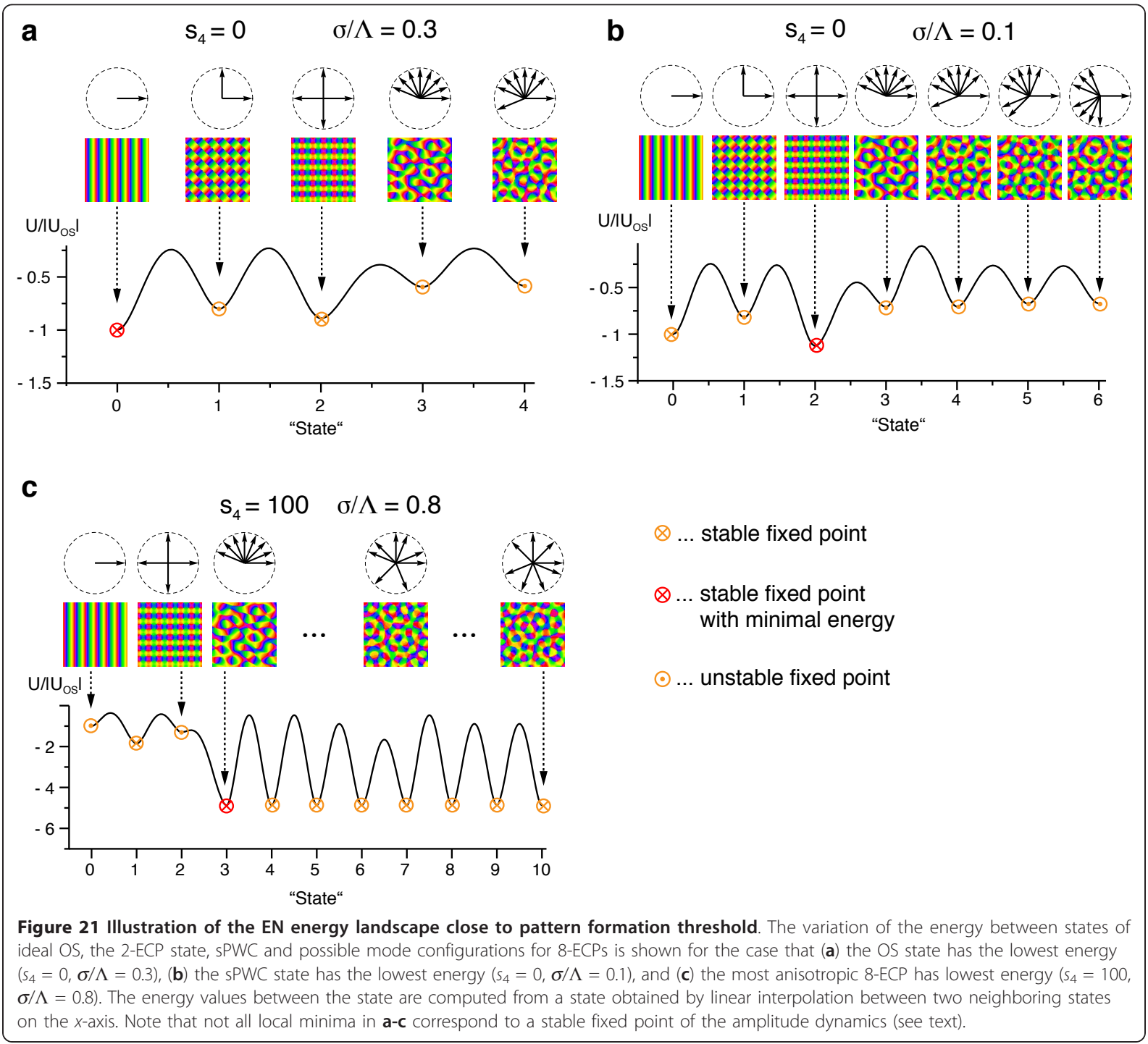

description of cortical selectivity patterns that is common to these approaches. Firstly, equally simplistic order parameter models for OPM development with long-range interactions have been shown to reproduce spatially irregular map layouts $[35,38,93]$. The occurrence of periodic optimal solutions is thus not a necessity in this model class. Secondly, pinwheel crystallization has also been observed in detailed network models for the development of OPMs, notably in the first ever model for the self-organization of orientation selectivity by von der Malsburg in 1973 [14,118]. Thus, on the one hand the phenomenon of pinwheel crystallization is thus not restricted to simple order parameter models and on the other hand abstract and mathematically relatively simple models can exhibit complex and biologically realistic optimal solutions.

\section{Map rearrangement and layout optimization}

Irrespective of the optimization principle invoked to describe the structure of visual cortical maps, several common features of the resulting dynamics have been observed. The dynamics of optimization models usually starts with a phase of pattern emergence, where selectivity to visual features arises from an initially homogeneous unselective or weakly selective state. As we and others have shown, feature maps in these models continue to evolve after single cell selectivities reach mature levels. In fact, the phase of initial pattern emergence is typically followed by a prolonged phase of rearrangement of selectivities and preferences until a stable configuration is reached that represents a genuine optimum. This is not an exceptional type of dynamics but rather constitutes the generic expectation for a spatially extended system $[83,84]$. 
What drives the second phase of map rearrangement? The initial emergence of feature selectivity is predominantly a local process in which merely neighboring units interact with each other to roughly match their selectivities. In the resulting spatial layout, selectivities are therefore far from being optimally arranged in space with respect to the global organization of selectivities on larger scales. Depending on the interactions incorporated in the model, local matching processes may (i) effectively propagate through space optimizing the pattern over gradually increasing spatial scales or (ii) distant sites may start to directly interact with each other to guide a rearrangement toward a globally optimized pattern after their initial emergence.

An illustrative example is provided by the emergence of pinwheel-sparse orientation stripes. Qualitatively, it is easy to see that a pattern of orientation stripes satisfies the continuity constraint very well. In a stripe pattern, preferred orientations are constant along one direction in space, realizing the absolute minimum of the orientation gradient in this direction. Reaching such a configuration obviously requires to select the preferred orientation at widely separated sites (along the stripe axis) to be identical. Because initially such sites develop independent preferred orientations, the optimized column layout can only emerge through a secondary rearrangement process. If the dominant low energy state has low pinwheel density, the later phase is governed by pinwheel motion and pairwise pinwheel annihilation. If this state is pinwheel-rich, e.g., a pinwheel crystal or an aperiodic pinwheel-rich state, both pinwheel annihilation and pinwheel creation together with a coordinated rearrangement of pinwheels are expected to occur.

The local, essentially random processes during the initial emergence of a fist pattern are in principle incapable of directly generating an optimized layout. In fact, it has been established that this initial so-called symmetry breaking phase will in general produce a random arrangement of selectivities of model-insensitive statistics $[25,32,36]$. The occurrence of some form of secondary reorganization is thus a qualitative prediction of any optimization model, provided that the optimal map is not seeded by an innate mechanism. The results presented in this study and many reports demonstrate that Hebbian plasticity is capable and often expected to achieve such rearrangements.

In gradient descent dynamics simulations of the EN model for retinotopy and stimulus orientation with conventional stimulus ensembles, pinwheel densities were found to be strongly time-dependent after the initial column formation (see e.g. Figures 6, 7). In particular, the timescale for the establishment of full orientation selectivity and the time needed for either annihilation of a substantial fraction of pinwheels or their crystallization into periodic pinwheel crystals are in the same range of tens of tau. A similar time dependence of pinwheel density has also been observed in other models for OPM development with periodic optima $[35,38]$. Pinwheel annihilation in the EN can be slightly slowed down by additional features such as retinotopy Figures 10 and 17 or OD [25,37] but not by orders of magnitude. For this reason, signatures of the periodic optima of a developmental dynamics become visible at rather early simulation stages. Long-term minimization is apparently not essential to express the main layout features of the global minimum.

Because the main features of the dominant optimal solutions become apparent immediately after orientation selectivity saturates it appears not easy to reproduce the species-independent map layout in models with periodic crystalline optima by pattern freezing. In our simulations to match even only the pinwheel density, a very precise timing of the freezing point would be required. There is currently no evidence for such a freezing mechanism in early development. In cats and ferrets, cortical maps for $\mathrm{OD}$, orientation or direction arise on a timescale between hours and a few days (e.g., $[13,119,120])$. The underlying circuits can be rapidly modified, e.g. by deprivation experiments, even on the timescale of hours $[121,122]$ weeks after full selectivity has been established. Recently, evidence for long-term visual cortical circuit reorganization after the emergence of feature selectivity during normal development has emerged in diverse systems. In mouse, for example, activity-dependent changes induced by normal visual experience during the critical period, i.e., long after the primary emergence of orientation selectivity, have been shown to gradually match eye-specific inputs in the cortex [123]. Specifically, the data from mouse indicates that preferred orientations in the two eyes initially often emerge unmatched and subsequently change toward one binocularly matched orientation preference. Because preferred orientations in the two eyes initially are statistically independent, this suggests that neurons can rotate their orientation preferences up to at least $45^{\circ}$ during postnatal development. This is reminiscent of pairing experiments in kitten visual cortex in which Frégnac and coworkers induced neurons to changed their preferred orientation by up to $90^{\circ}$ after pairing of a visual stimulus with intracortical stimulation [124,125] (see also [126]). Also in the cat, visual cortical orientation columns in visual areas V1 and V2 have been found to undergo rearrangement during the late phase of the critical period [41]. In this process, columns in mutually connected regions of areas V1 and V2 or in retinotopically matched regions in left and right hemisphere areas become progressively better matched in size. In the same species, a systematic reorganization of OD columns during postnatal development has been observed [69]. Essential features of this columnar rearrangement are reproduced by the EN model for OD patterns simulated in a growing domain. 
In view of these observations, it seems unlikely that aperiodic orientation maps in the visual cortex represent frozen transient states of a developmental dynamics whose attracting layouts are pinwheel crystals or pinwheel free states. In fact, models for the activity-dependent development of OPMs with aperiodic optima predict only subtle changes of the OPM layout during the convergence after the establishment of selectivity $[35,38]$. This might also explain the apparent stability of cortical maps during normal development over short periods [119]. Further studies of the long-term rearrangement and stabilization of cortical functional architecture are needed to exhaustively characterize such processes. Given the fundamental role of map reorganization for any optimization theory of visual cortical development, chronic imaging experiments tracking the spatial arrangement of feature selectivities in individual animals beyond the emergence of selectivity and through later developmental stages are expected to be highly informative about fundamental principles of visual cortical optimization.

\section{Conclusions}

Together with recent progress on the quantitative characterization of cortical functional architecture [38,69,93], this study lays the foundation for a mathematically rigorous and biologically informative search for optimization principles that successfully explain the architecture of columnar contour representations in the primary visual cortex. A mathematically controlled and quantitatively precise determination of the predictions of candidate optimization principles is demanded by accumulating evidence indicating that geometrical features of visual cortical representations are biologically laid down with a precision in the range of a few percent $[38,127,128]$. Such data is expected to substantially reduce the range of candidate optimization principles that are consistent with biological observations. In particular, for the principle that cortical orientation maps are designed to optimally compromise stimulus coverage and feature continuity, our analysis demonstrates that the classical EN model for orientation preference and retinotopy essentially fails at explaining the biologically observed architecture. Our finding that the EN model exhibits biologically realistic optima only in a limit in which point-like stimuli are represented by complex spatially extended activity patterns corroborates that large-scale interactions are essential for the stabilization of OPM layouts with realistic geometry $[35,39,87,93]$. In the light of these results, principles for the optimal representation of entire visual scenes by extended cortical activity patterns appear as promising candidates for future studies (see also [54]). In fact, there is recent evidence that visual cortical activity becomes progressively better matched to the statistics of natural stimuli but not to simplistic artificial stimulus ensembles [129]. We expect the methods developed here to facilitate a comprehensive characterization of such candidate principles.

\section{Methods}

Expansion of EN equation

In order to analytically calculate the approximate optimal dimension-reducing mappings in the EN model with fixed retinotopy, an expansion of the nonlinear EN OPM dynamics (Equation (3)) up to third-order around the unselective fixed point has to be derived. This expansion is briefly sketched in the following. Equation (3) with $\mathbf{r}(\mathbf{x})=\mathbf{0}$ is of the form

$$
\partial_{t} z(\mathbf{x}, t)=\mathcal{N}_{\mathbf{x}}[z]+\eta \Delta z(\mathbf{x}, t)
$$

where $\mathcal{N}_{\mathbf{x}}[z]$ is a nonlinear functional of $z(\cdot, t)$, parameterized by the position $\mathbf{x}$. Clearly, the diffusion term contains no nonlinear terms in $z(\cdot, t)$ and therefore third order terms of the dynamics $\partial_{t} z(\mathbf{x}, t)$ exclusively stem from third order terms of the Volterra series expansion of the functional $\mathcal{N}_{\mathbf{x}}[z]$ around the fixed point $z(\mathbf{x}, t) \equiv$ 0 . By the Shift symmetry (Equation (8)), only third-order contributions of the form $N_{3}[z, z, \bar{z}]$ are allowed, i.e.,

$$
N_{3}[z, z, \bar{z}]=\left.\frac{1}{2} \iiint d^{2} \gamma d^{2} w d^{2} v \frac{\delta^{3} \mathcal{N}_{\mathbf{x}}[z]}{\delta z(\mathbf{y}) \delta z(\mathbf{w}) \delta \bar{z}(\mathbf{v})}\right|_{z \equiv 0} z(\mathbf{y}) z(\mathbf{w}) \bar{z}(\mathbf{v}) .
$$

Collecting all the terms yields

$$
N_{3}[z, z, \bar{z}]=\sum_{j=1}^{11} a_{j} N_{3}^{j}[z],
$$

where

$$
\begin{aligned}
N_{3}^{1}[z] & =|z(\mathbf{x})|^{2} z(\mathbf{x}) \\
N_{3}^{2}[z] & =|z(\mathbf{x})|^{2} \int d^{2} \gamma K_{2}(\mathbf{y}-\mathbf{x}) z(\mathbf{y}) \\
N_{3}^{3}[z] & =z(\mathbf{x})^{2} \int d^{2} y K_{2}(\mathbf{y}-\mathbf{x}) \bar{z}(\mathbf{y}) \\
N_{3}^{4}[z] & =z(\mathbf{x}) \int d^{2} K_{2}(\mathbf{y}-\mathbf{x})|z(\mathbf{y})|^{2} \\
N_{3}^{5}[z] & =\bar{z}(\mathbf{x}) \int d^{2} \gamma K_{2}(\mathbf{y}-\mathbf{x}) z(\mathbf{y})^{2} \\
N_{3}^{6}[z] & =\int d^{2} y K_{2}(\mathbf{y}-\mathbf{x})|z(\mathbf{y})|^{2} z(\mathbf{y}) \\
N_{3}^{7}[z] & =z(\mathbf{x}) \iint d^{2} y d d^{2} w K_{3}(\mathbf{y}-\mathbf{x}, \mathbf{w}-\mathbf{x}, \mathbf{y}-\mathbf{w}) \bar{z}(\mathbf{w}) z(\mathbf{y}) \\
N_{3}^{8}[z] & =\bar{z}(\mathbf{x}) \iint d^{2} y d^{2} w K_{3}(\mathbf{y}-\mathbf{x}, \mathbf{w}-\mathbf{x}, \mathbf{y}-\mathbf{w}) z(\mathbf{w}) z(\mathbf{y}) \\
N_{3}^{9}[z] & =\iiint d^{2} y d^{2} w d^{2} v K_{4}(\mathbf{y}-\mathbf{x}, \mathbf{w}-\mathbf{x}, \mathbf{v}-\mathbf{x}, \mathbf{y}-\mathbf{w}, \mathbf{v}-\mathbf{w}, \mathbf{y}-\mathbf{v}) \bar{z}(\mathbf{y}) z(\mathbf{w}) z(\mathbf{y}) \\
N_{3}^{10}[z] & =\iint d^{2} y d^{2} w K_{3}(\mathbf{y}-\mathbf{x}, \mathbf{w}-\mathbf{x}, \mathbf{y}-\mathbf{w})|z(\mathbf{w})|^{2} z(\mathbf{y}) \\
N_{3}^{11}[z] & =\iint d^{2} y d^{2} w K_{3}(\mathbf{y}-\mathbf{x}, \mathbf{w}-\mathbf{x}, \mathbf{y}-\mathbf{w}) z(\mathbf{w})^{2} \bar{z}(\mathbf{y})
\end{aligned}
$$

and

$$
\begin{aligned}
K_{2}(\mathbf{x}) & =e^{-\mathbf{x}^{2} /\left(4 \sigma^{2}\right)} \\
K_{3}\left(\mathbf{x}_{1}, \mathbf{x}_{2}, \mathbf{x}_{3}\right) & =e^{-\left(\mathrm{x}_{1}^{2}+\mathrm{x}_{2}^{2}+\mathrm{x}_{3}^{2}\right) /\left(6 \sigma^{2}\right)} \\
K_{4}\left(\mathbf{x}_{1}, \mathbf{x}_{2}, \mathbf{x}_{3}, \mathbf{x}_{4}, \mathbf{x}_{5}, \mathbf{x}_{6}\right) & =e^{-\left(\mathrm{x}_{1}^{2}+\mathbf{x}_{2}^{2}+\mathrm{x}_{3}^{2}+\mathrm{x}_{4}^{2}+\mathbf{x}_{5}^{2}+\mathbf{x}_{6}^{2}\right) /\left(8 \sigma^{2}\right)} .
\end{aligned}
$$


The coefficients $a_{j}$ for various orientation stimulus ensembles are given in 'Results' section.

\section{Adiabatic elimination of $r(x, t)$}

In order to analytically calculate the approximate optimal dimension-reducing mappings in the EN model with variable retinotopy, an expansion of the nonlinear EN retinotopy and orientation map dynamics (Equations $(3,4)$ ) up to third-order around the nonselective fixed point has to be derived and retinotopic distortions have to be adiabatically eliminated. Both of these calculations are briefly sketched in the following. Equation (3) is of the form

$$
\partial_{t} z(\mathbf{x}, t)=\mathcal{N}_{\mathbf{x}}[z, \mathbf{r}]+\eta \Delta z(\mathbf{x}, t),
$$

where $\mathcal{N}_{\mathbf{x}}[z, \mathbf{r}]$ is a nonlinear functional of $z(\cdot, t)$ and $\mathbf{r}$ $(\cdot, t)$, parameterized by the position $\mathbf{x}$. The diffusion term contains no nonlinear terms in $z(\cdot, t)$ and therefore third order terms of the dynamics of $z(\mathbf{x}, t)$ exclusively stem from third-order terms of the Volterra series expansion of the functional $\mathcal{N}_{\mathbf{x}}[z, \mathbf{r}]$ around the fixed point $\{z(\mathbf{x}, t) \equiv 0, \mathbf{r}(\mathbf{x}, t) \equiv 0\}$. By the shift symmetry (Equation (8)), only terms in form of a cubic operator $N_{3}[z, z, \bar{z}]$ and a quadratic operator $Q^{z}[\mathbf{r}, z]$ are allowed when expanding up to third order. $N_{3}[z, z, \bar{z}]$ is given in Equation (37). $Q[z, \mathbf{r}]$ can be calculated via

$$
\left.Q^{z}[\mathbf{r}, z]=\left.\iint d^{2} y d^{2} w\left(\frac{\delta^{2}}{\delta z(\mathbf{y}) \delta r_{1}(\mathbf{w})} \mathcal{N} \mathbf{x}|z, \mathbf{r}|_{z=0, \mathbf{r}=0} r_{1}(\mathbf{w})+\frac{\delta^{2}}{\delta z\left(\mathbf{y} \delta r_{2}(\mathbf{w})\right.} \mathcal{N} \mathbf{x} \mid z, \mathbf{r}\right]\right|_{z=0, \mathbf{r}=0} r_{2}(\mathbf{w})\right) z(\mathbf{y})
$$

and this yields

$$
\begin{aligned}
Q^{z}[\mathbf{r}, z] & =\frac{\left(\left\langle\left|s_{z}\right|^{2}\right\rangle-2 \sigma^{2}\right)}{16 \pi \sigma^{6}} z(\mathbf{x}) \int d^{2} \gamma\left\langle\mathbf{r}(\mathbf{y}), \mathbf{K}_{2}^{r}(\mathbf{y}-\mathbf{x})\right\rangle \\
& -\frac{\left\langle\left|s_{z}\right|^{2}\right\rangle}{16 \pi \sigma^{6}} \int d^{2} \gamma\left\langle\mathbf{r}(\mathbf{x}), z(\mathbf{y}) \mathbf{K}_{2}^{r}(\mathbf{y}-\mathbf{x})\right\rangle+\frac{\left\langle\left|s_{z}\right|^{2}\right\rangle}{16 \pi \sigma^{6}} \int d^{2} \gamma\left\langle\mathbf{r}(\mathbf{y}), z(\mathbf{y}) \mathbf{K}_{2}^{r}(\mathbf{y}-\mathbf{x})\right\rangle \\
& -\frac{\left\langle\left|s_{z}\right|^{2}\right\rangle}{36 \pi^{2} \sigma^{8}} \iint d^{2} \gamma d^{2} w z(\mathbf{y})\left\langle\mathbf{r}(\mathbf{w}), \mathbf{K}_{3}^{r}(\mathbf{y}-\mathbf{x}, \mathbf{w}-\mathbf{x}, \mathbf{y}-\mathbf{w})\right\rangle,
\end{aligned}
$$

where $\langle\cdot, \cdot\rangle$ denotes the scalar product between two vectors and

$$
\begin{aligned}
& \mathbf{K}_{2}^{r}(\mathbf{x})=e^{-\mathbf{x}^{2} / 4 \sigma^{2}} \mathbf{x} \\
& \mathbf{K}_{3}^{r}\left(\mathbf{x}_{1}, \mathbf{x}_{2}, \mathbf{x}_{3}\right)=e^{-\frac{\mathbf{x}_{1}^{2}+\mathbf{x}_{2}^{2}+\mathbf{x}_{3}^{2}}{6 \sigma^{2}}}\left[\mathbf{x}_{1}+\mathbf{x}_{3}\right]
\end{aligned}
$$

In complete analogy, by expanding the right hand side of the dynamical equation for the retinotopic distortions (Equation (4)) up to second-order, the vector-valued quadratic operator $\mathbf{Q}^{r}[z, \bar{z}]$ can be obtained as

$$
\begin{aligned}
\mathbf{Q}^{r}[z, \bar{z}] & =-\Re\left(\frac{\left\langle\left|s_{z}\right|^{2}\right\rangle}{16 \pi \sigma^{6}} \bar{z}(\mathbf{x}) \int d^{2} \gamma \mathbf{K}_{2}^{r}(\mathbf{y}-\mathbf{x}) z(\mathbf{y})\right) \\
& +\frac{2 \sigma^{2}-\left\langle\left|s_{z}\right|^{2}\right\rangle}{32 \pi \sigma^{6}} \int d^{2} \gamma \mathbf{K}_{2}^{r}(\mathbf{y}-\mathbf{x})|z(\mathbf{y})|^{2} \\
& +\frac{\left\langle\left|s_{z}\right|^{2}\right\rangle}{72 \pi^{2} \sigma^{8}} \iint d^{2} \gamma d^{2} w \mathbf{K}_{3}^{r}(\mathbf{y}-\mathbf{x}, \mathbf{y}-\mathbf{w}, \mathbf{w}-\mathbf{x}) z(\mathbf{w}) \bar{z}(\mathbf{y})
\end{aligned}
$$

Inserting $\mathbf{r}(\mathbf{x})=-\mathbf{L}_{r}^{-1}\left[\mathbf{Q}^{r}[z, \bar{z}]\right]$ into $Q^{z}[\mathbf{r}, z]$ and using the linearity of $\mathbf{L}_{r}^{-1}$ as well as the bilinearity of both, $\mathbf{Q}^{r}[z, \bar{z}] \quad$ and $\quad Q^{z}[\mathbf{r}, \quad z], \quad$ yields a sum $N_{3}^{r}[z, z, \bar{z}]=\sum_{j=1}^{12} a_{r}^{j} N_{r}^{j}$, with

$$
\begin{aligned}
& N_{r}^{1}=z(\mathbf{x}) \int d^{2} \gamma / \mathbf{L}_{r}^{-1}\left[\Re\left(z\left(\bar{z}(\mathbf{y}) \int d^{2} w K_{2}(\mathbf{w}-\mathbf{y}) z(\mathbf{w})\right)\right], \mathbf{K}_{2}^{r}(\mathbf{y}-\mathbf{x})\right) \\
& N_{r}^{2}=z(\mathbf{x}) \int d^{2} y\left|\mathbf{L}_{r}^{-1}\left[\int d^{2} w K_{2}(\mathbf{w}-\mathbf{y})|z(\mathbf{w})|^{2}\right], \mathbf{K}_{2}^{\leftarrow}(\mathbf{y}-\mathbf{x})\right\rangle \\
& N_{r}^{3}=z(\mathbf{x}) \int d^{2} y\left(\mathbf{L}_{r}^{\mathbf{L}_{r}^{-1}}\left[\iint d^{2} w d^{2} \mathbf{V}_{3}^{\mathbf{K}}(\mathbf{w}-\mathbf{y}, \mathbf{w}-\mathbf{v}, \mathbf{v}-\mathbf{y})(\mathbf{w}) \overline{(v)}\right], \mathbf{K}_{2}^{T}(\mathbf{y}-\mathbf{x})\right) \\
& N_{r}^{4}=\left\langle\mathbf{L}_{r}^{-1}\left[\Re\left(\tilde{z}(\mathbf{z}) \int d^{2} \gamma \mathbf{K}_{2}^{t}(\mathbf{y}-\mathbf{x}) z(\mathbf{y})\right)\right] \cdot \int d^{2} \gamma z(\mathbf{y}) \mathbf{K}_{2}^{t}(\mathbf{y}-\mathbf{x})\right\rangle \\
& N_{r}^{5}=\left\langle\mathbf{L}_{r}^{-1}\left[\int d^{2} \gamma \mathbf{K}_{2}^{\tau}(\mathbf{y}-\mathbf{x})|z(\mathbf{y})|^{2}\right], \int d^{2} \gamma z(\mathbf{y}) \mathbf{K}_{2}^{\tau}(\mathbf{y}-\mathbf{x})\right\rangle \\
& N_{r}^{6}=\left\langle\mathbf{L}_{r}^{-1}\left[\iint d^{2} \gamma d^{2} w \mathbf{K}_{3}^{K}(\mathbf{y}-\mathbf{x}, \mathbf{y}-\mathbf{w}, \mathbf{w}-\mathbf{x}) \bar{z}(\mathbf{w}) \bar{z}(\mathbf{y})\right] \cdot \int d^{2} y z(\mathbf{y}) \mathbf{K}_{2}^{t}(\mathbf{y}-\mathbf{x})\right\rangle \\
& N_{r}^{7}=\int d^{2} \gamma z(\mathbf{y})\left\langle\mathbf{L}_{r}^{-1}\left[\Re\left(\tilde{z}(\mathbf{y}) \int d^{2} w K_{2}(\mathbf{w}-\mathbf{y}) z(\mathbf{w})\right)\right], K_{2}^{\mathrm{r}}(\mathbf{y}-\mathbf{x})\right\rangle \\
& N_{r}^{8}=\int d^{2} \gamma z(\mathbf{y})\left(\mathbf{L}_{r}^{-1}\left[\int d^{2} w \mathbf{K}_{2}^{\tau}(\mathbf{w}-\mathbf{y})|z(\mathbf{w})|^{2}\right], \mathbf{K}_{2}^{\tau}(\mathbf{y}-\mathbf{x})\right) \\
& N_{r}^{9}=\int d^{2} y z(\mathbf{y})\left|\mathbf{L}_{r}^{-1}\left[\iint d^{2} v^{2} w \mathbf{K}_{3}^{\tau}(\mathbf{w}-\mathbf{y}, \mathbf{w}-\mathbf{v}, \mathbf{v}-\mathbf{y})(\mathbf{w}) \bar{z}(\mathbf{y})\right], \mathbf{K}_{2}^{\tau}(\mathbf{y}-\mathbf{x})\right\rangle \\
& N_{r}^{10}=\iint d^{2} y d^{2} w z(\mathbf{y})\left(\mathbf{L}_{r}^{-1}\left[\Re\left(z(\mathbf{z}) \int d^{2} v \mathbf{K}_{2}^{\tau}(\mathbf{v}-\mathbf{w}) z(\mathbf{v})\right)\right], \mathbf{K}_{3}^{r}(\mathbf{y}-\mathbf{x}, \mathbf{y}-\mathbf{w}, \mathbf{w}-\mathbf{x})\right) \\
& N_{r}^{11}=\iint d^{2} y d^{2} w z(\mathbf{y})\left\langle\mathbf{L}_{r}^{-1}\left[\int d^{2} v \mathbf{K}_{2}^{\tau}(\mathbf{v}-\mathbf{w})|z(\mathbf{v})|^{2}\right], \mathbf{K}_{3}^{t}(\mathbf{y}-\mathbf{x}, \mathbf{y}-\mathbf{w}, \mathbf{w}-\mathbf{x})\right\rangle \\
& N_{r}^{12}=\iint d^{2} y d^{2} w z(\mathbf{y})\left|\mathbf{L}_{r}^{-1}\left[\iint d^{2} v d^{2} u \mathbf{K}_{3}^{5}(\mathbf{v}-\mathbf{w}, \mathbf{v}-\mathbf{u}, \mathbf{u}-\mathbf{w}) z(\mathbf{v}) \bar{z}(\mathbf{u})\right], \mathbf{K}_{3}^{\mathrm{K}}(\mathbf{y}-\mathbf{x}, \mathbf{y}-\mathbf{w}, \mathbf{w}-\mathbf{y})\right|
\end{aligned}
$$

The coefficients $a_{r}^{j}$ are given by

$$
\begin{aligned}
& a_{r}^{1}=-\frac{\left(\left\langle\left|s_{z}\right|^{2}\right\rangle-2 \sigma^{2}\right)\left\langle\left|s_{z}\right|^{2}\right\rangle}{256 \pi^{2} \sigma^{12}}=-\frac{\left(1-\sigma^{2}\right)}{64 \pi^{2} \sigma^{12}} \\
& a_{r}^{2}=\frac{\left(\left\langle\left|s_{z}\right|^{2}\right\rangle-2 \sigma^{2}\right)^{2}}{512 \pi^{2} \sigma^{12}}=\frac{\left(1-\sigma^{2}\right)^{2}}{128 \pi^{2} \sigma^{12}} \\
& a_{r}^{3}=\frac{\left(\left\langle\left|s_{z}\right|^{2}\right\rangle-2 \sigma^{2}\right)\left\langle\left|s_{z}\right|^{2}\right\rangle}{1152 \pi^{3} \sigma^{14}}=\frac{\left(1-\sigma^{2}\right)}{288 \pi^{3} \sigma^{14}} \\
& a_{r}^{4}=-\frac{\left\langle\left|s_{z}\right|^{2}\right\rangle^{2}}{256 \pi^{2} \sigma^{12}}=-\frac{1}{64 \pi^{2} \sigma^{12}} \\
& a_{r}^{5}=-\frac{\left(\left\langle\left|s_{z}\right|^{2}\right\rangle-2 \sigma^{2}\right)\left\langle\left|s_{z}\right|^{2}\right\rangle}{512 \pi^{2} \sigma^{12}}=-\frac{\left(1-\sigma^{2}\right)}{128 \pi^{2} \sigma^{12}} \\
& a_{r}^{6}=\frac{\left\langle\left|s_{z}\right|^{2}\right\rangle^{2}}{1152 \pi^{3} \sigma^{14}}=\frac{1}{288 \pi^{3} \sigma^{14}} \\
& a_{r}^{7}=-\frac{\left\langle\left|s_{z}\right|^{2}\right\rangle^{2}}{256 \pi^{2} \sigma^{12}}=-\frac{1}{64 \pi^{2} \sigma^{12}} \\
& a_{r}^{8}=-\frac{\left(\left\langle\left|s_{z}\right|^{2}\right\rangle-2 \sigma^{2}\right)\left\langle\left|s_{z}\right|^{2}\right\rangle}{512 \pi^{2} \sigma^{12}}=-\frac{\left(1-\sigma^{2}\right)}{128 \pi^{2} \sigma^{12}} \\
& a_{r}^{9}=\frac{\left\langle\left|s_{z}\right|^{2}\right\rangle^{2}}{1152 \pi^{3} \sigma^{14}}=\frac{1}{288 \pi^{3} \sigma^{14}} \\
& a_{r}^{10}=\frac{\left\langle\left|s_{z}\right|^{2}\right\rangle^{2}}{576 \pi^{3} \sigma^{14}}=\frac{1}{144 \pi^{3} \sigma^{14}} \\
& a_{r}^{11}=\frac{\left(\left\langle\left|s_{z}\right|^{2}\right\rangle-2 \sigma^{2}\right)\left\langle\left|s_{z}\right|^{2}\right\rangle}{1152 \pi^{3} \sigma^{14}}=\frac{\left(1-\sigma^{2}\right)}{288 \pi^{3} \sigma^{14}} \\
& a_{r}^{12}=-\frac{\left\langle\left|s_{z}\right|^{2}\right\rangle^{2}}{2592 \pi^{4} \sigma^{16}}=-\frac{1}{648 \pi^{4} \sigma^{16}}
\end{aligned}
$$

where the second equal sign is valid for $\left\langle\left|s_{z}\right|^{2}\right\rangle=2$. 
Amplitude equations from $N_{3}^{z}[z, z, \bar{z}]$

We catalog the numerous stationary solutions of Equation (13) following [35], by considering planforms

$$
z(\mathbf{x}, t)=\sum_{j=1}^{N} A_{j}(t) e^{i \mathbf{k}_{j} \mathbf{x}}
$$

with an even number $N$ of modes with amplitudes $A_{j}$ and $\mathbf{k}_{j}=k_{c}(\cos (2 \pi j / N), \sin (2 \pi j / N))$. In the vicinity of a finite wavelength instability, where the nonselective state $z(\mathbf{x})=0$ becomes unstable with respect to a band of Fourier modes around a finite wave number $k_{c}$-by symmetry, the dynamics of the amplitudes $A_{j}$ at threshold has the form

$$
\dot{A}_{i}=A_{i}-A_{i} \sum_{j=1}^{N} g_{i j}\left|A_{j}\right|^{2}-\sum_{j=1}^{N} f_{i j} A_{j} A_{j^{-}} \bar{A}_{i^{-}},
$$

where $j$ denotes the index of the mode antiparallel to the mode $j, \mathbf{k}_{j}=-\mathbf{k}_{j^{-}}$, and the coefficients $g_{i j}=\left(1-\frac{1}{2} \delta_{i j}\right) g\left(\left|\alpha_{i}-\alpha_{j}\right|\right)$ and $f_{i j}=\left(1-\delta_{i j}-\delta_{i^{-} j}\right) f\left(\left|\alpha_{i}-\alpha_{j}\right|\right)$ only depend on the angle $\left|\alpha_{i}-\alpha_{j}\right|$ between mode $i$ and $j$. The angle-dependent interaction functions $g(\alpha)$ and $f(\alpha)$ are obtained from Equation (13) by a multi scale expansion $[35,83,84,87]$ as

$$
\begin{aligned}
g(\alpha)= & -e^{-i \mathbf{k}_{0} \mathbf{x}}\left[N_{3}^{z}\left(e^{i \mathbf{k}_{0} \mathbf{x}}, e^{i \mathbf{h}(\alpha) \mathbf{x}}, e^{-i \mathbf{h}(\alpha) \mathbf{x}}\right)\right. \\
& \left.+N_{3}^{z}\left(e^{i \mathbf{h}(\alpha) \mathbf{x}}, e^{-i \mathbf{h}(\alpha) \mathbf{x}}, e^{i \mathbf{k}_{0} \mathbf{x}}\right)\right] \\
f(\alpha)= & -\frac{1}{2} e^{-i \mathbf{k}_{0} \mathbf{x}}\left[N_{3}^{z}\left(e^{i \mathbf{h}(\alpha) \mathbf{x}}, e^{-i \mathbf{h}(\alpha) \mathbf{x}}, e^{i \mathbf{k}_{0} \mathbf{x}}\right)\right. \\
& \left.+N_{3}^{z}\left(, e^{-i \mathbf{h}(\alpha) \mathbf{x}}, e^{i \mathbf{h}(\alpha) \mathbf{x}}, e^{i \mathbf{k}_{0} \mathbf{x}}\right)\right],
\end{aligned}
$$

where $\mathbf{k}_{0}=k_{c}(1,0)$ and $\mathbf{h}(\alpha)=k_{c}(\cos \alpha$, sin $\alpha)$. $f(\alpha)$ is $\pi$-periodic, since the right hand side of Equation (44) is invariant with respect to the transformation $\mathbf{h}(\alpha) \rightarrow \mathbf{h}(\alpha$ $+\pi)=-\mathbf{h}(\alpha) \cdot g(\alpha)$ is $2 \pi$-periodic in general. If, however, the nonlinearity is permutation symmetric (Equation (34)) it can be seen from Equation (43) that $g(\alpha)$ is $\pi$ periodic as well.

\section{Stability of stationary planform solutions}

To determine the stability of fixed points of the amplitude equations (42), the eigenvalues of their stability matrices have to be determined. In general, for any fixed point $\mathbf{A}=\mathbf{A}^{0}$ of the dynamical system $\dot{A}=\mathbf{F}(\mathbf{A})$ with complex-valued $\mathbf{A}$ and $\mathbf{F}$, we have to compute the eigenvalues of the Hermitian $2 N \times 2 N$ matrix

$$
\mathbf{M}=\left.\left(\begin{array}{ll}
\frac{\partial \mathbf{F}}{\partial A} & \frac{\partial F}{\partial \overline{\bar{A}}} \\
\frac{\partial \overline{\mathbf{F}}}{\partial \mathrm{A}} & \frac{\partial \mathbf{F}}{\partial \mathrm{A}}
\end{array}\right)\right|_{\mathrm{A}=\mathrm{A}_{0}} .
$$

For the system of amplitude equations, we obtain

$$
\begin{aligned}
& \frac{\partial F_{i}}{\partial A_{k}}=r \delta_{i k}-\delta_{i k}\left(\sum_{j}^{N} g_{i j}\left|A_{j}\right|^{2}\right)-A_{i} g_{i k} \bar{A}_{k}-\bar{A}_{i^{-}} f_{i k}\left(A_{k^{-}}+A_{k}\right) \\
& \frac{\partial F_{i}}{\partial \bar{A}_{k}}=-A_{i} g_{i k} A_{k}-\delta_{i^{-} k}\left(\sum_{j}^{N} f_{i j} A_{j} A_{j^{-}}\right) .
\end{aligned}
$$

Stability of a solution, or more precise intrinsic stability is given, if all eigenvalues of $\mathbf{M}$ are negative definite. Extrinsic stability is given, if the growth of additional Fourier modes is suppressed. To test whether a planform solution is extrinsically stable, we introduce a test mode $T$ such that

$$
z(\mathbf{x})=T e^{i \mathbf{k}_{\beta} \mathbf{x}}+\sum_{j}^{N} A_{j} e^{i \mathbf{k}_{j} \mathbf{x}},
$$

with $\mathbf{k}_{\beta}=(\cos \beta, \sin \beta) k_{c}$. We insert this ansatz into Equation 15 and obtain

$$
\partial_{t} T=r T-\sum_{j}^{N} g\left(\beta-\beta_{j}\right)\left|A_{j}\right|^{2} T+\mathcal{O}\left(T^{2}\right)
$$

as the dynamics of the test mode $T$, where $g(\beta)$ is the angle-dependent interaction function corresponding to $N_{3}[z, z, \bar{z}]$. For the solution $T=0$ to be stable, we therefore obtain the condition

$$
r-\sum_{j}^{N} g\left(\beta-\beta_{j}\right)\left|A_{j}\right|^{2}<0, \quad \forall \alpha \in[0,2 \pi],
$$

where we assumed $\mathbf{k}_{\beta} \neq \mathbf{k}_{j}, \mathbf{k}_{j}^{-}$. These conditions for intrinsic and extrinsic stability were numerically evaluated to study the stability of $n$-ECPs and rPWCs.

\section{Coupled essentially complex planforms}

In 'Results' section, we presented a closed form expression for the retinotopic distortions associated via Equation (28) with stationary planform solutions of Equation (29). Here, we sketch how to explicitly calculate this representation. We start with the ansatz

$$
z(\mathbf{x})=\sum_{j}^{n} A_{j} e^{i \mathbf{k}_{j} \mathbf{x}} \quad\left|\mathbf{k}_{j}\right|=k_{c}
$$

for the OPM $z(\mathbf{x})$. Note that this general ansatz includes essentially complex planforms as well as 
rPWCs. To simplify notation, we denote the individual terms in Equation (41)

$$
\begin{aligned}
& \mathbf{Q}_{1}[z, \bar{z}]=-\Re\left(\frac{\left\langle\left|s_{z}\right|^{2}\right\rangle}{16 \pi \sigma^{6}} \bar{z}(\mathbf{x}) \int d^{2} \gamma \mathbf{K}_{2}^{r}(\mathbf{y}-\mathbf{x}) z(\mathbf{y})\right) \\
& \mathbf{Q}_{2}[z, \bar{z}]=\frac{2 \sigma^{2}-\left\langle\left|s_{z}\right|^{2}\right\rangle}{32 \pi \sigma^{6}} \int d^{2} \gamma \mathbf{K}_{2}^{r}(\mathbf{y}-\mathbf{x})|z(\mathbf{y})|^{2} \\
& \mathbf{Q}_{3}[z, \bar{z}]=\frac{\left\langle\left|s_{z}\right|^{2}\right\rangle}{72 \pi^{2} \sigma^{8}} \iint d^{2} \gamma d^{2} w \mathbf{K}_{3}^{r}(\mathbf{y}-\mathbf{x}, \mathbf{y}-\mathbf{w}, \mathbf{w}-\mathbf{x}) z(\mathbf{w}) \bar{z}(\mathbf{y}) .
\end{aligned}
$$

Each of the $\mathbf{Q}_{i}[z, \bar{z}], i=1,2,3$ can be evaluated for the planform ansatz in Equation (45) and we obtain

$$
\begin{aligned}
& \begin{array}{c}
\mathbf{Q}_{1}\left[\sum_{j}^{n} A_{j} e^{i \mathbf{k}_{j}, \mathbf{x}}, \sum_{k}^{n} \bar{A}_{k} e^{-i \mathbf{k}_{k} \mathbf{x}}\right]=\frac{\left\langle\left|s_{z}\right|^{2}\right\rangle e^{-k_{k}^{2} \sigma^{2}}}{2 \sigma^{2}} \sum_{k_{j}<k}^{n}\left\{\Re\left(A_{j} \bar{A}_{k}\right)\left(\mathbf{k}_{j}-\mathbf{k}_{k}\right) \sin \left(\left(\mathbf{k}_{j}-\mathbf{k}_{k}\right) \mathbf{x}\right)\right. \\
\left.+\Im\left(A_{j} \bar{A}_{k}\right)\left(\mathbf{k}_{j}-\mathbf{k}_{k}\right) \cos \left(\left(\mathbf{k}_{j}-\mathbf{k}_{k}\right) \mathbf{x}\right)\right\}
\end{array} \\
& \mathbf{Q}_{2}\left[\sum_{j}^{n} A_{j} e^{i \mathbf{k}_{j} \mathbf{x}}, \sum_{k}^{n} \bar{A}_{k} e^{-i \mathbf{k}_{k} \mathbf{x}}\right]=-\frac{\left(2 \sigma^{2}-\left\langle\left|s_{z}\right|^{2}\right\rangle\right)}{2 \sigma^{2}} \sum_{j<k}^{n} e^{-\left(\mathbf{k}_{j}-\mathbf{k}_{k}\right)^{2} \sigma^{2}}\left(\mathbf{k}_{j}-\mathbf{k}_{k}\right)\left\{\Re\left(A_{j} \bar{A}_{k}\right) \sin \left(\left(\mathbf{k}_{j}-\mathbf{k}_{k}\right) \mathbf{x}\right)\right. \\
& \begin{array}{c}
\mathbf{Q}_{3}\left[\sum_{j}^{n} A_{j} e^{i \mathbf{k}_{j} \mathbf{x}}, \sum_{k}^{n} \bar{A}_{k} e^{-i \mathbf{k}_{k} \mathbf{x}}\right]=-\frac{\left\langle\left.||_{z}\right|^{2}\right\rangle e^{-\frac{k_{c}^{2} \sigma^{2}}{2}}}{\sigma^{2}} \sum_{j=k}^{n}\left(\mathbf{k}_{j}-\mathbf{k}_{k}\right) e^{-\frac{\left(\mathbf{k}_{\mathbf{j}}-\mathbf{k}_{k}\right)^{2} \sigma^{2}}{2}}\left\{\Re\left(A_{j} \bar{A}_{k}\right) \sin \left(\left(\mathbf{k}_{j}-\mathbf{k}_{k}\right) \mathbf{x}\right)\right. \\
\left.+\Im\left(A_{j} \bar{A}_{k}\right) \cos \left(\left(\mathbf{k}_{j}-\mathbf{k}_{k}\right) \mathbf{x}\right)\right\} .
\end{array}
\end{aligned}
$$

All resulting terms are proportional to either $\left(\mathbf{k}_{i}-\mathbf{k}_{j}\right)$ $\sin \left(\left(\mathbf{k}_{i}-\mathbf{k}_{j}\right) \mathbf{x}\right)$ or $\left(\mathbf{k}_{i}-\mathbf{k}_{j}\right) \cos \left(\left(\mathbf{k}_{i}-\mathbf{k}_{j}\right) \mathbf{x}\right) i \neq j$. These functions are longitudinal modes (see Figure $3 \mathrm{~b}$ ) which have been identified as eigenfunctions of the linearized dynamics of retinotopic distortions $\mathbf{L}_{r}[\mathbf{r}]$ with eigenvalue

$$
\lambda_{L}^{r}\left(\left|\mathbf{k}_{i}-\mathbf{k}_{j}\right|\right)=-\left|\mathbf{k}_{i}-\mathbf{k}_{j}\right|^{2}\left(\eta_{r}+e^{-\sigma^{2}\left|\mathbf{k}_{i}-\mathbf{k}_{j}\right|^{2}} \sigma^{2}\right)
$$

Hence, they are eigenfunctions of $\mathbf{L}_{r}^{-1}[\mathbf{r}]$ with eigenvalue $1 / \lambda_{L}^{r}\left(\left|\mathbf{k}_{i}-\mathbf{k}_{\mathbf{j}}\right|\right)$. Using this when inserting in Equation (28) and setting $\left\langle\left|s_{z}\right|^{2}\right\rangle=2$, we obtain expression (31) for the retinotopic distortions belonging to an arbitrary planform.

\section{Phase diagrams}

To compute the regions of minimal energy shown in Figures 6, 10, 12, 15, and 16 as well as Figures 23, 25 in Appendix 1, we first computed the fixed points of Equation (42) at each point in parameter space. For $n$-ECPs, we constructed the coupling matrix $\mathbf{g}$ in Equation (22) for all mode configurations not related by any combination of the symmetry operations: (i) Translation: $A_{j} \rightarrow A_{j} e^{i \mathbf{k}_{j} \mathbf{y}}$, (ii) Rotation: $A_{j} \rightarrow A_{j+\Delta j}$, (iii) Parity: $A_{j} \rightarrow \bar{A}_{(N-j)^{-}}$. By Equation (22), we then computed the absolute values of the corresponding amplitudes. If $\sum_{j=1}^{n}\left(\mathbf{g}^{-1}\right)_{i j} \geq 0$ for all $i$, a valid $n$-ECP fixed point of Equation 42 was identified. Its energy was then computed by Equation (23). For orientation stripes and rPWCs, the derived analytical expressions for their energy (Equations $(18,20)$ ) were evaluated. To analyze the stability of the fixed points, the conditions for intrinsic and extrinsic stability (see above) were numerically evaluated.

\section{Numerical procedures-gradient descent simulations}

To test our analytical calculations and explore their range of validity, we simulated Equations (3) and (4) on a $64 \times 64$ grid with periodic boundary conditions. Simulated systems were spatially discretized with typically 8 grid points per expected column spacing $\Lambda_{\max }$ of the orientation preference pattern (see 'Results' section) to achieve sufficient resolution in space. Test simulations with finer discretization (16 and 32 grid points per $\Lambda_{\max }$ ) did not lead to different results. Progression of time was measured in units of the intrinsic timescale $\tau$ (see 'Results' section) of the pattern formation process. The integration time step $\delta t$ was bounded by the relevant decay time constant of the Laplacian in Equation (3) around $k_{c}$ and by the intrinsic timescale $\tau$ of the system, using $\delta t=\min \left\{1 /\left(20 \eta k_{c}^{2}\right), \tau / 10\right\}$. This ensured good approximation to the temporally continuous changes of the patterns. We used an Adams-Bashforth scheme for the first terms on the respective r.h.s. of Equations $(3,4)$. The second terms (diffusion) were treated by spectral integration, exhibiting unconditional numerical stability. The stimulus positions $\mathbf{s}_{r}$ were chosen to be uniformly distributed in retinal coordinates. The stimulus averages in Equations $(3,4)$ were approximated by choosing a random representative sample of $N_{s}$ stimuli at each integration time step, with

$$
N_{s}=\max \left\{10^{5}, \frac{N_{0} \Gamma^{2}}{\left(\varepsilon_{s}\right)^{n}} \frac{\delta t}{\tau}\right\},
$$

where $n$ corresponds to the dimensions of the feature space in addition to the two retinal positions (in our case, $n=2), \Gamma^{2}=\left(L / \Lambda_{\max }\right)^{2}$ the squared aspect ratio of the simulated system in units of $\Lambda^{2}, \varepsilon_{s}$ the resolution in feature space, $N_{0}$ the number of stimuli we required to approximate the cumulative effect of the ensemble of stimuli within each feature space voxel $\varepsilon^{n+2}$. With $N_{0}=$ 100 and $\varepsilon_{s}=0.05$, we ensured a high signal-to-noise ratio for all the simulations. Typical values for $N_{s}$ were between $2.5 \times 10^{5}$ and $4 \times 10^{6}$. All simulations were initialized with $z(\mathbf{x}, t=0)=10^{-6} e^{i 2 \pi \xi(\mathbf{x})}$ and $\mathbf{r}(\mathbf{x}, t=0)=$ $\mathbf{0}$, where the $\xi(\mathbf{x})$ are independent identically distributed random numbers uniformly in $[0,1]$. Different realizations were obtained by using different stimulus samples.

Stimuli were drawn from different distributions, each with $\left\langle\left|s_{z}\right|^{2}\right\rangle=2$. We considered (i) stimuli uniformly distributed on a ring with $\left|s_{z}\right|^{2}=\sqrt{2}$ (circular stimulus ensemble), (ii) stimuli uniformly distributed within a circle $\left\{s_{z},\left|s_{z}\right| \leq 2\right\}$ (uniform stimulus ensemble), and (iii) a 
Gaussian stimuli ensemble with $\rho_{s_{z}}=1 /(2 \pi) \exp \left(-\left|s_{z}\right|^{2} / 2\right)$. In addition, we considered mixtures of a high-fourth moment Pearson type VII distribution and the circular stimulus ensemble. The Pearson distribution is given by

$$
\rho_{s_{z}}=\frac{1}{\alpha B\left(m-\frac{1}{2}, \frac{1}{2}\right)}\left[1+\frac{\left|s_{z}\right|^{2}}{\alpha^{2}}\right]^{-m},
$$

where $B(\cdot, \cdot)$ is the Beta function [130] and $\alpha=\sqrt{2 m-3}$, and $m=\frac{5}{2}+\frac{12}{\gamma-12}$ such that $\left\langle\left|s_{z}\right|^{2}\right\rangle=2$, \langle| $\left.\left.s_{z}\right|^{4}\right\rangle=\gamma$ or equivalently $s_{4}=\gamma-4$.

In addition to simulations in which independent sets of stimuli were used for evaluating the stimulus average in Equations $(3,4)$ for every time step, we also performed simulations in which the same fixed set of $N$ stimuli was used (see 'Results' section). To determine the time step $\delta t$ in these simulations, we first calculated

$$
N_{\tau}=\frac{N_{0} \Gamma^{2}}{\left(\varepsilon_{s}\right)^{n}}
$$

(parameters as in regular simulations) which yields the number of stimuli presented to the model in one intrinsic time unit $\tau$ in regular simulations. To subject the network to the same number of stimuli per intrinsic time scale $\tau$ in fixed stimulus set simulations, the integration time step $\delta t$ was in this case chosen as

$$
\delta t=\min \left\{\frac{N}{N_{s}} \tau, \frac{1}{20 \eta k_{c}^{2}}, \frac{\tau}{10}\right\} .
$$

For small $N$, this resulted in very small integration steps. For very large $N$, time steps were identical to the regular simulations. Different realizations were obtained by different but fixed stimulus sets. In all simulations with fixed stimulus sets, stimuli were drawn from the circular stimulus ensemble. All other numerical methods were chosen as in regular simulations.

\section{Numerical procedures - solving the EN model with deterministic annealing}

A large body of previous study has solved the EN models for various aspects of visual cortical architecture for discrete fixed sets of stimuli and using deterministic annealing. We therefore also used deterministic annealing with fixed discrete sets of stimuli to solve the EN model for the most frequently used stimulus distribution. This allowed us to better compare our analytical and numerical results based on the gradient descent dynamics for a continuous stimulus with prior results. For the discrete deterministic annealing approach, cortical maps are described by a collection of $M$ centroids $\left\{\mathbf{y}_{m}\right\}_{m=1}^{M} \subset \mathbb{R}^{d}$ that can be represented as a $D \times M$ matrix $\mathbf{Y}=\left(\mathbf{y}_{1}, \ldots, \mathbf{y}_{M}\right)$. Maps forming a compromise of coverage and continuity are obtained for $\left\{\mathbf{x}_{n}\right\}_{n=1}^{N} \subset \mathbb{R}^{d}$ represented as a $D \times$ $N$ matrix $\mathbf{X}=\left(\mathbf{x}_{1}, \ldots, \mathbf{x}_{M}\right)$. In our case, $d=4$. The trade-off between coverage and continuity is formulated by the energy function

$$
E(\mathbf{Y}, \sigma)=-\alpha \sigma \sum_{n=1}^{N} \log \sum_{m=1}^{M} e^{-\frac{1}{2}\left\|\frac{\mathbf{x}_{n}-\mathbf{y}_{m}}{\sigma}\right\|^{2}}+\frac{\beta}{2} \operatorname{tr}\left(\mathbf{Y}^{T} \mathbf{Y S}\right) .
$$

The matrix $\mathbf{S}$ determines the topology of the network as well as the boundary conditions and is typically derived from a discretized derivative based on a finite difference scheme or stencil (see below). For large $N$ and $M$, the energy function in Equation (46) is equivalent to the energy functional of the continuum formulation given in Equation (1) for $\beta=\eta N$ and $\mathrm{S}$ implementing the discretized Laplacian operator in two dimensions.

Following [62-65,71] we minimized the EN energy function (Equation (46)) by an iterative deterministic annealing algorithm, starting with a minimization for large $\sigma$ and tracking this minimum to a small value of $\sigma$. As in [4], we reduced $\sigma$ from $\sigma_{\text {init }}=0.2$ to the point at which the amplitude of the orientation maps saturate ( $\sigma \approx 0.03$ ), following $\sigma=\sigma_{\text {init }} \times \chi^{j}$ where $j$ counts the annealing step. This choice tracks stationary solutions of the EN to parameters that are very far from threshold. For high precision tracking of solutions, we used an annealing rate of $\chi=0.999$.

At each value of $\sigma$, setting the gradient of Equation (46) to zero yields a nonlinear system of equations

$$
\mathbf{Y A}=\mathbf{X W} \quad \text { with } \quad \mathbf{A}=\mathbf{G}+\sigma \beta\left(\frac{\mathbf{S}+\mathbf{S}^{T}}{2}\right),
$$

Here, the $N \times M$-matrix $W$ is given by

$$
w_{n m}=\frac{e^{-\frac{1}{2}\left\|\frac{\mathbf{x}_{n}-\mathbf{y}_{m}}{\sigma}\right\|^{2}}}{\sum_{m^{\prime}=1}^{M} e^{-\frac{1}{2}\left\|\frac{\mathbf{x}_{n}-\mathbf{y}_{m^{\prime}}}{\sigma}\right\|^{2}}}
$$

and the $M \times M$-matrix $G$ is

$$
g_{i j}=\delta_{i j} \sum_{n=1}^{N} w_{n i} .
$$

A is a symmetric positive-definite $M \times M$ matrix. The $M \times M$ matrix A is symmetric and positive-definite. Since both $G$ and $W$ depend on $Y$, this equation is nonlinear in $Y$ and has to be solved iteratively. Following 
[62-65,71], we solved Equation (47) at each value of $\sigma$ and for each iteration directly via Cholesky-factorization.

We implemented periodic and nonperiodic boundary conditions by appropriate choice of the matrix $\mathbf{S}$. $\mathbf{S}$ must be positive (semi)definite for the energy to be bounded from below. We used the canonical 2D Laplacian stencil of order 2, to construct the $M \times M$ matrix

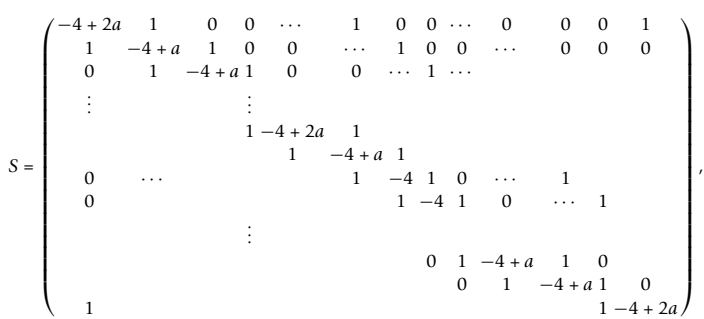

Here, $a=0$ for periodic boundary conditions and $a=$ 1 for nonperiodic boundary conditions. In Appendix 3, we also present simulation results for a fourth derivative stencil, in which $S^{2}$ was used for the continuity term. We used random stimulus positions and orientations as well as stimuli arranged on a grid in feature space. For random stimuli, positions were drawn from a uniform distribution in $[0,1] \times[0,1]$. Orientations $s_{z}$ were drawn from the circular stimulus ensemble, with $\left|s_{z}\right|=$ 0.08 as in [65]. Stimuli from grid-like ensembles were distributed evenly-spaced in $[0,1] \times[0,1]$ and contained $2^{k}$ evenly space orientations with $\left|s_{z}\right|=0.08$.

To compute the energy of pinwheel-free configuration, we initiated simulations with a stripe-like orientation preference pattern with the same typical spacing as the observed orientation maps and annealed from $\sigma=0.035$ to $\sigma=0.03$.

To enable comparison between simulations with different numbers of stimuli, we scaled the continuity parameter proportionally to $N$ such that the equivalent $\eta$ was approximately constant. The simulated domain then contained a comparable number of hyper columns for all stimulus numbers.

\section{Pinwheel density from simulations}

Pinwheels locations in models were identified by the crossings of the zero contour lines of real and imaginary parts of the orientation map. Estimation of local column spacing $\Lambda(\mathbf{x})$ was done using the wavelet analysis introduced in $[127,128]$. In short, an overcomplete basis of complex Morlet wavelets at various scales and orientations was compared to the OPM pattern at each location. $\Lambda(\mathbf{x})$ was estimated by the scale of the best matching wavelet. The mean column spacing $\langle\Lambda(\mathbf{x})\rangle_{x}$ of a given map was then calculated from the local column spacing by spatial averaging. For details we refer to $[38,127,128]$. Given $N_{p w}$ pinwheels in a simulated cortical area of size $L^{2}$, we defined the pinwheel density $[25,38,102]$

$$
\rho=N_{p w} \frac{\langle\Lambda\rangle_{x}^{2}}{L^{2}}
$$

The pinwheel density $\rho$ is a dimensionless quantity and depends only on the layout of orientation columns. The pinwheel density defined in this way is large for patchy and small for more band-like columnar layouts.

\section{Appendix 1}

\section{The impact of nonoriented stimuli}

The main text of this article contains a complete analysis of optimal dimension-reducing mappings of the EN model with a circular ensemble of orientation stimuli. These optima are simple regular orientation stripes or square pinwheel crystals. The circular orientation stimulus ensemble, however, contains only stimuli with a fixed and finite 'orientation energy' or elongation $\left|s_{z}\right|$. This raises the question of whether the simple nature of the circular stimulus ensemble might restrain the realm of complex dynamics in the EN model. The EN dynamics are expected to depend on the characteristics of the activity patterns evoked by the stimuli and these will be more diverse and complex with ensembles containing a greater diversity of stimuli. Therefore, we also examined the EN model in detail for a richer ensemble of stimuli. In this ensemble, called a uniform stimulus ensemble in the following, orientation stimuli are uniformly distributed on the disk $\left\{s_{z},\left|s_{z}\right| \leq 2\right\}$, a choice common to many previous studies, e.g., $[19,25,81]$. The uniform ensemble in particular contains unoriented stimuli with $\left|s_{z}\right|=0$. Intuitively, the presence of these unoriented stimuli might be expected to fundamentally change the importance of pinwheels in the optimal OPM layout. Pinwheels' population activity is untuned for orientation. Pinwheel centers may therefore acquire a key role for the representation of unoriented stimuli. As such an effect should be independent of retinotopic distortions and to aid comparison with our previous results, we will again start with a fixed uniform retinotopy $\mathbf{r}(\mathbf{x})=\mathbf{0}$.

The linear stability properties of the unselective fixed point are independent of the ensemble of orientation stimuli $\left(\left\langle\left|s_{z}\right|^{2}\right\rangle=2\right.$ throughout this article). The coefficients in Equation (14), however, of course depend on the fourth moment of the stimulus distribution. Inserting $\left\langle\left|s_{z}\right|^{4}\right\rangle=16 / 3$ into Equation (32), we obtain

$$
\begin{array}{lll}
a_{1}=\frac{1}{3 \sigma^{6}}-\frac{1}{\sigma^{4}}+\frac{1}{2 \sigma^{2}} & a_{2}=\frac{1}{4 \pi \sigma^{6}}-\frac{1}{6 \pi \sigma^{8}} & a_{3}=-\frac{1}{12 \pi \sigma^{8}}+\frac{1}{8 \pi \sigma^{6}} \\
a_{4}=-\frac{1}{6 \pi \sigma^{8}}+\frac{1}{4 \pi \sigma^{6}}-\frac{1}{8 \pi \sigma^{4}} & a_{5}=-\frac{1}{12 \pi \sigma^{8}} & a_{6}=\frac{1}{8 \pi \sigma^{6}}-\frac{1}{12 \pi \sigma^{8}} \\
a_{7}=\frac{1}{9 \pi^{2} \sigma^{10}}-\frac{1}{12 \pi^{2} \sigma^{8}} & a_{8}=\frac{1}{18 \pi^{2} \sigma^{10}} & a_{9}=-\frac{1}{16 \pi^{3} \sigma^{12}} \\
a_{10}=\frac{1}{9 \pi^{2} \sigma^{10}}-\frac{1}{12 \pi^{2} \sigma^{8}} & a_{11}=\frac{1}{18 \pi^{2} \sigma^{10}} . &
\end{array}
$$


The angle-dependent interaction functions are then given by

$$
\begin{aligned}
g(\alpha) & =\frac{1}{\sigma^{4}}\left(1-2 e^{-k_{c}^{2} \sigma^{2}}-e^{2 k_{c}^{2} \sigma^{2}(\cos \alpha-1)}\left(1-2 e^{-k_{c}^{2} \sigma^{2} \cos \alpha}\right)\right) \\
& +\frac{1}{2 \sigma^{2}}\left(e^{2 k_{c}^{2} \sigma^{2}(\cos \alpha-1)}-1\right)+\frac{32}{3 \sigma^{6}} e^{-2 k_{c}^{2} \sigma^{2}} \sinh ^{4}\left(1 / 2 k_{c}^{2} \sigma^{2} \cos \alpha\right) \\
f(\alpha) & =\frac{1}{\sigma^{4}}\left(1-e^{-2 k_{c}^{2} \sigma^{2}}\left(\cosh \left(2 k_{c}^{2} \sigma^{2} \cos \alpha\right)+2 \cosh \left(k_{c}^{2} \sigma^{2} \cos \alpha\right)\right)+2 e^{-k_{c}^{2} \sigma^{2}}\right) \\
& +\frac{1}{2 \sigma^{2}}\left(e^{-2 k_{c}^{2} \sigma^{2}} \cosh \left(2 k_{c}^{2} \sigma^{2} \cos \alpha\right)-1\right)+\frac{16}{3 \sigma^{6}} e^{-2 k_{c}^{2} \sigma^{2}} \sinh ^{4}\left(1 / 2 k_{c}^{2} \sigma^{2} \cos \alpha\right) .
\end{aligned}
$$

Both functions are depicted in Figure 22 for two different values of the effective intracortical interaction range $\sigma / \Lambda$. They qualitatively resemble the functions depicted in Figure 5. Figure 23 displays the phase diagram of the EN model with uniform stimulus ensemble. As summarized in the main part of this article, it is almost identical to that obtained for the circular stimulus ensemble (Figure 6). Two different optimal states are found, square pinwheel crystals (sPWCs) and orientation stripes (OSs) separated by a phase border at $\sigma / \Lambda \simeq 0.15$. Both fixed points are stable for all $\sigma / \Lambda$. Figure $23 \mathrm{~b}-\mathrm{k}$ demonstrates, that these analytical results are confirmed by direct numerical simulations of Equation (3) with $\mathbf{r}$ $(\mathbf{x})=\mathbf{0}$. As for the circular stimulus ensemble, we also tested the stability of stationary $n$-ECP solutions with 2 $\leq n \leq 20$ by numerical evaluation of the criteria for intrinsic and extrinsic stability (see 'Methods' section). We find all $n$-ECPs with $2 \leq n \leq 20$ intrinsically unstable for all interaction ranges $\sigma / \Lambda$. The simple phase space structure furthermore apparently remains unchanged if we consider the model far from pattern formation threshold as shown in Figure 24. Simulations bear a close resemblance to the simulations with circular orientation stimulus ensemble (Figure 7). Either convergence to SPWC-like patterns or patterns with large orientation stripe domains is observed. Again, pinwheel annihilation in the case of large $\sigma / \Lambda$ is less rapid than close to threshold (Figure 24a,b). The linear pinwheel- free zones increase their size over the time course of the simulations, eventually leading to a stripe pattern. For smaller interaction ranges $\sigma / \Lambda$, the OPM layout rapidly converges toward a crystal-like rhombic arrangement of pinwheels with dislocations and pinwheel density close to 4 .

Figure 25 shows that taking retinotopic distortions into account yields an almost identical picture compared to the circular stimulus ensemble. For small interaction range $\sigma / \Lambda$, the analytically predicted optimum is a quadratic pinwheel crystal with pinwheel density $\rho=4$. For larger $\sigma / \Lambda$, the analytically predicted optimum is an orientation stripe pattern with pinwheel density $\rho=0$. Our results are confirmed by direct simulations of Equations $(3,4)$ (Figure $25 \mathrm{~b}-\mathrm{e})$. The simulation results are virtually indistinguishable from the circular stimulus ensemble.

All together, the EN dynamic given by Equations $(3,4)$ and in particular the set of ground states of the EN model and their stability regions appear almost identical when considering either a circular or a uniform orientation stimulus ensemble. We found two different optima depending on the parameter regime, orientation stripes for larger interaction ranges and quadratic pinwheel crystals for shorter interaction ranges. In addition, the EN dynamics appears to be unchanged by the presence of unoriented stimuli.

\section{Appendix 2}

\section{Strength of retinotopic coupling}

In our manuscript, we have shown that retinotopic distortions only have a weak influence on the optima of the EN model as well as its dynamics (see Figures 10 and 12). Here, we quantify the influence of retinotopic distortions on the pattern formation process by comparing the angle-dependent interaction function for
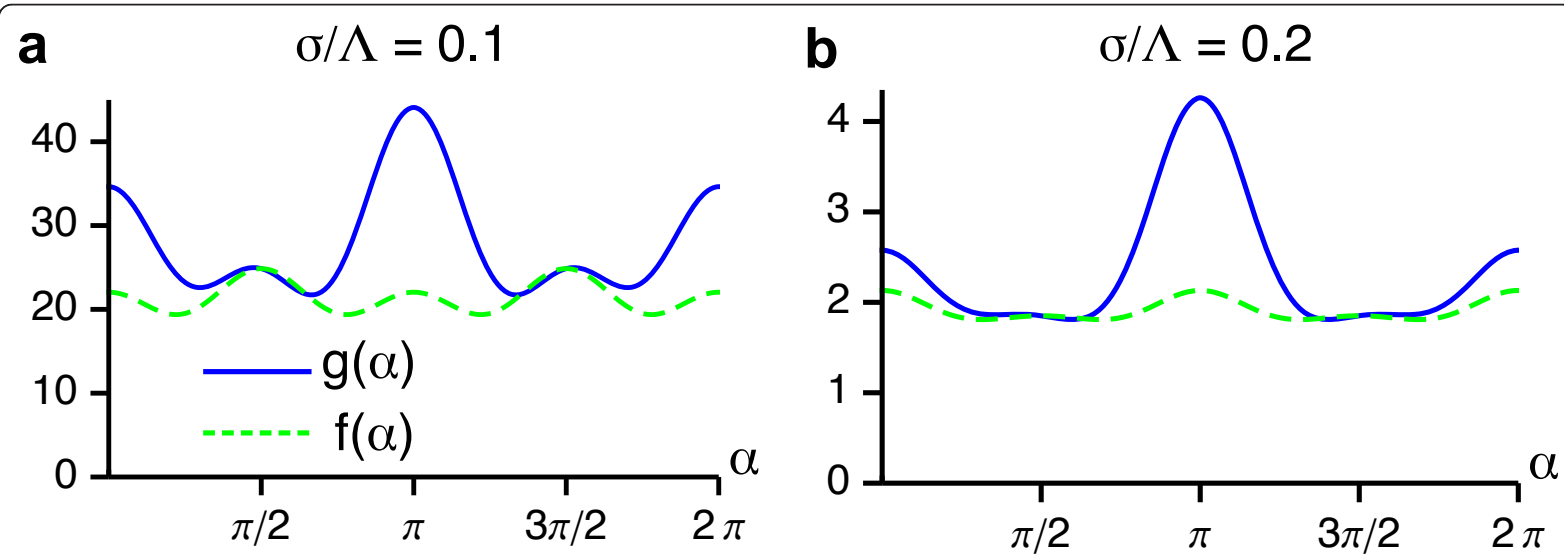

Figure 22 Angle-dependent interaction functions for the EN model with fixed retinotopy and uniform orientation stimulus ensemble. $(\mathbf{a}, \mathbf{b}) g(\alpha)$ and $f(\alpha)$ for $\sigma / \Lambda=0.1(\mathbf{a})$ and $\sigma / \Lambda=0.2(\mathbf{b})$. 


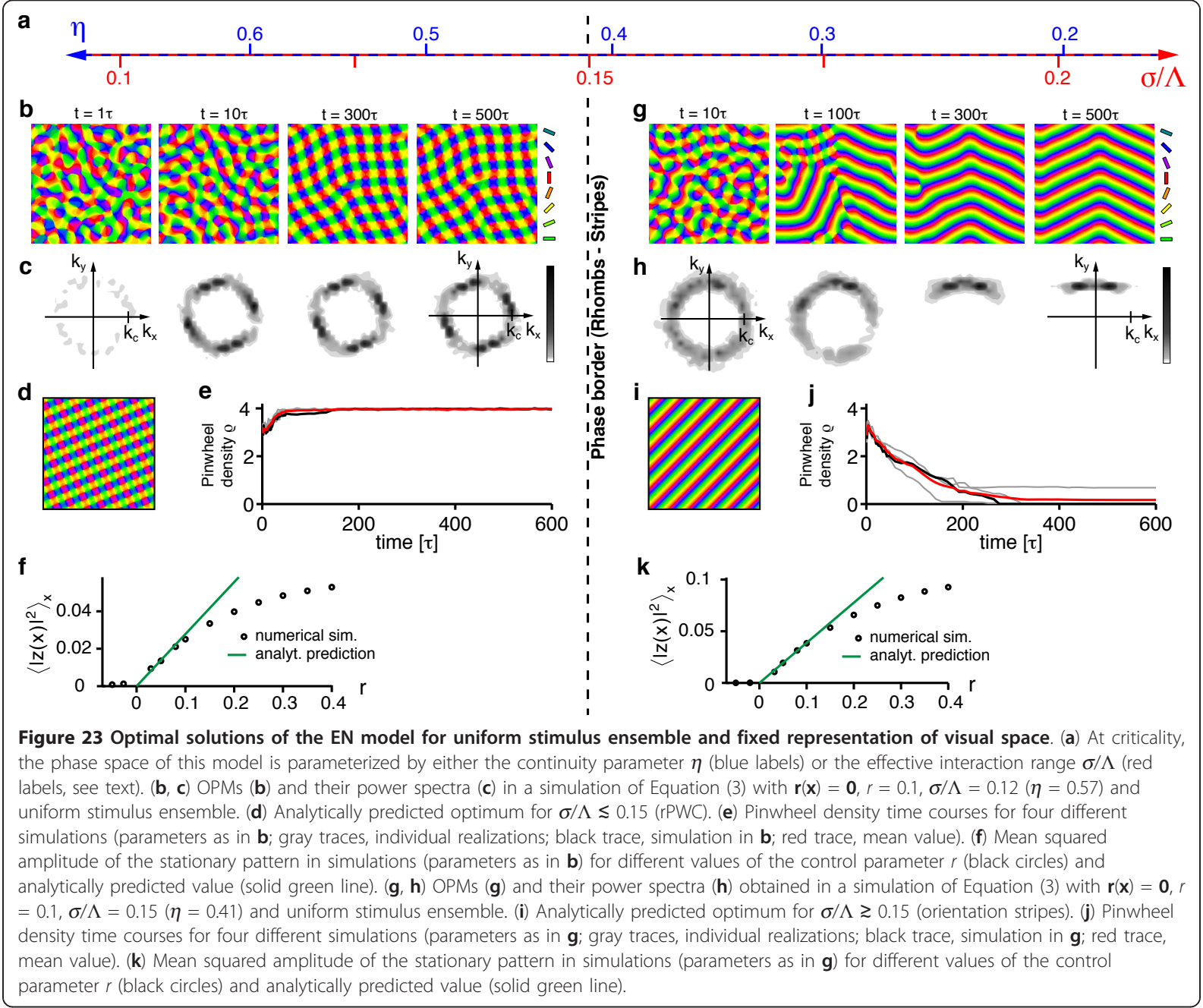

retinotopic coupling $g_{r}(\alpha)$ with angle-dependent interaction function of the EN model with fixed retinotopy. We use the ratio

$$
c=\frac{\left\|g_{r}(\cdot)\right\|_{2}}{\|g(\cdot)\|_{2}}
$$

as a measure to quantify the influence of retinotopic distortions. $\|\cdot\|_{2}$ denotes the 2-Norm,

$$
\|f(\cdot)\|_{2}=\int_{0}^{2 \pi} f^{2}(\alpha) d \alpha .
$$

If $c$ is larger than one, $g_{r}(\alpha)$ dominates the total interaction function $g_{r}(\alpha)+g(\alpha)$ and retinotopic distortions may strongly influence the layout and stability of solutions of the EN model. On the other hand, if $c$ is small, the solutions and their stability properties are expected to not change substantially when including variable retinotopy into the EN model. Figure 26 displays the parameter $c$ in the $s_{4}-\sigma / \Lambda$-plane for the EN model at threshold for two different conditions, $\eta=\eta_{r}$ and $\eta_{r}=0$. In the latter case, retinotopic distortions are expected to have the strongest impact. However, in both cases, $c \ll 1$, in almost all of the parameter space, implying little influence of retinotopic deviations. Only for small $\sigma / \Lambda$ and small $s_{4}, c$ is larger than one. As shown in Figure 12, this leads to slight deformations of the stability regions for rhombs, and stripes in this region of parameter space but does not result in novel optimal solutions.

\section{Appendix 3}

Grid-like stimulus ensembles

References $[64,65])$ performed simulations with stimuli distributed in regular intervals in feature space, called grid-like ensemble. For comparison, we also performed deterministic annealing simulations with grid-like 


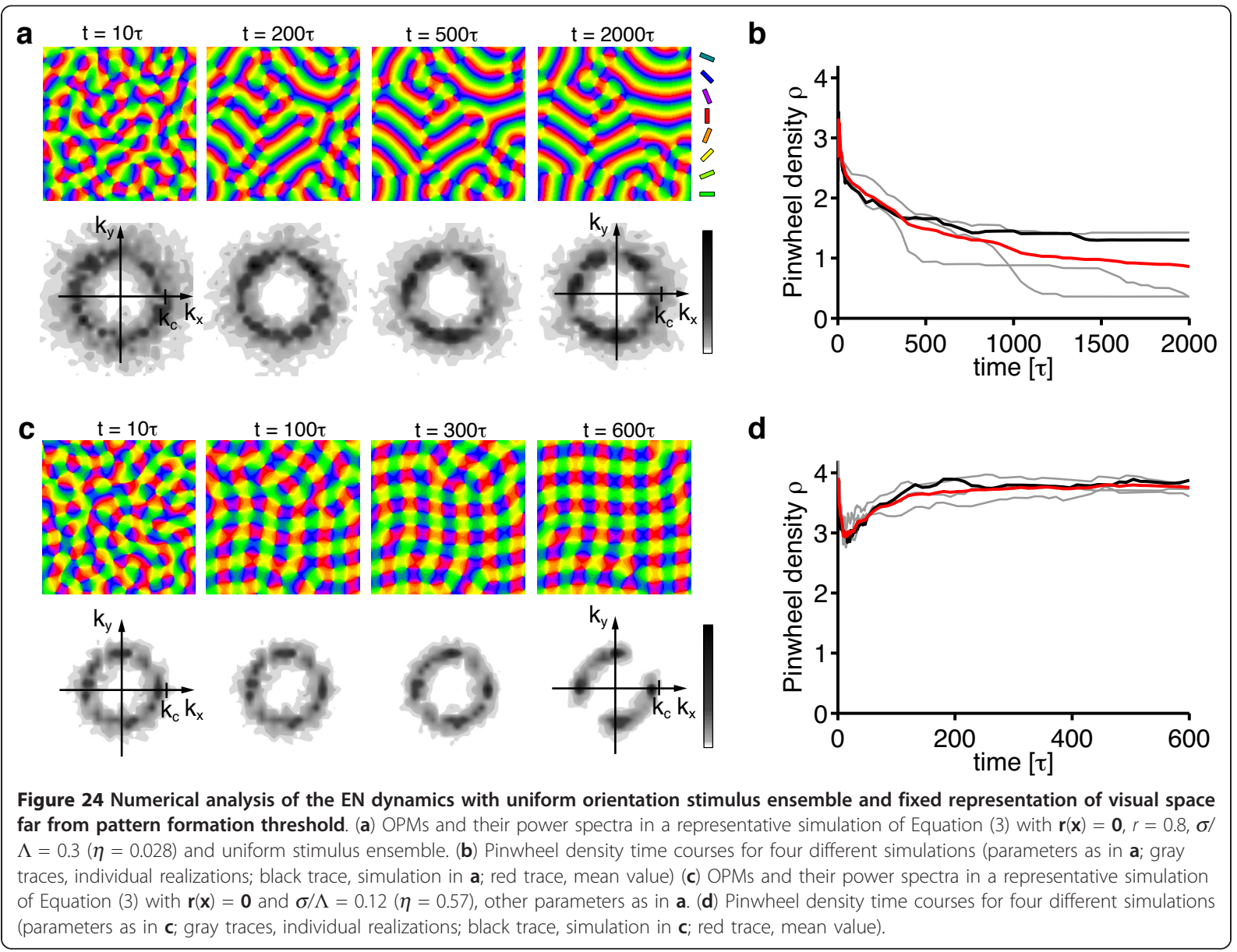

stimulus sets of varying size with nonperiodic boundary conditions (see 'Methods' section). For these grid-like stimulus patterns, a competition between stripes and rhombs is observed (Figure 27a). Notably, these are the only two stable states identified by our analysis for the circular stimulus ensemble. For nonperiodic boundary conditions, rhombic pinwheel arrangements seem energetically favored for grid-like stimuli, almost independently of the size of the stimulus set. The average pinwheel density for $N=100 \times 100 \times 8$ stimuli was $\rho=$ 3.4 (Figure 27b). As expected from the predominantly rhombic arrangement of pinwheels, NN-pinwheel distances concentrate around half the typical column spacing and pinwheel pairs at short distances are not observed (Figure 27c). With these features, the maps obtained substantially differ from the experimentally observed pinwheel statistics [38].

The discrete EN model with fourth derivative In previous studies of the EN model, alternative definitions of the continuity term in the EN model have been explored [64]. A general continuity term for the spatially continuous formulation of the EN for OPM and retinotopy is a linear differential operator which will suppress the emergence of high-frequencies during the EN dynamics. A finite-wavelength instability is expected in this case, although the precise expressions for the critical $\sigma$ and the typical wavelength will differ. As linear terms do not enter in the higher-order derivatives of the EN functional, changing the continuity term is not expected to alter the stability results obtained in this study.

To numerically test this expected robustness of our results for the EN model with discrete fixed sets of stimuli (see Figures 18 and 19), we also performed simulations using deterministic annealing with a fourth derivative stencil (see 'Methods' section). Figure 28 illustrates that the results almost perfectly match the ones for the second-order derivative, considered in the main part of this article (Figures 18, 19 and Figure 27).

When annealing with periodic boundary conditions, the solutions very much resemble our gradient descent 

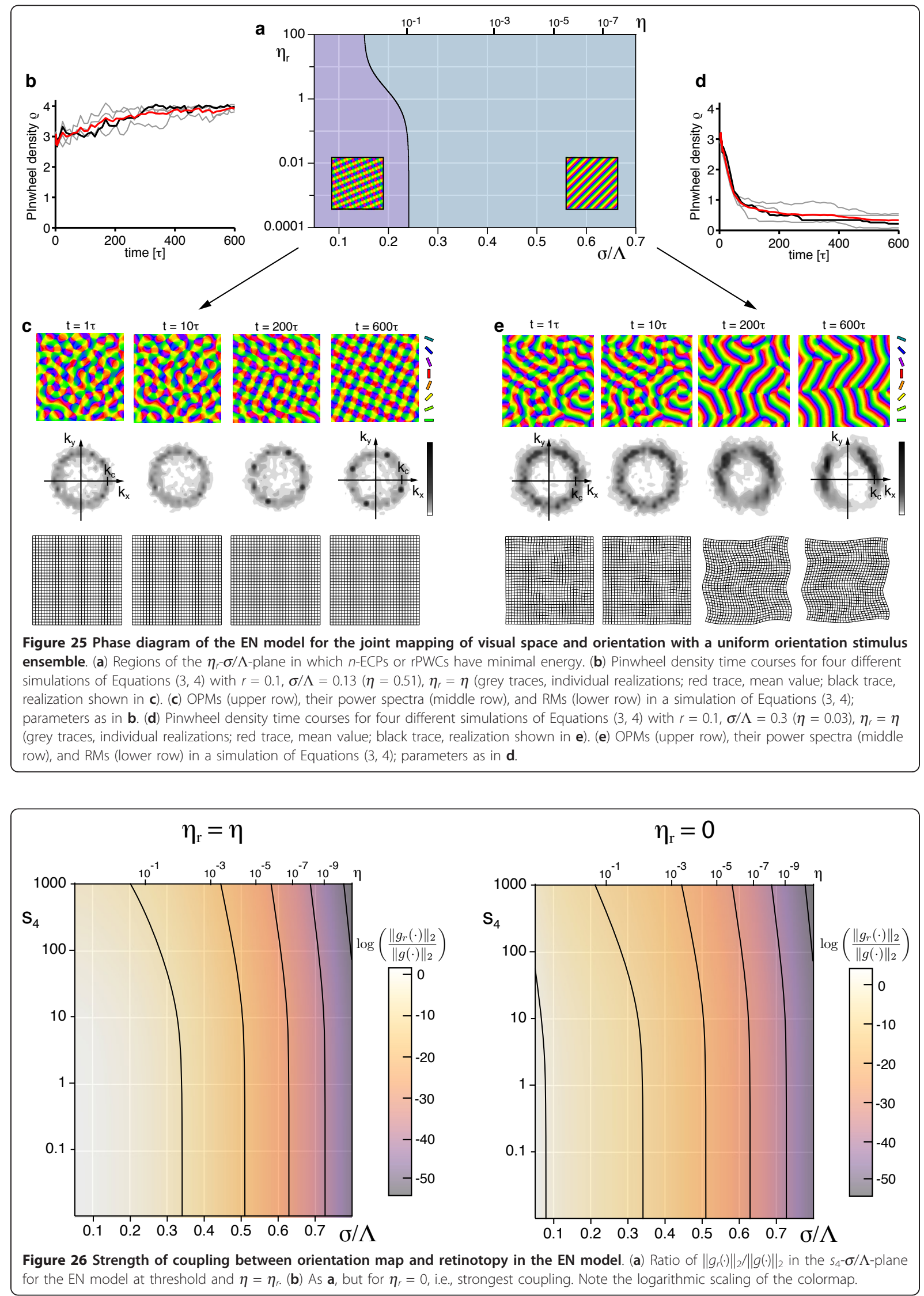


\section{Nonperiodic boundary conditions (grid-like ensemble)}
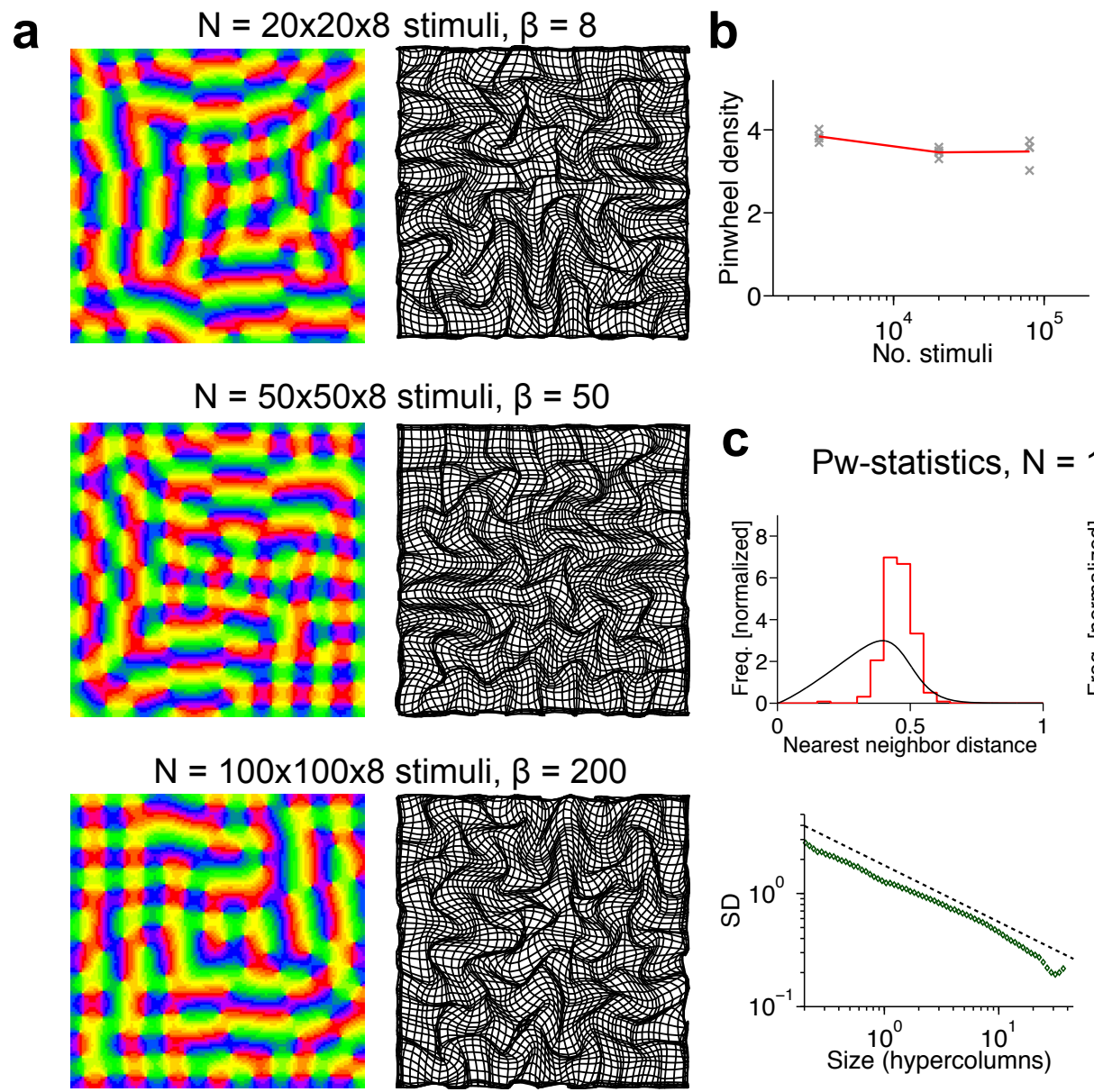

Figure 27 The EN model with deterministic annealing and stimuli, distributed on a grid in feature space. (a) OPMs (left) and RMs (right) for $N=20 \times 20 \times 8$ (upper row), $N=50 \times 50 \times 8$ (middle row) and $N=100 \times 100 \times 8$ (lower row) stimuli and nonperiodic boundary conditions (annealing rate $\chi=0.999$ ). $\beta$ is the continuity parameter in the conventional definition of the EN model (see 'Methods' section, Equation (46)) and is scaled, such that a comparable number of columns emerges in all simulation for each N. (b) Pinwheel densities of EN solutions for different numbers of stimuli (annealing rate $\chi=0.999$ ). Crosses mark individual simulations, red line indicates average values. (c) Statistics of nearest neighbor pinwheel distances for pinwheels of (upper left) arbitrary and (upper right) opposite and equal charge for $100 \times 100 \times 8$ stimuli and nonperiodic boundary conditions, averaged over four simulations (red curves). Black curves represent fits to the experimental data from [38]. Lower left: SD of pinwheel densities estimated from randomly selected regions in the OPM. Black dashed curve indicates SD for a two-dimensional Poisson process of equal density.

dynamics simulations with Laplacian term. The larger the set of stimuli, the more stripe-like are the OPMs obtained (Figure 28a) and consequently pinwheel densities decrease (Figure 28b, upper right). The exponent for the SD is considerably lower than for the Poisson process (Figure 28b, upper right).Typical NN-pinwheel distances concentrate around half the typical column spacing and in particular pinwheel pairs with short distances lack completely (Figure 28b, lower left and right).
For nonperiodic boundary conditions and random stimuli, we found that retinotopic distortions are much more pronounced. They however decreased with increasing number of stimuli. For large stimulus numbers, we observed stripe-like orientation preference domains which are interspersed with lattice-like pinwheel arrangements (see Figure 28c), lower row, upper left corner of the OPM). Similarly to the periodic boundary conditions, short distance pinwheel pairs 


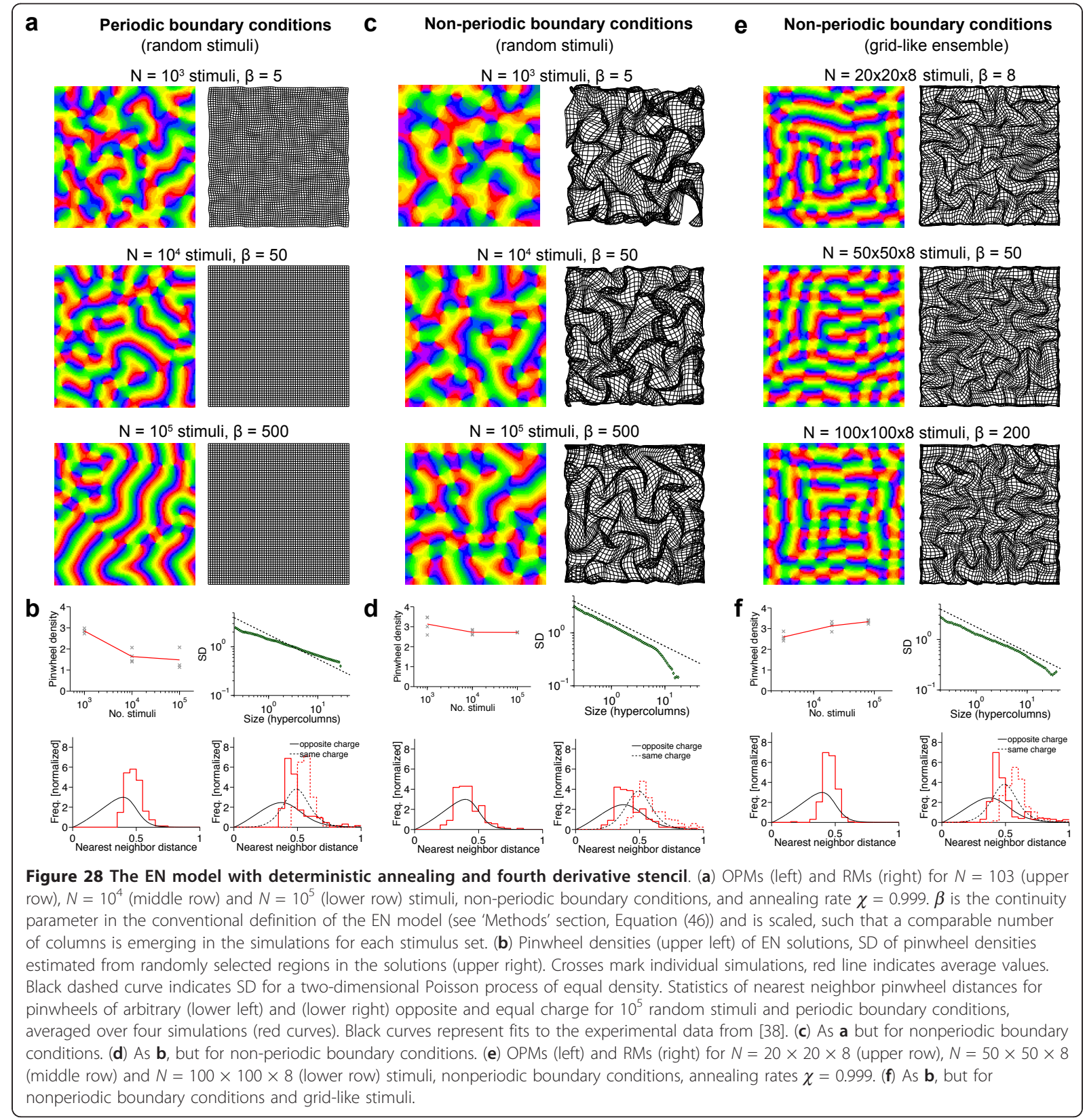

occur much less frequently than in the experimentally observed maps, indicating an increased regularity in the pinwheel arrangements compared to realistic OPMs (Figure 28d, lower left and right). This regularity also manifests itself in a smaller exponent of the SD compared to the Poisson process (Figure 28d).

Simulations with grid-like stimulus as, e.g., used in $[64,65]$ displayed a strong tendency toward rhombic pinwheel arrangements analogous to the second derivative case (Figure 27e,f)

\section{Additional material}

Additional file 1: Rhombic pinwheel crystallization in the EN model The movie shows OPMs (left) as well as their power spectrum (right). In the left panel, colors encode preferred orientation and brightness orientation selectivity. The simulation of the EN model was obtained by gradient descent dynamics with circular stimulus ensemble and fixed retinotopy. The simulation was started from the unselective fixed point $z$ $(\mathbf{x}, t=0)=0$ (parameters: $r=0.1, \sigma / \Lambda=0.1(\eta=0.67))$.

Additional file 2: Pinwheel annihilation in the EN model. The movie shows OPMs (left) as well as their power spectrum (right). In the left panel, colors encode preferred orientation and brightness orientation 
selectivity. The simulation of the EN model was obtained by gradient descent dynamics with circular stimulus ensemble and fixed retinotopy. The simulation was started from the unselective fixed point $z(\mathbf{x}, t=0)=$ 0 (parameters: $r=0.1, \sigma / \Lambda=0.3(\eta=0.028)$ ).

Additional file 3: Convergence to fractured stripes in the EN model. The movie shows OPMs (left) as well as their power spectrum (right). In the left panel, colors encode preferred orientation and brightness orientation selectivity. The simulation of the EN model was obtained by gradient descent dynamics with fixed retinotopy. The simulation was started from the unselective fixed point $z(\mathbf{x}, t=0)=0$ (parameters: $r=$ $\left.0.1, \sigma / \Lambda=0.2(\eta=0.2), s_{4}=6\right)$

Additional file 4: Hexagonal pinwheel crystallization in the EN model. The movie shows OPMs (left) as well as their power spectrum (right). In the left panel, colors encode preferred orientation and brightness orientation selectivity. The simulation of the EN model was obtained by gradient descent dynamics with fixed retinotopy. The simulation was started from the unselective fixed point $z(\mathbf{x}, t=0)=0$ (parameters: $\left.r=0.1, \sigma / \Lambda=0.3(\eta=0.028), s_{4}=8\right)$.

\section{Acknowledgements}

We thank E. Bodenschatz, D. Coppola, J. Crowley, A. Gail, T. Geisel, G. Goodhill, M. Huang, M. Kaschube, C. Kirst, S. Löwel, J. Metzger, T. Mooser, L. Reichl, M. Schnabel, D. Tsigankov, and L. White for many inspiring discussions and the Kavli Institute for Theoretical Physics for its hospitality. This study was supported by Grant Nos. 01GQ0922 (Bernstein Focus Learning) and 01GQ1005B (Bernstein Center for Computational Neuroscience, Göttingen) from the German Ministry for Education and Science (BMBF), Grant WWZN2632 from the Volkswagen Foundation, HFSP Grant Nos. RGP63/2003 and RGY0065/2007 as well as the NSF Grant No. NSF PHY05-51164.

\section{Author details}

${ }^{1}$ Max-Planck-Institute for Dynamics and Self-organization, Am Fassberg 17, D 37077 Göttingen, Germany. ${ }^{2}$ Bernstein Center for Computational Neuroscience, Am Fassberg 17, D-37077 Göttingen, Germany. ${ }^{3}$ GeorgAugust-University, Faculty of Physics, Friedrich-Hund-Platz 1, D-37077 Göttingen, Germany. ${ }^{4}$ Kavli Institute for Theoretical Physics, Santa Barbara, CA 93106-4030, USA

\section{Authors' contributions}

WK performed analytical calculations and numerical simulations. FW designed the study and performed analytical calculations. All authors read and approved the final manuscript.

\section{Competing interests}

The authors declare that they have no competing interests.

Received: 2 March 2011 Accepted: 29 December 2011 Published: 29 December 2011

\section{References}

1. Hubel DH, Wiesel TN: Shape and arrangement of columns in cats striate cortex. J Neurophysiol 1963, 160:559.

2. Hubel DH, Wiesel TN: Ferrier lecture. Functional architecture of macaque monkey visual cortex. Proc R Soc Lond B 1977, 198:1-59.

3. Grinvald A, Lieke E, Frostig RD, Gilbert CD, Wiesel TN: Functional architecture of cortex revealed by optical imaging of intrinsic signals. Nature 1986, 324(6095):361-364

4. Blasdel GG, Salama G: Voltage-sensitive dyes reveal a modular organization in monkey striate cortex. Nature 1986, 321(6070):579-585.

5. Bonhoeffer T, Grinvald A: Iso-orientation domains in cat visual cortex are arranged in pinwheel-like patterns. Nature 1991, 353(6343):429-431.

6. Blasdel GG: Orientation selectivity, preference, and continuity in monkey striate cortex. J Neurosci 1992, 12:3139-3161.

7. Bonhoeffer T, Grinvald A: The layout of iso-orientation domains in area 18 of cat visual cortex: optical imaging reveals a pinwheel-like organization. J Neurosci 1993, 13:4157-4180.
8. Bartfeld E, Grinvald A: Relationships between orientation-preference pinwheels, cytochrome oxidase blobs, and ocular-dominance columns in primate striate cortex. Proc Natl Acad Sci, USA 1992, 89:11905-11909.

9. Blasdel G, Obermayer K, Kiorpes L: Organization of ocular dominance and orientation columns in the striate cortex of neonatal macaque monkeys. Vis Neurosci 1995, 12(3):589-603.

10. Bosking WH, Zhang Y, Schofield B, Fitzpatrick D: Orientation selectivity and the arrangement of horizontal connections in tree shrew striate cortex. Neurosci 1997, 17(6):2112-2127.

11. White LE, Coppola DM, Fitzpatrick D: The contribution of sensory experience to the maturation of orientation selectivity in ferret visual cortex. Nature 2001, 411(6841):1049-1052.

12. Ohki K, Chung S, Kara P, Hübener M, Bonhoeffer T, Reid C: Highly ordered arrangement of single neurons in orientation pinwheels. Nature 2006 442:925-928.

13. White LE, Fitzpatrick D: Vision and cortical map development. Neuron 2007, 56(2):327-338.

14. von der Malsburg C: Self-organization of orientation sensitive cells in the striate cortex. Kybernetik 1973, 14(2):85-100.

15. Swindale NV: A model for the formation of orientation columns. Proc $R$ Soc Lond B 1982, 215:211-230.

16. Braitenberg V: An isotropic network which implicitly defines orientation columns: discussion of an hypothesis. In Models of the visual cortex. Edited by: Rose D, Dobson VG. John Wiley and Sons; 1985:

17. Linsker R: From basic network principles to neural architecture: emergence of orientation-selective cells. Proc Natl Acad Sci USA 1986, 83(21):8390-8394.

18. Linsker R: From basic network principles to neural architecture: emergence of orientation columns. Proc Natl Acad Sci USA 1986, 83(22):8779-8783.

19. Obermayer $K$, Ritter $H$, Schulten $K$ : A principle for the formation of the spatial structure of cortical feature maps. Proc Natl Acad Sci USA 1990 87(21):8345-8349.

20. Tanaka S: Theory of self-organization of cortical maps: mathematical framework. Neural Netw 1990, 3(6):625-640.

21. Durbin R, Mitchison G: A dimension reduction framework for understanding cortical maps. Nature 1990, 343(6259):644-647.

22. Wörgötter F, Koch C: A detailed model of the primary visual pathway in the cat: comparison of afferent excitatory and intracortical inhibitory connection schemes for orientation selectivity. J Neurosci 1991, 11(7):1959-1979.

23. Miller KD: A model for the development of simple cell receptive fields and the ordered arrangement of orientation columns through activitydependent competition between ON- and OFF-center inputs. J Neurosc 1994, 14:409-441.

24. Stetter M, Müller A, Lang E: Neural network model for the coordinated formation of orientation preference and orientation selectivity maps. Phys Rev E 1994, 50(5):4167-4181.

25. Wolf $F$, Geisel T: Spontaneous pinwheel annihilation during visual development. Nature 1998, 395(6697):73-78.

26. Wimbauer S, Gerstner W, van Hemmen JL: Analysis of a correlation-based model for the development of orientation-selective receptive fields in the visual cortex. Network 1998, 9(4):449-466.

27. Koulakov AA, Chklovskii DB: Orientation preference patterns in mammalian visual cortex: a wire length minimization approach. Neuron 2001, 29(2):519-527.

28. Ernst UA, Pawelzik KR, Sahar-Pikielny C, Tsodyks MV: Intracortical origin of visual maps. Nat Neurosci 2001, 4(4):431-436.

29. Mayer N, Herrmann J, Geisel T: Curved feature metrics in models of visual cortex. Neurocomputing 2002, 44:533-539.

30. Kang K, Shelley M, Sompolinsky H: Mexican hats and pinwheels in visual cortex. Proc Natl Acad Sci USA 2003, 100(5):2848-2853.

31. Lee HY, Yahyanejad M, Kardar M: Symmetry considerations and development of pinwheels in visual maps. Proc Natl Acad Sci USA 2003, 100(26):16036-16040.

32. Wolf F, Geisel T: Universality in visual cortical pattern formation. J Physiol 2003, 97(2-3):253-264.

33. Thomas PJ, Cowan JD: Symmetry induced coupling of cortical feature maps. Phys Rev Let 2004, 92(18):188101-188104.

34. Bressloff PC: Spontaneous symmetry breaking in self-organizing neural fields. Biol Cybern 2005, 93:256-274. 
35. Wolf F: Symmetry, multistability, and long-range interactions in brain development. Phys Rev Let 2005, 95(20):208701-208704.

36. Schnabel M, Kaschube M, Löwel S, Wolf F: Random waves in the brain: Symmetries and defect generation in the visual cortex. Eur Phys $J 2007$ 145:137-157.

37. Reichl $L$, Löwel $S$, Wolf F: Pinwheel stabilization by ocular dominance segregation. Phys Rev Lett 2009, 102:208101-208104

38. Kaschube M, Schnabel M, Löwel S, Coppola DM, White LE, Wolf F Universality in the evolution of orientation columns in the visual cortex. Science 2010, 330(6007):1113-1116

39. Schnabel M, Kaschube M, Wolf F: Pinwheel stability, pattern selection and the geometry of visual space. arXiv:0801.3832v2 [a-bio.NC] 2008.

40. Hensch T, Stryker MP: Columnar architecture sculpted by GABA circuits in developing cat visual cortex. Science 2004, 303:1678-1681.

41. Kaschube M, Schnabel M, Wolf F, Löwel S: Interareal coordination of columnar architectures during visual cortical development. Proc Nat Acad Sci USA 2009, 106(40):17205-17210.

42. Erwin E, Obermayer K, Schulten K: Models of orientation and ocular dominance columns in the visual cortex: a critical comparison. Neural Comp 1995, 7(3):425-468.

43. Horton JC, Hocking DR: An adult-like pattern of ocular dominance columns in striate cortex of newborn monkeys prior to visual experience. I Neurosci 1996, 16(5):1791-1807.

44. Horton JC, Hocking DR: Monocular core zones and binocular border strips in primate striate cortex revealed by the contrasting effects of enucleation, eyelid suture, and retinal laser lesions on cytochrome oxidase activity. J Neurosci 1998, 18(14):5433-5455.

45. Lund JS, Angelucci A, Bressloff PC: Anatomical substrates for functional columns in macaque monkey primary visual cortex. Cereb Cortex 2003, 13:15-24

46. Muir DR, da Costa NMA, Girardin CC, Naaman S, Omer DB, Ruesch E, Grinvald A, Douglas RJ: Embedding of cortical representations by the superficial patch system. Cereb Cortex 2011, 21(10):2244-2260.

47. Kaas JH: Reconstructing the organization of neocortex of the first mammals and subsequent modifications. In Evolution of Nervous Systems: A Comprehensive Reference. Volume 3. Edited by: Kaas JH. Amsterdam: Elsevier; 2006:27-48.

48. Kriegs JO, Churakov G, Kiefmann M, Jordan U, Brosius J, Schmitz J: Retroposed elements as archives for the evolutionary history of placental mammals. PLoS Biol 2008, 4:537-544.

49. Kriegs JO, Churakov G, Jurka J, Brosius J, Schmitz J: Evolutionary history of 7SL RNA-derived SINEs in supraprimates. Trends Genet 2007, 4:158-161.

50. Kielian-Jaworowsk Z, Cifelli RL, Luo ZX: Mammals from the Age of Dinosaurs New York: Columbia University Press; 2004.

51. Bininda-Emonds OR, Cardillo M, Jones KE, MacPhee RD, Beck RM, Grenyer R Price SA, Vos RA, Gittleman JL, Purvis A: The delayed rise of present-day mammals. Nature 2007, 446:507-512.

52. Wible JR, Rougier GW, Novacek MJ, Asher RJ: Cretaceous eutherians and Laurasian origin for placental mammals near the $\mathrm{K} / \mathrm{T}$ boundary. Nature 2007, 447:1003-1006

53. Hooser SDV: Similarity and diversity in visual cortex: is there a unifying theory of cortical computation? Neuroscientist 2007, 13:639-656.

54. Kaschube M, Schnabel M, Löwel S, Coppola DM, White LE, Wolf F: Universality in the evolution of orientation columns in the visual cortex Supporting Online Material. Science 2010, 330(6007):1113-1116.

55. Swindale NV, Shoham D, Grinvald A, Bonhoeffer T, Hübener M: Visual cortex maps are optimized for uniform coverage. Nat Neurosci 2000 3(8):822-826.

56. Swindale NV: How many maps are there in visual cortex? Cereb Cortex 2000, 10(7):633-643

57. Swindale NV, Bauer HU: Application of Kohonen's self-organising feature map algorithm to cortical maps of orientation and direction preference. Proc R Soc Lond B 1998, 265:827-838.

58. Goodhill GJ: Contributions of theoretical modeling to the understanding of neural map development. Neuron 2007, 56:301-311.

59. Kohonen T: Self-organized formation of topologically correct feature maps. Biol Cybern 1982, 43:59-69.

60. Obermayer K, Ritter $\mathrm{H}$, Schulten K: A model for the development of the spatial structure of retinotopic maps and orientation columns. IEICE Trans Fund 1992, E75(A):537-545

61. Kohonen T: Self-Organizing Maps Heidelberg: Springer-Verlag; 1995.
62. Cimponeriu A, Goodhill G: Dynamics of cortical map development in the elastic net model. Neurocomputing 2000, 32:83-90.

63. Goodhill GJ, Cimponeriu A: Analysis of the elastic net model applied to the formation of ocular dominance and orientation columns. Network 2000, 11(2):153-168.

64. Carreira-Perpiñán MA, Goodhill GJ: Influence of lateral connections on the structure of cortical maps. J Neurophysio/ 2004, 92(5):2947-2959.

65. Carreira-Perpiñán MA, Lister RJ, Goodhill GJ: A computational model for the development of multiple maps in primary visual cortex. Cereb Cortex 2005, 15(8):1222-1233.

66. Swindale NV: How different feature spaces may be represented in cortical maps. Network 2004, 15(4):217-242.

67. Yu H, Farley BJ, Jin DZ, Sur M: The coordinated mapping of visual space and response features in visual cortex. Neuron 2005, 47(2):267-280.

68. Farley BJ, Yu H, Jin DZ, Sur M: Alteration of visual input results in a coordinated reorganization of multiple visual cortex maps. J Neurosc 2007, 27(38):10299-10310

69. Keil W, Schmidt KF, Löwel S, Kaschube M: Reorganization of columnar architecture in the growing visual cortex. Proc Natl Acad Sci USA 2010 107(27):12293-12298.

70. Giacomantonio CE, Goodhill GJ: The effect of angioscotomas on map structure in primary visual cortex. J Neurosci 2007, 27(18):4935-4946.

71. Giacomantonio CE, Ibbotson MR, Goodhill GJ: The influence of restricted orientation on map structure in primary visual cortex. Neuroimage 2010 52:875-883.

72. Hoffsümmer $F$, Wolf $F$, Geisel $T$, Löwel $S$, Schmidt K: Sequential bifurcation of orientation-and ocular dominance maps. ICANN95. Proc Int'l Conf Art Neur Net 1995, 535

73. Ringach DL: Haphazard wiring of simple receptive fields and orientation columns in visual cortex. J Neurophysiol 2004, 92:468-476.

74. Chiu C, Weliky M: Spontaneous activity in developing ferret visual cortex in vivo. J Neurosci 2001, 21(22):8906-8914.

75. Spinelli DN, Jensen FE: Plasticity: the mirror of experience. Science 1979 203(4375):75-78

76. Ritter H, Marinetz T, Schulten K: Neural Computation and Self-Organizing Maps New York: Addison-Wesley; 1992

77. Durbin R, Willshaw D: An analogue approach to the traveling salesman problem using an elastic net method. Nature 1987, 326:689-691.

78. Applegate DL, Bixby RE, Chvatal V, Cook WJ: The Traveling Salesman Problem: A Computational Study NJ: Princeton University Press: 2006.

79. Carreira-Perpiñán MA, Goodhill GJ: Generalized elastic nets. arXiv:1108.2840v1 [q-bio.NC] 2011

80. Ritter H, Schulten K: Convergence properties of Kohonen's topology conserving maps: fluctuations, stability, and dimension selection. Bio Cybern 1988, 60:59-71.

81. Obermayer K, Blasdel G, Schulten K: Statistical-mechanical analysis of selforganization and pattern formation during the development of visual maps. Phys Rev A 1992, 45(10):7568-7589.

82. Beaulieu C, Colonnier M: Number of neurons in individual laminae of areas $3 \mathrm{~B}, 4$ gamma, and $6 \mathrm{a}$ alpha of the cat cerebral cortex: a comparison with major visual areas. J Comp Neurol 1989, 279.228-234.

83. Cross MC, Hohenberg PC: Pattern formation outside of equilibrium. Rev Mod Phys 1993, 65(3):854-1086.

84. Manneville P: Dissipative Structures and Weak Turbulence UK: Elsevier Science \& Technology; 1990

85. Cross MC, Greenside H: Pattern Formation and Dynamics in Nonequilibrium Systems Cambridge: Cambridge University Press; 2009.

86. Turing A: The chemical basis of morphogenesis. Philos Trans $R$ Soc Lond $B$ 1952, 237(641):37-72

87. Wolf F: Symmetry breaking and pattern selection in visual cortical development. In École d'Été de Physique Les Houches, 2003, Methods and Models in Neurophysics. Edited by: Chow C, Gutkin B, Hansel D, Maunier C Dalibard J. Elsevier Press; 2005:575-639.

88. Schnabel M: A symmetry of the visual world in the architecture of the visual cortex. PhD thesis University of Göttingen, Department of Physics; 2009

89. Mermin N, Troian S: Mean-field theory of quasicrystalline order. Phys Rev Lett 1985, 54(14):1524-1527.

90. Newell A, Pomeau Y: Turbulent crystals in macroscopic systems. J Phys A 1993, 26:LA29-LA43. 
91. Swindale NV: A model for the coordinated development of columnar systems in primate striate cortex. Biol Cybern 1992, 66(3):217-230.

92. Thomas PJ, Cowan JD: Simultaneous constraints on pre- and postsynaptic cells couple cortical feature maps in a $2 D$ geometric model of orientation preference. Math Med Biol 2006, 23:119-138.

93. Kaschube M, Schnabel M, Wolf F: Self-organization and the selection of pinwheel density in visual cortical development. New J Phys 2008, 10:015009.

94. Reichl L: Symmetry breaking and pattern selection in models of visual development. PhD thesis, Georg August University Göttingen 2010.

95. Yu H, Schummers J, Sur M: The relationship between multiple feature maps in ferret visual cortex at single cell resolution. Soc Neurosci Abstracts 2008, 769.2/KK8

96. Das A, Gilbert CD: Distortions of visuotopic map match orientation singularities in primary visual cortex. Nature 1997, 387(6633):594-598.

97. Goodhill GJ, Willshaw DJ: Application of the elastic net algorithm to the formation of ocular dominance stripes. Network 1990, 1:41-59.

98. Simpson H, Mortimer D, Goodhill GJ: Theoretical models of neural circuit development. Curr Top Dev Biol 2009, 87:1-51.

99. Bauer HU, Pawelzik K: Quantifying the neighborhood preservation of selforganizing feature maps. IEEE Trans Neur Net 1992, 3:570-579.

100. Pawelzik K, Bauer HU, Wolf F, Geisel T: Precise restoration of cortical orientation maps explained by Hebbian dynamics of geniculocortical connections. In ICANN 1996: Art Neur Net. Edited by: von der Malsburg C von Seelen W, Vorbrüggen JC. New York: Springer; 1996:415-419.

101. Rose K: Deterministic annealing for clustering, compression, classification, regression, and related optimization problems. Proc IEEE 1998, 86:2210-2239.

102. Swindale NV: The development of topography in the visual cortex: a review of models. Network 1996, 7(2):161-247.

103. Obermayer K, Schulten GG, Schulten K: Neural network model for the formation and for the spatial structure of retinotopic maps, orientationand ocular dominance columns. In Artificial Neural Networks. Edited by: Kohonen T, Makisara K, Simula O, Kangas J. Elsevier; 1991:501-511.

104. Goodhill GJ, Willshaw DJ: Elastic net model of ocular dominance: overall stripe pattern and monocular deprivation. Neural Comput 1994, 6:615-621.

105. Durbin R, Szeliski R, Yuille A: An analysis of the Elastic Net Approach to the Traveling Salesman Problem. Neural Comput 1989, 1:348-358.

106. Dayan P: Arbitrary elastic topologies and ocular dominance. Neural Comput 1993, 5:392-401.

107. Reichl L, Heide D, Löwel S, Crowley J, Kaschube M, FWolf: Symmetry-based analysis of the coordinated optimization of visual cortical maps. arXiv:1102.3353v2 [q-bio.NC] 2011.

108. Bosking WH, Crowley JC, Fitzpatrick D: Spatial coding of position and orientation in primary visual cortex. Nat Neurosci 2002, 5(9):874-882.

109. Hetherington PA, Swindale NV: Receptive field and orientation scatter studied by tetrode recordings in cat area 17. Vis Neurosci 1999, 4:637-652

110. Buzas P, Volgushev M, Eysel UT, Kisvarday ZF: Independence of visuotopic representation and orientation map in the visual cortex of the cat. Eur $J$ Neurosci 2003, 18:957-968.

111. Wolf F, Bauer HU, Pawelzik K, Geisel T: Organization of the visual cortex. Nature 1996, 382:306.

112. Bressloff PC, Cowan JD, Golubitsky M, Thomas PJ, Wiener MC: Geometric visual hallucinations, Euclidean symmetry and the functional architecture of the striate cortex. Philos Trans R Soc Lond B 2001 356:299-330.

113. Field DJ: Relations between the statistics of natural images and the response properties of cortical cells. J Opt Soc Am A 1987, 4(12):2379-2394.

114. Lee HC, Choe Y: Detecting salient contours using orientation energy distribution. In IEEE IJCNN New York Edited by: Wang D 2003, 206-211.

115. Erwin $E$, Obermayer K, Schulten K: Self-organizing maps: ordering, convergence properties and energy functions. Biol Cybern 1992, 67(1):47-55.

116. Huang M: Spatio-temporal dynamics of pattern formation in the cerebral cortex: visual maps, population response and action potential generation. PhD thesis Georg August University Göttingen; 2009.

117. Mayer NM, Herrmann M, Asada M, Geisel T: Pinwheel stability in a nonEuclidean model of pattern formation in visual cortex. J Korean Phys Soc 2007, 50(S01):150-157.
118. Grabska-Barwinska A, von der Malsburg C: Establishment of a scaffold for orientation maps in primary visual cortex of higher mammals. J Neurosci 2008, 28:249-257.

119. Chapman B, Stryker MP, Bonhoeffer T: Development of orientation preference maps in ferret primary visual cortex. J Neurosci 1996, 16(20):6443-6453.

120. Crowley JC, Katz LC: Early development of ocular dominance columns. Science 2000, 395(5495):1321-1324.

121. Blakemore C, van Sluyters RC: Reversal of the physiological effects of monocular deprivation in the kitten: further evidence for a sensitive period. J Physiol 1974, 237:195-216.

122. Antonini A, Stryker MP: Rapid remodeling of axonal arbors in the visual cortex. Science 1993, 260(5115):1819-1821.

123. Wang BS, Sarnaik R, Cang J: Critical period plasticity matches binocular orientation preference in the visual cortex. Neuron 2010, 65(2):246-256.

124. Frégnac $Y$, Shulz $D$, Thorpe $S$, Bienenstock E: A cellular analogue of visual cortical plasticity. Nature 1988, 333:367-370.

125. Frégnac $Y$, Shulz D, Thorpe S, Bienenstock E: Cellular analogues of visual cortex epigenesis: I. Plasticity of orientation selectivity. J Neurosci 1992, 12:1280-1300.

126. Schuett S, Bonhoeffer T, Hübener M: Pairing-induced changes of orientation maps in cat visual cortex. Neuron 2001, 32:325-337.

127. Kaschube M, Wolf F, Geisel T, Löwel S: Genetic influence on quantitative features of neocortical architecture. J Neurosci 2002, 22(16):7206-7217.

128. Kaschube M, Wolf F, Puhlmann M, Rathjen S, Schmidt KF, Geisel T, Löwel S: The pattern of ocular dominance columns in cat primary visual cortex: intra- and interindividual variability of column spacing and its dependence on genetic background. Eur J Neurosci 2003, 18(12):3251-3266.

129. Berkes $P$, Orbán G, Lengyel M, Fiser J: Spontaneous cortical activity reveals hallmarks of an optimal internal model of the environment. Science 2011, 331:83-87.

130. Abramowitz M, Stegun IA: Handbook of mathematical functions National Bureau of Standards; 1964

doi:10.1186/2042-1001-1-17

Cite this article as: Keil and Wolf: Coverage, continuity, and visual cortical architecture. Neural Systems \& Circuits 2011 1:17.

\section{Submit your next manuscript to BioMed Central and take full advantage of:}

- Convenient online submission

- Thorough peer review

- No space constraints or color figure charges

- Immediate publication on acceptance

- Inclusion in PubMed, CAS, Scopus and Google Scholar

- Research which is freely available for redistribution

Submit your manuscript at www.biomedcentral.com/submit
C BioMed Central 


\section{Chapter 4}

\section{Orientation maps lack hexagonal organization}

\section{Citation}

Keil W, Schnabel M, Coppola D M, Löwel S, White L E, Kaschube M, and Wolf F Orientation maps lack hexagonal organization. Under review.

(C2012 Keil, Schnabel, Coppola, Löwel, White, Kaschube, and Wolf

\section{Original contribution}

Conception and design of the study together with M. Kaschube and F. Wolf. I performed the entire data analysis and wrote the manuscript as well as the Supporting Information together with M. Kaschube. 



\title{
Orientation maps lack hexagonal order
}

\author{
Wolfgang Keil ${ }^{1}$, Michael Schnabel ${ }^{1,2}$, David M. Coppola ${ }^{3}$, Siegrid Löwel ${ }^{4}$, Leonard E. White ${ }^{5}$, \\ Matthias Kaschube $^{6 *}$, and Fred Wolf ${ }^{1}$ \\ ${ }^{1}$ Max Planck Institute for Dynamics and Self-organization, Bernstein Center for Computational Neuroscience, \\ Faculty of Physics, Göttingen University, Göttingen, Germany \\ 2 Physical Sciences-Oncology Center, Northwestern Institute on Complex Systems, Departments of Applied \\ Mathematics and Physics, Northwestern University, Chicago, IL, USA \\ ${ }^{3}$ Department of Biology, Randolph-Macon College, Ashland, VA, USA \\ ${ }^{4}$ Bernstein Center for Computational Neuroscience, Bernstein Focus for Neurotechnology, School of Biology, \\ Göttingen University, Göttingen, Germany \\ ${ }^{5}$ Department of Community and Family Medicine, Duke Institute for Brain Sciences, Duke University, Durham, \\ NC, USA \\ ${ }^{6}$ Frankfurt Institute for Advanced Studies, Faculty of Computer Science and Mathematics, Frankfurt University, \\ Frankfurt, Germany; Max Planck Institute for Dynamics and Self-organization, Göttingen, Germany \\ * Correspondence should be addressed to Matthias Kaschube (kaschube@,fias.uni-frankfurt.de).
}

In recent years, D. Ringach (Ringach, 2004; 2007) has extended an intriguing hypothesis put forward by Soodak (1987) to account for the organization of iso-orientation domains in orientation preference maps (OPMs) that are present in the visual cortex of primates and carnivores. The essence of this hypothesis is that the structure of OPMs is constrained by a sparse sampling of ON and OFF retinal ganglion cell (RGC) mosaics by subcortical visual pathways that provide input to the visual cortex. In a new paper, Paik and Ringach (2011) claimed that iso-orientation domains in the visual cortex are locally organized into hexagonal lattices (Paik \& Ringach, 2011) and that this finding provides strong evidence in support of this hypothesis. We appreciate the merits of this hypothesis and we commend Paik and Ringach for emphasizing the potential contributions of subcortical structures to the organization of cortical maps, a consideration that is widely neglected in studies of developing cortical networks. However, here we show that what Paik and Ringach presented as evidence for hexagonal order in cortical OPMs simply results from an elementary fact of planar geometry and is even found in isotropic random maps that, by construction, are unconstrained by any RGC mosaic and lack any hexagonal organization. By testing for signatures of a retinal constraint towards hexagonal organization in cortical OPMs of large datasets from three species ( $>90$ maps) we found that indicators of hexagonal order were statistically indistinguishable from isotropic random maps. We therefore conclude that the spatial structure of OPMs as assessed from current experimental data does not support the model proposed by Paik and Ringach (Paik \& Ringach, 2011).

Paik and Ringach calculated a spatial auto-correlation function from small patches of OPMs and used the relative angular positions of its secondary peaks as an indicator of hexagonal order (called PRanalysis in the following; see Fig. 3a in Paik \& Ringach, 2011). However, when reproducing the PRanalysis, we found similar peaks not only in the correlation function of experimental maps (Fig. 1a and b), but also of isotropic control maps (Fig. 1, c and d), despite the fact that they lack any hexagonal order. These control maps were drawn from the ensemble of Gaussian random orientation maps (Schnabel et al., 2007) with rotation symmetric auto-correlation function and marginal power distribution matching tree shrew OPMs. This control ensemble is the maximum entropy ensemble consistent with the null hypothesis tested in Paik \& Ringach (2011), critically making fewer additional assumptions than the control ensemble Paik and Ringach used. 
We also applied PR-analysis to known solutions of self-organization models previously shown to accurately reproduce many spatial statistics of OPMs in the visual cortex (Kaschube, Schnabel, Löwel, Coppola, White, \& Wolf, 2010), statistical features that are not addressed in Paik \& Ringach (2011). These maps are roughly repetitive, but spatially irregular (i.e., non-hexagonal), and by construction completely unconstrained by a hexagonal RGC mosaic (Fig. 1e). The PR-analysis, however, again yielded secondary peaks suggestive of an apparent hexagonal order (Fig. 1f).

For our non-hexagonal test maps (c-f), PR-analysis generates these peaks because i) it is based on relatively small map regions (identical to the maps shown in a, c, e), and ii) these maps, despite being spatially irregular, exhibit a typical column spacing (periodicity), i.e. their amplitude spectrum is concentrated on a narrow frequency band. Because of the latter, adjacent iso-orientation columns keep a typical distance from each other. Hence, the most likely arrangement of a set of three neighboring iso-orientation columns is an equilateral triangle (Fig. 1e for an example). Because of this triangular arrangement, the auto-correlation calculated for small subregions preferentially exhibits a roughly sixfold modulation (Fig. 1f). When calculating the auto-correlation over larger map regions, this modulation becomes weaker, since the triangles from different subregions are not in register and their contributions cancel out (Suppl. Fig. 1). For maps not dominated by one typical scale, triangular arrangements are less likely even within small subregions and therefore the six-fold modulation is weak or absent (Suppl. Fig. 2). This explains why Paik and Ringach report absence of significant peaks for one particular model of activity-dependent development (Miller, 1994). This particular model is known to generate maps lacking a typical column spacing (Miller, 1994). Thus, we conclude that even in isotropic maps, PR-analysis produces an apparent hexagonal structure in the autocorrelation function if the subregions that the analysis is based on are sufficiently small and the maps are dominated by a typical column spacing.

Can this effect of featuring a typical column spacing already account for the peaks observed for experimental maps or are these significantly larger than those found for controls indicating a real underlying hexagonal organization? To answer these questions, we assessed whether the average autocorrelation function at 60 and 120 degrees (at a distance of one column spacing) is significantly larger in tree shrews $(\mathrm{N}=21$; Fig. 1g) than in groups of isotropic control maps, each consisting of $\mathrm{N}=21$ Gaussian random maps with matched marginal power spectrum ( $n=500$ groups in total; Fig. 1h). Since experimental OPMs and controls have identical spectral widths, they have identical properties in terms of a dominant spatial scale and therefore its effect on the auto-correlation function does not affect the comparison. We found that average auto-correlation functions were very similar for tree shrew and control maps (Fig. 1g, h). In fact, when comparing average values in tree shrews to the distribution obtained by the $n=500$ control groups, they were statistically indistinguishable at both angles 60 and 120 degrees ( $\mathrm{p}=0.38, \mathrm{p}=0.47$, respectively, Suppl. Fig. 3). We obtained similar results when applying the same analysis to OPMs in both galagos $\left(\mathrm{N}=6, \mathrm{p}_{60^{\circ}}=0.06, \mathrm{p}_{120^{\circ}}=0.13\right)$ and ferrets $\left(\mathrm{N}=71, \mathrm{p}_{60} 0^{\circ}=0.35\right.$, $\mathrm{p}_{120^{\circ}}=0.68$ ). These analyses demonstrate that the peaks obtained by the PR-analysis of experimental maps are not indicative of a hexagonal organization of OPMs. Even with our relatively large data set of $\mathrm{N}=98$ hemispheres (Paik \& Ringach (2011) used $\mathrm{N}=8$ hemispheres), we could not reveal any evidence for hexagonal organization that is not common to isotropic (non-hexagonal) maps.

As a second indicator for hexagonal order in OPMs, Paik and Ringach calculated the histogram of peak angles (Fig. 3, b and d, in Paik \& Ringach, 2011). For perfectly hexagonal OPMs, this histogram exhibits sharp peaks at 60 and 120 degrees with height 0.5 . For non-hexagonal maps, peak heights at 60 and 120 degrees are reduced. When applied to measured OPMs, Paik and Ringach found that the distribution of angles exhibited peaks at roughly 60 and 120 degrees with heights around 0.25 . We performed the same analysis in a large database of OPMs from three species ( $>90$ maps). We 
confirmed the presence of such peaks for tree shrew maps ( $\mathrm{N}=21$; Fig. 1g). However, when applying the identical analysis to the isotropic Gaussian control maps (Fig. 1h) and self-organization model maps (Fig. 1j), we found that their histograms exhibited peaks at multiples of 60 degrees that were both comparable in height to those reported in Paik \& Ringach (2011) (Fig.1k, 1) and statistically indistinguishable from those observed for tree shrews ( $\mathrm{p}=0.37$ (Fig. 1k), and $\mathrm{p}=0.72$ (Fig. 11), respectively). Consistently, we found that neither galagos nor ferrets differed from control or model maps $\left(p_{\text {galagos }}=0.16, p_{\text {galagos }}=0.23\right.$ (Fig. $\left.1 \mathrm{~m}\right) ; p_{\text {ferrets }}=0.69, p_{\text {ferrets }}=0.97$ (data not shown)). In sum, the PR analysis did not distinguish real OPMs from non-hexagonal control or model maps. Thus, we did not observe any evidence for hexagonal order in cortical OPMs.

It is possible that hexagonal lattice is a transitory form in map formation that could be demonstrated in PR-analysis of maps from immature animals. In particular, if RGC mosaics would seed the initial OPM one might suspect stronger signatures of hexagonal organization in younger animals that are undergoing map formation. We therefore checked whether the apparent lack of specificity might be due to animal age by performing the PR-analysis in a subsample of immature ferrets (younger than postnatal day 43). Neither were differences between younger and older ferrets $(\mathrm{p}=0.23$, Fig. $1 \mathrm{n})$ nor between young ferrets and their isotropic controls $(\mathrm{p}=0.77)$ or the model $(\mathrm{p}=0.95)$ significant. We thus conclude that hexagonal lattice structure is also not evident in the available examples of maps from developing ferret visual cortex.

What can explain the present discrepancy with Paik \& Ringach (2011), which reported that experimental maps exhibit larger secondary peaks in the auto-correlation than isotropic control maps? First of all, in their study, Paik and Ringach did not compare correlations of real and control maps at 60 and 120 degrees. Instead they compared values at peaks in the auto-correlation of experimental maps (which are often not at 60 and 120 degrees, see Paik \& Ringach (2011) Fig. 3a) to values attained at the same location in control auto-correlations. Thus, they compared an extreme value of the data (the peak) to arbitrary values of the control family (not necessarily peaks). Consequently, this statistical test is heavily biased towards low p-values. For instance, when applying this test to the isotropic control ensemble, we found that $20 \%$ of control maps have "significant" peaks with p-values of 0.01 or smaller (Suppl. Fig. 4). Thus, according to this test, a considerable fraction of the control maps differs "significantly" from the ensemble from which they were drawn. In contrast, a sound hypothesis test applied to a control group should attest such significance to only $1 \%$ of the control maps (Suppl. Fig. 5). A second possible source for the discrepancy might be that the control maps used by Paik and Ringach were confounded by noise to a larger degree than their experimental maps (see Suppl. Fig. 3 in Paik \& Ringach (2011) for example maps). This could be due to the heuristic algorithm they used to generate control maps and the fact that their analysis is based on angle maps (as opposed to difference maps used in our study, see Suppl. Fig. 6). Noise reduces secondary peaks in the auto-correlation function, which could also explain why they appeared smaller in their controls then in the experimental data (see Suppl. Fig. 3 in Paik \& Ringach (2011)).

Taken together, our results show that there is currently no empirical evidence for hexagonal organization of OPMs in the visual cortex and the mechanism proposed in Paik \& Ringach (2011), at least for three of the four species investigated in their study. There is ample evidence that activitydependent mechanisms are shaping the layout of OPMs in the visual cortex and are involved already during the initial emergence of orientation selectivity (White \& Fitzpatrick, 2007). Recent studies of the development of orientation selectivity in cat and mouse visual cortex indicate that the preferred orientations of cortical neurons undergo significant reorganization over the critical period (Kaschube, Schnabel, Wolf, \& Löwel, 2009, Wang, Sarnaik, \& Cang, 2010). In mice, for instance, preferred orientations of binocular neurons emerge statistically independently for the left and the right eye and 
are then progressively matched (Wang, Sarnaik, \& Cang, 2010). Such evidence highlights that it is crucial for explaining the design of orientation selective circuits to understand their stabilization in the presence of ongoing developmental plasticity. As recently shown, the dynamics of network selforganization can successfully and quantitatively account for the spatially complex arrangement of OPMs in the visual cortex (Kaschube, Schnabel, Löwel, Coppola, White, \& Wolf, 2010). It remains to be determined whether and how the self-organization of visual cortical circuits might be constrained by RGC mosaics.

\section{Works Cited}

Kaschube, M., Schnabel, M., Löwel, S., Coppola, D. M., White, L. E., \& Wolf, F. (2010). Universality in the Evolution of Orientation Columns in the Visual Cortex. Science, 330, 1113-1115. Kaschube, M., Schnabel, M., Wolf, F., \& Löwel, S. (2009). Interareal coordination of columnar architectures during visual cortical development. PNAS, 106 (40), 17205-11.

Miller, K. D. (1994). A Model for the Development of Simple Cell Receptive Fields and the Ordered Arrangement of Orientation Columns through Activity-dependent Competition between ON- and OFFCenter Inputs. J Neurosci, 14 (1), 409-41.

Paik, S. B., \& Ringach, D. L. (2011). Retinal origin of orientation maps. Nat Neurosci, 14 (7), 919925.

Ringach, D.L. (2004). Haphazard wiring of simple receptive fields and orientation columns in visual cortex. J Neurophysiol, 92(1), 468-76.

Ringach, D.L. (2007). On the origin of the functional architecture of the cortex. PLoS One, 2(2), e251. Schnabel, M., Kaschube, M., Löwel, S. and F. Wolf (2007). Random Waves in the Brain: Symmetries and Defect Generation in the Visual Cortex. Europhys $J$, 145, 137-157.

Soodak, R. E. (1987). The retinal ganglion cell mosaic defines orientation columns in striate cortex. PNAS, 84(11), 3936-40.

Wang, B.-S., Sarnaik, R., \& Cang, J. (2010). Critical Period Plasticity Matches Binocular Orientation Preference in the Visual Cortex. Neuron, 65, 245-56.

White, L. E., \& Fitzpatrick, D. (2007). Vision and Cortical Map Development. Neuron, 56, 327-338.

\section{Acknowledgements}

We thank J. Crowley, C. Dempsey, and D. Whitney for discussions; B. Bosking for acquiring and processing imaging data; D. Fitzpatrick for support of imaging experiments (NIH/National Eye Institute (NEI) EY06821 and EY11488); and the Kavli Institute for Theoretical Physics for its hospitality. This work was funded by grants from the Human Frontier Science Program and Bundesministerium für Bildung, Wissenschaft, Forschung und Technologie to S.L. and F.W. (01GQ0430 and 01GQ0922); NSF to D.M.C. (0641433); NIH/National Institute of General Medical Sciences to M.K. (P50 GM071508); and the Whitehall Foundation to L.E.W. and was supported in part by the National Science Foundation (NSF PHY05-51164). 


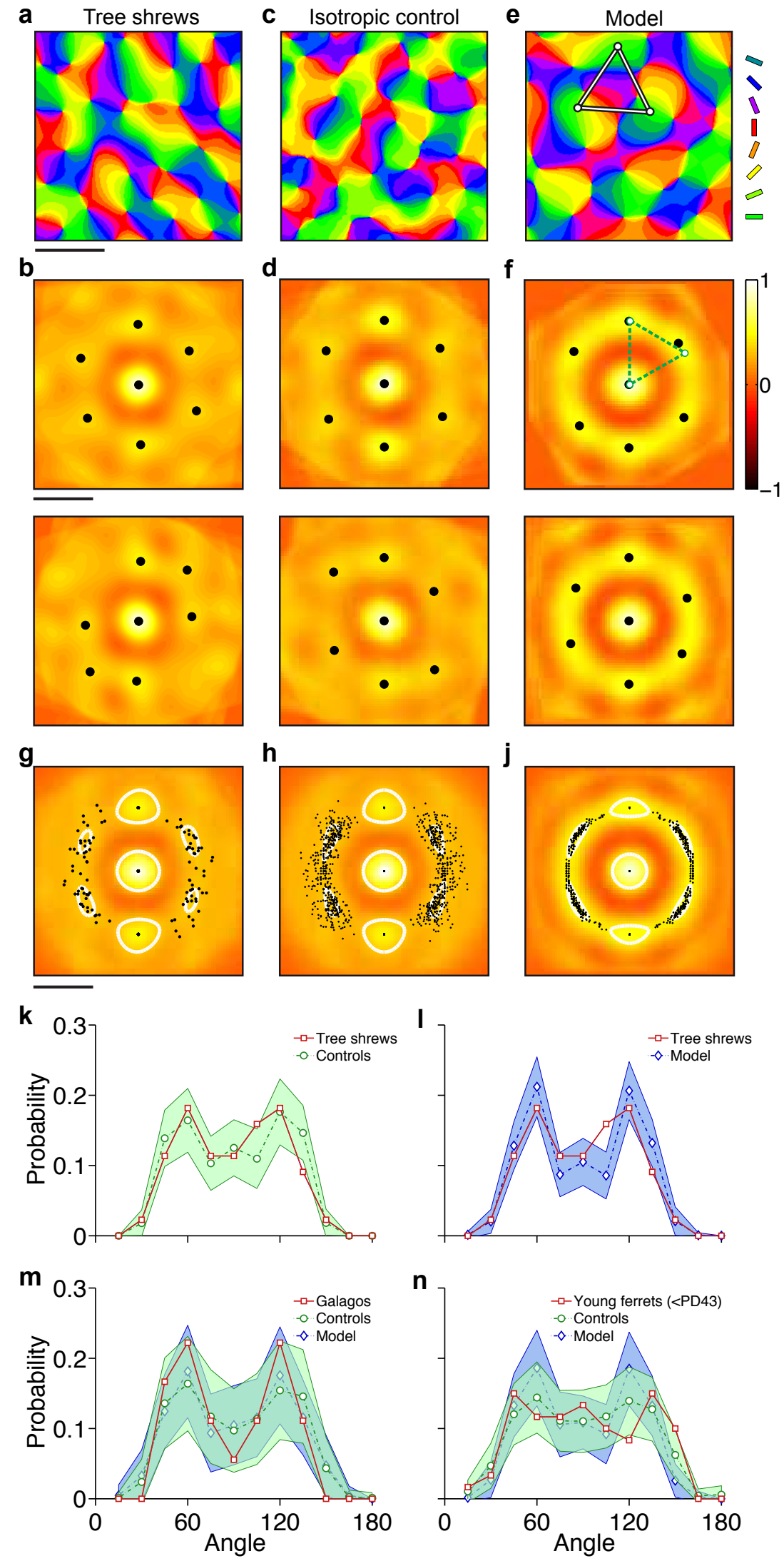




\section{Figure 1:}

(a) Example of a tree shrew orientation map inset of size $3 \times 3 \Lambda$ ( $\Lambda$ is the column spacing, i.e. map periodicity). (b) Auto-correlation of two tree shrew orientation maps following the analysis by Paik $\&$ Ringach (2011), Fig. 3 (PR-analysis). The black dots mark peaks within [0.66 1.33] mean column spacing ( $\sim$ map periodicity) from the origin. (c) $3 \times 3 \Lambda$-inset of an isotropic Gaussian random orientation map with marginal spatial power distribution matching the tree shrew orientation map in a. (d) Same as in $\mathbf{b}$ for two such isotropic random maps. (e) $3 \times 3 \Lambda$-inset of a solution of the long-range interaction model (8-ECP, see Kaschube et al., 2010). The most likely arrangement of a set of three neighboring orientation columns of the same preferred orientation in $\mathbf{a}, \mathbf{c}$ and $\mathbf{e}$ is an equilateral triangle with edge size of one column spacing $\Lambda$ (black-white lines show an example). (f) Same as in b for two model maps. Despite the absence of hexagonal symmetry in the maps in $\mathbf{c}$ and $\mathbf{e}, \mathrm{PR}$-analysis generates peaks in the auto-correlation function at multiples of roughly 120 degrees in $\mathbf{d}$ and $\mathbf{f}$, respectively. Green dashed lines reproduce the triangle from e. (g) Average tree shrew auto-correlation function based on PR-analysis ( $\mathrm{N}=21$ hemispheres). The solid dots mark the peak locations in all individual correlations. (h) Same as in $\mathbf{g}$ for the isotropic random maps in $\mathbf{c}(\mathrm{N}=500)$, randomly generated from the $\mathrm{N}=21$ tree shrew maps. The solid black circles mark the peak locations for a subset of $\mathrm{N}=200$ maps. (j) As in $\mathbf{g}$ for the model maps in $\mathbf{e}(\mathrm{N}=500)$. The solid black circles mark the peak locations for a subset of $\mathrm{N}=100$ maps. White contour lines are plotted at a correlation coefficient of $0.32 \mathrm{in} \mathbf{g}$ and $\mathbf{h}$ and at 0.46 in $\mathbf{j}$ to illustrate the separation of local peaks. (k) Histogram of angular locations of peaks in individual autocorrelations from tree shrews (red squares, $\mathrm{N}=21$ ) and from the isotropic random maps in $\mathbf{c}$ (green circles, $\mathrm{N}=500$ ). Error margins indicate standard deviation of bootstrap distribution obtained with subsamples of size $\mathrm{N}=21$. (l) As in $\mathbf{k}$, but for the model maps in e (blue diamonds, $\mathrm{N}=500$ ).

(m) Same as in $\mathbf{k}$ but for galagos $(\mathrm{N}=6)$, isotropic Gaussian random maps with matched marginal spatial power distribution (green circles, $\mathrm{N}=500$ ), and model solutions (blue diamonds, $\mathrm{N}=500$ ). (n) As $\mathbf{m}$, but for young ferrets $(\mathrm{N}=13)$ imaged before postnatal day 43 . Scale bars in $\mathbf{a}, \mathbf{b}$ and $\mathbf{g}$ equal mean column spacing $\Lambda$. 


\section{Orientation maps lack hexagonal order - Supporting Information}

Wolfgang Keil ${ }^{1}$, Michael Schnabel ${ }^{1,2}$, David M. Coppola ${ }^{3}$, Siegrid Löwel ${ }^{4}$, Leonard E. White ${ }^{5}$, Matthias Kaschube $^{6 *}$, and Fred Wolf ${ }^{1}$

${ }^{1}$ Max Planck Institute for Dynamics and Self-organization, Bernstein Center for Computational Neuroscience, Faculty of Physics, Göttingen University, Göttingen, Germany

${ }^{2}$ Physical Sciences-Oncology Center, Northwestern Institute on Complex Systems, Departments of Applied Mathematics and Physics, Northwestern University, Chicago, IL, USA

${ }^{3}$ Department of Biology, Randolph-Macon College, Ashland, VA, USA

${ }^{4}$ Bernstein Center for Computational Neuroscience, Bernstein Focus for Neurotechnology, School of Biology, Göttingen University, Göttingen, Germany

${ }^{5}$ Department of Community and Family Medicine, Duke Institute for Brain Sciences, Duke University, Durham, NC, USA

${ }^{6}$ Frankfurt Institute for Advanced Studies, Faculty of Computer Science and Mathematics, Frankfurt University, Frankfurt, Germany; Max Planck Institute for Dynamics and Self-organization, Göttingen, Germany

* Correspondence should be addressed to Matthias Kaschube (kaschube@fias.uni-frankfurt.de). 


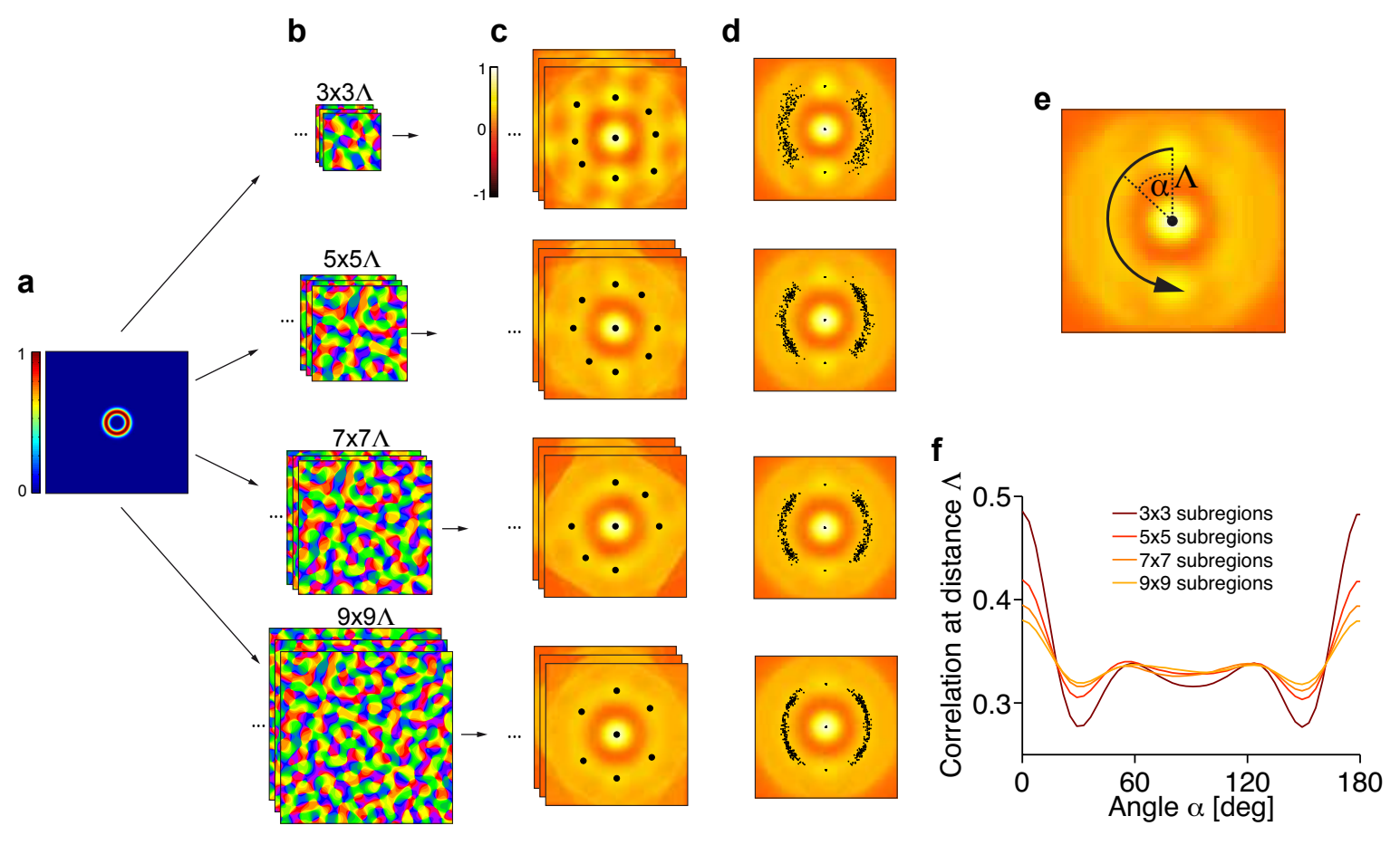

\section{Suppl. Figure 1:}

Six-fold modulation of the auto-correlation function increases with decreasing size of subregion. PRanalysis in Paik \& Ringach (2011) was based on relatively small subregions of 3x3 column spacings $\Lambda$.

(a-d) $\mathrm{PR}$ analysis was performed on $\mathrm{N}=500$ synthesized random isotropic maps based on the methods in Schnabel et al $2007(\beta=10)$ (a), using subregions of size $3 \times 3 \Lambda, 5 \times 5 \Lambda, 7 \times 7 \Lambda$, and $9 \times 9 \Lambda(\mathbf{b})$.

(c) Individual auto-correlation functions for isotropic maps obtained for different subregion sizes. Black dots mark the peak locations. (d) Auto-correlation function averaged over N=500 maps. Black dots mark the peak locations for a subset of $\mathrm{N}=200$ maps. (e) Extraction of the six-fold modulation from the average correlation functions in $\mathbf{d}$. Average correlation values at a distance $\Lambda$ were extracted for $0^{\circ} \leq \alpha \leq 180^{\circ}$. (f) Six-fold modulation for averaged auto-correlation functions from d. Modulation around $60^{\circ}$ and $120^{\circ}$ becomes small only for large subregion sizes. 

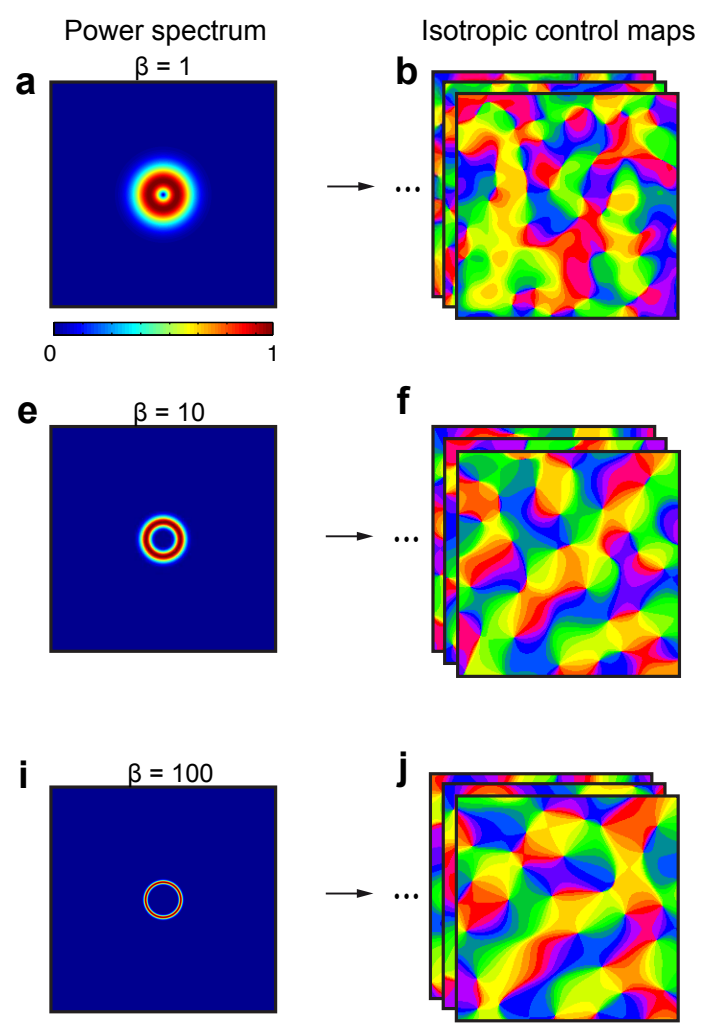

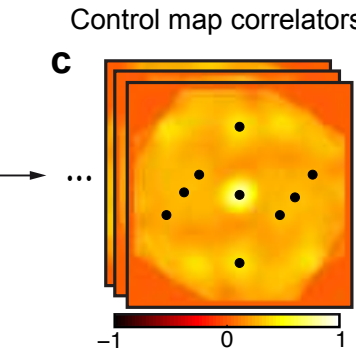

g
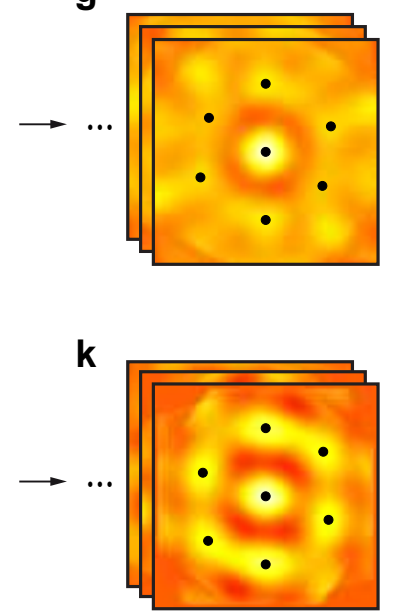

Angular histogram of peaks

d

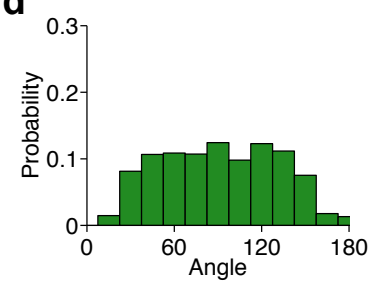

h

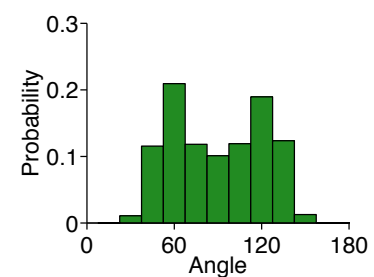

I

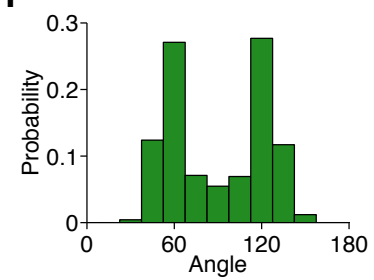

\section{Suppl. Figure 2:}

A six-fold modulation of the auto-correlation function as reported in Paik \& Ringach (2011) arises in isotropic random maps if dominated by a typical column spacing $\Lambda$ (i.e., if the power spectrum is nearly mono frequent).

(a) Isotropic marginal power spectrum (see Schnabel et al. (2007)). The parameter $\beta=1$ controls the radial width of the spectrum. (b) $3 \times 3 \Lambda$ insets of orientation maps, generated with the power spectrum in a. (c) Auto-correlation functions obtained by PR-analysis of the synthesized random isotropic maps in b. Black dots mark peaks in auto-correlation function. (d) Histogram of angular locations of peaks in $\mathbf{c}(\mathrm{N}=500$ maps). (e-h) Same as a-d, but for $\beta=10$. (i-I) Same as $\mathbf{a}-\mathbf{d}$, but for $\beta=100$.

The larger the width of the spectrum, the more dominant is the typical scale in the isotropic maps $(\mathbf{b}, \mathbf{f}$, j). For the ensemble with $\beta=1$, the histogram of angular peak locations is unimodal (d). For $\beta=10$ and $\beta=100$, histograms of angular peak locations exhibit peaks at $60^{\circ}$ and $120^{\circ}(\mathbf{h}, \mathbf{l})$. The more prominent the typical scale is, the more bimodal is the angular histogram $(\mathbf{h}, \mathbf{l})$. For real maps the spectral width corresponds to $\beta \approx 10$. 
Hypothesis test for hexagonal organization used in the present study

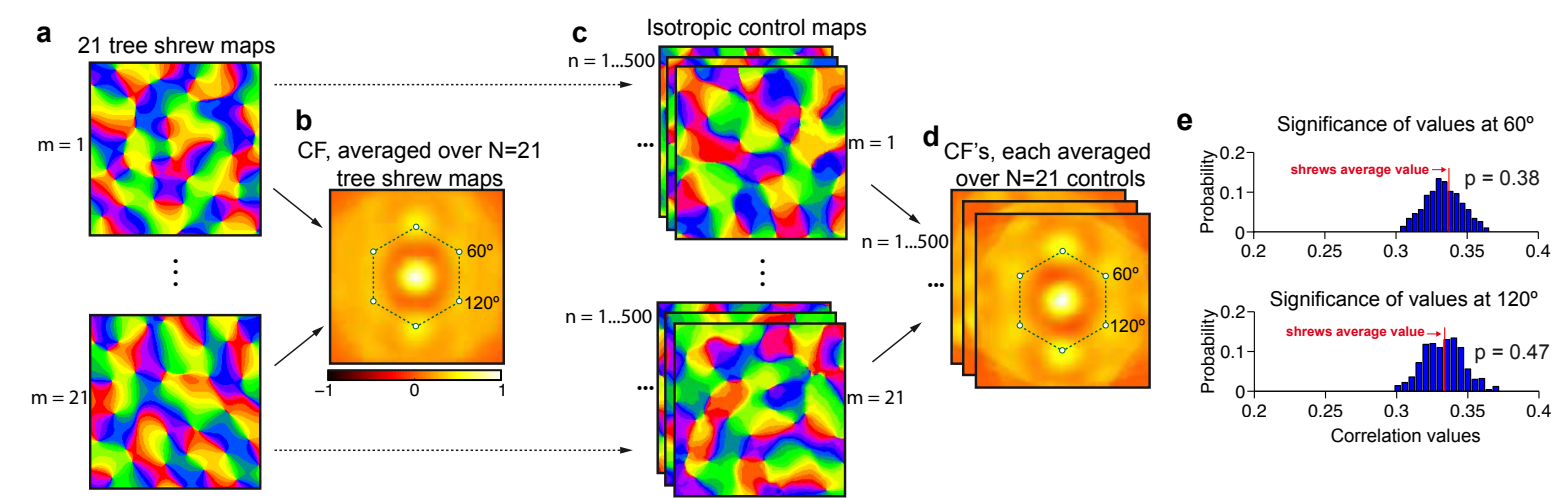

\section{Suppl. Figure 3:}

Illustration of the hypothesis test for hexagonal organization used in the present study. (a-e) For each of the $\mathrm{N}=21$ tree shrew maps (a, $3 \times 3 \Lambda$ insets), an auto-correlation function according to PR analysis was obtained. These auto-correlation functions were averaged to obtain the average tree shrew autocorrelation function in b. Correlation values at an angle of $60^{\circ} / 120^{\circ}$ and a distance of $1 \Lambda$ from the origin were extracted (b). For each tree shrew map, 500 isotropic Gaussian control maps with identical marginal power spectrum were generated (c). 500 average auto-correlation functions were computed, each obtained by averaging over $\mathrm{N}=21$ auto-correlation functions, drawn from the tree shrew map controls (d). (e) Histogram of correlation values of the control maps at $60^{\circ} / 120^{\circ}$ (upper/lower panel). To obtain the two p-values the values attained in the control average autocorrelation functions were compared to the values in the average tree shrew auto-correlation function (red lines). 
Hypothesis test by Paik \& Ringach, 2011
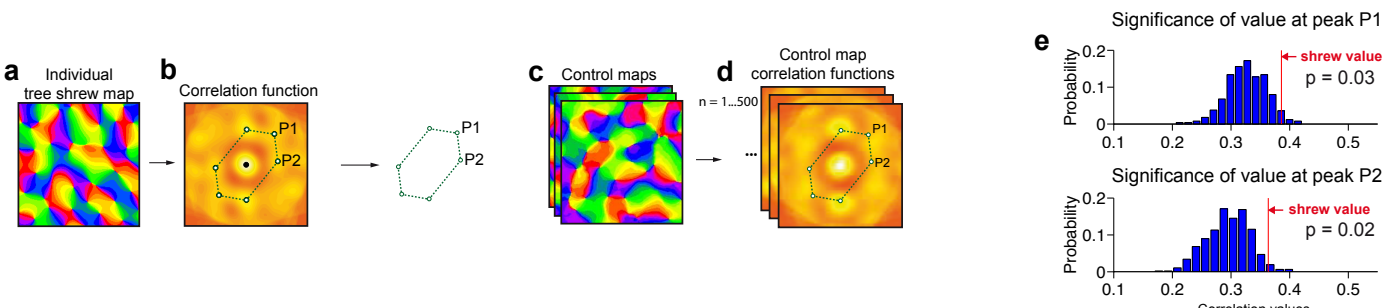

Hypothesis test by Paik \& Ringach, 2011, applied to a control map ensemble with Gaussian statistics

f
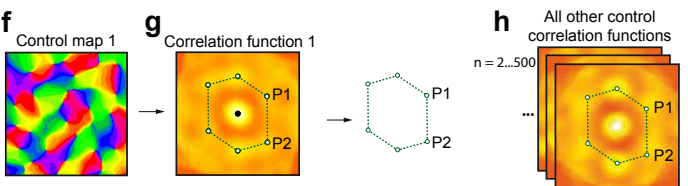

500 p-values for $P 1$
500 p-values for P2
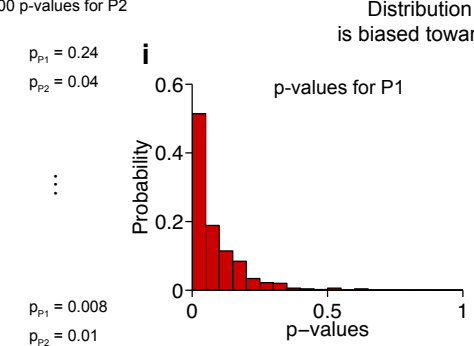

Distribution of p-values
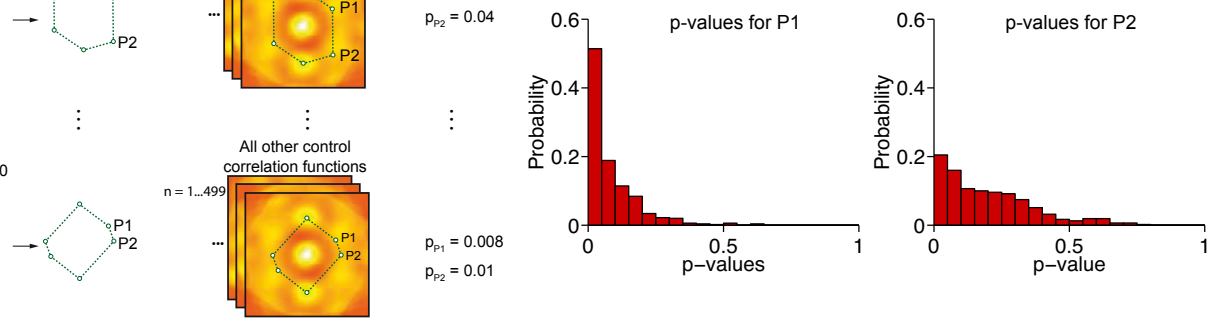

\section{Suppl. Figure 4:}

The hypothesis test in Paik \& Ringach (2011) is biased toward low p-values.

(a-e) Illustration of the hypothesis test used in Paik \& Ringach (2011). For each measured orientation map (a), the auto-correlation function is computed by PR-analysis (based on $3 \times 3 \Lambda$ insets). The positions of the two highest secondary peaks within a range $0.66 \Lambda-1.33 \Lambda$ from the origin (apart from the main peak at $(0,1)$ to which auto-correlation functions were aligned) are extracted. At these positions, named P1 and P2, the correlation values are obtained (b). 500 isotropic control maps with marginal power spectrum matching the spectrum of the measured map are generated (c), their autocorrelation functions computed (d) and the values at the same locations, $\mathrm{P} 1$ and P2, extracted (e) Histogram of the values at P1 (upper panel) and P2 (lower panel) for the controls. Comparing these to the values for the measured map at P1 and P2, respectively (red lines), yields the p-values. Note that the positions P1 and P2 do not necessarily correspond to peak positions in the control maps (see d).

(f-i) Assessment of bias of this hypothesis test.

We generated 500 control maps with Gaussian statistics and marginal power-spectrum matching the tree shrew orientation map power-spectrum in a. Their auto-correlation functions were computed by PR-analysis and peak positions P1 and P2 of the highest and second highest secondary peaks as well as the correlation values attained at $\mathrm{P} 1$ and $\mathrm{P} 2$ were extracted (g).

The significance test used by Paik \& Ringach (2011) (a-e) was performed for each control map, using the remaining 499 control maps as control ensemble (h). By doing so, $500 \mathrm{p}$-values for each of the two peaks were obtained. (i) The histograms of these p-values are strongly skewed toward low p-values, demonstrating that the hypothesis test is biased and results in erroneous significance levels. In contrast, a sound hypothesis test applied to this control ensemble should result in a uniform distribution of pvalues (see Suppl. Fig. 5). 
Hypothesis test for hexagonal organization
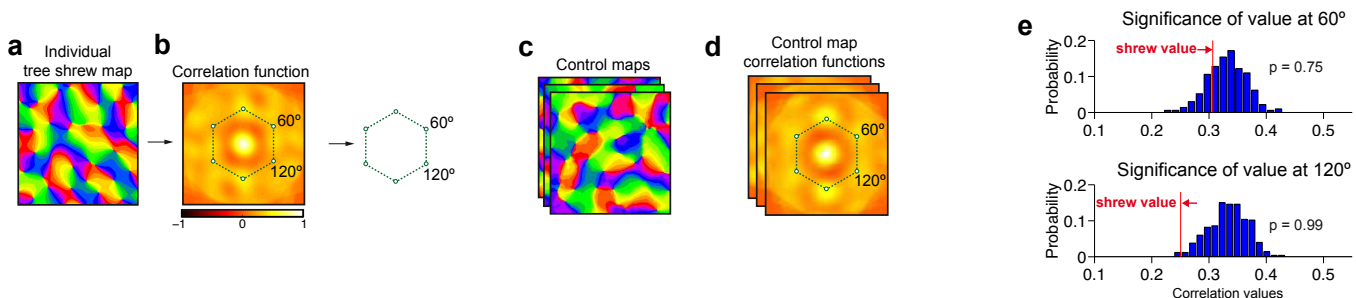

Hypothesis test for hexagonal organization, applied to a control map ensemble with Gaussian statistics

$\mathbf{f}$

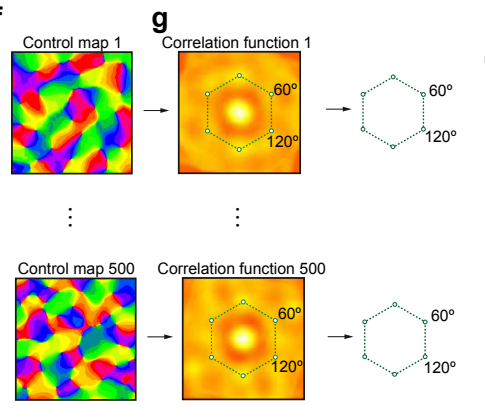

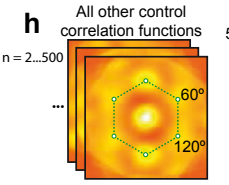

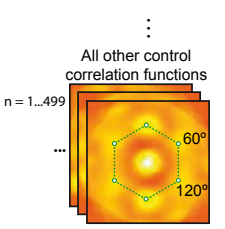

500 p-values at $60^{\circ}$

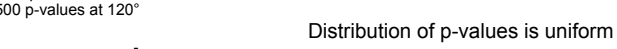

i
(1)

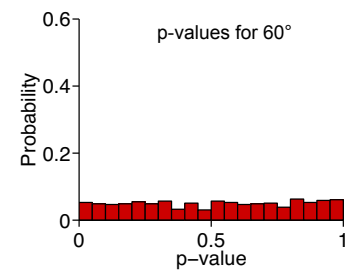

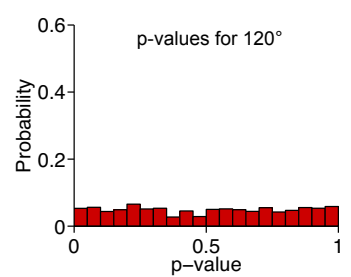

\section{Suppl. Figure 5:}

An unbiased hypothesis test for hexagonal organization (compare with Suppl. Fig. 4).

(a-e) Illustration of a hypothesis test for hexagonal organization of an individual orientation map. For each measured orientation map (a), the auto-correlation function is computed by PR-analysis and correlation values attained at $60^{\circ} / 120^{\circ}$ are extracted (b). 500 isotropic control maps with marginal power spectrum matching the spectrum of the measured map are generated (c) and their autocorrelation function is computed (d). (e) Histogram of the values attained at $60^{\circ} / 120^{\circ}$ (lower/upper panel) in the family of control auto-correlations. These values are compared to values attained at $60^{\circ} / 120^{\circ}$ in correlation function of the measured map (red lines) to obtain p-values.

(f-i) Assessment of bias of the hypothesis test for hexagonal organization.

We generated 500 control maps with Gaussian statistics and marginal power-spectrum matching the tree shrew orientation map power-spectrum in a. Their auto-correlation functions were computed by PR-analysis and correlation values at $60^{\circ} / 120^{\circ}$ were extracted (g). The hypothesis test for hexagonal organization (a-e) was performed for each control map, using the remaining 499 control maps as control ensemble (h). In this way, $500 \mathrm{p}$-values for each of the two peaks were obtained. (i) Histogram of p-values obtained for the correlation value at $60^{\circ} / 120^{\circ}$ (left/right panel). Both distributions are uniform as expected for an unbiased hypothesis test (compare to Suppl. Fig. 4, i). 
a

Original Tree Shrew map

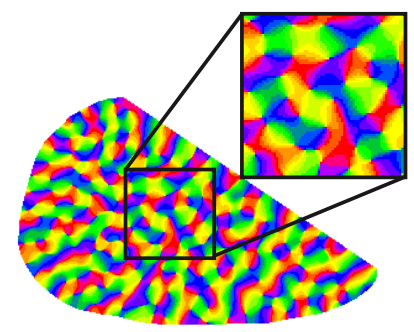

b

Isotropic Gaussian control

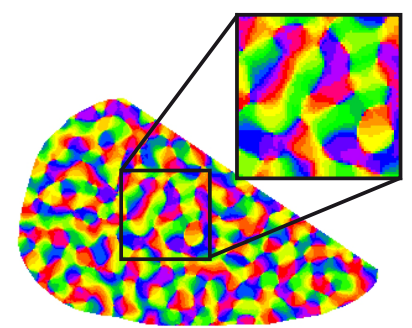

C Control map as in Paik and Ringach, 2011 based on angular map

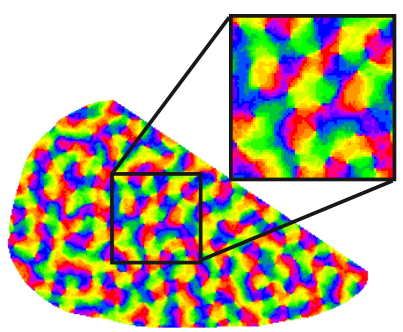

\section{Suppl. Figure 6:}

Control maps based on angular maps as used in Paik \& Ringach (2011) are confounded by highfrequency noise.

(a) Tree shrew angular map obtained from single-condition maps. (b) Isotropic control maps used in the present study are calculated from single-condition maps. (c) Isotropic control maps used in Paik \& Ringach (2011) were calculated from angular maps. Insets in a-c are $3 \times 3 \Lambda$. Note the pronounced contamination by high-frequency noise in $\mathbf{c}$ compared to $\mathbf{a}$ and $\mathbf{b}$. 



\section{Chapter 5}

\section{Robustness of pinwheel stabilization by long-range interactions}

\subsection{Motivation}

The interplay between the emergence of cortical maps and the emergence and refinement of clustered long-range horizontal connections has been a matter of long-standing debate $[41,44,180]$. In cats horizontal connections are unclustered during the first postnatal week prior to eye-opening. Around the time of eye-opening, crude clusters start emerging which are progressively refined and elaborated over the following weeks [76, 80,181]. This refinement of horizontal connections has been shown to be influenced by alteration of activity patterns during this period $[79,81]$. Similarly, in ferrets the system of orientation-specific patches begins to develop around postnatal day 34 (P34), that is very shortly after eye-opening [77], and refines in subsequent weeks. In both cats and ferrets, postnatal cortical maturation, which is characterized by the rapid construction of neural circuits in supragranular layers of V1 and the refinement of clustered connections, occurs synchronously with the maturation of orientation-selective responses and the emergence of orientation preference maps (OPMs) [41-44, 78, 182, 183].

Is it a mere coincidence that these two features emergence in parallel during normal postnatal development? Or do long-range connections and OPMs mutually influence each other during their emergence and refinement? Can the simultaneous development of tangential connections and orientation maps be viewed as a dynamical optimization process? In this chapter, I shed light on these issues by studying optimization models for the coordinated development of long-range tangential connections and orientation maps. Previously, Wolf demonstrated the existence of a class of simple order parameter models in which orientation column patterns converge toward realistic non-crystalline, spatially quasi-periodic arrangements. Pinwheel rich configurations in this model class are stabilized by suppressive long-range interactions [123]. Wolf and Kaschube et al. showed that the order parameter model analyzed by Wolf represents a one-parametric limit of a model for the simultaneous development of tangential connections and orientation preference patterns [11, 159]. In this chapter, I generalize these theoretical results by studying models with different types of biologically plausible long-range interactions and investigate how these interactions might mediate the stabilization of pinwheels in aperiodic irregular arrays. Two main causes 


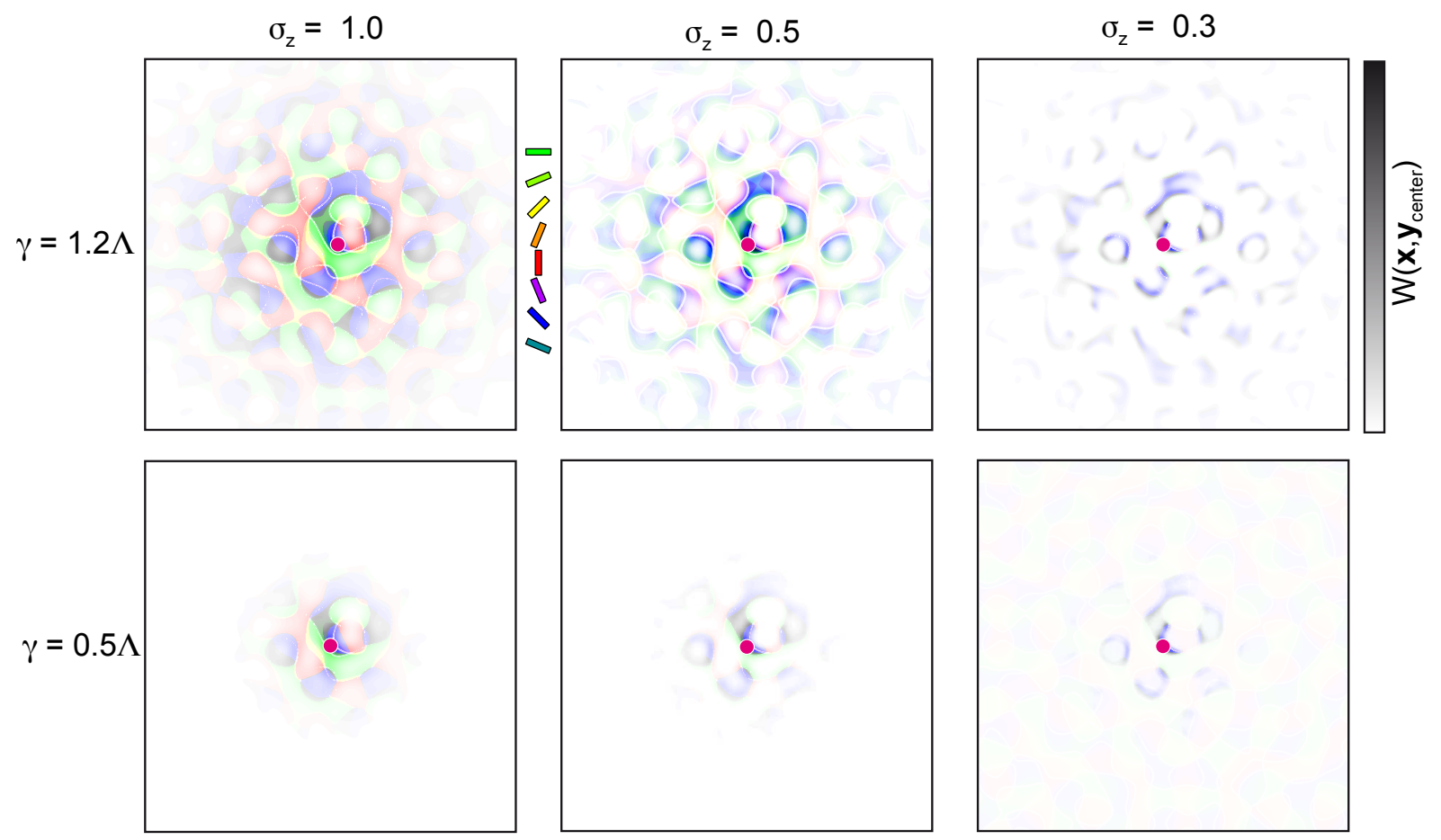

Figure 5.2.1: Orientation-selective long-range connectivity as implemented by the function $W\left(\mathbf{x}, \mathbf{y}_{\text {center }}\right)$ (Eq. (5.2.5)) with exponentially decaying kernel $K(\mathbf{x}) \sim \exp (-|\mathbf{x}| / \gamma)$ for different spatial ranges of interaction $\gamma$ and orientation specificities of the lateral connections $\sigma_{z}$. The red spot in the center is at $\mathbf{y}_{c e n t e r}$ with orientation preference of $135^{\circ}$ (blue color). Brightness encodes absolute value of $W\left(\mathbf{x}, \mathbf{y}_{\text {center }}\right)$. For $\sigma_{z}=1$ connections are nearly unspecific with respect to the difference in preferred orientation. For $\sigma_{z}=0.3$ they almost exclusively connect domains with similar orientation preference around $135^{\circ}$.

may lead to a coordination of column development at widely separated sites in V1: (i) interactions mediated by the above-mentioned long-range horizontal connections [21, 158] or (ii) long-range correlations of visual input due to the statistics of natural scenes [184-187]. These two interaction types may exhibit different spatial dependencies and respond differently to experimental manipulations. To assess whether long-range interactions of different kinds can, in principle, mediate pinwheel stabilization, I construct a variety of analytically solvable model instances in which longrange interactions spatially decay either exponentially or with power-law dependence. I present complete phase diagrams of stable solutions near the pattern formation threshold for these models. In addition, extensive numerical simulations of the different models are carried out to assess potential differences in their dynamics during the emergence of orientation selectivity as well as in the reorganizations taking place during later stages.

\subsection{The development of orientation preference maps and long-range connections as dynamical optimization process}

The pattern of long-range tangential connections can be described by a positive-definite function $W(\mathbf{x}, \mathbf{y})$. Here, $\mathbf{x}$ and $\mathbf{y}$ represent the positions of two columns within the cortex and $W(\mathbf{x}, \mathbf{y})$ gives 
the strength of connection between the neurons at $\mathbf{x}$ and $\mathbf{y}$. As a function of $\mathbf{x}, W(\mathbf{x}, \mathbf{y})$ describes the area of projection of the neurons at location $\mathbf{y}$. As a function of $\mathbf{y}, W(\mathbf{x}, \mathbf{y})$ describes areas of the regions of the visual cortex from which the neurons at location $\mathbf{x}$ receive synaptic inputs. I will assume in the following that the coupling between the sites $\mathbf{x}$ and $\mathbf{y}$ is symmetric, i.e. $W(\mathbf{x}, \mathbf{y})=$ $W(\mathbf{y}, \mathbf{x})$. Three phenomenological requirements are made in order to define a suitable variational dynamics: (1) Both the system of tangential connections and the system of orientation preference columns converge to a stationary state during development. (2) The development of orientation preferences at distant locations within the cortex is coupled via long-range interactions. (3) In the absence of these long-range interactions, the orientation preference pattern should converge to a stable stripe pattern. The first requirement should ensure that the model reproduces the experimentally observed structure of tangential connections. The second requirement ensures causality. The last requirement implies that the model reproduces the behavior of models that have local interactions only.

I start by proposing an energy functional $F[z, W]$ from which the dynamics for the coupled dynamics of the connections $W(\mathbf{x}, \mathbf{y})$ and the orientation preference pattern $z(\mathbf{x})$ can be derived via gradient descent

$$
\begin{aligned}
\partial_{t} z(\mathbf{x}) & =-\frac{\delta F[z, W]}{\delta \bar{z}(\mathbf{x})} \\
\partial_{t} W(\mathbf{x}) & =-\frac{\delta F[z, W]}{\delta W(\mathbf{x}, \mathbf{y})} .
\end{aligned}
$$

As an energy functional to be minimized, I propose

$$
\begin{aligned}
\mathcal{F}[z, W]= & -\int d^{2} \mathbf{p} r|z(\mathbf{p})|^{2}+\int d^{2} \mathbf{p}\left|\left(\Delta+k_{c}^{2}\right) z(\mathbf{p})\right|^{2}-\frac{1}{2} g_{s r} \int d^{2} \mathbf{p}|z(\mathbf{p})|^{4} \\
& +\frac{1}{2 \tau} \iint d^{2} \mathbf{p} d^{2} \mathbf{q} W^{2}(\mathbf{p}, \mathbf{q})-h_{l r} \frac{\sigma_{z}^{2}}{\tau} \iint d^{2} p d^{2} q W(\mathbf{p}, \mathbf{q}) \mathcal{K}(\mathbf{p}, \mathbf{q}) e^{-|z(\mathbf{p})-z(\mathbf{q})|^{2} / 2 \sigma_{z}^{2}},
\end{aligned}
$$

with positive and symmetric kernel $\mathcal{K}(\mathbf{p}, \mathbf{q})=\mathcal{K}(\mathbf{q}, \mathbf{p})$, and a time constant $\tau$. From Equations (5.2.1,5.2.2), the coupled dynamics of $W(\mathbf{x}, \mathbf{y})$ and $z(\mathbf{x})$ is obtained:

$$
\begin{aligned}
\partial_{t} z(\mathbf{x})= & L_{S H}[z]+g_{s r} z(\mathbf{x})|z(\mathbf{x})|^{2} \\
& +\frac{h_{l r}}{\tau} \int d^{2} y[z(\mathbf{y})-z(\mathbf{x})] \mathcal{K}(\mathbf{x}, \mathbf{y}) W(\mathbf{x}, \mathbf{y}) e^{-|z(\mathbf{x})-z(\mathbf{y})|^{2} / 2 \sigma_{z}^{2}} \\
\tau \partial_{t} W(\mathbf{x}, \mathbf{y})= & -W(\mathbf{x}, \mathbf{y})+h_{l r} \sigma_{z}^{2} \mathcal{K}(\mathbf{x}, \mathbf{y}) e^{-|z(\mathbf{x})-z(\mathbf{y})|^{2} / 2 \sigma_{z}^{2}},
\end{aligned}
$$

with $L_{S H}$ being the Swift-Hohenberg operator $L_{S H}=r-\left(\Delta+k_{c}^{2}\right)^{2}$. It follows that the parameter $h_{l r}$ is a coupling constant which describes the strength and sign of the interaction given by $W(\mathbf{x}, \mathbf{y})$. If $h_{l r}=0$, the orientation preference pattern develops freely according to a standard Swift-Hohenberg equation [153, 154]. For $r>0$ the homogeneous state $z(\mathbf{x})=0$ is unstable with respect to a finite band of Fourier modes. After the emergence of an orientation preference pattern with numerous pinwheels, convergence toward stripe patterns via pinwheel annihilation is the expected model behavior. The system of long-range connections relaxes toward $W(\mathbf{x}, \mathbf{y})=0$, i.e. no tangential connections, with time constant $\tau$. If $h_{l r} \neq 0$ in Equations (5.2.3,5.2.4), $W(\mathbf{x}, \mathbf{y})$ and 
$z(\mathbf{x})$ mutually influence each other during development. It will now be investigated whether this mutual influence is able to stabilize the pinwheel-rich layouts, reproducing the experimentally observed common design of orientation preference maps in V1 [11]. In order to do so, I introduce the simplifying assumption that the tangential connections change much faster than the pattern of preferred orientations. In this case, while the connections attain equilibrium, the pattern of orientation preferences changes only slightly. The variable $W(\mathbf{x}, \mathbf{y})$ can then be adiabatically eliminated from the system of equations. From experimental data, this adiabatic elimination can be justified by the fact that the tangential connections appear to link columns with similar orientation preferences early in the development of selective columns [76]. Such early selectivity would not be expected if the orientation preference pattern changed faster than $W(\mathbf{x}, \mathbf{y})$. The pattern of connections in the stationary state reads

$$
W(\mathbf{x}, \mathbf{y})=\mathcal{K}(\mathbf{x}, \mathbf{y}) e^{-|z(\mathbf{x})-z(\mathbf{y})|^{2} / 2 \sigma_{z}^{2}} .
$$

In the following, I assume translational and rotational invariance of $\mathcal{K}(\mathbf{x}, \mathbf{y})$, i.e. $\mathcal{K}(\mathbf{x}, \mathbf{y})=\mathcal{K}(\mathbf{x}-$ $\mathbf{y})$. For spatially decaying interaction kernel $\mathcal{K}(|\mathbf{x}|)$, Equation (5.2.5) realizes the experimental observation that the mature pattern of the long-range tangential connections selectively connects orientation columns with similar orientation preferences if within a certain distance $[21,156,158]$. With the assumption of rotational symmetry effects of axial selectivity [21, 156] are neglected. The system of long-range tangential connections given by Equation (5.2.5) links an orientation column at a location $\mathbf{y}$ with the orientation columns in the area determined by the function $\mathcal{K}(\mathbf{x}, \mathbf{y})$ that have a similar preferred orientation as the position $\mathbf{y}$. The parameter $\sigma_{z}$ determines the selectivity of the connections in Equation (5.2.5). The smaller $\sigma_{z}$ is, the more orientation-selective they are. The choice of the kernel $\mathcal{K}(\mathbf{x})$ will play the key role in the present chapter. As the model is rather general, the spatial dependency of the lateral interactions is not easily inferred from experiment. The main goal of the following analysis is therefore to investigate the influence of different choices of $\mathcal{K}(\mathbf{x})$ on the dynamics of the model as well as its attractor states. Typical examples of $W(\mathbf{x}, \mathbf{y})$ with an exponential kernel $\mathcal{K}(|\mathbf{x}|)=1 /\left(2 \pi \gamma^{2}\right) e^{-|\mathbf{x}| / \gamma}$ are shown in Figure 5.2.1 for different spatial ranges $\gamma$ and connection specificities $\sigma_{z}$.

With the above "slaving" of $W(\mathbf{x}, \mathbf{y})$ to the dynamics of $z(\mathbf{x})$, the coupled system of equations (Eqs. (5.2.3,5.2.4)), reduces to an effective dynamics of $z(\mathbf{x})$, given by

$$
\partial_{t} z(\mathbf{x})=L_{S H} z(\mathbf{x})+g_{s r} z(\mathbf{x})|z(\mathbf{x})|^{2}+g_{l r} \int d^{2} y[z(\mathbf{y})-z(\mathbf{x})] K(|\mathbf{x}-\mathbf{y}|) e^{-|z(\mathbf{x})-z(\mathbf{y})|^{2} / \sigma_{z}^{2}},
$$

with $g_{l r}=\frac{h_{l r}^{2} \sigma_{z}^{2}}{\tau}$ and $K(|\mathbf{x}-\mathbf{y}|)=\mathcal{K}^{2}(|\mathbf{x}-\mathbf{y}|)$. Note that the long-range interactions which enter in the effective equation for $z(\mathbf{x})$ involve the squared spatial dependence of $W(\mathbf{x}, \mathbf{y})$. It is worth mentioning that this effective dynamics for the orientation preference map is again variational, and can be derived via

$$
\partial_{t} z(\mathbf{x})=-\frac{\delta \mathcal{G}[z]}{\delta \bar{z}(\mathbf{x})}
$$


from the energy functional

$$
\begin{aligned}
\mathcal{G}[z]= & -\int d^{2} x\left(r|z(\mathbf{x})|^{2}+\left|\left(\Delta+k_{c}^{2}\right) z(\mathbf{x})\right|^{2}-\frac{1}{2} g_{s r} \int d^{2} \mathbf{x}|z(\mathbf{x})|^{4}\right) \\
& -\frac{1}{2} g_{s r} \int d^{2} \mathbf{x}|z(\mathbf{x})|^{4}+g_{l r} \sigma_{z}^{2} \iint d^{2} \mathbf{w} d^{2} \mathbf{y} K(|\mathbf{w}-\mathbf{y}|) e^{-\frac{|z(\mathbf{w})-z(\mathbf{y})|^{2}}{\sigma_{z}^{2}}} .
\end{aligned}
$$

\subsubsection{Weakly nonlinear analysis of the long-range interaction model}

\section{Dynamics up to third-order terms in $z(\mathbf{x})$}

In the vicinity of the bifurcation point of the orientation preference pattern, the steady states of the model Equation (5.2.6) are determined by the third-order $z(\mathbf{x})$-terms [153, 154]. In order to determine these third-order terms, the right hand side of Equation (5.2.6) has to be rewritten as a Volterra series with respect to $z(\mathbf{x})$, relative to the homogeneous state $z(\mathbf{x})=0$ by series expansion of the integrand of the interaction term in $(z(\mathbf{x}), z(\mathbf{y}))$. For small $z(\mathbf{x})$ or relatively weak orientation selectivity $\sigma_{z} \sim \mathcal{O}(z)$ of the tangential connections, Equation (5.2.5) becomes

$$
W(\mathbf{x}, \mathbf{y} \mid z) \approx g_{l r} K(\mathbf{x}-\mathbf{y})\left(1-\frac{|z(\mathbf{x})-z(\mathbf{y})|^{2}}{\sigma_{z}^{2}}\right)
$$

and consequently, the terms up to third order in $z(\mathbf{x})$ of Equation (5.2.6) which originate from the non-local lateral interactions are given by

$$
\begin{aligned}
N_{\text {nonlocal }} & \approx g_{l r} \int d^{2} y[z(\mathbf{y})-z(\mathbf{x})] K(\mathbf{x}-\mathbf{y})\left(1-\frac{|z(\mathbf{x})-z(\mathbf{y})|^{2}}{\sigma_{z}^{2}}\right) \\
& =g_{l r} L[z]+g_{l r} N_{3}[z, z, \bar{z}] .
\end{aligned}
$$

Thus, one obtains a non-local linear operator

$$
L[z]=-z(\mathbf{x})+\int d^{2} y K(\mathbf{x}-\mathbf{y}) z(\mathbf{y})
$$

and a non-local cubic operator

$$
\begin{aligned}
N_{3}[z, z, \bar{z}]= & \frac{1}{\sigma_{z}^{2}}|z(\mathbf{x})|^{2} z(\mathbf{x})+2 \frac{z(\mathbf{x})}{\sigma_{z}^{2}} \int d^{2} y K(\mathbf{x}-\mathbf{y})|z(\mathbf{y})|^{2} \\
& -2 \frac{|z(\mathbf{x})|^{2}}{\sigma_{z}^{2}} \int d^{2} y K(\mathbf{x}-\mathbf{y}) z(\mathbf{y})-\frac{z(\mathbf{x})^{2}}{\sigma_{z}^{2}} \int d^{2} y K(\mathbf{x}-\mathbf{y}) \bar{z}(\mathbf{y}) \\
& -\frac{1}{\sigma_{z}^{2}} \int d^{2} y K(\mathbf{x}-\mathbf{y}) z(\mathbf{y})|z(\mathbf{y})|^{2}+\frac{\bar{z}(\mathbf{x})}{\sigma_{z}^{2}} \int d^{2} y K(\mathbf{x}-\mathbf{y}) z^{2}(\mathbf{y})
\end{aligned}
$$

The linear term $L[z]$ has a spectrum of eigenvalues

$$
\lambda(k)=-1+F_{0}(k),
$$


where

$$
F_{0}(k)=\int_{0}^{\infty} d r(r K(r)) J_{0}(r k)
$$

is the Hankel transform of the kernel $K(r)\left(J_{0}(x)\right.$ is the zeroth-order Bessel-function of the first kind). The first term in Equation (5.2.9) is just a shift of the entire spectrum. The second term depends on $k$ and thus changes the form of the spectrum. However, if the kernel $K(r)$ mediating intracortical interactions is sufficiently long-range, its Hankel transform $F_{0}(k)$ is a rapidly decaying function in $k$ and $F_{0}\left(k=k_{c}\right) \approx 0$. Thus, this term does not alter the linear growth rates for sufficiently long-range interactions. To describe the formation of a repetitive orientation map, the complete linear operator $L_{S H}[z]+g_{l r} L[z]$ must exhibit a single finite-wavelength maximum in its spectrum. Near pattern formation onset, the precise shape of the spectrum does not impact on the solutions and their stability $[153,160]$. The third-order approximation of the model may thus be written as

$$
\partial_{t} z(\mathbf{x})=L_{S H}[z]++g_{s r} N_{\text {local }}[z]+g_{l r} N_{3}[z, z, \bar{z}] .
$$

The above approximations result in partial integro-differential equation with non-local cubic nonlinearities which is valid in the vicinity of the bifurcation point. As explained in Chapter 1, approximate non-trivial fixed points of such dynamics as well as their stability can be determined via weakly nonlinear analysis by calculating the so-called angle-dependent interaction functions corresponding to the cubic nonlinearities (Sec. 1.5.1). This is carried out in the following.

\section{Mapping the long-range interaction model to a one-parametric model}

The nonlinearities in Equation (5.2.10) consist of a local and a non-local part which depend on three parameters $g_{l r}, \sigma_{z}^{2}$, and $g_{s r}$ in addition to possible parameters of the interaction kernel $K(|\mathbf{x}|)$. To facilitate a complete characterization of the fixed point of this dynamics, a reduction of the number of parameters is desirable. In fact, as the following calculation shows, for sufficiently long-range kernel the number of parameters in addition to the kernel parameters can be effectively reduced to one.

The six terms of the cubic part (Eq. (5.2.8)) are given by

$$
\begin{aligned}
& N_{3}^{1}[z, z, \bar{z}]=|z(\mathbf{x})|^{2} z(\mathbf{x}) \frac{1}{\sigma_{z}^{2}} \\
& N_{3}^{2}[z, z, \bar{z}]=2 \frac{z(\mathbf{x})}{\sigma_{z}^{2}} \int d^{2} y K(\mathbf{x}-\mathbf{y})|z(\mathbf{y})|^{2} \\
& N_{3}^{3}[z, z, \bar{z}]=-\frac{|z(\mathbf{x})|^{2}}{\sigma_{z}^{2}} \int d^{2} y K(\mathbf{x}-\mathbf{y}) z(\mathbf{y}) \\
& N_{3}^{4}[z, z, \bar{z}]=-\frac{z(\mathbf{x})^{2}}{\sigma_{z}^{2}} \int d^{2} y K(\mathbf{x}-\mathbf{y}) \bar{z}(\mathbf{y}) \\
& N_{3}^{5}[z, z, \bar{z}]=-\frac{1}{\sigma_{z}^{2}} \int d^{2} y K(\mathbf{x}-\mathbf{y}) z(\mathbf{y})|z(\mathbf{y})|^{2} \\
& N_{3}^{6}[z, z, \bar{z}]=+\frac{\bar{z}(\mathbf{x})}{\sigma_{z}^{2}} \int d^{2} y K(\mathbf{x}-\mathbf{y}) z^{2}(\mathbf{y}) .
\end{aligned}
$$


According to Equations $(1.5 .8,1.5 .9)$ the angle-dependent interaction functions corresponding to these operators are given by

$$
\begin{aligned}
& g_{1}(\alpha)=-\frac{2}{\sigma_{z}^{2}} \\
& g_{2}(\alpha)=-\frac{2}{\sigma_{z}^{2}}\left(1+F_{0}\left(k_{c} \sqrt{(2(1-\cos \alpha))}\right)\right) \\
& g_{3}(\alpha)=\frac{4}{\sigma_{z}^{2}} F_{0}\left(k_{c}\right) \\
& g_{4}(\alpha)=\frac{2}{\sigma_{z}^{2}} F_{0}\left(k_{c}\right) \\
& g_{5}(\alpha)=\frac{2}{\sigma_{z}^{2}} F_{0}\left(k_{c}\right) \\
& g_{6}(\alpha)=-\frac{2}{\sigma^{2}}\left(F_{0}\left(k_{c} \sqrt{(2(1+\cos \alpha))}\right)\right) \\
& f_{1}(\alpha)=-\frac{1}{\sigma_{z}^{2}} \\
& f_{2}(\alpha)=-\frac{1}{\sigma_{z}^{2}}\left(F_{0}\left(k_{c} \sqrt{(2(1+\cos \alpha))}\right)+F_{0}\left(k_{c} \sqrt{(2(1-\cos \alpha))}\right)\right) \\
& f_{3}(\alpha)=\frac{2}{\sigma_{z}^{2}} F_{0}\left(k_{c}\right) \\
& f_{4}(\alpha)= \frac{1}{\sigma_{z}^{2}} F_{0}\left(k_{c}\right) \\
& f_{5}(\alpha)= \frac{1}{\sigma_{z}^{2}} F_{0}\left(k_{c}\right) \\
& f_{6}(\alpha)=-\frac{1}{\sigma_{z}^{2}} .
\end{aligned}
$$

The complete angle-dependent g-function of Equation (5.2.10) then reads

$$
g(\alpha)=g_{2}\left(\frac{g_{1}}{g_{2}}+\left(F_{0}\left(k_{c} \sqrt{(2(1+\cos \alpha))}\right)+F_{0}\left(k_{c} \sqrt{(2(1-\cos \alpha))}\right)\right),\right.
$$

with effective coefficients (see also [159])

$$
\begin{aligned}
& g_{2}=-2 \hat{g}_{l r} \\
& g_{1}=-2 g_{s r}-4 \hat{g}_{l r}\left(1-2 F_{0}\left(k_{c}\right)\right) .
\end{aligned}
$$

and $\hat{g}_{l r}=g_{l r} / \sigma_{z}^{2}$. Only the shape and not the absolute value of $g(\alpha)$ is relevant to pattern selection, i.e. the determination of stable solutions near threshold [153,154,160,161]. The shape of $g(\alpha)$ only depends on the ratio of $g_{1}$ and $g_{2}$. Furthermore, one of the effective coefficients $g_{1}, g_{2}$ can always be absorbed by a rescaling of the field $z(\mathbf{x})$. One can thus, without loss of generality, reparametrize the model by introducing a single effective coupling parameter $g$, i.e. $2-g=g_{2}$ and $g=g_{1}$, such 
that

$$
\begin{aligned}
& g(\alpha) \approx g+(2-g)\left(F_{0}\left(k_{c} \sqrt{(2(1+\cos \alpha))}\right)+F_{0}\left(k_{c} \sqrt{(2(1-\cos \alpha))}\right)\right) \\
& f(\alpha)=g(\alpha) / 2
\end{aligned}
$$

with $0 \leq g \leq 2$. If the spatial kernel $K(r)$ mediating intracortical interactions is sufficiently longrange, its Hankel transform $F_{0}(k)$ is a rapidly decaying function in $k$, and $F_{0}\left(k=k_{c}\right) \approx 0$. Hence, with the above choice of parametrization, the parameter $g$ is the minimal value of the angledependent interaction function for large interaction ranges. The above results establish that the complete set of attractor states of the long-range interaction model (Eq. (5.2.6)) is approximately determined by a single parameter in addition to the parameters of the interaction kernel $K(r)$. From $2-g=-2 \hat{g}_{l r}$, it is obvious that long-range interactions in the model are always suppressive, as $\hat{g}_{l r}$ has to be smaller than zero. The smaller the effective parameter $g$, the more strongly suppressive are the long-range interactions. The equation $g_{s r}=\frac{3}{2}\left(\frac{4}{3}-g\right)-2(2-g) F_{0}\left(k_{c}\right)$, implies that for large interaction ranges, i.e. $F_{0}\left(k_{c}\right) \approx 0$, the coefficient of the local term $g_{s r}$ becomes positive if $g<4 / 3$. Hence, for $g<4 / 3$ and large interaction ranges, the dynamics is exclusively stabilized by the non-local interactions.

In the following, I analyze specific models with biologically plausible interaction kernels of different spatial dependance. The main question is, whether and how different types of long-range interaction are able to stabilize pinwheel-rich aperiodic OPM layouts. This question is answered in analytical calculations as well as numerical simulations. In the remainder of this chapter, phase diagrams are obtained analytically for the one-parametric limit corresponding to each of the specific models. Weakly nonlinear analysis is always used to determine the essentially complex planform (ECP) fixed points of such dynamics as well as their stability properties via the angle-dependent interaction functions (cf. Chap. 1, Sec. 1.5.1). Numerical results, however, are obtained by simulating all of the cubic nonlinearities (Eq. (5.2.8)) in the effective dynamics of $z(\mathbf{x})$ (Eq. (5.2.10)).

\subsection{Numerical procedures}

The methods used to numerically solve Equation (5.2.10) were adapted from [125, 188] and are only briefly summarized in the following. A fully implicit integrator was used to avoid numerical instabilities and to enable the use of increasing step sizes when approaching an attractor state. The equation

$$
\partial_{t} z(\mathbf{x}, t)=L_{S H} z(\mathbf{x}, t)+N[z(\mathbf{x}, t)], \quad L_{S H}=r-\left(k_{c}^{2}+\Delta\right)^{2}
$$

was discretized in time using a Crank-Nicolson scheme:

$$
\frac{z_{t+1}-z_{t}}{\Delta t}=\frac{\left(L_{S H} z_{t+1}+N\left[z_{t+1}\right]\right)+\left(L_{S H} z_{t}+N\left[z_{t}\right]\right)}{2} .
$$


This nonlinear difference equation was iteratively solved for $z_{t+1}$ by finding the root of the function

$$
G\left[z_{t+1}\right]=\left(-L_{S H}+\frac{2}{\Delta t}\right) z_{t+1}-N\left[z_{t+1}\right]-\left(\left(L_{S H}+\frac{2}{\Delta t}\right) z_{t}+N\left[z_{t}\right]\right)
$$

with Newton's method. 2-dimensional space was discretized with $N \times N$ grid points leading to an $N \times N$ dimensional state vector $\mathbf{u}$. The Newton iteration at step $k$ is then given by

$$
D G\left(u^{k}\right) \Delta \mathbf{u}^{k}=-G\left(\mathbf{u}^{k}\right), \quad \mathbf{u}^{k+1}=\mathbf{u}^{k}+\Delta \mathbf{u}^{k},
$$

with $D G$ being the Jacobian of $G$. With $A=D G\left(\mathbf{u}^{k}\right), \mathbf{b}=-G\left(\mathbf{u}^{k}\right)$, Equation (5.3.1) is a linear equation of the form $A \mathbf{x}=\mathbf{b}$. $D G$ was not explicitly computed and instead the action of $D G$ on $\Delta \mathbf{u}^{k}$ was approximated using finite differences (matrix-free method). To solve the linear system (5.3.1), a Generalized Minimum Residual (GMRES) algorithm [189] combined with a preconditioner to improve convergence was used. A preconditioner $M$ is multiplied to $A \mathbf{x}=\mathbf{b}$ such that $M^{-1} A$ is close to unity. A preconditioner suitable for the Swift-Hohenberg model is the inverse of the SwiftHohenberg operator in Fourier space with a small shift $0<\epsilon \ll 1$ in order to avoid singularities, i.e.

$$
M=\left(\epsilon+\left(k^{2}-k_{c}^{2}\right)^{2}+\frac{2}{\Delta t}\right)^{-1} .
$$

In the integration scheme, a line search method was used to ensure global convergence of Newton's method. Equation (5.3.1) is thus modified to $\mathbf{u}^{k+1}=\mathbf{u}^{k}+\lambda \Delta \mathbf{u}^{k}$, where the function $f\left(\mathbf{u}^{k}\right)=$ $\frac{1}{2} G\left(\mathbf{u}^{k}\right) G\left(\mathbf{u}^{k}\right)$ is iteratively minimized with respect to $\lambda$. To increase speed, in particular when the dynamics approached attractor states, an adaptive step size control was implemented as described in [190]. The above integration scheme was implemented in c++ using the PetSc library.

\subsection{Specific models and their phase diagrams}

\subsubsection{Gaussian interactions}

Wolf as well as Kaschube et al. [11, 123] assumed the long-range interaction to decay as a Gaussian, i.e.

$$
K(r)=\frac{e^{-r^{2} / 2 \sigma^{2}}}{2 \pi \sigma^{2}}
$$

(cf. Fig. 5.4.1a). The Hankel transform $F_{0}(k)$ of this kernel is $F_{0}(k)=e^{-k^{2} \sigma^{2} / 2}$ and, hence, the angle-dependent interaction function of this model according to Equation (1.5.8) is given by

$$
g(\alpha)=g+(2-g) 2 e^{-k_{c}^{2} \sigma^{2}} \cosh \left(k_{c}^{2} \sigma^{2} \cos \alpha\right) .
$$

The one-parametric limit of the long-range interaction model with Gaussian interactions corresponds to the Wolf model [123] introduced in Chapter 1. The angle-dependent interaction function $g(\alpha)$ is depicted in Figure 5.4.1b for different interaction ranges. The function is parametrized by only two variables, namely the coupling parameter $g$ and the interaction range compared to the size of the typical column spacing $\sigma / \Lambda$. According to weakly nonlinear analysis (cf. Sec. 1.5.1), pattern selection in this model can thus be summarized in a two-dimensional phase dia- 
a

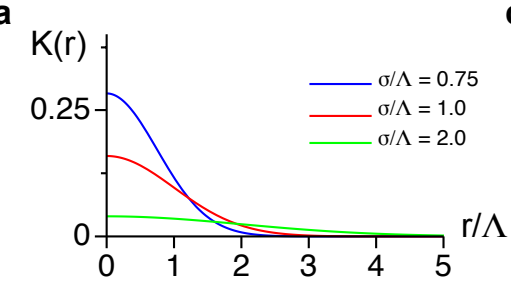

b

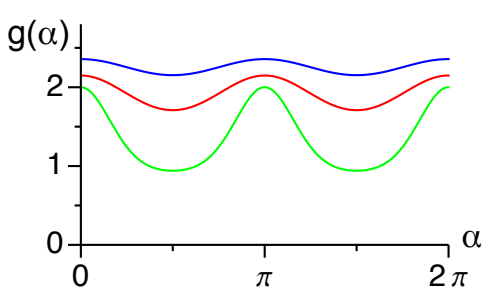

C

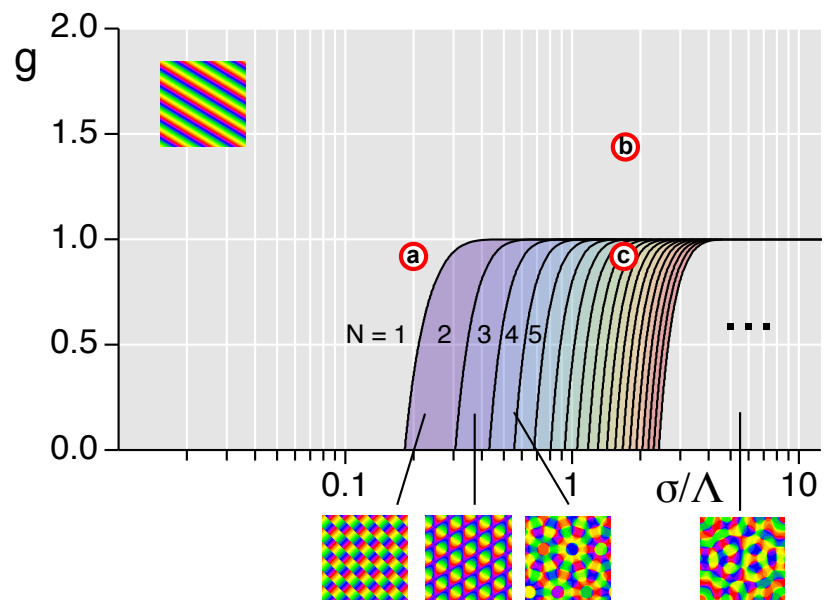

Figure 5.4.1: Phase diagram of the one-parametric limit of the long-range interaction model with interactions decaying as $K(r)=e^{-r^{2} / 2 \sigma^{2}}$. (a) Gaussian interaction kernels for different width of the intracortical interaction $\sigma / \Lambda=0.75$ (blue line), $\sigma / \Lambda=1.0$ (red line), and $\sigma / \Lambda=2.0$ (green line). (b) Angle-dependent interaction functions for different interaction ranges (colors as in a) and $g=0.9$. (c) Phase diagram of the model. The graph shows the regions in the g$\sigma / \Lambda$-plane in which $n$-ECPs have minimal energy $(n=1-20, n>20$ dots). The letters a-c in the phase diagram mark the values of $g$ and $\sigma / \Lambda$ that correspond to the parameters $g_{s r}, g_{l r}$, and $\sigma / \Lambda$ at which the numerical solutions in Figure 5.4.2 for the complete third-order approximation of the long-range interaction model (Eq. (5.2.10)) were obtained.

gram which depicts the regions in the g- $\sigma / \Lambda$-Plane in which $n$-ECPs have minimal energy. In doing so, one exactly recovers the phase diagram obtained in [123] (Fig. 5.4.1c). Whereas for $g>1$ and for short-range interactions pinwheel-free stripe patterns exhibit minimal energy, they lose stability for $g<1$ and sufficiently large $\sigma$. In this regime, $n$-ECPs with several active modes exhibit minimal energy. Solutions with the same number of active modes are energetically degenerate due to permutation symmetry [123]. For large $n, n$-ECP solutions quantitatively resemble the experimentally observed common design of orientation preference maps.

Figure 5.4.2 depicts the dynamics of the long-range interaction model with Gaussian interactions as well as the pinwheel distance statistics for states reached long after pattern emergence. For strongly suppressive but short-range interactions (Fig. 5.4.2a) pinwheels generated during the phase of pattern emergence annihilate quickly during development. The pattern becomes stripelike. Pinwheel density time courses for individual simulations display a fast decrease toward pinwheel densities around zero. At $t=500 \tau$, the statistics of nearest neighbor pinwheel distances strongly deviates from the experimentally observed curves (Fig. 5.4.2a, lower row, middle and right). Pinwheels occur essentially along the domain boundaries between stripes of different orientations. As a consequence, they most frequently have a distance of roughly half the column spacing to their next neighbors. Very similar dynamics arise when the model is considered for long-range but only weakly suppressive interactions (Fig. 5.4.2b). This picture completely changes, when the model is considered with strongly-suppressive and long-range interactions (Fig. 5.4.2c). Rearrangements after the initial map emergence phase are subtle and pinwheels are present in abundance in the later stages of development. Pinwheel density time courses for individual simulations display little variation. Their values remain close to the experimentally observed value of 3.14 [11]. At $t=500 \tau$, pinwheel nearest neighbor statistics (Fig. 5.4.2c, lower 
row, middle and right, red lines) match the fits to the experiments from Kaschube et al. [11] (Fig. 5.4.2c, lower row, middle and right, black lines).

These results not only confirm the results from [11, 123]. Strongly suppressive long-range interactions are able to stabilize pinwheel-rich OPM layouts during development, with pinwheelstatistics matching the common design found in experimental data. If suppression is not strong enough or, alternatively, interactions are not sufficiently long-range, pinwheels either annihilate in pairs or arrange in periodic crystals. They also corroborate the strong correspondence between the full effective dynamics of the orientation preference pattern given by Equation (5.2.6) and the corresponding one-parametric limit.

\subsubsection{Interactions derived from the statistics of long-range horizontal connections}

The assumption in $[11,123]$ of long-range interactions spatially decaying as a Gaussian function was introduced ad hoc, mainly for reasons of analytical tractability. I now consider a different functional form for the long-range interactions which is derived from the statistics of synaptic connections of the system of tangential connections in V1. The resulting model will turn out to be equally analytically tractable.

In a seminal publication in 1997, Bosking et al. analyzed the arrangement of orientation maps and horizontal connections in tree shrew striate cortex by combining optical imaging of intrinsic signals with small injections of biocytin [21]. The biocytin injections resulted in the labeling of neuronal processes and boutons of a small number of cell bodies thereby revealing the prominent long-range patchy connectivity. Figure 5.4.3a shows the labeling obtained in a representative example of such an experiment. Chisum et al. then quantitatively analyzed these data to assess the statistics of the bouton density in relation to receptive field properties in V1 as well as cortical distances [158]. In particular, these authors analyzed the number of boutons as a function of cortical distance (Fig. 5.4.3b,c). As can be inferred from the semi-logarithmic plot in Figure 5.4.3c, the density decays roughly exponentially $\sim \exp (-r / \gamma)$ (see also [180]). It seems therefore promising to consider a model with exponentially decaying long-range interactions:

$$
K(r)=\frac{e^{-r / \gamma}}{2 \pi \gamma^{2}}
$$

(cf. Fig. 5.4.4a). The Hankel transform $F_{0}(k)$ of this kernel then is given by $F_{0}(k)=\left(1+k^{2} \gamma^{2}\right)^{-3 / 2}$ and, hence, according to Equation (5.2.12), the angle-dependent interaction function $g(\alpha)$ of the one-parametric limit of the model reads

$$
g(\alpha)=g+(2-g)\left(\left(1+2 k_{c}^{2} \gamma^{2}(1-\cos \alpha)\right)^{-3 / 2}+\left(1+2 k_{c}^{2} \gamma^{2}(1+\cos \alpha)\right)^{-3 / 2}\right) .
$$

This function is plotted in Figure 5.4.5b for different interaction ranges. Again, it depends on only two variables, namely the coupling parameter $g$ and the interaction range $\gamma / \Lambda$ which, like in the Gaussian case, enables the calculation of a two-dimensional phase diagram. This phase diagram is depicted in Figure 5.4.5c. It is virtually indistinguishable from the phase diagram obtained in the 
a short-range / strongly suppressive
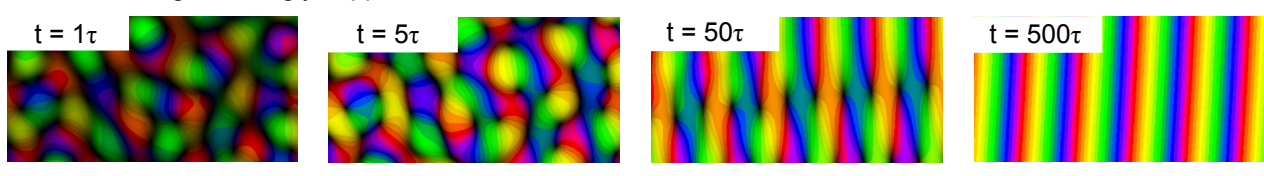

$t=500 \tau$
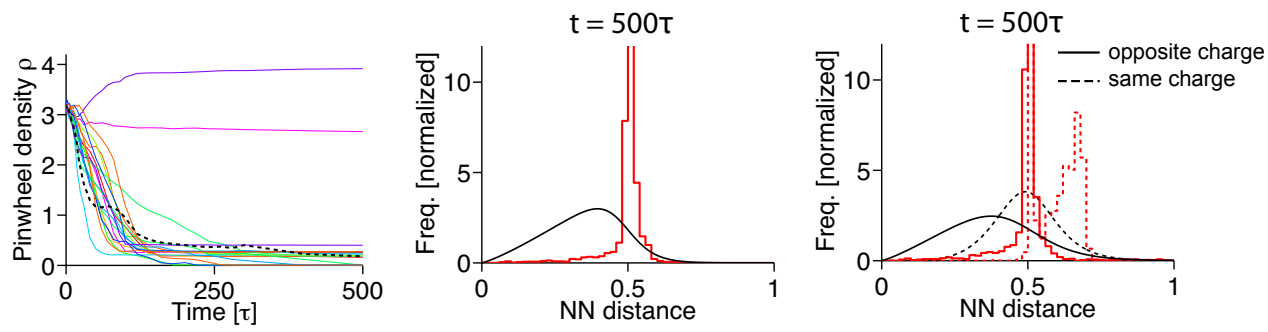

b long-range / weakly suppressive
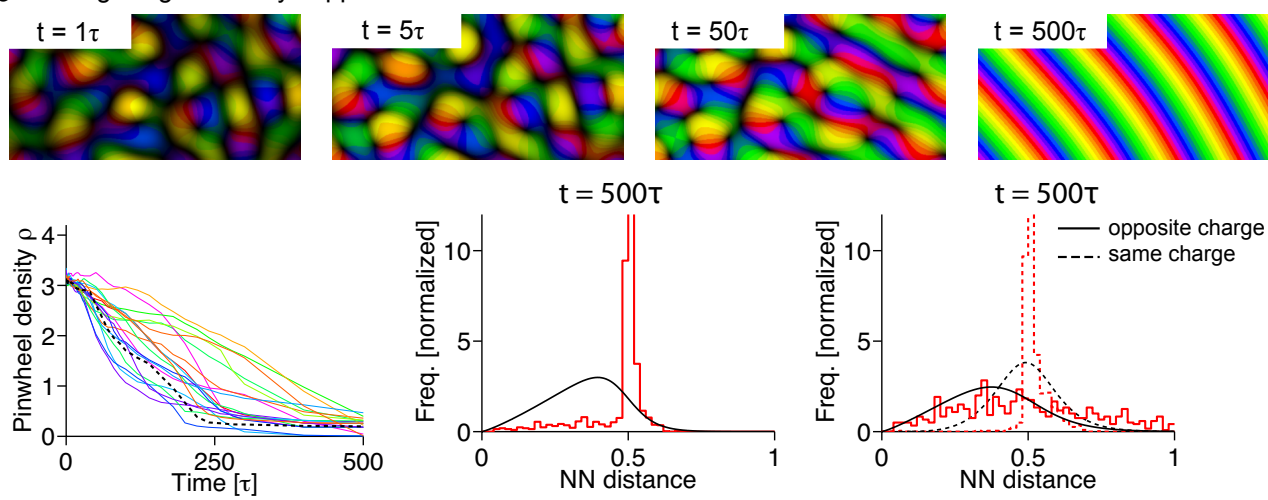

C long-range / strongly suppressive
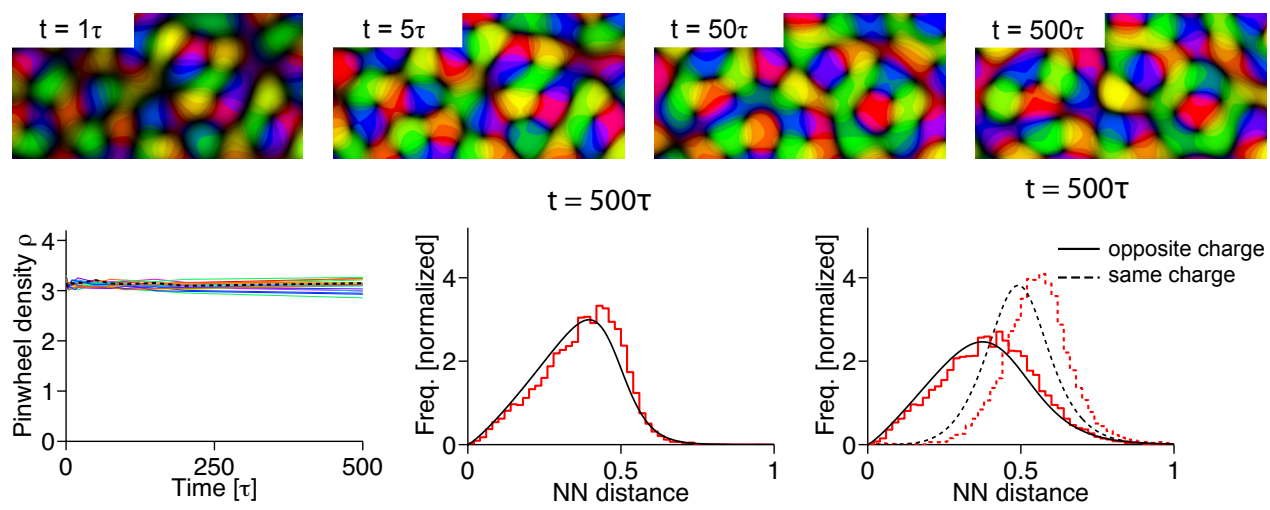

Figure 5.4.2: Dynamics of the long-range interaction model (Eq. (5.2.10)) with interaction strength decaying as a Gaussian (cf. Fig. 5.4.1). (a) Upper row: Snapshots of a numerically obtained solution of the model for short-range strongly suppressive interactions $\left(g_{s r}=-0.39, g_{l r}=-2.04, \sigma / \Lambda=0.2\right)$ with weakly orientation-selective random initial condition. Color encodes preferred orientation, brightness encodes selectivity. Lower row, left: Pinwheel density time courses for $\mathrm{N}=20$ simulations (colored solid traces, individual simulations; black dashed trace, simulation in upper row; parameters as in upper row). Middle and right: Statistics of nearest neighbor pinwheel distances at $t=500 \tau$, averaged over $\mathrm{N}=20$ simulations for pinwheels pairs of arbitrary (middle), opposite and equal sign (right) and parameters as in upper row. (b) As a, but for long-range weakly suppressive interactions $\left(g_{s r}=-0.1, g_{l r}=-1.2, \sigma / \Lambda=1.7\right)$. (c) As a, but for long-range and strongly suppressive interactions $\left(g_{s r}=0.53, g_{l r}=-2.04, \sigma / \Lambda=1.7\right)$. All snapshots are insets from simulations with $22 \Lambda \times 22 \Lambda$ aspect ratio on a $128 \times 128$ mesh. Initial conditions are the same in $\mathbf{a}, \mathbf{b}$, and $\mathbf{c}$. 


\section{a}

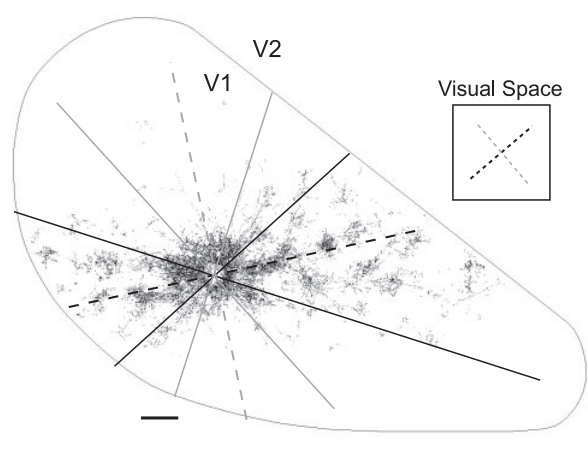

b
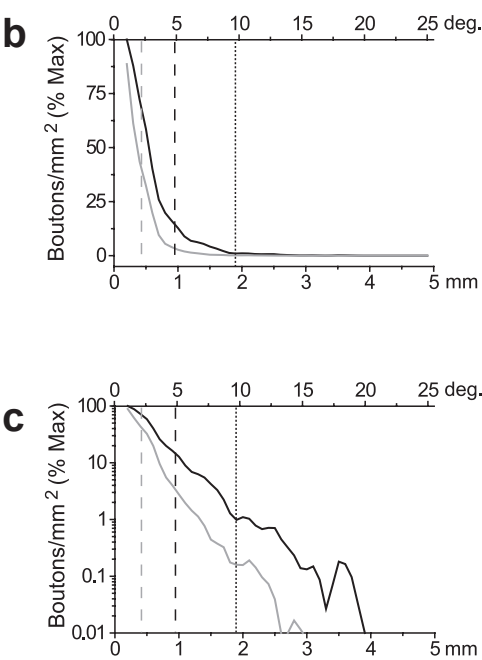

Figure 5.4.3: Horizontal connections in layer $2 / 3$ of tree shrew V1. (a) A plot of labeled boutons within layer 2/3 of area V1 resulting from a biocytin injection into a site with an orientation preference of $40^{\circ}$ (mod. from [21]). The dorsal surface of $\mathrm{V} 1$ is outlined. The axes of preferred $\left(40^{\circ}\right)$ and orthogonal $\left(130^{\circ}\right)$ orientation are indicated with black and gray dashed lines, respectively, both in the icon of visual space to the right and translated onto the cortical surface. Solid black and gray lines show the areas over which boutons were counted ( $\pm 30^{\circ}$ from the two axes). Scale bar, 500 $\mu \mathrm{m}$. (b) Fall-off in bouton density with distance for the preferred (black) and orthogonal (gray) axes as a percentage of maximum density; distance is shown both in millimeters across cortex and degrees of visual space (average of 10 cases) (mod. from [158]). (c) The same data as $\mathbf{b}$ shown with a logarithmic y-scale.

a

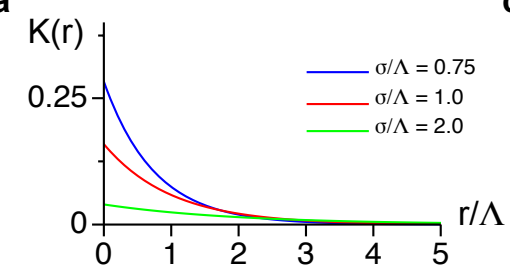

b

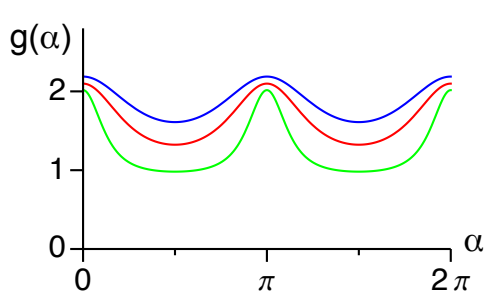

C

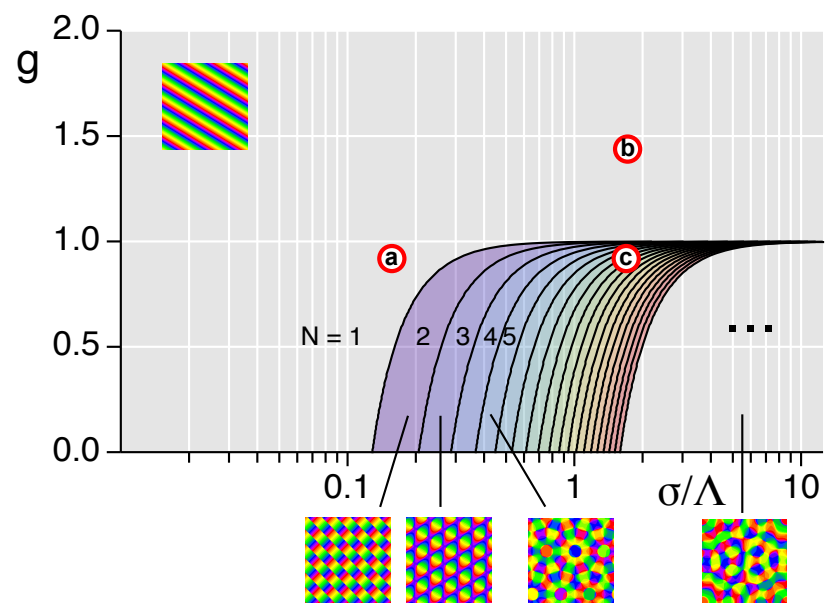

Figure 5.4.4: Phase diagram of the one-parametric limit of the long-range interaction model with interactions decaying as $K(r)=e^{-r / \gamma}$. (a) Exponentially decaying interaction kernels for different widths $\gamma / \Lambda=0.75$ (blue line), $\gamma / \Lambda=1.0$ (red line), and $\gamma / \Lambda=2.0$ (green line). (b) Angle-dependent interaction functions for different interaction ranges (colors as in a) and $g=0.9$. (c) Phase diagram of the model with exponentially decaying interactions. The graph shows the regions in the g- $\gamma / \Lambda$-plane in which $n$-ECPs have minimal energy $(n=1-20, n>20$ dots). The letters a-c in the phase diagram mark the values of $g$ and $\gamma / \Lambda$ that correspond to the parameters $g_{s r}, g_{l r}$, and $\gamma / \Lambda$ at which the numerical solutions in Fig. 5.4.5 for the full third-order approximation of the long-range interaction model (Eq. (5.2.10)) were obtained. 
Gaussian case (Fig. 5.4.1c). Again, whereas for $g>1$ and for short-range interactions pinwheelfree stripe patterns exhibit minimal energy, they lose stability for $g<1$ and sufficiently large $\gamma / \Lambda$. Aperiodic $n$-ECPs with several active modes exhibit minimal energy in this regime. With the data obtained by Bosking et al., one can roughly estimate the decay constant $\gamma$ for the bouton density as a function of cortical distance in tree shrew V1 with $\gamma \approx \Lambda$ (for the preferred orientation). Surprisingly, this value perfectly fits into a regime, where aperiodic pinwheel-rich orientation preference patterns realizing the experimentally observed common design are predicted by the model.

Strong similarity with the Gaussian case is also observed in the numerically obtained solutions. For strongly suppressive but short-range interactions (Fig. 5.4.5a) as well as weakly suppressive long-range interactions (Fig. 5.4.5b), pinwheels generated during the phase of pattern emergence annihilate quickly during subsequent simulation stages. Pinwheel density time courses for individual simulations display a fast decrease towards almost zero pinwheel densities (Figs. 5.4.5a,b, lower rows, left) and the patterns become stripe-like. The statistics of nearest neighbor pinwheel distances exhibits strong peaks at a distance of roughly half the column spacing to their next neighbors (Figs. 5.4.5a,b, lower rows, middle and right). Only when the model is considered with strongly suppressive and long-range exponentially decaying interactions (Fig. 5.4.5c) pinwheels are present in abundance in the later simulation stages. Pinwheel density time courses remain close to the experimentally observed value of 3.14 and pinwheel nearest neighbor statistics at $t=500 \tau$ agree well with experimental data (Fig. 5.4.5c, lower row, middle and right, red lines).

In summary, the model behaviors for Gaussian and exponential interactions are strikingly similar. Neither in the phase diagram of the one-parametric limit nor in the dynamics of orientation preference patterns, substantial differences could be observed.

\subsubsection{Non-orientation-selective local connectivity}

So far, I have examined two types of biologically plausible orientation-selective interactions which mainly differed in their spatial asymptotic behavior. Common to both of these long-range interactions considered is that they most strongly connected sites at short distances. It is however unclear at present and also difficult to extract from biocytin labeling experiments due to the extremely high labeling density close to the injections sites, to which extent the connections to nearby sites are in fact orientation-selective at all. Figure 5.4.6 illustrates this point for two different bouton-labeling experiments in ferret and tree shrew visual cortex (redrawn from [44] and [21]). While at large distances from the injection site (center of the red region), bouton density exhibits clear patches, bouton density close to the injection site appears rather be isotropic and, thus, non-orientationselective.

To investigate the influence of this potential feature of the system of tangential connections, I now consider an analytically tractable model in which the Kernel $K(r)$ vanishes at $r=0$, namely

$$
K(r)=\frac{1}{2 \pi \gamma^{2} \Gamma(m+1)}\left(\frac{r}{\gamma}\right)^{m-1} e^{-r / \gamma} .
$$

Local interactions in such a model are exclusively mediated by the linear term $L_{S H}$ and the nonlinear term $\sim|z(\mathbf{x})|^{2} z(\mathbf{x})$ in Equation (5.2.6). These interactions are independent of the local dif- 
a short-range / strongly suppressive
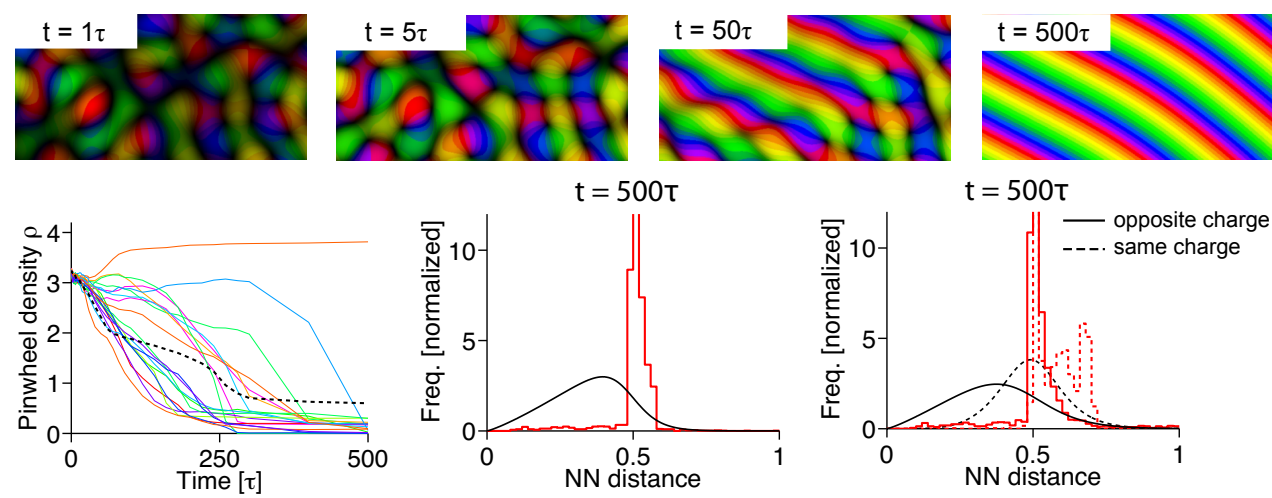

b long-range / weakly suppressive
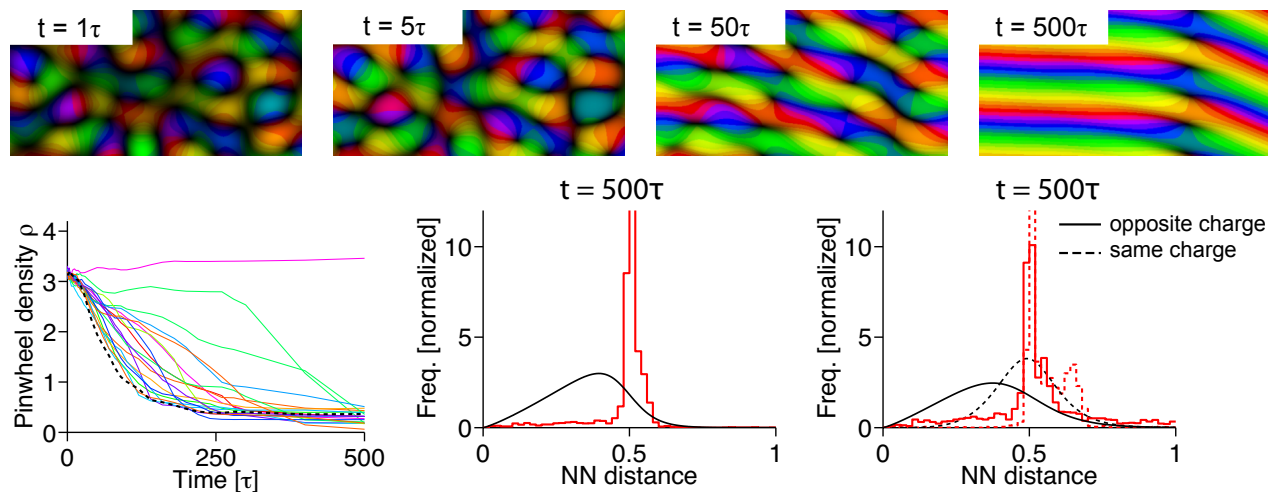

C long-range / strongly suppressive
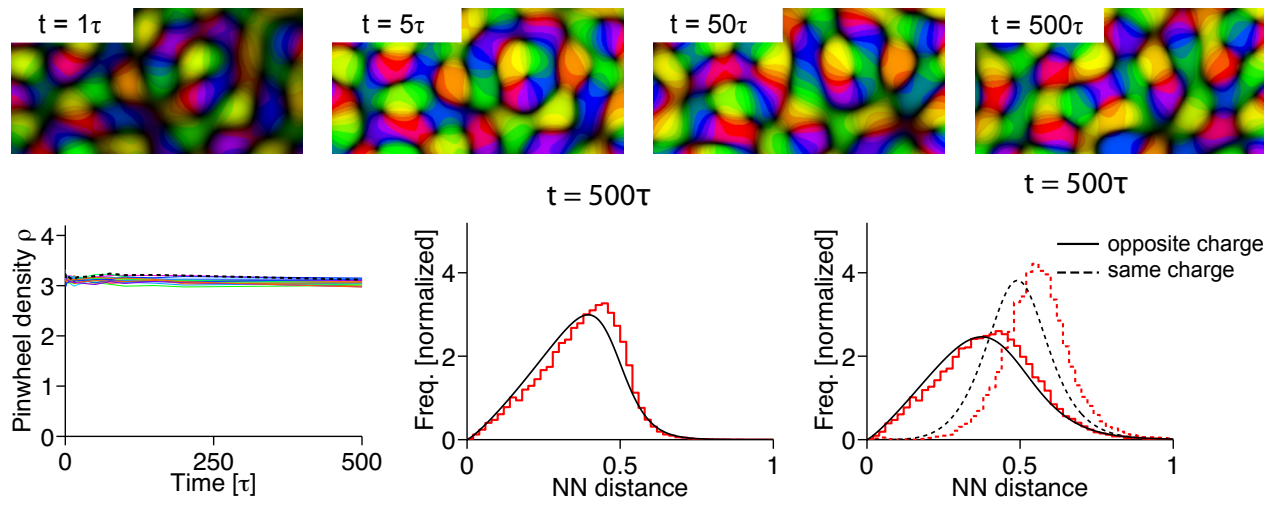

Figure 5.4.5: Dynamics of the long-range interaction model (Eq. (5.2.10)) with exponentially decaying interactions (cf. Fig. 5.4.4). (a) Upper row: Snapshots of a numerically obtained solution of the model for short-range strongly suppressive interactions $\left(g_{s r}=-0.23, g_{l r}=-2.1, \gamma / \Lambda=0.15\right)$ with weakly orientation-selective random initial condition. Lower row, left: Pinwheel density time courses for $\mathrm{N}=20$ simulations (colored solid traces, individual simulations; black dashed trace, simulation in upper row; parameters as in upper row). Middle and right: Statistics of nearest neighbor pinwheel distances at $t=500 \tau$, averaged over $\mathrm{N}=20$ simulations for pinwheels pairs of arbitrary (middle), opposite and equal sign (right) and parameters as in upper row. (b) As a, but for long-range weakly suppressive interactions $\left(g_{s r}=-0.1, g_{l r}=-1.2, \gamma / \Lambda=2.2\right)$. (c) As a, but for long-range and strongly suppressive interactions $\left(g_{s r}=0.53\right.$, $\left.g_{l r}=-2.04, \gamma / \Lambda=2.2\right)$. All snapshots are insets from simulations with $22 \Lambda \times 22 \Lambda$ aspect ratio on a $128 \times 128$ mesh. Initial conditions are the same in $\mathbf{a}, \mathbf{b}$, and $\mathbf{c}$. 

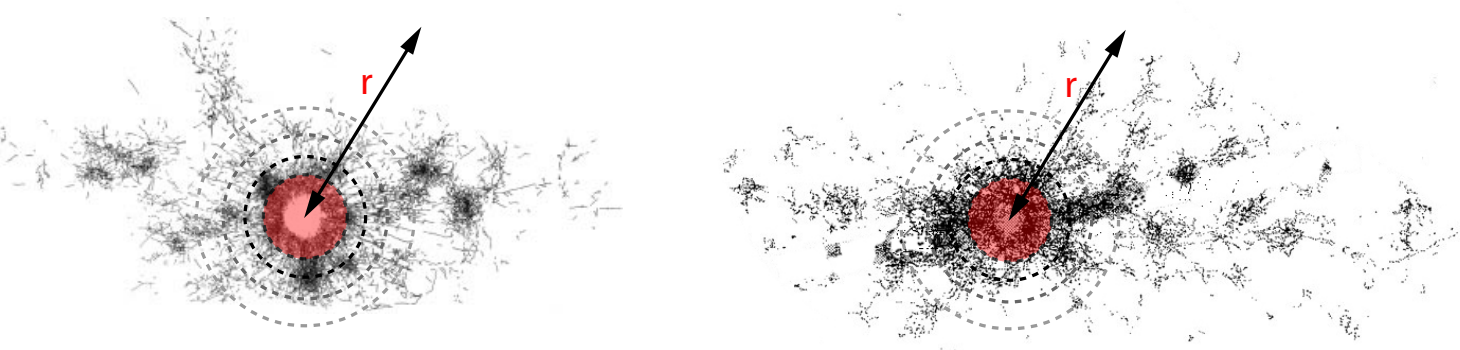

Figure 5.4.6: Plots of labeled boutons within layer $2 / 3$ of resulting from a biocytin injection in ferret (left, mod. from [44]) and tree shrew (right, mod. from [21]) area V1. The local bouton densities within the red regions appear isotropic and connectivity at short distances may not depend on the difference in preferred orientation between connected sites.

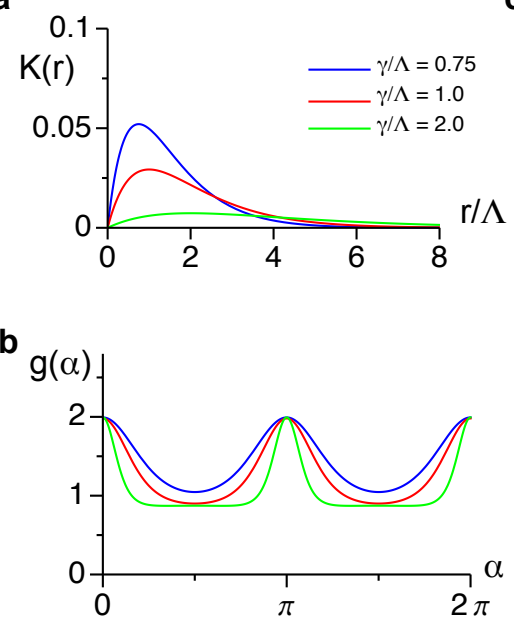

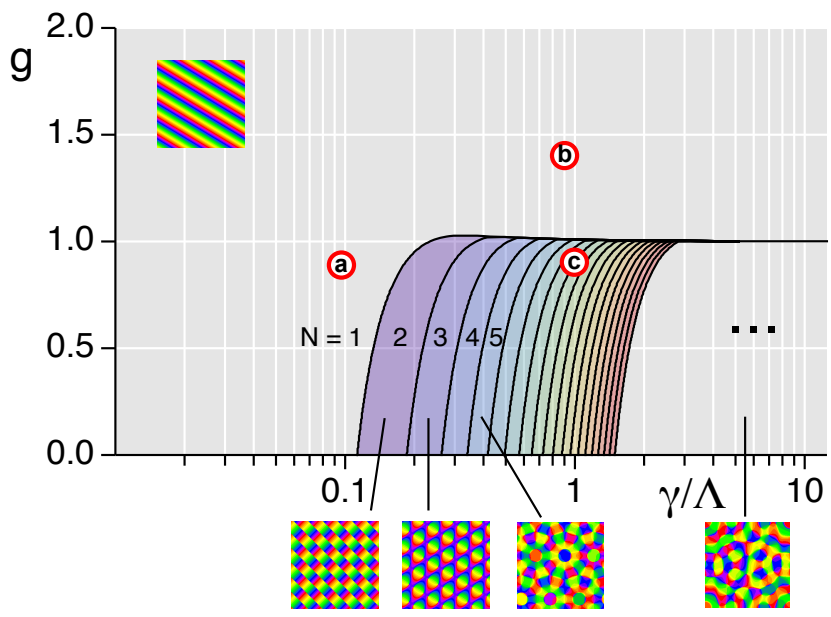

Figure 5.4.7: Phase diagram of the one-parametric limit of the long-range interaction model (Eq. (5.2.10)) with locally non-orientation-selective interactions. (a) Interaction kernels (Eq. (5.4.1)) for different interaction ranges $\gamma / \Lambda=0.75$ (blue line), $\gamma / \Lambda=1.0$ (red line), and $\gamma / \Lambda=2.0$ (green line). (b) Angle-dependent interaction functions for different interaction ranges (colors as in a) and $g=0.9$. (c) Phase diagram of the model. The graph shows the regions in the g- $\gamma / \Lambda$-plane in which $n$-ECPs have minimal energy $(n=1-20, n>20$ dots). The letters a-c in the phase diagram mark the values of $g$ and $\gamma / \Lambda$ that correspond to the parameters $g_{s r}, g_{l r}$, and $\gamma / \Lambda$ at which the numerical solutions in Fig. 5.4.8 for the full third-order approximation of the long-range interaction model (Eq. (5.2.10)) were obtained. 
ferences in the preferred angle. As for the other two model instances, $\gamma$ mediates the range of the long-range interactions. For large distances, interactions still decay exponentially as suggested by the bouton density analysis of Chisum et al. ([158], cf. Fig. 5.4.3).

For $m=2,3, \ldots$, the angle-dependent interaction functions of the one-parametric limit can be computed analytically and read

$$
\begin{aligned}
g(\alpha)= & g+(2-g)\left(\frac{1}{\left(1+2 k_{c}^{2} \gamma^{2}(1-\cos \alpha)\right)^{1 / 2 m+1 / 2}} P_{m}\left[\frac{1}{\left(1+2 k_{c}^{2} \gamma^{2}(1-\cos \alpha)\right)^{1 / 2}}\right]\right. \\
& \left.+\frac{1}{\left(1+2 k_{c}^{2} \gamma^{2}(1+\cos \alpha)\right)^{1 / 2 m+1 / 2}} P_{m}\left[\frac{1}{\left(1+2 k_{c}^{2} \gamma^{2}(1+\cos \alpha)\right)^{1 / 2}}\right]\right),
\end{aligned}
$$

where $P_{m}(x)$ denotes the Legendre polynomial of $m$ th-degree. Note that it is possible to generalize this model to arbitrary $m \in \mathbb{R}^{+}$. However, no analytical results regarding the angle-dependent interaction functions can be obtained in this case. In the following, I focus on the case $m=1$.

The kernel $K(r)$ for $m=1$ is depicted in Figure 5.4.7a for various values of the parameter $\gamma$. The angle-dependent interaction function $g(\alpha)$ for this model is plotted in Figure 5.4.7b for different interaction ranges $\gamma$. This function is again very similar to the ones of the previously considered models (cf. Figs. 5.4.1b,5.4.4b). The same holds for the phase diagram (Fig. 5.4.7c). Again, whereas for $g>1$ and for short-range interactions pinwheel-free stripe patterns exhibit minimal energy, they lose stability for $g<1$ and sufficiently large $\gamma$. Aperiodic $n$-ECPs with several active modes exhibit minimal energy in this regime. Finally, as Figure 5.4.8 shows, the dynamics of the model with only non-orientation-selective local connections is very similar to the model instances considered above.

\subsubsection{Power-law interactions mediated by visual input?}

So far, I have investigated two biologically plausible types of long-range interactions, Gaussian and exponential decay. I have shown that both types of interaction lead to virtually indistinguishable dynamics and phase diagrams. The functional form of these interactions was, in particular in the exponential case, inspired by the statistics of the system of long-range tangential intracortical connections. The class of pattern formation theories considered in this chapter, however, is so general that it can also be applied to study alternative mechanisms by which long-range interaction could be mediated. One such mechanism is an interaction mediated by long-range spatial correlations in the input patterns which activate the primary visual cortex during map development. The pattern of activity evoked by visual experience has been shown to affect the architecture of intracortical circuits and to be a critical factor in the maturation of orientation selectivity in the developing visual cortex [44]. In fact, abnormal patterns of visually driven activity are far more disruptive to the maturation of orientation-selective responses than the total absence of visually driven activity [44, 78]. The normal visual inputs patterns to V1 are elicited by natural scenes. The spatial statistics of natural scenes might therefore be a crucial determinant to understand visual cortical development. In fact, there is recent evidence that visual cortical activity becomes progressively better matched to the statistics of natural stimuli but not to simplistic artificial stimulus ensembles [191]. The statistics of natural images has been shown to exhibit long-range scale-free spatial correlations decaying with power-law dependance[185-187, 192]. In view of this, it seems 
a short-range / strongly suppressive
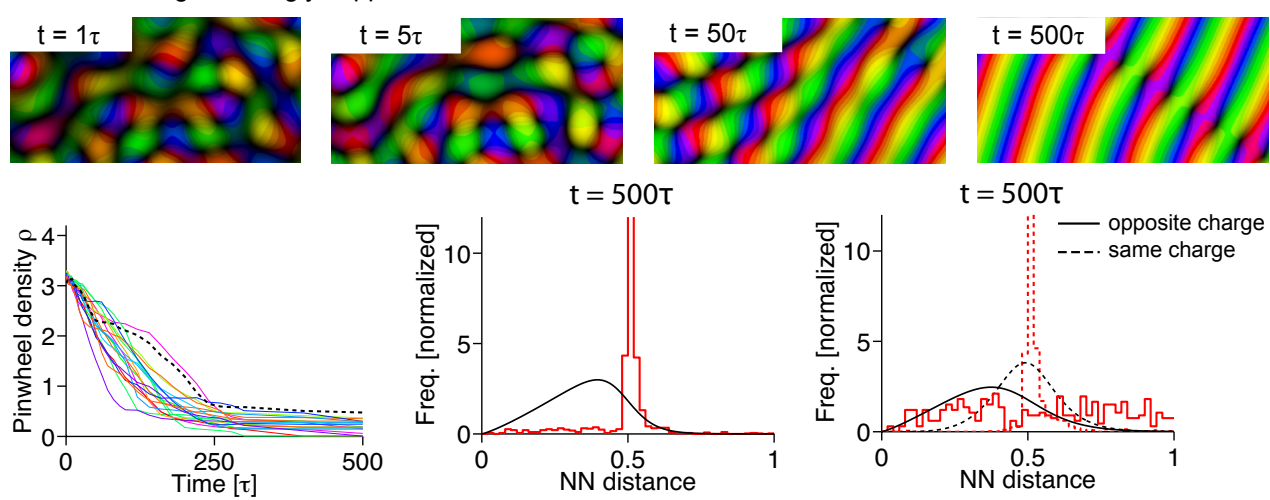

b long-range / weakly suppressive
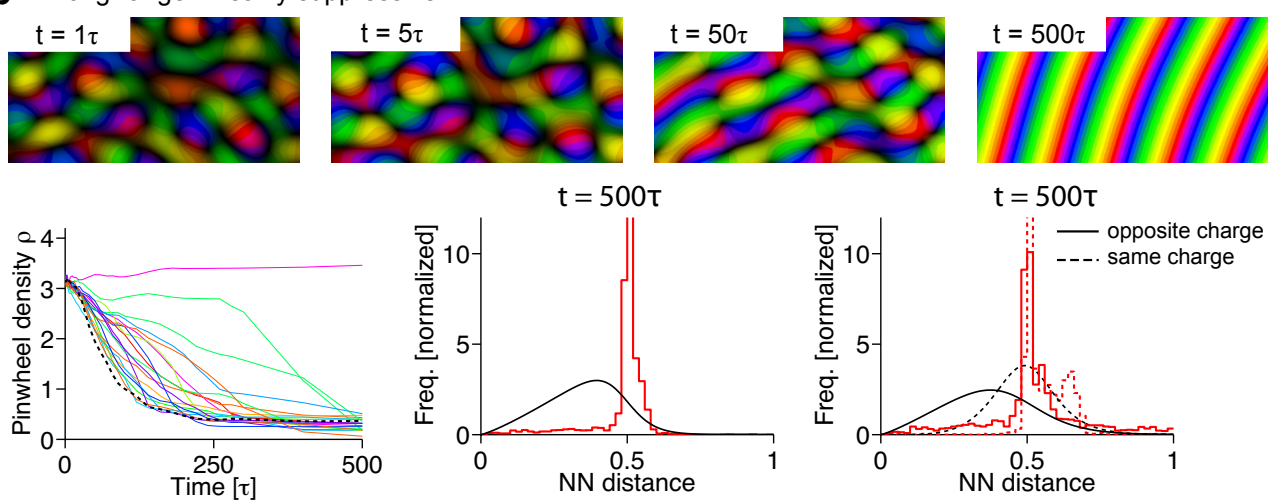

C
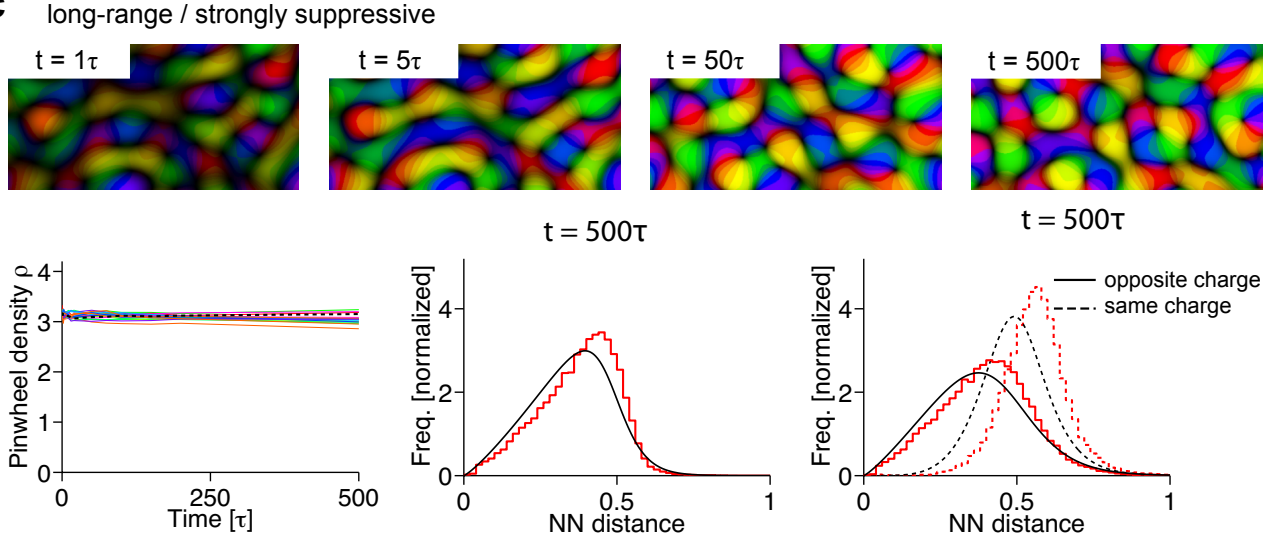

Figure 5.4.8: Dynamics of the long-range interaction model with non-orientation-selective short-range interactions (cf. Fig. 5.4.7). (a) Upper row: Snapshots of a numerically obtained solution of the model Equation (5.2.10) for shortrange strongly suppressive interactions $\left(g_{s r}=-0.23, g_{l r}=-2.1, \gamma=0.15\right)$ with weakly orientation-selective random initial condition. Lower row, left: Pinwheel density time courses for $\mathrm{N}=20$ simulations (left, gray traces, individual simulations; black trace, simulation in upper row; parameters as in upper row). Middle and right: Statistics of nearest neighbor pinwheel distances at $t=500 \tau$, averaged over $\mathrm{N}=20$ simulations for pinwheels pairs of arbitrary (middle), opposite and equal sign (right) and parameters as in upper row. (b) As a, but for long-range weakly suppressive interactions $\left(g_{s r}=-0.1, g_{l r}=-1.2, \gamma=2.2\right)$. (c) As a, but for long-range and strongly suppressive interactions $\left(g_{s r}=0.53, g_{l r}=-2.04, \gamma=2.2\right)$. All snapshots are insets from simulations with $22 \Lambda \times 22 \Lambda$ aspect ratio on a $128 \times 128$ mesh. Initial conditions are the same in $\mathbf{a}, \mathbf{b}$, and $\mathbf{c}$. 
a

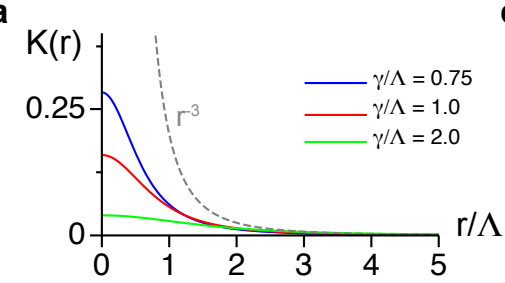

b

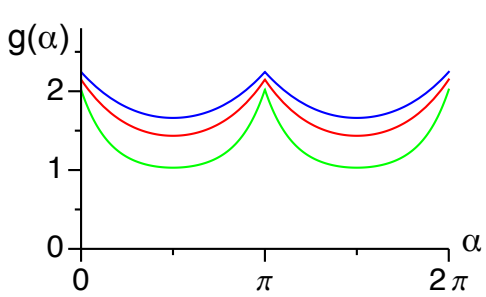

C

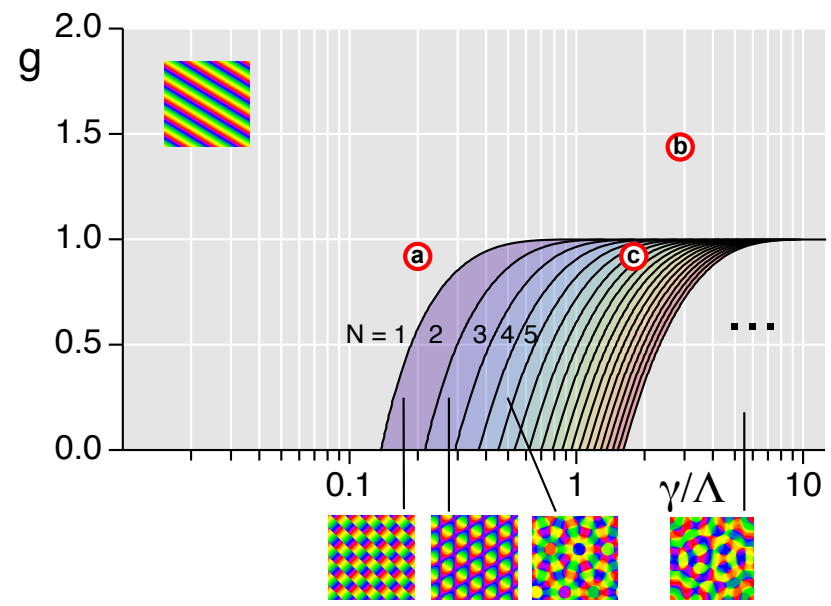

Figure 5.4.9: Phase diagram of the one-parametric limit of the long-range interaction model with interactions decaying as $r^{-3}$. (a) Interaction kernels decaying as $r^{-3}$ for different interaction ranges $\gamma / \Lambda=0.75$ (blue line), $\gamma / \Lambda=1.0$ (red line), and $\gamma / \Lambda=2.0$ (green line). (b) Angle-dependent interaction functions for different interaction ranges (colors as in a) and $g=0.9$. (c) Phase diagram of the model. The graph shows the regions in the g- $\gamma / \Lambda$-plane in which $n$-ECPs have minimal energy $(n=1-20, n>20$ dots). The letters a-c in the phase diagram mark the values of $g$ and $\gamma / \Lambda$ that correspond to the parameters $g_{s r}, g_{l r}$, and $\gamma / \Lambda$ at which the numerical solutions in Fig. 5.4.10 for the full third-order approximation of the long-range interaction model (Eq. (5.2.10)) were obtained.

worthwhile investigating whether realistic OPM layouts can as well be stabilized by interactions decaying as power-laws. In the following, I study two analytically tractable model instances in which interactions decay as either $\sim r^{-3}$ or $\sim r^{-4}$ for sufficiently large interaction ranges.

The first model instance I consider is a model with long-range interactions of the form

$$
K(r)=\frac{1}{2 \pi \gamma^{2}}\left(1+\left(\frac{r}{\gamma}\right)^{2}\right)^{-3 / 2} .
$$

Similarly to the exponential decay, the parameter $\gamma$ determines the range of interactions. For large distance, these interactions decay asymptotically proportionally to $\sim r^{-3}$ (Fig. 5.4.9a). The angle-dependent interaction function $g(\alpha)$ of the one-parametric model limit according to Equation (5.2.12) then reads

$$
g(\alpha)=g+(2-g)\left(e^{-k_{c} \sqrt{2(1-\cos \alpha)} \gamma}+e^{-k_{c} \sqrt{2(1+\cos \alpha)} \gamma}\right) .
$$

This function is plotted in Figure 5.4.9b for different interaction ranges. It bears close resemblance with the angle-dependent interaction functions for Gaussian and exponentially decaying interaction (Figs. 5.4.1b, 5.4.5b, 5.4.4b, and 5.4.7b). This similarity also manifests itself in the 2dimensional phase diagram of the model (Fig. 5.4.9c). It is strikingly similar to the phase diagram obtained in the Gaussian (Fig. 5.4.1c) as well as the exponential cases (Figs. 5.4.5c, 5.4.8c). In the same line, as Figure 5.4.10 illustrates, the dynamics of the model with power-law interactions $\sim r^{-3}$ is also not different from the three model instances considered above.

The second analytically tractable model instance with power-law interactions which I consider is 
a short-range / strongly-suppressive
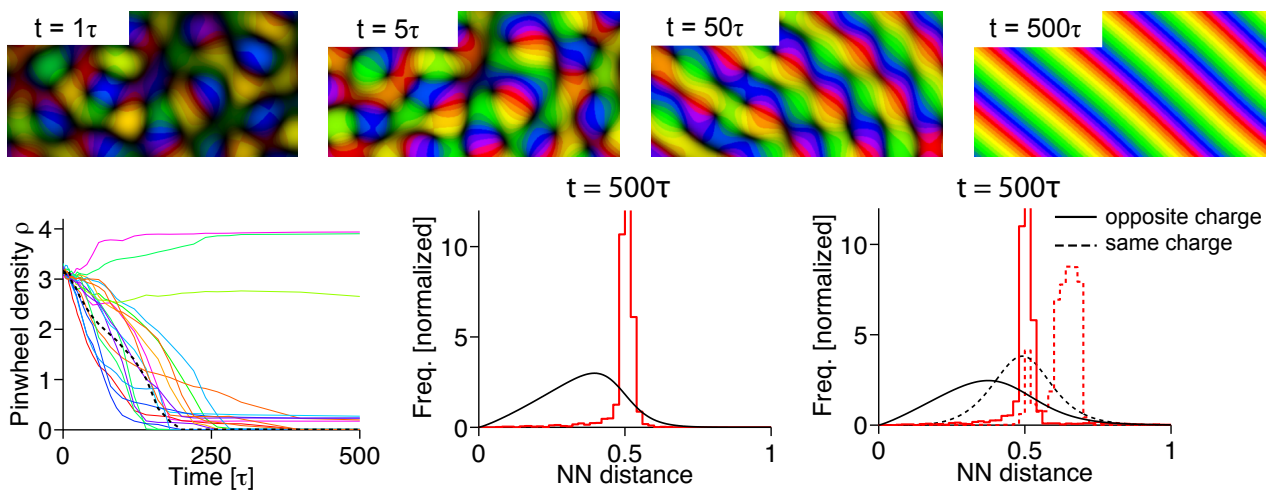

b long-range / weakly-suppressive
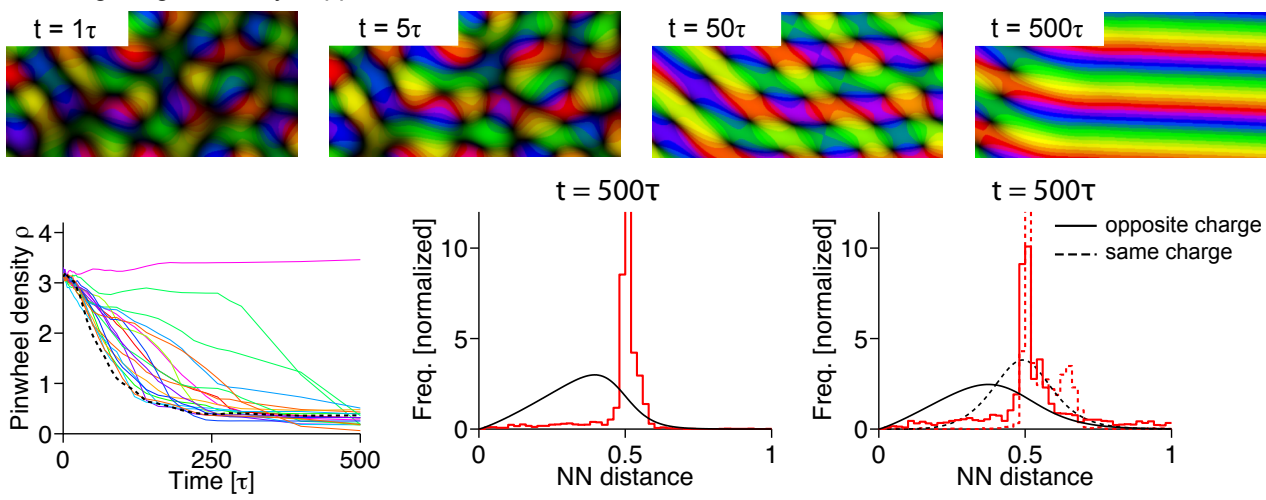

C long-range / strongly-suppressive
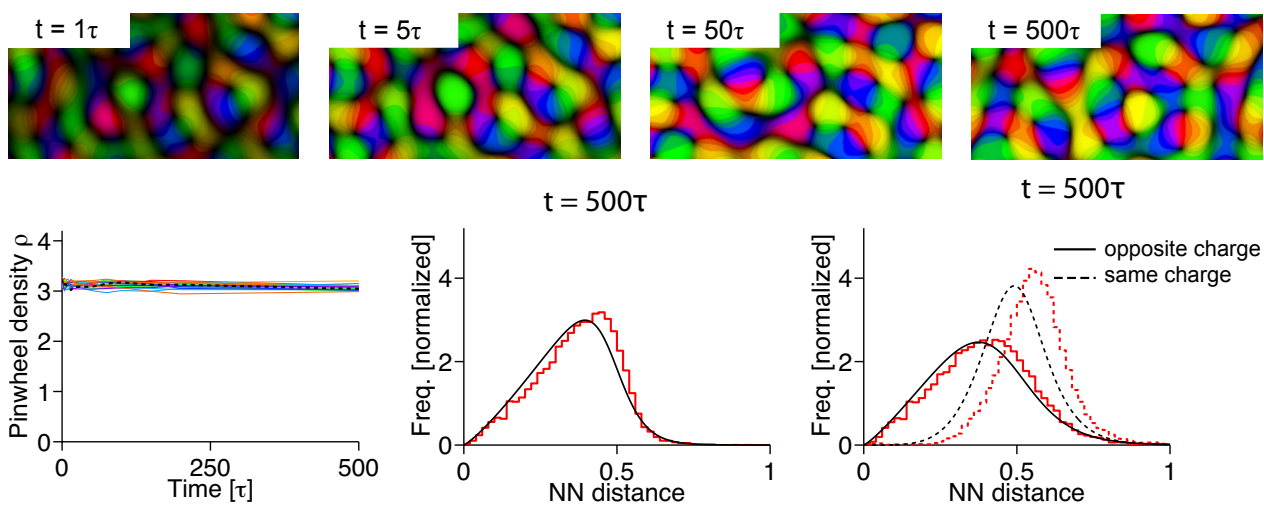

Figure 5.4.10: Dynamics of the long-range interaction model (Eq. (5.2.10)) with interaction strength decaying as $r^{-3}$ (cf. Fig. 5.4.9). (a) Upper row: Snapshots of a numerically obtained solution of the model for short-range strongly suppressive interactions $\left(g_{s r}=-0.05, g_{l r}=-2.04, \gamma / \Lambda=0.2\right)$ with weakly orientation-selective random initial condition. Lower row, left: Pinwheel density time courses for $\mathrm{N}=20$ simulations (colored solid traces, individual simulations; black dashed trace, simulation in upper row; parameters as in upper row). Middle and right: Statistics of nearest neighbor pinwheel distances at $t=500 \tau$, averaged over $\mathrm{N}=20$ simulations for pinwheels pairs of arbitrary (middle), opposite and equal sign (right) and parameters as in upper row. (b) As a, but for long-range weakly suppressive interactions $\left(g_{s r}=-0.1, g_{l r}=-1.2, \gamma / \Lambda=3.0\right)$. (c) As a, but for long-range and strongly suppressive interactions $\left(g_{s r}=0.55\right.$, $g_{l r}=-2.04, \gamma / \Lambda=2.2$. All snapshots are insets from simulations with $22 \Lambda \times 22 \Lambda$ aspect ratio on a $128 \times 128$ mesh. Initial conditions are the same in $\mathbf{a}, \mathbf{b}$, and $\mathbf{c}$. 
a

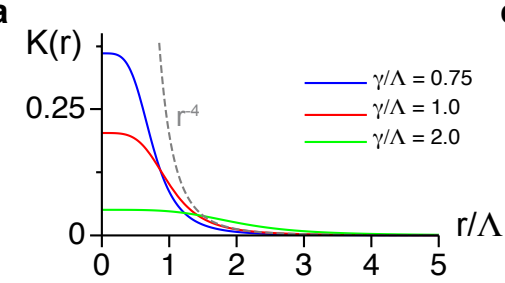

b

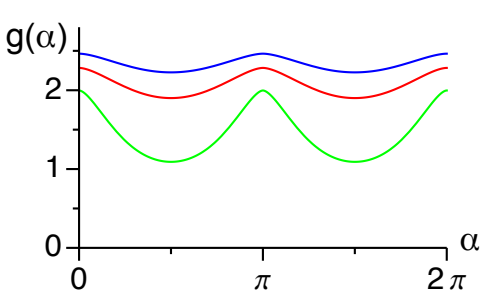

C

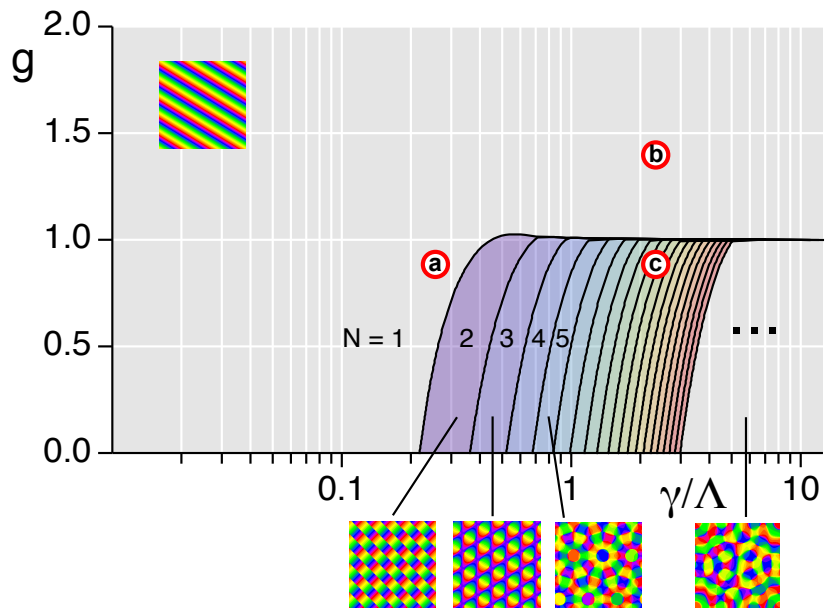

Figure 5.4.11: Phase diagram of the one-parametric limit of the long-range interaction model with interactions decaying as $r^{-4}$. (a) Interaction kernels decaying as $r^{-4}$ for different interaction ranges $\gamma / \Lambda=0.75$ (blue line), $\gamma / \Lambda=1.0$ (red line), and $\gamma / \Lambda=2.0$ (green line). (b) Angle-dependent interaction functions for different interaction ranges (colors as in a) and $g=0.9$. (c) Phase diagram of the model. The graph shows the regions in the g- $\gamma / \Lambda$-plane in which $n$-ECPs have minimal energy $(n=1-20, n>20$ dots). The letters a-c in the phase diagram mark the values of $g$ and $\gamma / \Lambda$ that correspond to the parameters $g_{s r}, g_{l r}$, and $\gamma / \Lambda$ at which the numerical solutions in Fig. 5.4.12 for the full third-order approximation of the long-range-interaction model (Eq. (5.2.10)) were obtained.

defined by a kernel

$$
K(r)=\frac{2}{\pi^{2} \gamma^{2}}\left(1+\left(\frac{r}{\gamma}\right)^{4}\right)^{-1}
$$

for the spatial decay of interactions. The parameter $\gamma$ determines the range of interactions (Fig. 5.4.11a). For large distance, these interactions decay asymptotically proportionally to $\sim r^{-4}$. The angle-dependent interaction function of the one-parametric limit of this model reads

$$
\left.\left.g(\alpha)=g-(2-g) \frac{4}{\pi}\left(\operatorname{kei}_{0}\left(\gamma k_{c} \sqrt{2(1-\cos \alpha)}\right)\right)+\operatorname{kei}_{0}\left(\gamma k_{c} \sqrt{2(1+\cos \alpha)}\right)\right)\right),
$$

where $\operatorname{kei}_{0}(x)$ denotes the Kelvin function of zeroth order, i.e. the imaginary part of $K_{0}\left(x e^{i 3 \pi / 4}\right)$, where $K_{0}(z)$ is the zeroth-order modified Bessel function of the second kind. This function is plotted in Figure 5.4.11b for different interaction ranges. As expected from the results obtained so far, the 2-dimensional phase diagram of the model (Fig. 5.4.9c) is very similar to the phase diagram obtained in the Gaussian (Fig. 5.4.1c), the exponential case (Fig. 5.4.5c) as well as the $\sim r^{-3}$-case (Fig. 5.4.9c). As depicted in Figure 5.4.10, the dynamics of the model with power-law interaction $\sim r^{-4}$ is not different from the four previously considered model instances.

\subsection{Simultaneous development of horizontal connections and OPMs}

At the beginning of this chapter, I started out by proposing an optimization model for the joint development of the system of tangential connections and OPMs. However, so far, we have exclu- 
a short-range / strongly suppressive
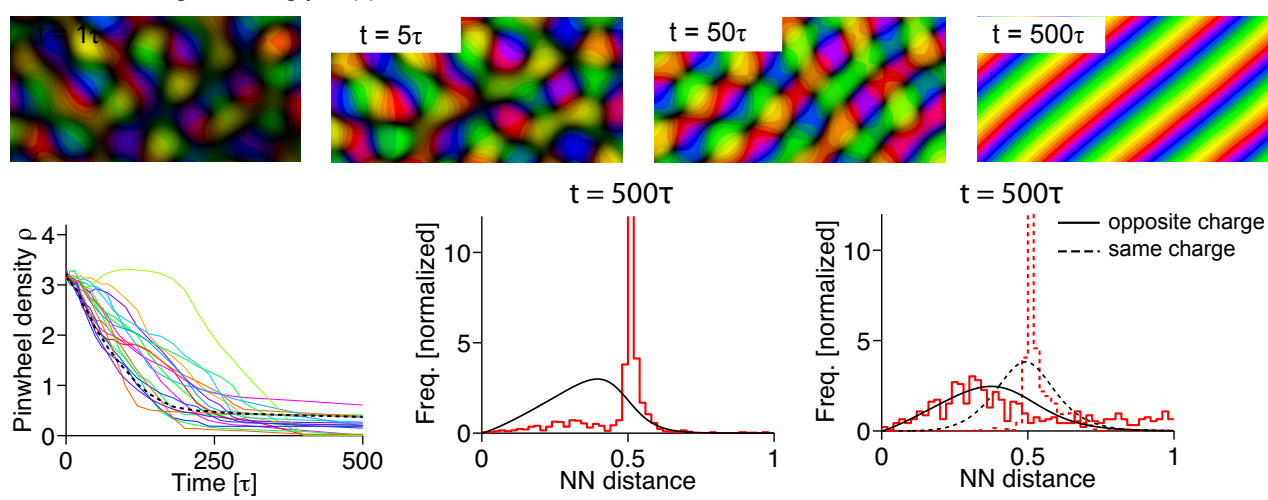

b long-range / weakly suppressive
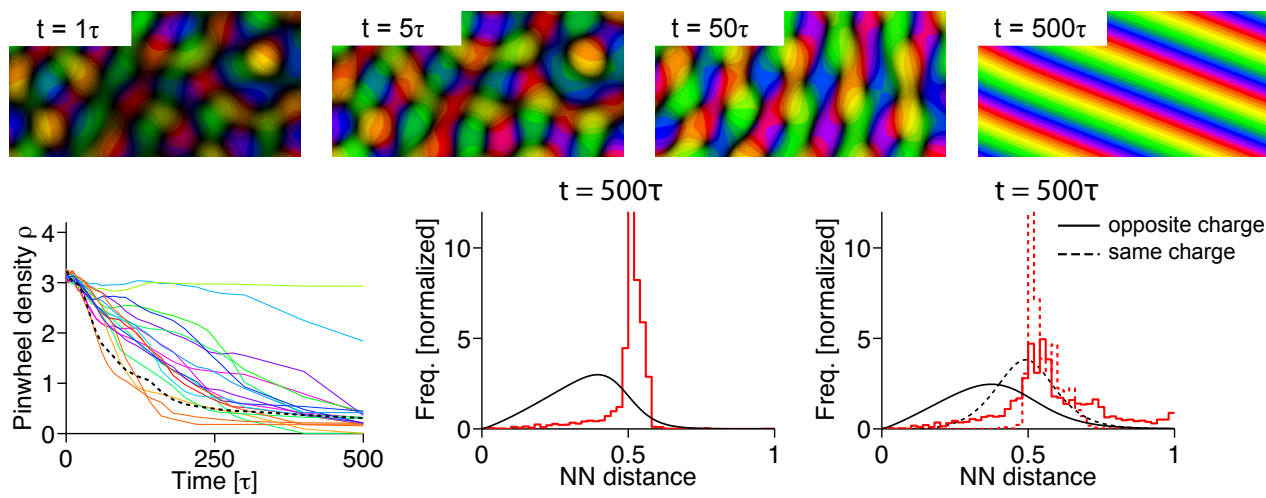

C
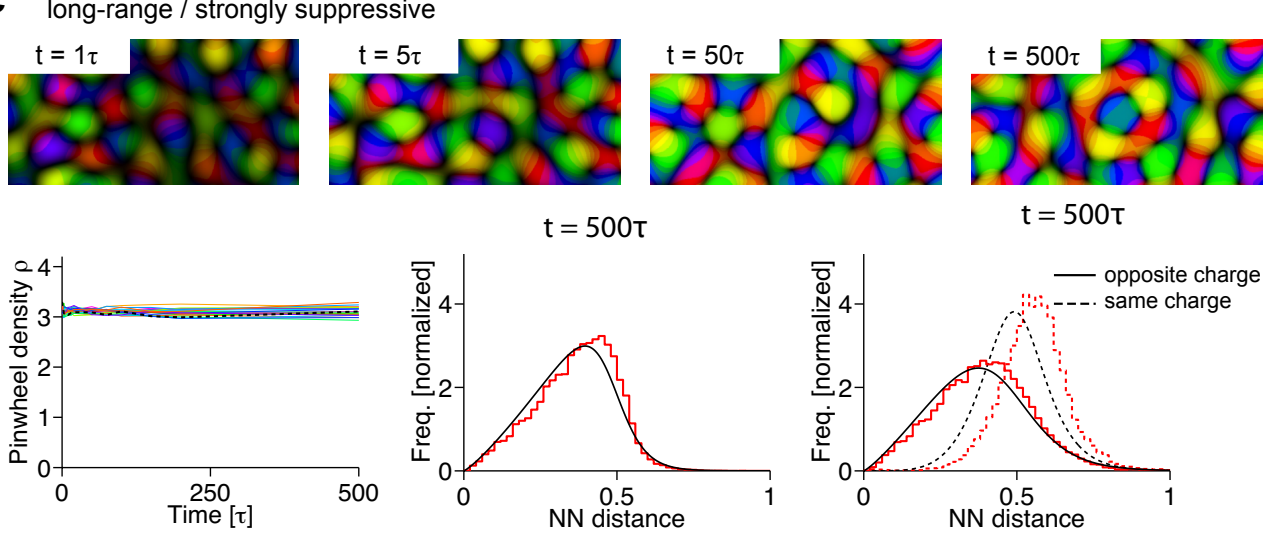

Figure 5.4.12: Dynamics of the long-range interaction model (Eq. (5.2.10)) with interaction strength decaying as $r^{-4}$ (cf. Fig. 5.4.11). (a) Upper row: Snapshots of a numerically obtained solution of the model for short-range strongly suppressive interactions $\left(g_{s r}=-0.27, g_{l r}=-2.04, \gamma / \Lambda=0.25\right)$ with weakly orientation-selective random initial condition. Lower row, left: Pinwheel density time courses for $\mathrm{N}=20$ simulations (left, gray traces, individual simulations; black trace, simulation in upper row; parameters as in upper row). Middle and right: Statistics of nearest neighbor pinwheel distances at $t=500 \tau$, averaged over $\mathrm{N}=20$ simulations for pinwheels pairs of arbitrary (middle), opposite and equal sign (right) and parameters as in upper row. (b) As a, but for long-range weakly suppressive interactions $\left(g_{s r}=-0.1, g_{l r}=-1.2, \gamma / \Lambda=2.2\right)$. (c) As a, but for long-range and strongly suppressive interactions $\left(g_{s r}=0.55\right.$, $g_{l r}=-2.04, \gamma / \Lambda=2.2$. All snapshots are insets from simulations with $22 \Lambda \times 22 \Lambda$ aspect ratio on a $128 \times 128$ mesh. Initial conditions are the same in $\mathbf{a}, \mathbf{b}$, and $\mathbf{c}$. 

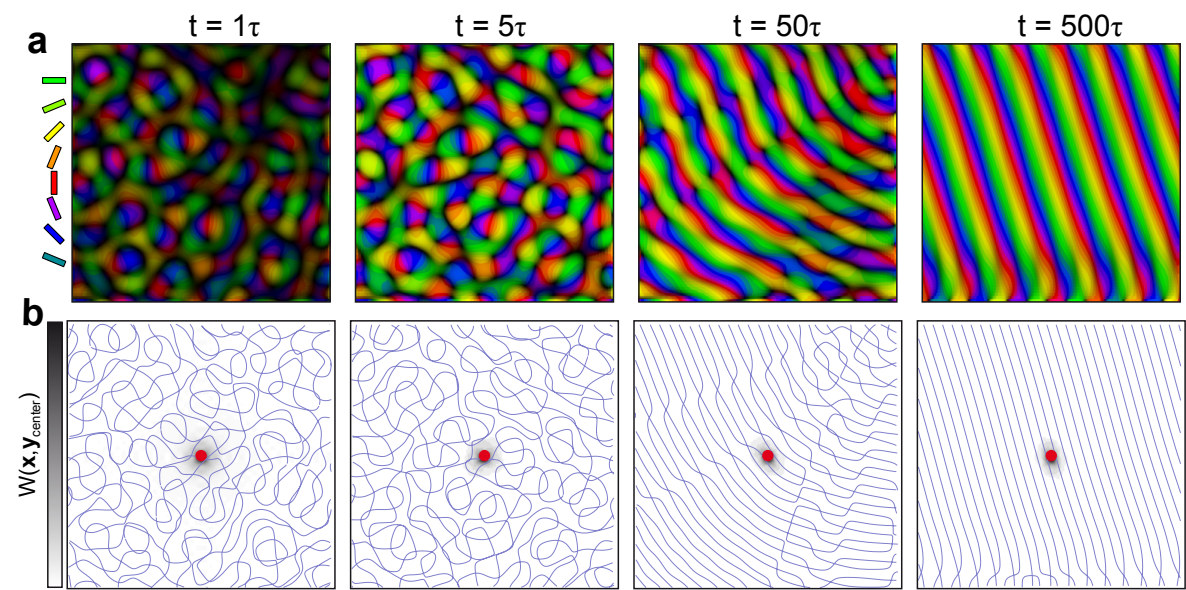

C
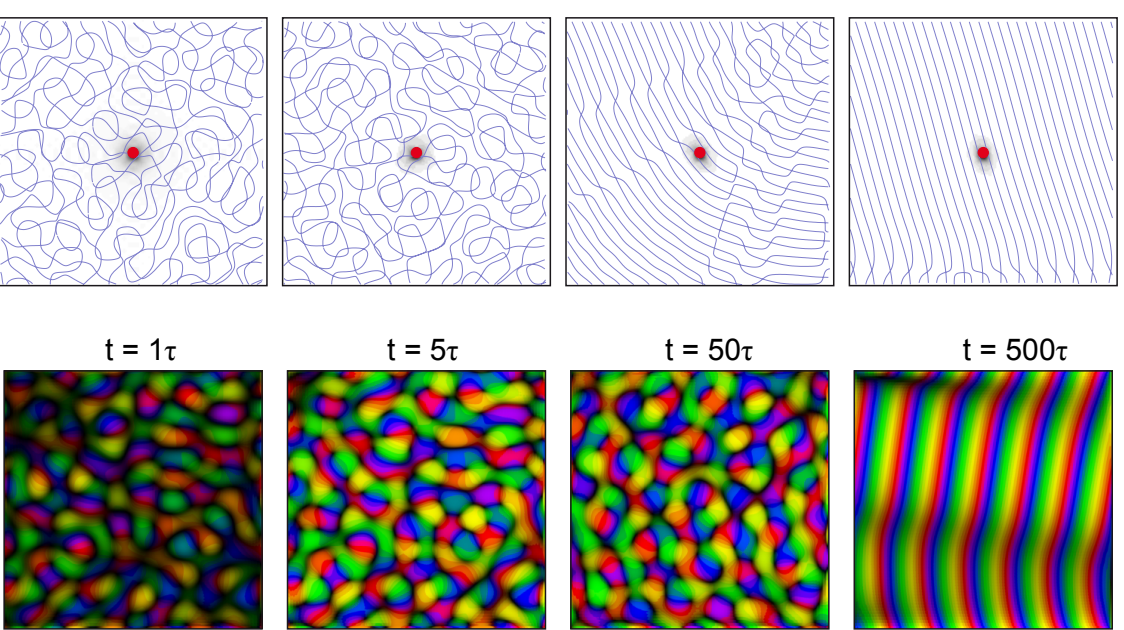

d
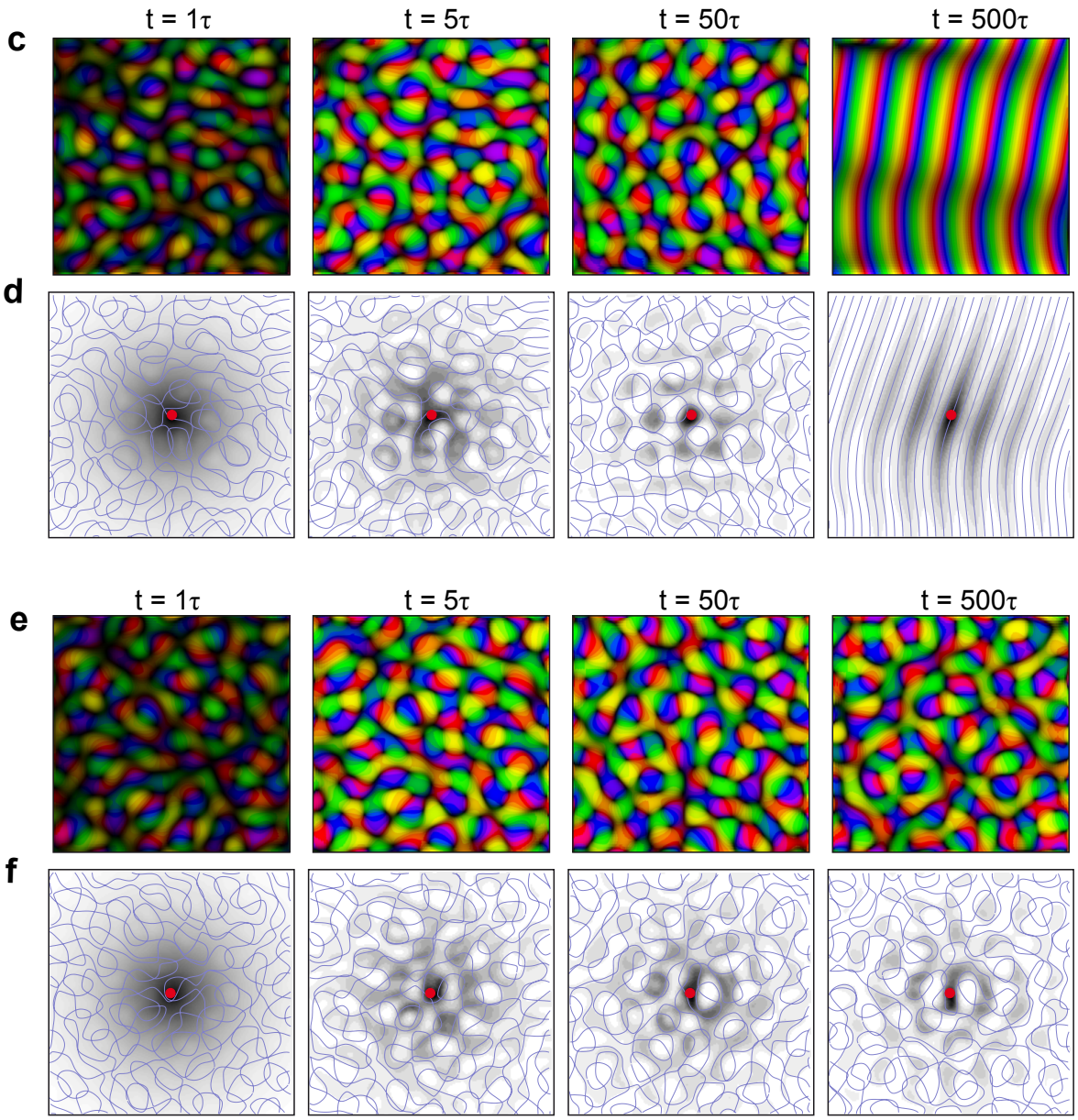

Figure 5.5.1: Simultaneous development of OPMs and tangential connections in the model. (a, b) Snapshots of OPM development (a) and the corresponding tangential connections $W\left(\mathbf{x}, \mathbf{y}_{\text {center }}\right)$ according to Equation (5.2.5) (b) for shortrange strongly suppressive connections decaying as a Gaussian function (parameters as in Fig. 5.4.2a). The red dot in $\mathbf{b}$ indicates the position of $\mathbf{y}_{\text {center }}$. (c, $\left.\mathbf{d}\right)$ As $\mathbf{a}, \mathbf{b}$ but for long-range weakly suppressive interactions (parameters as in Fig. 5.4.2b). (e, f) As a, b but for long-range strongly suppressive interactions (parameters as in Fig. 5.4.2c). 
sively studied the dynamics of the OPM in the presence and absence of long-range interactions of different types after the dynamics of the connections has been adiabatically eliminated (cf. Sec. 5.2). In the model, long-range suppressive interactions are critical where suppressive means suppressing the development of the same orientation as the projecting neurons. What happens to the tangential connections in this adiabatic limit during orientation preference map development? Figure 5.5.1 qualitatively illustrates the types of connectivity rearrangements that occur in the three main regimes of the model considered in this chapter. If connections are short-range (Fig. 5.5.1a,b), rearrangement during the convergence to stripe-like OPM patterns are subtle. The connection profile slightly elongates along the axis of the stripes during the developmental dynamics (Fig. 5.5.1b, most right). In the case of long-range but weakly suppressive interactions (Fig. 5.5.1c,d), connectivity sparsifies from an initially nearly isotropic system of connections and develops into a Gabor-function-like arrangement, in which stripe-like domains of the same orientation preference are preferentially connected. In fact, for $z(\mathbf{x}) \sim e^{i \mathbf{k} \mathbf{x}}$, Gaussian kernel $K(|\mathbf{x}|)$, and fixed $\mathbf{y}$, Equation (5.2.5) describes a Gabor function. For long-range and strongly suppressive interactions, the main change between the initially isotropic connectivity profile and the connections at later stages is obtained through selective pruning of connections (Fig. 5.5.1e,f). This process very much resembles the refinement of horizontal connections observed in experiment $[76,77,80,181]$.

\subsection{General stability ranges for the one-parametric long-range interac- tion models}

In the following two sections, I generalize the results obtained in [193] and compute the extrinsic, intrinsic, and asymptotic stability ranges of $n$-ECPs in order parameter models with long-range interactions of arbitrary spatial dependance.

\subsubsection{Intrinsic and extrinsic stability boundaries}

In all of the models considered so far, the angle-dependent interaction function $g(\alpha)$ is $\pi$-periodic (Eq. (5.2.11)) and, hence, the stability properties of $n$-ECPs do not depend on the specific configuration of active modes [123, 161]. Following [161, 193], I refer to the stability of an $n$-ECP within its system of modes as intrinsic stability. The stability against other Fourier modes on the critical circle is referred to as extrinsic stability. To analyze the intrinsic stability of an ECP with $n$ active modes $A_{i}$ and $n$ inactive modes $B_{i}$, I linearize (1.5.7) around the fixed point $\left|A_{i}\right|=\left|A_{0}\right|=\left(\sum_{j} g_{0 j}\right)^{-1 / 2}$ and $B_{i}=B_{0}=0$ for small deviations $a_{i}$ and $b_{i}$. This results in the linear dynamics

$$
\begin{aligned}
\partial_{t} a_{i} & =-\frac{2}{\sum_{j} g_{0 j}} \sum_{j} g_{i j} a_{j} \\
\partial_{t} b_{i} & =\frac{1}{\sum_{k} g_{0 k}}\left(1-\sum_{k}\left(1+\delta_{i k}\right) g_{i k}\right) b_{i}-2 \sum_{j} f_{i j} A_{j} \bar{A}_{i} b_{j} .
\end{aligned}
$$



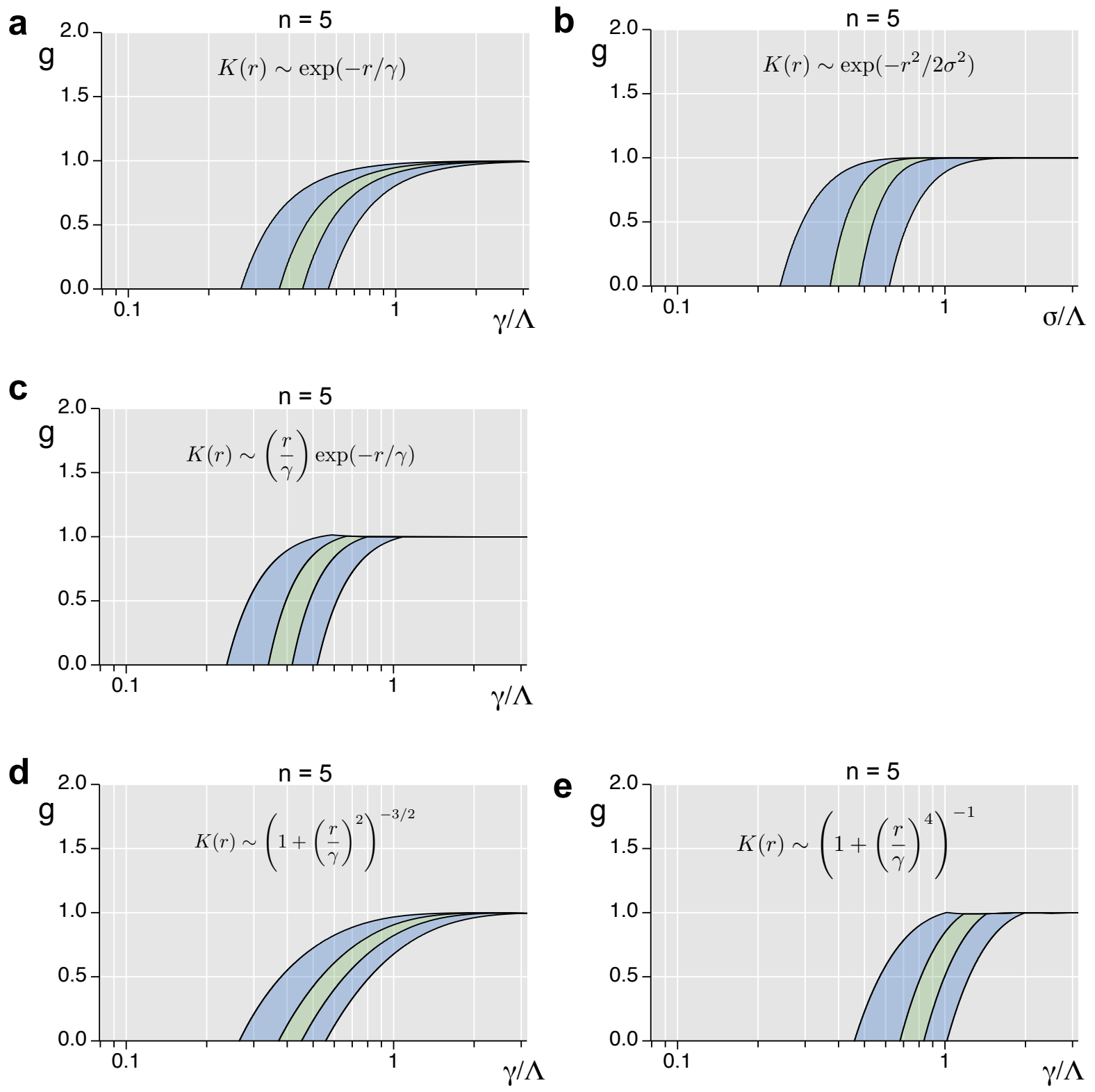

Figure 5.6.1: Extrinsic and intrinsic stability ranges in the one-parametric model limits with different types of biologically plausible long-range interactions. Depicted are the stability ranges for a planform with five active modes for (a) exponentially decaying interactions, (b) Gaussian interactions, (c) locally non-orientation-selective interactions $K(r) \sim r e^{-r / \gamma}$, (d) interactions, decaying as $r^{-3}$, and (e) interactions, decaying as $r^{-4}$. The left/right border of the blue region is given by the condition for intrinsic/extrinsic stability. In the green region, the ECP with 5 active modes possesses minimal energy among all other ECPs. 
The stability of the amplitudes $A_{i}$ is ensured by requiring that the matrix $g_{i j}$ has only positive eigenvalues. The stability of the $B_{i}$ s requires that the matrix

$$
\begin{aligned}
F_{i j} & =\frac{1}{\sum_{k} g_{0 k}}\left(1-\sum_{k}\left(1+\delta_{i k}\right) g_{i k}\right)-2 f_{i j} A_{j} \bar{A}_{i} \\
& =-\frac{g_{i i}}{\sum_{k} g_{0 k}}-2 f_{i j} A_{j} \bar{A}_{i}
\end{aligned}
$$

has only negative eigenvalues. According to [193], the condition $g<1$ together with the minimal eigenvalue $\omega_{\min }^{n}$ of $g_{i j}$ being larger than zero, are, thus, necessary and sufficient for the intrinsic stability of an $n$-ECPs provided that the interaction large is sufficiently large.

An $n$-ECPs is extrinsically stable if $[161,193]$

$$
g(0) / 2-g\left(\alpha-\alpha_{0}\right)+\sum_{j=1}^{n-1}\left(g\left(\alpha_{j}\right)-g\left(\alpha_{j}-\alpha\right)\right)<0,
$$

with $\alpha_{j}=j \pi / n$ for all angles $0<\alpha<2 \pi$. The above condition is equivalent to

$$
g(0) / 2-g\left(\frac{\pi}{2 n}\right)+\sum_{j=1}^{n-1}\left(g\left(\frac{j \pi}{2 n}\right)-g\left(\frac{\pi}{2 n}(1-2 j)\right)\right)<0 .
$$

In summary, for any given number of active modes $n$ the conditions for extrinsic and intrinsic stability define two stability borders $g_{n}^{* i}\left(\gamma k_{c}\right)$ and $g_{n}^{* e}\left(\gamma k_{c}\right)$ of an $n$-ECP ${ }^{1}$ :

$$
\begin{aligned}
& 0=\left.\omega_{\min }^{n}\right|_{\gamma, g=g_{n}^{* i}\left(\gamma k_{0}\right)} \\
& 0=g(0) / 2-g\left(\frac{\pi}{2 n}\right)+\left.\sum_{j=1}^{n-1}\left(g\left(\frac{j \pi}{2 n}\right)-g\left(\frac{\pi}{2 n}(1-2 j)\right)\right)\right|_{\gamma, g=g_{n}^{* e}\left(\gamma k_{0}\right)} .
\end{aligned}
$$

The resulting extrinsic and intrinsic stability ranges for the one-parametric limit of all model instances considered in this chapter are depicted in Figure 5.6.1 in the case of an ECP with five active modes.

\subsubsection{Asymptotic stability boundaries}

In this section, I show that the stability boundaries asymptotically scale as

$$
g_{n}^{* i / e}\left(\gamma k_{0}\right)=g^{* i / e}\left(\frac{\gamma k_{0}}{n}\right)
$$

and derive approximate expressions for the asymptotic stability boundaries $g^{* i / e}$ of an $n$-ECP in the limit of large $n$, and large interaction width $\gamma / \Lambda$. The derivation closely follows [193].

\footnotetext{
${ }^{1}$ All angle-dependent interaction functions depend on the product of $k_{c} \gamma$. In the Gaussian case, $\gamma$ is replaced by $\sigma$.
} 

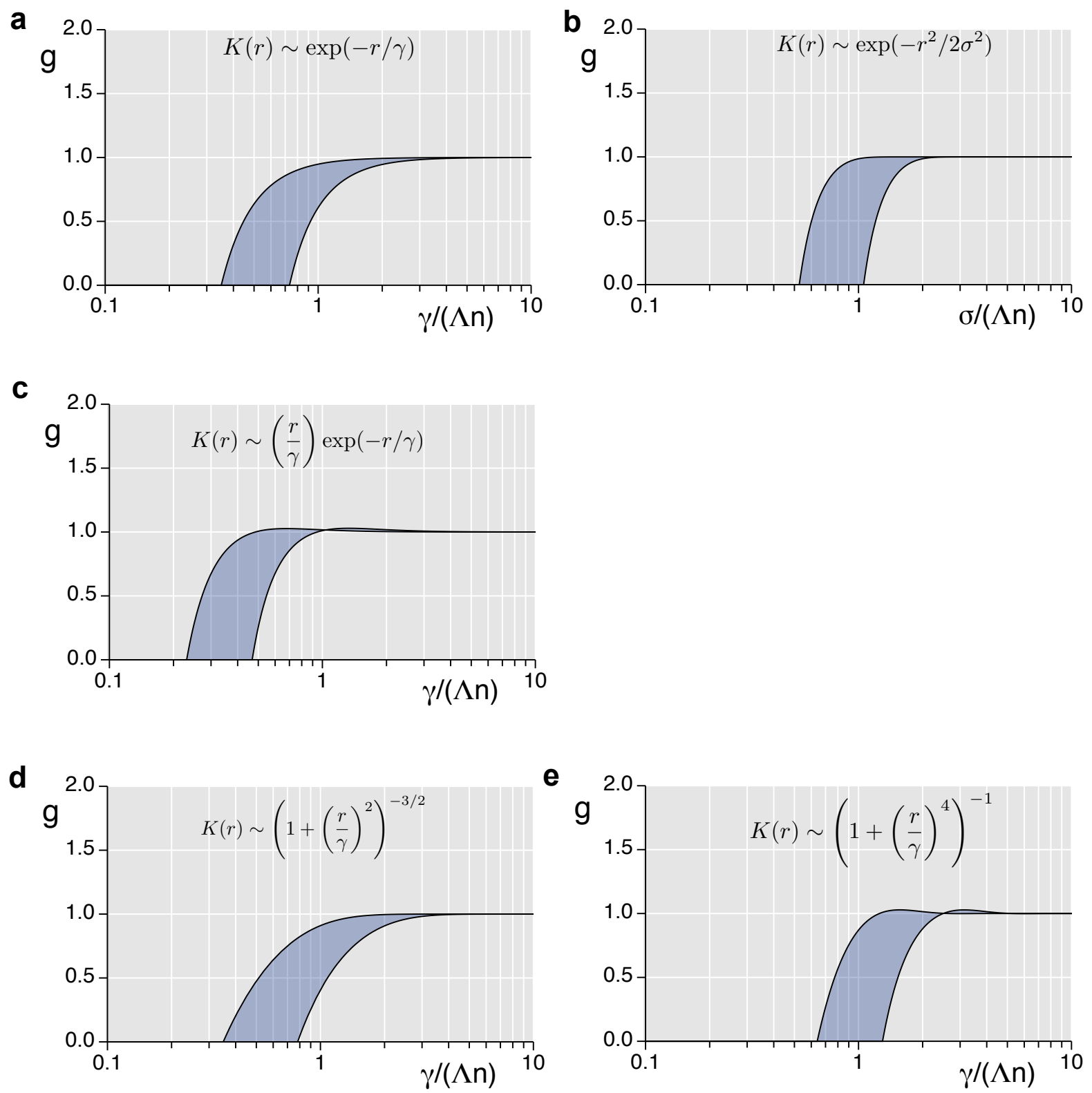

Figure 5.6.2: Asymptotic stability ranges of $n$-ECPs in long-range interaction models with (a) exponentially decaying interactions (b) Gaussian interactions (c) locally non-orientation-selective interactions $K(r) \sim r e^{-r / \gamma}$ (d) interactions decaying as $r^{-3}(\mathbf{e})$ interactions decaying as $r^{-4}$. 
I start by restating the angle-dependent interaction functions:

$$
\begin{aligned}
& g(\alpha)=g+(2-g)\left(F_{0}\left(k_{c} \sqrt{(2(1+\cos \alpha))}\right)+F_{0}\left(k_{c} \sqrt{(2(1-\cos \alpha))}\right)\right) \\
& f(\alpha)=g(\alpha) / 2
\end{aligned}
$$

with $F_{0}$ denoting the Hankel transform of the interaction kernel $K(r)^{2}$. For large interaction width $\gamma$, Equation (5.6.2) is approximately given by

$$
g(0) / 2+g(\pi / n)+2 g(\pi /(2 n))=0
$$

since in this case

$$
\sum_{j=2}^{n-1}\left(g\left(\frac{j \pi}{2 n}\right)-g\left(\frac{\pi}{2 n}(1-2 j)\right)\right) \simeq \sum_{j=1}^{n-1}(g-g)=0 .
$$

Furthermore, for $n$ large, $g(\alpha)$ is evaluated in Equation (5.6.3) only for very small angles, meaning

$$
g(\alpha) \simeq g+(2-g)\left(F_{0}\left(k_{c} \sqrt{2}\right)+F_{0}\left(k_{c} \alpha\right)\right)
$$

and again, for $\left(k_{c} \gamma\right)$ large,

$$
g(\alpha) \simeq g+(2-g) F_{0}\left(k_{c} \alpha\right) .
$$

Thus, the condition for extrinsic stability reduces to

$$
1-g+(2-g)\left(F_{0}\left(k_{c} \frac{\pi}{n}\right)-2 F_{0}\left(k_{c} \frac{\pi}{2 n}\right)\right)=0 .
$$

For rapidly decaying $F_{0}$, one ensures

$$
F_{0}\left(k_{c} \frac{\pi}{n}\right)-2 F_{0}\left(k_{c} \frac{\pi}{2 n}\right)>-1
$$

and finds the condition for extrinsic stability by solving for $g$ :

$$
g_{n}^{* e}\left(\gamma k_{c}\right)=1+\frac{F_{0}\left(k_{c} \frac{\pi}{n}\right)-2 F_{0}\left(k_{c} \frac{\pi}{2 n}\right)}{1+F_{0}\left(k_{c} \frac{\pi}{n}\right)-2 F_{0}\left(k_{c} \frac{\pi}{2 n}\right)} .
$$

This function only depends on $k_{c} \gamma / n$ and, thus, confirms the above hypothesized scaling. The border of intrinsic stability is given by the minimal eigenvalue of the cyclic matrix $g_{i j}$, which in the limit $k_{c} \gamma$ being large can be approximated by

$$
\begin{aligned}
g_{0 j} & =-\delta_{0 j}+g+(2-g)\left(F_{0}\left(k_{c} \sqrt{2\left(1-\cos \left(\frac{\pi}{n} j\right)\right)}\right)+F_{0}\left(k_{c} \sqrt{2\left(1+\cos \left(\frac{\pi}{n} j\right)\right)}\right)\right) \\
& \simeq-\delta_{0 j}+g+(2-g)\left(F_{0}\left(k_{c} \frac{\pi j}{n}\right)+F_{0}\left(k_{c} \frac{\pi}{n}(n-j)\right)\right) .
\end{aligned}
$$

\footnotetext{
2 It has been have shown above that $F_{0}\left(k_{c}\right)$ is always only a function of the product $k_{c} \gamma$. Thus, in the following, wherever it is written $F_{0}\left(k_{c}\right)$ a dependence $F_{0}\left(k_{c} \gamma\right)$ is assumed. In the Gaussian case, $\gamma$ is replaced by $\sigma$.
} 
For the eigenvalues of $g_{i j}$ one obtains

$$
\begin{aligned}
\omega_{l}^{n} & =\sum_{j=0}^{n-1} \cos \left(\frac{2 \pi}{n} j l\right) g_{0 j} \\
& =-1+n g \delta_{l 0}+(2-g) \underbrace{\sum_{j=0}^{n-1} \cos \left(\frac{2 \pi}{n} j l\right)\left(F_{0}\left(k_{c} \frac{\pi j}{n}\right)+F_{0}\left(k_{c} \frac{\pi}{n}(n-j)\right)\right)}_{\Omega_{l}^{n}} .
\end{aligned}
$$

The task now is to find the minimal eigenvalue $\omega_{m i n}^{n}$, i.e. to minimize the function

$$
\Omega_{l}^{n}=\sum_{j=0}^{n-1} \cos \left(\frac{2 \pi}{n} j l\right)\left(F_{0}\left(k_{c} \frac{\pi j}{n}\right)+F_{0}\left(k_{c} \frac{\pi}{n}(n-j)\right)\right) \simeq \sum_{j=-\infty}^{\infty} \cos \left(\frac{2 \pi}{n} j l\right) F_{0}\left(k_{c} \frac{\pi j}{n}\right)
$$

with respect to $l$. Solving

$$
\frac{\partial \Omega_{l}^{n}}{\partial l} \simeq \sum_{j=-\infty}^{\infty} \sin \left(\frac{2 \pi}{n} j l\right) \frac{2 \pi}{n} j F_{0}\left(k_{c} \frac{\pi j}{n}\right)=0,
$$

yields the two solutions $l=0$ and $l=n / 2$ for $l \in[0, n-1]$. Since

$$
\frac{\partial^{2} \Omega_{l}^{n}}{\partial l^{2}} \simeq-\sum_{j=-\infty}^{\infty} \cos \left(\frac{2 \pi}{n} j l\right)\left(\frac{2 \pi}{n} j\right)^{2} F_{0}\left(k_{c} \frac{\pi j}{n}\right)
$$

these two solutions can be expressed as

$$
\left.\frac{\partial^{2} \Omega_{l}^{n}}{\partial l^{2}}\right|_{l=0}=-\sum_{j=0}^{\infty}\left(\frac{2 \pi}{n} j\right)^{2} F_{0}\left(k_{c} \frac{\pi j}{n}\right)<0
$$

and

$$
\left.\frac{\partial^{2} \Omega_{l}^{n}}{\partial l^{2}}\right|_{l=n / 2}=-\sum_{j=\infty}^{\infty}(-1)^{j}\left(\frac{2 \pi}{n} j\right)^{2} F_{0}\left(k_{c} \frac{\pi}{n} j\right),
$$

always assuming that $F_{0}(k)$ decays faster than $k^{-3}$ for $k \rightarrow \infty$. In this case, it is not difficult to show that $\Omega_{l}^{n}$ has indeed a minimum at $l=n / 2$. Thus, the minimal eigenvalue of $g_{i j}$ can be approximately given by

$$
\omega_{\text {min }}^{n} \simeq \Omega_{n / 2}^{n}=-1+(2-g) \sum_{j=-\infty}^{\infty}(-1)^{j} F_{0}\left(k_{c} \frac{\pi j}{n}\right)
$$

and again scales with $k_{c} \gamma / n$ as assumed above. The resulting asymptotic stability ranges for all model instances considered in this chapter are depicted in Figure 5.6.2. They demonstrate that also the asymptotic stability ranges for the various types of biologically plausible interactions are almost independent of the precise spatial dependency of the long-range interactions. 


\subsection{Summary}

In this chapter, I have examined a variety of optimization models for the joint development of the system of long-range tangential connections and the pattern of orientation preferences. The main questions were whether the parallel development of the system of tangential connections and the OPM can be viewed as a dynamical optimization process and whether and how different types of long-range interactions like those mediated in the visual cortex by the system of tangential connections or mediated by visual inputs with long-range correlations are able to stabilize orientation preference patterns with realistic pinwheel-rich layouts. I proposed a variational model for the coupled dynamics of connections and OPM. Following [193], I adiabatically eliminated the time-dependent system of tangential connections to obtain an effective integro-differential equation for the development of the OPM that includes non-local interactions. Series expansion of this equation in the vicinity of the bifurcation point together with weakly nonlinear analysis led to amplitude equations with which pattern selection in these models could be analyzed. I calculated the complete phase diagram of stable solutions near the pattern formation threshold for models with Gaussian, exponential, power-law interactions as well as for models in which local connections are non-orientation-selective. I found that

- the spatial organization of stationary maps is entirely insensitive to the structure of longrange coupling;

- all types of long-range interactions are capable of stabilizing realistic patterns of orientation columns, provided the relative strength of long-range interactions compared to local interactions exceeds a critical strength;

- pinwheel stabilization by long-range intracortical interactions is highly robust and thus the spatial layout of orientation columns is expected to be fairly insensitive to the nature of long-range interactions.

The robustness of pinwheel stabilization could explain why in experiments orientation maps appear to be almost unaltered by dark-rearing or abnormal visual experience. Only genetic or experimental perturbations that are able to confine the lateral interactions in the visual cortex to a range smaller than a hypercolumn are predicted to induce a breakdown of spatially complex orientation maps into pinwheel sparse or crystal-like patterns [11]. According to the model, neither dark-rearing nor any other experimental manipulation has so far been able to drive visual cortical development into such a regime. 


\section{Chapter 6}

\section{Stimulus-driven optimization models for rodent visual cortical development}

\subsection{Motivation}

Since the discovery of orientation selectivity in cat V1 by Hubel and Wiesel in the early 1960s $[27,28,108]$, electrophysiologists have dedicated little attention to uncovering the organization, development and plasticity of the functional visual cortical architecture in Glires, i.e. rodents and lagomorphs, compared to primates and carnivorans. The reason for this negligence is probably threefold. Firstly, primates, in particular, are considered closer relatives of humans and therefore properties of primate brains are expected to be most easily transferable to the human nervous system. Secondly, while the columnar system of receptive field properties in primates and carnivorans has turned out to be a fruitful playground for the study of plasticity and development $[27,28,45,194]$, physiologists have failed to discover a similarly ordered selectivity organization in rodents and lagomorphs $[14,23,65]$. Thirdly, carnivorans and primates are thought to crucially depend on sharp and binocular vision whereas most rodents such as mice and rats as nocturnal animals are believed to rely more on tactile information and olfactory cues. This situation has almost reversed over the last few years, due to the advent of two-photon imaging techniques $[62,63,195-197]$ and transgenic mouse technology $[198,199]$ up to the point that the mouse visual cortex is currently considered one of the most versatile model systems for the study of cortical plasticity and critical periods $[102,104,105,200]$.

Several studies in the primary visual cortex of Glires species have revealed all the major receptive field types that have been described in cats, ferrets or monkeys [13, 15, 23, 65-67, 201, 202]. Many V1 neurons are orientation-selective, and, among these, simple and complex cells can be distinguished. The spatial scale of receptive fields in mouse, for instance, is up to one or two orders of magnitude larger, but at the same time neurons show selectivity for stimulus parameters such as orientation and spatial frequency close to that found in the cat or monkey [66]. Despite these highly selective receptive fields, no apparent spatial clustering of response properties could be identified [13-15, 65]. Instead, selectivities appear as if randomly distributed across the cortex (cf. Chap. 1, Sec. 1.3). Additionally, connection probabilities between orientation-selective neurons have been found to be only weakly dependent on the difference in the preferred orientations of 
connected neuron pairs $[15,203]$. The lack of columnar organization the primary visual cortex of in mice and rats has previously been hypothesized to stem from their relatively poor visual acuity and contrast sensitivity as well as their poorly laminated visual brain areas. But the fact that squirrels as highly visual animals with clear lamination of V1 and V2 [204] lack orientation maps suggests that an elaborated visual system is neither sufficient for nor dependent on the development of orientation maps in mammals [14]. In view of the uttermost confusing randomness exhibited by rodent and lagomorph V1 circuitry, Priebe and Ferster were even tempted to doubt that classical mechanisms such as Hebbian learning are at work in shaping such circuits during postnatal development: "If this were so, during development, there would be no need for 'Hebbian Plasticity' and instead neurons could wire together whether they fired together or not." they wrote in a review paper in 2010 [205]. In fact, currently no model exists that could explain the development of the observed disordered organizations of selectivities in terms of classical Hebbian paradigms.

In this chapter, I propose a simple optimization model for the activity-dependent development of orientation selectivity to explain the disordered selectivity organizations observed in Glires V1. The model is based on a simple and well-established Hebbian learning rule together with a normalization of the total amount of cortical activity, which induces a soft competition for activity among the neurons in the cortical layer. These two ingredients are shown to be sufficient to account for (1) the emergence of orientation selectivity and (2) the establishment of a spatially disordered organization of these selectivities. This clearly demonstrates that the apparent randomness and non-specificity in rodent and lagomorph visual cortical circuits is not at all in conflict with classical Hebbian plasticity paradigms.

\subsection{Model outline}

The model proposed in this chapter mathematically idealizes two processes that are widely accepted to be critical for activity-dependent cortical development: Firstly, afferent activity pattern from subcortical structures excite neurons in the cortical layer via feedforward connections and secondly, the selectivities of neurons in the target layer are modified as a function of pre- and postsynaptic activity via Hebbian learning. Similar to dimension reduction approaches for modeling columnar organizations in V1, e.g. [142, 144, 146, 166], the model formalizes these two processes on an intermediate level of description. Afferent activity patterns, called stimuli in the following, are represented by only two variables - their position in visual field coordinates as well as an orientation toward which the selectivities of units in the target layer are modified when being activated by the stimulus. As throughout this thesis, the spatial organization of orientation preference is described by a complex-valued field $z(\mathbf{x})$ and the preferred angle $\vartheta(\mathbf{x})$ of a cell at cortical position $\mathbf{x}$ is obtained from $z(\mathbf{x})$ via

$$
\vartheta(\mathbf{x})=\frac{1}{2} \arg (z(\mathbf{x}))
$$

The model of stimulus-driven visual cortical development in Glires V1 is based on the modification rule [166]

$$
\delta z(\mathbf{x})=\varepsilon\left(s_{z}-z(\mathbf{x})\right) e(\mathbf{x}, \mathbf{S}, z(\cdot)),
$$


a

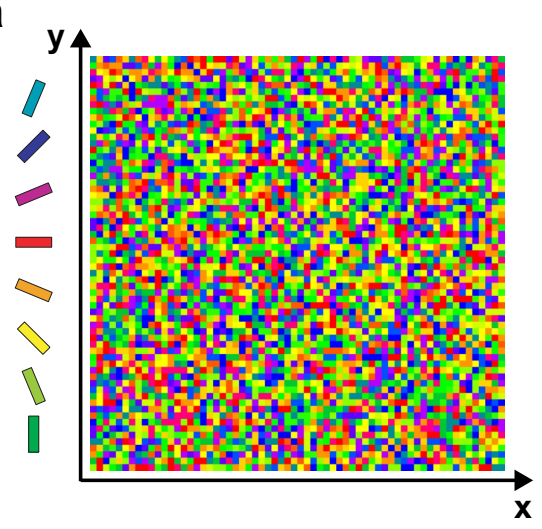

C

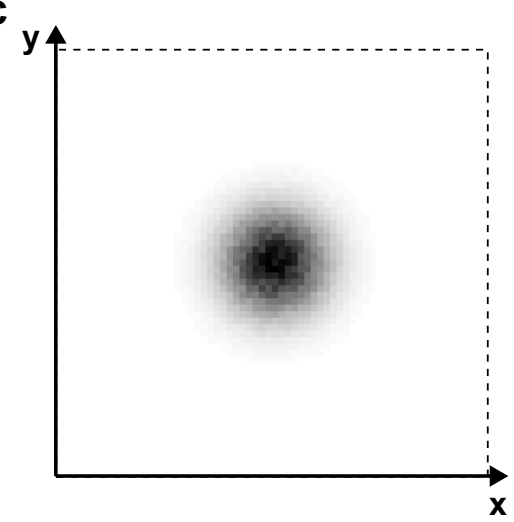

b

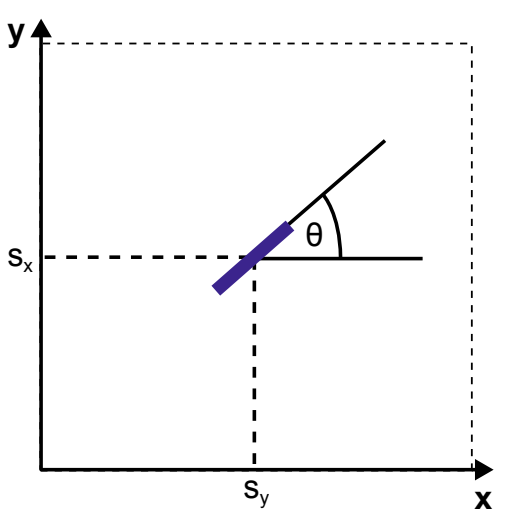

d

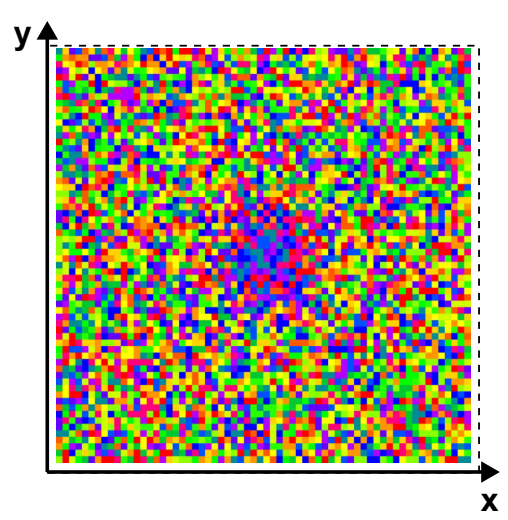

Figure 6.2.1: Schematic illustration of the stimulus-driven model. (a) Example of a random orientation preference pattern (color code) $z(\mathbf{x})=10^{-2} e^{i 2 \pi \xi(\mathbf{x})}$, where the $\xi(\mathbf{x})$ are independent random numbers uniformly distributed in $[0,1]$. (b) Position $\mathbf{s}_{r}=\left(s_{x}, s_{y}\right)$ and orientation $\theta$ of a 'point-like' stimulus. (c) Cortical activity, evoked by the stimulus in $\mathbf{b}$ for the orientation preference pattern in a. Dark regions are activated. Note that neighboring neurons are coactivated. (d) Orientation preference pattern $z(\mathbf{x})+\delta z(\mathbf{x})$ after modification, caused by the stimulus in b (cf. Eq. (6.2.1)). Orientation preferences in the vicinity of the stimulus position are adjusted to better match the orientation of the stimulus. A very high learning rate $(\varepsilon=1.5)$ was chosen for illustration purposes.

which specifies how the pattern of orientation selectivity $z(\mathbf{x})$ is changed through a stimulus $\mathbf{S}=$ $\left\{\mathbf{s}_{r}, s_{z}\right\}$, with $s_{z}=\left|s_{z}\right| e^{2 i \theta}$, and $\mathbf{s}_{r}=\left(r_{x}, r_{y}\right) . \theta$ denotes the orientation of a stimulus and $\mathbf{s}_{r}$ its position. Every individual stimulus $\mathbf{S}$ is assumed to cause a plastic change in the receptive field parameters at activated locations, which is proportional to their response $e(\mathbf{x}, \mathbf{S}, z(\cdot))$ and which enhances the matching between receptive fields and activity patterns. For simplicity and in good agreement with experimental observations in mouse V1 [23, 24, 200], I assume an ordered one-toone topographic mapping between retinal and cortical coordinates. The activation rule is exactly chosen as for the Elastic Network model with fixed retinotopy in Chapter 3 [142]:

$$
e(\mathbf{x}, \mathbf{S}, z(\cdot))=\frac{e^{-\left(\left|\mathbf{s}_{r}-\mathbf{x}\right|^{2}\right) / 2 \sigma^{2}} e^{-\left(\left|s_{z}-z(\mathbf{x})\right|^{2}\right) / 2 \sigma^{2}}}{\int d^{2} y e^{-\left(\left|\mathbf{s}_{r}-\mathbf{y}\right|^{2}\right) / 2 \sigma^{2}} e^{-\left(\left|s_{z}-z(\mathbf{y})\right|^{2}\right) / 2 \sigma^{2}}} .
$$

The parameter $\sigma$ is the receptive field size measured in stimulus parameter space. Neurons which are close together in the cortical target layer have similar spatial receptive fields and are therefore likely to be co-activated by an afferent activity pattern. The integral term in the denominator leads to a normalization of the total amount of activity, and, hence, to a so-called soft competition in the target layer [116]. This divisive form of the normalization can be derived from detailed network models with recurrent inhibitory interactions [206-208]. Figure 6.2.1 illustrates the general features of this model for a random pattern of orientation preferences (Fig. 6.2.1a). A single pointlike, oriented stimulus $\mathbf{S}=\left(\mathbf{s}_{r}, s_{z}\right)$ with position $\mathbf{s}_{r}=\left(s_{x}, s_{y}\right)$ and orientation $\theta=1 / 2 \arg \left(s_{z}\right)$ (Fig. 6.2.1b) evokes a cortical activity pattern $e(\mathbf{x}, \mathbf{S}, z(\cdot))$ (Fig. 6.2.1c). The stimulus and the evoked 
activity pattern induce a modification of the orientation preferences. Orientation preference in the activated regions is shifted towards the orientation of the stimulus (Fig. 6.2.1d). As outlined in Chapter 3, the repeated presentation of stimuli which each are assumed to have a very small impact on the orientation preferences in the target layer leads to an effective developmental dynamics of the form (cf. [166])

$$
\partial_{t} z(\mathbf{x})=F[z(\cdot)]=\left\langle\left[s_{z}-z(\mathbf{x})\right] e(\mathbf{x}, \mathbf{S}, z(\cdot))\right\rangle_{\mathbf{S}} .
$$

The only difference to the conventional Elastic Network model for the formation of ordered columnar selectivity layouts is the lack of the phenomenological excitation term, usually a Laplacian $[99,142,166]$ or some other differential operator $[142,209]$. It is known that these models exhibit a phase transition for decreasing $\sigma$, by which roughly periodic stimulus representations emerge on a characteristic timescale $\tau[99,142,166]$. In this chapter, I study the dynamics given by Equation (6.2.3), i.e. the Elastic Network model without lateral excitation term. The only lateral interaction present in the model is therefore mediated by the soft-competition for activity between the units. One of the key questions will be: What type of selectivity organization emerges in the absence of lateral excitation? Again, it is critical to emphasize that neighboring neurons are likely to be co-activated by an afferent activity patterns and intuitively one might expect ordered selectivity organizations also in the absence of lateral excitation.

As in Chapter 3, I make three basic and widely accepted assumptions about the set of stimuli: (i) The stimulus locations $\mathbf{s}_{r}$ are uniformly distributed across visual space. (ii) For the distribution of stimulus orientations, $\left|s_{z}\right|$ and $\theta$ are independent. (iii) Stimulus orientations $\theta$ are distributed uniformly in $[0, \pi]$. As outlined in Chapter 3, these assumptions imply translational, rotational and Shift-symmetry of the model dynamics. Thus, two patterns are equivalent solutions of the model, if their layout is identical up to translations (Eq. (1.5.2)) and/or rotations (Eq. (1.5.3)) as well as if their layout is identical but the preferred orientations differ everywhere by the same constant angle (Eq. (1.5.5)). Without loss of generality, I set $\left\langle\left|s_{z}\right|^{2}\right\rangle=2$. This normalization can always be restored by a rescaling of $z(\mathbf{x})$ (cf. $[99,166]$ ). Finally, I note that the model given by Equation (6.2.3) is variational, with energy functional

$$
\mathcal{C}[z]=-2 \sigma^{2}\left\langle\ln \int d^{2} y e^{-\left(\left|s_{z}-z(\mathbf{y})\right|^{2}+\left\|\mathbf{s}_{r}-\mathbf{y}\right\|^{2}\right) / 2 \sigma^{2}}\right\rangle_{\mathbf{S}}
$$

since

$$
\partial_{t} z(\mathbf{x})=-\frac{\delta \mathcal{C}[z]}{\delta \bar{z}(\mathbf{x})}
$$

This free energy term corresponds to the measure of stimulus space coverage in dimension reduction approaches to cortical map development, e.g. [134, 138, 139, 143, 166]. The developmental dynamics given by Equation (6.2.3) can therefore be interpreted as a dynamical optimization process to minimize (i.e. to optimize) coverage.

In the next paragraph, I perform a linear stability analysis of the homogeneous non-selective state. This stability analysis reveals the regions of parameter space, in which patterns of orientation preference emerge via spontaneous symmetry breaking. Using these insights, I then numerically solve the developmental dynamics (Eq. (6.2.3)). 


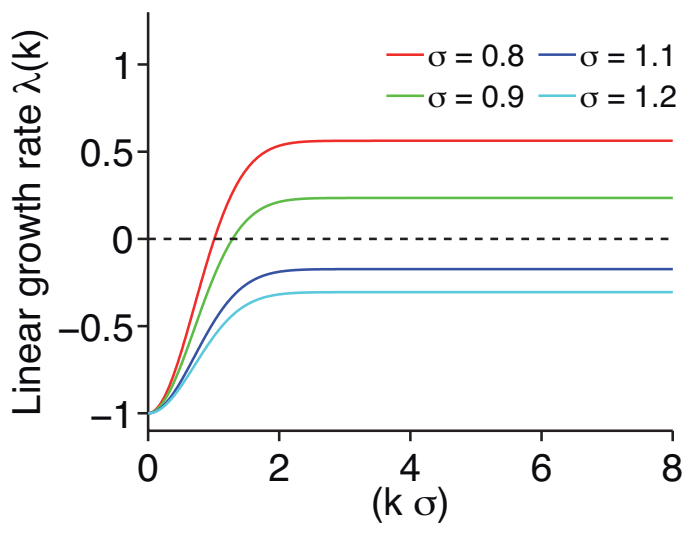

Figure 6.3.1: Linear growth rates (Eq. (6.3.2)) of Fourier modes $\sim e^{i \mathbf{k x}}$ as a function of the normalized wave number $k \sigma=|\mathbf{k}| \sigma$ as calculated from linear stability analysis of the homogeneous fixed point $z(\mathbf{x})=0$ in the model dynamics given by Equation (6.2.3) for four different receptive field widths $\sigma$. For $\sigma<1$, the homogeneous non-selective state loses stability with respect to all Fourier modes with wave vectors $k$ larger than a critical value $k_{c} \sigma=\sqrt{-\log \left(1-\sigma^{2}\right)}$.

\subsection{Linear stability analysis}

The Shift symmetry property (Eq. (1.5.5)) implies that the non-orientation-selective state $z(\mathbf{x})=0$ is a fixed point of Equation (6.2.3). Whether from this homogeneous non-selective state a pattern of orientation preferences emerges by spontaneous symmetry breaking is determined by the properties of the linearized dynamics of small deviations $z(\mathbf{x})$ around this state. This linearized dynamics reads

$$
\partial_{t} z(\mathbf{x}) \simeq L_{z}[z]=\left(\frac{1}{\sigma^{2}}-1\right) z(\mathbf{x})-\frac{1}{4 \pi \sigma^{4}} \int d^{2} y e^{-\frac{(x-y)^{2}}{4 \sigma^{2}}} z(\mathbf{y}) .
$$

By translational symmetry, the eigenfunctions of the linearization $L_{z}[z]$ are Fourier modes $\sim e^{i \mathbf{k x}}$. By rotational symmetry, their eigenvalues (or equivalently linear growth rates) only depend on the wave number $k=|\mathbf{k}|$. They are given by

$$
\lambda(k)=-1+\frac{1}{\sigma^{2}}\left(1-e^{-k^{2} \sigma^{2}}\right) .
$$

These linear growth rates are depicted in Figure 6.3.1 for four different values of $\sigma$. For all $\sigma>0$, the Fourier mode with infinite wave number possesses the largest eigenvalue

$$
r_{\text {control }}=-1+\frac{1}{\sigma^{2}} .
$$

For $\sigma>1$, this largest growth rate is negative, and, hence, the non-selective state is a stable fixed point of Equation (6.2.3). For $\sigma<1$, the maximal eigenvalue $r_{\text {control }}$ is positive, and the nonselective state is unstable with respect to all Fourier modes $\sim e^{i \mathbf{k x}}$ with wave numbers $|\mathbf{k}|$ larger than a finite critical value $k_{c}$ (cf. Fig. (6.3.1))

$$
k_{c}(\sigma)=\frac{1}{\sigma} \sqrt{-\log \left(1-\sigma^{2}\right)} .
$$

This instability predicts the spontaneous emergence of orientation selectivity from the non-selective state on a characteristic timescale $\tau=1 / r_{\text {control }}$. Note, however, that the instability found in this model is not an instability of Turing type $[154,210]$, because an infinite range of wave numbers is 
unstable for any finite value of $r_{\text {control }}>0$. Methods such as weakly nonlinear analysis (cf. Chapter 1) to analyze the behavior of the model in the nonlinear regime can therefore not be applied. Therefore, the precise arrangement of the emerging selectivities and in particular their long-term development has to be studied numerically.

\subsection{The dynamics of the model}

\subsubsection{Methods}

Adapting the methods from Chapter 3, I used a fourth-order Runge-Kutta scheme [190] to obtain numerical solutions of the dynamics given in Equation (6.2.3). Simulations were performed on $32 \times 32,64 \times 64,96 \times 96$, and $128 \times 128$ grids with periodic boundary conditions. Progression of time was measured in units of the intrinsic timescale $\tau=1 / r_{\text {control }}$ defined by the maximum growth rate of the linearization (cf. Eq. (6.3.3)). The integration time step was chosen as $\delta t=\tau / 20$. This ensured good approximation to the temporally continuous changes of the patterns. The stimulus average in Equation (6.2.3) was approximated by choosing a random representative sample of $N_{\mathcal{S}}$ stimuli at each integration time step with

$$
N_{s}=\max \left\{10^{5}, \frac{N_{0} \Gamma^{2}}{\left(\varepsilon_{s}\right)^{n}} \frac{\delta t}{\tau}\right\}
$$

where $n$ corresponds to the dimensions of the feature space in addition to the two retinal positions (in our case, $n=2), \Gamma^{2}=(L / \sigma)^{2}$ the squared aspect ratio of the simulated system in units of the squared width of the activation function $\sigma^{2}, \varepsilon_{s}$ the resolution in feature space, $N_{0}$ the number of stimuli that were required to approximate the cumulative effect of the ensemble of stimuli within each feature space voxel $\varepsilon^{n+2}$. The choices $N_{0}=100$ and $\varepsilon_{s}=0.05$ ensured a high signal-to-noise ratio for all the simulations. A high signal to noise ratio in the simulations is particularly important as the noise stemming from the finite sample stimulus averages is expected to have low-pass statistics [148]. The noise statistics thus may result in an artificial tendency for neighboring units to align their selectivities apart from potential alignments stemming from the model dynamics. Typical values for $N_{s}$ were between $2.5 \times 10^{5}$ and $4 \times 10^{6}$. To simulate the emergence of orientation selectivity, simulations were initialized with a random weakly selective state, $z(\mathbf{x}, t=0)=10^{-2} e^{i 2 \pi \xi(\mathbf{x})}$, where the $\xi(\mathbf{x})$ are independent identically distributed random numbers uniform in $[0,1]$. Different realizations were obtained by using different stimulus samples. When starting from ordered initial conditions, e.g. Figure 6.4.2, the amplitude $\langle|z(\mathbf{x})|\rangle_{x}$ of the initial states was set to the average value obtained in a simulation starting from the non-selective state with the same model parameters. Stimuli were drawn from two different distributions, each with $\left\langle\left|s_{z}\right|^{2}\right\rangle=2$ : (i) stimuli uniformly distributed on a ring with $\left|s_{z}\right|^{2}=\sqrt{2}$ (circular stimulus ensemble), (ii) stimuli uniformly distributed within a circle $\left\{s_{z},\left|s_{z}\right| \leq 2\right\}$ (uniform stimulus ensemble). Similar to Chapter 3, the results for both stimulus ensembles were virtually indistinguishable. All results that follow were obtained with the circular stimulus ensemble. 


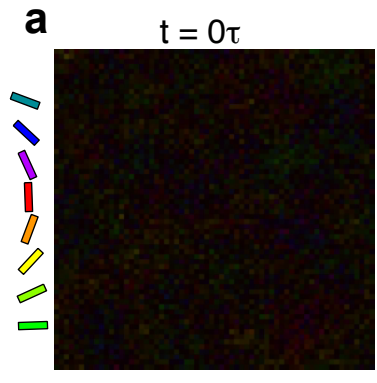

b

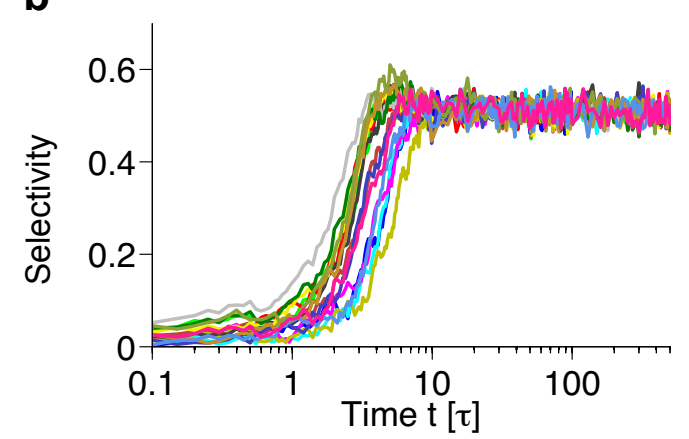

$t=5 \tau$

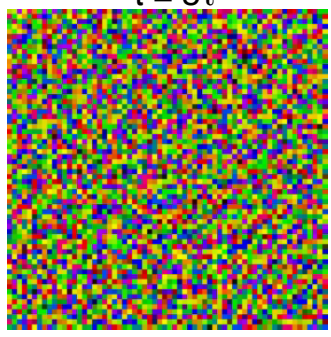

C

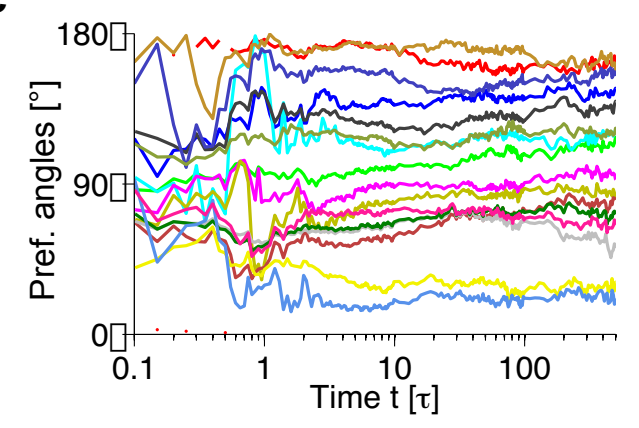

Figure 6.4.1: Dynamics of orientation preference layouts in the optimization model (Eq. (6.2.3)), starting from an initial condition with small random selectivities. (a) Snapshots of a simulation with $r_{\text {control }}=0.1$, circular stimulus ensemble (cf. Chap. 3 and Sec. 6.4.1) on a $64 \times 64$ mesh. Color encodes the preferred orientation, brightness the selectivity of the units. (b) Development of selectivities of 12 randomly chosen individual units in the simulation in a. (c) Orientation preferences of the units in $\mathbf{b}$.

\subsubsection{The spontaneous emergence of disordered selectivity organizations}

Firstly, the simulations confirmed the spontaneous emergence of selectivity for $\sigma<1$ (Fig. 6.4.1a,b), as expected from linear stability analysis. Figure 6.4.1a shows snapshots of a representative example of a simulation, starting from an initially spatially uncorrelated pattern of orientation preference with low selectivity (cf. Sec. 6.4.1 above). Selectivities rapidly increase during the first $10 \tau$ until they saturate, fluctuating around a constant value of 0.5 (Fig. 6.4.1b). Orientation preferences change substantially only within the first few $\tau$ and reach an almost stationary value thereafter (Fig. 6.4.1c). Most interestingly, however, despite the tendency for neighboring neurons to be co-activated (cf. Fig. 6.2.1), no ordered columnar structure develops. Instead, at first sight, the numerically stationary state of the simulation at large values of $\tau$ appears to be a random spatial arrangement of selectivities. This is very reminiscent of the selectivity layouts observed in rodents (cf. Fig. 1.3.1 and [13, 14, 211]). It needs to be emphasized at this point that the disordered stationary states observed in the numerics are not a consequence of disorder in the dynamical equations, for instance random coupling between the units on the 2-dimensional lattice. Unlike in spin glass models, e.g. [212-214], where the disorder results from disordered connections between the spins, Equation (6.2.3) is translation and rotation invariant. The spatial disorder in the numerically stationary states arises by inhibitory interactions between the sites. The effectively suppressive interactions, mediated by the soft-competition for activity among the units, lead to a separation of orientation preferences despite initial co-activation of neighboring units. These interactions favor 
the separation (misalignment) of selectivities of a pair of neighboring units. However, similar to classical geometrically frustrated systems such as antiferromagnetically coupled spins on triangular and face-centered cubic lattices [215, 216], disorder arises when the preferred orientations of multiple sites (within a range $\sigma$ ) attempt to simultaneously misalign due to the inhibitory interactions during development. I conclude that Hebbian learning of feedforward connections together with soft competition in the cortical layer naturally generates a salt-and-pepper layout of orientation selectivities via a type of geometric frustration during the developmental dynamics.

\subsubsection{Starting from ordered initial conditions}

In the last paragraph, a simple Hebbian learning scheme together with normalization of the total V1 activity has been demonstrated to be sufficient to explain the spontaneous emergence of salt-and-pepper orientation selectivity layouts when initializing simulations with random initial conditions. However, by the shift and translational symmetry alone, the orientation stripe pattern (cf. Fig. 1.5.1) is a stationary solution of the developmental dynamics given by Equation (6.2.3) (cf. Chapter 3). Furthermore, crystalline patterns of orientation preferences such as rhombic pinwheel crystals and orientation stripes have appeared in numerous models for OPM development $[109,118,122,123,125,133]$. They have also been identified as optimal dimension reducing mappings in the Elastic Network model (see Chapter 3), which bears striking similarity to the model treated in this chapter. For these reasons, it is crucial to test whether ordered states are stable stationary states of the model dynamics (Eq. (6.2.3)). In order to do so, I initialized simulations with the most widely found crystalline states: (i) orientation stripes (OS), (ii) rhombic pinwheel crystals (rPWC), and (iii) aperiodic pinwheel rich layouts resembling experimentally observed maps (for Methods see Sec. 6.4.1) with aspect ratios ranging from $6 \Lambda \times 6 \Lambda$ to $12 \Lambda \times 12 \Lambda$ on numerical grids with $64 \times 64$ and $128 \times 128$ grid points. Figure 6.4.2a shows snapshots of a representative simulation starting from an OS initial condition. The OS pattern rapidly decays into an almost randomly looking pattern, were neighboring neurons have very different orientation preferences. A salt-and-pepper layout is dynamically reached from an ordered initial condition. A very similar picture emerges when rPWC and aperiodic orientation maps layouts are considered as initial conditions (Fig. 6.4.2b,c). Hence, none of the canonical solutions of models for the development of columnar orientation selectivity layouts appears to be stable with respect to the dynamics of the model presented so far.

Finally, simulations were initialized with salt-and-pepper states obtained from simulations as in Figure 6.4.1, but where a square subregion of neurons was set to have the same preferred orientation (Fig. 6.4.2d, left). Such an initial configuration mimics a rodent or lagomorph orientation preference layout after the preferred orientations in a small cortical subregion have been artificially aligned, e.g. by a pairing experiment [217-219]. In the model, the neurons in the aligned subregion dynamically spread their preferred orientations to form part of the overall salt-andpepper arrangement (Fig. 6.4.2d). These changes occur on larger timescales compared to the timescale of emergence of orientation selectivity from random initial conditions (Fig. 6.4.1). Such long-term rearrangements after a pairing protocol represent perhaps the most easily testable prediction of the stimulus-driven model presented in this chapter. 
a
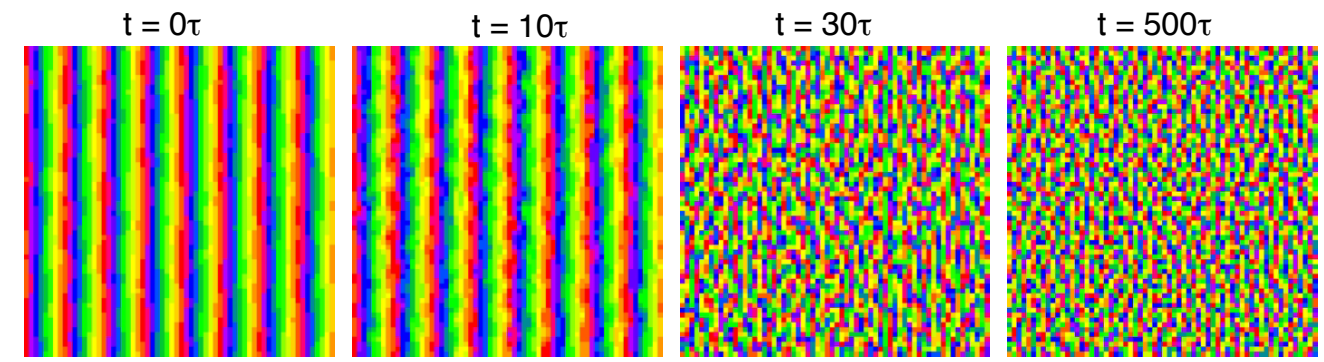

b
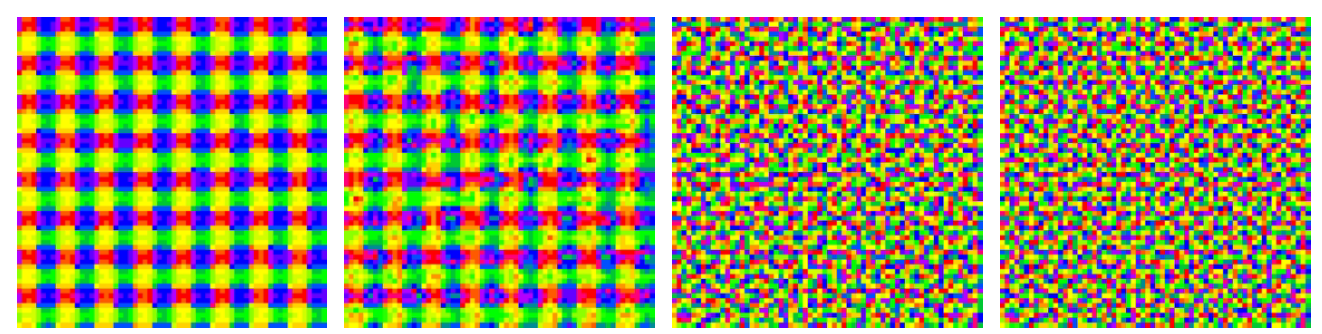

C
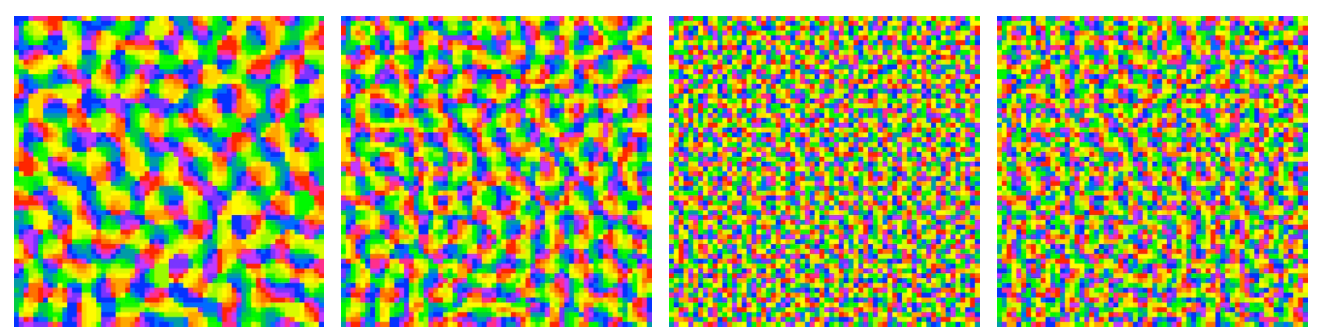

\section{d}
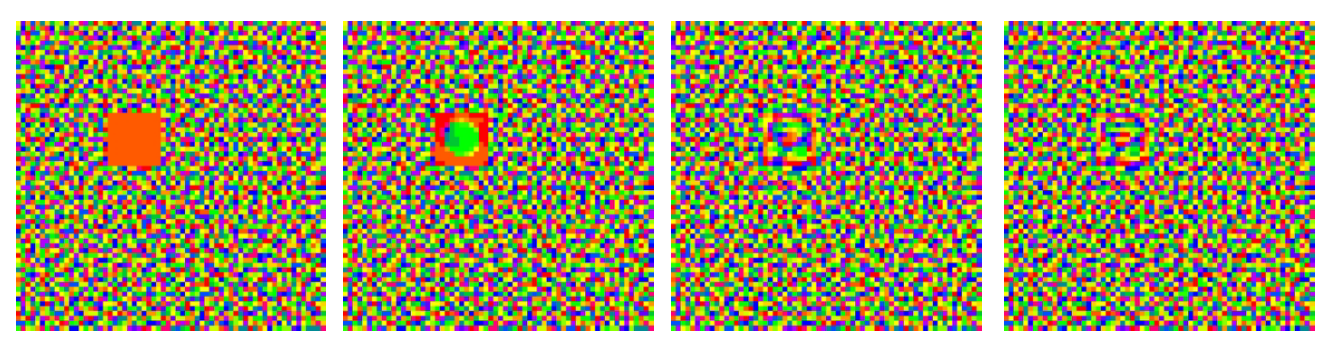

Figure 6.4.2: Dynamically obtained salt-and-pepper layouts in a stimulus-driven optimization model for visual cortical development. (a) Orientation preference patterns obtained in a simulation of Equation (6.2.3) $\left(r_{\text {control }}=0.1\right)$ starting from pinwheel-free orientation stripes. (b) As a but starting from a rhombic pinwheel crystals. (c) As a, but starting from an aperiodic pinwheel-rich state with spatial layout similar to experimentally observed orientation maps in carnivoran and primate V1. (d) As a, but starting from a dynamically obtained salt-and-pepper state as in Figure (6.4.1) with a subregion in which selectivities are clustered (cf. Sec. 6.4.3). 
Figure 6.4.3: Illustration of the discrepancy measure. Right: The discrepancy of a sequence of $N$ random numbers distributed in $[0, \pi]$ is defined as the maximum distance between the cumulative distribution of these numbers and the distribution function $P(x)=x / \pi$. Left: Schematic illustration for the extraction of the distancedependent discrepancy (cf. Sec. 6.4.4).
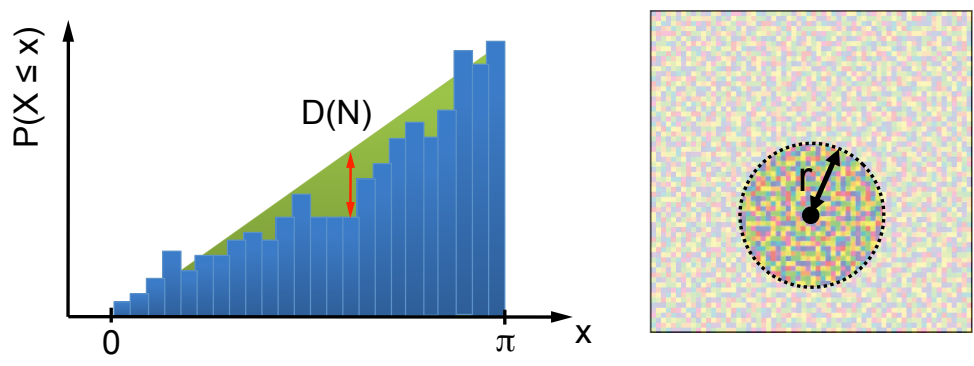

\subsubsection{Quantifying the spatial structure of orientation preference patterns in the model}

In the previous sections, I have studied a model for the development of orientation selectivity in Glires visual cortex in which disordered states are dynamically reached as equilibria when starting from random weakly orientation-selective states as well as from ordered initial conditions. In the following, I address the question as to whether the disordered states obtained in our simulations are quantitatively different from just random spatially uncorrelated layouts.

I used three different measures to quantitatively access the structure of the apparently random stationary patterns obtained in numerical simulations. As a first measure, I used the radial part of the conventional spatial correlation function

$$
C(r)=\frac{1}{2 \pi} \int d \phi C(\mathbf{r}),
$$

with

$$
C(\mathbf{r})=\frac{\langle z(\mathbf{x}) \bar{z}(\mathbf{x}+\mathbf{r})\rangle_{\mathbf{x}}}{\left\langle|z(\mathbf{x})|^{2}\right\rangle_{\mathbf{x}}}
$$

To assess higher-order spatial statistical properties not captured by the correlation function, I used the so-called root mean square discrepancy as a function of distance. The root mean square discrepancy $D_{N}$, simply referred to as "discrepancy" in the following, has been found to be a useful measure of the equidistribution of a random sequence of numbers $a_{1}, a_{2}, \ldots a_{N}$ in some interval. The discrepancy with respect to the interval $[0, \pi]$ is defined as

$$
D_{N}=\sup _{0 \leq a<b \leq \pi}\left|\frac{\left\{a_{1}, a_{2}, \ldots a_{N}\right\} \cap[a, b]}{N}-\frac{(b-a)}{\pi}\right| .
$$

The smaller the value of $D_{N}$, the more uniform the sequence fills the interval $[0, \pi]$. Note that $D_{N}$ does not depend on the order of the sequence. This fact was employed to obtain the discrepancy as a function of distance, $D(r)$, for a state reached by the numerical simulations. To calculate $D(r)$, I first constructed a sequence which consisted of all orientation preferences of units within a distance $r$ from the unit $(i, j)$ (cf. Fig. 6.4.3) and computed its discrepancy. These discrepancies were then averaged over all units $(i, j)$ to obtain $D(r)$. To reveal higher-order effects, discrepancies of states obtained in the simulations were compared to the discrepancies of randomized states with identical second-order statistics.

Finally, I adopted Swindale's measure of stimulus coverage for cortical selectivity layouts. This 
measure of coverage is based on the variation of the total amount of neural activity $A$, evoked in the striate cortex by a point-like stimulus $[136,220]$. Swindale hypothesized that the columnar architecture of the primary visual cortex of carnivorans and primates is designed in such a way as to minimize the local variation in $A$. As a simple measure of non-uniformity he chose the ratio between the standard deviation of the total amount of activity and the activity itself, i.e.

$$
c=\frac{\sqrt{\left\langle A^{2}\right\rangle}}{\langle A\rangle}
$$

where the mean is taken over all contour orientations and spatial positions. When $c$ is large, coverage is bad, i.e. $A$ is uneven. According to Swindale, coverage uniformity, as defined by Equation (6.4.3), is a measure of one source of uncertainty in the amount of neural activity evoked in V1 by a contour of a particular orientation lying in a particular receptive field position [220].

If the function $e(\mathbf{x}, \mathbf{S}, z(\cdot))$ in Equation (6.2.3) was chosen as the expression for the neural activity $A$, the total amount of neural activity $\int d^{2} x e(\mathbf{x}, \mathbf{S}, z(\cdot))$ evoked by any stimulus $\mathbf{S}$ would be equal to one for all the stimuli. Hence, with an activity definition including soft competition via divisive normalization, coverage is zero by definition, i.e. perfect, for any map in the model. To nevertheless analyze the coverage of the simulated orientation preference patterns, I adopted Swindale's definition of evoked neural activity [220]. For a contour of a particular orientation $\theta$ present in a particular visual field location $(\psi, \phi)$ he defines the activity $A(\theta, \psi, \phi)$. This activity is computed via the so-called cortical point image $P_{c}(x, y)$, the distribution of activity in the cortex evoked by stimulation of a point $(\psi, \phi)$ in visual space. Let $\theta(x, y)$ be the preferred orientation as a function of cortical position and let $\Omega\left(\theta-\theta_{c}\right)$ be an averaged orientation tuning curve. Assuming that the map of visual space is isotropic and the magnification factors relating $(\psi, \phi)$ to $(x, y)$ are both unity, one can define the activity $A$ as

$$
A(\theta, \psi, \phi)=\int d x^{\prime} d y^{\prime} \Omega\left(\theta-\theta_{c}\left(x^{\prime}, y^{\prime}\right)\right) P_{c}\left(\psi-x^{\prime}, \phi-y^{\prime}\right) .
$$

Following Swindale, I chose

$$
P_{c}(x, y)=\frac{1}{2 \pi \sigma^{2}} e^{-\left(x^{2}+y^{2}\right) / 2 \sigma^{2}},
$$

with the $\sigma$ as given by the model parameters. For the Tuning Curves, I chose a von-Mises-function

$$
\Omega\left(\theta-\theta_{c}\right)=\frac{e^{\kappa \cos \left(2\left(\theta-\theta_{c}\right)\right)}}{2 \pi I_{0}(\kappa)}
$$

with $\kappa=\pi / 8$ and $I_{0}(\kappa)$ being the modified Bessel function of first kind. All other parameters were chosen as in [220].

Figure 6.4.4 demonstrates how these measures distinguish the stationary states obtained in simulations of Equation (6.2.3) from random arrangements. Figure 6.4.4a again depicts the emergence of an orientation-selective salt-and-pepper layout. In Figure $6.4 .4 \mathrm{~b}$, the correlation functions corresponding to the states in Figure 6.4.4a are depicted. During the simulation, the uncorrelated random initial arrangement of weakly selective units develops into a pattern with strong local anti-correlation. This quantitatively demonstrates that the effectively suppressive interactions, mediated by the soft competition for activity among the units in the model, in fact lead to a local 

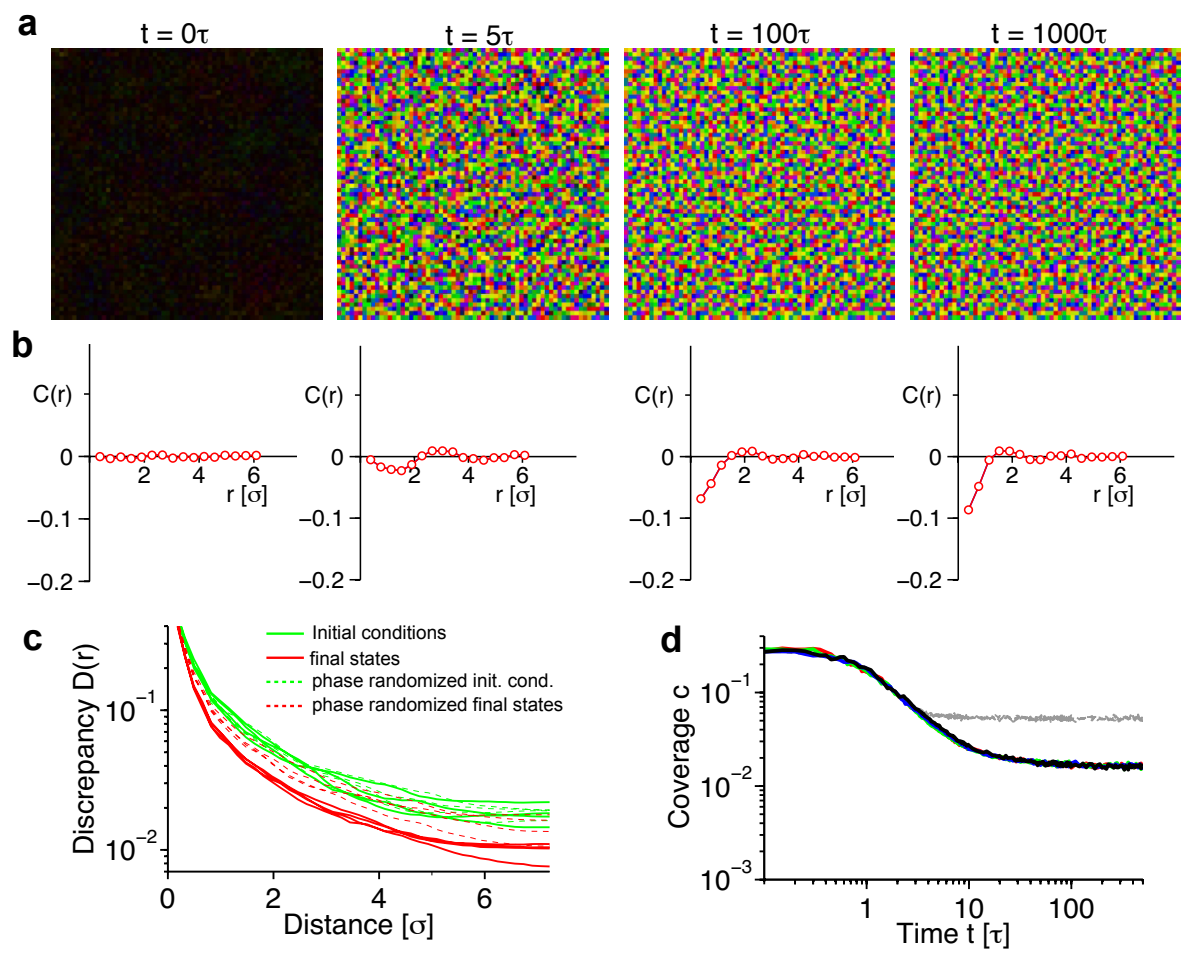

Figure 6.4.4: Quantifying salt-and-pepper layouts obtained in the model. (a) Redrawn from Fig. 6.4.1a. (b) Spatial correlation function $C(r)$ (Eq. (6.4.1)) for the maps depicted in a. (c) Discrepancy (Eq. (6.4.2)) as a function of distance for $N=4$ simulations with random weakly selective initial conditions (solid green lines) as well as for the final states after $t=1000 \tau$ (solid red lines). The dashed green and red lines represent discrepancies for randomized initial states and final states respectively (cf. Sec. 6.4.1). (d) Swindale coverage for $N=4$ simulations (parameters as in a). The grey lines represent Swindale coverage of phase randomized time courses with identical second-order statistics.

'repulsion' (or misalignment) of orientation preferences. Units in the immediate vicinity tend to avoid each others preferred orientations. In Figure 6.4.4c, the discrepancies $D(r)$ as a function of distance for the initial and the final states at $t=1000 \tau$ are shown for four simulations. The discrepancy of the final states is always lower than that of the uncorrelated initial conditions. Discrepancies for randomized final states are considerably larger than those of their simulation counterparts. This illustrates that the local anti-correlation (Fig. 6.4.4b) containing two-point statistics only, does not fully account for the spatial structure of the salt-and-pepper layout of the model. It is intuitively clear that a layout of orientation preferences with locally anti-correlated preferred orientations achieves an excellent coverage of the space of stimuli. Units selective to all possible orientation can be encountered within a very small subregion. Indeed, the development of the pronounced anti-correlation is accompanied with a drop in coverage during development (Fig. 6.4.4d). However, again this drop is much more pronounced compared to the randomized states with identical second-order statistics (Fig. 6.4.4d).

Equation (6.2.3), which describes the dynamics of orientation preference layouts as a function of time, has only a single parameter, i.e., the width of the activation function $\sigma$. This parameter modulates the distance to the instability of the homogeneous non-selective state. However, as revealed by numerical simulations (Figs. 6.4.1,6.4.2,6.4.4), orientation preference layouts in the 

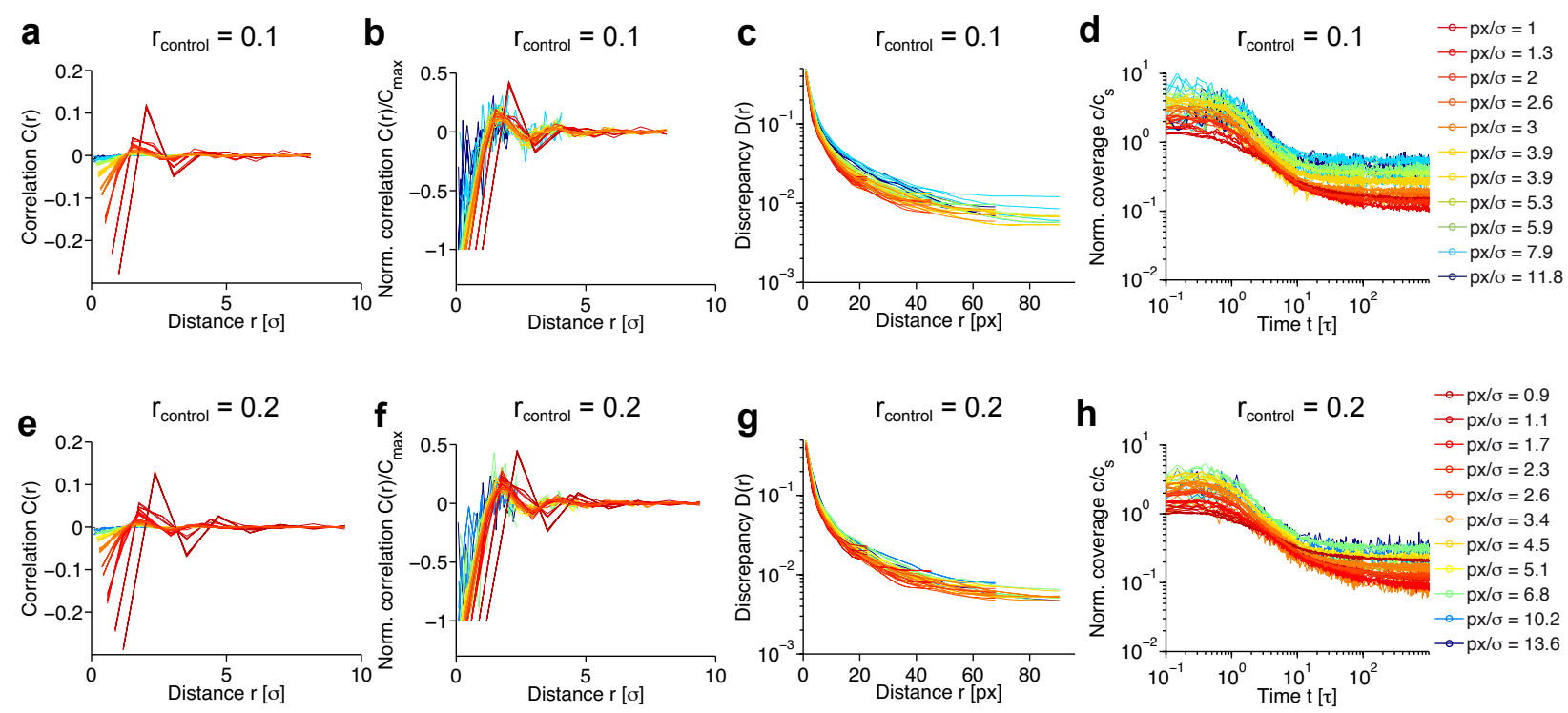

Figure 6.4.5: Characterization of numerically stationary salt-and-pepper states of the model as a function of the numerical discretization. (a-d) Correlation functions (a), correlation functions normalized by their maximum value (b), discrepancies as a function of distance (c), and Swindale coverage as a function of simulation time (d) for simulations with control parameter $r_{\text {control }}=0.1$ and different ratios $p x / \sigma$ (line colors, see legend on the right). (e-h) As a-d but for $r_{\text {control }}=0.2$. To enable comparison among different simulations, coverage values $c$ in the simulations were normalized with the values $c_{S}$, obtained for a spatially randomized arrangement of the preferred orientations in the simulations. For each set of parameter, $N=4$ simulations are shown.

model rapidly exhibit spatial modulations on the scale of the discretization mesh used. It is therefore essential to clarify, whether the statistics of the solutions of the model critically depend on the chosen grid size. To answer this question, I simulated Equation (6.2.3) for different numbers of grid points $p x$ per width of the activation function $\sigma$, keeping $\sigma$ at the same numerical value. In all of the simulations, when starting from a weakly selective state (see Methods above), numerically stationary orientation selectivity layouts were of apparently random nature very similar to Figure 6.4.1. Nevertheless, a quantification of the layouts for different values of $p x / \sigma$ revealed substantial differences between them (Fig. 6.4.5). Local anti-correlations in the numerically stationary states decrease with increasing $p x / \sigma$ (Fig. 6.4.5a). Normalizing the correlations by the maximum absolute value $C_{\max }$ acquired outside of $r=0$, reveals that the range of the correlation, measured in units of $\sigma$, is rather invariant (with the exception of simulations with very few grid points within a $\sigma$ range ( $p x / \sigma<1.5$, cf. Fig. 6.4.5b)) and essentially set by the range of soft competition. Only the magnitude of the anti-correlations decreases with increasing number of grid points per $\sigma$. According to these observations, one would expect a spatially uncorrelated pattern for $p x / \sigma \rightarrow \infty$. The decrease in anti-correlation strength is not surprising, given the invariance of its range as illustrated by the following simple calculation using the $n \times n$ covariance matrix $M$,

$$
(M)_{i j}=\left\langle z\left(\mathbf{x}_{i}\right) \bar{z}\left(\mathbf{x}_{j}\right)\right\rangle,
$$

$i, j=0 \ldots n-1$ for the preferred orientations of the $n-1$ nearest neighbor grid points. Approximating the anti-correlation as a constant around $-\gamma$ in a certain range, then discretizing with $n$ 
grid points within this range leads to the cyclic covariance matrix

$$
M=\left(\begin{array}{ccccc}
1 & -\gamma & -\gamma & \cdots & -\gamma \\
-\gamma & 1 & -\gamma & \ddots & -\gamma \\
-\gamma & -\gamma & \ddots & -\gamma & \vdots \\
\vdots & \ddots & -\gamma & 1 & -\gamma \\
-\gamma & -\gamma & -\gamma & -\gamma & 1
\end{array}\right)
$$

for the $n-1$ units within the range of anti-correlation. This cyclic matrix has eigenvalues

$$
\lambda_{m}= \begin{cases}1-(n-1) \gamma & m=1 \\ 1+\gamma & m=2 \ldots n\end{cases}
$$

and positive semi-definiteness of the covariance matrix implies that

$$
\gamma \leq \frac{1}{n-1}
$$

With increasing number of grid points within the range of the local anti-correlation, the value of the negative correlation necessarily has to decrease. In two dimensions, the strength of the local anti-correlations should decrease $\sim 1 /(p x / \sigma)^{2}$, which is indeed observed.

Similarly to the second-order statistics, also the higher-order statistical quantities that have been introduced above demonstrate that the numerically stationary states come closer to purely random layouts with increasing $p x / \sigma$. The discrepancy for the stationary states as a function of distance, now measured in $p x$ to enable comparison between the simulations, tend to increase with increasing $p x$ to $\sigma$ ratio (Fig. 6.4.5c). Swindale's measure of coverage shows a much stronger decrease with respect to spatially randomized arrangement of the preferred orientations in the simulations when $p x / \sigma$ is small (Fig. 6.4.5d). None of the above properties depends on the distance to instability threshold (compare Fig. 6.4.5a-d with Fig. 6.4.5e-g).

To summarize, the numerically stationary states obtained in simulations of a stimulus-driven model for Glires cortical development were found to be distinct from random arrangement. They could be characterized by a local anti-correlation of preferred orientations and a decreased discrepancy and coverage compared to random selectivity arrangements as well as randomized states with identical correlations. For increasing number of grid points per range of activation in the model, the observed numerically stationary states become more similar to spatially random layouts.

\subsubsection{Modeling an initial bias in preferred orientations}

Recent data suggests an interesting difference of the development of orientation selectivity between Glires on the one hand and carnivorans or primates on the other. Rochefort et al. discovered that in mice, prior to any visual experience, a fraction of around $12 \%$ of neurons in V1 were orientation-selective [107]. Furthermore, almost all of these neurons appeared to preferentially responded to anterodorsal directions. This corresponds to anterior and dorsal motion 

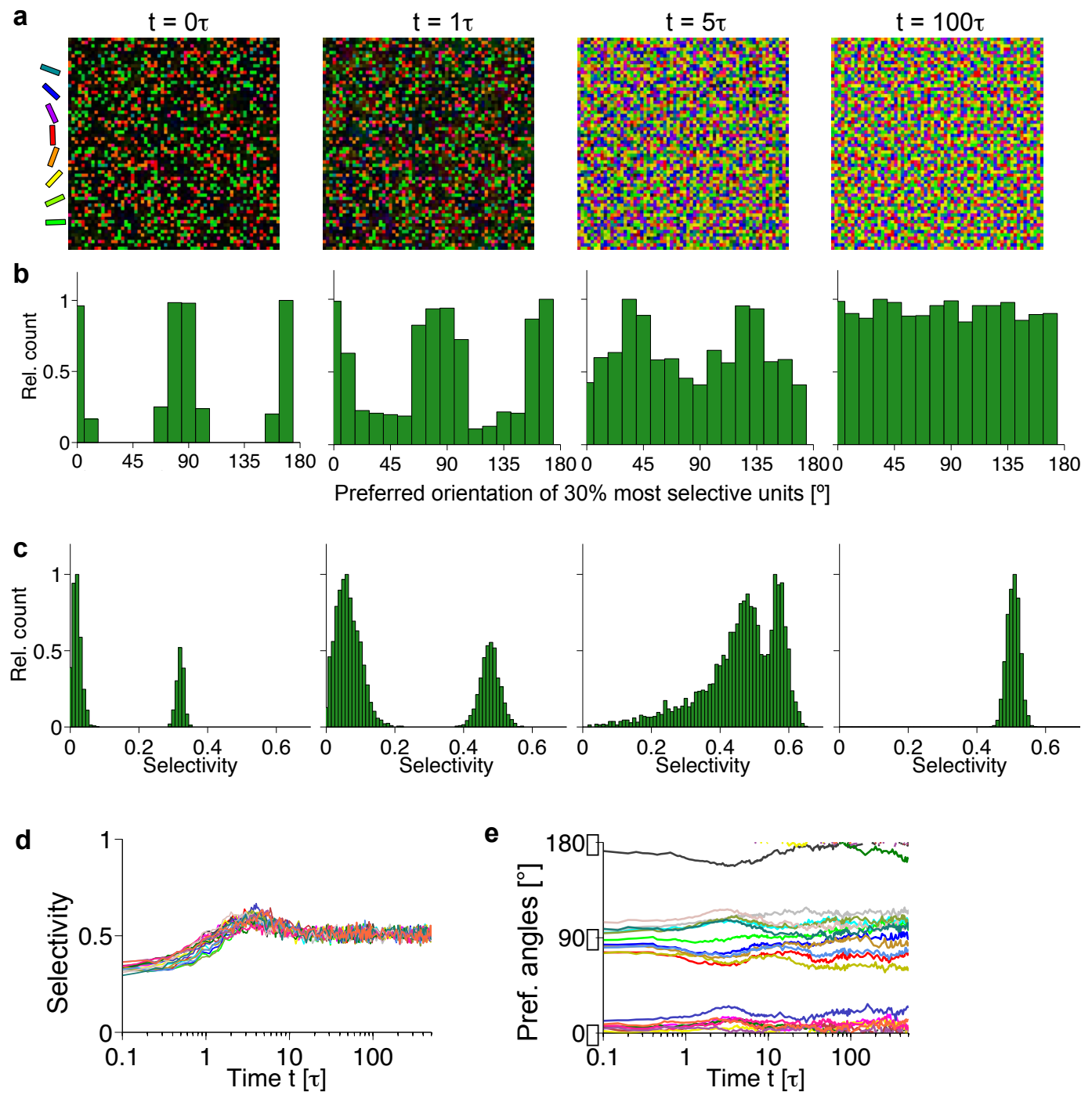

Figure 6.4.6: Initial bias of preferred orientation in a subpopulation of units vanishes via active self-organization of salt-and-pepper layouts. (a) Snapshots of a simulation with $r_{\text {control }}=0.1$, circular stimulus ensemble (cf. Chap. 3) on a $64 \times 64$ mesh and initial condition mimicking the experimentally observed initial bias toward a preference for anterodorsal directions [107] (cf. Sec. 6.4.5) (b) Histograms of preferred orientations of the 30\% most selective cells for the simulation in a. (c) Histograms of selectivities for the simulation in a. (d, e) Selectivities (d) and preferred orientations (e) of initially orientation-selective units. 
Figure 6.5.1: Convolution kernels given by Equation (6.5.2). For $S=0$, the kernel is negative and the model thus contains only inhibitory interactions. For increasing $S$, an excitatory component is added to the kernel. For $S=1$, the convolution kernel is purely excitatory. However, the model still contains both types of interactions, excitation mediated by the convolution and inhibition mediated by soft competition for activity which is induced by the normalization of the total amount of activity in the cortical layer.

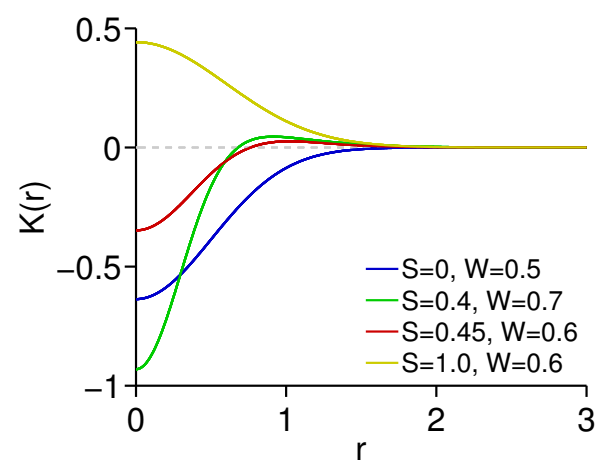

direction, i.e. $0^{\circ}$ and $90^{\circ}$ orientation, in visual coordinates. During later development, the fraction of orientation-selective neurons increased to about 33\% and the initial bias in preferred orientations vanished. Orientation selectivity development and bias were found to be unaltered in dark-reared mice, indicating that the early development of direction and orientation selectivity is independent of visual experience. The authors speculate that such bias might originate from the early formation of direction-selective retinal ganglion cells via a genetically predetermined and experience independent asymmetric inhibition [221,222]. In this view, the subcortical inputs driven by direction-selective retinal ganglion cells with strong preference for motion toward either the temporal or the ventral pole of the retina could be conceived as providing a seed for the emergence of orientation selectivity in V1 [107, 221].

To include these findings into the model, I initialized simulations with states in which $20 \%$ of the units had either $0^{\circ}$ or $90^{\circ}$ orientation preference with $50 \%$ of the expected selectivity of the final states (cf. Fig. 6.4.6a-c). All other units had small random selectivities with $\sim 10^{-2} e^{i 2 \pi \xi(\mathbf{x})}$, where the $\xi(\mathbf{x})$ are independent identically distributed random numbers uniform in $[0,1]$ (Fig. 6.4.6a). When initialized with these conditions, the self-organization dynamics of the model almost exactly reproduces the findings by Rochefort et al.. Within a few $\tau$, the initially unselective neurons reach mature selectivities (Fig. 6.4.6a,c) and the distribution of preferred angles for the most selective units quickly loses its bias to achieve a uniform distribution (Fig. 6.4.6b, most right). During this dynamics, the preferred orientations and selectivities of the units seeded with strong preferences exhibit only small changes (Fig. 6.4.6d,e). This, in turn, means that the preferences of the other units literally fill in the remaining angles to obtain an equal representation of the space of orientations. Such type of dynamics represents an interesting prediction of the model, testable by repeated 2-photon-imaging of the same group of cells in developing mice.

\subsection{Adding lateral excitation to the model}

The previous sections introduced a stimulus-driven model for Glires visual cortical architecture, based on a Hebbian learning scheme and an activation rule normalization of the total amount of V1 activity. Mathematically, the model dynamics has great similarities with classical feature map models such as the Elastic Network (cf. Chap. 3), Kohonen's self-organizing feature map [145] or the convolution model by Scherf et al. [144]. These models have previously been used to describe the development of continuous feature representation in the primary visual cortices 
of carnivorans and primates. The main difference between the model for rodent and lagomorph cortical development studied so far in this chapter and conventional feature models is the lack of any lateral intracortical excitation. In the model as introduced in Section 6.2, lateral interactions in the cortical layer are exclusively mediated by the soft competition mechanism. This mechanism effectively provides solely inhibitory intracortical interactions, and it is these interactions that lead to the salt-and-pepper states during the self-organizing dynamics. However, certainly both lateral excitation and inhibition play a role in shaping the activity in rodent V1 [223]. It is therefore of fundamental importance to clarify, whether salt-and-pepper states continue to exist as numerically stationary states of the dynamics of the model when lateral excitation is explicitly included into the model and to work out the precise conditions for a potential transition from ordered selectivity layouts to salt-and-pepper states.

One of the simplest ways of including different types of lateral interactions into models with activity-dependent Hebbian learning and soft competition for activity is by introducing a convolution of the competition term with a cooperative lateral neighborhood function:

$$
\partial_{t} z(\mathbf{x})=\left\langle\left[s_{z}-z(\mathbf{x})\right] \int d^{2} y K(\mathbf{x}-\mathbf{y}) e(\mathbf{y}, \mathbf{S}, z(\cdot))\right\rangle_{\mathbf{s}^{\prime}}
$$

with $e(\mathbf{x}, \mathbf{S}, z(\cdot))$ given by Equation (6.2.2). For a Gaussian kernel $K(\mathbf{x})$, the resulting model is analogous to the model by Scherf et al. for the formation of ocular dominance columns [144]. This model was shown to generalize several well-known feature map models, such as Kohonen's self-organizing feature map [145, 146] and the Elastic Network model [134, 143]. I now consider Equation (6.5.1) with a more general convolution kernel of the form

$$
K(\mathbf{x})=\frac{(1-S)}{2 \pi} \delta(|\mathbf{x}|)-\frac{(1-S)}{2 \pi(1-W)^{2}} e^{-\mathbf{x}^{2} /\left(2(1-W)^{2}\right)}+\frac{S}{2 \pi W^{2}} e^{-\mathbf{x}^{2} /\left(2 W^{2}\right)},
$$

where $0 \leq S, W \leq 1$ and $\delta(x)$ being Dirac's delta function. For $S=1$, the original model considered by Scherf et al. with a Gaussian kernel mediating the local spread of activation from neighboring neurons is recovered and map-like selectivity arrangements are expected [144]. For $S<1$, both lateral excitation and inhibition are present. Auto-inhibition is prevented by the first term of the kernel in Equation (6.5.2). Figure 6.5.1 depicts this convolution kernel for different parameters $S$ and $W$. Note that the above model (Eq. (6.5.1)) is non-potential.

There are three key questions to investigate now: (i) Is there a transition to a salt-and-pepper-like spatial pattern of neural selectivities at a finite value of $S<1$, i.e. when increasing lateral inhibition but with non-vanishing excitation? (ii) If yes, are the resulting states statistically similar to the states observed in the absence of excitation? (iii) What do the stationary orientation map patterns in this model look like ${ }^{1}$ ? In order to answer these questions, I first analyze the dynamics of the model (Eq. (6.5.1)), linearized around the homogeneous non-selective fixed point. Its asymptotic behavior will then be studied by numerical simulations adapting the methods presented in Section 6.4.1.

\footnotetext{
${ }^{1}$ Scherf et al. did not use a numerical method suitable to investigate long-term pattern dynamics.
} 
a

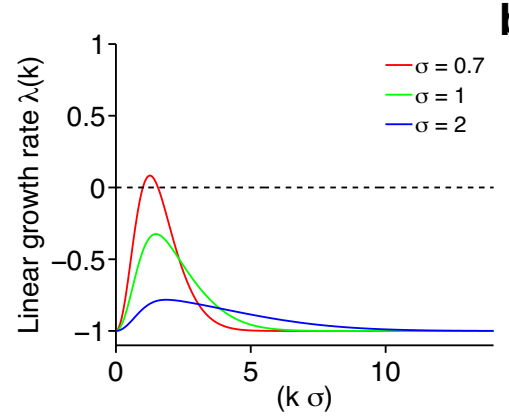

b

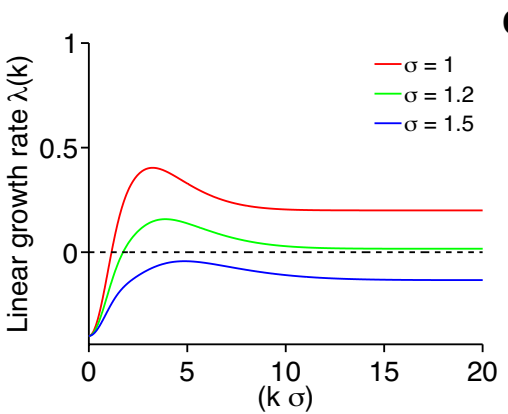

C

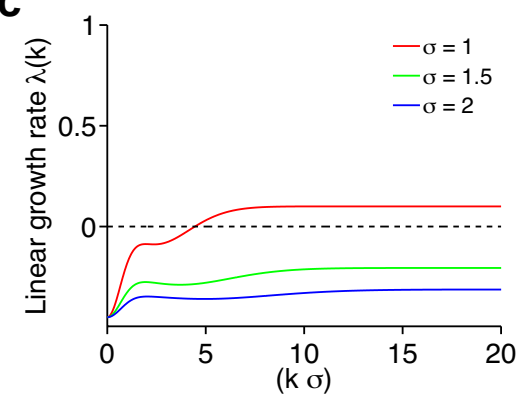

Figure 6.5.2: Linear growth rates (Eq. (6.3.2)) of Fourier modes $\sim e^{i \mathbf{k x}}$ as a function of the normalized wave number $k \sigma$ in the model Equation (6.5.1) for different receptive field widths $\sigma$ and different kernel parameters $S$ and $W$. (a) Linear growth rates for $S=1$ (purely excitatory convolution kernel) and $W=0.7$. For decreasing $\sigma$, the model undergoes a Turing Bifurcation where the homogeneous non-selective fixed point becomes unstable and the spontaneous emergence of ordered orientation selectivity layouts with a typical scale is predicted. (b) Linear growth rates for $S=0.4, W=0.7$ (short-range excitation plus strong but relatively long-range inhibition). For decreasing $\sigma$, the model first undergoes a Turing Bifurcation. Further decreasing $\sigma$ destabilizes all Fourier modes above a critical $k$-value. The maximum growth rate, however, is acquired for finite $k$. (c) Linear growth rates for $S=0.45, W=0.6$ (weak long-range excitation plus strong short-range inhibition). For decreasing $\sigma$, the homogeneous state loses stability with the maximum growth rate acquired for $k \rightarrow \infty$.

\subsubsection{Linear stability analysis and numerical simulations}

For rotationally symmetric kernel $K(\mathbf{x})=K(r)$, the spectrum of eigenvalues of the linearized dynamics of the convolution model model reads

$$
\lambda(k)=-\int d^{2} y K(\mathbf{y})+\frac{\left\langle\left|s_{z}\right|^{2}\right\rangle}{2 \sigma^{2}}\left(1-e^{-k^{2} \sigma^{2}}\right) F_{0}(k),
$$

where $F_{0}(k)$ is the Hankel transform of the kernel $K(r)$. With the ansatz in Equation (6.5.2) and the normalization $\left\langle\left|s_{z}\right|^{2}\right\rangle=2$, one obtains

$$
\lambda(k)=-S+\frac{1}{\sigma^{2}}\left(1-e^{-k^{2} \sigma^{2}}\right)\left((1-S)-(1-S) e^{-k^{2}(1-W)^{2} / 2}+S e^{-k^{2} W^{2} / 2}\right) .
$$

The growth rate at $k \rightarrow \infty$ equals

$$
\lambda_{\infty}=-S+\frac{1}{\sigma^{2}}(1-S)
$$

from which follows that, provided a certain strength of inhibitory interactions and sufficiently small $\sigma$, the growth rate at infinity can be larger than zero. It is not possible to analytically determine the maximum growth rate as well as the corresponding wave number $k_{\max }$. Figure 6.5.2 depicts the linear growth rates of the convolution model for various parameter regimes. In all three depicted regimes, the homogeneous non-selective states loses stability when $\sigma$ is lowered beyond a critical value. For purely excitatory kernel $K(\mathbf{x})(S=1$, Fig. 6.5.2a), the linear instability is of the classical Turing type [210], i.e. the homogeneous state gets unstable with respect to Fourier modes with a finite wave number $k$. For intermediate values of $W$ and $S$-values close to one, the instability is again of Turing type, but above a certain value of $\sigma$, all Fourier modes with 
wave numbers larger than a critical value $k_{c}$ lose stability (Fig. 6.5.2b). The fastest growing mode still has a finite wave number $k$. If $S$ is further decreased, the first Fourier mode to lose stability when decreasing $\sigma$ is at $k \rightarrow \infty$. In this case, it is also the fastest growing mode (Fig. 6.5.2c). This instability is of the same type as in the original model with pure inhibition by soft competition. Importantly, the transition between the two types of bifurcations generally occurs at a finite value of $S$. According to the above considerations, the linearized dynamics of the convolution model can be classified into four categories: (1) The non-selective state is stable. (2) The non-selective state is unstable with respect to a finite band of Fourier modes. (3) The non-selective state is unstable with respect to all Fourier modes with wave numbers larger than a critical value $k_{c}$. The fastest growing mode has a finite wave number. (4) The non-selective state is unstable with respect to all Fourier modes with wave numbers larger than a critical value $k_{c}$. The fastest growing mode has infinite wave number. Figure 6.5.3 depicts each of these linear regimes as a function of the kernel parameters $S$ and $W$ of excitation for $\sigma=1$. For small $S$ and large $W$, the linearity is of salt-and-pepper type, i.e. (4). In such a regime, excitation is long-range but weak and short-range strongly suppressive interactions dominate the model dynamics. For small $S$ and small $W$, the linearity is of type (1). The range of inhibitory interactions in this parameter regime is apparently to large to result in disordered selectivity layouts. For large $S$ and small $W$, the linearity is of type (2). For large $S$ and large $W$, the non-selective state is stable.

It is now particularly interesting to study the behavior of the model for times much larger than the intrinsic timescale $\tau$, i.e. beyond the linear regime, by numerical simulation. First, the stationary states in regime (2) have to be determined. Do they resemble experimentally observed orientation preference maps? Second, it is unclear how the nonlinear interactions between Fourier modes alter the model dynamics in the regime (3). Third, it has to be analyzed whether the transition to the disordered states in regime (4) as predicted by linear stability analysis is really observed in the numerically stationary states of the model.

Figure 6.5.3b-d depicts two representative simulations in each of the three regimes where spontaneous symmetry is expected. In regime (4), numerically stationary states in the model are indeed disordered (Fig. 6.5.3b) and show similar properties as previously observed in the $S=1, W=0$ limiting case, as exemplified by the local anti-correlation in the final state $(t=500 \tau)$ of the simulation (Fig. 6.5.3b, most right). The dynamics in regime (2) and (3) appear very similar (Fig. 6.5.3c,d). In both cases, the emergence of a pinwheel-rich map-like orientation preference layout can be observed. Via pairwise pinwheel annihilation, these acquire large stripe-like regions during the later simulation stages. These dynamics strongly resemble the behavior of the Elastic Network model (cf. $[142,166]$ and Chap. 3). In fact, for a different set of parameters $(\sigma=0.7, S=1, W=0.5)$ a convergence toward rhombic pinwheel patterns was also observed.

\subsection{Summary}

In this chapter, I studied stimulus-driven models for the activity-dependent development of orientation selectivity in the primary visual cortex of rodents and lagomorphs. I first considered a simple optimization model describing the emergence of orientation selectivity by the interplay between Hebbian learning and soft competition for activity within the cortical layer. Linear stability analysis of the non-selective state revealed a novel type of instability in which the first Fourier 


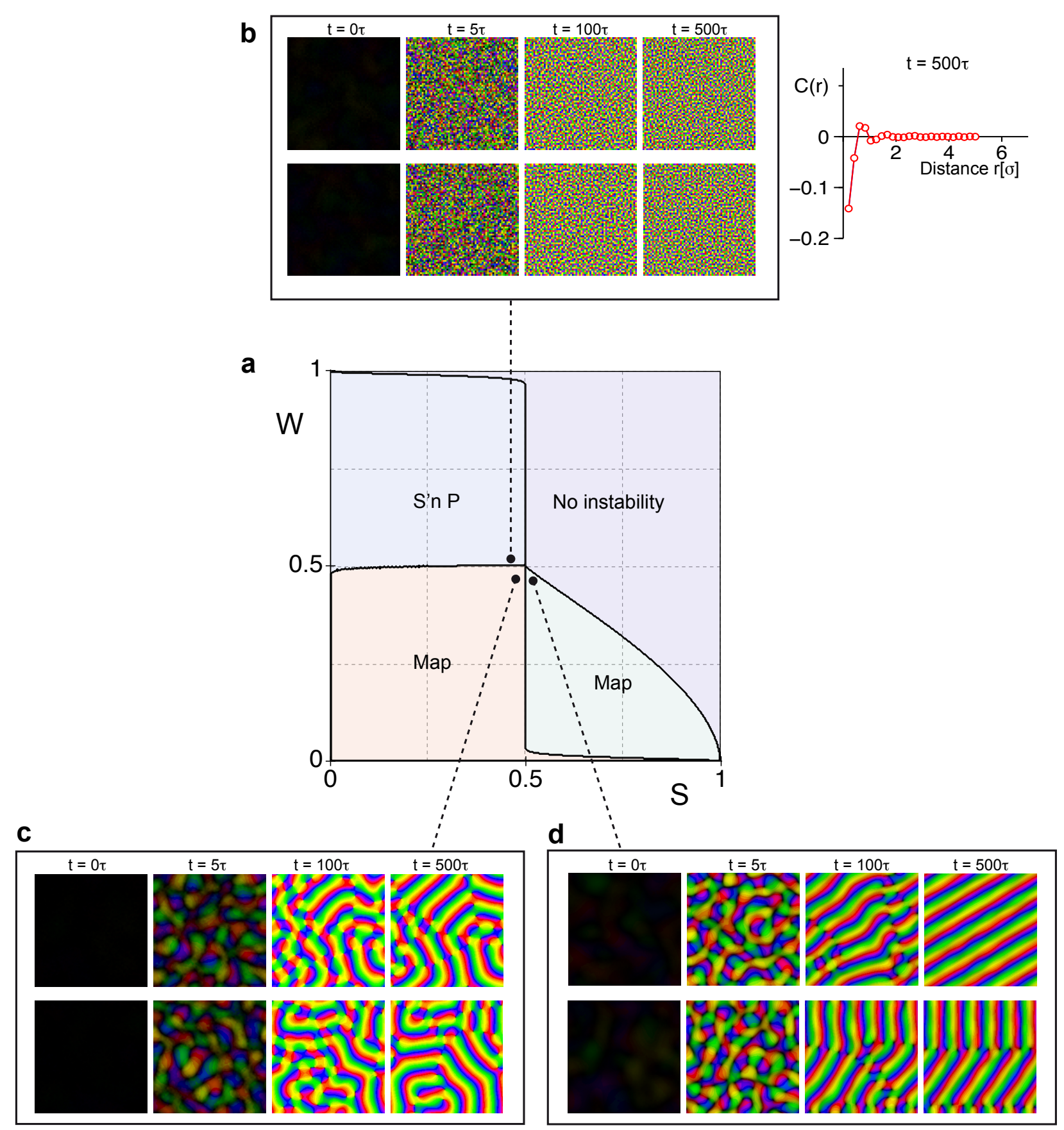

Figure 6.5.3: Types of linear instabilities as well as numerical solutions of the model defined by Equation (6.5.1). (a) Diagram showing the different types of instabilities in the model including lateral excitation and inhibition with $\sigma=1$. In the blue region $\left(\mathrm{S}^{\prime} \mathrm{nP}\right)$, the maximum linear growth rate is at $k \rightarrow \infty$; in the red region, the linear growth rate is maximal for a finite $k_{c}$ and $\lim _{k \rightarrow \infty} \lambda(k)<0$; in the green region, the linear growth rate is maximal for a finite $k_{c}$ and $\lim _{k \rightarrow \infty} \lambda(k)>0$; in the violet region, the homogeneous non-selective state is stable. (b) Snapshots of two representative simulations of the convolution model with linearity of salt-and-pepper type (Fig. 6.5.2c, $\sigma=1, S=0.45$, $W=0.5$ ). Right: Correlation function at $t=500 \tau$ for the simulation in the upper panels. (c) Snapshots of two representative simulations of the Scherf model in the red phase with linear growth rates similar to Figure 6.5.2a, red curve $(\sigma=1, S=0.48, W=0.45)$. (d) As $\mathbf{c}$ but for the green phase with linearity similar to Figure 6.5.2b, red curve $(\sigma=1, S=0.51, W=0.45)$. Note that the distance to the instability threshold differs in $\mathbf{c}$ and $\mathbf{d}$. 
mode to lose stability has infinite wave number. For finite control parameter, i.e. growth rate of the infinite wave number Fourier mode, all Fourier modes above a certain wave number $k_{c}$ are unstable. Due to this particular type of instability, in the linear regime, the emergence of patterns without any apparent structure or typical scale is expected. This was indeed confirmed by direct numerical integration of the model equations. In simulations, layouts continued to exhibit a disordered structure for large simulation times, compared to the timescale of the orientation selectivity emergence. These features of the model dynamics are very reminiscent of what has been observed in the rodent visual cortex [13-15]. Next, I numerically investigated the dynamics of the model, for initial conditions that matched the stationary solutions classically observed in similar approaches to the emergence of orientation selectivity in primates and carnivorans. None of the ordered patterns seemed to be stable solutions of the model. In all cases, salt-and-pepper layouts in which neighboring units show very different orientation preferences were dynamically reached.

To quantify the apparently random numerically stationary patterns, I used three different measures: the marginal correlation function, the discrepancy as a function of distance, and Swindale's measure of stimulus space coverage. Stationary solutions of the model were found to be distinct from spatially uncorrelated layouts. Orientation preferences were locally anti-correlated roughly within the scale of the activation function width in the model. Both, discrepancy as function of distance as well as Swindale's coverage measure were considerably lower even when compared to random arrangements with spatial correlations identical to observed solutions. The number of pixels per width of the activation function was found to be the critical parameter for the strength of distinction between the obtained solutions and truly random layouts.

As a second step, I generalized the model to include lateral excitation and inhibition of varying strength and width by introducing a convolution term. Linear stability analysis uncovered that the generalized model now in addition to the above linear instability also exhibits a classical Turing instability, which is usually invoked to model the emergence of patterns with a typical scale. In fact, the model now exhibits two distinct and sharply separated phases: (1) a phase in which the homogeneous non-selective state is stable and, thus, orientation selectivity does not emerge (2) a phase in which orientation maps emerge. Notably, these are also the two fundamentally different visual cortical architectures found in Glires on the one hand and carnivorans and primates on the other. Key ingredient for the transition from map-like to salt-and-pepper-like functional architecture is the dominance of effective short-range inhibition over the excitatory interactions in the dynamics.

The models considered in this chapter clearly show that the apparent randomness of spatial selectivity arrangements and the lack of specificity in the lateral connections found in Glires primary visual cortex is not at all in conflict with the classical Hebbian plasticity paradigms. As a matter of fact, large parts of our current knowledge about the precise mechanisms of synaptic plasticity has been derived from electrophysiological data obtained in slice preparations and in-vivo recordings from rat and mouse cortex. It would have been more than surprising if the functional cortical architecture in these paradigmatic model organisms could not be explained by the same mechanisms that have been shown to critically determine the feature maps found in carnivorans and primates. In the map regime, the dynamics of the model is very similar to the EN model dynamics studied in detail in Chapter 3. In fact, both models are based on a local activation of the cortical layer by idealized point-like stimuli leading to effectively short-range interactions. As outlined in 
Chapter 5 and already pointed out in $[11,123]$, in such a regime, pinwheel annihilation and crystallization represent the canonical dynamics. A suitable stimulus-driven model for the transition from rodent-like arrangements to ordered layouts realizing the common design of experimentally observed orientation preference maps [11] is yet to be found. 


\section{Chapter 7}

\section{Summary and Outlook}

I now summarize the results of the present dissertation and discuss potential future lines of research that, in my view, would be worth pursuing.

Chapter 2 was devoted to the analysis of the interaction between postnatal brain growth and the development of ocular dominance (OD) columns in cat primary visual cortex (V1). By analyzing data from numerous imaging experiments, I was able to show that the naively expected expansion of columns during cortical growth does not occur. Instead, columnar layouts reorganize over the considered period and become more isotropic in older animals. Driven by these experimental findings, I analytically and numerically analyzed the predicted reorganization in a widely used optimization model for the activity-dependent formation of OD columns which, originally, was conceived for non-growing cortical domains. Based on general properties of this model, I developed a novel scenario of growth-induced cortical reorganization based on the zigzag instability $[160,224,225]$, a generic secondary instability which has been observed in many inanimate pattern-forming systems. I found characteristic features of this reorganization as well as the time scale on which it evolves to be in good agreement with the changes in columnar layout that are observed during postnatal growth in cat V1. Most importantly, these results suggest a novel function of plasticity in normal development, namely that cortical circuits remain plastic for an extended period during development in order to facilitate the modification of neuronal circuits to adjust for cortical growth.

Postnatal growth constitutes a naturally occurring constraint to cortical development, since the addition of vasculature, blood vessels, and glial cells represents a necessity for ensuring nutrition and homeostasis in the adult brain. However, the strategies chosen by different species to adapt to cortical growth may vary greatly [226]. In fact, developmental programs of the central nervous system and, in particular, the amount of postnatal brain growth varies considerably between mammalian species [227]. Therefore, considering different and possibly evolutionarily widely separated species that potentially utilize different strategies to account for brain growth represents a crucial step to extend the results of Chapter 2. As a matter of fact, preliminary experimental results in ferret V1 indicate a different spatially anisotropic growth scenario for this species [228]. Additionally, the investigation of growth-related reorganizations of other stimulus feature representations, such as stimulus orientation and direction, seems highly promising. The mathematical description of orientation preference, for instance, differs fundamentally from OD 
and different theoretical predictions are therefore likely. If the scenario of anisotropic growth in ferret V1 consolidates, novel modeling approaches to pattern formation on anisotropically growing domains will be required to better understand possible modes of reorganization in orientation preference and OD layouts induced by postnatal brain growth.

The remainder of the present dissertation is dedicated to optimization principles and constraints that potentially govern the emergence of orientation selectivity and its spatial organization. In carnivorans and primates, orientation selectivity is arranged in columnar patterns, so-called orientation preference maps (OPMs, cf. Figs. 1.2.1 and 1.2.2) with apparently universal spatial statistics among species with highly different genetic background ([11] and Chapter 1). In Chapter 3, I studied one of the most widely used optimization hypotheses for the design of orientation maps and retinotopic maps, namely that they incorporate the most uniform, yet smooth representation of two visual feature combinations: (i) position in visual space and (ii) line orientation. Mathematically, this would imply that the apparently universal OPM layout can be interpreted as ground states of a smooth mapping of a 4-dimensional space of visual stimulus features to an effectively 2dimensional array of neurons (dimension reduction hypothesis). I used a perturbative approach to compute the ground states of perhaps the most prominent dimension reduction model, the Elastic Network (EN) model, for the joint optimization of retinotopy and OPM. I showed that the dynamics of both feature representations can be treated within a general theory for the stability of OPMs. I found various ground states in different regions of the parameter space of the model. However, in all relevant parameter regimes, the ground states of the EN model are either stripe-like, or crystalline representations of the two visual features. Summarizing pattern selection, I calculated a complete phase diagram of the model where additional quasi-crystalline ground states with low pinwheel densities are found in extreme parameter regimes. All analytical predictions were confirmed by direct numerical simulations. These results strongly question previous numerical studies concluding that dimension reduction models correctly reproduce the spatially aperiodic arrangement of visual cortical orientation columns.

One of the most encouraging results of the first two chapters of this thesis, together with recent work on Kohonen's self-organizing feature map algorithm [229, 230], is the striking analogy between the dynamics of optimization models for cortical representations and the dynamics of the classical pattern generating systems of physics. The zigzag instability in response to growth, nonlinear mode competition, the occurrence of dislocations and defects in patterns far from the instability threshold - all these features have long been studied in many models for pattern formation in inanimate systems phenomenologically described by equations of the Ginzburg-Landau type. This raises hope that the lessons learned from much simpler and analytically more tractable models still hold, if less idealized optimization approaches are to be considered.

Aperiodic pinwheel-rich OPMs are nearly impossible to obtain in the classical Elastic Network model, but is the statistics of real OPMs really aperiodic and spatially isotropic? A recent study by Paik and Ringach indeed questioned the findings in [11] and identified a hexagonal arrangement underlying the layout of orientation columns in ferret, macaque monkey, cat, and tree shrew [168]. The study explains this hexagonal arrangement by constraints arising from a limited number of feedforward inputs to V1 cells provided by the regular mosaics of retinal ganglion cells (RGCs) and relayed by the lateral geniculate nucleus. To reproduce OPMs with hexagonal order and typical column spacing, the proposed model crucially relies on the presence of an almost perfectly hexagonal lattice of RGC cells together with a so-called dipole assumption, i.e. the assumption 
that the input to a cortical cell is dominated by a single pair of one ON and one OFF cell, that are one another's nearest neighbors. Chapter 4 resolved the apparent controversy between the finding of a universal aperiodic OPM statistics by Kaschube et al. [11] and the regular hexagonal pinwheel lattices found by Paik and Ringach. In the search for hexagonal organization, I analyzed a data set of experimentally measured OPMs which is more than 10 times larger than the set analyzed in [168]. In addition, I tested different sets of control maps, including the maximum entropy ensemble, i.e. the most general ensemble consistent with the null-hypothesis formulated by Paik and Ringach. Importantly, the isotropic control ensemble used in their analysis is not the most general one and is based on additional implicit assumptions.

First, by applying the exact same analysis as in [168] to essentially complex planforms ${ }^{1}$ that lack any hexagonal symmetry by definition, I revealed that it is a general problem of Paik and Ringach's indicator for hexagonal order that it can suggest some degree of "hexagonality" even if there is none at all. Hence, one has to act with great caution when interpreting the results of such analysis applied to experimentally measured maps. Additionally, I identified a strong bias in the hypothesis test for hexagonal order used in [168]. On the basis of these findings, I developed a modified and unbiased hypothesis test to search for true signatures of an underlying hexagonal organization in OPMs. According to this novel indicator, the degree of hexagonal order in real maps is statistically indistinguishable from the one in artificial maps such as essentially complex planforms. Experimental maps are also statistically indistinguishable from maximum entropy control maps with completely isotropic power spectra. Thus, in contrast to the central claim of Paik and Ringach, the degree of hexagonal order is not larger in real maps than in isotropic maps. These results demonstrate that there is currently no evidence for hexagonal order in visual cortical OPMs and, hence, clearly refute the central conclusions in [168]. But the importance of the findings in Chapter 4 goes beyond the now again debatable contribution of RGC mosaics to OPM development since hexagonal arrangements of orientation columns are not a distinctive signature of such subcortical constraints. In particular it is known that a strong role of lateral intracortical connections [11, 118], interactions between orientation and eye dominance column formation [125] or even the mere coupling of contour orientation and retinotopic organization implied by Euclidean symmetry [129] can give rise to hexagonal arrangements of orientation columns. Notably, already the first theoretical study of the activity-dependent formation of orientation maps ever published 4 decades ago - found maps of hexagonal organization [109]. Not least, as shown in Chapter 3, the Elastic Network model for the joint optimization of retinotopic distortions and OPMs also shows a parameter region in which hexagonal OPMs are optimal. Consequently, hexagonal structures are abundant and can arise by very different mechanisms. The findings in Chapter 4 about the lack of such hexagonal order in real OPMs thus also show that any of the above alternative mechanisms for hexagonal orientation column arrangements plays, at most, a small role in shaping the layout of $\mathrm{V} 1$ orientation maps.

Despite the lack of hexagonal organization in experimentally measured OPMs and the methodological problems identified in Chapter 6, many interesting questions are raised and remain unanswered by the study of Paik and Ringach. Presumably, the placement of ON- and OFF-center retinal ganglion cells in the mammalian retina is better described by a pairwise interacting point process (PIPP) than by a noisy hexagonal lattice [231]. When using such a description, the interac-

\footnotetext{
${ }^{1}$ Note that essentially complex planforms (ECPs) are solutions of the self-organization models presented in Chapters 3 and 5.
} 
tions underlying the PIPP have been shown to exclusively arise from homotypic constraints (i.e. constraints concerning the placement of cells of the same type) and the physical constraint that no two somas of opposite type can occupy the same position [171, 231, 232]. Such placement of model cells in the retina has been previously used by Ringach together with multiple (more than two) LGN inputs to V1 cells [176, 177]. These approaches predicted spatially organized patterns of orientation selectivity that appear to lack a typical scale much like those predicted by the model of Miller [115]. It is unclear at present, whether OPMs exhibiting a typical column spacing can be obtained when considering realistic placements of RGCs together with a dipole assumption, or whether alternative point processes need to be invoked to explain the presence of such column spacing in purely feedforward models.

There are certainly constraints on the layout of feature representations in V1 that originate from the retina. Probably the most striking experimental evidence for retinal constraints on the layout of OD columns was found by Adams and Horton in the V1 of the squirrel monkey [54, 233]. These authors revealed that the pattern of retinal blood vessels can specifically determine the layout of OD columns in squirrel monkey visual cortex. A modeling study using the Elastic Network model indicates that such angioscotomas strongly influence the optimal solutions of the model as far as OD is concerned but have little effect on OPMs [140].

Adopting the self-organization perspective, it remains to be determined whether and how the selforganization of visual cortical circuits might be constrained by RGC mosaics. Such constraints are expected to vary strongly with the number of LGN inputs. While in cat, this number has been estimated to be around $30[174,234]$ there is currently little knowledge about the LGN to V1 connectivity in rodents and lagomorphs [235]. To investigate the precise nature of subcortical constraints and to include them into self-organization models for the emergence and refinement of orientation-selective response and orientation preference layouts in carnivorans, primates, and Glires (rodents and lagomorphs) represents an exciting route of research for future studies.

Chapter 3 also revealed a key mechanism in stimulus-driven models for stabilizing realistic OPM layouts, namely long-range suppressive interactions. In the classical Elastic Network model, such long-range interactions occur only in a limit in which point-like stimuli are represented by complex spatially extended activity patterns. Despite its lack of biological realism, this regime is highly informative. The fact that realistic aperiodic OPM layouts are stabilized in such a regime, corroborates that large-scale interactions are essential for the stabilization of OPM layouts with realistic geometry. Intuitively, in such a regime, the dynamics of dimension reduction models is governed by long-range, effectively suppressive interactions, very similar to the interactions phenomenologically incorporated into the order parameter equations studied in $[11,123]$ as well as Chapter 5. How could such types of interactions be mediated in stimulus-driven optimization models? Activity patterns evoked by entire extended stimuli, such as natural visual scenes, are necessarily extended. Therefore, it seems an obvious direction for future studies to investigate principles for the optimal representation of entire visual scenes by extended cortical activity patterns. It is an intriguing hypothesis that the common design of orientation preference maps in carnivorans and primates might be optimally shaped for the mapping of contours of natural visual scenes onto neural activity. In fact, recent data suggests that neural activity in the visual cortex becomes progressively adapted to the statistics of natural stimuli but not to artificial stimuli such as drifting gratings $[191,236]$. In the same line, orientation columns appear to be strategically positioned for a reduction of redundancy in visual information [124]. It remains an exciting question, in what 
sense optimality is achieved by visual cortical representations, whether it is maximum capacity, reduction of redundancies, minimal information loss, or some other principle yet to be discovered. The analysis of optimization models for cortical development based on the representation of random point-stimuli carried out in this thesis ask for a generalization to extended randomi stimuli to understand these questions. While assuming Gaussian statistics for these extended stimuli appears crucial for obtaining analytical results, numerical investigations of representative model dynamics should be used to identify essential statistical features of natural images necessary for stabilizing aperiodic pinwheel-rich layouts in the various optimization principles. The mathematical methods developed in this thesis are expected to strongly facilitate a comprehensive characterization of candidate optimization principles that aim at explaining the geometric features of visual cortical architecture, even if they involve extended stimuli.

Chapter 5 represents a further step in this direction. There, I generalized previous results on the stabilization of aperiodic pinwheel-rich orientation map layouts by long-range suppressive interactions. In particular, I considered an optimization model for the coordinated development of the system of tangential connections in V1 and the orientation preference map. Different biologically plausible interactions, for instance interactions mediated by long-range horizontal connections [21, 158] or by correlations of visual input due to long-range correlations in natural scenes $[185,192]$, may lead to different effective types of long-range interactions with distinct spatial dependencies. I constructed a variety of analytically solvable models in which long-range interactions decay either exponentially or with power-law dependence and calculated the complete phase diagram of stable solutions near the pattern formation threshold. I found that pinwheel stabilization by long-range intracortical interactions is highly robust and thus the spatial layout of orientation columns is expected to be fairly insensitive to the nature of long-range interactions. In view of this robustness, any altering of the horizontal connectivity pattern, e.g. by genetic means, has to be sufficiently strong to result in a measurable difference in the statistics of OPM layouts. An interesting direction for future studies will be the inclusion of spatial heterogeneities into models for OPM development that are derived from biologically plausible energy functionals. Kaschube et al. have reported that the column spacing of cat orientation domains can vary within up to $35 \%$ of the mean over the entire V1 [94, 237]. Such fluctuations in column spacing within V1 might originate from heterogeneities in lateral interactions within the primary cortical area or from interareal coordination of columnar development [94]. In a simple Swift-Hohenberg model with linearity $L_{S H}[z]=r-\left(k_{c}^{2}+\Delta\right)^{2}$, as for instance Equation (1.5.6), an intuitive way of incorporating such heterogeneities is by assuming a wave number field $k_{c}(\mathbf{x})$ that varies with a certain correlation function around a mean wave number $\left\langle k_{c}\right\rangle$. Inevitably, such a description entails a spatial variation in the effective control parameter, $r \rightarrow r(\mathbf{x})$. Such spatial variations of the control parameter have been examined in simple examples of order parameter equations and were found to qualitatively change the type of the bifurcation and the nature of the unstable modes [238-240]. It will be crucial to investigate the robustness of the selection of aperiodic pinwheel-rich layouts with respect to spatial disorder in models with long-range suppressive interactions. The present thesis, in which several perfectly homogeneous and isotropic optimization principles for neural circuit self-organization are analytically studied for the first time, hopefully provides a solid ground for disentangling the specific contributions of self-organization and constraints arising from spatial heterogeneities.

The findings in Chapter 3 on the crystalline optima of the EN model together with the numerical results in the same line on Kohonen's self-organizing feature map [229] essentially rule out dimen- 
sion reduction as a potential optimization theory for explaining the complex spatial layout of orientation preference maps. The second major hypothesis, i.e. wiring-length minimization, results in similar crystalline and perfectly periodic optima [133]. As a consequence, since the two leading models which assign a functional significance to OPMs seem to fail in reproducing their universal statistics, their role in visual information processing still remains elusive. The gap between explaining the emergence of the structure of OPM in V1 and understanding their functional role in visual information processing has not been bridged yet. But why should there be a single principle underlying the organization of orientation maps in V1? One of the main arguments in favor of such a principle is evolutionary convergence. The lineages of primates and carnivoran species diverged more than 65 million years ago [241-243] (cf. Fig. 7.0.1) and their last common ancestors were small-brained, nocturnal, squirrel-like animals of reduced visual abilities [243, 244]. Analyses of endocranial cavities of a representative stem eutherian from the late cretaceous around $85 \mathrm{mya}$ ago indicates a total anterior-posterior extent of $4 \mathrm{~mm}$ for its entire neocortex (cf. Fig. 7.0.1, [245-247]). Similarly, the tenrec (Echinops telfari), one of the closest living relatives of the boreoeutherian ancestor [248,249], has a neocortex of essentially the same size and a visual cortex that totals only $2 \mathrm{~mm}^{2}$ [243]. Since the neocortex of early mammals was subdivided into several cortical areas $[243,250]$ and orientation hypercolumns measure between 0.4 and $1.4 \mathrm{~mm}^{2}[11,237]$, it is difficult to imagine ancestral eutherians with a system of orientation columns. In fact, no extant mammal with a visual cortex of such size is known to possess orientation columns ([251], cf. Fig. 7.0.2). Therefore, it is very likely that systems of orientation columns independently evolved in Laurasiatheria (such as carnivorans) and in Euarchonta (such as tree shrews and primates). In this case, the quantitative precision with which orientation maps in both clades match strongly implicates a common developmental mechanism. Evolutionary convergence of biological structures is often assumed to provide a way to identify features of of such mechanisms. For instance, common aspects of habitats and habits of the organisms involved may reveal insights into the functional significance of the structure under consideration [252]. In view of this, it is even more puzzling that the three species studied by Kaschube et al. widely differ in the their habits and habitats, for instance galagos being nocturnal whereas tree shrews and ferrets are diurnal. To understand potential developmental mechanisms that might have driven the different lineages to adopt quantitatively similar layouts, it appears crucial to characterize other mammalian species with regard to their primary visual cortical architecture. Visual acuity and receptive field properties of neurons in the V1 of several species belonging to the marsupial clade appear comparable to those of some Glires and Scandentia species [253-255] and orientation selectivity has been found in all mammals so far investigated [251]. But the spatial organization of the selectivities in representative species of the Marsupialia, Monotremata, Afrotheria, and Xenarthra clades is still completely unexplored (cf. Fig. 7.0.1). Furthermore, it is yet unexplored whether V1s in large rodent brains such as the brains of agouti or capybara (cf. Fig. 7.0.2) that even show gyrification [256] exhibit ordered selectivity layouts. Is mere V1 size key for the transition to columnar architecture? The likely answer is no, because squirrels and ferrets have almost equal V1 sizes (Fig. 7.0.2). A quantitative understanding of the differences and similarities between V1 functional architecture from the extremes of body and brain sizes of the various mammalian clades might ultimately clarify to what extend the design of orientation column layouts is really universal.

The study of V1 circuitry in "exotic" mammalian species is also of general importance for investigating the evolutionary origin of the neocortical column in general. The dorsal pallium in reptiles as well as the allocortex in mammals is entered horizontally by thalamic inputs $[257,258]$. In con- 


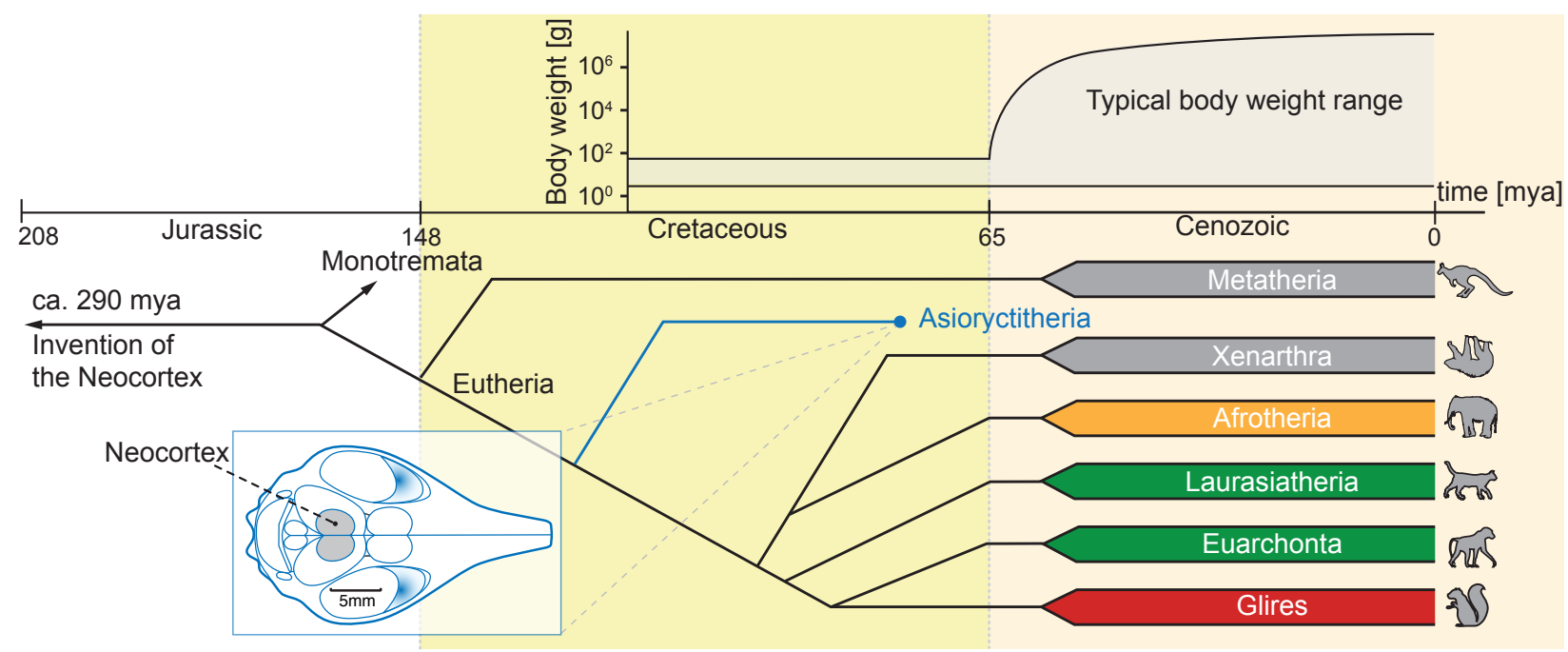

Figure 7.0.1: Columnar architectures for V1 in modern mammals may have independently evolved several times. Upper right: Evolution of typical body weight range in eutherian mammals [259, 260]. After the extinction of dinosaurs at the Cretaceous/Tertiary $(\mathrm{K} / \mathrm{T})$ boundary around 65 mya ago a near-exponential increase of the upper body weight range is observed. Phylogenetic relationships between ancestors of eutherian mammals [227, 241, 243, 261]. All species from Laurasiatheria, Scandentia and Euarchonta (green) that have so far been investigated possess orientation columns. In Glires (red) the organization of orientation selectivity is spatially disordered. Nothing is know about the spatial organization of orientation selectivity in Metatheria, Monotremata (gray), Xenarthra (gray), and Afrotheria (yellow). Lower left: The anatomical scheme depicts the cranium of a representative late cretaceous stem eutherian (Asioryctitherian). This close relative to the last common ancestor to extant mammals had a small V1, presumably lacking orientation columns (mod. from [246]).

trast, afferents vertically enter the mammalian neocortex. This difference is known to originate from genetic programs during embryonic development $[257,258]$. Super and Uylings hypothesize that vertical entrance of afferent fibers may have relieved a functional constraint limiting early expansion of the cortex, cortical laminar differentiation as well as the physical segregation into discrete axonal-dendritic systems which underly the neocortical column [258]. In this respect, it is a conondrum why such columnar representations did not evolve in Glires, at least not on the microscopic scale.

One might argue that the universality of orientation column layouts is already challenged by the lack of any horizontal columnar organization in all Glires species so far investigated (Figs. 7.0.1, 7.0.2). Studies as long as to the 1970s showed that rabbits [67], hamsters [201], mice [66], rats [13], and squirrels [14] exhibit orientation-selective responses in V1 without any apparent ordering and apparently the size of V1 surface area is not key for possessing orderly arrangements of orientation selectivity (cf. Fig. 7.0.2). The optimization approach introduced in Chapter 6 demonstrates for the first time that the development of salt-and-pepper layouts in rodent and lagomorph visual cortex can be modeled with very similar concepts as the emergence of columnar representations and, hence, this approach may be viewed as a first step towards a "unifying theory of cortical computation" [251] or at least of mammalian primary visual cortical architecture. Much like in the Elastic Network model, in the model for salt-and-pepper arrangements of orientation selectivity, visual stimuli are represented by their position and orientation in visual field coordinates. Activity patterns in the cortical layer are determined according to a simple activation rule by which neighbor- 
Figure 7.0.2: Both columnar and saltand-pepper arrangements of orientation preference appear over wide and overlapping ranges of V1 surface area and body weight. The two design-types are indicated by schemes: Upper scheme, columnar; lower scheme, salt-and-pepper. Organization of orientation preference in Laurasiatherian and Euarchonta (red), and Glires (green). Filled bars indicate columnar (green) and saltand-pepper (red) organization respectively. Hatched bars indicate unknown spatial organization of orientation preference (data collected from $[24,74,250,251,256,262-264])$.

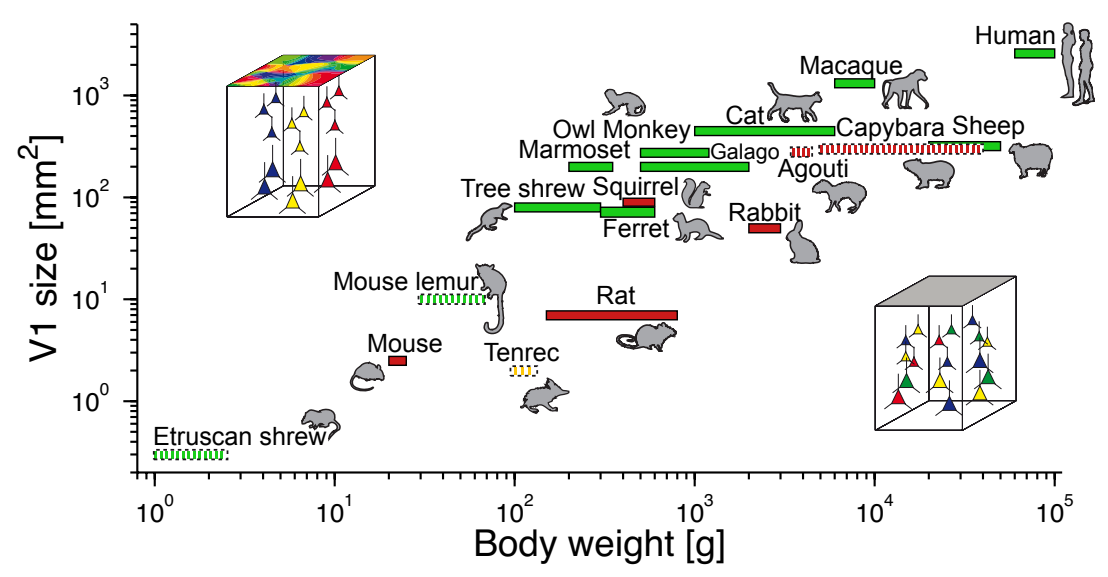

ing units tend to be co-activated. The selectivities of activated units are modified with a Hebbian learning rule to better match the parameters of the stimuli. First, I showed that, by a symmetry argument, the model has two stationary solutions: (i) the non-selective homogeneous state and (ii) the pinwheel-free orientation stripe state. Linear stability analysis of the non-selective fixed point showed that by a non-Turing-type instability, the non-selective state becomes unstable, leading to the spontaneous emergence of orientation selectivity from the non-selective state in the model. By extensive simulations, it was shown that the emerging states exhibit a salt-and-pepper arrangement of orientation selectivities, despite the tendency for co-activation of neighboring units during the learning process. The spatial layout of numerically stationary solutions is characterized by a local anti-correlation of preferred orientations, a high stimulus coverage, and a low discrepancy. Both high coverage and low discrepancy are more pronounced compared to a Gaussian random field with negative correlations alone. These results indicate that the apparently random arrangement of orientation selectivity in Glires might be the results of a dynamical activity-dependent process that optimizes coverage of a space of local stimulus features. By including lateral excitation into the model via a convolution term, I was able to show that a dominant and short-ranged inhibition is critical for stabilizing Glires-like selectivity layouts.

This key factor is highly noteworthy in view of recent findings on the spatial distribution of excitation and inhibition in mouse somatosensory cortex. Activity in layer $2 / 3$ of the juvenile mouse barrel cortex was shown to generate strong lateral suppression of spiking in layer $2 / 3$ pyramidal cells together with feedforward facilitation of layer 5 pyramidal cells [223]. As a possible reason for this suppressive effect, the small ratio of excitation to inhibition in layer $2 / 3(<0.2)$ was identified. In the visual cortex it has been shown that the spatial firing patterns of fast-spiking broadly tuned inhibitory interneurons were only weakly modified by visual stimulation [203]. This indicates dense, strong, and unspecific inputs from nearby pyramidal cells with a wide range of selectivity properties. The dense network of inhibitory interneurons in which sharply orientationtuned pyramidal cells are embedded might mediate the local suppressive interaction that, according to the model, are essential to stabilize salt-and-pepper layouts of selectivities.

Numerically stationary states of the model are distinct from random spatial organization as revealed by several statistical measures. The degree of distinction however was shown to critically 
depend on the number of simulated units within the spatial range of inhibition. This range is hard to estimate experimentally. If it only was to linearly extend over more than 10 cells, the arrangement of cortical selectivities predicted by the model would already be hard to distinguish from a random spatial distribution. Therefore, the precise organizations of selectivities in the model are not a promising prediction to test experimentally.

Probably the most easily testable prediction of the model is the reestablishing of a disordered selectivity layout after orientation selectivity in a small subregion has been aligned by a pairing experiment, as e.g. [217, 218], in which a very brief visual stimulus is repeatedly paired with stimulation within the visual cortex. With optogenetic tools in mice [265-267] as well as two-photon-imaging techniques [62, 63, 195-197], that have become available in recent years, a selective activation of a small set of neurons by photo-stimulation paired with the presentation of a grating of one orientation and subsequent chronic imaging constitutes a feasible experiment to test the predictions of the model.

As the discovery of the disordered salt-and-pepper-like functional layouts in rodents and lagomorphs was made possible only by the advent of novel imaging techniques, a complete theoretical understanding of these layouts will almost certainly require different mathematical tools than the analysis of ordered columnar layouts. The theoretical analyses presented in Chapter 6 are far from being a complete description of the novel type of instability characterized by an infinite wave number to first lose stability. An analytical treatment of the stability of patterns in 2-dimensional or even 3-dimensional models of visual cortical architecture exhibiting such types of instability does not appear to be not feasible at this moment. After all, the assumption of a columnar structure that has originally motivated a 2-dimensional description of visual cortical architecture seems inappropriate for Glires V1. As a necessary and interesting step toward an understanding of the novel phenomena expected, it appears promising to analyze simplified 1-dimensional order parameter models for Glires cortical development. In such models, an analytical study of the stability of periodic solutions in the nonlinear regime using Bloch-Floquet theory [268] seems possible.

In the parameter range in which the model introduced in Chapter 6 exhibits ordered selectivity layouts, its dynamics strongly resembles the dynamics of the Elastic Network model (cf. Chap. 3). Initially aperiodic layouts develop into either pinwheel-sparse layouts with large stripe-like domains or rhombic pinwheel crystals not resembling the experimentally observed common OPM layout. In fact, due to the point-like stimuli which only locally activate the cortical layer, interactions in the model are, similarly to the EN model, short-range. An investigation of the transition from salt-and-pepper layouts to ordered selectivity organizations in the presence of long-range interactions, for instance mediated by spatially extended stimuli, represents an interesting direction for future studies.

Specifically, the data from mouse suggest a further obvious route for generalizations of the model. Wang and co-workers discovered that in mice preferred orientations in the two eyes initially often emerge unmatched and subsequently change toward one binocularly matched orientation preference [106]. Because preferred orientations in the two eyes initially are statistically independent, this suggests that neurons can rotate their orientation preferences up to at least $45^{\circ}$ during postnatal development. To understand these findings, it seems natural to generalize the present model to include two orientation selectivity variables, each for one of the two eyes. The matching of the preferred orientations is then expected to depend on the correlation between the stimulus orientations coming from each of the two eyes. How the interplay between the potentially ordering 
force induced by a positive correlation of the inputs from the two eyes and the disordering force induced by the dominant local inhibition alters the solutions of the model is an interesting open question.

A salt-and-pepper-like arrangement of cellular response properties in the neocortex of rodents and lagomorphs seems neither restricted to orientation selectivity nor to the primary visual cortex. For instance, although there is gross retinotopy on the macroscopic scale in mouse V1 [24, 269, 270], on the scale of neighboring neurons retinotopic scattering is substantial [271]. Heterogeneously distributed local population of layer $2 / 3$ neurons can exhibit precisely overlapping spatial receptive fields. Larger receptive field subregions appear to be composed of non-overlapping smaller subregions. Similarly, in rat and mouse auditory cortex, despite a large-scale tonotopic organization [272, 273], local populations in A1 exhibit highly heterogeneous response properties [274-276].

Even more intriguing are the recent findings in mouse barrel cortex. There, neurons are selective for the direction of vibrissa motion [277]. While in juvenile mice the spatial organization of these direction selectivities is disordered [277], a late emergence of a direction selectivity map with a pinwheel-like structure has been observed although substantial scattering of direction selectivity between neighboring neurons appears to remain [278, 279]. The pinwheel structure in adult mouse barrel cortex emerges from a salt-and-pepper layout with highly selective individual cells. In a detailed network model of layers $2 / 3$ and layer 4 of the barrel cortex, this late transition to a pinwheel-like map organization was attributed to a particular stimulations protocol, which deflected vibrissae in a regular spatio-temporal pattern [278]. However, the initial formation of the spatially disordered directional selectivities has not been modeled in this study. It is therefore currently unclear what factors determine the apparent transition from salt-and-pepper in juvenile mice to maps in the adult mouse barrel cortex. A measurable shift in the relative strengths and/or ranges of excitation and inhibition during development would constitute a confirmation of the predictions of the model developed in the present dissertation.

The development of the organization of direction selectivity in rodents appears to be an ideal model system to study optimization principles that may govern the self-organization of neuronal circuits together with constraints imposed by a genetically predetermined developmental program. Direction-selective responses of retinal ganglion cells largely develop independently of visual experience and activity-dependent mechanisms, potentially as a result of complex molecular interactions [221, 222]. While in adult mice these direction-selective responses cluster into the four cardinal directions (dorsal, ventral, temporal, and nasal), the temporal and ventral directions seem highly overrepresented in visually inexperienced mice [221]. In mouse primary visual cortex, direction selectivity also emerges without visual experience and selectivities in juvenile mice are clustered around the dorsal and anterior directions which correspond to the temporal and nasal directions in the retina. These results suggest that in the juvenile mouse visual system, direction selectivity emerges in the retina and is relayed to the visual cortex. However, direction preferences in the adult mice do not cluster around cardinal directions [107]. The findings in Chapter 6 provide an explanation for this in terms of circuit self-organization. The direction selectivities relayed from the juvenile, visually inexperienced mouse retina might serve as a "seed" or "drive" to increase robustness of the activity-dependent self-organized emergence of selectivities at later stages in development. 
To summarize, in this dissertation I analyzed the reorganization of V1 columnar architecture during postnatal brain growth and suggested a new role for the so-called critical period: the enhanced plasticity in V1 observed over this period might facilitate the modification of neuronal circuits to adjust for cortical growth. Furthermore, I developed a general mathematical formalism to analytically calculate OPMs predicted by optimization models for OPM development. Using this formalism I was able to obtain the first analytical expressions for the precise layouts of the predicted OPMs in the Elastic Network model, 22 years after it was introduced by Durbin and Mitchison. These results now set the stage for a mathematically rigorous and biologically informative search for optimization principles that more successfully explain the architecture of columnar contour representations in V1. In a subsequent study, I shed light on a still highly controversial aspect of visual neuroscience, namely to what degree V1 response properties and functional architecture can be explained by simple feedforward constraints arising from subcortical inputs. Surprisingly, by analyzing a large data set of experimentally measured OPMs, I found no empirical evidence for an influence of subcortical constraints on the layout of OPMs. Instead, I demonstrated that the common design of experimentally observed OPM layouts is very robustly obtained in selforganization models for the coordinated development of OPMs and the system of long-range tangential connections in V1. This robustness offers an explanation for the strikingly precise agreement between the statistics of OPMs in evolutionarily widely separated species with very different habits and habitats. Finally, I developed and analyzed the first stimulus-driven model for the emergence of disordered selectivity organizations in Glires. I showed that, depending on the balance between local inhibitory and excitatory interactions, both types of experimentally observed functional architectures, salt-and-pepper layouts and smooth OPMs, appear generically as solutions of optimization models for the self-organization of cortical circuits.

The dichotomy of visual cortical architectures between Glires on the one hand and carnivorans and primates on the other might turn out to be a real blessing not only for sensory neuroscience but also for evolutionary neurobiology. Individual neurons in both functional layouts have many response properties in common, which suggests the existence of general building principles for local elements involved and that they are potentially optimized for processing visual information. Yet, in the course of evolution nature has found two completely different solutions for connecting these elements in order to construct functional circuits. Uncovering how these different network structures might be specialized to serve specific computational tasks and how they adapt to a changing environment during brain development may not only help us better understand the visual system. Very likely, it will reveal fundamental optimization principles of evolutionary cortical circuit design and information processing in the brain that can also be applied to other sensory areas. 


\section{Bibliography}

[1] Vogel S: Twist-to-bend ratios of woody structures. J Exp Bot 1995, 46:981-985.

[2] Kamiya A, Togawa T: Optimal branching structure of the vascular tree. Bull Math Biophys 1972, 34(4):431-438.

[3] Weibel ER: Fractal geometry: a design principle for living organisms. Am J Physiol 1991, 261(6):L361-L369.

[4] Weibel ER, Taylor CR, Bolis L: Principles of animal design: the optimization and symmorphosis debate. Cambridge University Press, 1st edition 1998.

[5] Weibel ER, Gomez DM: Architecture of the human lung. Use of quantitative methods establishes fundamental relations between size and number of lung structures. Science 1962, 137(3530):577-585.

[6] Cherniak C: Local optimization of neuron arbors. Biol Cybern 1992, 66(6):503-510.

[7] Cherniak C, Changizi M, Kang D: Large-scale optimization of neuron arbors. Phys Rev E 1999, 59(5):6001-6009.

[8] Chen BL, Hall DH, Chklovskii DB: Wiring optimization can relate neuronal structure and function. Proc Natl Acad Sci USA 2006, 103(12):4723-4728.

[9] Rivera-Alba M, Vitaladevuni SN, Mischenko Y, Lu Z, Takemura SY, Scheffer L, Meinertzhagen IA, Chklovskii DB, de Polavieja GG: Wiring economy and volume exclusion determine neuronal placement in the Drosophila brain. Curr Biol 2011, 21(23):2000-2005.

[10] Cherniak C, Mokhtarzada Z, Rodriguez-Esteban R, Changizi K: Global optimization of cerebral cortex layout. Proc Natl Acad Sci USA 2004, 101(4):1081-1086.

[11] Kaschube M, Schnabel M, Löwel S, Coppola DM, White LE, Wolf F: Universality in the evolution of orientation columns in the visual cortex. Science 2010, 330(6007):1113-1116.

[12] Muir DR, Da Costa NMA, Girardin CC, Naaman S, Omer DB, Ruesch E, Grinvald A, Douglas RJ: Embedding of cortical representations by the superficial patch system. Cereb Cortex 2011, 21(10):2244-2260.

[13] Ohki K, Chung S, Ch'ng Y, Kara P, Reid RC: Functional imaging with cellular resolution reveals precise micro-architecture in visual cortex. Nature 2005, 433(7026):597-603. 
[14] Hooser SV, Heimel J, Chung S, Nelson S, Toth L: Orientation selectivity without orientation maps in visual cortex of a highly visual mammal. J Neurosci 2005, 25:19-28.

[15] Ko H, Hofer SB, Pichler B, Buchanan KA, Sjöström PJ, Mrsic-Flogel TD: Functional specificity of local synaptic connections in neocortical networks. Nature 2011, 473(7345):87-91.

[16] Chalupa LM, Werner JS: The visual neurosciences, Volume 1. MIT Press, 1st edition 2004.

[17] Hannula DE, Simons DJ, Cohen NJ: Imaging implicit perception: promise and pitfalls. Nat Rev Neurosci 2005, 6(3):247-255.

[18] Hubel DH, Wiesel TN: Integrative action in the cat's lateral geniculate body. J Physiol 1961, 155:385-398.

[19] Kaplan E, Marcus S, So YT: Effects of dark adaptation on spatial and temporal properties of receptive fields in cat lateral geniculate nucleus. J Physiol 1979, 294:561-580.

[20] Tavazoie SF, Reid RC: Diverse receptive fields in the lateral geniculate nucleus during thalamocortical development. Nat Neurosci 2000, 3(6):608-616.

[21] Bosking WH, Zhang Y, Schofield B, Fitzpatrick D: Orientation selectivity and the arrangement of horizontal connections in tree shrew striate cortex. J Neurosci 1997, 17(6):21122127.

[22] Bosking WH, Crowley JC, Fitzpatrick D: Spatial coding of position and orientation in primary visual cortex. Nat Neurosci 2002, 5(9):874-882.

[23] Dräger U: Receptive fields of single cells and topography in mouse visual cortex. J Comp Neurol 1975, 160:269-290.

[24] Schuett S, Bonhoeffer T, Huebener M: Mapping retinotopic structure in mouse visual cortex with optical imaging. J Neurosci 2002, 22(15):6549-6559.

[25] Mallot HA: An overall description of retinotopic mapping in the cat's visual cortex areas 17, 18, and 19. Biol Cybern 1985, 52:45-51.

[26] Tootell RB, Switkes E, Silverman MS, Hamilton SL: Functional anatomy of macaque striate cortex. II. Retinotopic organization. J Neurosci 1988, 8(5):1531-1568.

[27] Hubel DH, Wiesel TN: Receptive fields, binocular interaction and functional architecture in cats visual cortex. J Physiol 1962, 160:106-154.

[28] Hubel DH, Wiesel TN: Shape and arrangement of columns in cats striate cortex. J Neurophysiol 1963, 160:559-568.

[29] Kaschube M, Wolf F, Puhlmann M, Rathjen S, Schmidt KF, Geisel T, Löwel S: The pattern of ocular dominance columns in cat primary visual cortex: intra- and interindividual variability of column spacing and its dependence on genetic background. Eur J Neurosci 2003, 18(12):3251-3266.

[30] Horton J, Hocking D: An adult-like pattern of ocular dominance columns in striate cortex of newborn monkeys prior to visual experience. J Neurosci 1996, 16(5):1791-1807. 
[31] Adams DL, Sincich LC, Horton JC: Complete pattern of ocular dominance columns in human primary visual cortex. J Neurosci 2007, 27(39):10391-10403.

[32] Obermayer K, Blasdel GG: Singularities in primate orientation maps. Neural Comput 1997, 9(3):555-575.

[33] Hubel DH, Wiesel TN, Stryker MP: Orientation columns in macaque monkey visual cortex demonstrated by the 2-deoxyglucose autoradiographic technique. Nature 1977, 269(5626):328-330.

[34] Hubel DH, Wiesel TN, Stryker MP: Anatomical demonstration of orientation columns in macaque monkey. J Comp Neurol 1978, 177(3):361-380.

[35] Ts'o DY, Frostig RD, Lieke EE, Grinvald A: Functional organization of primate visual cortex revealed by high resolution optical imaging. Science 1990, 249(4967):417-420.

[36] Malach R, Tootell RB, Malonek D: Relationship between orientation domains, cytochrome oxidase stripes, and intrinsic horizontal connections in squirrel monkey area V2. Cereb Cortex 1994, 4(2):151-165.

[37] Xu X, Bosking W, Sáry G, Stefansic J, Shima D, Casagrande V: Functional organization of visual cortex in the owl monkey. J Neurosci 2004, 24(28):6237-6247.

[38] Xu X, Bosking WH, White LE, Fitzpatrick D, Casagrande VA: Functional organization of visual cortex in the prosimian bush baby revealed by optical imaging of intrinsic signals. J Neurophysiol 2005, 94(4):2748-2762.

[39] Yacoub E, Harel N, Ugurbil K: High-field fMRI unveils orientation columns in humans. Proc Natl Acad Sci USA 2008, 105(30):10607-10612.

[40] Bonhoeffer T, Grinvald A: Iso-orientation domains in cat visual cortex are arranged in pinwheel-like patterns. Nature 1991, 353(6343):429-431.

[41] Weliky M, Katz LC: Functional mapping of horizontal connections in developing ferret visual cortex: experiments and modeling. J Neurosci 1994, 14(12):7291-7305.

[42] Chapman B, Stryker MP: Development of orientation selectivity in ferret visual cortex and effects of deprivation. J Neurosci 1993, 13(12):5251-5262.

[43] Chapman B, Stryker MP, Bonhoeffer T: Development of orientation preference maps in ferret primary visual cortex. J Neurosci 1996, 16(20):6443-6453.

[44] White LE, Coppola DM, Fitzpatrick D: The contribution of sensory experience to the maturation of orientation selectivity in ferret visual cortex. Nature 2001, 411(6841):1049-1052.

[45] Hubel DH, Wiesel TN: Ferrier lecture. Functional architecture of macaque monkey visual cortex. Proc R Soc Lond B 1977, 198(1130):1-59.

[46] Grinvald A, Lieke E, Frostig RD, Gilbert CD, Wiesel TN: Functional architecture of cortex revealed by optical imaging of intrinsic signals. Nature 1986, 324(6095):361-364. 
[47] Blasdel GG, Salama G: Voltage-sensitive dyes reveal a modular organization in monkey striate cortex. Nature 1986, 321(6070):579-585.

[48] Grinvald A, Frostig RD, Lieke E, Hildesheim R: Optical imaging of neuronal activity. Physiol Rev 1988, 68(4):1285-1366.

[49] Bonhoeffer T, Grinvald A: The layout of iso-orientation domains in area 18 of cat visual cortex: optical imaging reveals a pinwheel-like organization. J Neurosci 1993, 13(10):41574180 .

[50] Swindale NV: A model for the formation of orientation columns. Proc R Soc Lond B 1982, 215:211-230.

[51] Löwel S: Ocular dominance column development: strabismus changes the spacing of adjacent columns in cat visual cortex. J Neurosci 1994, 14(12):7451-7468.

[52] Löwel S, Singer W: The pattern of ocular dominance columns in flatmounts of the cat visual cortex. Exp Brain Res 1987, 68:661-666.

[53] Löwel S, Schmidt KE, Kim DS, Wolf F, Hoffsümmer F, Singer W, Bonhoeffer T: The layout of orientation and ocular dominance domains in area $\mathbf{1 7}$ of strabismic cats. Eur J Neurosci 1998, 10(8):2629-2643.

[54] Adams DL, Horton JC: Capricious expression of cortical columns in the primate brain. Nat Neurosci 2003, 6(2):113-114.

[55] Horton JC, Adams DL: The cortical column: a structure without a function. Phil Trans $R$ Soc Lond B 2005, 360(1456):837-862.

[56] Blasdel G, Obermayer K, Kiorpes L: Organization of ocular dominance and orientation columns in the striate cortex of neonatal macaque monkeys. Visual Neurosci 1995, 12(3):589-603.

[57] Wolf F, Geisel T: Universality in visual cortical pattern formation. J Physiol 2003, 97(23):253-264.

[58] Schnabel M, Kaschube M, Löwel S, Wolf F: Random waves in the brain: Symmetries and defect generation in the visual cortex. Eur Phys J 2007, 145:137-157.

[59] Erwin E, Obermayer K, Schulten K: Models of orientation and ocular dominance columns in the visual cortex: a critical comparison. Neur Comp 1995, 7(3):425-468.

[60] Lund JS, Angelucci A, Bressloff PC: Anatomical substrates for functional columns in macaque monkey primary visual cortex. Cereb Cortex 2003, 13:15-24.

[61] Ohki K, Chung S, Kara P, Hübener M, Bonhoeffer T, Reid R: Highly ordered arrangement of single neurons in orientation pinwheels. Nature 2006, 442(7105):925-928.

[62] Denk W, Strickler JH, Webb WW: Two-photon laser scanning fluorescence microscopy. Science 1990, 248:73-76. 
[63] Stosiek C, Garaschuk O, Holthoff K, Konnerth A: In vivo two-photon calcium imaging of neuronal networks. Proc Nat Acad Sci USA 2003, 100(12):7319-7324.

[64] Girman SV, Sauvé Y, Lund RD: Receptive field properties of single neurons in rat primary visual cortex. J Neurophysiol 1999, 82:301-311.

[65] Métin C, Godement P, Imbert M: The primary visual cortex in the mouse: receptive field properties and functional organization. Exp Brain Res 1988, 69(3):594-612.

[66] Niell CM, Stryker MP: Highly selective receptive fields in mouse visual cortex. J Neurosci 2008, 28(30):7520-7536.

[67] Murphy EH, Berman N: The rabbit and the cat: a comparison of some features of response properties of single cells in the primary visual cortex. J Comp Neurol 1979, 188(3):401-427.

[68] Jia H, Rochefort NL, Chen X, Konnerth A: Dendritic organization of sensory input to cortical neurons in vivo. Nature 2010, 464(7293):1307-1312.

[69] Sengpiel F, Kind PC: The role of activity in development of the visual system. Curr Biol 2002, 12(23):R818-R826.

[70] Crowley JC, Katz LC: Early development of ocular dominance columns. Science 2000, 290(5495):1321-1324.

[71] Crair MC, Horton JC, Antonini A, Stryker MP: Emergence of ocular dominance columns in cat visual cortex by 2 weeks of age. J Comp Neurol 2001, 430(2):235-249.

[72] Crowley JC, Katz LC: Ocular dominance development revisited. Curr Opin Neurobiol 2002, 12:104-109.

[73] Rathjen S, Löwel S: Early postnatal development of functional ocular dominance columns in cat primary visual cortex. Neuroreport 2000, 11(11):2363-2367.

[74] Rathjen S, Schmidt KE, Löwel S: Postnatal growth and column spacing in cat primary visual cortex. Exp Brain Res 2003, 149(2):151-158.

[75] Crair MC, Gillespie DC, Stryker MP: The role of visual experience in the development of columns in cat visual cortex. Science 1998, 279(5350):566-570.

[76] Catz LC, Callaway EM: Emergence and refinement of clustered horizontal connections in cat striate cortex. J Neurosci 1990, 10(4):1134-1153.

[77] Durack JC, Katz LC: Development of horizontal projections in layer 2/3 of ferret visual cortex. Cereb Cortex 1996, 6(2):178-183.

[78] White L, Fitzpatrick D: Vision and cortical map development. Neuron 2007, 56(2):327-338.

[79] Callaway EM, Katz LC: Effects of binocular deprivation on the development of clustered horizontal connections in cat striate cortex. Proc Natl Acad Sci USA 1991, 88(3):745-749.

[80] Lübke J, Albus K: Lack of exuberance in clustered intrinsic connections in the striate cortex of one-month-old kitten. Eur J Neurosci 1992, 4:189-192. 
[81] Löwel S, Singer W: Selection of intrinsic horizontal connections in the visual cortex by correlated neuronal activity. Science 1992, 255(5041):209-212.

[82] Hensch TK, Stryker MP: Columnar architecture sculpted by GABA circuits in developing cat visual cortex. Science 2004, 303(5664):1678-1681.

[83] Sengpiel F: The critical period. Curr Biol 2007, 17(17):R742-R743.

[84] Sengpiel F, Gödecke I, Stawinski P, Hübener M: Intrinsic and environmental factors in the development of functional maps in cat visual cortex. Neuropharmacology 1998, 37:607-621.

[85] Sengpiel F, Stawinski P, Bonhoeffer T: Influence of experience on orientation maps in cat visual cortex. Nat Neurosci 1999, 2(8):727-732.

[86] Antonini A, Stryker MP: Rapid remodeling of axonal arbors in the visual cortex. Science 1993, 260(5115):1819-1821.

[87] Antonini A, Stryker MP: Plasticity of geniculocortical afferents following brief or prolonged monocular occlusion in the cat. J Comp Neurol 1996, 369:64-82.

[88] LeVay S, Wiesel TN, Hubel DH: The development of ocular dominance columns in normal and visually deprived monkeys. J Comp Neurol 1980, 191:1-51.

[89] Wiesel TN: Postnatal development of the visual cortex and the influence of environment. Nature 1982, 299(5884):583-591.

[90] Sur M, Leamey CA: Development and plasticity of cortical areas and networks. Nat Rev Neurosci 2001, 2(4):251-262.

[91] Li Y, Fitzpatrick D, White LE: The development of direction selectivity in ferret visual cortex requires early visual experience. Nat Neurosci 2006, 9:676-681.

[92] Pettigrew JD: The paradox of the critical period for striate cortex. In Neuronal Plasticity. Edited by Cotman CW, New York Raven Press 1978:311-330.

[93] Ruthazer ES: You're perfect, now change-redefining the role of developmental plasticity. Neuron 2005, 45(6):825-828.

[94] Kaschube M, Schnabel M, Wolf F, Löwel S: Interareal coordination of columnar architectures during visual cortical development. Proc Natl Acad Sci USA 2009, 106(40):1720517210.

[95] Dekaban AS, Sadowksy D: Changes in brain weight during the span of a human life: relation of brain weight to body heights and body weights. Ann Neurol 1978, 4:345-356.

[96] Villabanca JR, Schmanke TD, Crutcher HA, Sung AC, Tavabi K: The growth of the feline brain from fetal into adult life I. A morphometric study of neocortex and white matter. Dev Brain Res 2000, 122:11-20.

[97] Duffy KR, Murphy KM, Jones DG: Analysis of the postnatal growth of visual cortex. Vis Neurosci 1998, 15(5):831-839. 
[98] Purves D: Neural activity and the growth of the brain. Lezioni Lincei, Academia Nazionale dei Lincei, Cambridge University Press 1994.

[99] Keil W, Schmidt KF, Löwel S, Kaschube M: Reorganization of columnar architecture in the growing visual cortex. Proc Nat Acad Sci USA 2010, 107:12293-12298.

[100] Fagiolini M, Pizzorusso T, Berardi N, Domenici L, Maffei L: Functional postnatal development of the rat primary visual cortex and the role of visual experience: dark rearing and monocular deprivation. Vision Res 1994, 34(6):709-720.

[101] Fagiolini M, Katagiri H, Miyamoto H, Mori H, Grant SGN, Mishina M, Hensch TK: Separable features of visual cortical plasticity revealed by $\mathrm{N}$-methyl-D-aspartate receptor $2 \mathrm{~A}$ signaling. Proc Natl Acad Sci USA 2003, 100(5):2854-2859.

[102] Tagawa Y, Kanold PO, Majdan M, Shatz CJ: Multiple periods of functional ocular dominance plasticity in mouse visual cortex. Nat Neurosci 2005, 8(3):380-388.

[103] Greifzu F, Schmidt S, Schmidt KF, Kreikemeier K, Witte OW, Löwel S: Global impairment and therapeutic restoration of visual plasticity mechanisms after a localized cortical stroke. Proc Natl Acad Sci USA 2011, 108(37):15450-15455.

[104] Lehmann K, Löwel S: Age-dependent ocular dominance plasticity in adult mice. PLoS ONE 2008, 3(9):e3120.

[105] Zhang J, Ackman JB, Xu HP, Crair MC: Visual map development depends on the temporal pattern of binocular activity in mice. Nat Neurosci 2011, 15(2):298-307.

[106] Wang BS, Sarnaik R, Cang J: Critical period plasticity matches binocular orientation preference in the visual cortex. Neuron 2010, 65(2):246-256.

[107] Rochefort NL, Narushima M, Grienberger C, Marandi N, Hill DN, Konnerth A: Development of direction selectivity in mouse cortical neurons. Neuron 2011, 71(3):425-432.

[108] Hubel DH, Wiesel TN: Receptive fields of cells in striate cortex of very young, visually inexperienced kittens. J Neurophysiol 1963, 26:994-1002.

[109] von der Malsburg C: Self-organization of orientation sensitive cells in the striate cortex. Kybernetik 1973, 14(2):85-100.

[110] von der Malsburg C: Development of ocularity domains and growth behaviour of axon terminals. Biol Cybern 1979, 32:243-264.

[111] Linsker R: From basic network principles to neural architecture: emergence of spatialopponent cells. Proc Natl Acad Sci USA 1986, 83(19):7508-7512.

[112] Linsker R: From basic network principles to neural architecture: emergence of orientation-selective cells. Proc Natl Acad Sci USA 1986, 83(21):8390-8394.

[113] Linsker R: From basic network principles to neural architecture: emergence of orientation columns. Proc Natl Acad Sci USA 1986, 83(22):8779-8783. 
[114] Miller KD, Keller JB, Stryker MP: Ocular dominance column development: Analysis and simulation. Science 1989, 245:605-615.

[115] Miller KD: A model for the development of simple cell receptive fields and the ordered arrangement of orientation columns through activity-dependent competition between $\mathrm{ON}$ and OFF-center inputs. J Neurosci 1994, 14:409-441.

[116] Miller KD, MacKay DJC: The role of constraints in Hebbian learning. Neur Comp 1994, 6:100-126.

[117] Kang K, Shelley M, Sompolinsky H: Mexican hats and pinwheels in visual cortex. Proc Natl Acad Sci USA 2003, 100(5):2848-2853.

[118] Grabska-Barwinska A, von der Malsburg C: Establishment of a scaffold for orientation maps in primary visual cortex of higher mammals. J Neurosci 2008, 28:249-257.

[119] Swindale NV: A model for the formation of ocular dominance stripes. Proc R Soc Lond B 1980, 208(1171):243-264.

[120] Bressloff PC, Cowan JD, Golubitsky M, Thomas PJ, Wiener MC: Geometric visual hallucinations, Euclidean symmetry and the functional architecture of striate cortex. Phil Trans $R$ Soc Lond B 2001, 356(1407):299-330.

[121] Bressloff PC, Cowan JD: SO(3) symmetry breaking mechanism for orientation and spatial frequency tuning in the visual cortex. Phys Rev Lett 2002, 88(7):078102.

[122] Lee HY, Yahyanejad M, Kardar M: Symmetry considerations and development of pinwheels in visual maps. Proc Nat Acad Sci USA 2003, 100:16036-11604.

[123] Wolf F: Symmetry, multistability, and long-range interactions in brain development. Phys Rev Lett 2005, 95(20):208701.

[124] Schnabel M: A symmetry of the visual world in the architecture of the visual cortex. PhD thesis, Göttingen University 2009.

[125] Reichl L, Löwel S, Wolf F: Pinwheel stabilization by ocular dominance segregation. Phys Rev Lett 2009, 102(20):208101.

[126] Schnabel M, Kaschube M, Wolf F: Pinwheel stability, pattern selection and the geometry of visual space. arXiv:0801.3832v2 [q-bio.NC].

[127] Bressloff PC, Cowan JD, Golubitsky M, Thomas PJ: Scalar and pseudoscalar bifurcations motivated by pattern formation in the visual cortex. Nonlinearity 2001, 14:739-775.

[128] Bressloff PC, Cowan JD: An amplitude equation approach to contextual effects in visual cortex. Neural Comp 2002, 14:493-525.

[129] Thomas PJ, Cowan JD: Symmetry induced coupling of cortical feature maps. Phys Rev Lett 2004, 92(18):188101.

[130] Thomas PJ: Simultaneous constraint on pre- and post-synaptic cells couple cortical feature maps in a 2D geometric model of orientation preference. Math Med Biol 2006, 1:1-20. 
[131] Stevens CF: A universal design principle for visual system pinwheels. Brain Behav Evol 2011, 77(3):132-135.

[132] Chklovskii D, Koulakov A: A wire length minimization approach to ocular dominance patterns in mammalian visual cortex. Physica A 2000, 284(1-4):318-334.

[133] Koulakov A, Chklovskii D: Orientation preference patterns in mammalian visual cortex: a wire length minimization approach. Neuron 2001, 29(2):519-527.

[134] Durbin R, Mitchison G: A dimension reduction framework for understanding cortical maps. Nature 1990, 343(6259):644-647.

[135] Swindale N, Bauer H: Application of Kohonen's self-organizing feature map algorithm to cortical maps of orientation and direction preference. Proc R Soc Lond B 1998.

[136] Swindale NV, Shoham D, Grinvald A, Bonhoeffer T, Hübener M: Visual cortex maps are optimized for uniform coverage. Nat Neurosci 2000, 3(8):822-826.

[137] Carreira-Perpiñán M, Goodhill GJ: Are visual cortex maps optimized for coverage? Neural Comp 2002, 14(7):1545-1560.

[138] Carreira-Perpiñán M, Goodhill GJ: Influence of lateral connections on the structure of cortical maps. J Neurophysiol 2004, 92(5):2947-2959.

[139] Carreira-Perpiñán M, Lister R, Goodhill G: A computational model for the development of multiple maps in primary visual cortex. Cereb Cortex 2005, 15(8):1222-1233.

[140] Giacomantonio CE, Goodhill GJ: The effect of angioscotomas on map structure in primary visual cortex. J Neurosci 2007, 27(18):4935-4946.

[141] Giacomantonio CE, Ibbotson MR, Goodhill GJ: The influence of restricted orientation rearing on map structure in primary visual cortex. Neuroimage 2010, 52(3):875-883.

[142] Keil W, Wolf F: Coverage, continuity, and visual cortical architecture. Neural Syst Circ 2011, 1:17.

[143] Goodhill G, Cimponeriu A: Analysis of the elastic net model applied to the formation of ocular dominance and orientation columns. Network 2000, 11(2):153-168.

[144] Scherf O, Pawelzik K, Wolf F, Geisel T: Theory of ocular dominance pattern formation. Phys Rev E 1999, 59(6):6977-6993.

[145] Kohonen T: Self-organized formation of topologically correct feature maps. Biol Cybern 1982, 43:59-69.

[146] Kohonen T: Self-organizing maps. Springer Verlag, 3rd edition 2000.

[147] Ritter H, Schulten K: On the stationary state of Kohonen's self-organizing sensory mapping. Biol Cybern 1986, 54(2):99-106.

[148] Ritter H, Schulten K: Convergence properties of Kohonen's topology conserving maps: fluctuations, stability, and dimension selection. Biol Cybern 1988, 60:59-71. 
[149] Hebb DO: The organization of behaviour: A neuropsychological theory. John Wiley \& Sons, 1st edition 1949.

[150] van Vreeswijk C, Sompolinsky H: Chaos in neuronal networks with balanced excitatory and inhibitory activity. Science 1996, 274(5293):1724-1726.

[151] van Vreeswijk C, Hansel D: Balanced network model of orientation selectivity in primary visual cortex without orientation map. Soc Neurosci, Abstr 2011, 175.12/LL31.

[152] Pehlevan C, Sompolinsky H: Sensory selectivity in random cortical circuits. Soc Neurosci, Abstr 2011, 624.09/ZZ31.

[153] Cross MC, Hohenberg PC: Pattern formation outside of equilibrium. Rev Mod Phys 1993, 65(3):854-1086.

[154] Cross MC, Greenside H: Pattern formation and dynamics in nonequilibrium systems. Cambridge University Press, 1st edition 2009.

[155] Swift J, Hohenberg PC: Hydrodynamic fluctuations at the convective instability. Phys Rev A 1977, 15:319-328.

[156] Schmidt KE, Goebel R, Löwel S, Singer W: The perceptual grouping criterion of colinearity is reflected by anisotropies of connections in the primary visual cortex. Eur J Neurosci 1997, 9(5):1083-1089.

[157] Kisvárday ZF, Tóth E, Rausch M, Eysel UT: Orientation-specific relationship between populations of excitatory and inhibitory lateral connections in the visual cortex of the cat. Cereb Cortex 1997, 7(7):605-618.

[158] Chisum HJ, Mooser F, Fitzpatrick D: Emergent properties of layer 2/3 neurons reflect the collinear arrangement of horizontal connections in tree shrew visual cortex. J Neurosci 2003, 23(7):2947-2960.

[159] Kaschube M, Schnabel M, Löwel S, Coppola D, White LE, Wolf F: Universality in the evolution of orientation columns in the visual cortex, Supporting Information. Science 2010, 330(6007):1113-1116.

[160] Manneville P: Dissipative structures and weak turbulence. Academic Press, 1st edition 1990.

[161] Wolf F: Symmetry breaking and pattern selection in visual cortical development. École d'Été de Physique Les Houches, 2003, Methods and Models in Neurophysics, Elsevier Press 2005.

[162] Reichl L: Symmetry breaking and pattern selection in models of visual development. $P h D$ thesis, Göttingen University 2010.

[163] Kaschube M, Schnabel M, Wolf F: Self-organization and the selection of pinwheel density in visual cortical development. New J Phys 2008, 10:015009.

[164] Hunt JJ, Giacomantonio CE, Tang H, Mortimer D, Jaffer S, Vorobyov V, Ericksson G, Sengpiel F, Goodhill GJ: Natural scene statistics and the structure of orientation maps in the visual cortex. Neuroimage 2009, 47:157-172. 
[165] Hunt JJ, Bosking WH, Goodhill GJ: Statistical structure of lateral connections in the primary visual cortex. Neural Syst Circ 2011, 1:3.

[166] Wolf F, Geisel T: Spontaneous pinwheel annihilation during visual development. Nature 1998, 395(6697):73-78.

[167] Wässle H, Boycott BB, Illing RB: Morphology and mosaic of ON- and OFF-beta cells in the cat retina and some functional considerations. Proc R Soc Lond B 1981, 212(1187):177-195.

[168] Paik SB, Ringach DL: Retinal origin of orientation maps in visual cortex. Nat Neurosci 2011, 14(7):919-925.

[169] Rodiek RW: Quantitative analysis of cat retinal ganglion cell response to visual stimuli. Vision Res 1965, 5:583-601.

[170] Wässle H, Peichl L, Boycott BB: Morphology and topography of ON- and OFF-alpha cells in the cat retina. Proc R Soc Lond B 1981, 212(1187):157-175.

[171] Rockhill RL, Euler T, Masland RH: Spatial order within but not between types of retinal neurons. Proc Natl Acad Sci USA 2000, 97(5):2303-2307.

[172] Soodak RE: Two-dimensional modeling of visual receptive fields using Gaussian subunits. Proc Nat Acad Sci USA 1986, 83:9259-9263.

[173] Soodak RE: The retinal ganglion cell mosaic defines orientation columns in striate cortex. Proc Nat Acad Sci USA 1987, 84:3936-3940.

[174] Jin J, Wang Y, Swadlow HA, Alonso JM: Population receptive fields of ON and OFF thalamic inputs to an orientation column in visual cortex. Nat Neurosci 2011, 14(2):232-238.

[175] Ringach DL: You get what you get and you don't get upset. Nat Neurosci 2011, 14(2):123124.

[176] Ringach DL: Haphazard wiring of simple receptive fields and orientation columns in visual cortex. J Neurophysiol 2004, 92:468-476.

[177] Ringach DL: On the origin of the functional architecture of the cortex. PLoS ONE 2007, 2(2):e251.

[178] Smith SL, Smith IT: Life imitates op art. Nat Neurosci 2011, 14(7):803-804.

[179] Amidror I: The Theory of the Moiré Phenomenon, Volume I: Periodic Layers. Springer Verlag, 2nd edition 2009.

[180] Ruthazer ES, Stryker MP: The role of activity in the development of long-range horizontal connections in area 17 of the ferret. J Neurosci 1996, 16(22):7253-7269.

[181] Lübke J, Albus K: Rapid rearrangement of intrinsic tangential connections in the striate cortex of normal and dark-reared kittens: Lack of exuberance beyond the second postnatal week. J Comp Neurol 1992, 323:42-58. 
[182] Gödecke I, Kim DS, Bonhoeffer T, Singer W: Development of orientation preference maps in area 18 of kitten visual cortex. Eur J Neurosci 1997, 9(8):1754-1762.

[183] Chapman B, Gödecke I, Bonhoeffer T: Development of orientation preference in the mammalian visual cortex. J Neurobiol 1999, 41:18-24.

[184] Field DJ: Relations between the statistics of natural images and the response properties of cortical cells. J Opt Soc Am A 1987, 4(12):2379-2394.

[185] Ruderman D, Bialek W: Statistics of natural images: Scaling in the woods. Phys Rev Lett 1994, 73(6):814-817.

[186] Simoncelli EP, Olshausen BA: Natural image statistics and neural representation. Ann Rev Neurosci 2001, 24:1193-1216.

[187] Simoncelli EP: Vision and the statistics of the visual environment. Curr Opin Neurobiol 2003, 13(2):144-149.

[188] Heide D: Nonlinear dynamics of large scale patterns in the visual cortex. Master's thesis, Frankfurt University 2005.

[189] Saad Y, Schultz MH: GMRES: A generalized minimal residual algorithm for solving nonsymmetric linear systems. SIAM J Sci Stat Comput 1986, 7:856-869.

[190] Press WH, Flannery BP, Teukolsky SA, Vetterling WT: Numerical recipes in C: the art of scientific computing. Cambridge University Press, 2nd edition 1992.

[191] Berkes P, Orbán G, Lengyel M, Fiser J: Spontaneous cortical activity reveals hallmarks of an optimal internal model of the environment. Science 2011, 331(6013):83-87.

[192] Sigman M, Cecchi GA, Gilbert CD, Magnasco MO: On a common circle: natural scenes and Gestalt rules. Proc Natl Acad Sci USA 2001, 98(4):1935-1940.

[193] Wolf F: Strukturbildung in der Entwicklung des visuellen Kortex. PhD thesis, Frankfurt University 1998.

[194] Hubel DH, Wiesel TN, LeVay S: Plasticity of ocular dominance columns in monkey striate cortex. Phil Trans R Soc Lond B 1977, 278(961):377-409.

[195] Kerr JND, Greenberg D, Helmchen F: Imaging input and output of neocortical networks in vivo. Proc Natl Acad Sci USA 2005, 102(39):14063-14068.

[196] Hübener M, Bonhoeffer T: Visual cortex: Two photon excitement. Curr Biol 2005, 15(6):205208.

[197] Greenberg DS, Houweling AR, Kerr JND: Population imaging of ongoing neuronal activity in the visual cortex of awake rats. Nat Neurosci 2008, 11(7):749-751.

[198] Capecchi MR: The new mouse genetics: altering the genome by gene targeting. Trends Genet 1989, 5(3):70-76. 
[199] Kumar TR, Larson M, Wang H, McDermott J, Bronshteyn I: Transgenic mouse technology: principles and methods. Methods Mol Biol 2009, 590:335-362.

[200] Hübener M: Mouse visual cortex. Curr Opin Neurobiol 2003, 13(4):413-420.

[201] Tiao YC, Blakemore C: Functional organization in the visual cortex of the golden hamster. J Comp Neurol 1976, 168(4):459-481.

[202] Mangini NJ, Pearlman AL: Laminar distribution of receptive field properties in the primary visual cortex of the mouse. J Comp Neurol 1980, 193:203-222.

[203] Hofer SB, Ko H, Pichler B, Vogelstein J, Ros H, Zeng H, Lein E, Lesica NA, Mrsic-Flogel TD: Differential connectivity and response dynamics of excitatory and inhibitory neurons in visual cortex. Nat Neurosci 2011, 14(8):1045-1052.

[204] Hall WC, Kaas JH, Killackey H, Diamond IT: Cortical visual areas in the grey squirrel (Sciurus carolinesis): a correlation between cortical evoked potential maps and architectonic subdivisions. J Neurophysiol 1971, 34(3):437-452.

[205] Priebe NJ, Ferster D: Neuroscience: Each synapse to its own. Nature 2010, 464(7293):12901291.

[206] Heeger DJ: Normalization of cell responses in cat striate cortex. Vis Neurosci 1992, 9(2):181197.

[207] Heeger DJ: Half-squaring in responses of cat striate cells. Vis Neurosci 1992, 9(5):427-443.

[208] Heeger DJ: Modeling simple-cell direction selectivity with normalized, half-squared, linear operators. J Neurophysiol 1993, 70(5):1885-1898.

[209] Carreira-Perpiñán MÁ, Goodhill GJ: Generalised elastic nets. arXiv:1108.2840v1 [q-bio.NC] 2011.

[210] Turing A: The chemical basis of morphogenesis. Phil Trans $R$ Soc Lond B 1952, 237(641):3772.

[211] Hooser SV, Heimel J, Chung S, Nelson S: Lack of patchy horizontal connectivity in primary visual cortex of a mammal without orientation maps. J Neurosci 2006, 26(29):7680-7692.

[212] Edwards SF, Anderson PK: Theory of spin glasses. J Phys F: Metal Phys 1975, 5:965-975.

[213] Edwards SF, Anderson PK: Theory of spin glasses II. J Phys F: Metal Phys 1976, 6:1927-1938.

[214] Sherrington D, Kirkpatrick S: Solvable model of a spin glass. Phys Rev Lett 1975, 35:17921795.

[215] Wannier G: Antiferromagnetism. The triangular Ising net. Phys Rev 1950, 79(2):357-364.

[216] Sadoc JF, Mosseri R: Geometrical Frustration. Cambridge University Press, 1st edition 1999.

[217] Fregnac $Y$, Shulz D, Thorpe S, Bienenstock E: A cellular analogue of visual cortical plasticity. Nature 1988, 333(6171):367-370. 
[218] Fregnac Y, Shulz D, Thorpe S, Bienenstock E: Cellular analogs of visual cortical epigenesis. I. Plasticity of orientation selectivity. J Neurosci 1992, 12(4):1280-1300.

[219] Schuett S, Bonhoeffer T, Hübener M: Pairing-induced changes of orientation maps in cat visual cortex. Neuron 2001, 32(2):325-337.

[220] Swindale NV: Coverage and the design of striate cortex. Biol Cybern 1991, 65(6):415-424.

[221] Elstrott J, Anishchenko A, Greschner M, Sher A, Litke AM, Chichilnisky EJ, Feller MB: Direction selectivity in the retina is established independent of visual experience and cholinergic retinal waves. Neuron 2008, 58(4):499-506.

[222] Wei W, Hamby AM, Zhou K, Feller MB: Development of asymmetric inhibition underlying direction selectivity in the retina. Nature 2011, 469(7330):402-406.

[223] Adesnik H, Scanziani M: Lateral competition for cortical space by layer-specific horizontal circuits. Nature 2010, 464(7292):1155-1160.

[224] Newell AC, Whitehead JA: Finite bandwidth, finite amplitude convection. J Fluid Mech 1969, 38(2):279-303.

[225] Busse F, Whitehead JA: Instabilities of convection rolls in a high Prandtl number fluid. J Fluid Mech 1971, 47:305-320.

[226] Charvet CJ, Striedter GF, Finlay BL: Evo-devo and brain scaling: candidate developmental mechanisms for variation and constancy in vertebrate brain evolution. Brain Behav Evol 2011, 78(3):248-257.

[227] Striedter GF: Principles of brain evolution. Sindauer Associates Inc., 1st edition 2005.

[228] Whitney D, Flynn CF, Keil W, Wolf F, Crowley JC: Age-dependent changes in the columnar organization and plasticity of the ferret visual cortex. Soc Neurosci, Abstr 2011, 270.10/II10.

[229] Huang M: Spatio-temporal dynamics of pattern formation in the cerebral cortex: visual maps, population response and action potential generation. PhD thesis, Göttingen University 2009.

[230] Huang M, Löwel S, Geisel T, Wolf F: Dimension suppression and pinwheel crystallization in competitive Hebbian models of visual cortical development. in prep.

[231] Eglen SJ, Diggle PJ, Troy JB: Homotypic constraints dominate positioning of on- and offcenter beta retinal ganglion cells. Vis Neurosci 2005, 22(6):859-871.

[232] Eglen SJ, Wong JCT: Spatial constraints underlying the retinal mosaics of two types of horizontal cells in cat and macaque. Vis Neurosci 2008, 25(2):209-214.

[233] Adams D, Horton JC: Shadows cast by retinal blood vessels mapped in primary visual cortex. Science 2002, 298(5593):572-576.

[234] Alonso JM, Usrey WM, Reid RC: Rules of connectivity between geniculate cells and simple cells in cat primary visual cortex. J Neurosci 2001, 21(11):4002-4015. 
[235] Sirotin YB, Das A: Zooming in on mouse vision. Nat Neurosci 2010, 13(9):1045-1046.

[236] Fiser J, Berkes P, Orbán G, Lengyel M: Statistically optimal perception and learning: from behavior to neural representations. Trends Cogn Sci 2010, 14(3):119-130.

[237] Kaschube M, Wolf F, Geisel T, Löwel S: Genetic influence on quantitative features of neocortical architecture. J Neurosci 2002, 22(16):7206-7217.

[238] Zimmermann W, Seesselberg M, Petruccione F: Effects of disorder in pattern formation. Phys Rev E 1993, 48(4):2699-2703.

[239] Zimmermann W, Painter B, Behringer R: Pattern formation in an inhomogeneous environment. Eur J Phys B 1998, 5:757-770.

[240] Hammele M, Schuler S, Zimmermann W: Effects of parametric disorder on a stationary bifurcation. Physica D 2006, 218(2):139-157.

[241] Kriegs J, Churakov G, Kiefmann M, Jordan U: Retroposed elements as archives for the evolutionary history of placental mammals. PLoS Biol 2006, 4(4):e91.

[242] Kriegs JO, Churakov G, Jurka J, Brosius J, Schmitz J: Evolutionary history of 7SL RNAderived SINEs in Supraprimates. Trends Genet 2007, 23(4):158-161.

[243] Kaas JH: Reconstructing the areal organization of the neocortex of the first mammals. Brain Behav Evol 2011, 78:7-21.

[244] Kielian-Jaworowsk Z, Cifelli RL, Luo ZX: Mammals from the age of dinosaurs. Columbia University Press, 1st edition 2004.

[245] Rosa MG, Krubitzer LA: The evolution of visual cortex: where is V2? Trends Neurosci 1999, 22(6):242-248.

[246] Kaas J: Reconstructing the organization of neocortex of the first mammals and subsequent modifications. In Evolution of nervous systems: A comprehensive reference. Edited by Kaas J, Academic Press 2007:27-48.

[247] Rowe TB, Macrini TE, Lou ZX: Fossil evidence on origin of the mammalian brain. Science 2011, 320(6032):955-957.

[248] Bininda-Emonds ORP, Cardillo M, Jones KE, MacPhee RDE, Beck RMD, Grenyer R, Price SA, Vos RA, Gittleman JL, Purvis A: The delayed rise of present-day mammals. Nature 2007, 446(7135):507-512.

[249] Wible JR, Rougier GW, Novacek MJ, Asher RJ: Cretaceous eutherians and Laurasian origin for placental mammals near the K/T boundary. Nature 2007, 447(7147):1003-1006.

[250] Rosa MG, Casagrande VA, Preuss T, Kaas JH: Visual field representation in striate and prestriate cortices of a prosimian primate (Galago garnetti). J Neurophysiol 1997, 77(6):31933217.

[251] Van Hooser SD: Similarity and diversity in visual cortex: is there a unifying theory of cortical computation? Neuroscientist 2007, 13(6):639-656. 
[252] Vogel S: Convergence as an analytical tool in evaluating design. In Principles of animal design. The optimization and symmorphosis debate. Edited by Weibel ER, Taylor CR, Bolis L, Cambridge University Press 1998.

[253] Hemmi JM, Mark RF: Visual acuity, contrast sensitivity and retinal magnification in a marsupial, the tammar wallaby (Macropus eugenii). J Comp Physiol A 1998, 183(3):379-387.

[254] Silveira L, Picanço-Diniz C: Contrast sensitivity function and visual acuity of the opossum. Vision Res 1982, 22:1371-1377.

[255] Arrese C, Dunlop S, Harman A, Braekevelt C, Ross W, Shand J, Beazley L: Retinal structure and visual acuity in a polyprotodont Marsupial, the fat-tailed dunnart (Sminthopsis crassicaudata). Brain Behav Evol 1999, 53(3):111-126.

[256] Campos GB, Welker WI: Comparisons between brains of a large and a small hystricomorph rodent: capybara, Hydrochoerus and guinea pig, Cavia; neocortical projection regions and measurements of brain subdivisions. Brain Behav Evol 1976, 13(4):243-266.

[257] Mulligan KA, Ulinski PS: Organization of geniculocortical projections in turtles: isoazimuth lamellae in the visual cortex. J Comp Neurol 1990, 296(4):531-547.

[258] Super H, Uylings HBM: The early differentiation of the neocortex: a hypothesis on neocortical evolution. Cereb Cortex 2001, 11:1101-1109.

[259] Alroy J: Cope's rule and the dynamics of body mass evolution in North American fossil mammals. Science 1998, 280(5364):731-734.

[260] Smith FA, Boyer AG, Brown JH, Costa DP, Dayan T, Ernest SKM, Evans AR, Fortelius M, Gittleman JL, Hamilton MJ, Harding LE, Lintulaakso K, Lyons SK, McCain C, Okie JG, Saarinen JJ, Sibly RM, Stephens PR, Theodor J, Uhen MD: The evolution of maximum body size of terrestrial mammals. Science 2010, 330(6008):1216-1219.

[261] Kaas JH: Neocortex in early mammals and its subsequent variations. Ann NY Acad Sci 2011, 1225:28-36.

[262] Frahm HD, Stephan H, Baron G: Comparison of brain structure volumes in insectivora and primates. V. Area striata (AS). J Hirnforsch 1984, 25(5):537-557.

[263] Fritsches KA, Rosa MG: Visuotopic organisation of striate cortex in the marmoset monkey (Callithrix jacchus). J Comp Neurol 1996, 372(2):264-282.

[264] Krubitzer L, Künzle H, Kaas J: Organization of sensory cortex in a Madagascan insectivore, the tenrec (Echinops telfairi). J Comp Neurol 1997, 379(3):399-414.

[265] Fenno L, Yizhar O, Deisseroth K: The development and application of optogenetics. Ann Rev Neurosci 2011, 34:389-412.

[266] Deisseroth K: Optogenetics. Nat Methods 2011, 8:26-29.

[267] Zariwala HA, Madisen L, Ahrens KF, Bernard A, Lein ES, Jones AR, Zeng H: Visual tuning properties of genetically identified layer $2 / 3$ neuronal types in the primary visual cortex of cre-transgenic mice. Front Syst Neurosci 2011, 4:162. 
[268] Floquet G: Sur les équations différentielles linéaires à coefficients périodiques. Ann École Norm Sup 1883, 12:47-88.

[269] Wagor E, Mangini NJ, Pearlman AL: Retinotopic organization of striate and extrastriate visual cortex in the mouse. J Comp Neurol 1980, 193:187-202.

[270] Kalatsky VA, Stryker MP: New paradigm for optical imaging: temporally encoded maps of intrinsic signal. Neuron 2003, 38(4):529-545.

[271] Smith SL, Häusser M: Parallel processing of visual space by neighboring neurons in mouse visual cortex. Nat Neurosci 2010, 13(9):1144-1149.

[272] Stiebler I, Neulist R, Fichtel I, Ehret G: The auditory cortex of the house mouse: left-right differences, tonotopic organization and quantitative analysis of frequency representation. J Comp Physiol A 1997, 181(6):559-571.

[273] Kalatsky VA, Polley DB, Merzenich MM, Schreiner CE, Stryker MP: Fine functional organization of auditory cortex revealed by Fourier optical imaging. Proc Natl Acad Sci USA 2005, 102(37):13325-13330.

[274] Hromádka T, Deweese MR, Zador AM: Sparse representation of sounds in the unanesthetized auditory cortex. PLoS Biol 2008, 6:e16.

[275] Rothschild G, Nelken I, Mizrahi A: Functional organization and population dynamics in the mouse primary auditory cortex. Nat Neurosci 2010, 13(3):353-360.

[276] Bandyopadhyay S, Shamma SA, Kanold PO: Dichotomy of functional organization in the mouse auditory cortex. Nat Neurosci 2010, 13(3):361-368.

[277] Kerr JND, de Kock CPJ, Greenberg DS, Bruno RM, Sakmann B, Helmchen F: Spatial organization of neuronal population responses in layer $2 / 3$ of rat barrel cortex. J Neurosci 2007, 27(48):13316-13328.

[278] Kremer Y, Leger JF, Goodman D, Brette R, Bourdieu L: Late emergence of the vibrissa direction selectivity map in the rat barrel cortex. J Neurosci 2011, 31(29):10689-10700.

[279] Andermann ML, Moore CI: A somatotopic map of vibrissa motion direction within a barrel column. Nat Neurosci 2006, 9(4):543-551. 


\section{Acknowledgements}

At this point, it is my pleasure to acknowledge the support of many people without whom this thesis would have never been completed.

First of all, let me thank Theo Geisel for giving me the opportunity to work in his department. I have visited a lot of research groups and institutes over the last years. But I have to say the scientific and personal environment in his department is truly unique. Your tireless efforts are the driving force behind both the scientific success and the familiar atmosphere of the NLD group.

I am deeply indebted to Fred Wolf for invaluable scientific guidance throughout my $\mathrm{PhD}$ time, and for being a role model in terms of endurance and patience. You, as a creative and innovative researcher, will be a lasting point of reference in my future academic live. Following your footsteps in terms of perseverance, assertiveness and scientific overview will be both a motivation and a challenge.

I would like to express my gratitude to my thesis committee members Eberhard Bodenschatz and Alexander Gail for encouraging comments and constructive criticism during sometimes unexpectedly long thesis committee meetings as well as strong support in all administrative issues which came up during my PhD time.

I thank Matthias Kaschube for giving me the chance to work with him in a unique environment at Princeton University. Thank you for teaching me how to extract precious information from the mist of noisy data and for assisting my first steps into academic writing. With your enthusiasm and faith in my skills, you have been able to push me beyond what I thought were my limits.

I am particularly grateful to my office mate and friend Jakob Metzger for providing tight beats accompanying my numerous ups and downs over the last years. I am well aware of the fact that it has not always been easy for your to endure my enthusiasm when something seemed to work out, and my disappointment about one minute later, when it finally did not.

I thank my former office mate Christoph "Leudde" Kirst for answering thousands of questions regarding Mathematica ${ }^{\odot}$ thereby compensating for what its developers did not consider important enough to document or what my mind was not able to grasp. Furthermore, I would like to thank you for being the rhythmical backbone in all the bands we have played in - the BCCN band, the NLD Jazz group, the MPIDS Think Tank just to name a few.

I am indebted to David Hofmann, not only for many stimulating discussions between systems theory, quantum physics, and politics, but also for introducing me to an addictive indoor/outdoor activity, sticking his neck out during my first attempts to belay him as a lead climber. The "Torri del Vajolet" are still awaiting us and we should not let them wait for too long! 
I thank Olav Stetter for legendary Mathematica ${ }^{\odot}$ hacks and for transferring his exhaustless enthusiasm to others thereby pushing the MPIDS Think Tank beyond its limits to the fields of "Volkstümliche Musik", Independent Rock and Boygroup Pop.

I thank Michael Schnabel for supervising my first steps into Neuroscience and "Pinwheelistics" during my diploma studies and the first years of my PhD as well as for countless after work bike trips that refreshed our minds after long working days.

I thank Michael Monteforte, aka Monte and Georg Martius for many short and long bike trips through the beautiful landscape around Göttingen. I did not make it to the top of the Brocken last time with you, but I sincerely hope that I will get a second chance.

I thank Mitya Tsigankov and Juan Daniel Florez Weidinger for a great collaboration on the saltand-pepper project which I sincerely hope we will continue and extend in the near future.

I am grateful to Ken Miller and Conor Dempsey for helpful comments on the manuscript on the lack of hexagonal order in orientation maps. Thank you as well, Conor, for eagerly learning how to safely belay a lead climber "the German way".

I am indebted to my experimental collaborators David Coppola, Justin Crowley, Siegrid Löwel, Karl-Friedrich Schmidt, Len White, and David Whitney not only for providing data of extraordinary quality, but also for being patient enough whenever something exceeded my knowledge in Neurobiology.

I thank Dominik Heide for lots of help with c++ programming. His first child, "Neksi", now "Neksi 2.0", has become an indispensable tool for all the map club people in the department and I hope I could add a useful part to this growing piece of software.

I thank Harold Gutch for providing the mathematician viewpoint in countless scientific discussions as well as for the restoration of corrupted filesystems on backup hard drives.

Special thanks go to Ragnar Fleischmann and Oliver Bendix for providing last minute expertise with pv-wave ${ }^{\odot}$.

York-Fabian Beensen, Markus Schwamberger and Denny Fliegner provided excellent computer support throughout my time at the MPIDS, even when I pushed our file servers beyond their limits.

I am grateful to Ayse Bolik, Viktoriya Novak, Regina Wunderlich, Tobias Niemann, and the GGNB office for all their support in administrative issues.

I thank Claudia and Ada Kaschube for letting me be part of their family life during my stay at Princeton University, patiently tolerating endless scientific discussions with Matthias at dinner, but also decidedly pointing out when enough was enough.

I am indebted to Saskia Eckhardt for her friendship throughout my PhD time and for her endurance while diligently proofreading these pages.

I thank my parents Sieglinde and Albrecht Keil for their love, trust, and support over the last years and my brother, soulmate and dear friend Reinhard Keil for regularly providing musical counterbalance to my scientific life and carefully proofreading these pages.

Last but not least, I would like to thank Katharina Rall for her love and constant support without reserve. Your contribution to all this was crucial. 


\section{Curriculum Vitae}

\section{Personal Details}

Date of birth $\quad 30.01 .1981$

Place of birth Karl-Marx-Stadt (Chemnitz), Germany

Nationality German

Address Calsowstr. 2, 37085 Göttingen, Germany

Marital status Single

\section{Education}

2008 - present $\quad$ Georg August University, Göttingen, Germany

PhD in Physics, Physics of Biological and Complex

Systems Program at the Göttingen Graduate School for

Neurosciences and Molecular Biosciences, Germany

Aug 2007 -Dec 2007 Princeton University, Princeton, NJ, USA

Visiting Exchange Student

Oct 2001 - Nov 2006 Friedrich Schiller University, Jena, Germany

"Diploma" (graduate studies) in Physics

Thesis: "Shift-Twist-Symmetrie und Musterbildung im visuellen Kortex" (Shift-Twist-Symmetry and Pattern

Formation in the Visual Cortex)

1995 - 2000 Immanuel-Kant-Gymnasium, Chemnitz, Germany

High school graduation ("Abitur")

\section{Fellowships}

Jan 2009 - Jan 2011 PhD Fellowship from the International Max Planck

Research School "Physics of Biological and Complex

Systems"

Oct 2003 - Nov 2006 Fellowship from the German National Academic

Foundation 
